\title{
Numerical study of wingtip shed vorticity reduction by wing Boundary Layer Control
}

Jose Alejandro Posada

West Virginia University

Follow this and additional works at: https://researchrepository.wvu.edu/etd

\section{Recommended Citation}

Posada, Jose Alejandro, "Numerical study of wingtip shed vorticity reduction by wing Boundary Layer Control" (2007). Graduate Theses, Dissertations, and Problem Reports. 2771.

https://researchrepository.wvu.edu/etd/2771

This Dissertation is protected by copyright and/or related rights. It has been brought to you by the The Research Repository @ WVU with permission from the rights-holder(s). You are free to use this Dissertation in any way that is permitted by the copyright and related rights legislation that applies to your use. For other uses you must obtain permission from the rights-holder(s) directly, unless additional rights are indicated by a Creative Commons license in the record and/ or on the work itself. This Dissertation has been accepted for inclusion in WVU Graduate Theses, Dissertations, and Problem Reports collection by an authorized administrator of The Research Repository @ WVU.

For more information, please contact researchrepository@mail.wvu.edu. 


\title{
Numerical Study of Wingtip Shed Vorticity Reduction by Wing Boundary Layer Control
}

\author{
Jose Alejandro Posada \\ at West Virginia University \\ in partial fulfillment of the requirements \\ for the degree of \\ Doctor of Philosophy in \\ Aerospace Engineering \\ Committee members: \\ John Loth, Ph.D., Chair \\ Mary Ann Clarke, Ph.D. \\ Gary Morris, Ph.D. \\ Wade Huebsch, Ph.D. \\ John Kuhlman, Ph.D.
}

Dissertation submitted to the College of Engineering and Mineral Resources

Department of Mechanical and Aerospace Engineering

Morgantown, WV

2007

Keywords: Boundary Layer Control, Wingtip Vortex, Ejectors, Induced Drag, Wake Survey Measurements.

Copyright 2007 Jose Alejandro Posada 


\title{
Abstract
}

\section{Numerical Study of Wingtip Shed Vorticity Reduction by Wing Boundary Layer Control}

\author{
Jose Alejandro Posada
}

Wingtip vortex reductions have been obtained by Boundary Layer Control application to an $\mathrm{AR}=1.5$ rectangular wing using a NACA 0012 airfoil. If wingtip shed vorticity could be reduced significantly, then so would induced drag resulting in improved cruise fuel economy. Power savings would be even more impressive at low flight speed or in climb.

A two dimensional wing produces lift without wingtip vorticity. Its bound vorticity, $\Gamma$, equals the contour integral of the boundary layer vorticity $\gamma$ or $\Gamma=\oint \gamma \cdot d l$. Where the upper and lower boundary layers meet at the cusped TE, their local static pressure $\mathrm{p}_{\mathrm{u}}=\mathrm{p}_{\mathrm{t}}$ then the boundary layer outer edge inviscid velocity $\mathrm{V}_{\text {upper }}=\mathrm{V}_{\text {lower }}$ and $\gamma_{\text {lower }}=-\gamma_{\text {upper }}$. This explains the 2-D wing self cancellation of the upper and lower surface boundary layer vorticity when they meet upon shedding at the trailing edge. In finite wings, the presence of spanwise pressure gradients near the wing tips misaligns $\gamma_{\text {lower }}$ and $\gamma_{\text {upper }}$ at the wingtip TE preventing the upper and lower surface boundary layers from completely canceling each other. To prevent them from generating wing tip vortices, the local boundary layers need to be captured in suction slots. Once vorticity is captured, it can be eliminated by viscous mixing prior to venting over board.

The objective of this dissertation was to use a commercial Computational Fluid Dynamics code (Fluent) to search for the best configuration to locate BLC suction slots to capture non-parallel boundary layer vorticity prior to shedding near the wingtips. The configuration selected for running the simulations was tested by trying to duplicate a 3D wing for which sufficient experimental and computational models by others are available. The practical case selected was done by Chow et al in the $32 \times 48$ in. low speed wind tunnel at the Fluid Mechanics Laboratory of NASA Ames Research Center, and computationally analyzed by Dacles-Mariani et al, and Khim and Rhee. The present computed pressure coefficient values compare very well (Figure 90).

The present simulations were also validated by comparison with wake survey and balance type experimental measurements done by Chometon and Laurent on a NACA $64_{3}-018$ wing. Lift, induced drag, and profile drag coefficients agree very well with Chometon and Laurent data.

More than one hundred simulations were performed with different BLC suction slot geometries. Suction slots were used in the chord-wise and span-wise locations near the wing tip region. Blowing slots were evaluated at the wing center line, the wing tip upper surface, and span-wise outside of the wing tip.

For an elliptically loaded wing, $50 \%$ of the bound vorticity is shed at the wing tips over a length of $7 \%$ of the wing span. The turbulent boundary layer thickness for a Cessna 206 aircraft at cruise is estimated as $0.09 \mathrm{ft}$. Theoretically the power required to remove by suction all the upper and lower surface boundary layer over the tip region for this aircraft at take-off is $2.6 \mathrm{HP}$, which would be very small compared to the $70 \mathrm{HP}$ induced drag power saved. This would only be true if $100 \%$ wingtip vortex elimination could be obtained. 


\section{Dedication}

To my lovely girlfriend and my parents - for their love, patience, support, and encouragement during this long journey. 


\section{Acknowledgments}

I would like to express my sincere gratitude to my research advisor, Dr. Loth, for his teaching, guidance, and support during this research. He has not been only my mentor, teacher, and advisor, but also my friend.

I would also like to thank the members of my committee, Dr. Mary Ann Clarke, Dr. Gary Morris, Dr. Wade Huebsch and Dr. John Kuhlman, for their invaluable suggestions, and their interest in this project. I would like to thanks especially to Dr. Clarke for letting me use the Math Department Cluster and Dr. Huebsch for letting me use the MAE CFD lab.

I would like to thank Dr. Jacky Prucz and Dr. Ever Barbero for their support and timely advice during my graduate program, and for allowing me to teach at West Virginia University (WVU). It was a great experience.

I would like to thank Pat Browning for helping me with the experimental set-up. I would also like to thank Lucas Shinkovich, and Damian Christey for their technical support, and Mrs. Debbie Willis for her timely information regarding matters related to the MAE department. 


\section{Table of Contents}

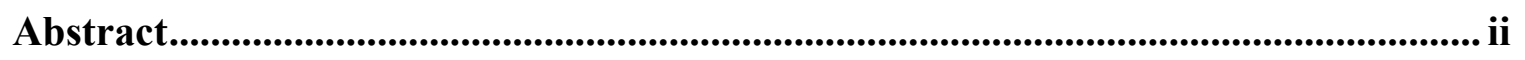

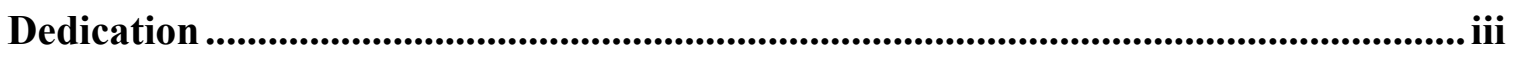

Acknowledgments .................................................................................................................. iv

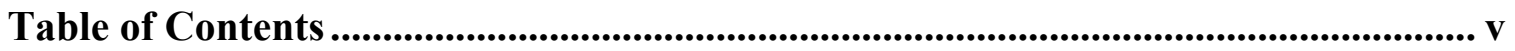

List of Figures............................................................................................................................ viii

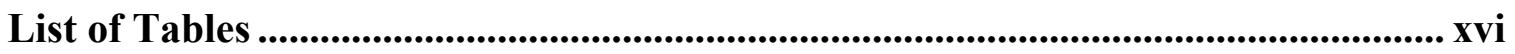

Nomenclature and Symbols .................................................................................................. xvii

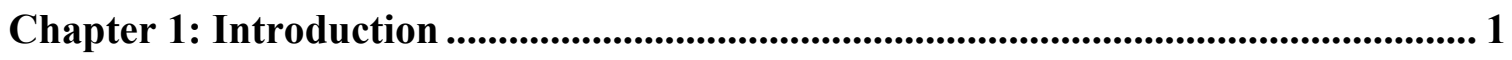

Potential benefits of wingtip wake minimization ............................................... 1

Flight at maximum L/D occurs when induced drag equals parasite drag ..................... 2

Boundary Layer Control technique for reducing wingtip shed vorticity ...................... 2

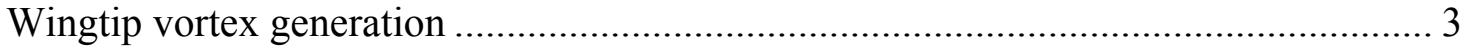

Estimate of minimum suction power required for wing tip BLC ............................ 4

Chapter 2: Literature Review ............................................................................................................ 7

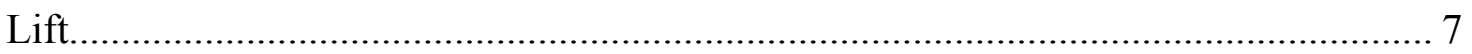

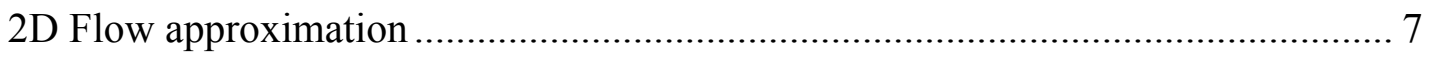

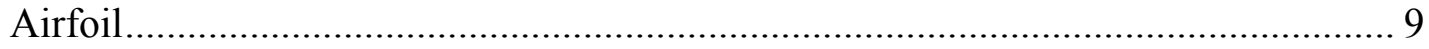

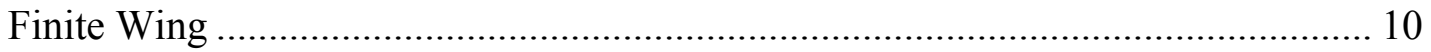

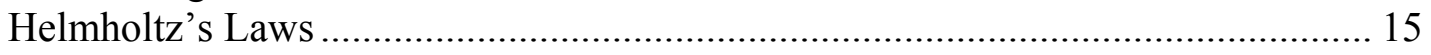

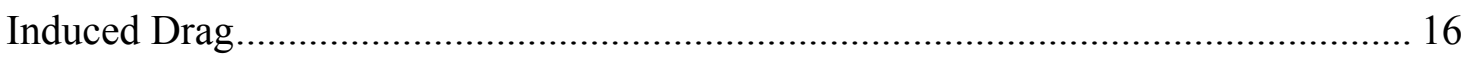

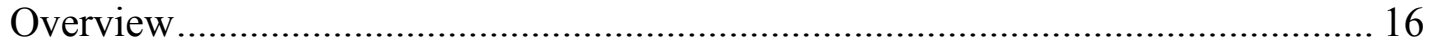

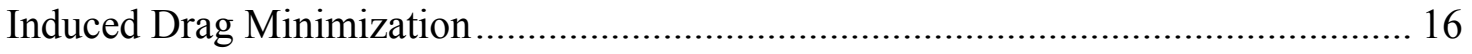

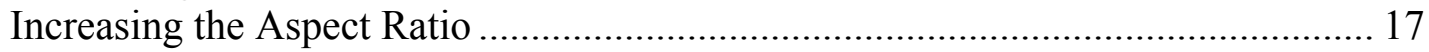

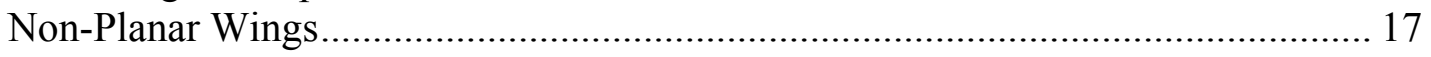

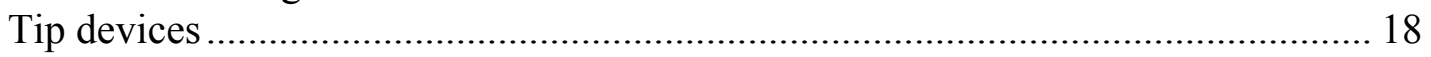

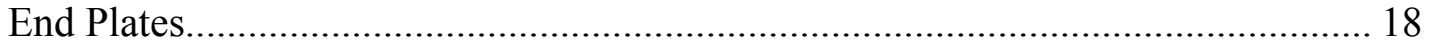

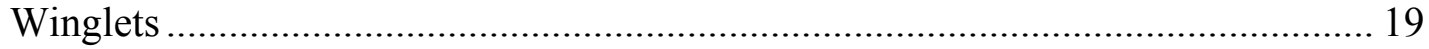

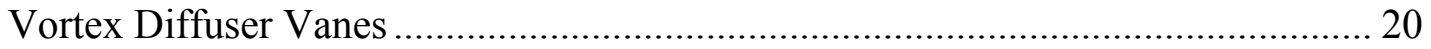

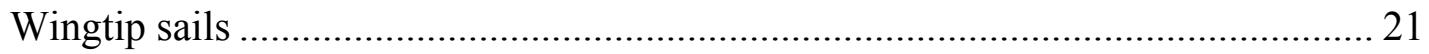

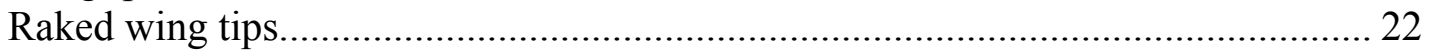

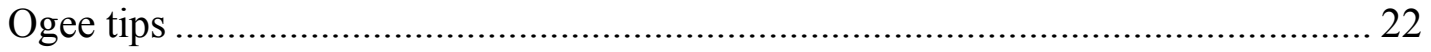

Crossed blade

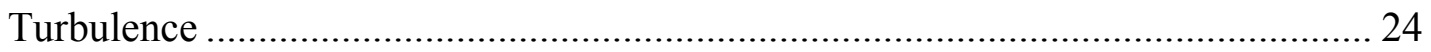




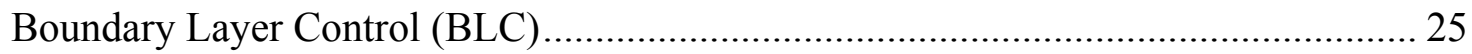

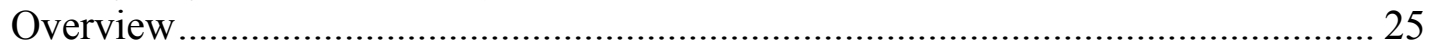

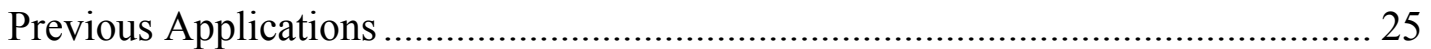

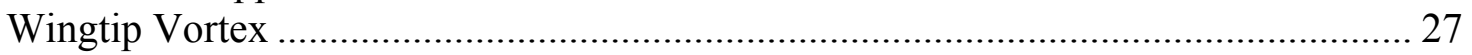

Wingtip Vortex Numerical Studies..................................................................... 27

Experimental Measurements in the Wingtip Vortex ............................................... 29

Chapter 3: Wingtip Vortex Formation .................................................................................... 30

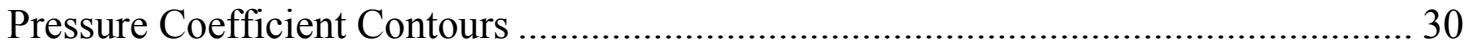

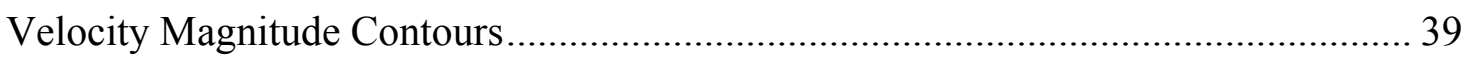

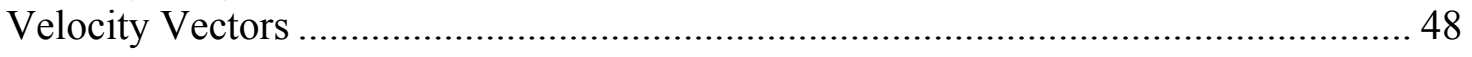

Chapter 4: Numerical Study of the Wingtip Vortex ........................................................ 60

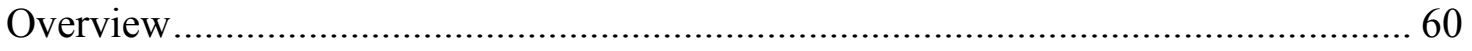

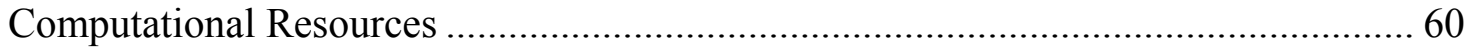

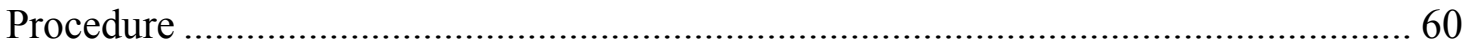

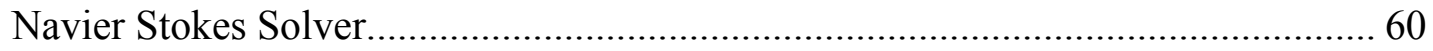

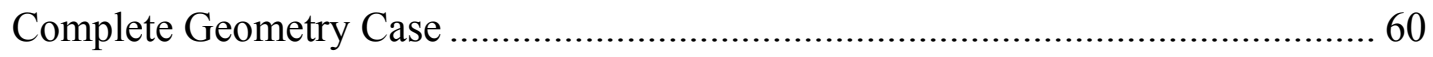

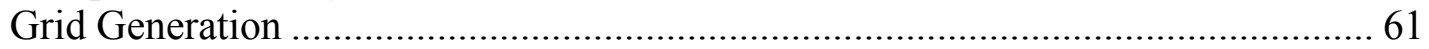

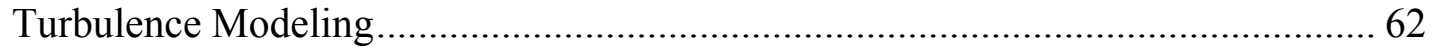

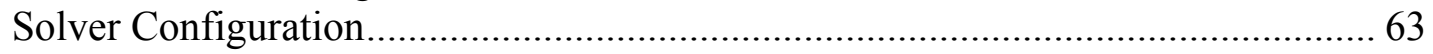

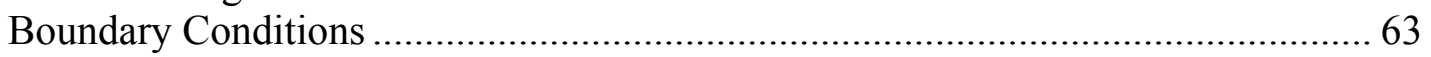

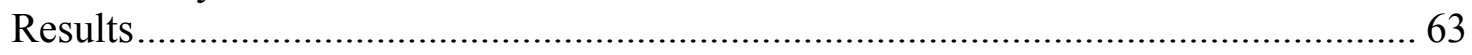

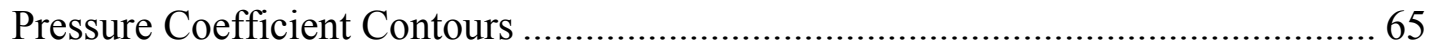

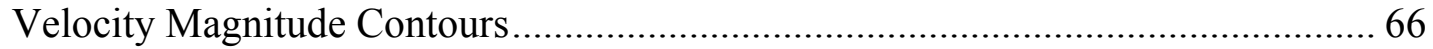

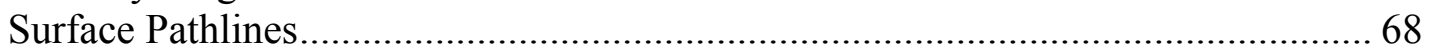

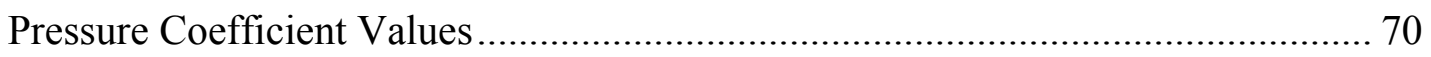

Circulation across the Wingtip Vortex Core........................................................... 71

Chapter 5: Application of Boundary Layer Control ........................................................... 81

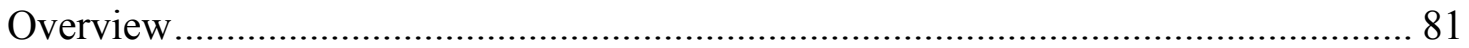

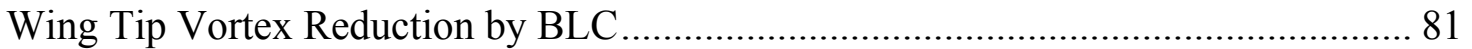

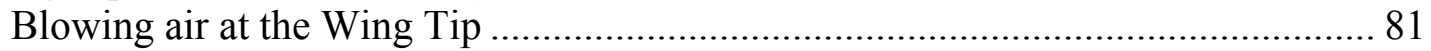

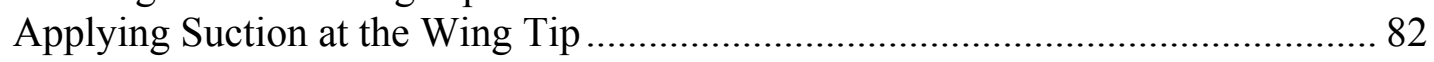

Suction on upper surface and blowing on lower surface ........................................ 84

Applying Suction on the Bottom wing surface....................................................... 85

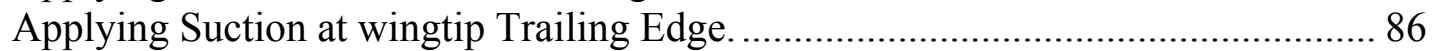

Applying Suction on the Wing Tip and on a Slot on the Bottom wing surface ....... 87

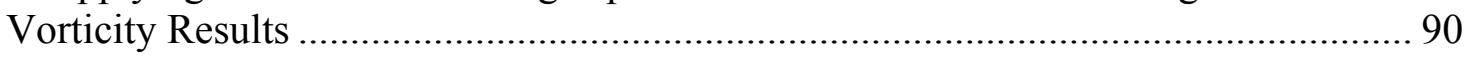

Chapter 6: Lift and Drag Prediction..................................................................................... 92

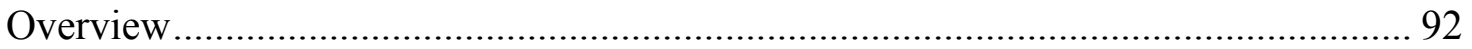

Drag Prediction using Wake Measurements........................................................... 92

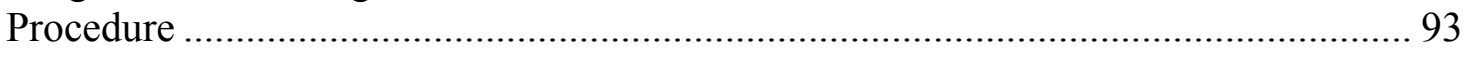


Lift and Drag Coefficients Results .................................................................... 94

BLC Application Results ................................................................................. 96

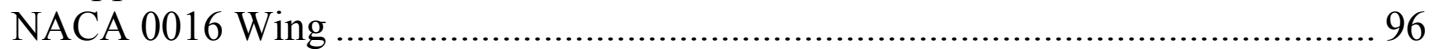

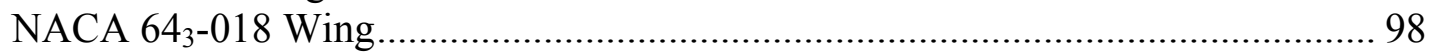

Grid Convergence Study.................................................................................. 108

Computation Domain, Meshes and Simulation Setup ..................................... 109

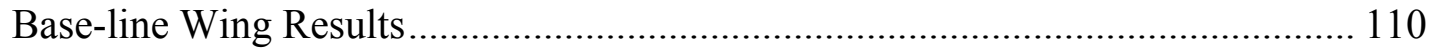

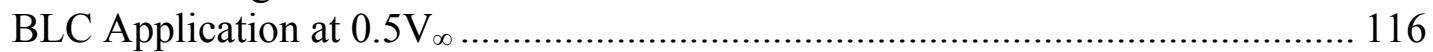

Grid Convergence Study Results .................................................................... 120

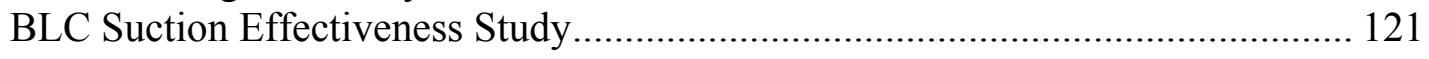

Top BLC Suction Velocity Study ..................................................................... 134

Chapter 7: Conclusions ................................................................................................................ 146

References .............................................................................................................................. 148

Appendixes......................................................................................................................... 152

Appendix A: Ejectors to Provide Wingtip Trailing Edge Suction............................ 152

Appendix B: Chow et al. [43] Pressure Coefficient Tables..................................... 166 


\section{List of Figures}

Figure 1. Cessna 206 total drag, induced drag, and parasite drag [6]........................... 2

Figure 2. Streamlines around a spinning cylinder [10] .................................................. 8

Figure 3. Mathematical representation of the flow around a spinning cylinder [10] ....... 9

Figure 4. Streamlines leaving smoothly at the trailing edge [10].................................. 10

Figure 5. Velocities at the trailing edge for finite angle and cusped TE [10] .................. 10

Figure 6. Prandtl's classical lifting-line theory [10]...................................................... 11

Figure 7. Biot-Savart law applied to a 2D wing. ........................................................ 11

Figure 8. Pressure equalization on the wing tip and curvature of the streamlines [11]... 12

Figure 9. Pressure and lift decreasing toward the tip [11] ........................................... 13

Figure 10. Trailing edge vortices shed behind a wing [10] .......................................... 13

Figure 11. Upwash and downwash behind a wing [13] ............................................... 14

Figure 12. Downwash due to bound and trailing vortices [13]...................................... 15

Figure 13. Drag distribution on an aircraft [11]...................................................... 16

Figure 14. Span efficiency for non planar wings $(\mathrm{h} / \mathrm{b}=0.2)$ [17] .................................. 18

Figure 15. Winglet thrust production mechanism [16] .................................................. 19

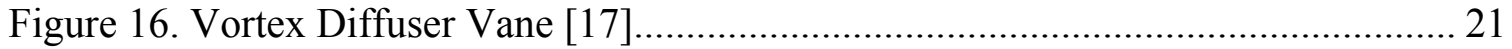

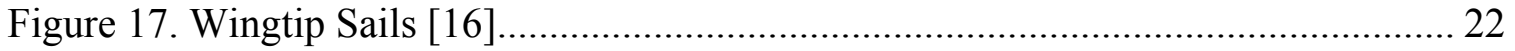

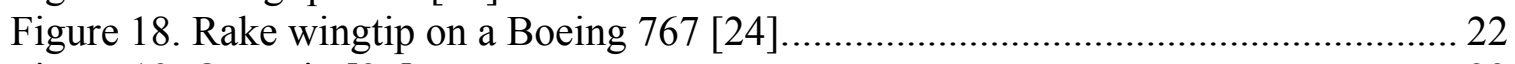

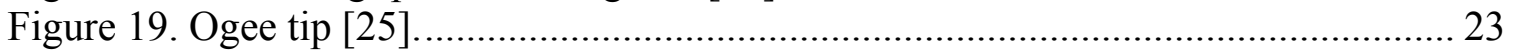

Figure 20. Effect of Ogee tip on vortex dissipation [25] ........................................... 23

Figure 21. Crossed blades [25] .......................................................................... 24

Figure 22. Effect of crossed blades on vortex velocity distribution [25] ........................ 24

Figure 23. Model used to test the effect of placing the engine at the tip [27]................ 26

Figure 24. Techniques used to blow air downward: a) chordwise, b) spanwise [25] ...... 26

Figure 25. Change in L/D ratio with spanwise blowing [25] ...................................... 26

Figure 26. Pressure coefficient contours at $\mathrm{x} / \mathrm{c}=0.25$, looking upstream from back [44]. 31

Figure 27. Pressure coefficient contours at $\mathrm{x} / \mathrm{c}=0.345$, looking upstream from back [44].

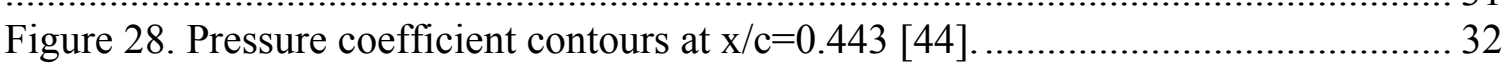

Figure 29. Pressure coefficient contours at $\mathrm{x} / \mathrm{c}=0.542$, looking upstream from back [44].

Figure 30. Pressure coefficient contours at $\mathrm{x} / \mathrm{c}=0.606$, looking upstream from back [44].

Figure 31. Pressure coefficient contours at $\mathrm{x} / \mathrm{c}=0.625$, looking upstream from back [44].

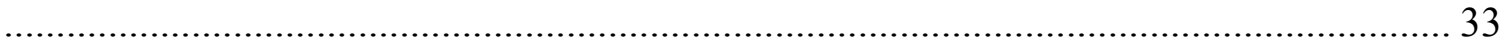

Figure 32. Pressure coefficient contours at $\mathrm{x} / \mathrm{c}=0.704$, looking upstream from back [44].

Figure 33. Pressure coefficient contours at $\mathrm{x} / \mathrm{c}=0.729$, looking upstream from back [44].

Figure 34. Pressure coefficient contours at $\mathrm{x} / \mathrm{c}=0.735$, looking upstream from back [44].

Figure 35. Pressure coefficient contours at $\mathrm{x} / \mathrm{c}=0.744$, looking upstream from back [44]. 
Figure 36. Pressure coefficient contours at $\mathrm{x} / \mathrm{c}=0.803$, looking upstream from back [44].

Figure 37. Pressure coefficient contours at $\mathrm{x} / \mathrm{c}=0.864$, looking upstream from back [44].

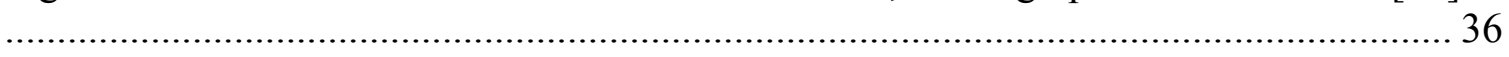

Figure 38. Pressure coefficient contours at $\mathrm{x} / \mathrm{c}=1.246$, looking upstream from back [44].

Figure 39. Pressure coefficient contours at $\mathrm{x} / \mathrm{c}=1.678$, looking upstream from back [44].

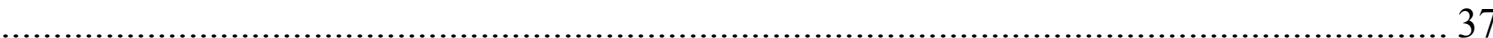

Figure 40. Pressure coefficient in the wing tip vortex core in the downstream direction. 38

Figure 41 . Velocity magnitude contours $(\mathrm{ft} / \mathrm{s})$ at $\mathrm{x} / \mathrm{c}=0.25$, looking upstream from back

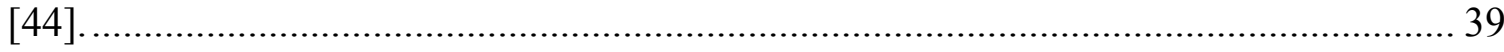

Figure 42 . Velocity magnitude contours $(\mathrm{ft} / \mathrm{s})$ at $\mathrm{x} / \mathrm{c}=0.345$, looking upstream from back

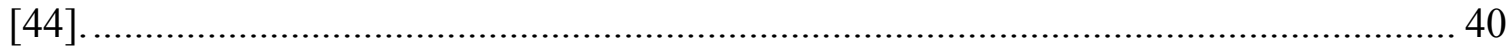

Figure 43 . Velocity magnitude contours $(\mathrm{ft} / \mathrm{s})$ at $\mathrm{x} / \mathrm{c}=0.443$, looking upstream from back

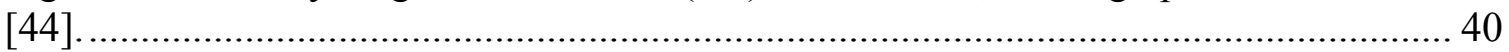

Figure 44. Velocity magnitude contours $(\mathrm{ft} / \mathrm{s})$ at $\mathrm{x} / \mathrm{c}=0.542$, looking upstream from back

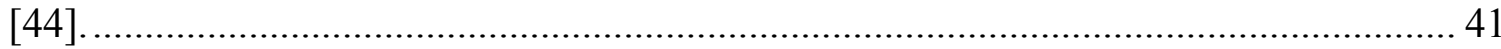

Figure 45 . Velocity magnitude contours $(\mathrm{ft} / \mathrm{s})$ at $\mathrm{x} / \mathrm{c}=0.606$, looking upstream from back

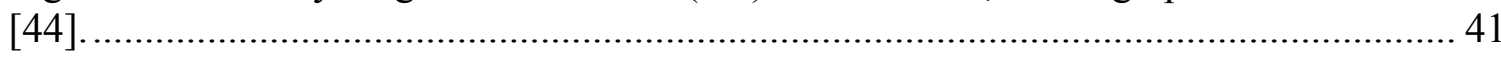

Figure 46 . Velocity magnitude contours $(\mathrm{ft} / \mathrm{s})$ at $\mathrm{x} / \mathrm{c}=0.625$, looking upstream from back

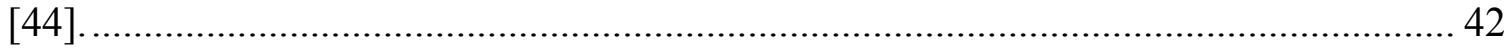

Figure 47 . Velocity magnitude contours $(\mathrm{ft} / \mathrm{s})$ at $\mathrm{x} / \mathrm{c}=0.704$, looking upstream from back

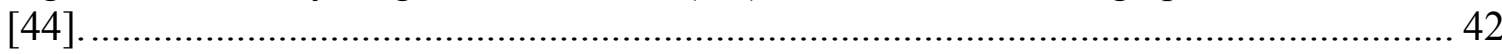

Figure 48 . Velocity magnitude contours $(\mathrm{ft} / \mathrm{s})$ at $\mathrm{x} / \mathrm{c}=0.729$, looking upstream from back

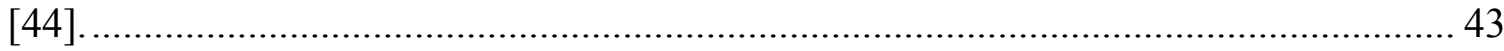

Figure 49 . Velocity magnitude contours $(\mathrm{ft} / \mathrm{s})$ at $\mathrm{x} / \mathrm{c}=0.735$, looking upstream from back

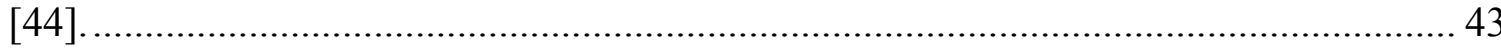

Figure 50. Velocity magnitude contours $(\mathrm{ft} / \mathrm{s})$ at $\mathrm{x} / \mathrm{c}=0.744$, looking upstream from back

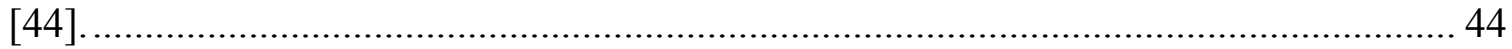

Figure 51 . Velocity magnitude contours $(\mathrm{ft} / \mathrm{s})$ at $\mathrm{x} / \mathrm{c}=0.803$, looking upstream from back

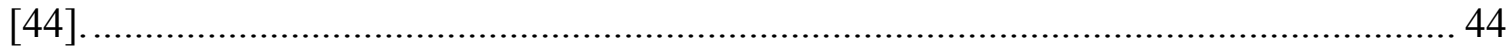

Figure 52. Velocity magnitude contours $(\mathrm{ft} / \mathrm{s})$ at $\mathrm{x} / \mathrm{c}=0.864$, looking upstream from back

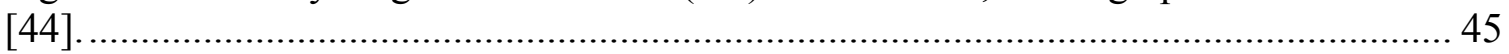

Figure 53. Velocity magnitude contours $(\mathrm{ft} / \mathrm{s})$ at $\mathrm{x} / \mathrm{c}=0.886$, looking upstream from back

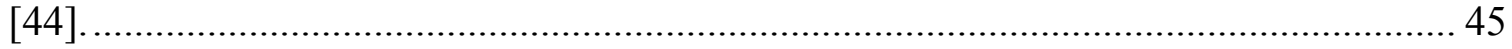

Figure 54 . Velocity magnitude contours $(\mathrm{ft} / \mathrm{s})$ at $\mathrm{x} / \mathrm{c}=0.985$, looking upstream from back

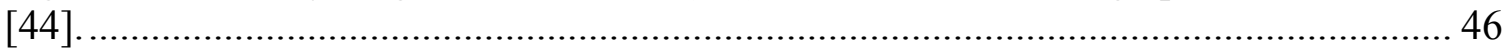

Figure 55. Velocity magnitude contours $(\mathrm{ft} / \mathrm{s})$ at $\mathrm{x} / \mathrm{c}=1.246$, looking upstream from back

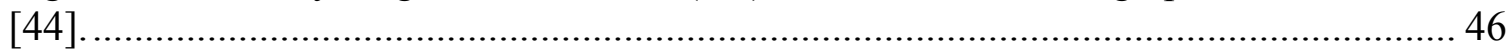

Figure 56. Velocity magnitude contours $(\mathrm{ft} / \mathrm{s})$ at $\mathrm{x} / \mathrm{c}=1.678$, looking upstream from back

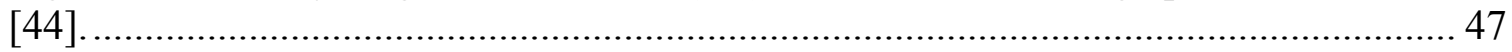

Figure 57. Wingtip vortex core maximum velocity variation in the downstream direction.

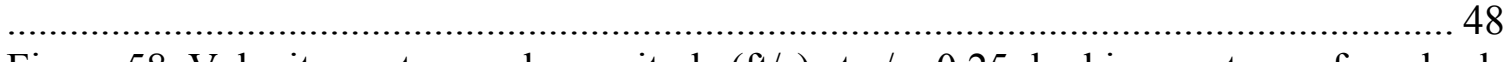

Figure 58. Velocity vectors and magnitude $(\mathrm{ft} / \mathrm{s})$ at $\mathrm{x} / \mathrm{c}=0.25$, looking upstream from back

[44]. 
Figure 59. Velocity vectors and magnitude $(\mathrm{ft} / \mathrm{s})$ at $\mathrm{x} / \mathrm{c}=0.345$, looking upstream from

back [44].

Figure 60 . Velocity vectors and magnitude $(\mathrm{ft} / \mathrm{s})$ at $\mathrm{x} / \mathrm{c}=0.443$, looking upstream from

back [44].

Figure 61 . Velocity vectors and magnitude $(\mathrm{ft} / \mathrm{s})$ at $\mathrm{x} / \mathrm{c}=0.542$, looking upstream from

back [44].

Figure 62 . Velocity vectors and magnitude $(\mathrm{ft} / \mathrm{s})$ at $\mathrm{x} / \mathrm{c}=0.542$, looking upstream from

back [44].

Figure 63. Velocity vectors and magnitude $(\mathrm{ft} / \mathrm{s})$ at $\mathrm{x} / \mathrm{c}=0.606$, looking upstream from

back [44].

Figure 64 . Velocity vectors and magnitude $(\mathrm{ft} / \mathrm{s})$ at $\mathrm{x} / \mathrm{c}=0.606$, looking upstream from

back [44].

Figure 65 . Velocity vectors and magnitude $(\mathrm{ft} / \mathrm{s})$ at $\mathrm{x} / \mathrm{c}=0.625$, looking upstream from

back [44].

Figure 66 . Velocity vectors and magnitude $(\mathrm{ft} / \mathrm{s})$ at $\mathrm{x} / \mathrm{c}=0.625$, looking upstream from

back [44].

Figure 67 . Velocity vectors and magnitude $(\mathrm{ft} / \mathrm{s})$ at $\mathrm{x} / \mathrm{c}=0.704$, looking upstream from back [44].

Figure 68 . Velocity vectors and magnitude $(\mathrm{ft} / \mathrm{s})$ at $\mathrm{x} / \mathrm{c}=0.704$, looking upstream from

back [44].

Figure 69 . Velocity vectors and magnitude $(\mathrm{ft} / \mathrm{s})$ at $\mathrm{x} / \mathrm{c}=0.729$, looking upstream from

back [44].

Figure 70 . Velocity vectors and magnitude $(\mathrm{ft} / \mathrm{s})$ at $\mathrm{x} / \mathrm{c}=0.729$, looking upstream from

back [44].

Figure 71 . Velocity vectors and magnitude $(\mathrm{ft} / \mathrm{s})$ at $\mathrm{x} / \mathrm{c}=0.735$, looking upstream from

back [44].

Figure 72 . Velocity vectors and magnitude $(\mathrm{ft} / \mathrm{s})$ at $\mathrm{x} / \mathrm{c}=0.744$, looking upstream from back [44].

Figure 73 . Velocity vectors and magnitude $(\mathrm{ft} / \mathrm{s})$ at $\mathrm{x} / \mathrm{c}=0.803$, looking upstream from

back [44].

Figure 74 . Velocity vectors and magnitude $(\mathrm{ft} / \mathrm{s})$ at $\mathrm{x} / \mathrm{c}=0.864$, looking upstream from back [44].

Figure 75 . Velocity vectors and magnitude $(\mathrm{ft} / \mathrm{s})$ at $\mathrm{x} / \mathrm{c}=0.886$, looking upstream from

back [44].

Figure 76 . Velocity vectors and magnitude $(\mathrm{ft} / \mathrm{s})$ at $\mathrm{x} / \mathrm{c}=1.005$, looking upstream from

back [44].

Figure 77 . Velocity vectors and magnitude $(\mathrm{ft} / \mathrm{s})$ at $\mathrm{x} / \mathrm{c}=1.678$, looking upstream from

back [44].

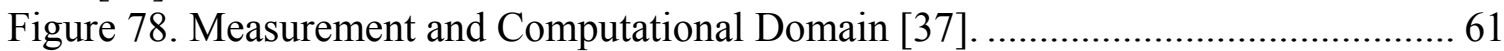

Figure 79. Grid Topology with two million cells and 0.4 million nodes...........................62

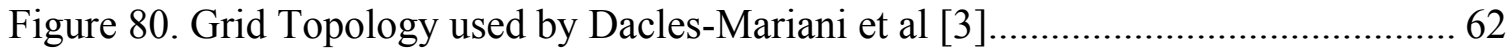

Figure 81. Residuals for mesh with 7 million cells and 1.3 million nodes........................ 64

Figure 82. Lift coefficient monitor for mesh with 7 million cells and 1.3 million nodes. 64

Figure 83. Drag coefficient monitor for mesh with 7 million cells and 1.3 million nodes. 
Figure 84. Pressure Coefficients measured and computed by Chow et al. [43] and

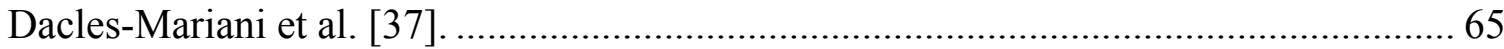

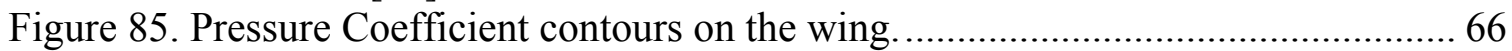
Figure 86. Measured and Computed Velocity Contours by Chow et al. [43] and Dacles-

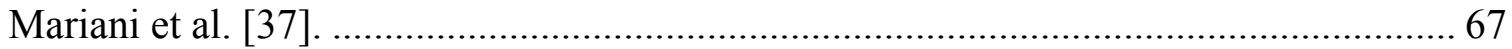

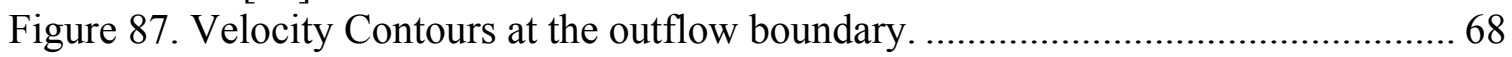

Figure 88. Surface Pathlines and Friction Coefficient measured and computed previously

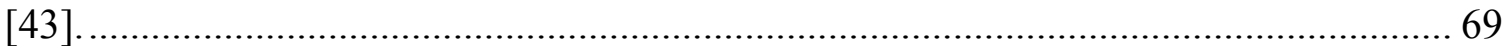

Figure 89. Pathlines computed on the top wing surface and the wing tip. ...................... 70

Figure 90. Comparison between experimental and CFD pressure coefficients at

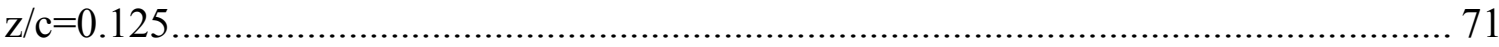

Figure 91. Square contours around the wingtip vortex core to compute the circulation

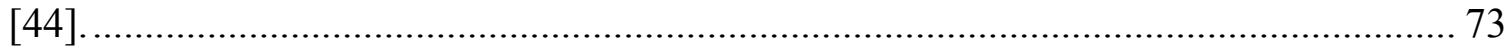

Figure 92. Wingtip vortex variation with distance from the vortex core........................ 75

Figure 93. Vorticity at one chord downstream of a NACA 0012 wing [44] .................. 75

Figure 94. Tangential velocity variation across the wingtip vortex core compared to an

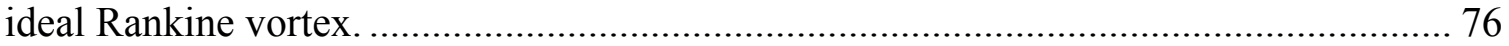

Figure 95. Rectangles drawn to the right of the vortex core to compute the circulation.. 79

Figure 96. Circulation at different areas at the right of a wingtip vortex core. ............... 80

Figure 97. Streamlines on a wing with blowing at $\mathrm{V}_{\infty}$ through a slot at the wing tip....... 82

Figure 98. Streamlines on a wing with suction at the wing tip, as viewed from

downstream.

Figure 99. Pressure coefficient contours on top of the wing when wing tip suction is

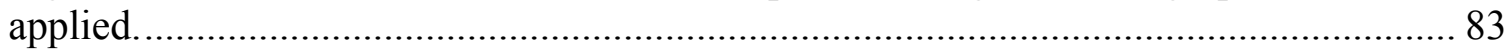

Figure 100. Streamlines on top of the wing when suction is applied at the wing tip...... 83

Figure 101. Streamlines on the bottom of the wing when suction is applied.................. 84

Figure 102. Grid when suction is applied on top and blowing on the bottom................. 84

Figure 103. Grid when a small suction slot is placed on the bottom of the wing............ 85

Figure 104. Bottom streamlines when suction at $\mathrm{V}_{\infty}$ is applied on a small slot. ............. 85

Figure 105. Grid used when suction and blowing are applied at the trailing edge.......... 86

Figure 106. Streamlines at the top of the wing when suction and blowing are applied at

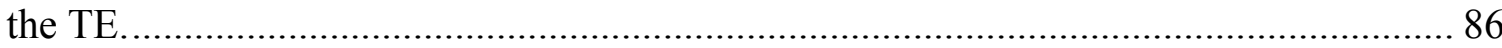

Figure 107. Streamlines at the bottom of the wing when suction and blowing are applied

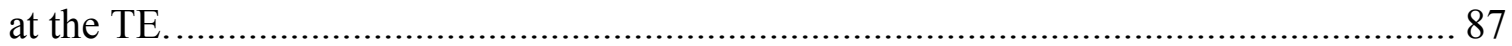

Figure 108. Streamlines when suction and blowing are applied at the TE..................... 87

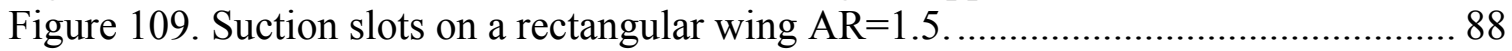

Figure 110. Wing tip vortex cancellation by wing tip suction and suction slot along the

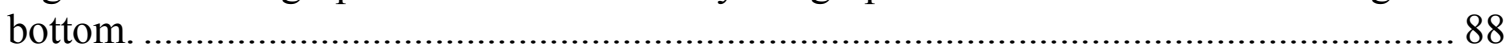

Figure 111. Bottom streamlines when suction is applied at the wing tip and along a slot

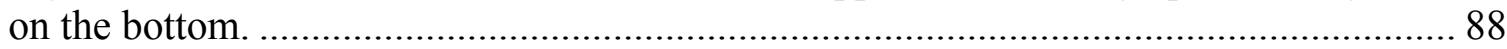

Figure 112. Streamlines when suction is applied at the wing tip and along the bottom of

the wing.....

Figure 113. Surface pressure contours on top of the wing when suction is applied on the

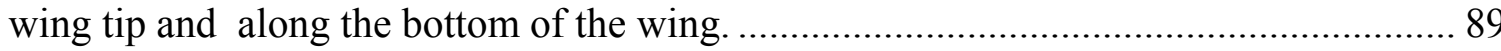

Figure 114. Surface pressure contours on the bottom of the wing when suction is applied

on the wing tip and along the bottom of the wing. 90 
Figure 115. Surface Integral of the X-Vorticity for three wings. ............................... 91

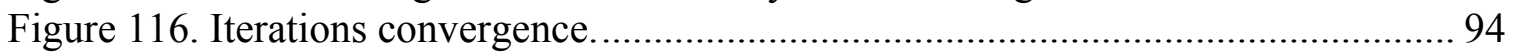

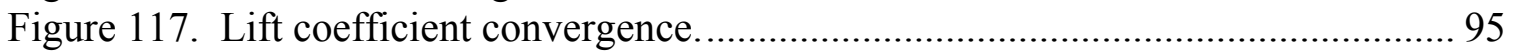

Figure 118. Drag Coefficient Convergence.................................................................. 95

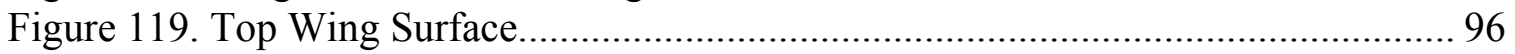

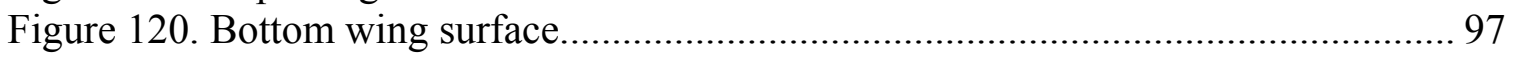

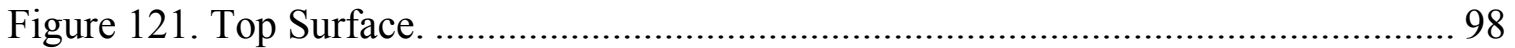

Figure 122. Bottom Surface..................................................................................... 98

Figure 123. Wing Top Surface with Span-wise Blowing.......................................... 100

Figure 124. Wing Bottom Surface with Span-wise Blowing ...................................... 100

Figure 125. NACA 64 ${ }_{3}-018$ Wing Top Surface with Span-wise Blowing.................... 101

Figure 126. NACA 64 ${ }_{3}-018$ Wing Bottom Surface with Span-wise Blowing ............... 101

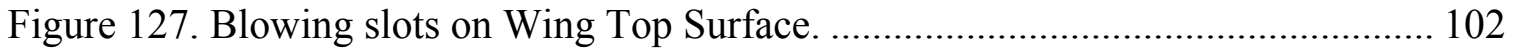

Figure 128. Suction slots on Wing Bottom surface................................................. 102

Figure 129. Vorticity contours behind a rectangular wing of $A R=4$ without $B L C$....... 103

Figure 130. Vorticity contours behind a rectangular wing of $A R=4$ with suction at the $\mathrm{TE}$

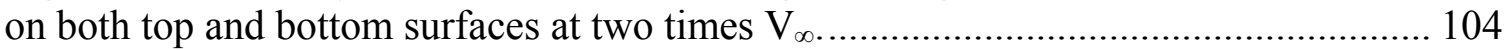

Figure 131 . Vorticity contours behind a rectangular wing of $A R=4$ with suction at the $T E$

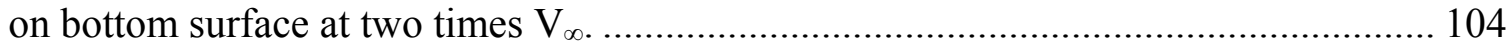

Figure 132. Bottom wing surface of a rectangular wing $A R=4$ with suction slots........ 105

Figure 133. Vorticity contours behind an $\mathrm{AR}=4$ rectangular wing without BLC. ......... 105

Figure 134 . Vorticity contours behind an $A R=4$ rectangular wing with suction on bottom

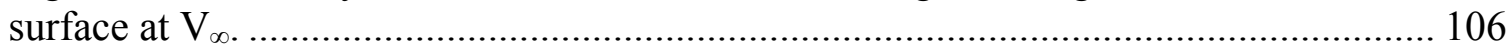

Figure 135 . Vorticity contours behind an $A R=4$ rectangular wing with suction on bottom

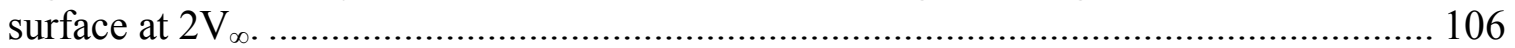

Figure 136. Vorticity contours on an $\mathrm{AR}=1.5$ rectangular wing without BLC suction.. 107 Figure 137. Vorticity contours on an $A R=1.5$ rectangular wing with suction at $0.5 \mathrm{~V}_{\infty}$ on

bottom TE and wing tip. ............................................................................... 107

Figure 138 . Vorticity contours on an $\mathrm{AR}=1.5$ rectangular wing with suction at $0.5 \mathrm{~V}_{\infty}$ on

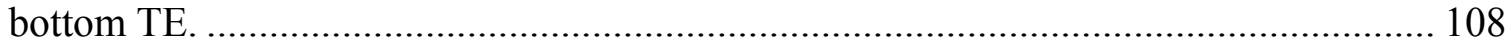

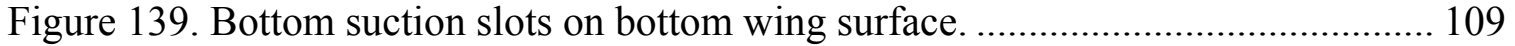

Figure 140. Top Suction slot on top wing surface................................................... 109

Figure 141. Solution Domain and Partial View of the Coarse mesh........................... 110

Figure 142. Continuity and Velocity residuals for the coarse mesh............................ 111

Figure 143. Drag Coefficient monitors for the coarse mesh........................................ 111

Figure 144. Pressure coefficient contours at a plane $\mathrm{x}=9 \mathrm{ft}$ for the coarse mesh. ........ 112

Figure 145 . Velocity magnitude ( $\mathrm{ft} / \mathrm{s})$ contours at a plane $\mathrm{x}=9 \mathrm{ft}$ for the coarse mesh.. 112

Figure 146 . Vorticity contours $(1 / \mathrm{s})$ at a plane $\mathrm{x}=9 \mathrm{ft}$. for the coarse mesh. ................ 113

Figure 147. Pressure coefficient contours at $\mathrm{x}=9 \mathrm{ft}$. for the fine mesh........................ 113

Figure 148. Velocity magnitude $(\mathrm{ft} / \mathrm{s})$ contours at $\mathrm{x}=9 \mathrm{ft}$ for the fine mesh................. 114

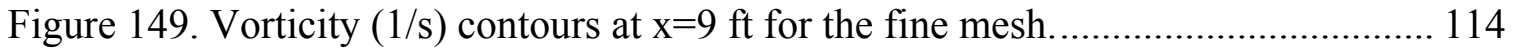

Figure 150. Pressure coefficient contours at $\mathrm{x}=9 \mathrm{ft}$. for the finest mesh..................... 115

Figure 151 . Velocity magnitude $(\mathrm{ft} / \mathrm{s})$ contours at $\mathrm{x}=9 \mathrm{ft}$ for the finest mesh. ............. 115

Figure 152 . Vorticity $(1 / \mathrm{s})$ contours at $\mathrm{x}=9 \mathrm{ft}$ for the finest mesh............................ 116

Figure 153. Pressure coefficient contours at $\mathrm{x}=9 \mathrm{ft}$. for the coarse mesh with suction $=0.5 \mathrm{~V}_{\infty}$ 
Figure 154 . Velocity magnitude $(\mathrm{ft} / \mathrm{s})$ contours at $\mathrm{x}=9 \mathrm{ft}$ for the coarse mesh with suction $=0.5 \mathrm{~V}_{\infty}$

Figure 155 . Vorticity $(1 / \mathrm{s})$ contours at $\mathrm{x}=9 \mathrm{ft}$ for the coarse mesh with suction $=0.5 \mathrm{~V}_{\infty} .117$ Figure 156. Pressure coefficient contours at $\mathrm{x}=9 \mathrm{ft}$. for the fine mesh with suction $=0.5 \mathrm{~V}_{\infty}$.

Figure 157. Velocity magnitude $(\mathrm{ft} / \mathrm{s})$ contours at $\mathrm{x}=9 \mathrm{ft}$ for the fine mesh with

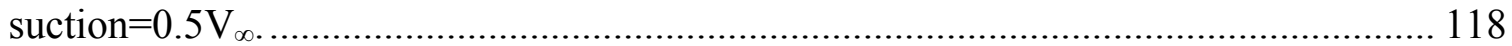

Figure 158 . Vorticity $(1 / \mathrm{s})$ contours at $\mathrm{x}=9 \mathrm{ft}$ for the fine mesh with suction $=0.5 \mathrm{~V}_{\infty} \ldots 119$ Figure 159. Pressure coefficient contours at $\mathrm{x}=9 \mathrm{ft}$. for the finest mesh with suction $=0.5 \mathrm{~V}_{\infty}$

Figure 160 . Velocity magnitude $(\mathrm{ft} / \mathrm{s})$ contours at $\mathrm{x}=9 \mathrm{ft}$ for the finest mesh with suction $=0.5 \mathrm{~V}_{\infty}$

Figure 161 . Vorticity $(1 / \mathrm{s})$ contours at $\mathrm{x}=9 \mathrm{ft}$ for the finest mesh with suction $=0.5 \mathrm{~V}_{\infty} .120$ Figure 162. Pressure coefficient contours at $x=9 \mathrm{ft}$. for the finest mesh with suction $=0.5 \mathrm{~V}_{\infty}$ on top slot.

Figure 163 . Velocity magnitude $(\mathrm{ft} / \mathrm{s})$ contours at $\mathrm{x}=9 \mathrm{ft}$ for the finest mesh with suction $=0.5 \mathrm{~V}_{\infty}$ on top slot.

Figure 164. Vorticity $(1 / \mathrm{s})$ contours at $\mathrm{x}=9 \mathrm{ft}$ for the finest mesh with suction $=0.5 \mathrm{~V}_{\infty}$ on

top slot.

Figure 165. Pressure coefficient contours at $\mathrm{x}=9 \mathrm{ft}$. for the finest mesh with suction $=\mathrm{V}_{\infty}$

on top slot. 123

Figure 166. Velocity magnitude $(\mathrm{ft} / \mathrm{s})$ contours at $\mathrm{x}=9 \mathrm{ft}$ for the finest mesh with suction $=\mathrm{V}_{\infty}$ on top slot.

Figure 167 . Vorticity $(1 / \mathrm{s})$ contours at $\mathrm{x}=9 \mathrm{ft}$ for the finest mesh with suction $=\mathrm{V}_{\infty}$ on top

slot.

Figure 168. Pressure coefficient contours at $\mathrm{x}=9 \mathrm{ft}$. for the finest mesh with suction $=0.5 \mathrm{~V}_{\infty}$ on bottom slots.

Figure 169 . Velocity magnitude $(\mathrm{ft} / \mathrm{s})$ contours at $\mathrm{x}=9 \mathrm{ft}$ for the finest mesh with suction $=0.5 \mathrm{~V}_{\infty}$ on bottom slots.

Figure 170 . Vorticity $(1 / \mathrm{s})$ contours at $\mathrm{x}=9 \mathrm{ft}$ for the finest mesh with suction $=0.5 \mathrm{~V}_{\infty}$ on bottom slots. 126 Figure 171. Pressure coefficient contours at $\mathrm{x}=9 \mathrm{ft}$. for the finest mesh with suction $=\mathrm{V}_{\infty}$ on bottom slots. 126

Figure 172 . Velocity magnitude $(\mathrm{ft} / \mathrm{s})$ contours at $\mathrm{x}=9 \mathrm{ft}$ for the finest mesh with suction $=\mathrm{V}_{\infty}$ on bottom slots.

Figure 173. Vorticity $(1 / \mathrm{s})$ contours at $\mathrm{x}=9 \mathrm{ft}$ for the finest mesh with suction $=\mathrm{V}_{\infty}$ on bottom slots.

Figure 174. Pressure coefficient contours at $\mathrm{x}=9 \mathrm{ft}$. for the finest mesh with

suction $=0.5 \mathrm{~V}_{\infty}$ on bottom front slot.

Figure 175 . Velocity magnitude $(\mathrm{ft} / \mathrm{s})$ contours at $\mathrm{x}=9 \mathrm{ft}$ for the finest mesh with suction $=0.5 \mathrm{~V}_{\infty}$ on bottom front slot.

Figure 176 . Vorticity $(1 / \mathrm{s})$ contours at $\mathrm{x}=9 \mathrm{ft}$ for the finest mesh with suction $=0.5 \mathrm{~V}_{\infty}$ on

bottom front slot.

Figure 177. Pressure coefficient contours at $\mathrm{x}=9 \mathrm{ft}$. for the finest mesh with suction $=\mathrm{V}_{\infty}$ on bottom front slot. 
Figure 178. Velocity magnitude ( $\mathrm{ft} / \mathrm{s})$ contours at $\mathrm{x}=9 \mathrm{ft}$ for the finest mesh with suction $=\mathrm{V}_{\infty}$ on bottom front slot.

Figure 179 . Vorticity $(1 / \mathrm{s})$ contours at $\mathrm{x}=9 \mathrm{ft}$ for the finest mesh with suction $=\mathrm{V}_{\infty}$ on bottom front slot.

Figure 180. Pressure coefficient contours at $\mathrm{x}=9 \mathrm{ft}$. for the finest mesh with

suction $=0.5 \mathrm{~V}_{\infty}$ on bottom rear slot.

Figure 181 . Velocity magnitude $(\mathrm{ft} / \mathrm{s})$ contours at $\mathrm{x}=9 \mathrm{ft}$ for the finest mesh with suction $=0.5 \mathrm{~V}_{\infty}$ on bottom rear slot.

Figure 182 . Vorticity $(1 / \mathrm{s})$ contours at $\mathrm{x}=9 \mathrm{ft}$ for the finest mesh with suction $=0.5 \mathrm{~V}_{\infty}$ on

bottom rear slot.

Figure 183. Pressure coefficient contours at $\mathrm{x}=9 \mathrm{ft}$. for the finest mesh with suction $=\mathrm{V}_{\infty}$

on bottom rear slot.

Figure 184. Velocity magnitude $(\mathrm{ft} / \mathrm{s})$ contours at $\mathrm{x}=9 \mathrm{ft}$ for the finest mesh with suction $=\mathrm{V}_{\infty}$ on bottom rear slot.

Figure 185 . Vorticity $(1 / \mathrm{s})$ contours at $\mathrm{x}=9 \mathrm{ft}$ for the finest mesh with suction $=\mathrm{V}_{\infty}$ on

bottom rear slot.

Figure 186. Pressure coefficient contours at $\mathrm{x}=9 \mathrm{ft}$. for the finest mesh with suction $=21$

$\mathrm{ft} / \mathrm{s}$ on top slot

Figure 187 . Velocity magnitude $(\mathrm{ft} / \mathrm{s})$ contours at $\mathrm{x}=9 \mathrm{ft}$ for the finest mesh with suction=21 ft/s on top slot.

Figure 188 . Vorticity $(1 / \mathrm{s})$ contours at $x=9 \mathrm{ft}$ for the finest mesh with suction $=21 \mathrm{ft} / \mathrm{s}$ on

top slot......

Figure 189. Pressure coefficient contours at $\mathrm{x}=9 \mathrm{ft}$. for the finest mesh with suction $=42$

$\mathrm{ft} / \mathrm{s}$ on top slot.

Figure 190 . Velocity magnitude $(\mathrm{ft} / \mathrm{s})$ contours at $\mathrm{x}=9 \mathrm{ft}$ for the finest mesh with suction $=42 \mathrm{ft} / \mathrm{s}$ on top slot.

Figure 191. Vorticity $(1 / \mathrm{s})$ contours at $\mathrm{x}=9 \mathrm{ft}$ for the finest mesh with suction $=42 \mathrm{ft} / \mathrm{s}$ on

top slot......

Figure 192. Pressure coefficient contours at $\mathrm{x}=9 \mathrm{ft}$. for the finest mesh with suction=63

$\mathrm{ft} / \mathrm{s}$ on top slot.

Figure 193. Velocity magnitude $(\mathrm{ft} / \mathrm{s})$ contours at $\mathrm{x}=9 \mathrm{ft}$ for the finest mesh with suction $=63 \mathrm{ft} / \mathrm{s}$ on top slot.

Figure 194. Vorticity $(1 / \mathrm{s})$ contours at $\mathrm{x}=9 \mathrm{ft}$ for the finest mesh with suction $=63 \mathrm{ft} / \mathrm{s}$ on

top slot......

Figure 195. Pressure coefficient contours at $\mathrm{x}=9 \mathrm{ft}$. for the finest mesh with suction $=106$

$\mathrm{ft} / \mathrm{s}$ on top slot.

Figure 196. Velocity magnitude $(\mathrm{ft} / \mathrm{s})$ contours at $\mathrm{x}=9 \mathrm{ft}$ for the finest mesh with

suction $=106 \mathrm{ft} / \mathrm{s}$ on top slot.

Figure 197 . Vorticity $(1 / \mathrm{s})$ contours at $\mathrm{x}=9 \mathrm{ft}$ for the finest mesh with suction $=106 \mathrm{ft} / \mathrm{s}$ on

top slot.

Figure 198. Pressure coefficient contours at $\mathrm{x}=9 \mathrm{ft}$. for the finest mesh with suction $=127$

$\mathrm{ft} / \mathrm{s}$ on top slot.

Figure 199. Velocity magnitude $(\mathrm{ft} / \mathrm{s})$ contours at $\mathrm{x}=9 \mathrm{ft}$ for the finest mesh with

suction $=127 \mathrm{ft} / \mathrm{s}$ on top slot.

Figure 200 . Vorticity $(1 / \mathrm{s})$ contours at $\mathrm{x}=9 \mathrm{ft}$ for the finest mesh with suction $=127 \mathrm{ft} / \mathrm{s}$ on

top slot. 
Figure 201. Pressure coefficient contours at $\mathrm{x}=9 \mathrm{ft}$. for the finest mesh with suction $=148$

$\mathrm{ft} / \mathrm{s}$ on top slot.

Figure 202. Velocity magnitude ( $\mathrm{ft} / \mathrm{s})$ contours at $\mathrm{x}=9 \mathrm{ft}$ for the finest mesh with suction $=148 \mathrm{ft} / \mathrm{s}$ on top slot.

Figure 203. Vorticity $(1 / \mathrm{s})$ contours at $\mathrm{x}=9 \mathrm{ft}$ for the finest mesh with suction $=148 \mathrm{ft} / \mathrm{s}$ on

top slot......

Figure 204. Wingtip vorticity variation with the BLC suction velocity.

Figure 205. WVU CC Technology Demonstrator STOL Aircraft in flight, 1974.

Figure 206. WVU CC Wind Tunnel Model A Wing, convertible form round to sharp

trailing edge.

Figure 207. WVU CC Technology Demonstrator STOL Aircraft Model B Wing [55]. 154

Figure 208. . WVU Model B CC flap with details of the internal ejector and Coanda surface [3].

Figure 209. One-dimensional ejector configuration with nozzle, ejector suction and exit.

Figure 210. Nozzles fabricated for the ejector tests.

Figure 211. Sketch of the test apparatus used for ejector experiments........................... 161

Figure 212. Test apparatus used for ejector experiments. .......................................... 161

Figure 213. Dimensionless exit velocity increases with an increase in nozzle-to-exit area

ratio.

Figure 214. Dimensionless suction velocity increases with an increase in nozzle-to-exit

area ratio.

Figure 215. Dimensionless total exit pressure increases with an increase in nozzle-to-exit

area ratio.

Figure 216. Dimensionless suction pressure decreases with an increase in nozzle-to-exit

area ratio.

164

Figure 217. Dimensionless suction volume flow rate decreases with an increase in nozzle-to-exit area ratio. 


\section{List of Tables}

Table 1. Pressure coefficient in the wing tip vortex core in the downstream direction.... 38 Table 2 . Wingtip vortex core maximum velocity variation with the downstream position.

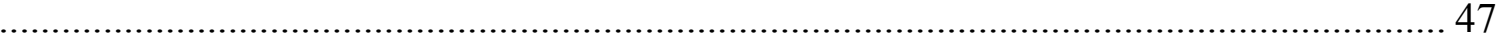

Table 3. Wingtip Vortex Circulation at various distances from the core. ....................... 74

Table 4. Circulation variation at different areas at the right of the vortex core............... 79

Table 5. Wake and Balance measurements for a NACA $64_{3}-018$ at $\mathrm{Re}=1.1 \times 10^{6}$ and $\alpha=10^{\circ}$

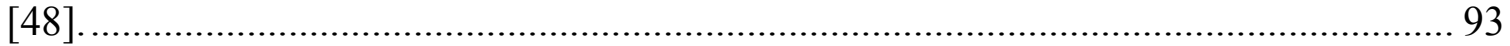

Table 6. Experimental and Numerical Drag Coefficients on a NACA 64 ${ }_{3}-018 \ldots \ldots \ldots \ldots . . . .96$

Table 7. Parametric Study of BLC application on a Wing. ......................................... 97

Table 8. Parametric Study of BLC application on a NACA 64 $3-018$ Wing. ................... 99

Table 9. Parametric Study of BLC application on a NACA 643-018 Wing with Span-wise

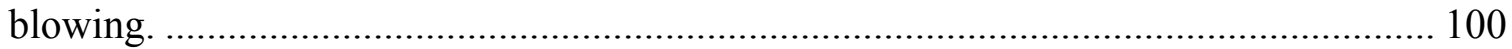

Table 10. Parametric Study of BLC application on a NACA 64 $3-018$ Wing with Span-

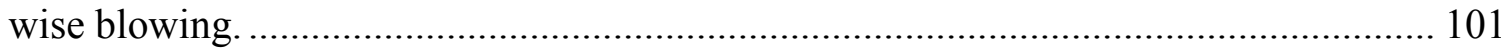

Table 11. Parametric Study of BLC application on a NACA $64_{3}-018$ Wing. ................ 102

Table 12. BLC suction on top and bottom surfaces of a rectangular wing with $A R=4 . .103$ Table 13. BLC suction on three slots on bottom surface of a rectangular wing of $A R=4$.

Table 14. BLC Suction on bottom surface and wingtip on an $\mathrm{AR}=1.5$ rectangular wing. 107

Table 15. Number of Cells and Nodes for the three grids used for the grid convergence study....................................................................................................... 110 Table 16. Grid Convergence Study Results with and without BLC application at $0.5 \mathrm{~V}_{\infty}$.

Table 17. Effectiveness Study Results of drag coefficient and vorticity.

Table 18. Drag Coefficient and Vorticity variation when the suction velocity on top slot increases.

Table 19. Theoretical non-dimensional parameters for one-dimensional incompressible flow ejectors at various area ratios 160 


\section{Nomenclature and Symbols}

\begin{tabular}{|l|l|l|}
\hline ABLC & $=$ & BLC suction area \\
\hline ACC & $=$ & CC blowing area \\
\hline$A_{\mathrm{e}}$ & $=$ & ejector exit area $=$ ACC \\
\hline$A_{\mathrm{n}}$ & $=$ & ejector nozzle area \\
\hline $\mathrm{A}_{\mathrm{s}}$ & $=$ & ejector suction area \\
\hline $\bar{A}_{\mathrm{s}}$ & $=$ & As/Ae, dimensionless suction area \\
\hline $\bar{A} \mathrm{n}$ & $=$ & An/Ae, dimensionless nozzle area \\
\hline $\mathrm{AR}$ & $=$ & Aspect ratio \\
\hline $\mathrm{b}$ & $=$ & span \\
\hline BLC & $=$ & Boundary layer control \\
\hline $\mathrm{CC}$ & $=$ & Circulation Control \\
\hline $\mathrm{C}_{\mathrm{D}}$ & $=$ & Drag coefficient \\
\hline $\mathrm{C}_{\mathrm{Di}}$ & $=$ & Induced drag coefficient \\
\hline $\mathrm{C}_{\mathrm{L}}$ & $=$ & Lift coefficient \\
\hline $\mathrm{C}_{\mathrm{p}}$ & $=$ & pressure coefficient \\
\hline $\mathrm{C}_{\mathrm{Q}}$ & $=$ & suction volume flow rate coefficient \\
\hline $\mathrm{D}$ & $=$ & drag \\
\hline $\mathrm{D}_{\mathrm{pp}}$ & $=$ & Profile drag due to pressure losses \\
\hline $\mathrm{D}_{\mathrm{pm}}$ & $=$ & Profile drag due to momentum deficit \\
\hline $\mathrm{D}_{\text {viscous }}$ & $=$ & Viscous drag \\
\hline $\mathrm{D}_{\mathrm{i}}$ & $=$ & Induced drag \\
\hline $\mathrm{e}$ & $=$ & Oswald efficiency factor \\
\hline $\mathrm{h}$ & $=$ & Height \\
\hline $\mathrm{H}$ & $=$ & Shape factor \\
\hline $\mathrm{L}$ & $=$ & lift \\
\hline $\mathrm{L}_{\mathrm{i}}$ & $=$ & Lift per unit span \\
\hline LE & $=$ & leading edge \\
\hline $\mathrm{p}_{\mathrm{e}}$ & $=$ & ejector exit static pressure \\
\hline
\end{tabular}




\begin{tabular}{|l|l|l|}
\hline $\mathrm{p}_{\mathrm{n}}$ & $=$ & fully expanded nozzle exit velocity equal $\mathrm{p}_{\mathrm{s}}$ \\
\hline $\mathrm{p}_{\mathrm{s}}$ & $=$ & suction side velocity inside ejector \\
\hline $\mathrm{p}_{\mathrm{oe}}$ & $=$ & total pressure at ejector exit \\
\hline $\mathrm{p}_{\mathrm{on}}$ & $=$ & total pressure of ejector nozzle flow \\
\hline $\mathrm{p}_{\mathrm{os}}$ & $=$ & total pressure of ejector secondary flow \\
\hline $\mathrm{p}_{\infty}$ & $=$ & free stream static pressure \\
\hline $\mathrm{Q}$ & $=$ & suction volume flow rate \\
\hline$q_{\infty}$ & $=$ & free stream dynamic pressure \\
\hline $\mathrm{S}_{\mathrm{w}}$ & $=$ & Wetted surface \\
\hline $\mathrm{T}$ & $=$ & Thrust \\
\hline $\mathrm{TE}$ & $=$ & trailing edge \\
\hline $\mathrm{U}$ & $=$ & Component of the velocity in the x-direction \\
\hline $\mathrm{V}$ & $=$ & Component of the velocity in the y-direction \\
\hline $\mathrm{VBLC}_{\mathrm{f}}$ & $=$ & BLC suction velocity \\
\hline $\mathrm{V}_{\mathrm{cc}}$ & $=$ & CC blowing velocity \\
\hline$V_{\infty}$ & $=$ & free stream velocity \\
\hline $\mathrm{V}_{\mathrm{e}}$ & $=$ & ejector exit velocity \\
\hline $\mathrm{V}_{\mathrm{i}}$ & $=$ & inviscid velocity \\
\hline $\mathrm{V}_{\text {inviscid }}$ & $=$ & Velocity in the inviscid region \\
\hline $\mathrm{V}_{\mathrm{n}}$ & $=$ & nozzle velocity \\
\hline $\mathrm{V}_{\mathrm{s}}$ & $=$ & Stall velocity \\
\hline $\mathrm{V}_{\mathrm{s}}$ & $=$ & ejector suction velocity \\
\hline $\mathrm{Vol}_{\mathrm{e}}$ & $=$ & volumetric ejector exit flow rate \\
\hline $\mathrm{Vol}_{\mathrm{n}}$ & $=$ & volumetric nozzle exit flow rate \\
\hline $\mathrm{Vol}_{\mathrm{s}}$ & $=$ & volumetric suction flow rate \\
\hline $\bar{V}_{\mathrm{s}}$ & $=$ & ejector secondary flow suction velocity to nozzle velocity ratio \\
\hline $\bar{V}_{n}$ & $=$ & Non dimensional nozzle velocity ratio equals 1. \\
\hline $\bar{V}_{e}$ & $=$ & ejector exit velocity to nozzle velocity ratio \\
\hline $\mathrm{W}$ & $\mathrm{W}$ & Coight \\
\hline
\end{tabular}




\begin{tabular}{|l|l|l|}
\hline WVU & $=$ & West Virginia University \\
\hline $\mathrm{x}$ & $=$ & Distance in the longitudinal direction \\
\hline$\Gamma$ & $=$ & Circulation within contour integral \\
\hline$\delta$ & $=$ & Boundary layer thickness \\
\hline$\delta^{*}$ & $=$ & Boundary layer displacement thickness \\
\hline $\mathrm{N}$ & $=$ & Viscosity \\
\hline$\rho$ & $=$ & Density \\
\hline$\gamma$ & $=$ & $2 \mathrm{D}$ local vortex sheet strength \\
\hline$\gamma_{\text {lower }}$ & $=$ & 2 D local vortex sheet strength on lower wing surface \\
\hline$\gamma_{\text {upper }}$ & $=$ & $2 \mathrm{D}$ local vortex sheet strength on upper wing surface \\
\hline
\end{tabular}




\section{Chapter 1: Introduction}

\section{Potential benefits of wingtip wake minimization}

Since the fuel crisis in the seventies and subsequent trend in fuel prices, aircraft drag reduction has become of prime importance for military and commercial aircraft manufacturers and operators. A $10 \%$ drag reduction on a large military transport aircraft is estimated to save up to 13 million gallons of fuel over its lifetime [1]. With current fuel prices, the savings is on the order of 60 million U.S. dollars (\$) per aircraft. The world total jet fleet is estimated to be around 17 thousand aircraft [2]. Such reduction in drag could result in fuel savings in fuel up to $1 \times 10^{10}$ U.S. dollars (\$).

Eliminating wake vorticity has military advantages for stealth operations because some aircraft detection instruments are based on locating wake vorticity to find the aircraft which produced it.

STOL operations are currently forced to fly on the backside of the power curve where slow level flight power required can be double that of level flight at minimum power, near maximum L/D. The WVU Circulation Control Technology Demonstrator STOL Aircraft, built and flight tested at WVU in 1974, experienced this maximum power required at minimum flight speed of 33 knots. Its performance is described in the Journal of Aircraft, 1976 [3]. Its slow flight performance is also compared to that of other STOL aircraft in Chapter One of the June 2006 edition of the AIAA volume 214 Progress in Astronautics and Aeronautics [4].

Besides the advantage of lowering operating costs, reducing wingtip shed vorticity, and therefore induced drag, may also reduce global warming because of the lower fuel consumption. The world's commercial jet aircraft generate more than 600 million tons of carbon dioxide per year [5]. Carbon dioxide is the major greenhouse gas. Ecologists are concerned about the nature of the emissions from aircraft that include carbon dioxide, nitric oxide, nitrogen dioxide, nitric oxide, and sulphur dioxide; they also worry about the high altitude at which these gases are spewed into the atmosphere. At 
these heights, these particular chemicals have twice the effect on global warming that they have near ground level [5].

\section{Flight at maximum L/D occurs when induced drag equals parasite drag}

Drag consists mainly of: parasite drag and induced drag. Parasite drag is the result of skin friction and pressure drag caused by boundary layer separation. Parasite drag increases with the square of velocity. However, induced drag coefficient decreases with the fourth power of velocity so induced drag decreases with the square of velocity. Figure 1 shows an example of the level-flight performance of a Cessna 206 with induced drag $\mathrm{D}_{\mathrm{i}}$ and parasite drag $\mathrm{D}_{\text {para }}$ plotted as a function of flight speed. Clearly shown is that the lowest value of the total drag occurs when these curves cross one another. Then, the lowest total drag $\mathrm{D}=\mathrm{D}_{\mathrm{i}}+\mathrm{D}_{\text {para }}=2 \mathrm{D}_{\text {para }}$ is obtained when $\mathrm{L} / \mathrm{D}$ is at its maximum. At the maximum $\mathrm{L} / \mathrm{D}$ velocity, induced drag is always $50 \%$ of the total drag.

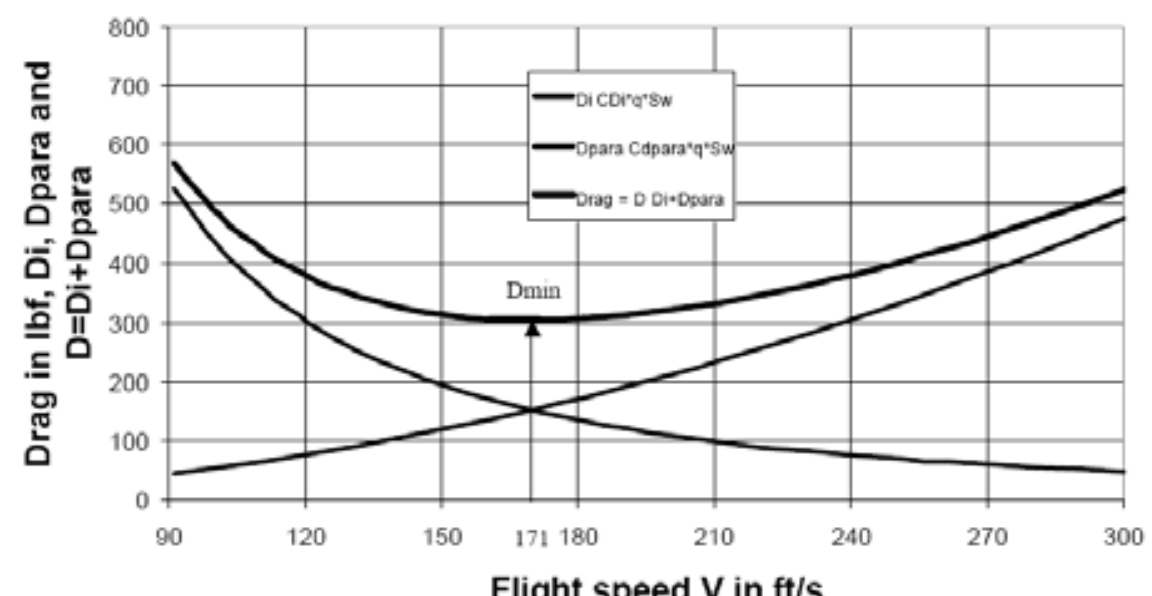

Figure 1. Cessna 206 total drag, induced drag, and parasite drag [6].

\section{Boundary Layer Control technique for reducing wingtip shed vorticity}

The technique investigated to reduce wingtip shed vorticity is capturing the wingtip upper and lower surface boundary layer vorticity prior to entering the wake. Intercepting the upper and lower surface boundary layer by suction along the trailing edge near the wing tips has been investigated herein. Additionally, chordwise suction may be beneficial to minimize spanwise pressure gradients in the wing outboard region. Along the inboard region of a cusped trailing edge, both upper and lower surface boundary layer vorticity meet at the same pressure and the same outer edge velocity. 
Then, the local boundary layer vorticity $\vec{\gamma}$ on the top and $-\vec{\gamma}$ on the bottom surfaces are equal in magnitude. Only when the vorticity vectors are exactly aligned, they can dissipate each other. Current wing tip devices, like winglets, try to catch some of the wing tip vortex energy to produce a thrust force. This attempts to capture the non-selfcanceling wing boundary layer vorticity; then the structure dissipates this captured vorticity by viscous mixing prior to discharging into the wake.

\section{Wingtip vortex generation}

Downstream of a conventional wing, the wing upper and lower surface boundary layer vorticity is only aligned with the free stream direction near the middle of the wing. The remainder rolls up to produce wing tip counter-rotating vortices. To produce lift, the wing produces a net upward pressure difference between the upper and lower wing surfaces. Near the wing tip, higher pressure air accelerates around the wing tip to the low pressure region on top, resulting in a misalignment between upper and lower surface boundary layer vorticity. The lack of self-cancellation of the boundary layer vorticity generates the wing tip vortex and the production of induced drag.

Induced drag is often considered the price one must pay for flying, but in reality, the induced drag can be reduced. New devices, like winglets, vortex diffuser vanes, and wing tip sails have reached reductions in induced drag by up to 30\% [1]. Munk [7], using inviscid induced flow analysis, showed how an elliptically loaded wing has the lowest induced drag for a planar configuration. However, in real air, vorticity can be dissipated by viscous effects to reduce induced drag (see non planar wings designed by Whitcomb [8]).

This research project concentrates on investigating different boundary layer suction configurations to reduce wingtip vorticity on subsonic aircraft. This effort concentrates on BLC suction at or near the trailing edge of the wing tips. With the aid of CFD codes which have been experimentally validated by NASA investigators, the author has analyzed how Boundary Layer Control (BLC) by suction can be used to reduce boundary layer vorticity shed at the trailing edge into the wake of the wing. 
The methodology for this thesis is to first discuss modeling of the wing viscous boundary layer using the CFD code Fluent, and then to investigate various boundary layer control configurations to minimize wake vorticity and associated downwash and induced drag. The code is validated against NASA experiments and codes published by others on the same configuration without BLC. Studying the changes in wake vorticity and associated downwash indicate the effectiveness of the configuration tested with the CFD code.

One type of vortex system is the "starting" vortex. This vortex appears only when there are changes in a wing's angle of attack. Changes in angle of attack have not been considered. Only steady state flow has been analyzed. Due to the nature of the wingtip vortex phenomena, this problem is obviously $3 \mathrm{D}$.

\section{Estimate of minimum suction power required for wing tip BLC}

In an elliptic loaded wing, $25 \%$ of the wake vorticity originates as wing tip boundary layer vorticity. The length of each wing tip responsible for $25 \%$ of the wake vorticity is only $7 \%$ of the wing span. At that location, the wing bound vorticity has already reduced to half its value from that at the wing centerline. All wake vorticity originates as shed upper and lower surface boundary layer vorticities, which fails to cancel one another, when merging at the trailing edge. Therefore wake vorticity might be reduced by up to $50 \%$, if all wing tip boundary layer vorticity could be captured by BLC suction, prior to shedding at the wing tip trailing edge. By viscous dissipation inside an ejector, its vorticity would not contribute to the wake vorticity.

Various wing tip BLC suction configurations have been investigated in this dissertation but none of them proved to be very effective in wake vorticity reduction. Prior to discussing the results it is important to compare the suction power required, relative to the potential induced drag power savings.

When cruising at maximum $\mathrm{L} / \mathrm{D}$, the parasite drag of the entire aircraft must equal the induced drag produced by vorticity inside the wing wake. Then, $25 \%$ of engine thrust is required to overcome the induced drag produced at both wing tips. At lower speeds, as in climb after take-off, up to $50 \%$ of the thrust required will be caused by induced drag which is generated by the outer $7 \%$ of the wing wake! 
The suction power required to intercept all boundary layer vorticity over the above mentioned wing tips is not insignificant. To investigate this consider, the characteristics of a Cessna 206 [6]. This aircraft, as delivered, has approximately the following characteristics: Maximum weight $3600 \mathrm{lbf}, \mathrm{S}_{\mathrm{w}}=175.5 \mathrm{ft}^{2}$, span $36.57 \mathrm{ft}$, chord $4.9 \mathrm{ft}, \mathrm{AR}=7.62,284 \mathrm{HP}$ engine, $\mathrm{e}=0.594, \mathrm{C}_{\mathrm{Dpara}}=0.025,72 \%$ propeller efficiency, and stall velocity $\mathrm{V}_{\mathrm{s}}=91 \mathrm{ft} / \mathrm{s}$.

When cruising at maximum $\mathrm{L} / \mathrm{D}$, the induced drag coefficient equals to the parasite drag coefficient $C_{\mathrm{Di}}=0.025$. The corresponding average wing lift coefficient equals $C_{L}=\sqrt{0.025 * \pi * 0.594 * 7.62}=0.6$. To fly at maximum $\mathrm{L} / \mathrm{D}$, the dynamic pressure $\mathrm{q}_{\infty}=34.4 \mathrm{psf}$, which at sea level requires a level flight speed of $171 \mathrm{ft} / \mathrm{s}$. The corresponding induced drag is $151 \mathrm{lbf}$ or induced drag power is $47 \mathrm{HP}$.

The wing Reynolds number at $\mathrm{V}_{\infty}=171 \mathrm{ft} / \mathrm{s}$, with a $4.9 \mathrm{ft}$ chord is $5.3 \times 10^{6}$ with corresponding flat plate turbulent boundary layer thickness $\delta=0.081 \mathrm{ft}$. Assuming this boundary layer does not separate from the wing surface prior to reaching the wing trailing edge, then its volume flow rate, over both $7 \%$ span wing tips equals $(2 * 0.081 * 171) *(2 * 0.07 * 36.57)=141 \mathrm{ft}^{3} / \mathrm{s}$.

If the suction slot absolute pressure equals $\left(\mathrm{p}_{\infty}-\mathrm{q}_{\infty}\right)$, then the ideal suction power required is $141 * 34.4=4850 \mathrm{ftlb} / \mathrm{s}=8.8 \mathrm{HP}$, which is lower than $50 \%$ of the possible reduction in induced drag power.

In take off conditions at a velocity of $109 \mathrm{ft} / \mathrm{s}$ and the induced drag is $355 \mathrm{lbf}$ as computed in reference 6 , or induced drag power is $70 \mathrm{HP}$.

The Reynolds number at take off is $3.4 \times 10^{6}$ so the turbulent boundary layer thickness is $0.09 \mathrm{ft}$. The boundary layer volume flow rate on both wing tips is $100 \mathrm{ft}^{3} / \mathrm{s}$. Then the suction power required is $2.6 \mathrm{HP}$ or significantly lower than half the induced power.

The rate of climb without suction is $1525 \mathrm{ft} / \mathrm{min}$. With a $50 \%$ reduction in induced drag, the maximum rate of climb could increase to $1982 \mathrm{ft} / \mathrm{min}$. This might be important to STOL take-off and landings. 
Upon completion of this BLC suction slot optimization by CFD, it was found that most of the wing tip boundary layer vorticity separates from the wing surface prior to being entrained into the suction slots. As a result the effectiveness in induced drag reduction is much lower than shown in the above estimates for a Cessna 206. 


\section{Chapter 2: Literature Review}

\section{Lift}

The aerodynamic force exerted by the airflow on the surface of a wing is found by integrating the distribution of:

1. Pressure on the surface, and

2. Shear stress (friction) on the surface [14].

Lift is defined as the component of force perpendicular to the relative wind. Drag is the component of force parallel to the relative wind.

When the flow dividing streamline is below the wing leading edge, then the velocity on the upper wing surface is higher than on its lower surface causing a pressure difference, computable using the aerodynamic equations of continuity, momentum, and energy plus the equation of state. As the velocity increases, the streamlines become closer together in subsonic flow and the static pressure drops in that region to obey the conservation of momentum [9].

The Bernoulli equation states that when fluid velocity increases, the static pressure decreases. Therefore, if the velocity over the top surface of a wing increases, then the pressure decreases. Bernoulli's equation is a special integrated form of the Euler equation.

The greater the wing angle of attack, the more lift is generated (below stall) causing the upstream stagnation point to move below the wing leading edge and upflow ahead of the leading edge. Simultaneously, the flow aft of the trailing edge will deflect downwards. The downflow momentum aft of the trailing edge contributes to the wing lift [9]. When creating lift, the average pressure force on the wing lower surface must be higher than that on its upper surface.

\section{D Flow approximation}

A two-dimensional spinning cylinder, in a uniform flow field, results in a lift force (Figure 2). This phenomena is called "The Magnus Effect" and can also be observed in a variety of real life situations, like spinning a baseball causing its path to 
curve, or spin on a golfball causing it to hook or slice [10]. The flow around a cylinder can be mathematically represented by the superposition of a uniform flow, a doublet, and a vortex of strength $\Gamma$ (Figure 3). This representation makes the lift per unit span (L') directly proportional to the circulation $(\Gamma)$, the freestream density $(\rho)$ and the velocity $\left(\mathrm{V}_{\infty}\right): \mathrm{L}$ ' $=\rho \mathrm{V}_{\infty} \times \Gamma$. This important relationship in aerodynamics is named the "KuttaJoukowski theorem". It can be seen from the flow field pictures, and shown mathematically, that the circulation $\Gamma$ induces an upwash in front of the cylinder and a downwash behind the cylinder, both with the same magnitude but opposite in direction.

Ideal uniform inviscid flow cannot generate a boundary layer, nor can it enter into circulation or shed vorticity into the wake. Circulation $\Gamma$ is the contour integral taken along the outside of the boundary layer and equals

$$
\Gamma=\oint \vec{V} \cdot d l
$$

$\Gamma$ is the same when taken along the outer edge of the boundary layer where the flow becomes nearly inviscid (actually $\mathrm{V}=99 \%$ of $\mathrm{V}_{\text {inviscid }}$ ). The boundary edge velocity $\mathrm{V}_{\text {edge }}$ for a $2 \mathrm{D}$ vortex sheet equals its vorticity $\gamma$, as on the surface $\mathrm{V}=0$ (due to the no slip condition) find

$$
\Gamma=\oint \overrightarrow{V_{e d g e}} \cdot d l=\oint \gamma \cdot d l
$$

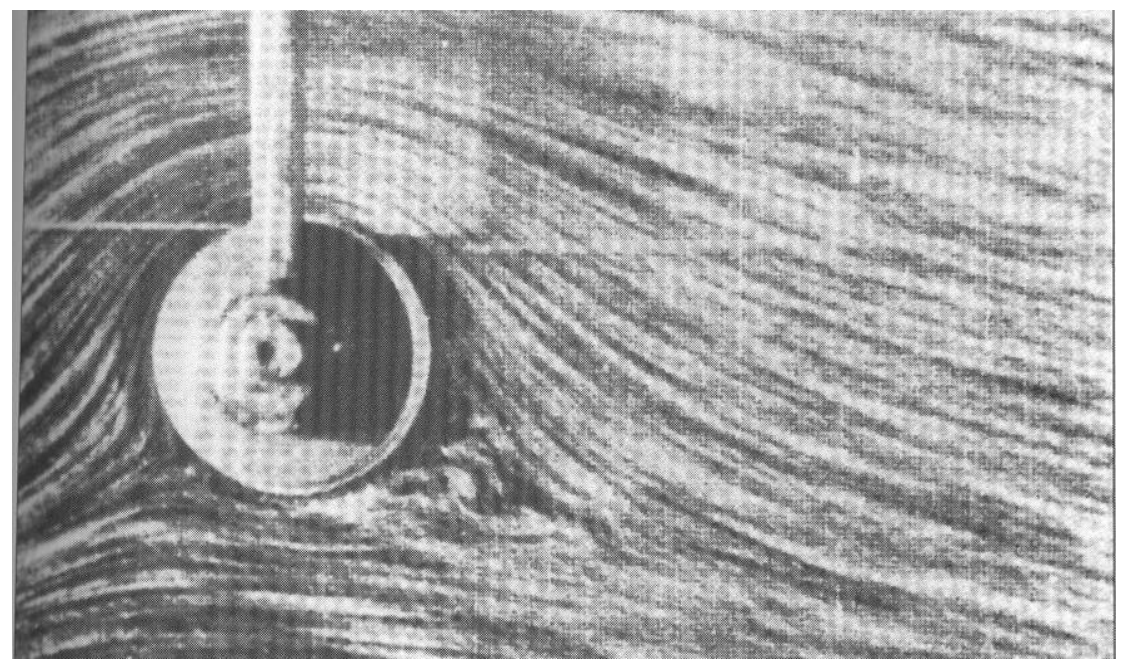

Figure 2. Streamlines around a spinning cylinder [10]. 


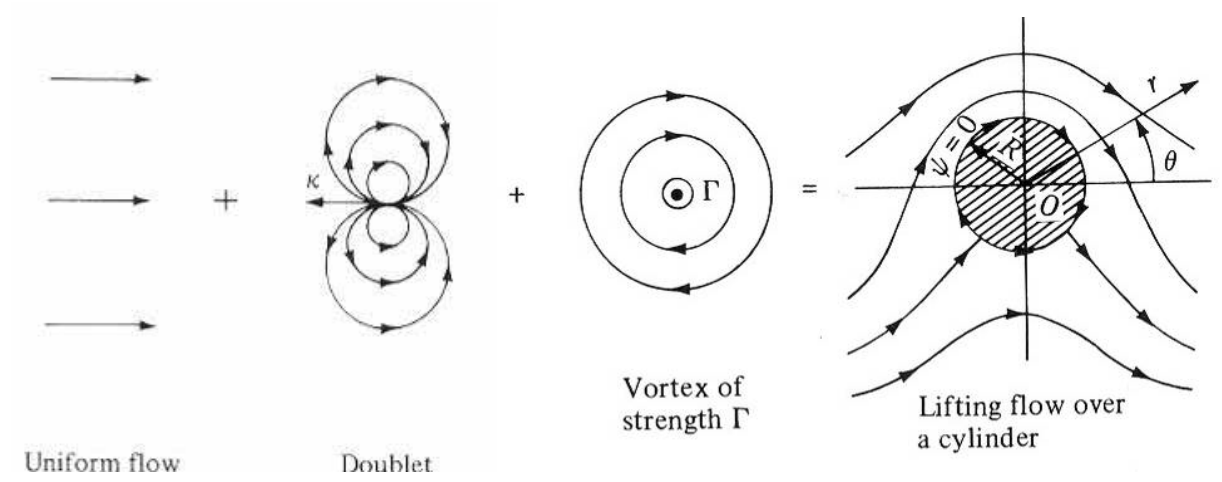

Figure 3. Mathematical representation of the flow around a spinning cylinder [10].

\section{Airfoil}

In flight, the flow around a wing generates mainly an inviscid flow region separated from the airfoil by a thin viscous region called the boundary layer (BL). Due to the lack of shear stress in the inviscid region, the fluid elements there have no angular velocity and their motion is purely irrotational [10]. Contrarily, the velocity gradient inside the boundary layer generates vorticity or rotational flow of the fluid elements.

In an irrotational flow field, the vorticity must be zero at every point within the flow. In such analysis the circulation $\Gamma$ within the viscous boundary layer is moved inside the boundary for analysis.

Photographs of the flow around an airfoil show that the flow smoothly leaves the top and the bottom surfaces of the airfoil at the trailing edge (Figure 4) [10]. The static pressure at the trailing edge has a unique value, and applying the Bernoulli equation at both the top and bottom surfaces yields that the velocities leaving the top and bottom surfaces are finite and equal in magnitude and direction if the trailing edge angle is cusped (Figure 5). If the trailing edge angle is finite, then the trailing edge is a stagnation point and the trailing edge velocity must reduce to zero. The boundary layer vorticity at the trailing edge of the top and bottom surfaces are of equal and opposite magnitude. Only if the upper and lower velocities are aligned, when reaching the trailing edge, will the associated boundary layer vorticity cancel each other. Then the net vorticity shed from the trailing edge goes to zero. 


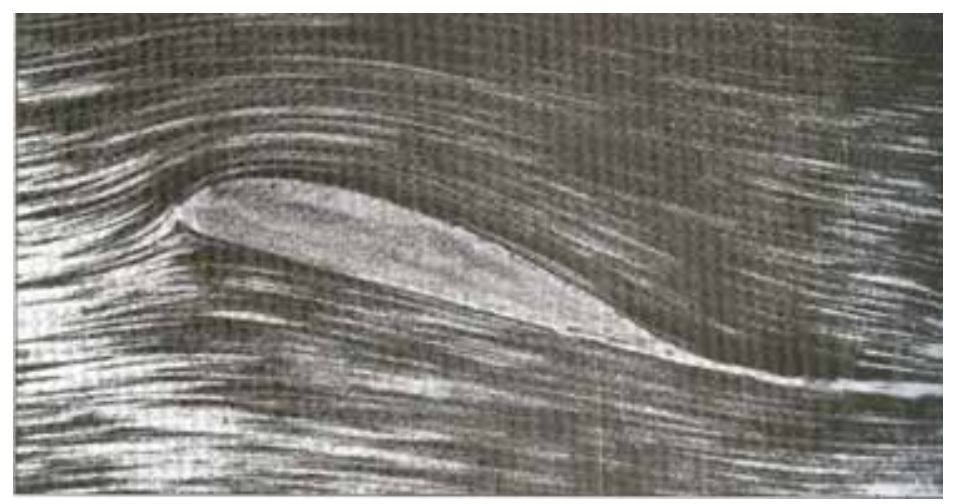

Figure 4. Streamlines leaving smoothly at the trailing edge [10].

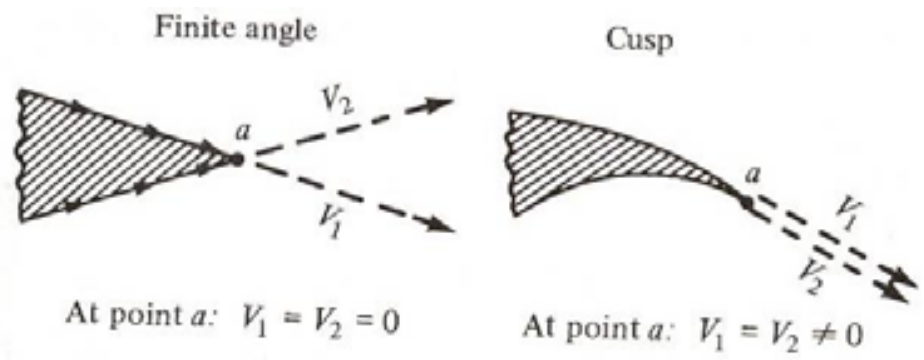

Figure 5. Velocities at the trailing edge for finite angle and cusped TE [10].

The flow over an airfoil is synthesized by distributing vortices either on the surface or inside the airfoil [10]. The contour integral of these vortex strengths $\gamma$, equals the circulation $\Gamma$ which is adjusted to satisfy the Kutta condition.

\section{Finite Wing}

Prandtl's theory of lift establishes a vortex system which consists of: the bound vortex system, a trailing vortex system, and a starting vortex (Figure 6) [11]. The bound vortex is a vortex filament of strength $\Gamma$ (the circulation) that is bound to a fixed location and will experience a force $\mathrm{L}^{\prime}=\rho_{\infty} \mathrm{V}_{\infty} \Gamma$ by the Kutta-Joukowski theorem [10]. An infinite wing has perfect alignment between the upper and lower surface trailing edge velocities together with equal magnitude in pressure (p), velocity (V), and local vortex strength $(\gamma)$; but $\gamma$ opposite in direction. Therefore, $\gamma_{\text {upper }}$ and $\gamma_{\text {lower }}$ cancel one another and there is no vorticity shed into the wake. Then the induced upwash and downwash velocities can be calculated by the Biot-Savart law (Figure 7). The flow is identical for each spanwise station. Therefore, the pressure differences between the lower surface and the upper surface of the wing, and circulation and the lift per unit span do not vary along the span [11]. 


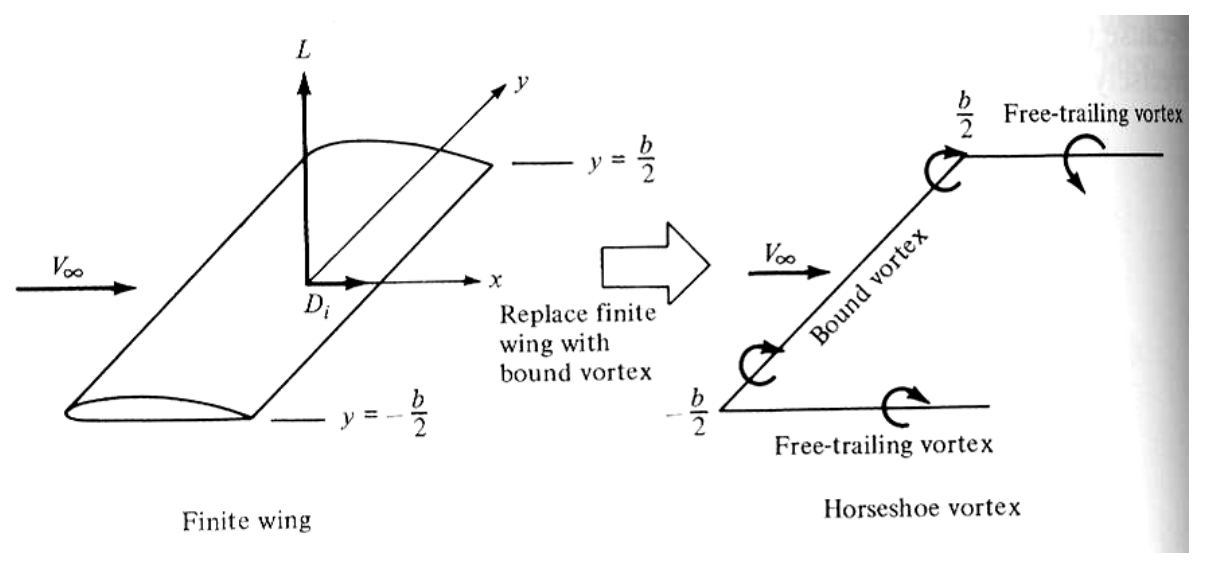

Figure 6. Prandtl's classical lifting-line theory [10].

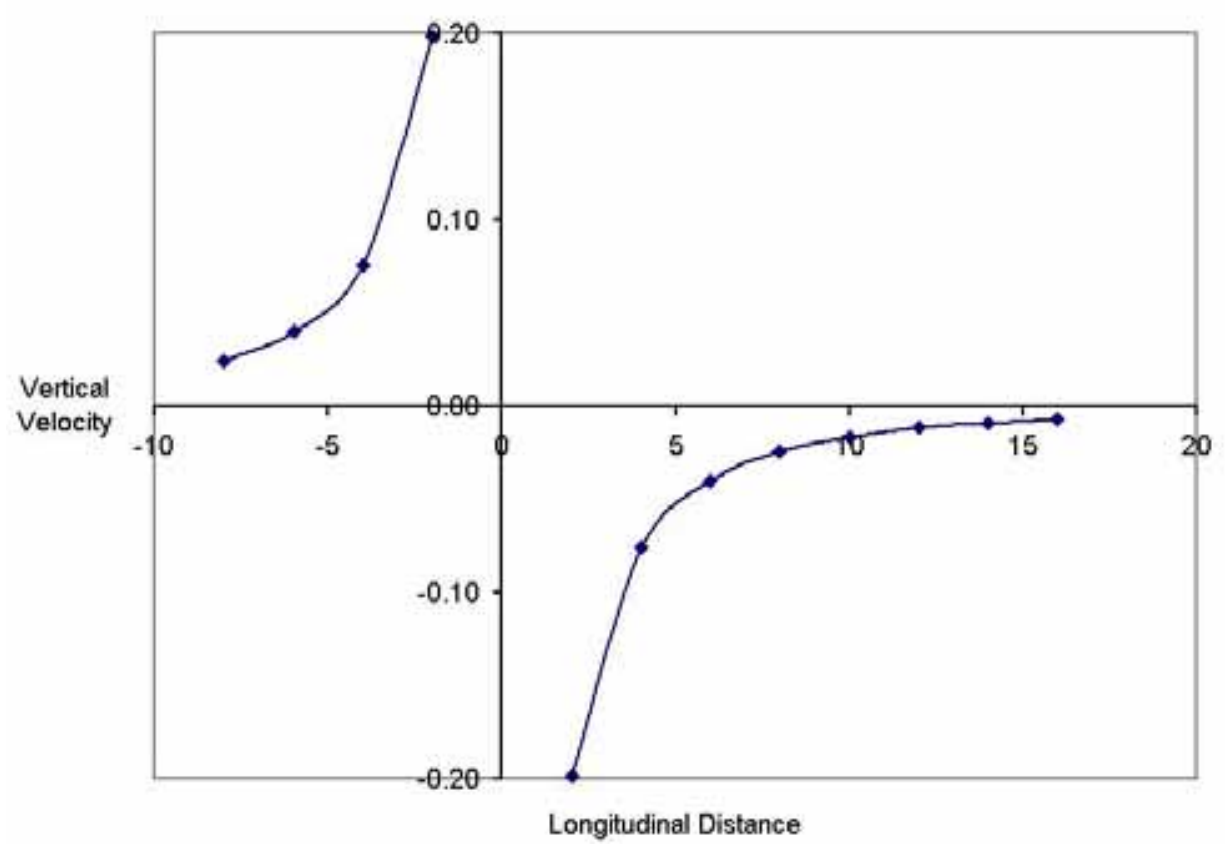

Figure 7. Biot-Savart law applied to a 2D wing.

In a finite wing, there is an opportunity for the pressures acting on the upper and lower surfaces to interact near the wing tip (Figure 8) [11]. The high-pressure air beneath the wing accelerates around the wing tips toward the low-pressure region above the wing, resulting in the initiation of two wing tip vortices. As a result, the spanwise pressure distribution decreases towards each tip, and likewise the lift (Figure 9). This spanwise pressure gradient causes inboard flow along the upper wing surface and outboard flow along the lower surface of the wing. The resulting spanwise flow component at the trailing edge prevents the boundary layer vorticities from canceling one another at the trailing edge. Misalignment between the boundary layer velocity and thus vorticity 
vector, causes shedding trailing edge vorticity. This is further downstream entrained into vorticity shed at the wing tips (Figure 10). The shorter the distance between the tip trailing wing tip vortices, the larger the downwash velocity and the induced drag will be [12].

When inviscid flow enters the flow field around the wing without vorticity, then by Helmholtz law it remains irrotational all the way downstream. In the absence of net downwash, then upstream the vertical momentum component upwards contributes to half the lift, while the downwash downstream of the wing contributes to the other half of the lift.

The following Figures are for real fluids, where the boundary layer vorticity induces wake vorticity downwash and induced drag.

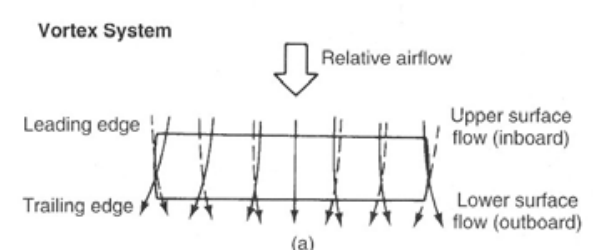

(a)

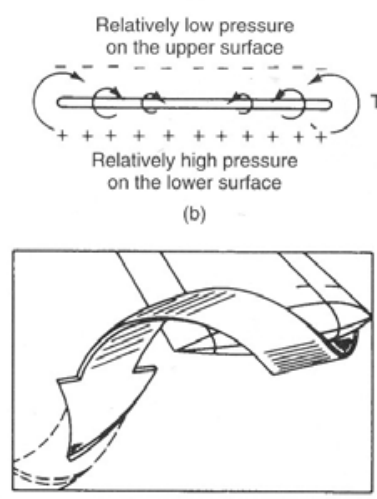

(c)

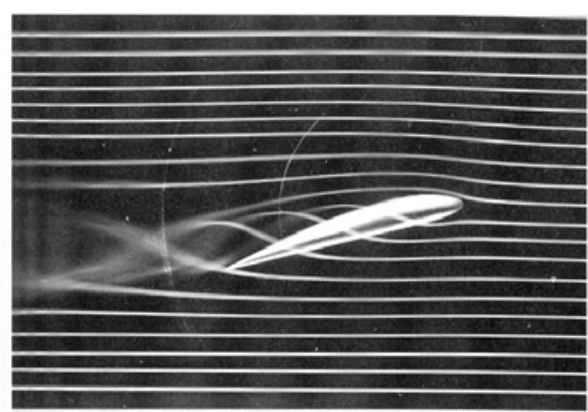

(d)

Figure 8. Pressure equalization on the wing tip and curvature of the streamlines [11]. 


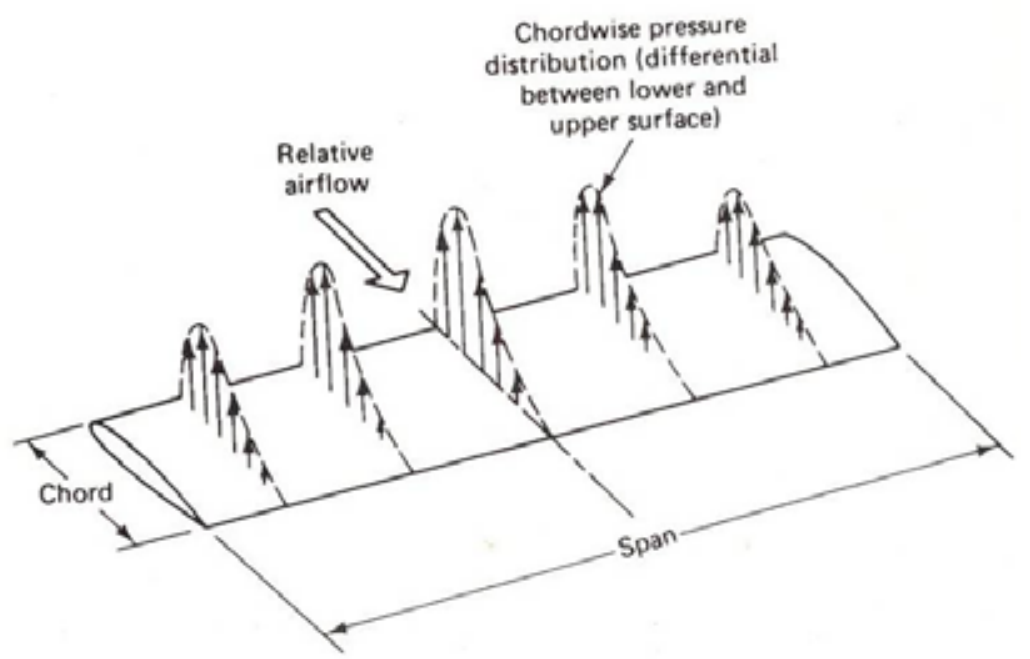

(a)

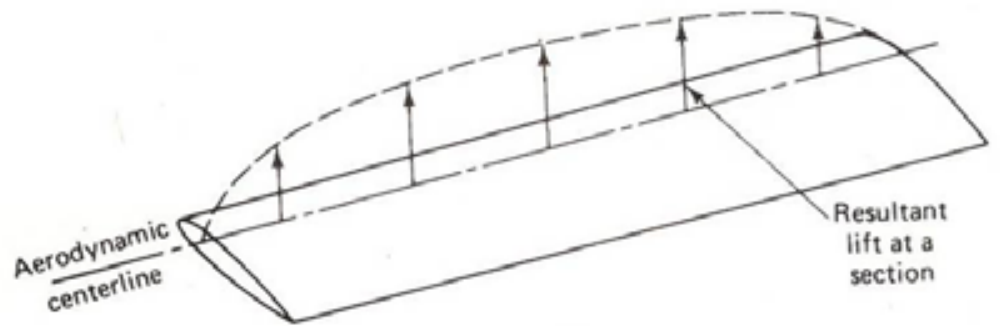

(b)

Figure 9. Pressure and lift decreasing toward the tip [11].

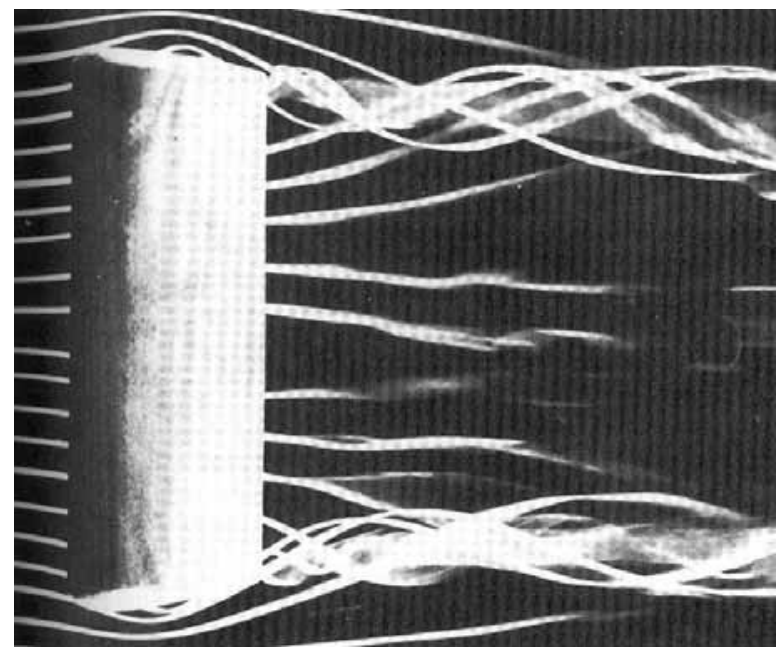

Figure 10. Trailing edge vortices shed behind a wing [10].

The trailing vortex system also generates an upwash in the regions beyond the wing span and a downwash inside the wing span (Figure 11). This downwash produced by the trailing vortex system adds to the downwash produced by the bound vortex system 
(Figure 12). The upwash outside the wing span is often taken advantage of by birds when they fly in V-formation. The wake shed vorticity is very stable and can last up to 5 miles downstream. It rotates the lift vector aft, thereby generating induced drag [10].

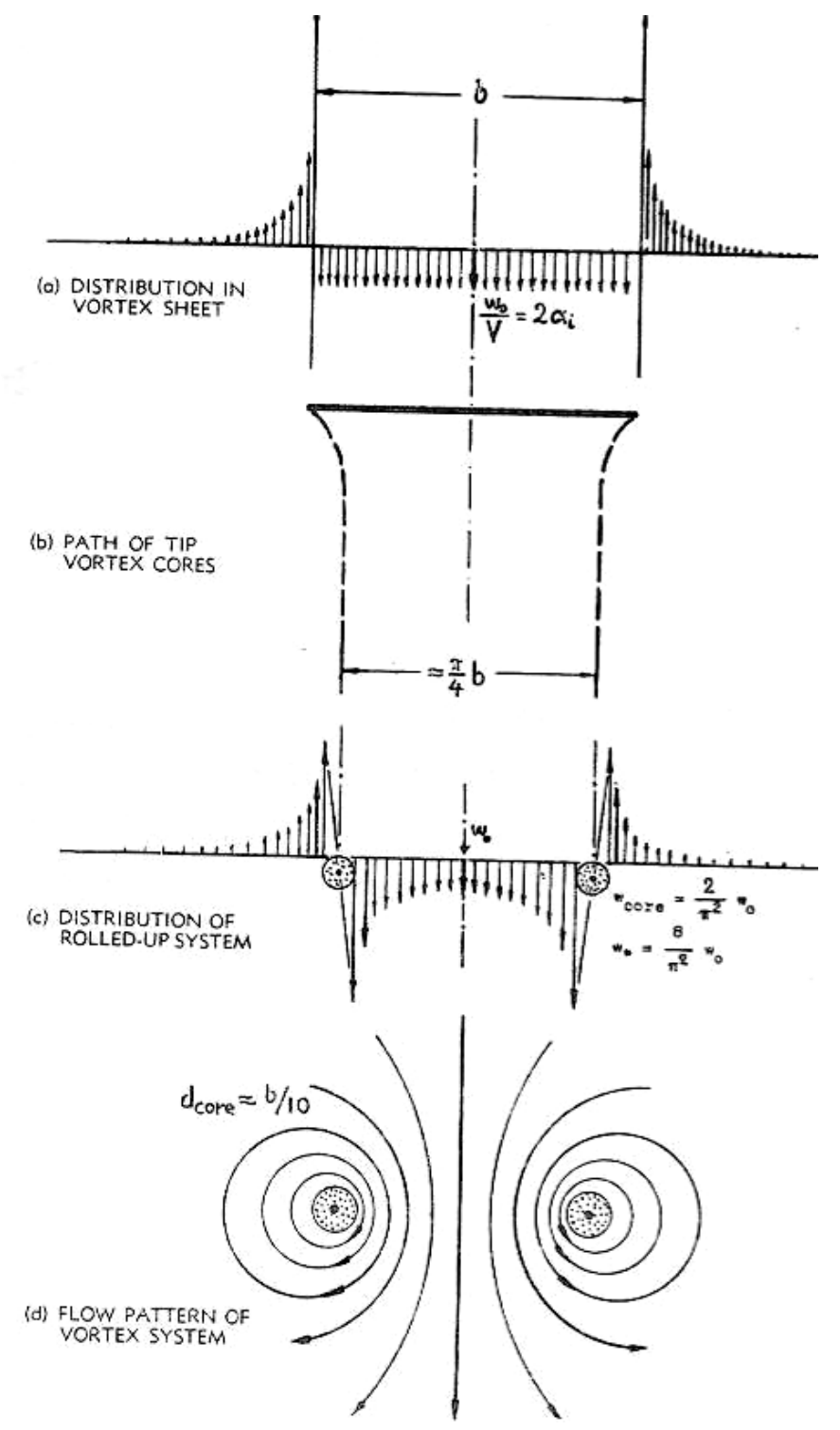

Figure 11. Upwash and downwash behind a wing [13]. 


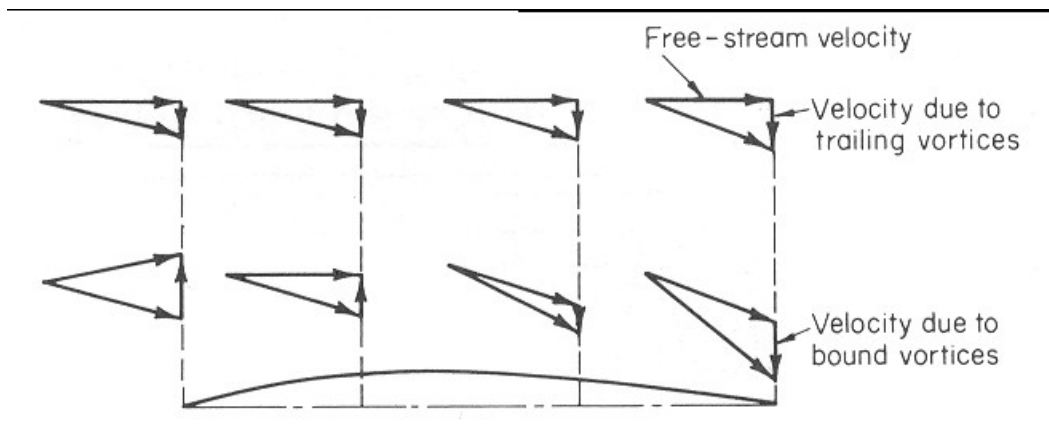

Figure 12. Downwash due to bound and trailing vortices [13].

\section{Helmholtz's Laws}

Helmholtz was the first to make use of the vortex filament concept in the analysis of fluid flow. He established several principles of vortex behavior which are limited to inviscid flow:

1. The strength of a vortex filament is constant along its length.

2. A vortex filament cannot end in a fluid: it must extend to the boundaries of the fluid, form a closed path, or terminate at a solid boundary $[10,14]$.

3. In the absence of rotational external forces, a fluid that is initially irrotational remains irrotational. Vortices are preserved with time. Only through the action of viscosity or other mechanism (detrainment) can they decay or disappear [10].

Munk at NASA has derived the requirement for minimum induced drag based on Helmholtz criteria for a wing inside an inviscid flow field and with the Kutta condition satisfied at the trailing edge, which for an elliptic loading planar wing is:

$$
C_{D i}=\frac{C_{L}^{2}}{\pi \cdot A R}
$$

To find $\mathrm{C}_{\mathrm{Di}}$ for the other wing a span loading efficiency factor $e$ is introduced to

$$
C_{D i}=\frac{C_{L}^{2}}{\pi \cdot e \cdot A R}
$$

The wing wake trailing vorticity satisfies Helmholtz's law, if viscous diffusion of vorticity is neglected, the fluid is barotropic, and external forces are conservative [15]. 
This wing theory is contradictory as wing circulation can only be generated by a viscous boundary layer. This limiting theory to inviscid flow makes it impossible to generate circulation and lift! This dissertation follows a different approach, where air is recognized as a viscous fluid and the Helmholtz criteria do not apply into a viscous domain.

It is well known that large scale vorticity, such as created in a centrifugal fan to power a wind tunnel, can be dissipated by passing the flow through multiple screens. This method of vorticity dissipation by separation in numerous separate vortex streams, goes at the expense of pressure loss.

\section{Induced Drag}

\section{Overview}

In cruise, induced drag can account for $33 \%$ of the total drag of the aircraft (Figure 13) [16]. This drag is even more significant at low speed, during take off conditions, where it can account for $80-90 \%$ of the aircraft drag [17]. The engine-out climb is often a critical constraint in the aircraft design [17].

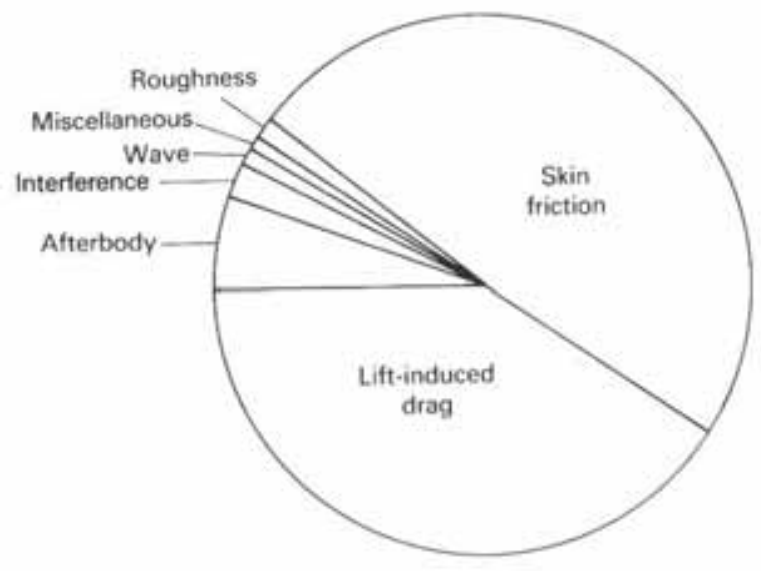

Figure 13. Drag distribution on an aircraft [11].

\section{Induced Drag Minimization}

The most basic way to decrease induced drag is by increasing the wing aspect ratio. The minimum induced drag for planar wings is achieved for an elliptical lift distribution across the span which produces a constant wing downwash according to Munk's theory [1]. New techniques for its reduction include winglets, vortex diffuser vanes, wingtip sails, and wingtip blowing. 


\section{Increasing the Aspect Ratio}

Increasing the aspect ratio of the aircraft has been the most known way to reduce induced drag. Large aspect ratio wings enable the tip vortex structures to be separated which reduces the strength of the induced flow between them [1]. However, for the same wing area, increasing the aspect ratio increases structural weight and decreases volume for fuel storage in an aircraft [16]. The increment in weight can offset the induced drag reduction [1]. A $10 \%$ increase in span causes a $17 \%$ reduction in induced drag at fixed speed and lift but increases the structural weight [17]. The selection of optimal aspect ratio is intimately tied to criteria used to define aircraft geometry during the design [1].

\section{Non-Planar Wings}

Non planar wings offer the possibility of greater reduction in drag compared with planar wings of the same span and lift $[17,18]$. Non planar configurations include biplanes, box planes, ring wings, joined wings and winglets. These wings reduce induced drag because each wing produces about half of the total lift so that the lift coefficient for each wing is half the lift coefficient for a monoplane wing. The induced drag coefficient is proportional to the square of the lift coefficient, thus the total induced drag coefficient for a biplane is half of the induced drag coefficient for a monoplane.

Span efficiency is defined as the ratio of the induced drag of an elliptically loaded planar wing to the induced drag of a non planar system of same span and lift. Figure 14 shows the span efficiency for various optimally loaded non planar wings with a ratio of height/span of 0.2 [17]. 


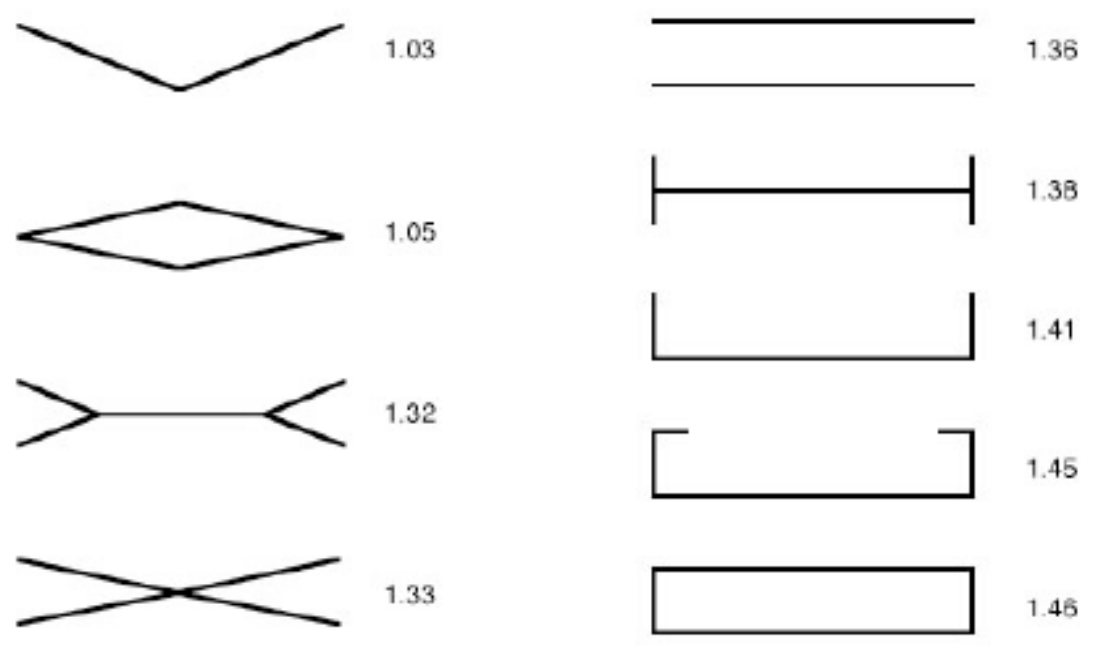

Figure 14. Span efficiency for non planar wings $(h / b=0.2)[17]$.

\section{Tip devices}

The addition of tip mounted surfaces to a wing has long been recognized as a way to reduce and diffuse the vortex structures arising from the tips [1]. Tip devices discharge trailing vorticity as a multiple vortex system, which dissipates quickly and therefore induces less downwash and less drag. Induced drag reduction may be offset by unfavorable interferences and viscous effects [1]. Adding a tip surface such as a winglet, increases nose down pitching moment, wing root bending moment, and skin friction; thereby increasing trim drag and interference drag from the junction between the winglet and the main wing [16]. But the net result is that tip devices are able to improve the lift/drag ratio in high lift configurations (second segment climb gradient) [16].

\section{End Plates}

Several authors have investigated the use of end plates to decrease induced drag. The first patent for vertical surfaces at wing tips was proposed by Lanchaster at 1897 [18]. An infinitely large end plate will make the flow two dimensional and theoretically eliminate vortex induced drag, but in practice, this drag reduction is offset by viscous drag on the increased wetted area [18]. Added to this is drag due to flow separation at the wing junction [16]. Therefore, the design of an efficient junction is very important if a net improvement in drag is to be realized [16]. 
End plates produce the same effect as increasing the span by $4 / 5$ of the total endplate height, but they are directionally destabilizing and the diameter for circular end plates should be equal to the wing chord to be effective [12].

\section{Winglets}

Small wings added to the wing tip almost vertically (Figure 15), called winglets, are the most promising of the tip mounted surfaces and can be considered as a device to increase the effective span of the wing [1]. Much of the development work for these devices was initiated by Whitcomb at NASA $[1,8,18]$. These winglets can be added to existing aircraft without major redesign [16]. Adding such surfaces to a wing can reduce and diffuse the vortex structure which originates at the tips [1, 16, 18]. The lift on the winglet acts inward towards the fuselage if properly designed. It also produces a thrust component in the upstream direction (Figure 15). The increase in vorticity present in the wing tip provides a highly angular flow field that permits the winglet to develop greater thrust [16]. These side forces counter the lift-induced inflow over the wing upper surface and the outflow over its lower surface [18]. Total drag reductions of up to $6 \%$ have been demonstrated [1].

Winglets develop a normal force that alters the span load configuration to diffuse the total circulation in the rolled-up wingtip vortex and reduce the total energy of the vortex. The basic physical effect of the winglets, which leads to drag reduction, is a vertical diffusion of the wing tip vortex flow which substantially reduces the magnitudes of the crossflow behind the tip [19].

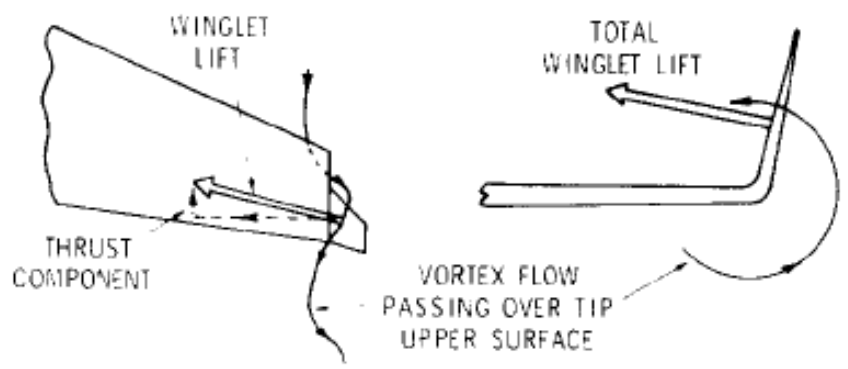

Figure 15. Winglet thrust production mechanism [16]. 
Some of the drag reductions are offset by unfavorable viscous effects and interference at the junction. Also, there will be an increase in wing root bending moment due to both the increased wing loading and the load on the winglet [1]. For moderate size winglets, an improvement in induced drag can be achieved with less root bending moment than by span extension [16]. If the winglet produced thrust component is above the center of gravity (CG), then it can produce a nose down pitching moment which leads to a trim drag penalty [1]. Winglets can not provide improved performance over all flight phases [20].

Proper design of the winglets is very important to obtain the best performance. The winglet should be tapered and swept aft for good supercritical performance. By mounting it behind the lowest pressure point on the wing and by canting it outward, the interference effects are minimized [1]. Some toe-out of the winglet is desirable since it reduces the likelihood of winglet stall during sideslip [1]. Drag reduction increases with winglet span, and also bending moments due to larger moment arms.

\section{Vortex Diffuser Vanes}

Such devices were designed at Lockheed-Georgia, in an attempt to extract energy from the available rotational kinetic energy inside the tip vortices (Figure 16). They are mounted in a region of intense vortex flow, where the flow total pressure is reduced by viscosity. Therefore, it is not possible to extract as much energy as from a uniform flow field [21]. However some unfavorable wing interference effects can be minimized by mounting the diffuser vanes in the rear [1]. Diffusing the wing tip vortex increases its dissipation; as a result, some measure of induced drag reduction is possible [16]. The total drag reduction achievable is $19 \%$ [1]. The outward canted vortex diffuser vane, the winglet, and tip extension produce comparable drag reductions with similar bending moment increment [16]. 


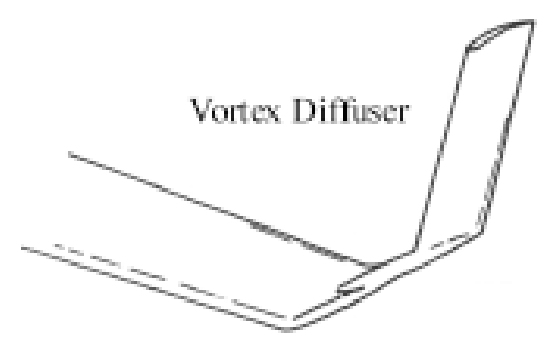

Figure 16. Vortex Diffuser Vane [17].

\section{Wingtip sails}

Wingtip sails are multiple high aspect ratio vanes mounted in a spiral array around the wing tip (Figure 17) [16]. They are similar to the tip feathers of some soaring birds. Birds suppress the tip vortices by the formation of a minor trailing vortex at the tip of each primary feather. The associated downwash, passing through the slot formed by the feather and the one following behind it, opposes leakage of air from the high pressure region below the wing, to the lower pressure upper side. In this case, the slotted tips of birds act to unwind tip vortices, thereby diffusing them [12]. Feathers twist aerodynamically so that each one adjusts itself to an optimum angle of attack. These wing tips generate a forward thrust component, which further reduces drag [12, 22]. Sails have demonstrated to provide the best ratio of drag reduction to root bending moment increase and also the most drag reduction, for a given area increase [16].

Define the angle between the local flow direction and the free stream by $\varphi$. Then when the angle of attack $\alpha$ increases, $\varphi$ also increases which causes flow separation and pressure drag [22]. Tip sails suffer from Reynolds number effects, e.g.: premature flow separation, because the Reynolds number is usually an order of magnitude lower than that of the wing [23]. A cascade of sails is used to reduce the ratio $\varphi / \alpha$ at every point [22]. Flight tests suggest that induced drag can be reduced up to $9 \%$ for a single sail and by up to $29 \%$ with three sails [22]. 


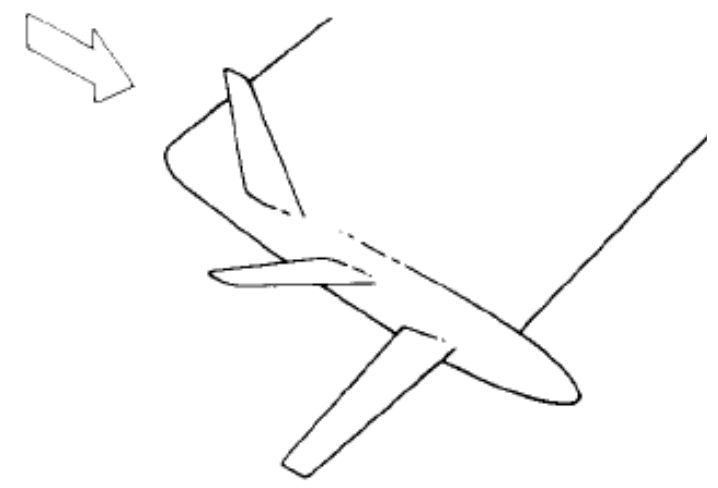

Figure 17. Wingtip Sails [16].

\section{Raked wing tips}

Raked wing tips have been used on the Boeing 767 and they are also going to be employed on the Boeing 787 Dreamliner (Figure 18). Such wing tips reduce leakage from the high pressure region below to the low pressure region above the wing [12].

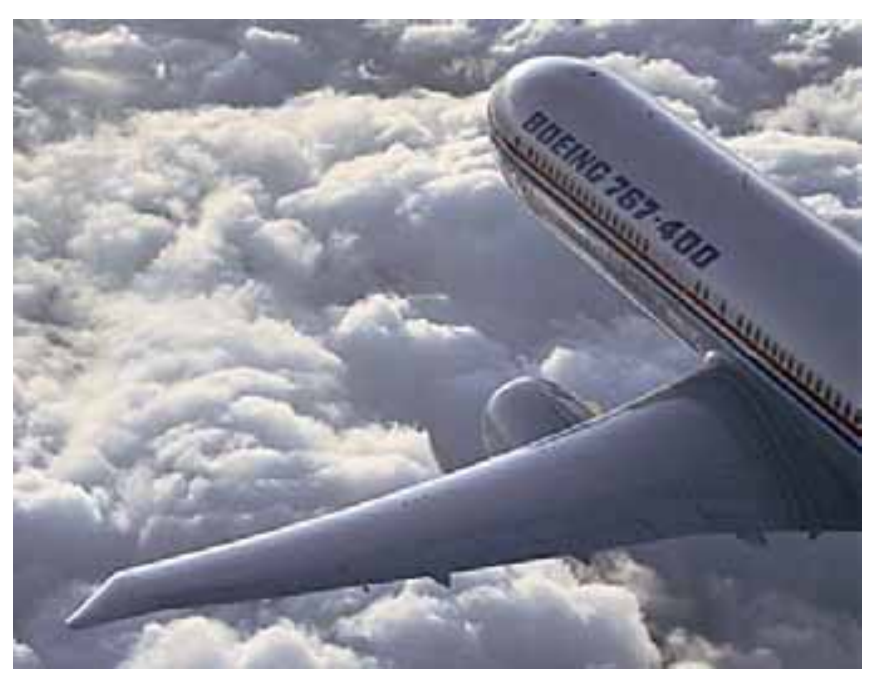

Figure 18. Rake wingtip on a Boeing 767 [24].

\section{Ogee tips}

Ogee tips show reduction in peak tangential velocity (Figure 19) [25]. Figure 20 shows the effect of Ogee tips on vortex dissipation compared to an equivalent rectangular tip. 


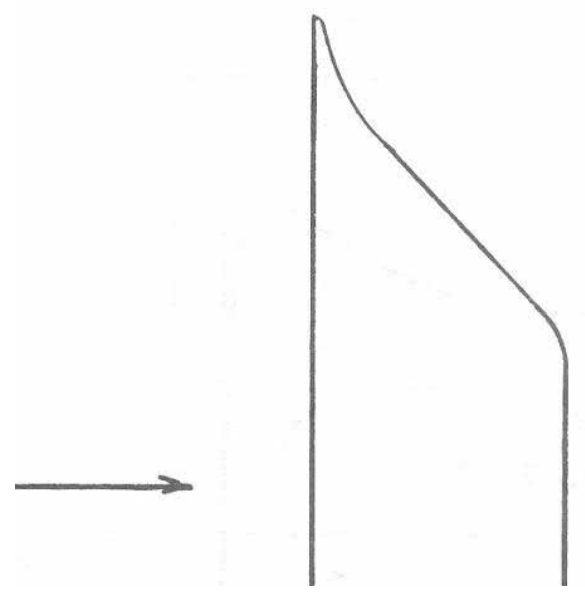

Figure 19. Ogee tip [25].

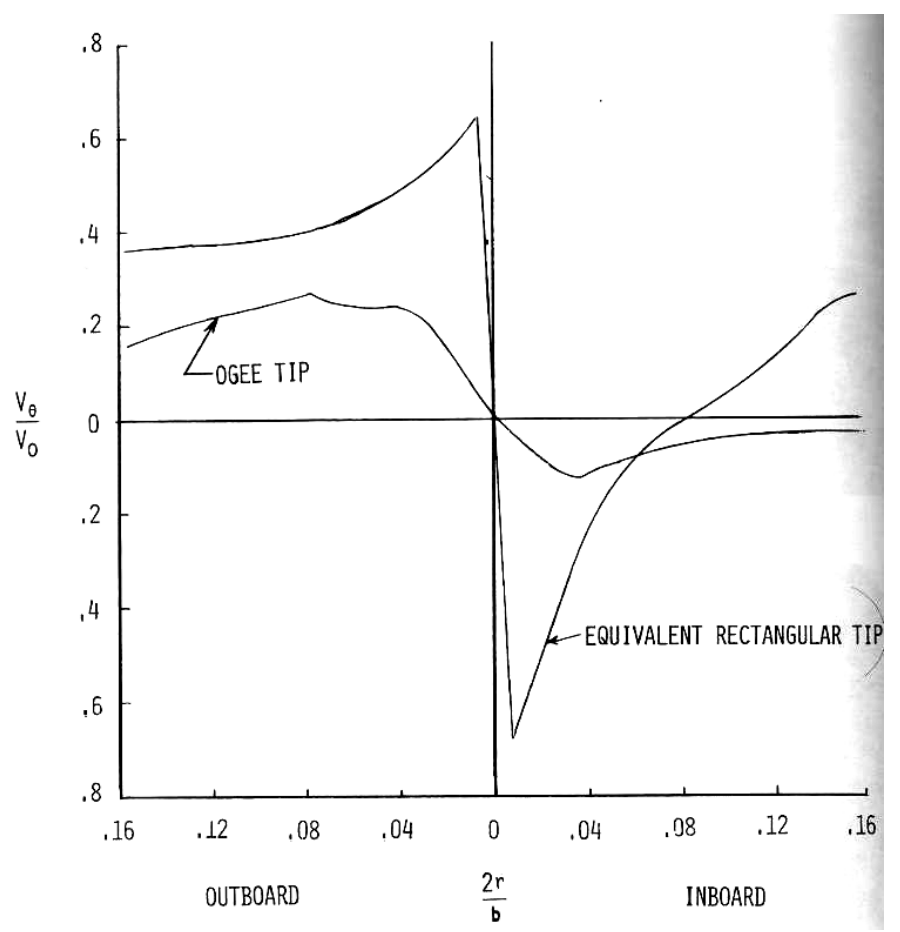

Figure 20. Effect of Ogee tip on vortex dissipation [25].

\section{Crossed blade}

Large fixed crossed blades added downstream of the wing tip, dissipate rotational energy within the trailing vorticity (Figure 21) [25]. Figure 22 shows the effect of crossed blades on the vortex velocity distribution. 


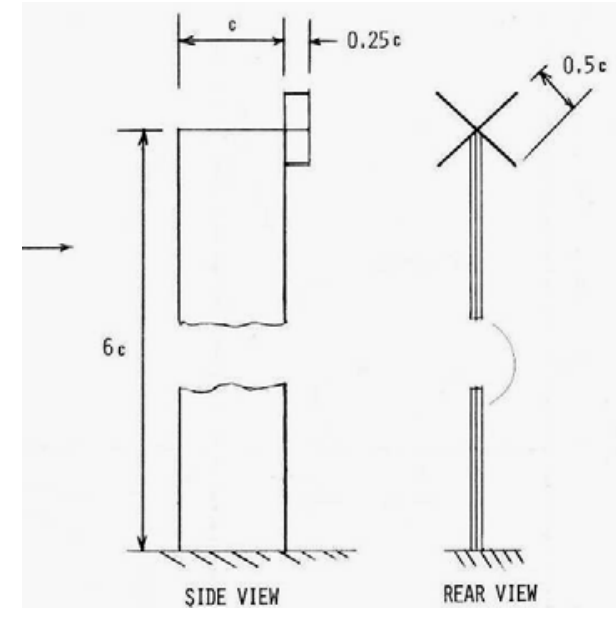

Figure 21. Crossed blades [25].

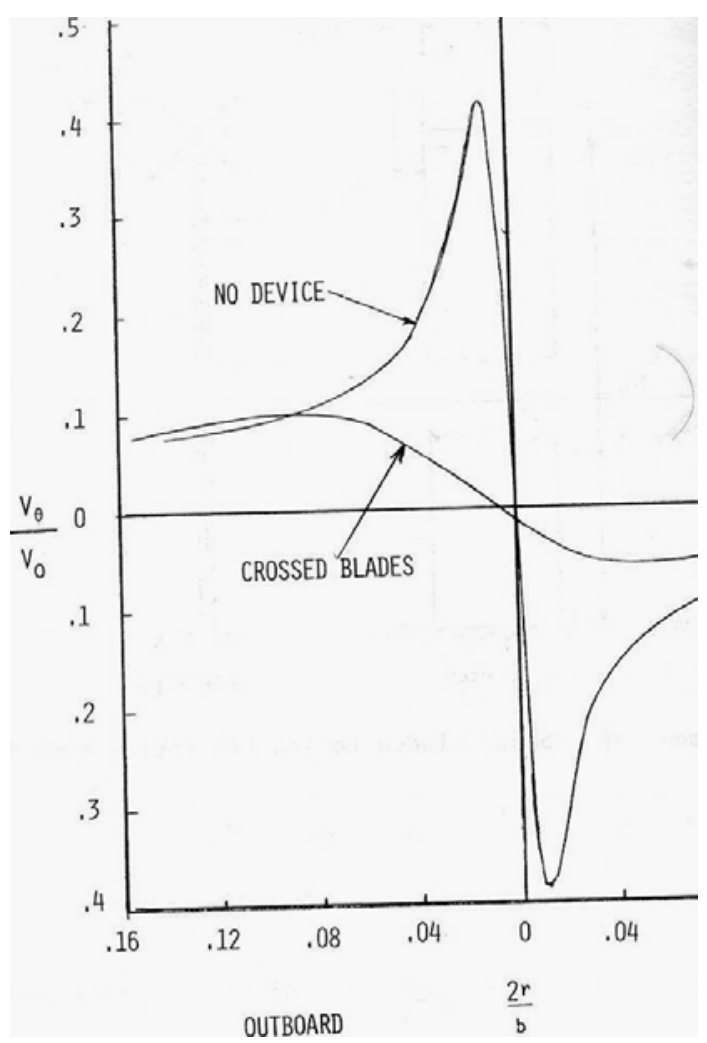

Figure 22. Effect of crossed blades on vortex velocity distribution [25].

\section{Turbulence}

Excess turbulence in the ambient fluid is known to disrupt or prevent the formation of lab vortices [26]. In fact, the injection of turbulence into the vortex has been shown to alter the vortex structure by premature aging and dissipation [25]. The 
combined effects of the turbulence and momentum impulse from jet engines have been shown to change the vortex structure [25].

\section{Boundary Layer Control (BLC)}

\section{Overview}

Boundary layer control is a technique used to prevent flow transition from laminar to turbulent, to energize the flow inside the boundary layer sub-layer, or to prevent separation by suction, air injection and many other techniques. BLC can also be used to counteract the formation of wing tip vorticity.

\section{Previous Applications}

Because of the poorer performance that is obtained from winglets at off-design conditions, an alternative that has been suggested is to use spanwise-blown jets of air to increase the effective span [1]. Improvements in lift/drag ratio (L/D) have been observed by the use of wing tip blowing [20]. The ability to adjust the blowing rate provides the best performance enhancement for any particular flight condition [20]. Some researchers have blown jets of air in the streamwise direction to breakup the tip vortex structure. Blowing air in the direction of the vortex axis significantly reduces its peak tangential velocity [25]. The benefits level off at high flow rates, where the blowing energy requirements override the drag reduction benefits.

Griswold caused the tip vortices to move outboard by blowing, thereby increasing the effective wing span [16].

Placing the engines at the wing tips, in order to use their exhaust velocity to breakup the shed wing tip vorticity has provided drag reduction on the order of one-third (Figure 23) [18]. The tip turbines are found to yield the largest drag reduction [28]. In this case a portion of the wing tip vortex energy is dissipated by entrainment into the nonrotating high-energy engine exhaust plume resulting in a reduction in downwash behind the wing and its associated induced drag [27]. This application may cause flutter and yawing moments upon engine failure, or other structural problems [18]. 


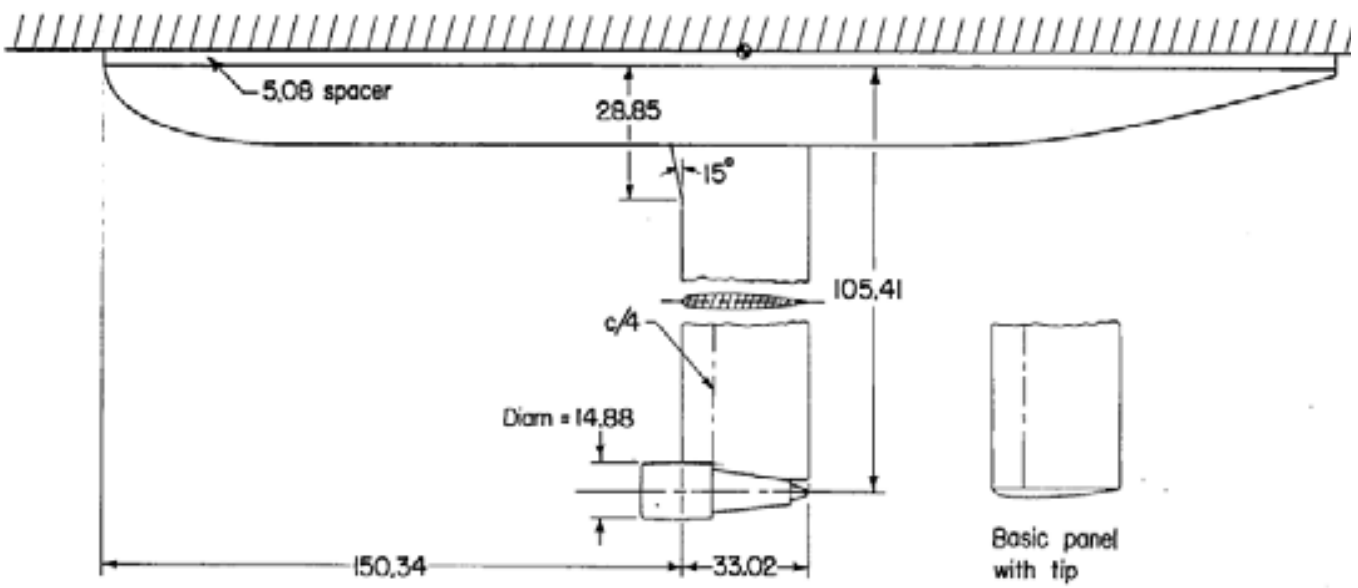

Figure 23. Model used to test the effect of placing the engine at the tip [27].

The use of BLC by blowing can increase the maximum L/D ratio, the span efficiency factor $e$ and the lift curve slope [29]. Spanwise tip blowing increases the effective span (Figure 24) [29]. Significant improvements in the wing L/D produced could be obtained by spanwise blowing (Figure 25) [25].

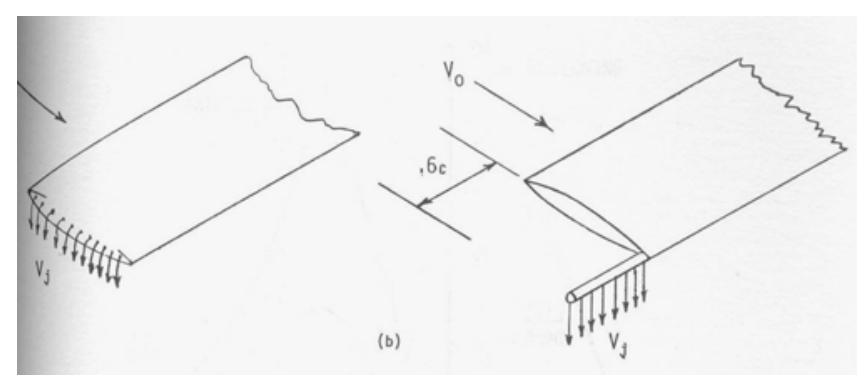

Figure 24. Techniques used to blow air downward: a) chordwise, b) spanwise [25].

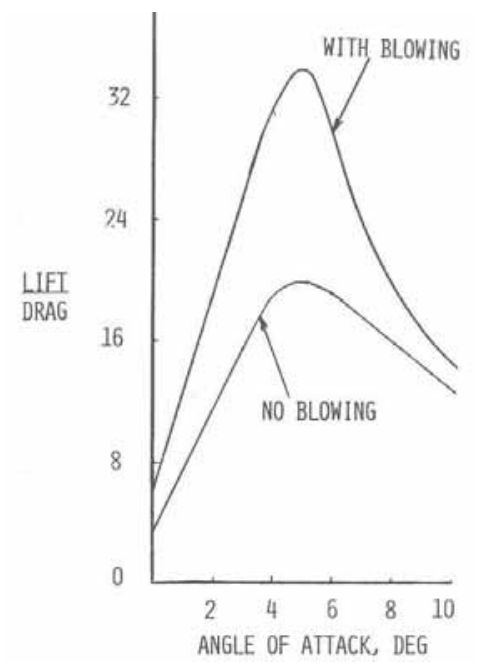

Figure 25. Change in L/D ratio with spanwise blowing [25]. 


\section{Wingtip Vortex}

Several numerical studies and experimental measurements have been conducted on wing tip vorticity. In the next sections, a review is presented of such studies.

\section{Wingtip Vortex Numerical Studies}

This literature review comprises the published numerical studies on wing tip vortex simulation. Some of these studies are based on Laplace's equation, some on Euler's equation, and the remaining one utilized the Navier Stokes equation. Laplace equations assume inviscid, irrotational, and incompressible flow and are used in techniques such as Panel methods, and Vortex Lattice Methods with special attention to control point locations. In order to get a better understanding of the formation of the tip vortex, a mathematical model is needed in which the appearance of the tip vortex is a result of the solution rather than an empirical input to the solution [30]. There are two popular simplifications to the Navier Stokes equations: the Parabolized Navier Stokes (PNS) model, and the Thin-Layer Navier Stokes (TLNS) model [31]. The simplest model at high Reynolds number seems to be the thin-layer Navier Stokes model [32]. Mansour [30] did a numerical study of the wing tip vortex, using thin-layer Navier Stokes equations for low aspect ratio swept wings at Mach 0.8. The turbulence viscosity model he used was the Two-Layer Baldwin-Lomax model. Srinavasan et al. [33] also solved the thin-layer Navier Stokes equations with a Baldwin-Lomax turbulence model, but they tried different wings and wing tips. Kramer et al. [34] solved the Euler equations for subsonic and transonic flow near the tip of a helicopter blade. Euler equations assume steady, inviscid flow with no body forces [10]. Since there is no viscosity, the mechanism for vortex formation was provided by numerical dissipation. Their results showed good agreement with experimental surface pressure distributions but they were not compared with experimentally measured tip vortex structure. Strawn [35] also solved the Euler equations on a NACA 0015 airfoil using an unstructured adaptive grid solver. These results were compared with experiments done by McAlister and Takahashi [36], showing good agreement inside the vortex core diameter up to 10 chords downstream but poor agreement on vortex peak swirl velocities. 
Full Navier Stokes equations were solved using the INS3D-UP code by DaclesMariani et al. [37] on a NACA 0012 airfoil. The convective term was modeled with fifth order differencing. The turbulence model used was the Baldwin-Barth [38] model with the production term modified to suppress the eddy viscosity in the vortex core. They concluded further improvements in the results could be obtained by incorporating a turbulence model of greater sophistication. In 1996, Dacles-Mariani et al [37] used a One-Equation Spalart-Allmaras [39] turbulence model, also modified in the production term. Surface pressures and streaklines were in good agreement with experiments but only within one chord length downstream, where the evolution of the vortex is incomplete. Hsiao and Pauley [31] solved the full Navier Stokes equation using the INS3D-UP code and the Baldwin-Barth [32] turbulence model but on a NACA 0015 hydrofoil. Their results were compared to the McAlister and Takahashi [36] experimental results at a Reynolds number of $1.5 \times 10^{6}$ and at an angle of attack of $12^{\circ}$. The simulation closely captured the initial rollup of the wing tip vortex, but the dissipative nature of the turbulence model caused a rapid decay of the vortex so a higher order turbulence model would be required to accurately account for the evolution of the vortex.

Higher order schemes based on Euler equations for studying the evolution of tip vortex on a NACA 0012 were used by Lockard and Morris [40]. A comparison to experimental measurements done by Devenport et al. [41] were done at distances up to nine chords downstream. Even with higher order schemes, effects of numerical dissipation were found to be significant on finest $\mathrm{H}-\mathrm{H}$ grids. Spall [42] employed a second order accurate pressure-based finite volume algorithm and well-designed computational grids to solve the Euler equations over a NACA 0012 rectangular wing of $\mathrm{AR}=8.6$. Comparisons with experimental data showed that the vortex strength and core radius were well preserved at a distance of ten chords downstream.

The choice between solving Euler equations and Navier-Stokes equations has been based on the computational power available to this research project. A multiblock zonal algorithm such as the one used by Srinivasan et al. [33] can be used to decrease the computational power requirements. This multiblock zonal algorithm uses the thin-layer Navier Stokes equation for the boundary layer, the wake and the wing tip vortex; and Euler equations for the rest of the flow domain. 


\section{Experimental Measurements in the Wingtip Vortex}

Chow et al [43], and McAlister and Takahashi [36] have obtained surface pressure measurements on the wing surface, and pressure and velocity measurements inside the flow field. McAlister used three NACA 0015 wings with AR of 6.6 and chord lengths of 12, 16.2, and 20.4 in. Pressure measurements at 320 locations on the upper and lower surface were realized. A two component laser velocimeter was used to measure the velocity across the vortex trailing up to $156 \mathrm{in}$. downstream. The experiments were done on the NASA $7 \times 10 \mathrm{ft}$ subsonic wind tunnel at Reynolds numbers between $1 \times 10^{6}$ and $3 \times 10^{6}$. No turbulence measurements were obtained. Chow et al. made turbulence measurements in the near field wing tip vortex of a rectangular NACA 0012 wing with rounded wingtip. The model had the following dimensions: $4 \mathrm{ft}$ chord and $3 \mathrm{ft}$. semispan. The experiments were done in a $32 \times 48$ in. low speed wind tunnel at Reynolds number $4.6 \times 10^{6}$. Velocity field measurements were obtained with a seven hole pressure probe, two-point single wire, and turbulence measurements with a three-wire probe. The model had 222 static pressure taps to record the surface pressure. The measurements done by Chow et al. are going to be used to compare the numerical results because of their turbulence measurements. Recently several new computational studies have used these measurements as a standard for comparison: e.g., Dacles-Mariani et al. [37], and Kim and Rhee [44]. 


\section{Chapter 3: Wingtip Vortex Formation}

High pressure air on the bottom surface of the lift producing wing accelerates toward the low pressure region on the top surface of the wing near the wingtip. Computed pressure coefficient contours in cross-flow planes at various locations in the downstream direction are shown in Figures 26 to 39 to show the wingtip vortex formation. These Figures were obtained from the simulations done by Kim and Rhee on a NACA 0012 wing with $\mathrm{AR}=1.5$ at $170 \mathrm{ft} / \mathrm{s}$ and $10^{\circ}$ angle of attack [43]. The Kim and Rhee study agrees very well with the Chow et al. experiments at the NASA wind tunnel [43].

\section{Pressure Coefficient Contours}

The high speed flow from the bottom surface to the top surface creates a low pressure region at the wingtip on the first quarter portion of the wing (Figure 26). This low pressure region is much lower than the pressure on top of the wing which creates a favorable pressure gradient in the front part but an adverse pressure gradient in the rear part. The adverse pressure gradient slows down the velocities inside the boundary layer. This causes the boundary layer to separate and form a clockwise recirculation region above the wingtip. At $\mathrm{x} / \mathrm{c}=0.542$ measured from the quarter chord (Figure 29), the recirculation zone separates to form a wingtip vortex. This wingtip vortex keeps growing as more and more air is accelerated from the bottom to the upper wing surface. Even after leaving the wing trailing edge, the wingtip vortex continues to grow as spanwise wing vorticity is entrained.

The lowest pressure coefficient in the wingtip vortex core is obtained at $\mathrm{x} / \mathrm{c}=0.864$ (Figure 37). Prior to this position the pressure coefficient increased and after $\mathrm{x} / \mathrm{c}=0.864$, it decreases. This position is $12 \%$ chord downstream of the trailing edge. 
$-1.28 \mathrm{e}-01$

$-1.78 \mathrm{e}-01$

$-2.29 \mathrm{e}-01$

$-2.79 \mathrm{e}-01$

$3.29 \mathrm{e}-01$

3. $79 \mathrm{e}-01$

$-4.29 \mathrm{e}-01$

$-4.80 \mathrm{e}-01$

$-5.30 \mathrm{e}-01$

$-5.80 \mathrm{e}-01$

$-6.30 e-01$

$-6.81 \mathrm{e}-01$

$-7.31 \mathrm{e}-01$

$-7.81 \mathrm{e}-01$

$8.31 \mathrm{e}-01$

$8.82 \mathrm{e}-01$

$-9.32 e-01$

$-9.82 \mathrm{e}-01$

$-1.03 \mathrm{e}+00$

$-1.08 \mathrm{e}+00$

$-1.13 e+00$

$-1.18 \mathrm{e}+00$

$-1.23 \mathrm{e}+0 \mathrm{OQ} \longrightarrow \mathrm{x}$

$-1.28 \mathrm{e}+00$

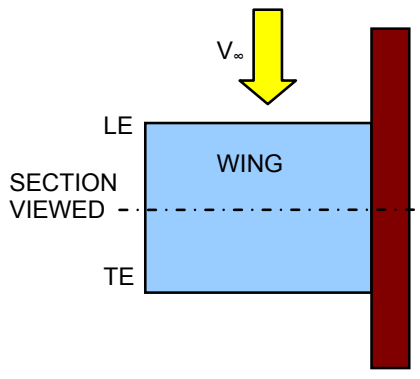

Figure 26. Pressure coefficient contours at $\mathbf{x} / \mathbf{c}=0.25$, looking upstream from back [44].

The low pressure region that was at the wing tip starts to move to the upper wing surface as can be seen in Figure 29.

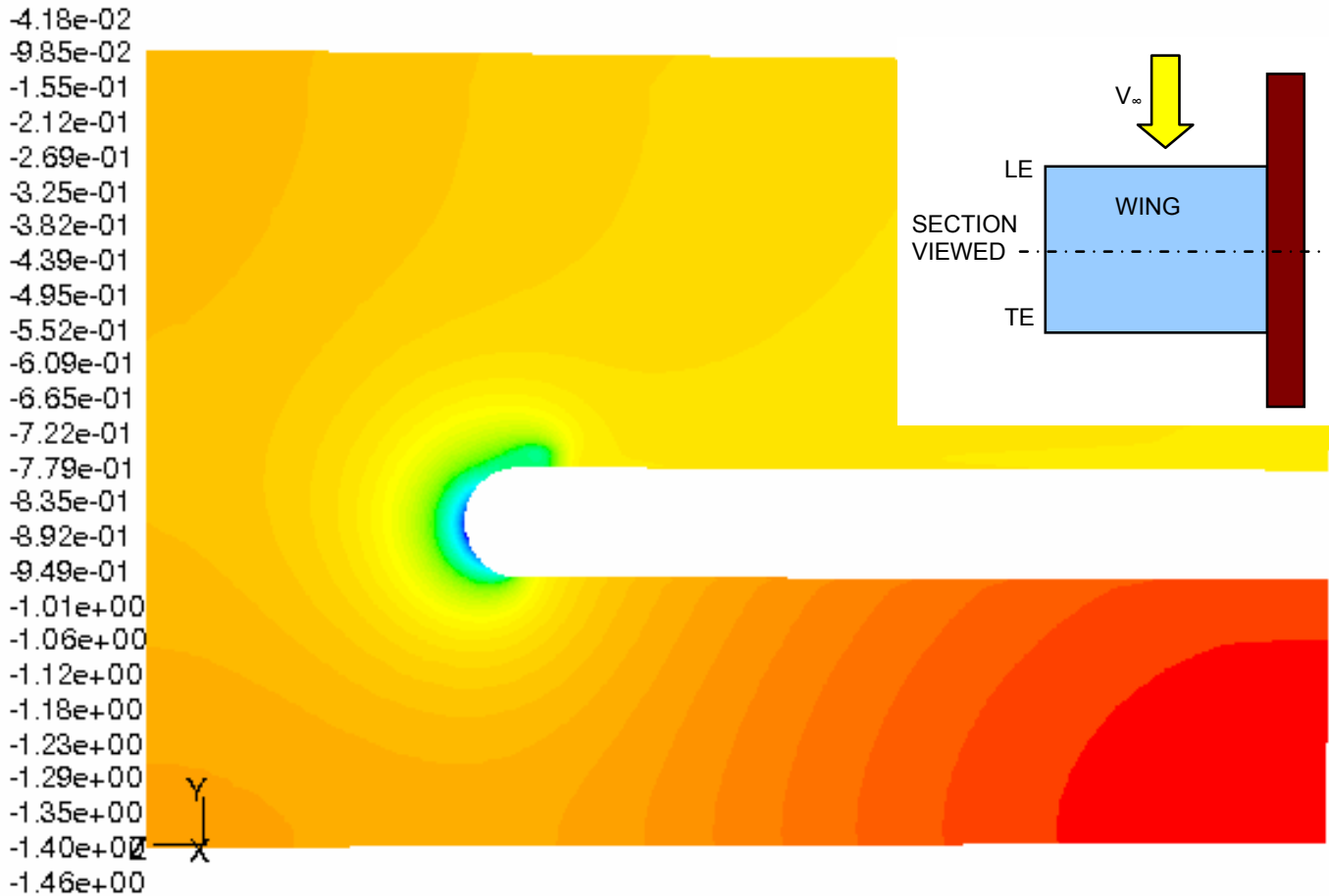

Figure 27. Pressure coefficient contours at $\mathbf{x} / \mathrm{c}=\mathbf{0 . 3 4 5}$, looking upstream from back [44]. 
The low pressure region in light blue in Figure 28 moves toward the wing root separating from the lowest pressure in the wing tip.

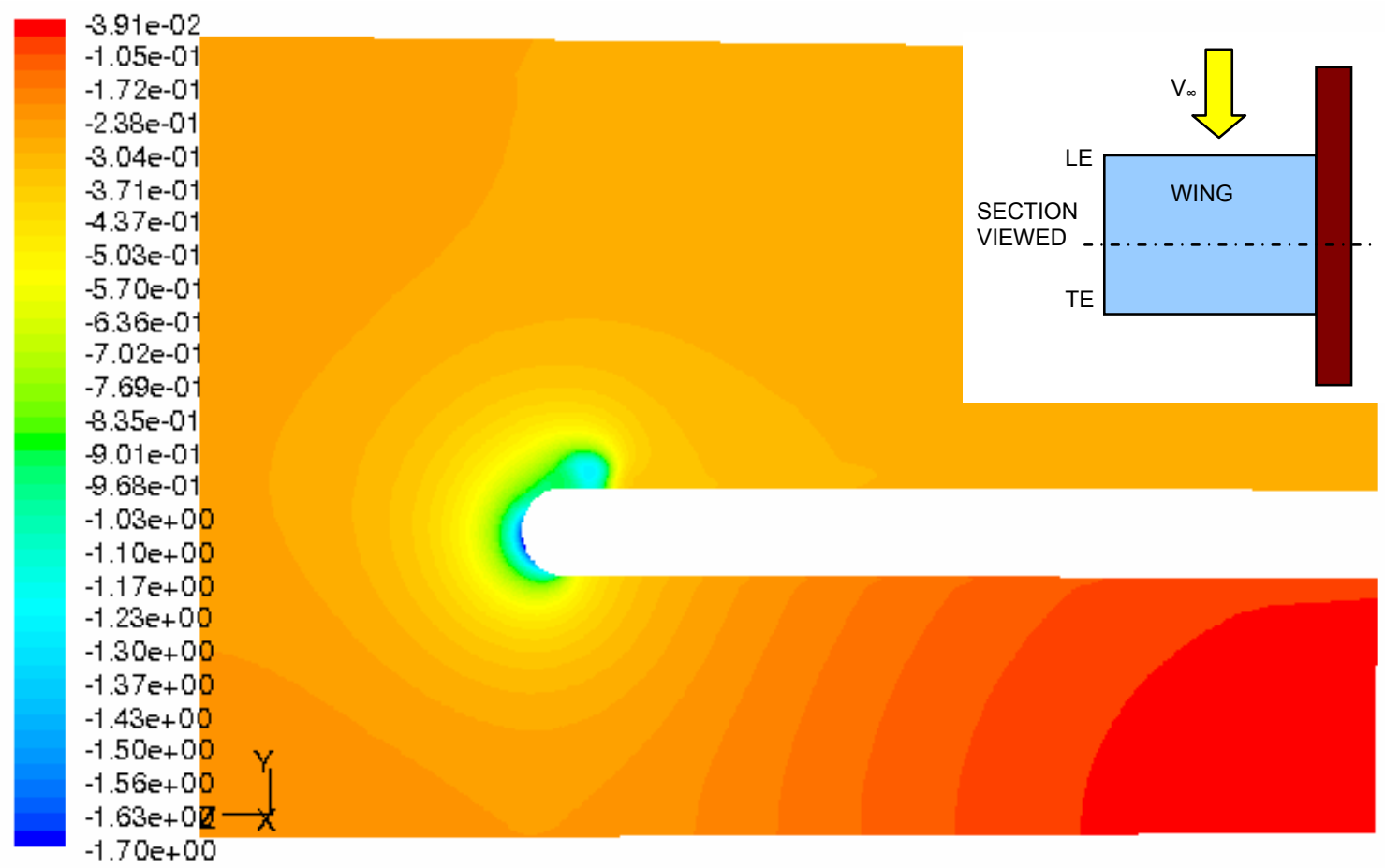

Figure 28. Pressure coefficient contours at $\mathrm{x} / \mathrm{c}=\mathbf{0 . 4 4 3}[44]$.

The low pressure region in light blue now becomes lower, generating a very low pressure vortex core (Figure 29).

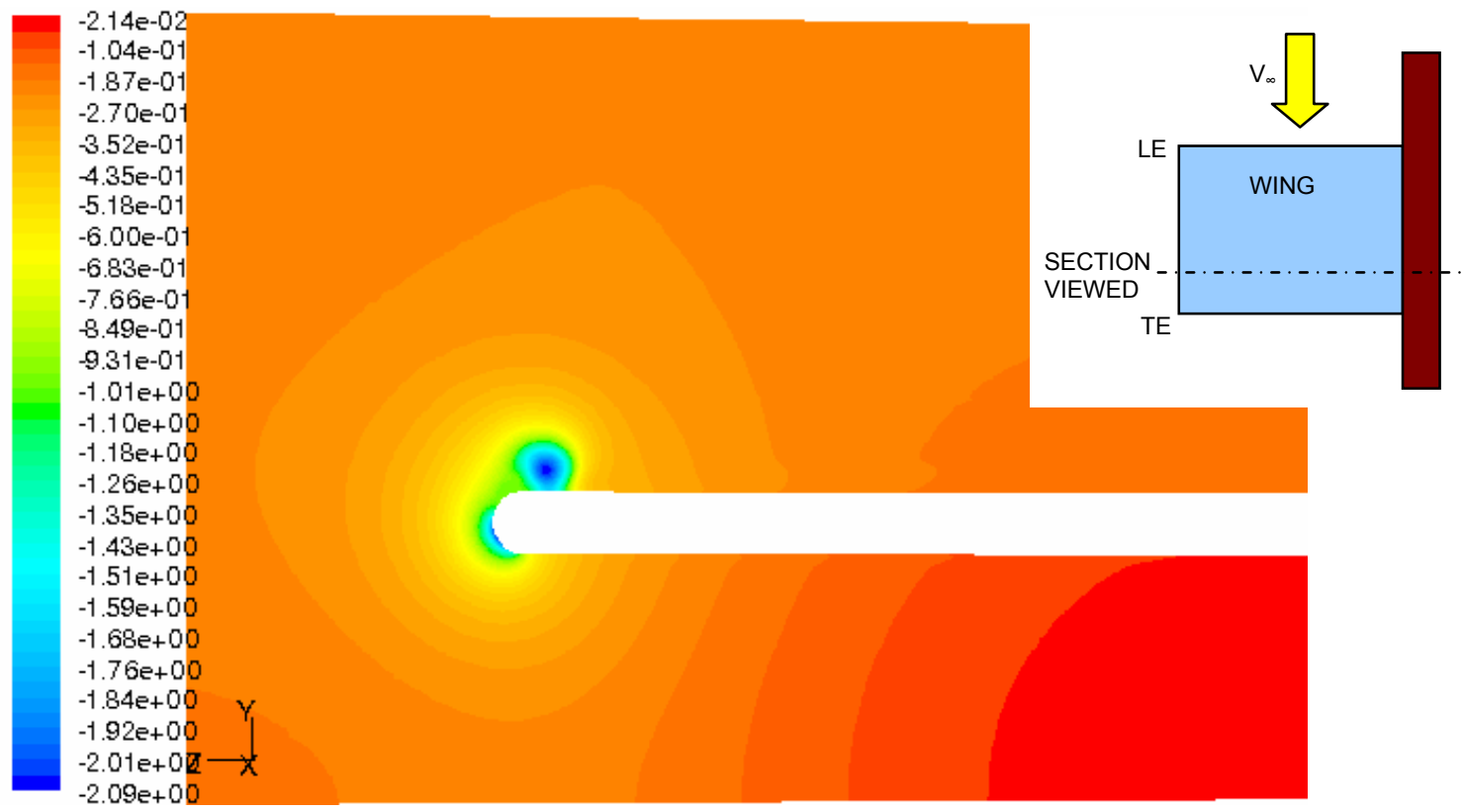

Figure 29. Pressure coefficient contours at $\mathrm{x} / \mathrm{c}=\mathbf{0 . 5 4 2}$, looking upstream from back [44]. 
The vortex keeps growing and now the pressure decreases even more (Figure 30).

It goes up and starts to separate from the top wing surface (Figure 31 ).

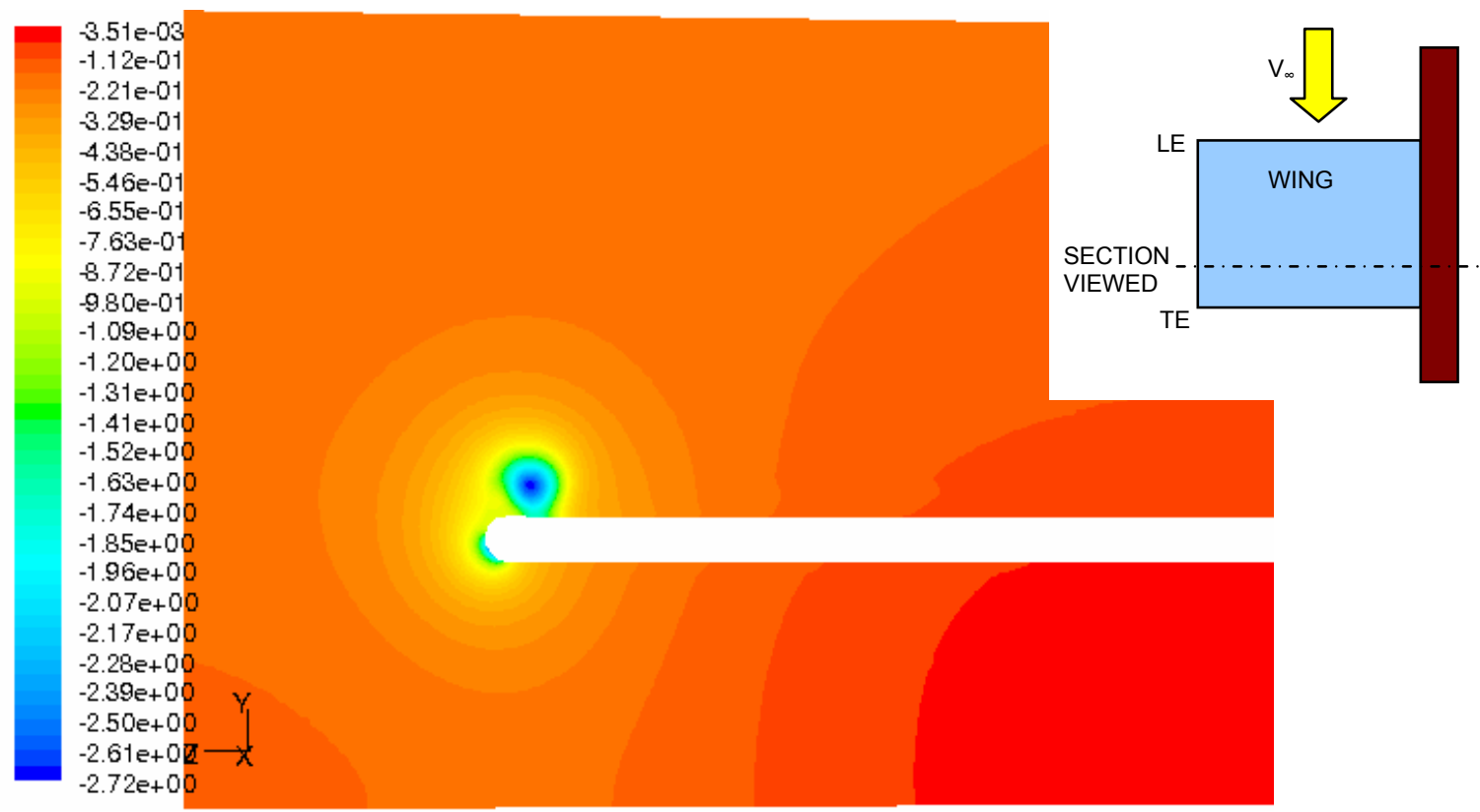

Figure 30. Pressure coefficient contours at $x / c=0.606$, looking upstream from back [44].

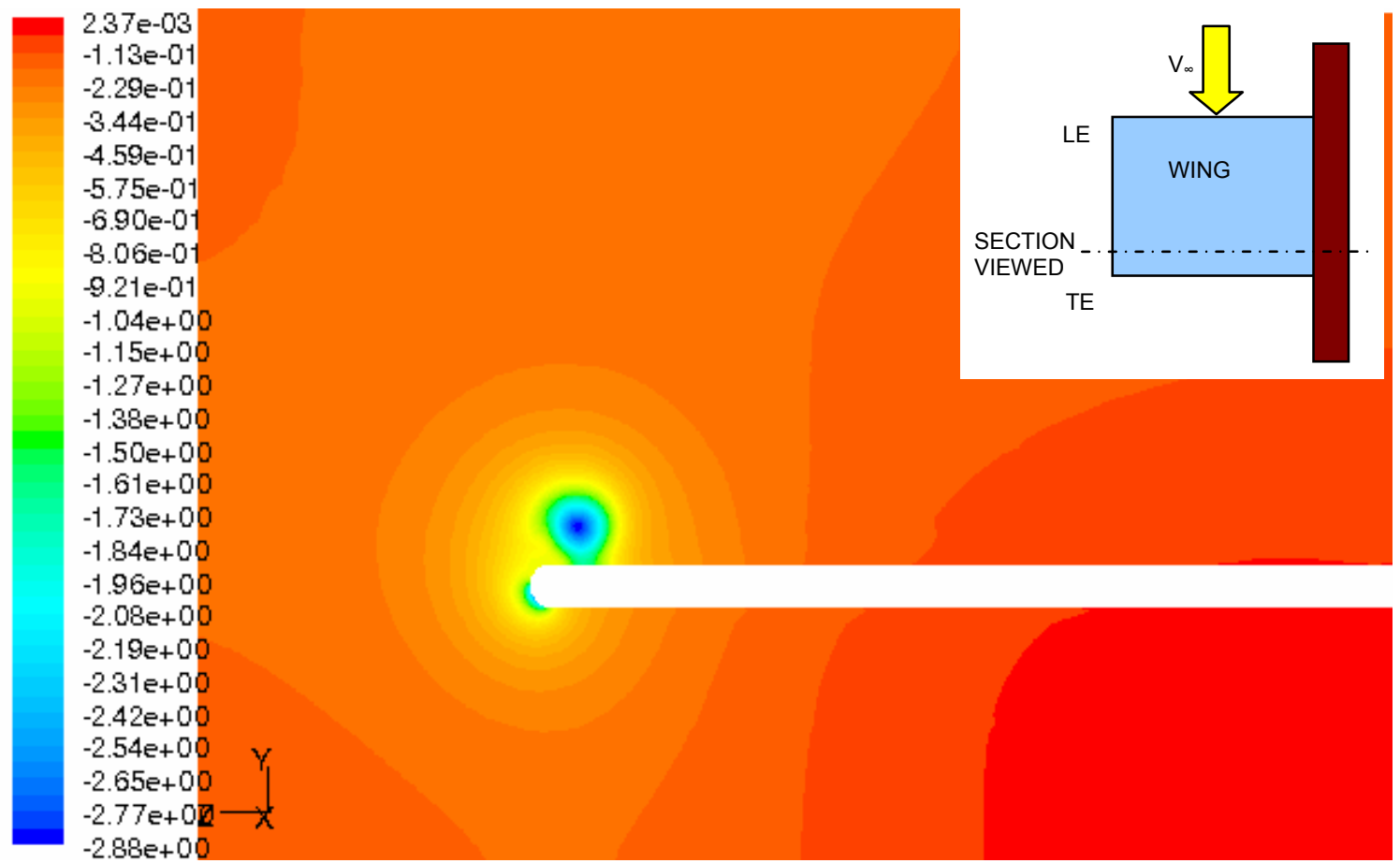

Figure 31. Pressure coefficient contours at $\mathrm{x} / \mathrm{c}=\mathbf{0 . 6 2 5}$, looking upstream from back [44].

Figure 32 shows the vortex completely separated from the upper wing surface. This is even more evident in Figure 33 at $\mathrm{x} / \mathrm{c}=0.729$. 


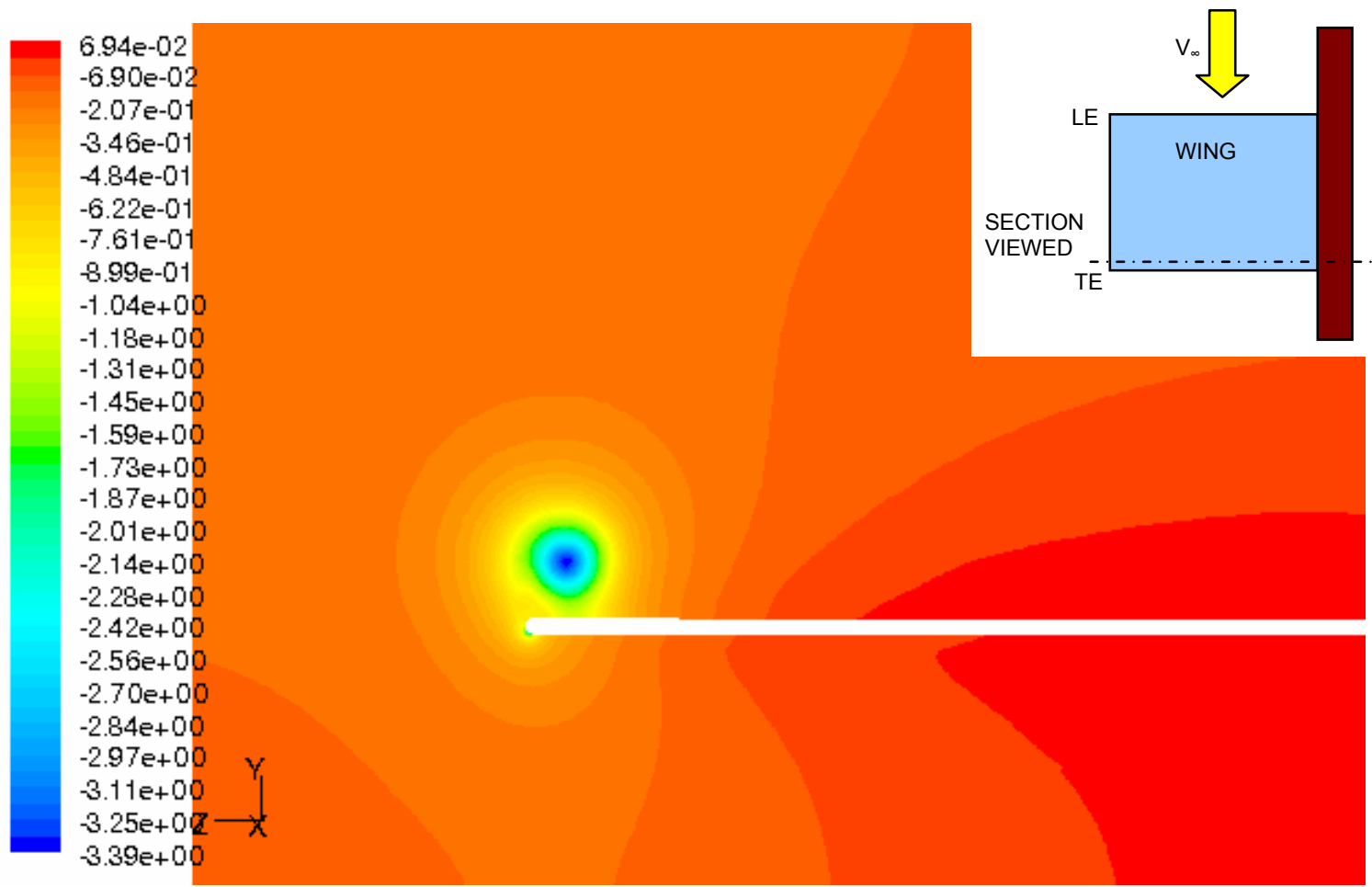

Figure 32. Pressure coefficient contours at $x / c=0.704$, looking upstream from back [44].

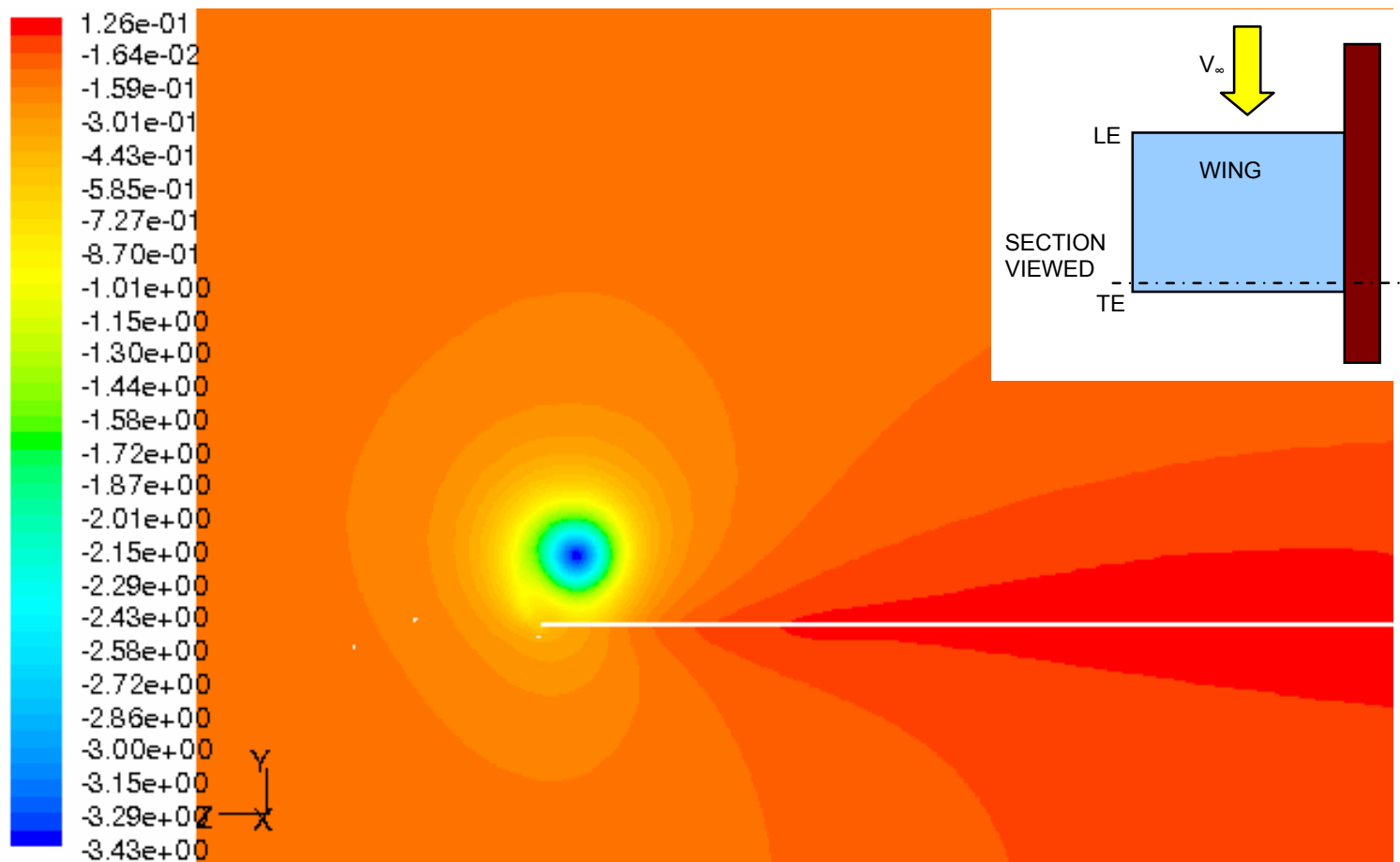

Figure 33. Pressure coefficient contours at $\mathbf{x} / \mathbf{c}=\mathbf{0 . 7 2 9}$, looking upstream from back [44].

Figures 34, 35, 36 and 37 shows a wingtip vortex of the same diameter even though, the low pressure vortex core is decreasing continuously. 


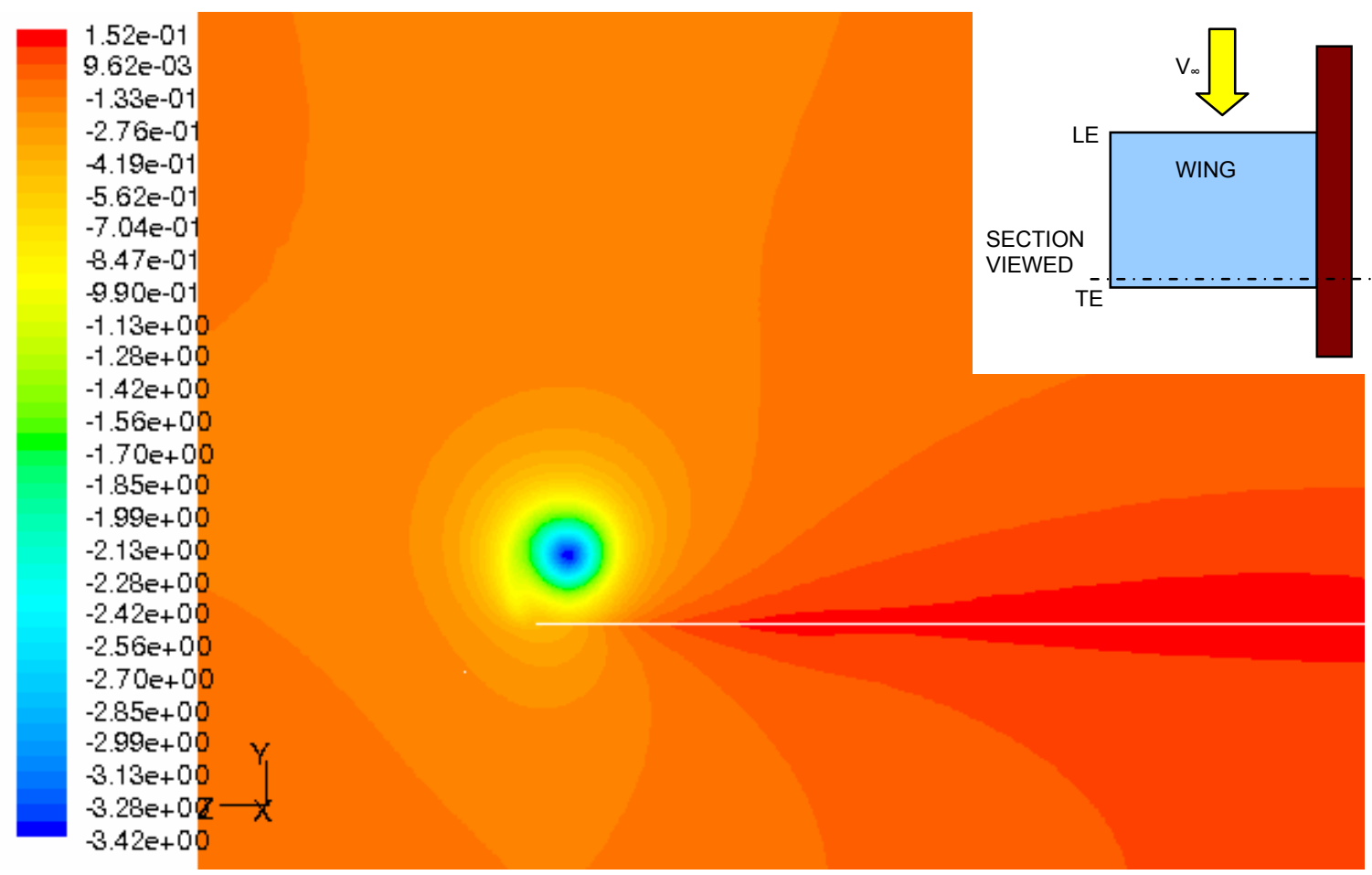

Figure 34. Pressure coefficient contours at $x / c=0.735$, looking upstream from back [44].

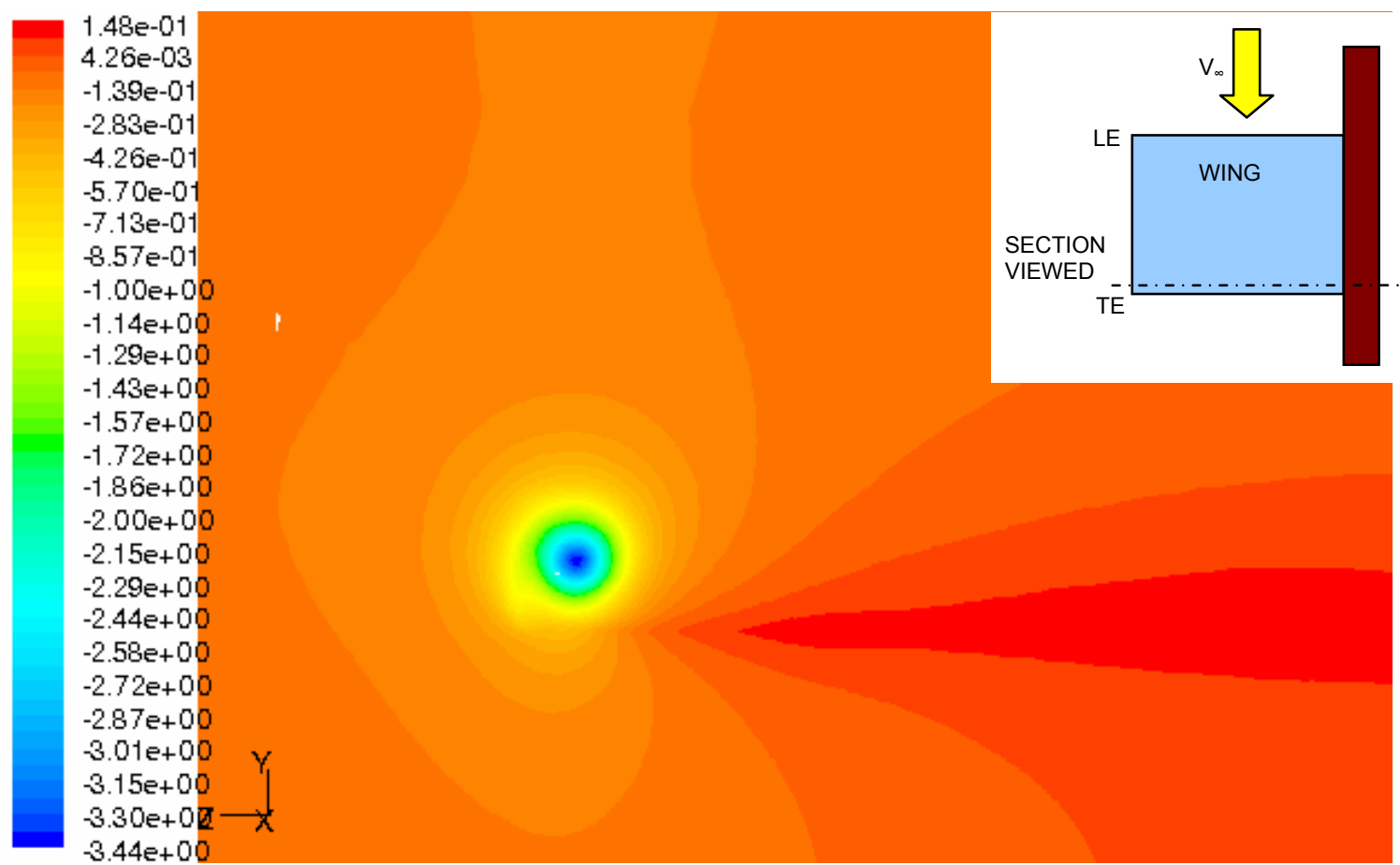

Figure 35. Pressure coefficient contours at $x / c=0.744$, looking upstream from back [44]. 


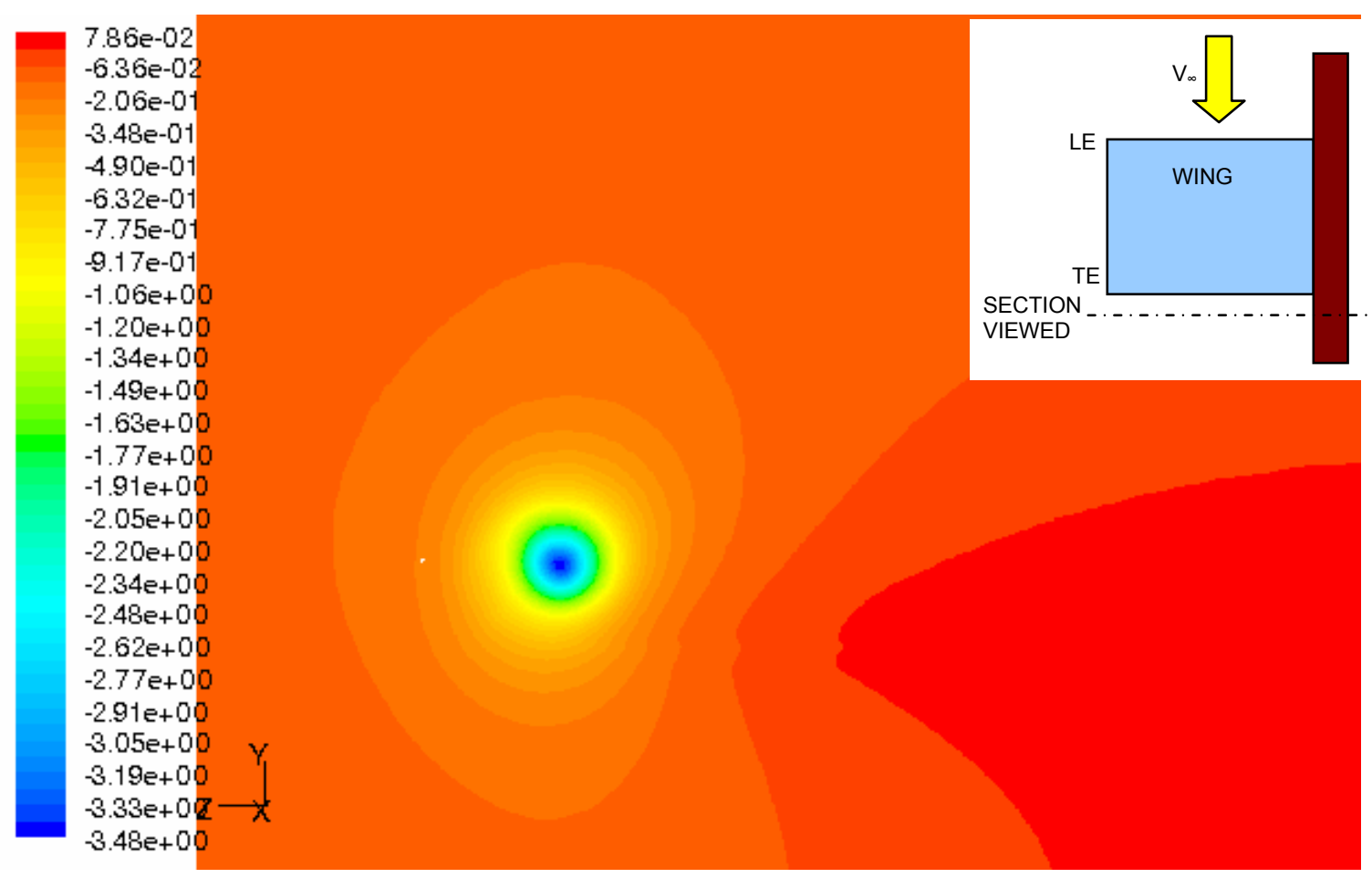

Figure 36. Pressure coefficient contours at $\mathbf{x} / \mathbf{c}=\mathbf{0 . 8 0 3}$, looking upstream from back [44].

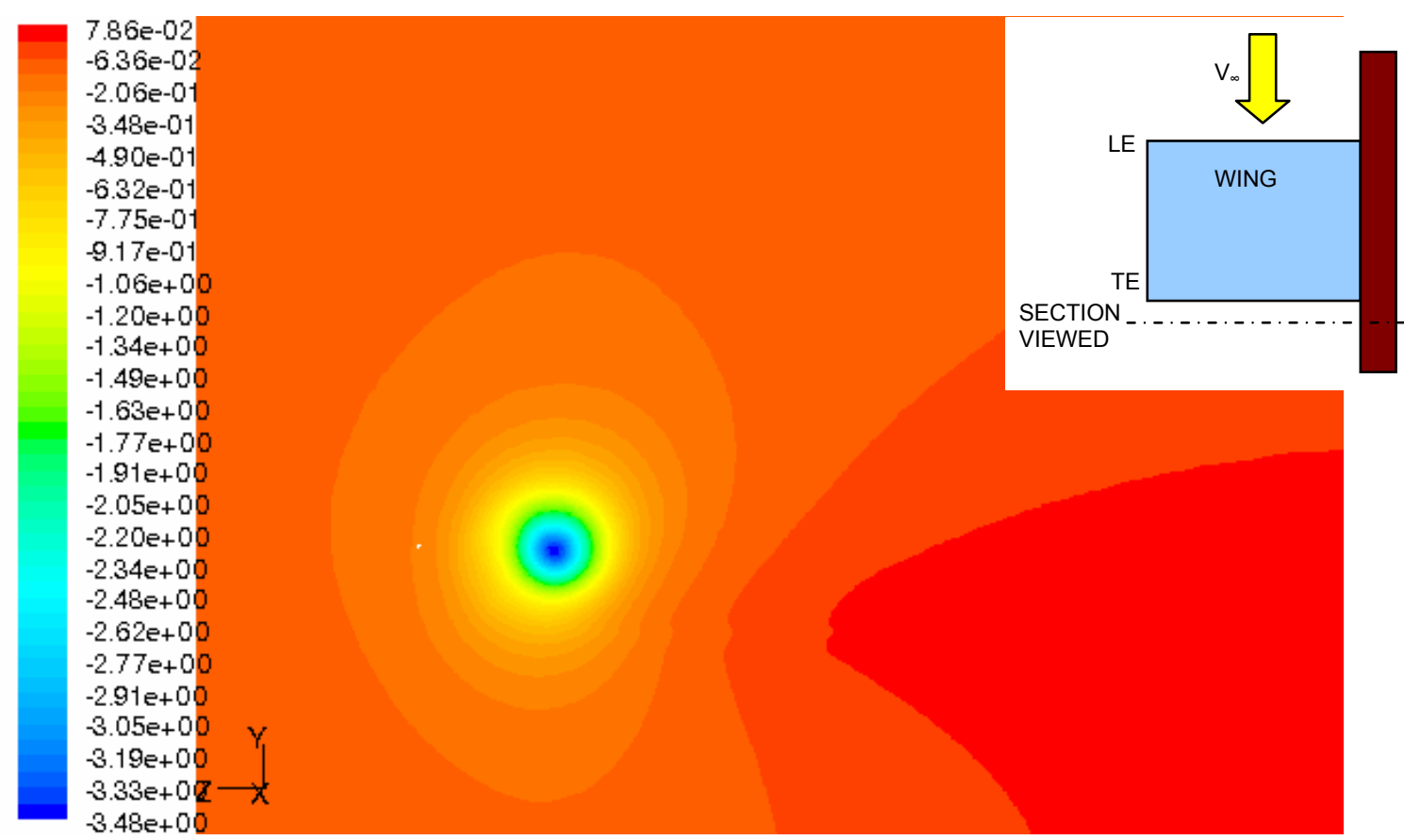

Figure 37. Pressure coefficient contours at $\mathrm{x} / \mathrm{c}=\mathbf{0 . 8 6 4}$, looking upstream from back [44].

Figure 38 shows the wingtip vortex to be bigger than the previous figures. However, the low pressure vortex core starts to decay and its pressure coefficient increases from -3.48 to -3.1 . 


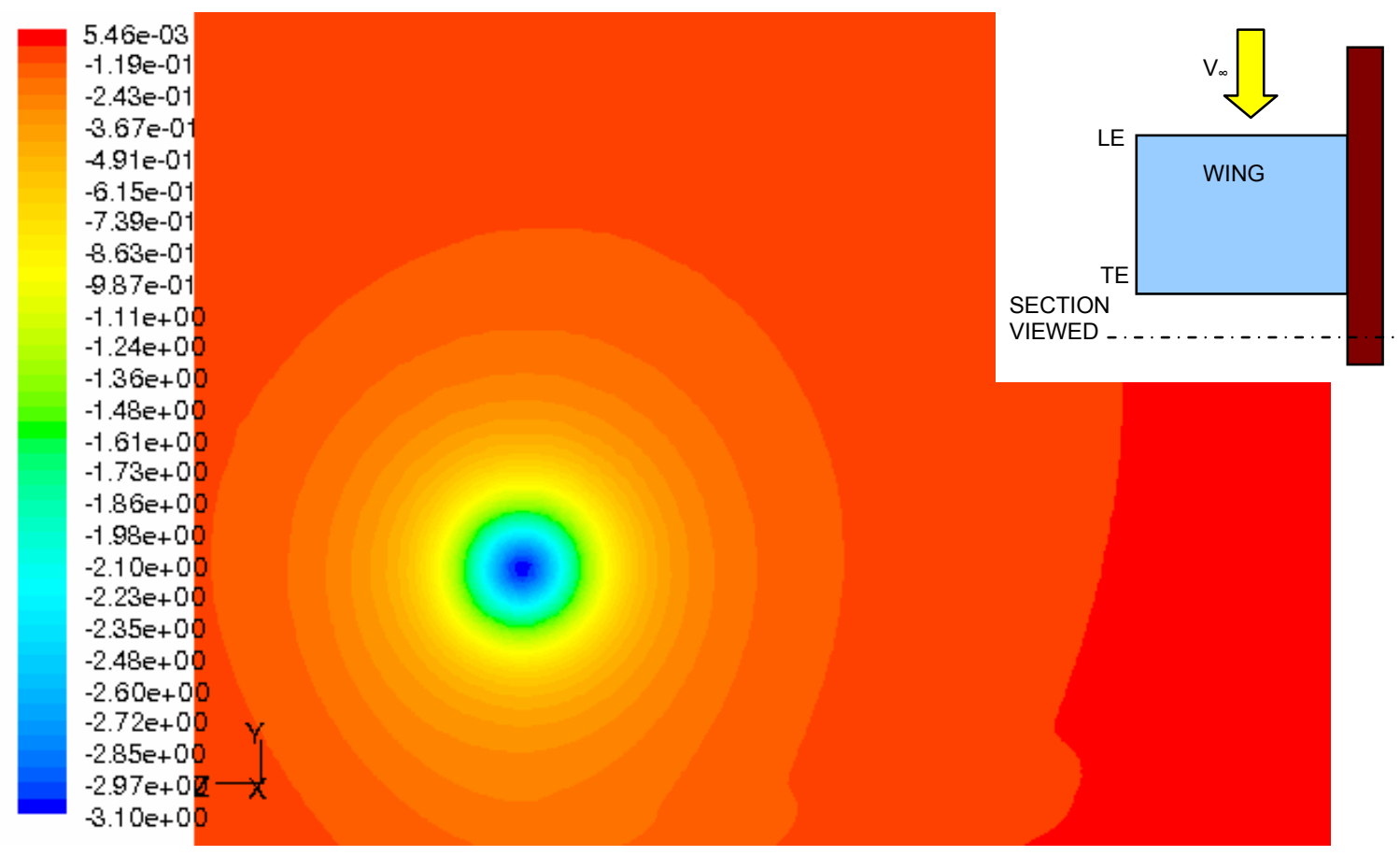

Figure 38. Pressure coefficient contours at $x / c=1.246$, looking upstream from back [44].

From distance $\mathrm{x} / \mathrm{c}=1.246$ to $\mathrm{x} / \mathrm{c}=1.678$, the wing tip vortex diameter increases considerably and the vortex core pressure coefficient increases from -3.1 to -2.7 .

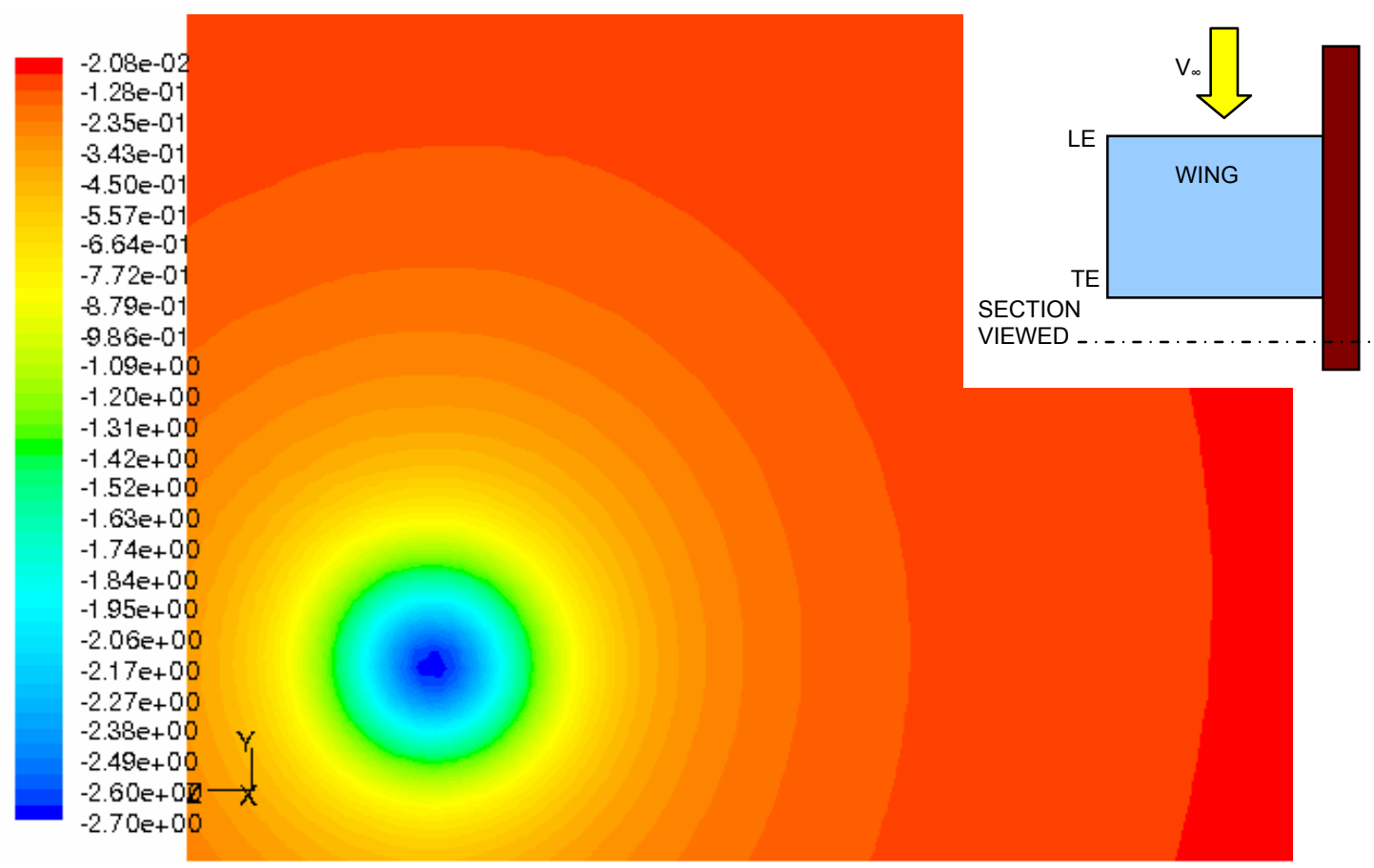

Figure 39. Pressure coefficient contours at $x / c=1.678$, looking upstream from back [44].

Table 1 shows the pressure coefficient in the wing tip vortex core for various locations in the flow direction. This table shows a minimum value around the 
downstream position $\mathrm{x} / \mathrm{c}=0.864$. Figure 40 shows how the pressure coefficient varies in the downstream direction. This figure shows the rapid decrease in wingtip vortex core pressure from the leading edge to the position $\mathrm{x} / \mathrm{c}=0.864$ followed by a slow increase in the pressure downstream. Note that this vortex core decay is overestimated by the Kim and Rhee simulations [44] compared to the Chow et al. experiments [43].

Table 1. Pressure coefficient in the wing tip vortex core in the downstream direction.

\begin{tabular}{|c|c|}
\hline $\mathbf{x} / \mathbf{c}$ & $\mathbf{C p}$ \\
\hline 0.443 & -1.30 \\
\hline 0.542 & -2.09 \\
\hline 0.606 & -2.72 \\
\hline 0.625 & -2.88 \\
\hline 0.704 & -3.39 \\
\hline 0.729 & -3.43 \\
\hline 0.735 & -3.42 \\
\hline 0.744 & -3.44 \\
\hline 0.803 & -3.48 \\
\hline 0.864 & -3.48 \\
\hline 1.246 & -3.10 \\
\hline 1.678 & -2.70 \\
\hline
\end{tabular}

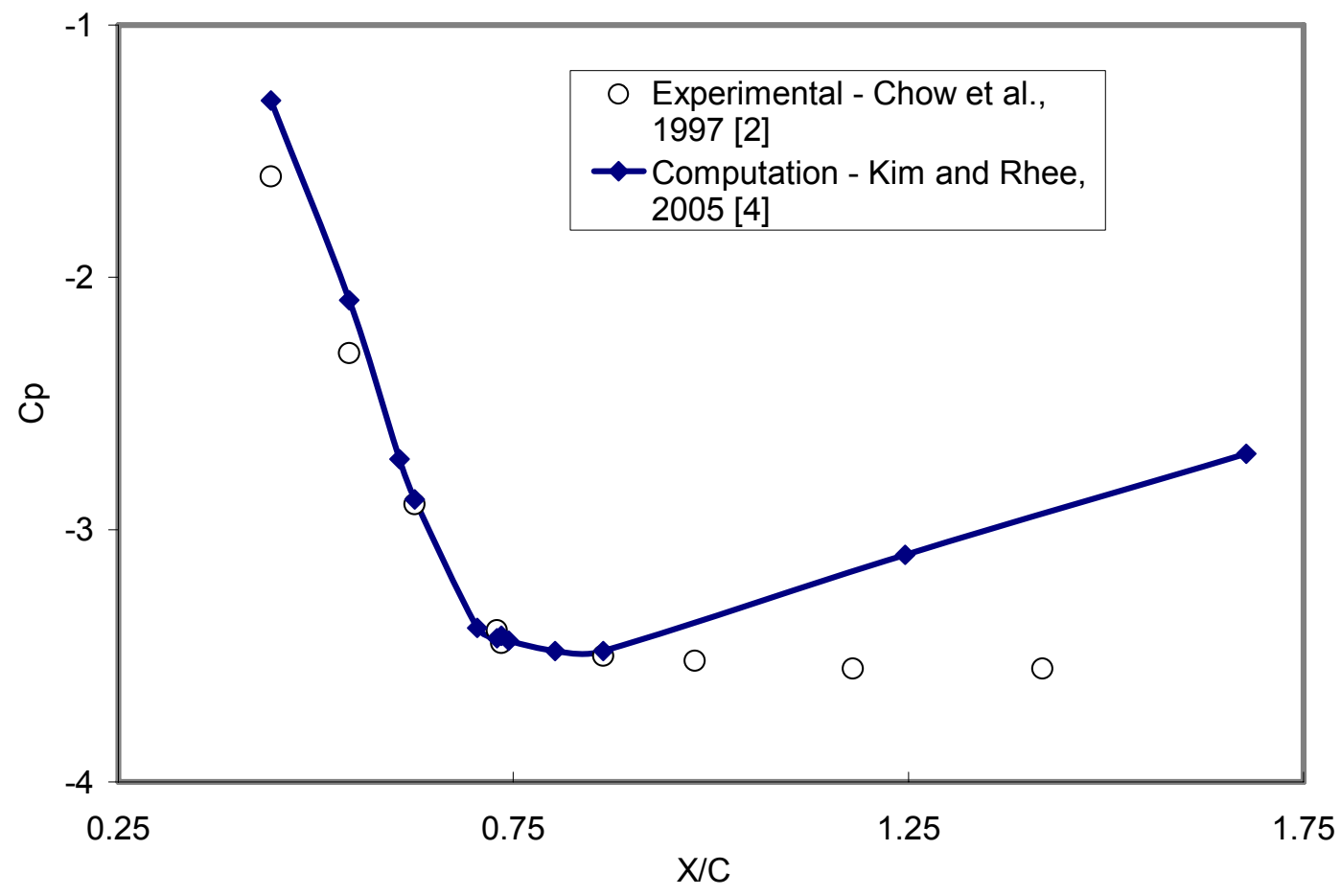

Figure 40. Pressure coefficient in the wing tip vortex core in the downstream direction. 


\section{Velocity Magnitude Contours}

Total velocity magnitude contours at different locations downstream are shown in Figures 41 to 56 . At $\mathrm{x} / \mathrm{c}=0.25$ (Figure 41 ), a high velocity region is seen near the wingtip due to the pressure difference between top and bottom wing surface.

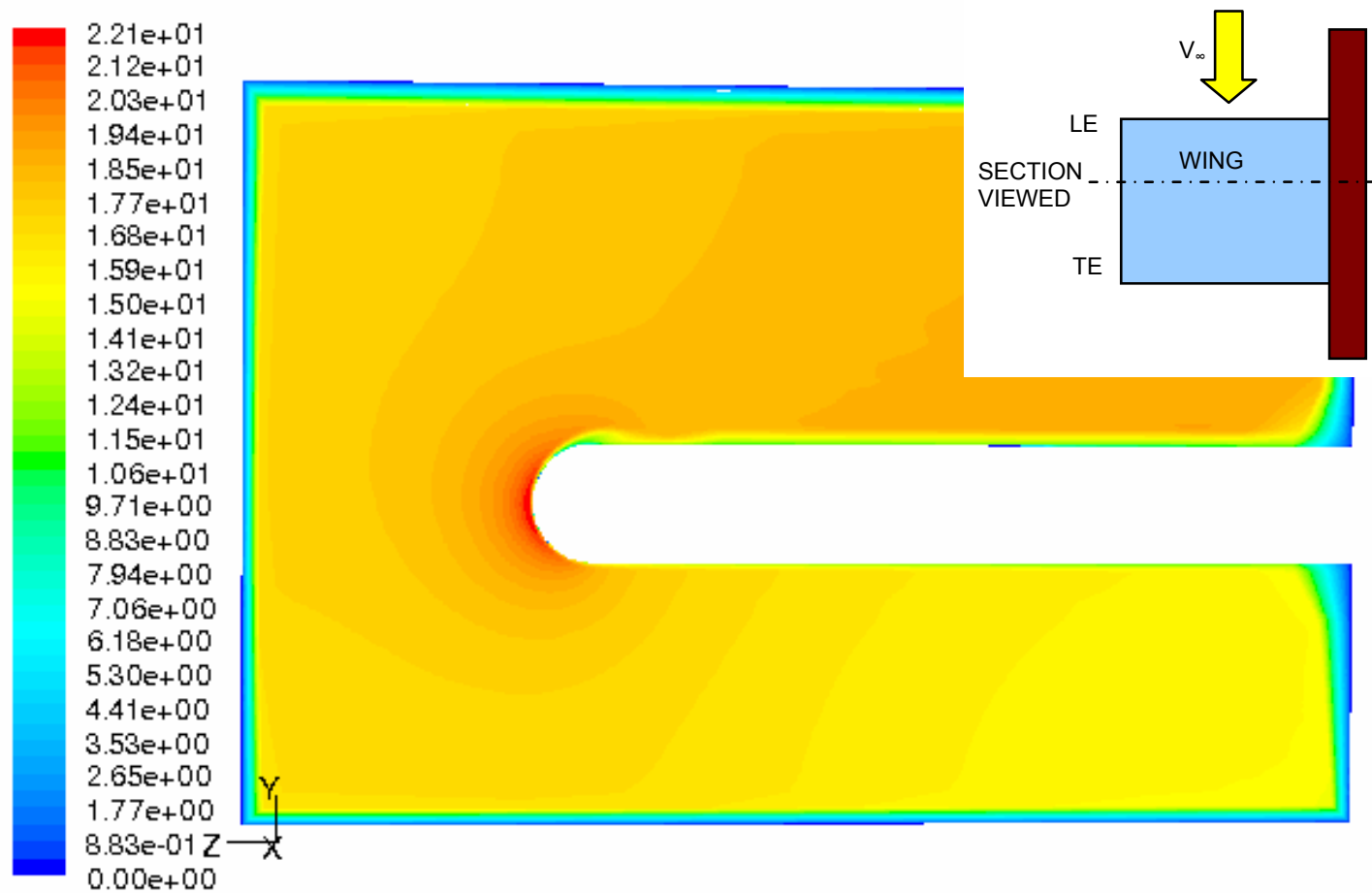

Figure 41. Velocity magnitude contours (ft/s) at $\mathbf{x} / \mathbf{c}=0.25$, looking upstream from back [44].

Figures 42 and 43 show how the boundary layer produced by the wingtip flow detaches from the top wing surface. 


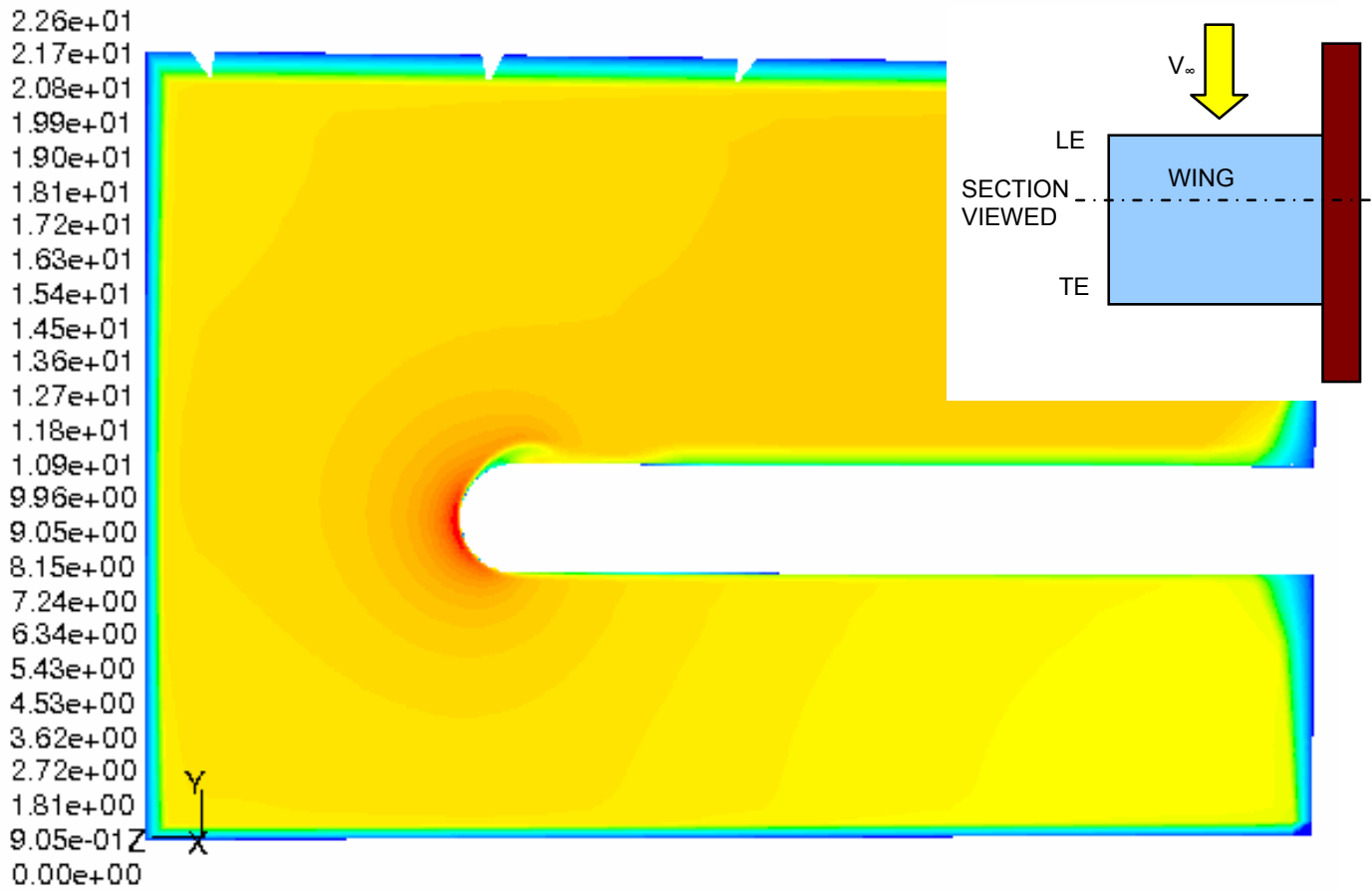

Figure 42. Velocity magnitude contours $(\mathrm{ft} / \mathrm{s})$ at $\mathrm{x} / \mathrm{c}=\mathbf{0 . 3 4 5}$, looking upstream from back [44].

The boundary layer detachment is clearly seen in Figure 43.

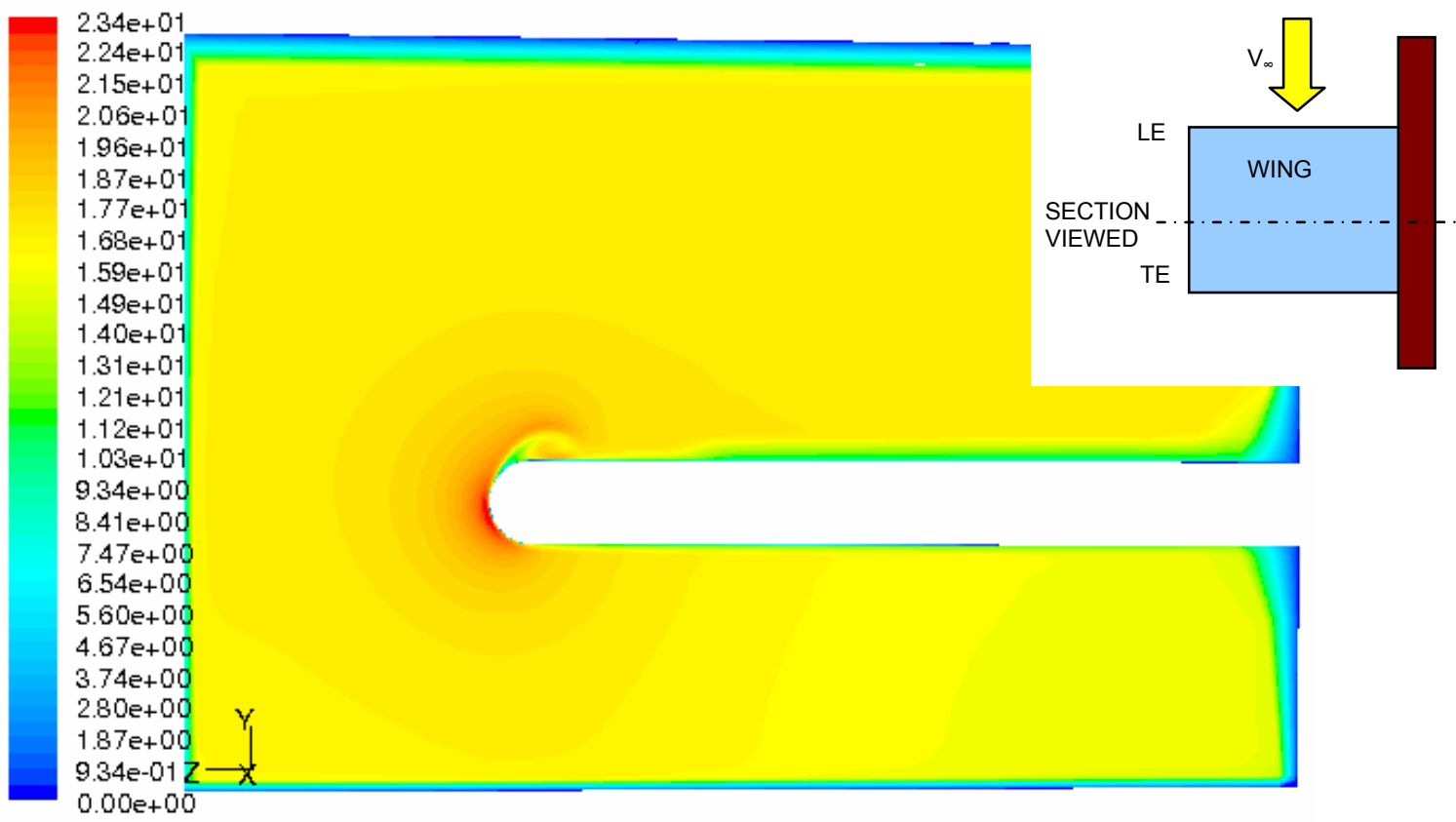

Figure 43. Velocity magnitude contours ( $(\mathrm{ft} / \mathrm{s})$ at $\mathrm{x} / \mathrm{c}=\mathbf{0 . 4 4 3}$, looking upstream from back [44].

Figure 44 shows the recirculation region generated by the boundary layer detachment. In the center of this region, a high velocity vortex core starts to form. There 
is also a high velocity area directly below the vortex core and next to the wing top surface.

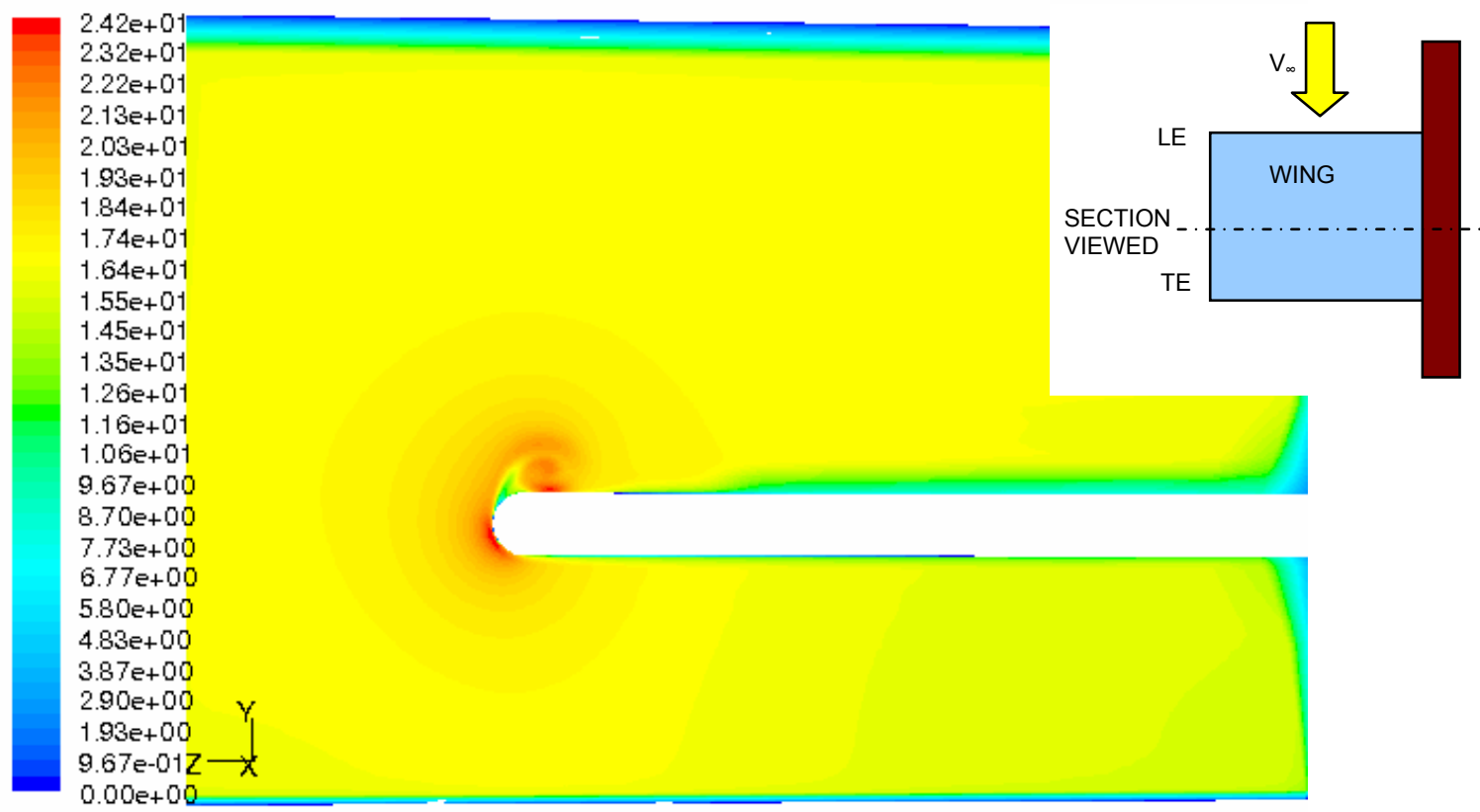

Figure 44. Velocity magnitude contours ( $\mathrm{ft} / \mathrm{s})$ at $\mathrm{x} / \mathrm{c}=\mathbf{0 . 5 4 2}$, looking upstream from back [44].

The vortex core is more evident at $\mathrm{x} / \mathrm{c}=0.606$ (Figure 45), where it gets a higher velocity $(25 \mathrm{ft} / \mathrm{s})$.

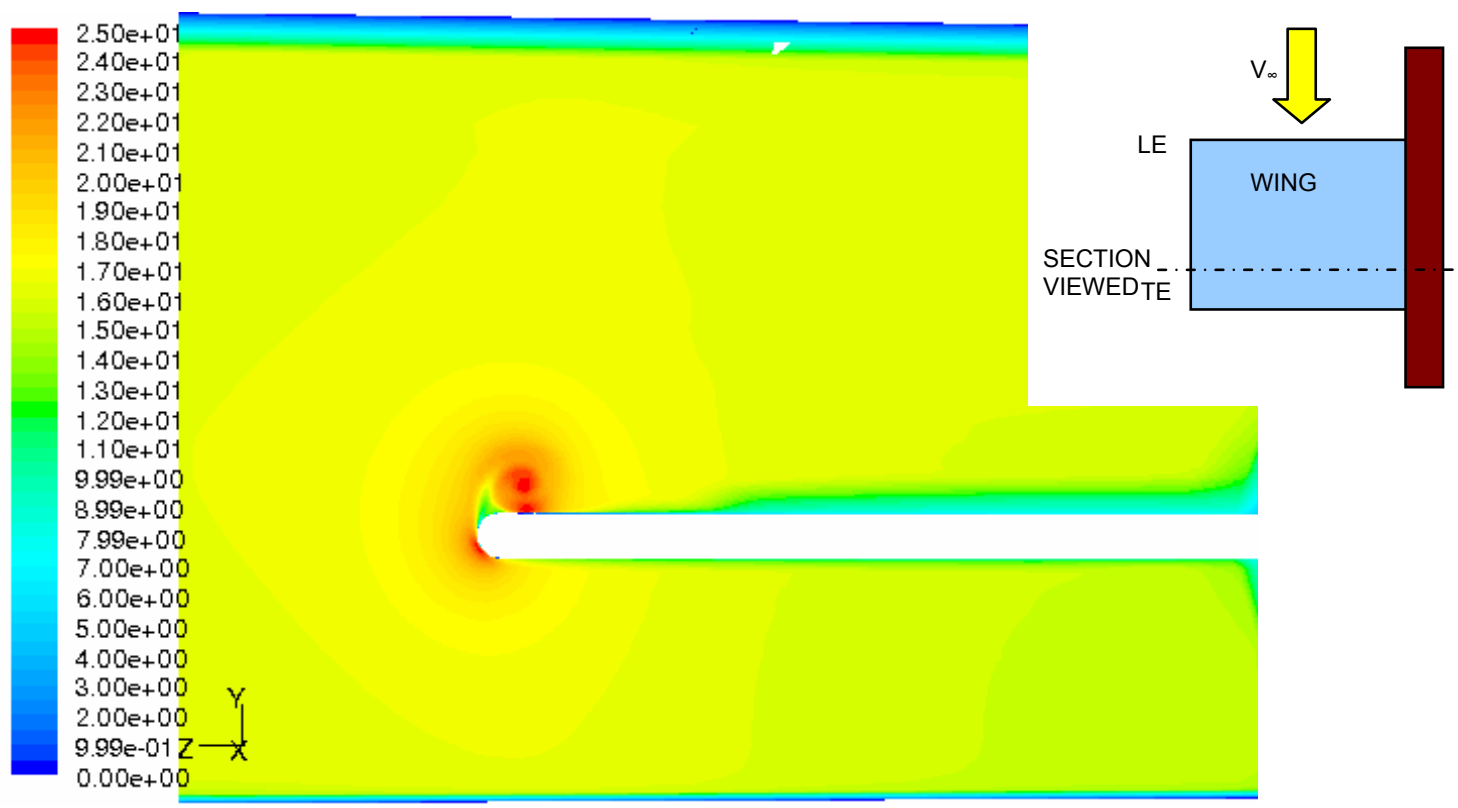

Figure 45. Velocity magnitude contours ( $\mathrm{ft} / \mathrm{s})$ at $\mathrm{x} / \mathrm{c}=\mathbf{0 . 6 0 6}$, looking upstream from back [44]. 
The distance between the vortex core and the wing top surface is increasing at $\mathrm{x} / \mathrm{c}=0.625$ (Figure 46). This causes that the high velocity region near the wing top surface at $\mathrm{x} / \mathrm{c}=0.606$ (Figure 45$)$ is decreasing.

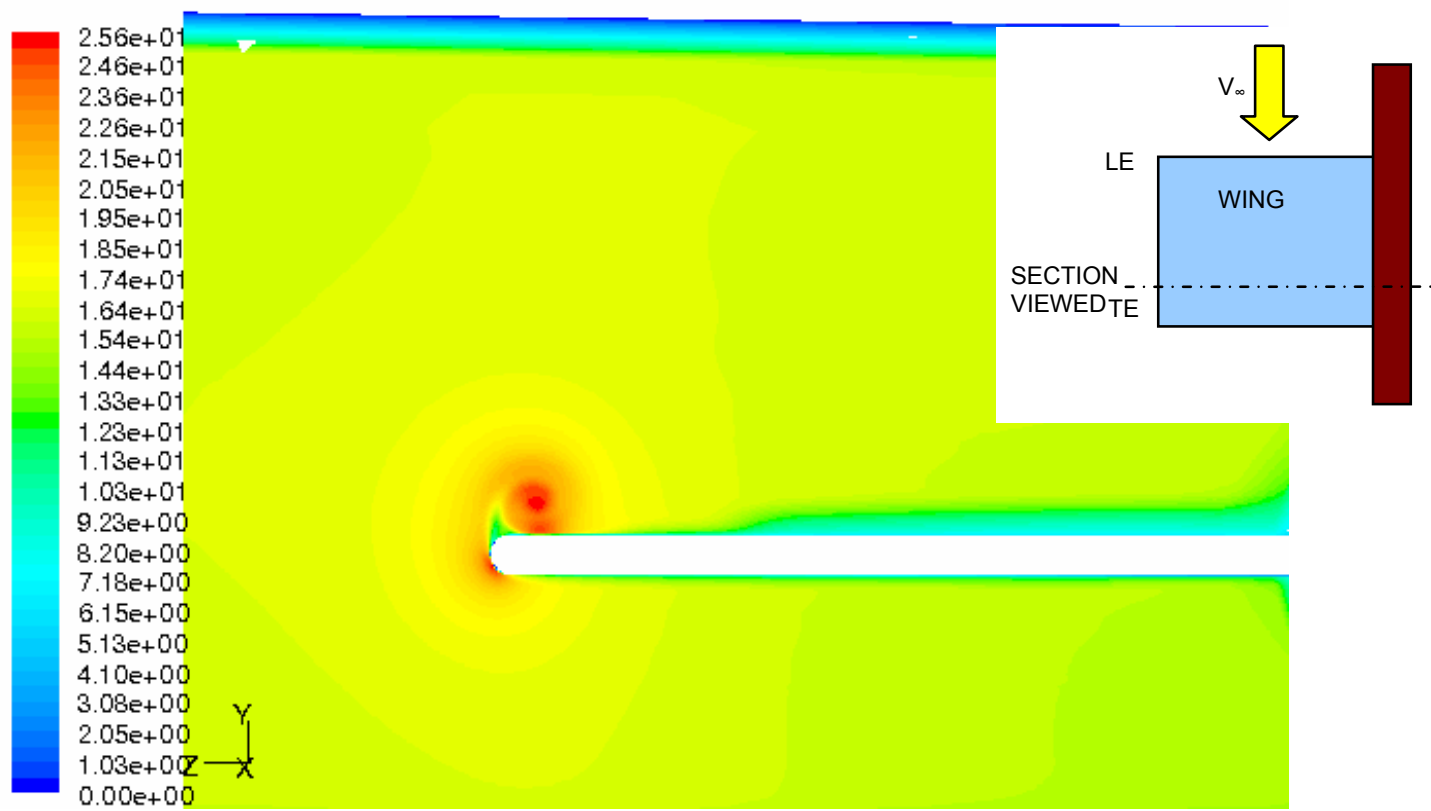

Figure 46. Velocity magnitude contours (ft/s) at $\mathrm{x} / \mathrm{c}=\mathbf{0 . 6 2 5}$, looking upstream from back [44].

At $\mathrm{x} / \mathrm{c}=0.704$ (Figure 47 ), there is no high velocity region close to the wing top surface.
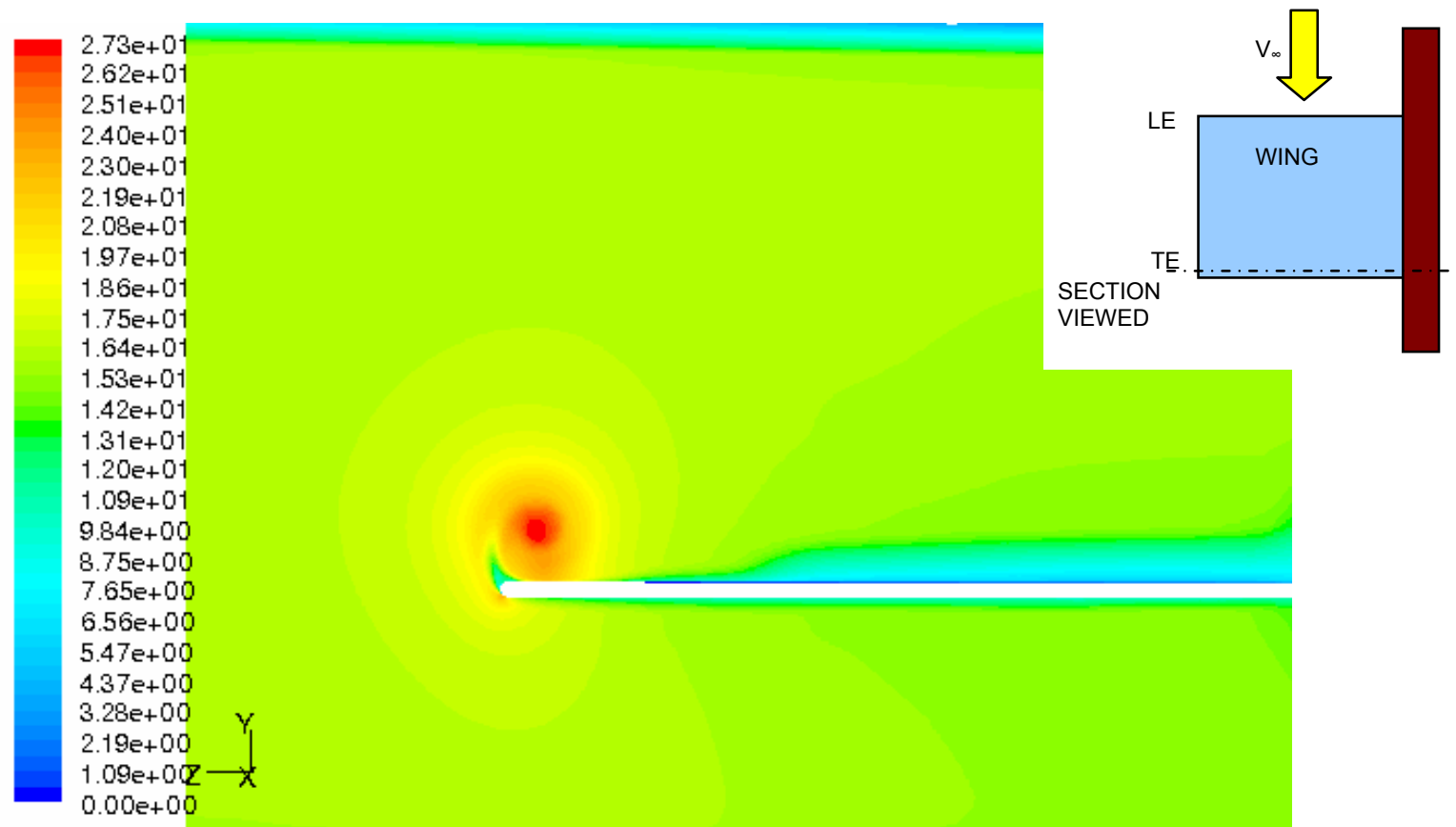

Figure 47. Velocity magnitude contours ( $\mathrm{ft} / \mathrm{s})$ at $\mathrm{x} / \mathrm{c}=\mathbf{0 . 7 0 4}$, looking upstream from back [44]. 
The high velocity in the wingtip vortex core remains almost constant $(27.4 \mathrm{ft} / \mathrm{s})$ from $\mathrm{x} / \mathrm{c}=0.704$ (Figure 47$)$ to $\mathrm{x} / \mathrm{c}=0.886$ (Figure 53).

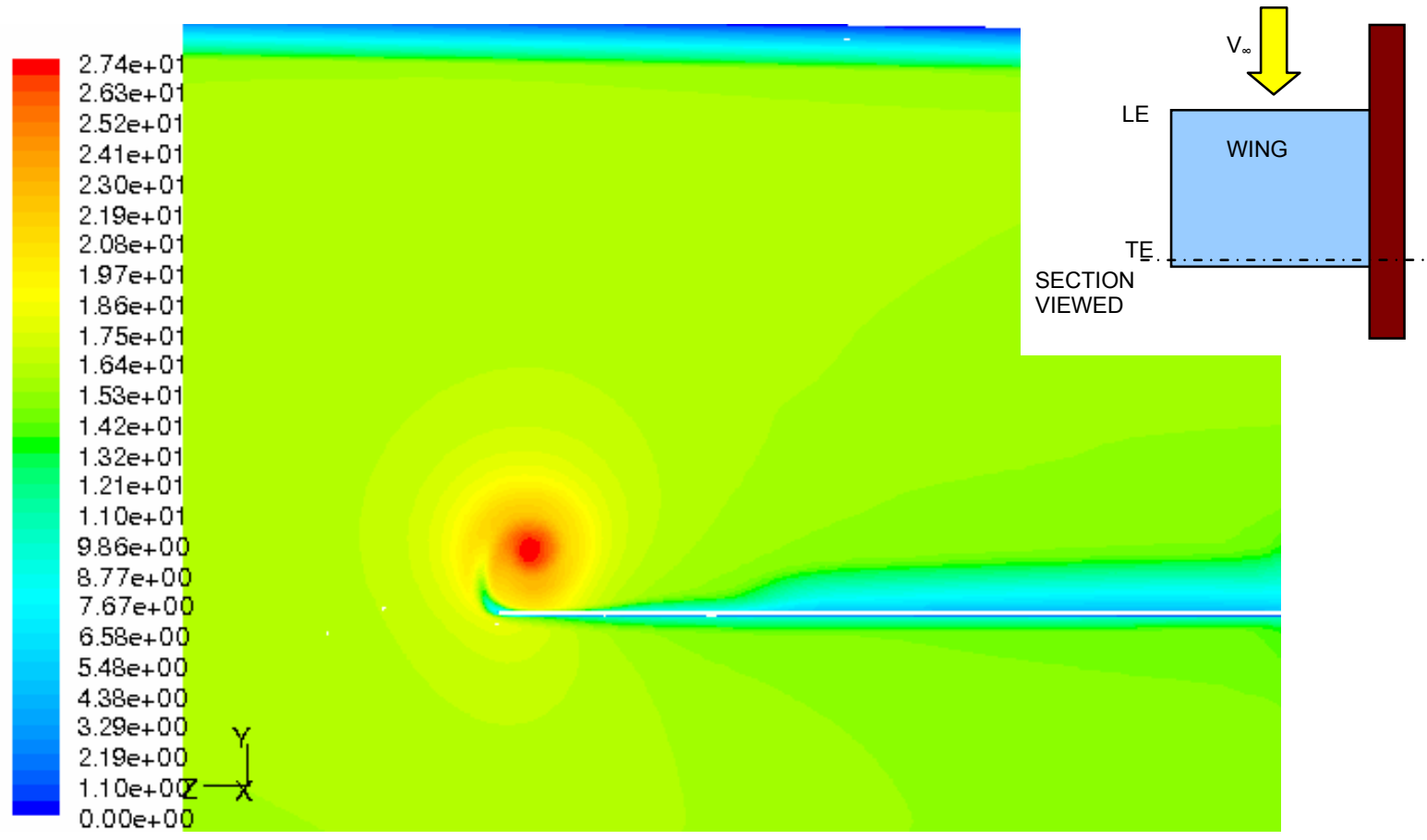

Figure 48. Velocity magnitude contours (ft/s) at $\mathrm{x} / \mathrm{c}=\mathbf{0 . 7 2 9}$, looking upstream from back [44].

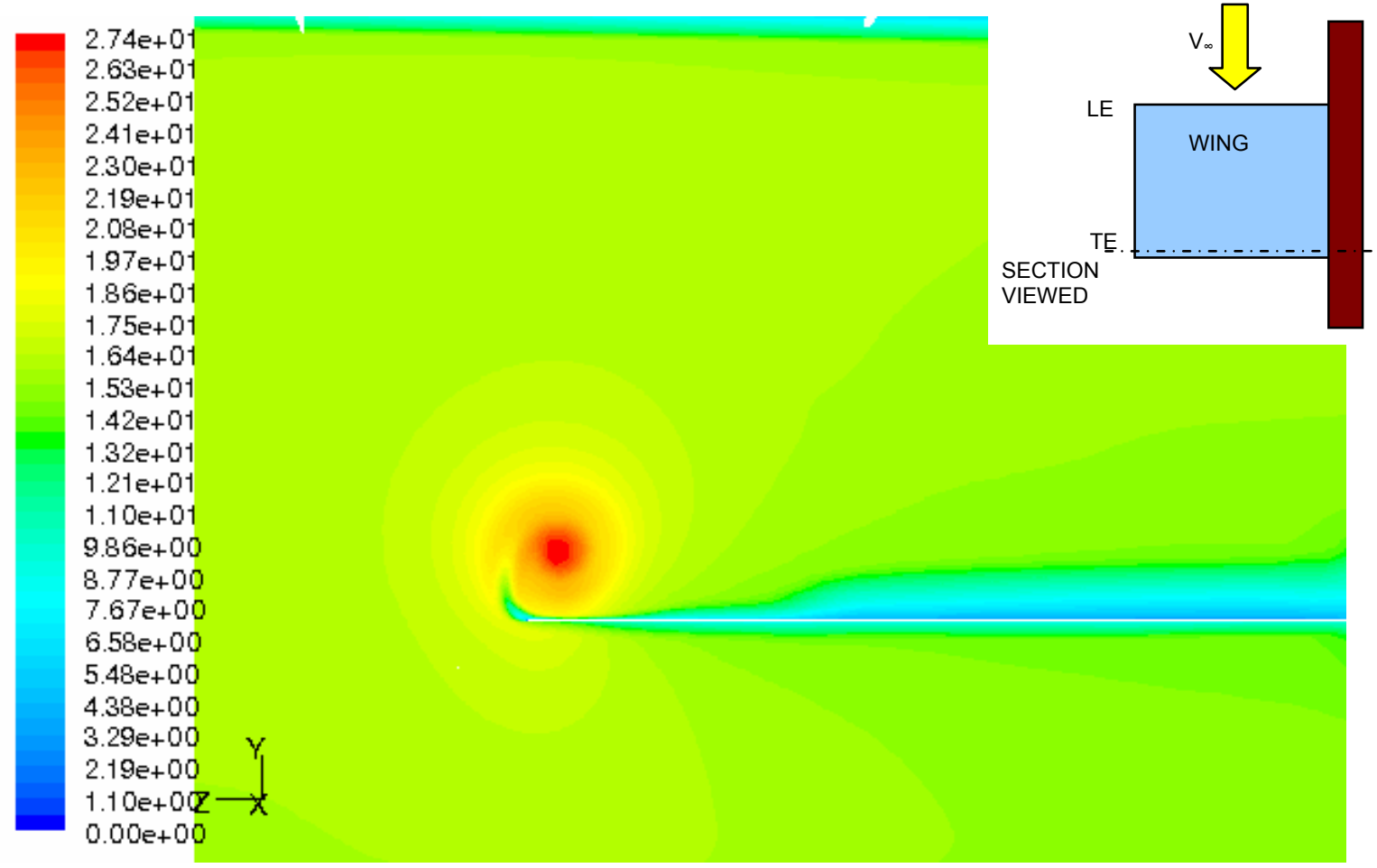

Figure 49. Velocity magnitude contours $(\mathrm{ft} / \mathrm{s})$ at $\mathrm{x} / \mathrm{c}=\mathbf{0 . 7 3 5}$, looking upstream from back [44]. 


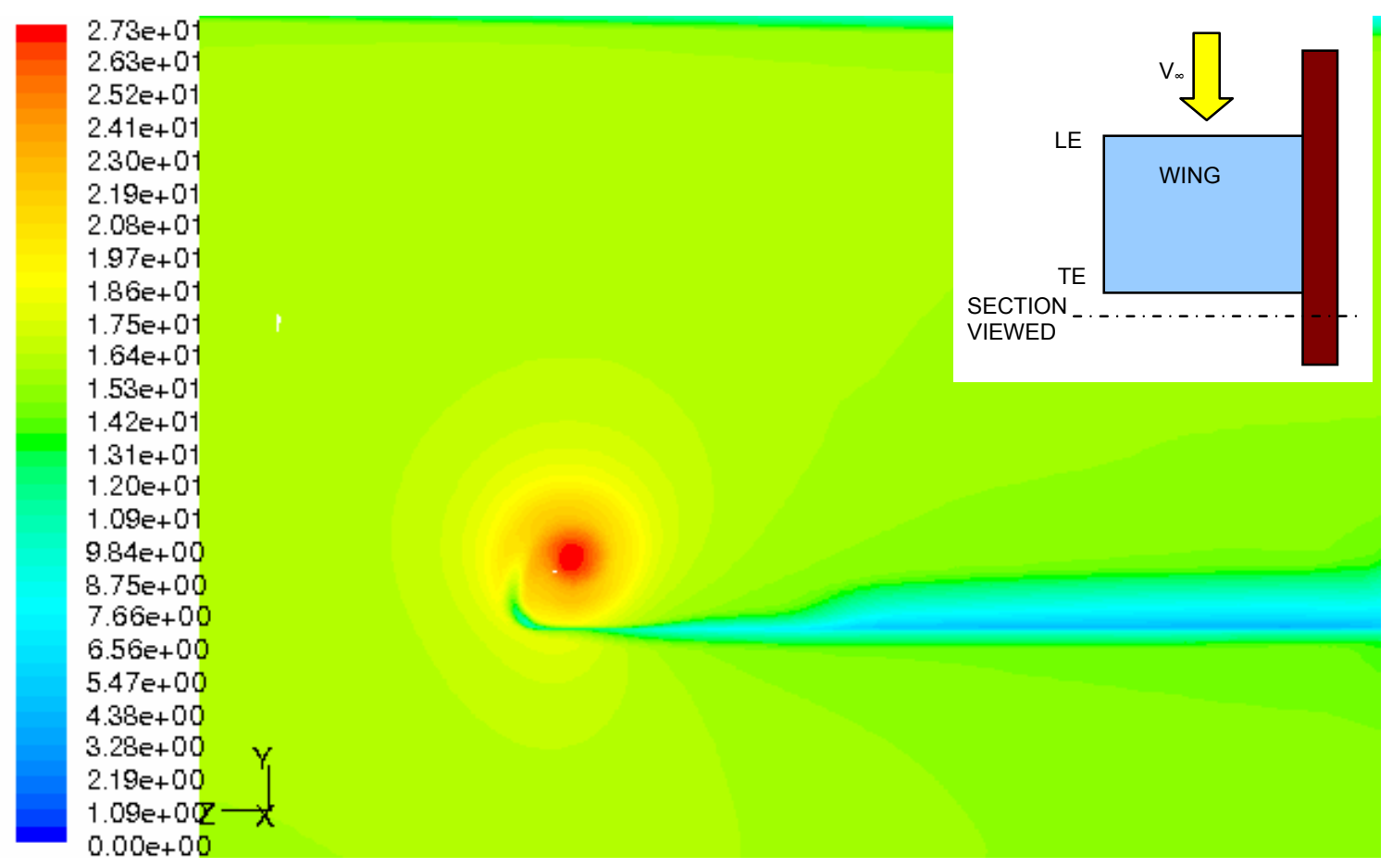

Figure 50. Velocity magnitude contours (ft/s) at $\mathrm{x} / \mathrm{c}=\mathbf{0 . 7 4 4}$, looking upstream from back [44].

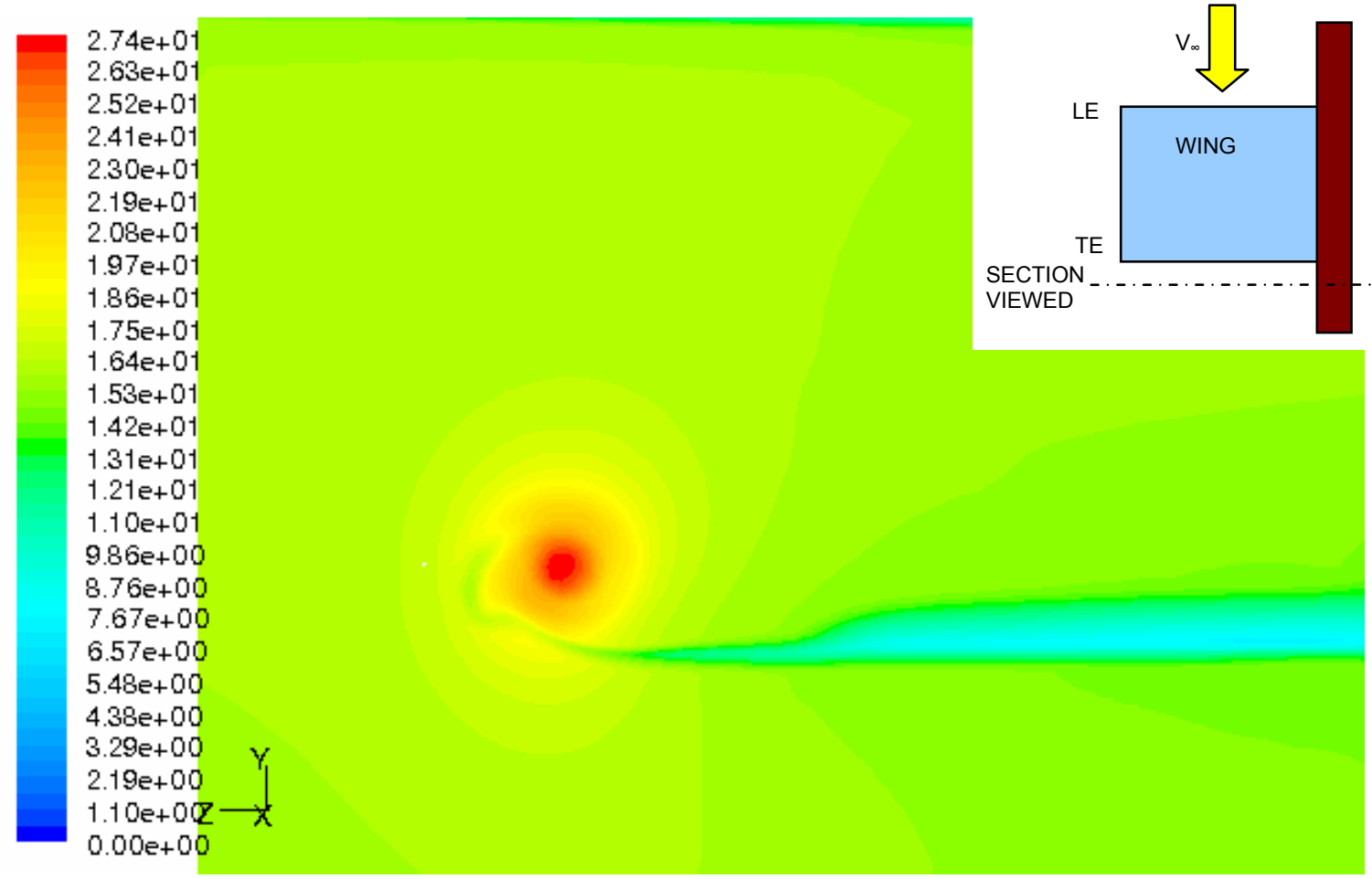

Figure 51. Velocity magnitude contours ( $(\mathrm{ft} / \mathrm{s})$ at $\mathrm{x} / \mathrm{c}=\mathbf{0 . 8 0 3}$, looking upstream from back [44]. 


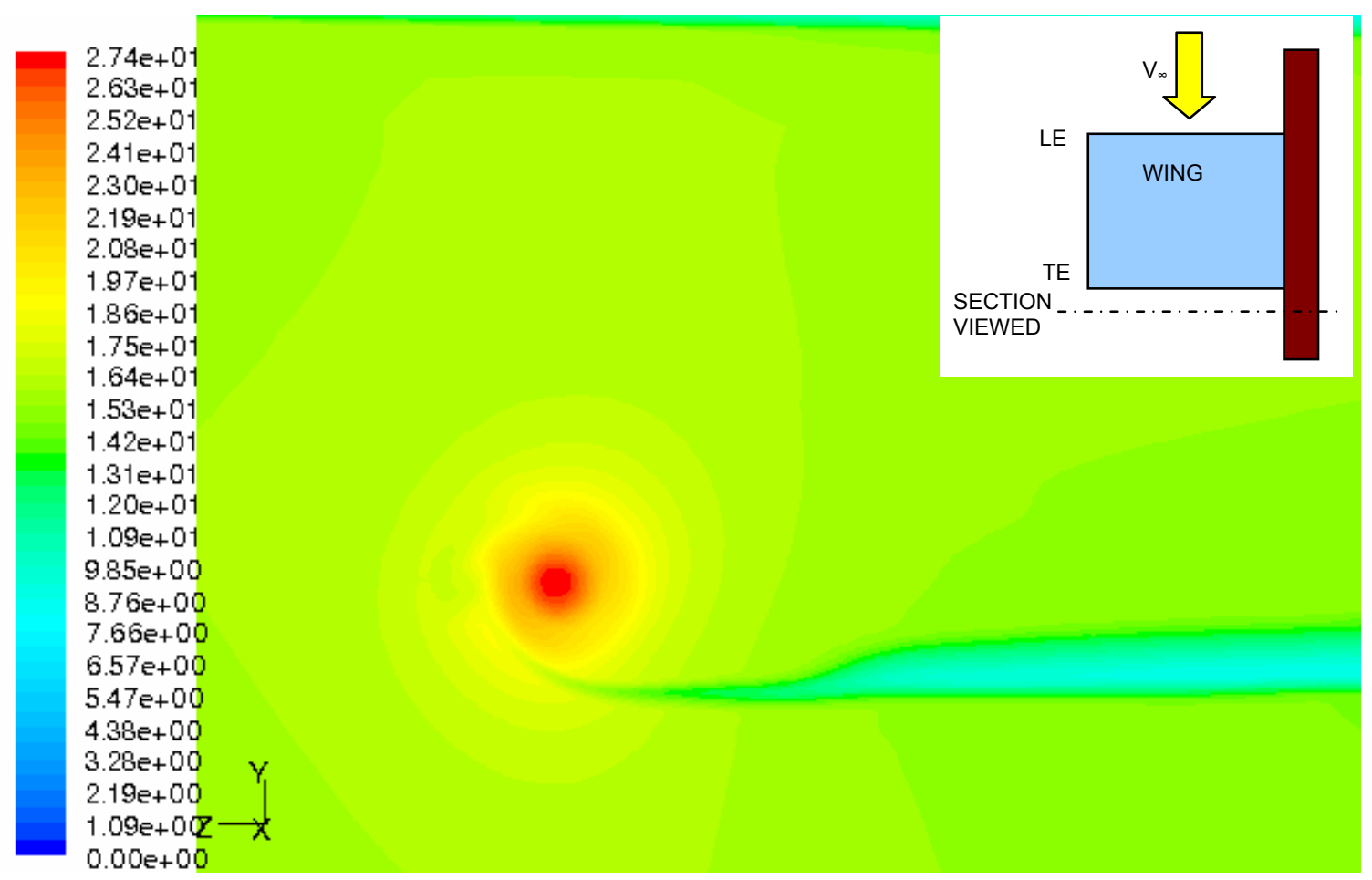

Figure 52. Velocity magnitude contours ( $(\mathrm{ft} / \mathrm{s})$ at $\mathrm{x} / \mathrm{c}=\mathbf{0 . 8 6 4}$, looking upstream from back [44].

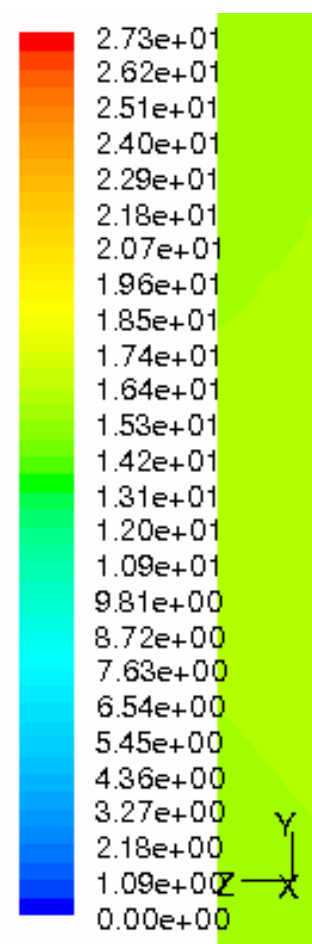

Figure 53. Velocity magnitude contours (ft/s) at $\mathrm{x} / \mathrm{c}=\mathbf{0 . 8 8 6}$, looking upstream from back [44].

Figures 54 to 56 show how the wingtip vortex increases its diameter with an accompanying decrease in the vortex core velocity. 


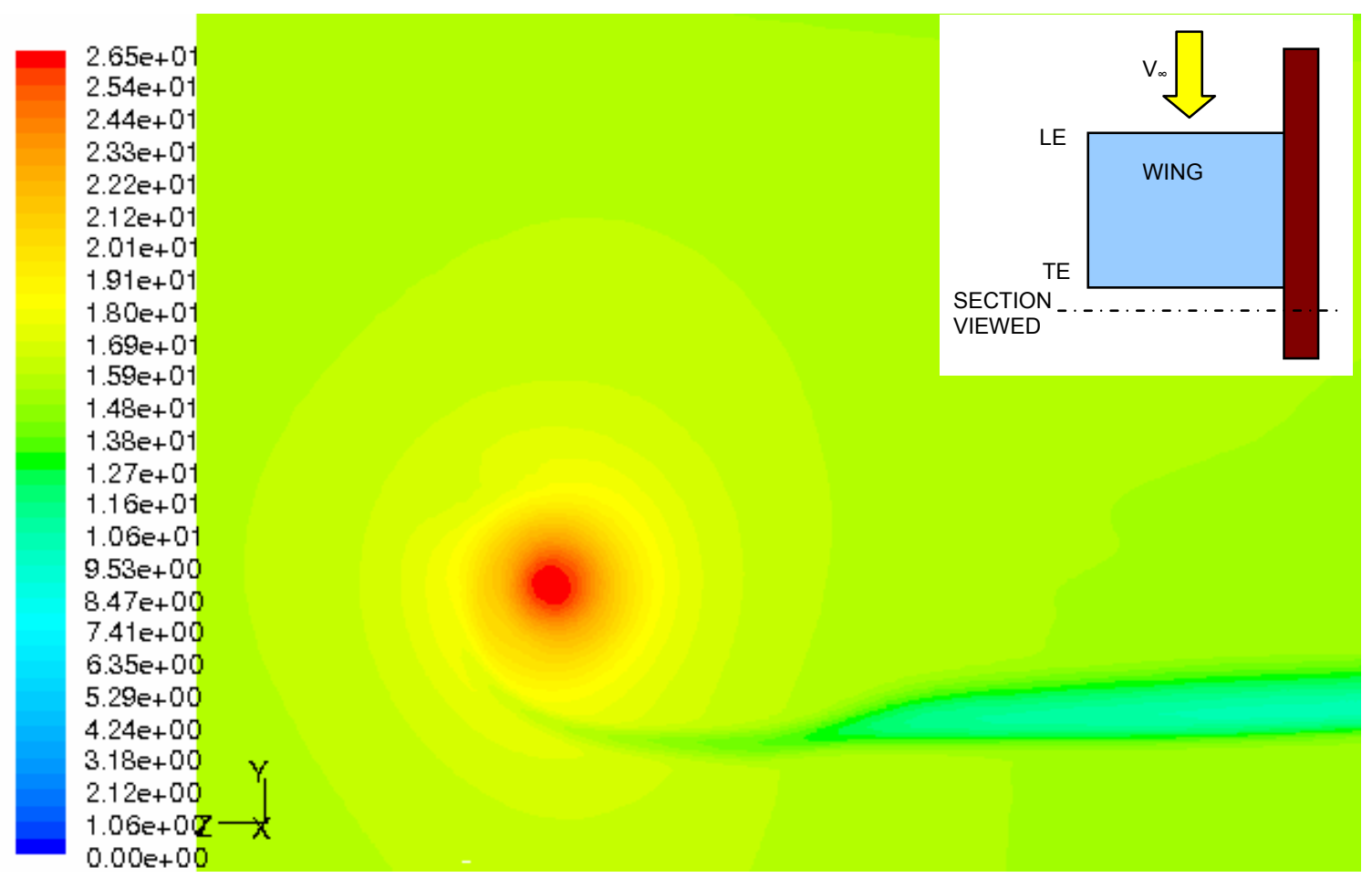

Figure 54. Velocity magnitude contours ( $(\mathrm{ft} / \mathrm{s})$ at $\mathrm{x} / \mathrm{c}=\mathbf{0 . 9 8 5}$, looking upstream from back [44].
$2.47 e+01$
$2.37 \mathrm{e}+01$
$2.27 e+01$
$2.17 \mathrm{e}+01$
$2.08 \mathrm{e}+01$
$1.98 \mathrm{e}+01$
$1.88 \mathrm{e}+01$
$1.78 \mathrm{e}+01$
$1.68 \mathrm{e}+01$
$1.58 \mathrm{e}+01$
$1.48 \mathrm{e}+01$
$1.38 \mathrm{e}+01$
$1.28 \mathrm{e}+01$
$1.19 \mathrm{e}+01$
$1.09 e+01$
$9.88 e+00$
$8.89 e+00$
$7.91 \mathrm{e}+00$
$6.92 \mathrm{e}+00$
$5.93 e+00$
$4.94 \mathrm{e}+00$
$3.95 e+00$
$2.96 \mathrm{e}+00$
$1.98 \mathrm{e}+00$
9.88e-01z $x$
$0.00 \mathrm{e}+00$

Figure 55. Velocity magnitude contours (ft/s) at $\mathrm{x} / \mathrm{c}=1.246$, looking upstream from back [44]. 
$2.26 \mathrm{e}+01$

$2.17 \mathrm{e}+01$

$2.08 \mathrm{e}+01$

$1.99 \mathrm{e}+01$

$1.90 e+01$

$1.81 \mathrm{e}+01$

$1.72 \mathrm{e}+01$

$1.63 e+01$

$1.53 e+01$

$1.44 \mathrm{e}+01$

$1.35 \mathrm{e}+01$

$1.26 \mathrm{e}+01$

$1.17 e+01$

$1.08 \mathrm{e}+01$

$9.93 \mathrm{e}+00$

$9.03 e+00$

$8.13 \mathrm{e}+00$

$7.22 \mathrm{e}+00$

$6.32 \mathrm{e}+00$

$5.42 e+00$

$4.51 \mathrm{e}+00$

$3.61 \mathrm{e}+00$

$2.71 \mathrm{e}+00$

$1.81 \mathrm{e}+00$

$9.03 \mathrm{e}-012 \longrightarrow x$

$0.00 e+00$

Figure 56. Velocity magnitude contours (ft/s) at $\mathrm{x} / \mathrm{c}=1.678$, looking upstream from back [44].

Table 2 shows the wingtip vortex core velocity variation with the downstream position. The maximum velocity magnitude in the wingtip vortex core is obtained at the downstream positions $\mathrm{x} / \mathrm{c}$ from 0.729 to 0.864 . Figure 57 shows the variation of this velocity with the downstream direction. It also shows a rapid increment in the velocity until $\mathrm{x} / \mathrm{c}=0.704$ when it reaches a constant value. After $\mathrm{x} / \mathrm{c}=0.886$, the wing tip vortex starts to dissipate shown by a slow decrease in velocity.

Table 2. Wingtip vortex core maximum velocity variation with the downstream position.

\begin{tabular}{|r|r|}
\hline $\mathbf{x} / \mathbf{c}$ & $\mathbf{V}$ (ft/s) \\
\hline 0.443 & 18.7 \\
\hline 0.542 & 22.2 \\
\hline 0.606 & 25.0 \\
\hline 0.625 & 25.6 \\
\hline 0.704 & 27.3 \\
\hline 0.729 & 27.4 \\
\hline 0.735 & 27.4 \\
\hline 0.744 & 27.3 \\
\hline 0.803 & 27.4 \\
\hline 0.864 & 27.4 \\
\hline 0.886 & 27.3 \\
\hline 0.985 & 26.5 \\
\hline 1.246 & 24.7 \\
\hline 1.678 & 20.8 \\
\hline
\end{tabular}




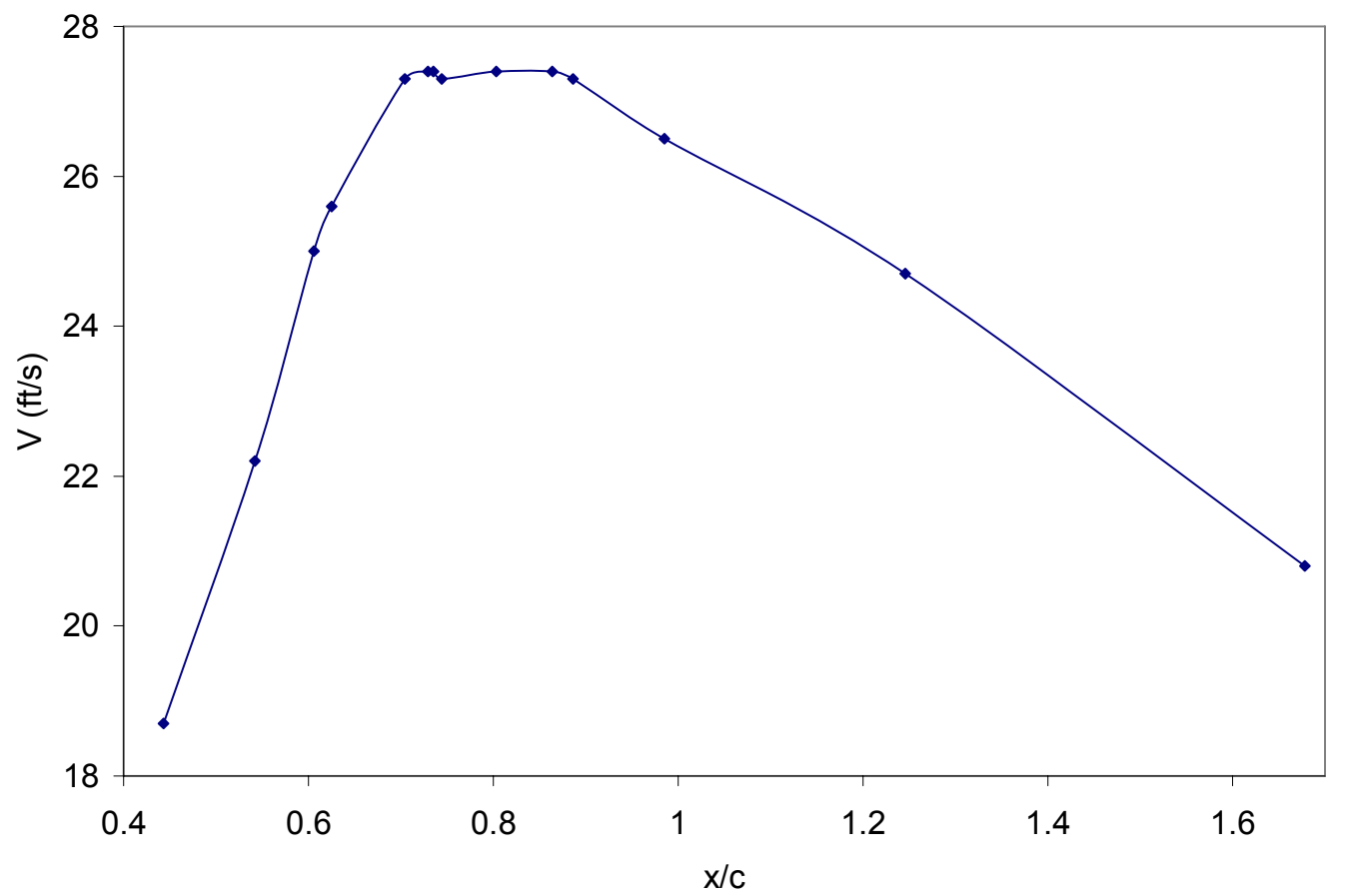

Figure 57. Wingtip vortex core maximum velocity variation in the downstream direction.

\section{Velocity Vectors}

Velocity vectors are plotted in Figures 58 to 77, again from Kim and Rhee [44], showing the details of the wingtip vortex formation. At $\mathrm{x} / \mathrm{c}=0.25$ (Figure 58), the streamlines flowing from the bottom wing surface to the top start to detach from the wing surface and flow almost vertically. 

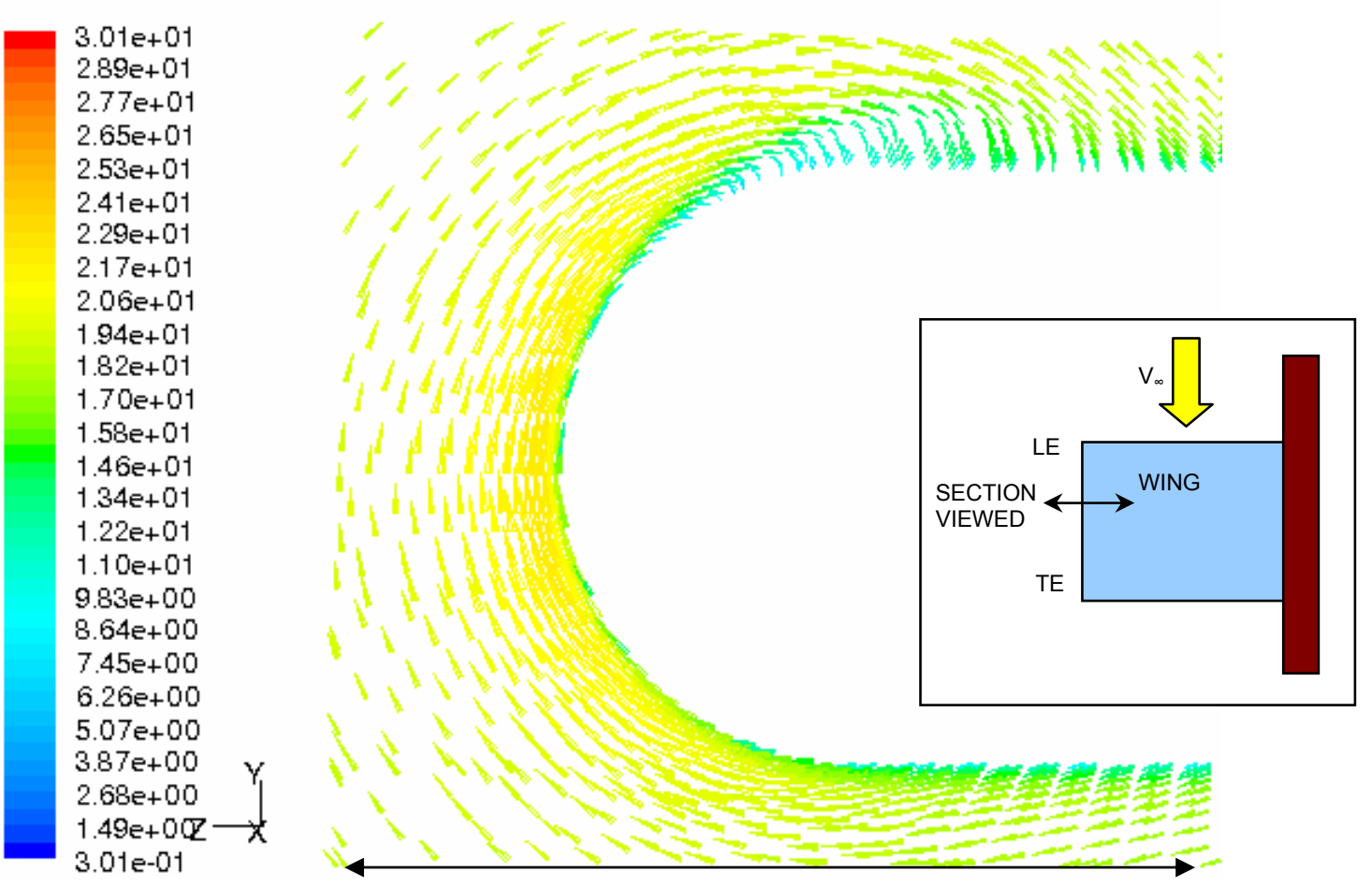

Figure 58. Velocity vectors and magnitude (ft/s) at $x / c=0.25$, looking upstream from back [44].

Figure 59 shows the boundary layer separation and a recirculation region on the top wing surface.
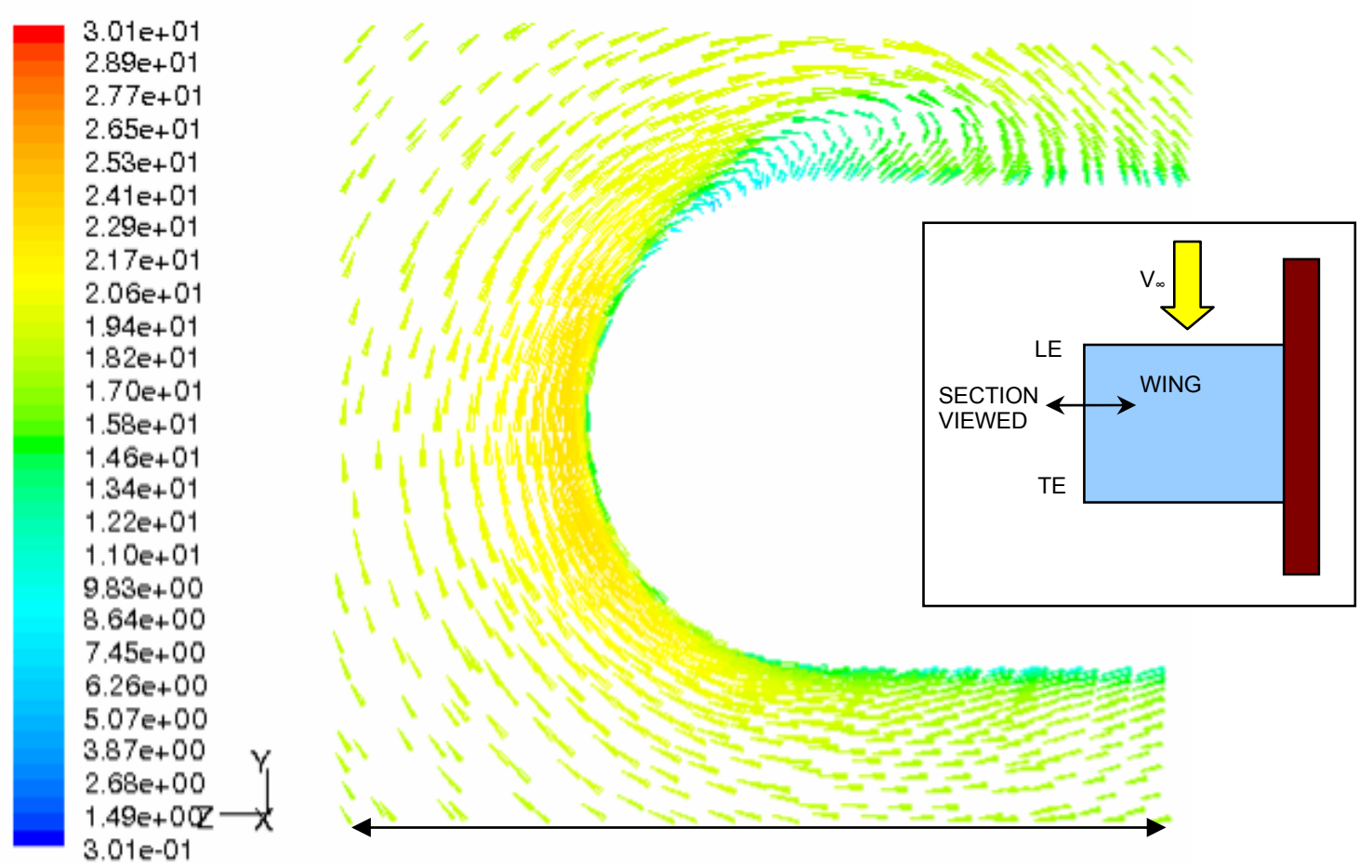

Figure 59. Velocity vectors and magnitude (ft/s) at $\mathrm{x} / \mathrm{c}=0.345$, looking upstream from back [44]. 
Figure 60 shows how this recirculation region is growing as more air comes from the bottom surface.
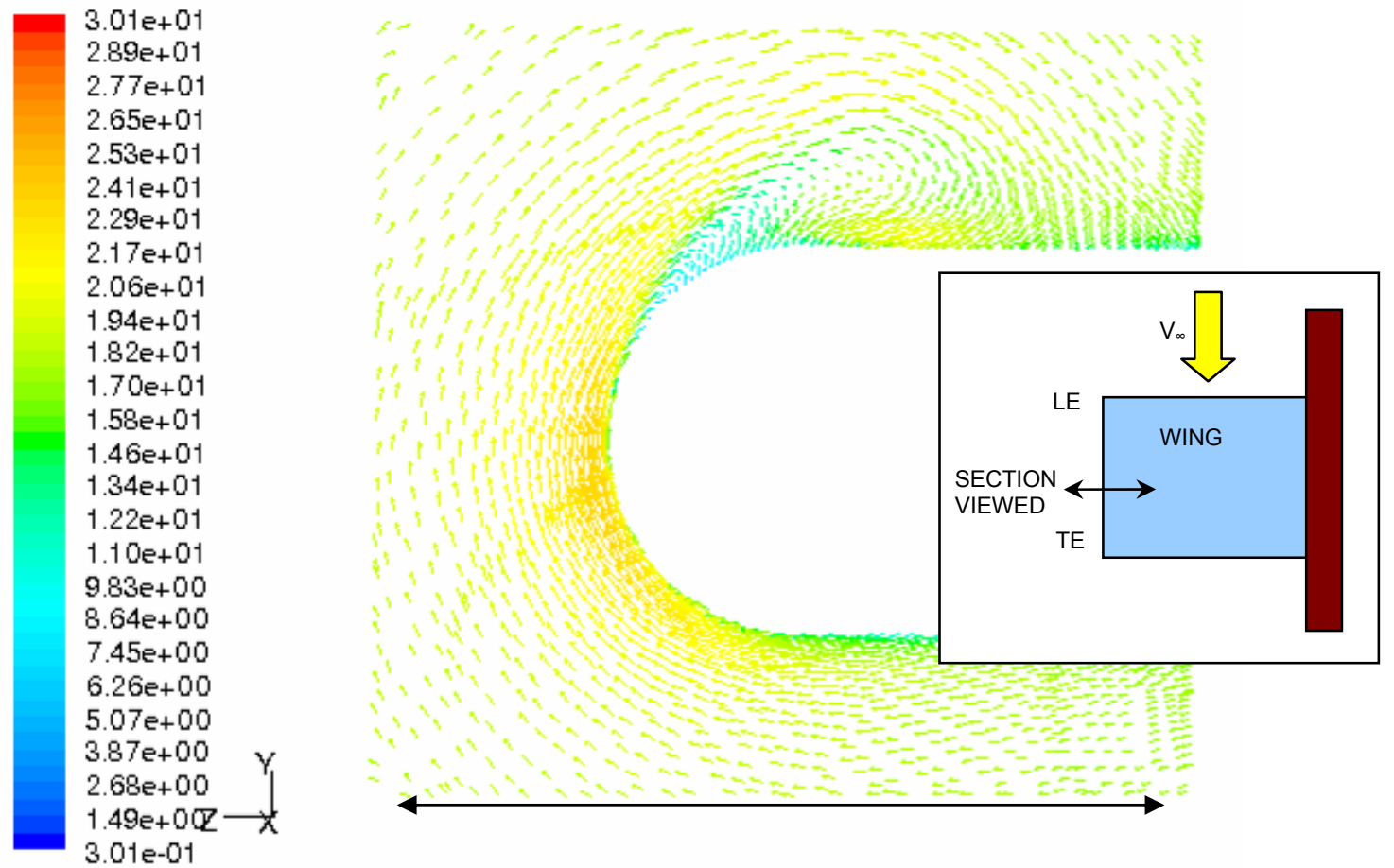

Figure 60. Velocity vectors and magnitude $(\mathrm{ft} / \mathrm{s})$ at $\mathrm{x} / \mathrm{c}=\mathbf{0 . 4 4 3}$, looking upstream from back [44].

$3.01 e+01$
$2.89 e+01$
$2.77 e+01$
$2.65 e+01$
$2.53 e+01$
$2.41 e+01$
$2.29 e+01$
$2.17 e+01$
$2.06 e+01$
$1.94 e+01$
$1.82 e+01$
$1.70 e+01$
$1.58 e+01$
$1.46 e+01$
$1.34 e+01$
$1.22 e+01$
$1.10 e+01$
$9.83 e+00$
$8.64 e+00$
$7.45 e+00$
$6.26 e+00$
$5.07 e+00$
$3.87 e+00$
$2.68 e+00$
$1.49 e+00 Z$
$3.01 e-01$

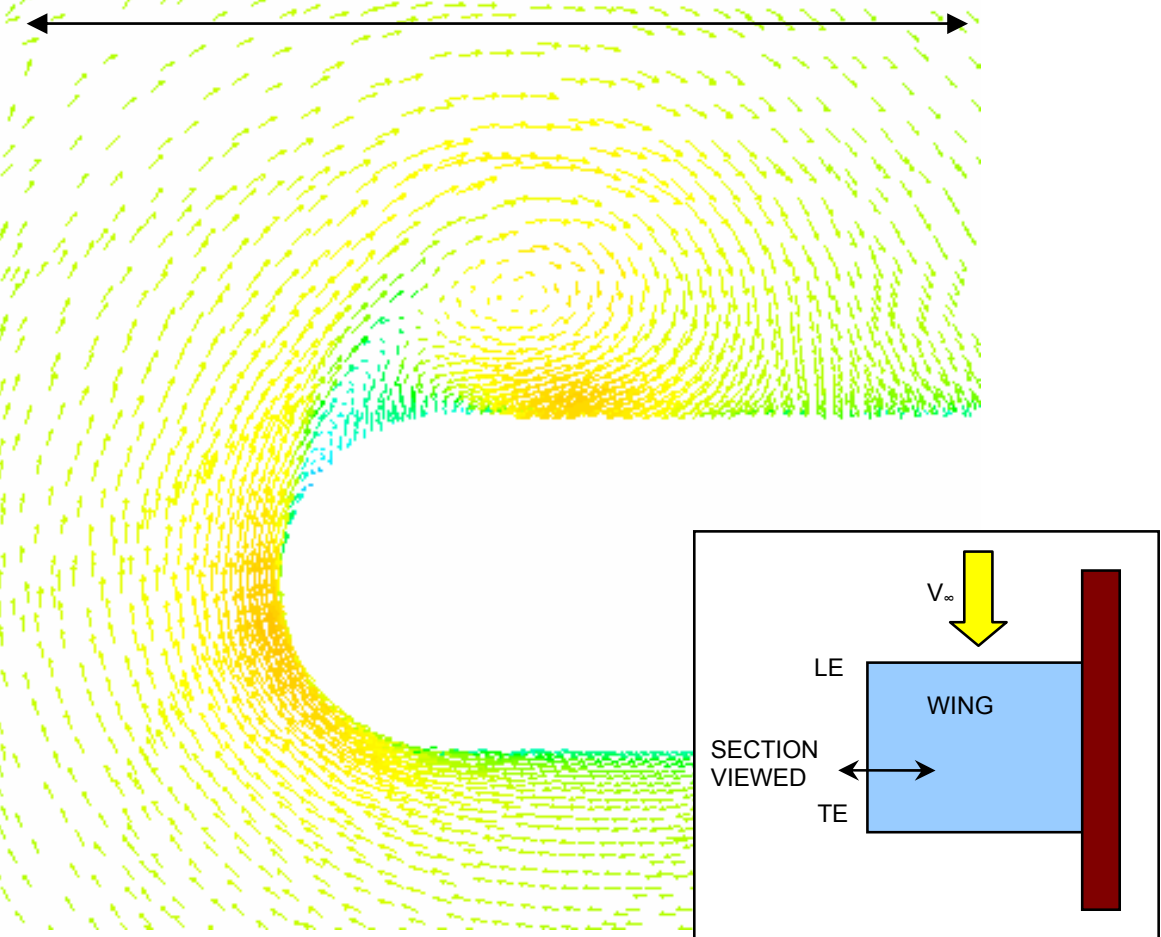

Figure 61. Velocity vectors and magnitude $(\mathrm{ft} / \mathrm{s})$ at $\mathrm{x} / \mathrm{c}=0.542$, looking upstream from back [44]. 
Figure 62 is a zoom into the vortex core shown in Figure 61.

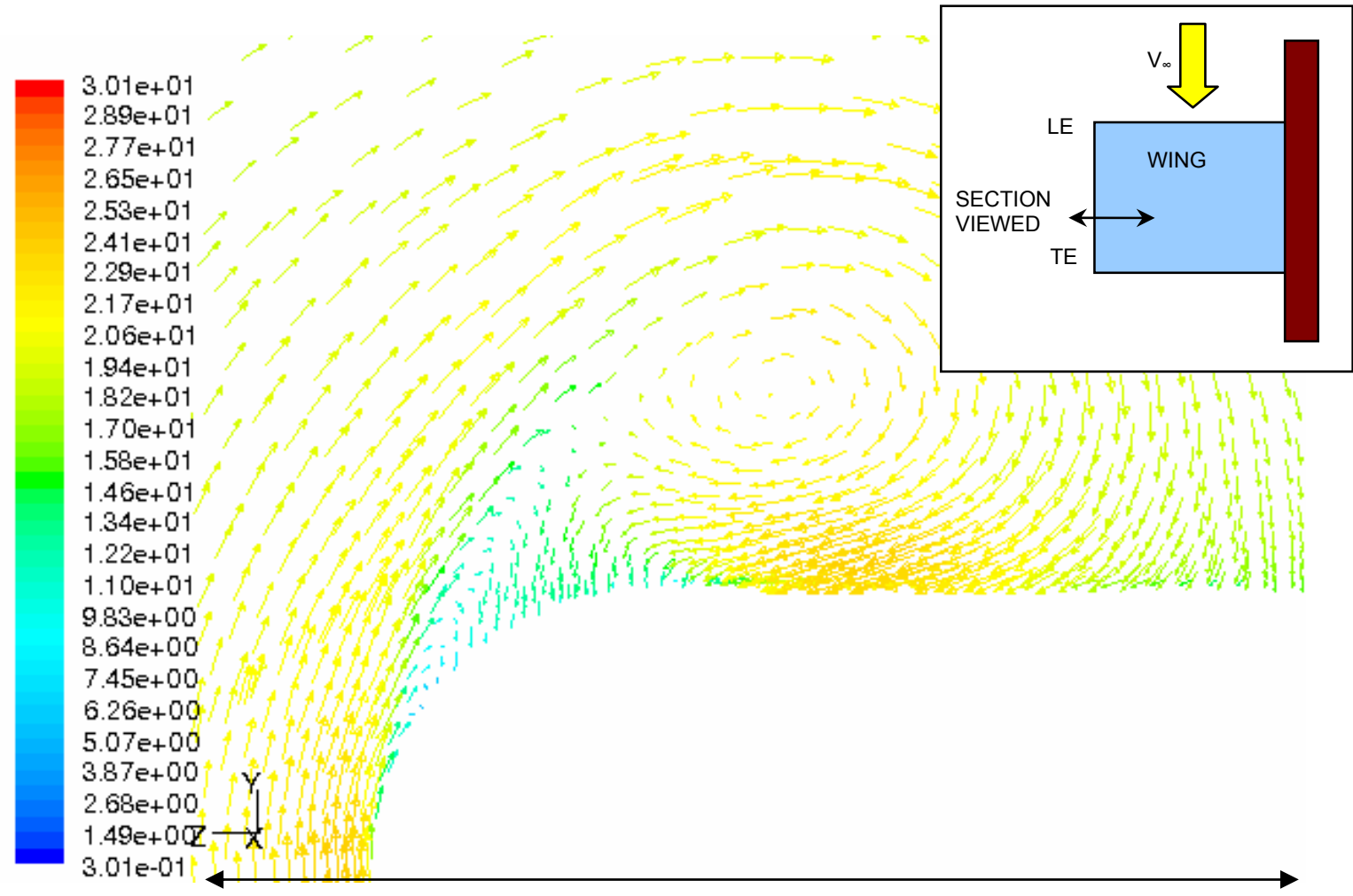

Figure 62. Velocity vectors and magnitude $(\mathrm{ft} / \mathrm{s})$ at $\mathrm{x} / \mathrm{c}=0.542$, looking upstream from back [44].
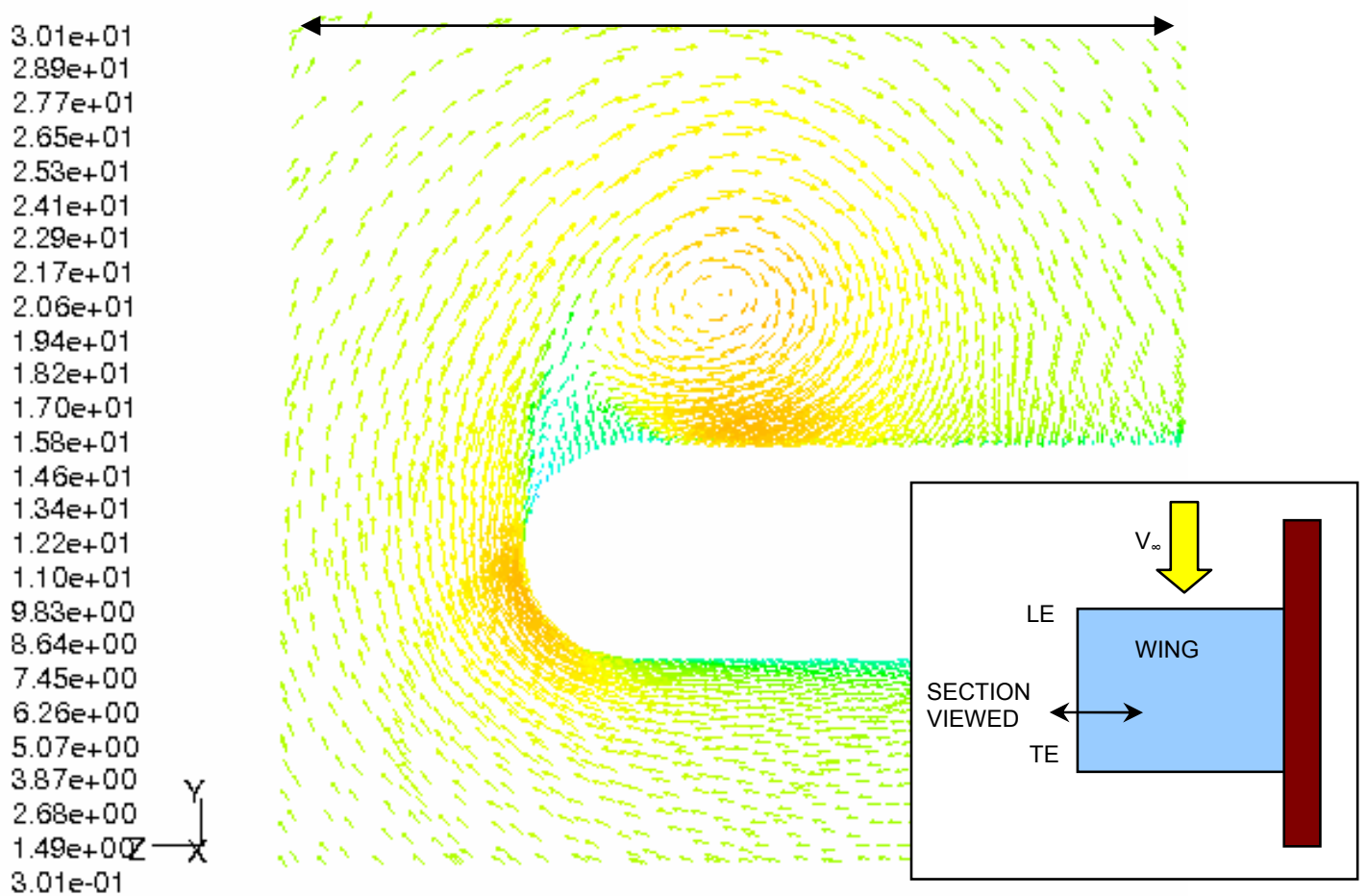

Figure 63. Velocity vectors and magnitude (ft/s) at $\mathrm{x} / \mathrm{c}=0.606$, looking upstream from back [44]. 
Figure 64 shows a zoom in of Figure 63. In this figure, the recirculation region is clearly shown.

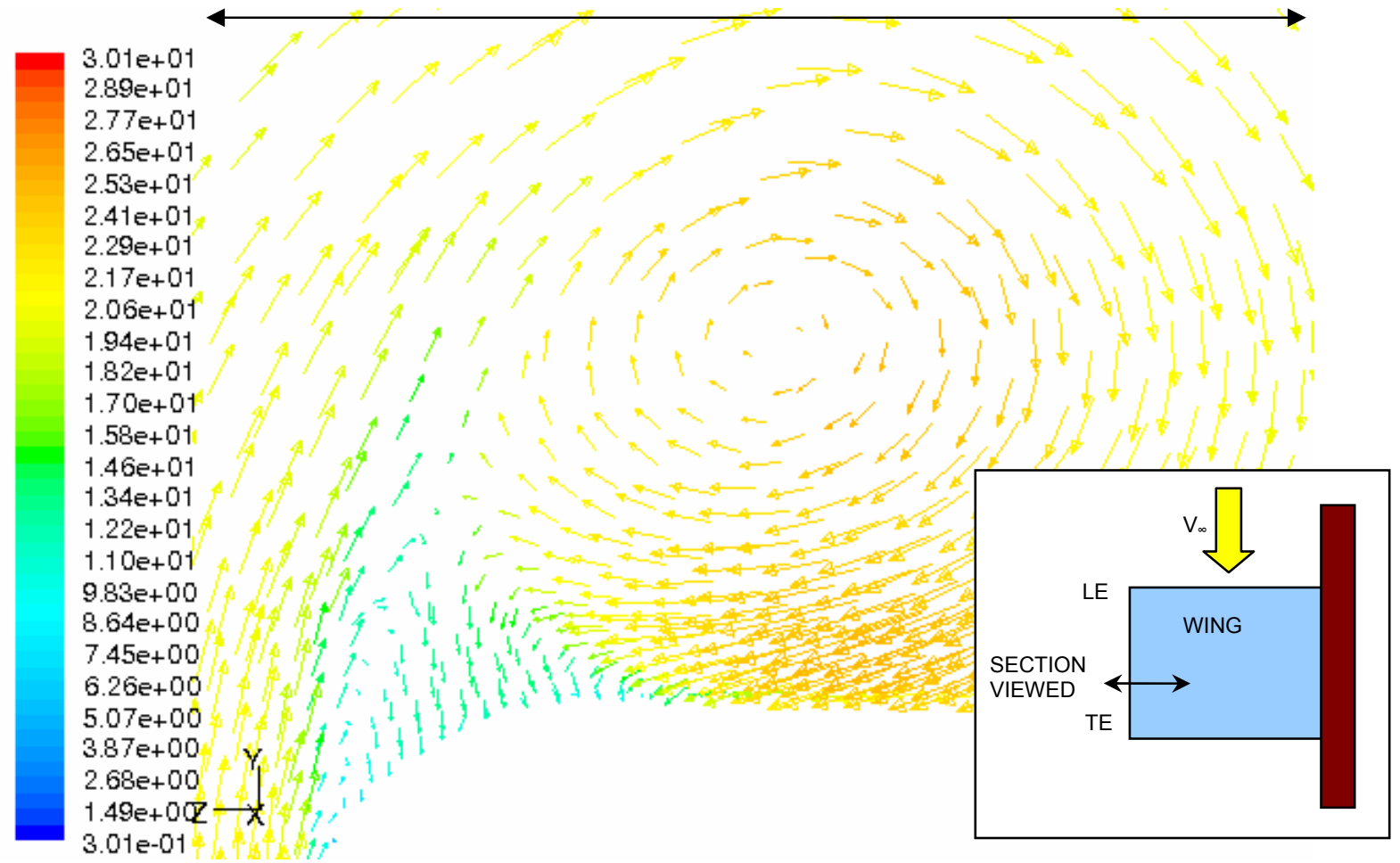

Figure 64. Velocity vectors and magnitude (ft/s) at $\mathrm{x} / \mathrm{c}=0.606$, looking upstream from back [44].

The wingtip vortex starts to move upward and to increase in diameter at $\mathrm{x} / \mathrm{c}=0.625$ (Figure 65). 
$3.01 \mathrm{e}+01$

$2.89 \mathrm{e}+01$

$2.77 e+01$

$2.65 e+01$

$2.53 \mathrm{e}+01$

$2.41 \mathrm{e}+01$

$2.29 \mathrm{e}+01$

$2.17 e+01$

$2.06 \mathrm{e}+01$

$1.94 \mathrm{e}+01$

$1.82 \mathrm{e}+01$

$1.70 \mathrm{e}+01$

$1.58 \mathrm{e}+01$

$146 e+01$

$1.34 \mathrm{e}+01$

$1.22 \mathrm{e}+01$

$1.10 \mathrm{e}+01$

$9.83 e+00$

$8.64 \mathrm{e}+00$

$7.45 \mathrm{e}+00$

$6.26 \mathrm{e}+00$

$5.07 e+00$

$3.87 e+00$

$2.68 \mathrm{e}+00$

$1.49 \mathrm{e}+0 \mathrm{QR}$

3.01e-01

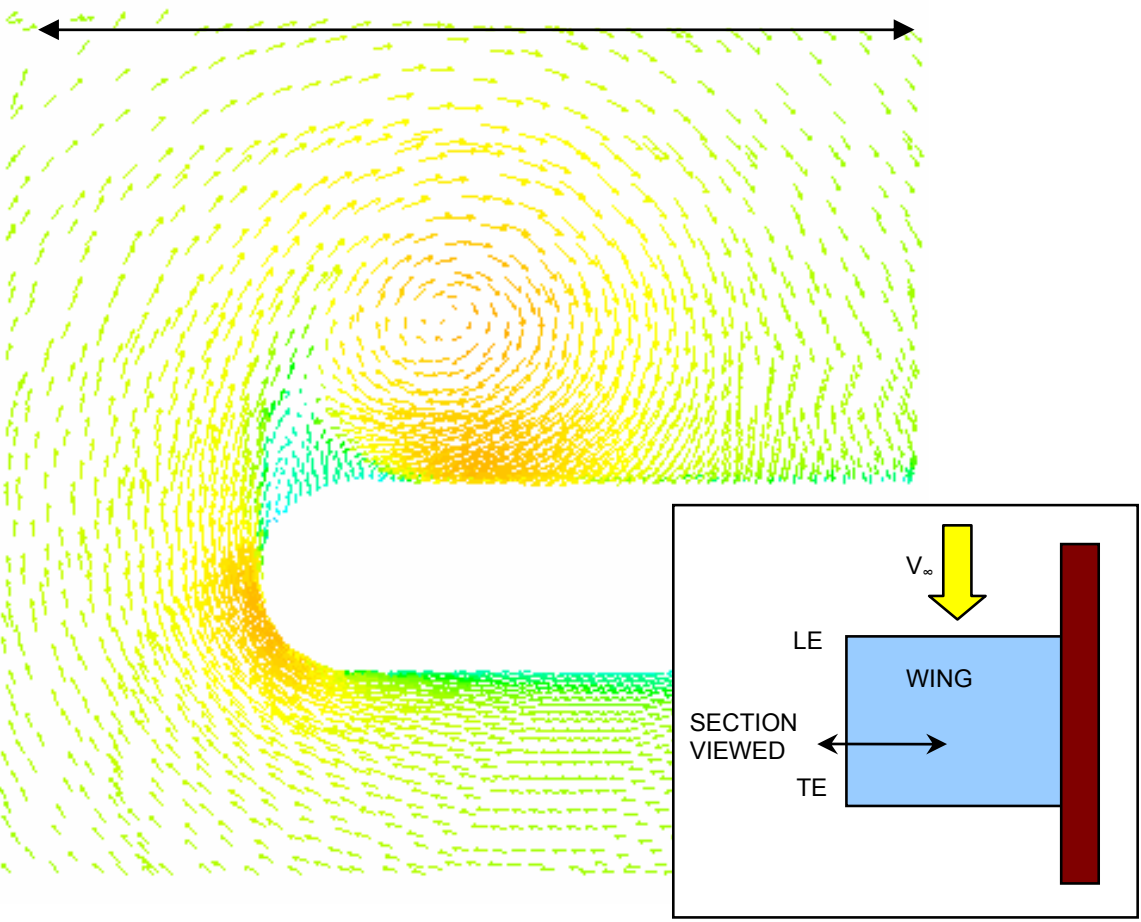

Figure 65. Velocity vectors and magnitude (ft/s) at $\mathrm{x} / \mathrm{c}=\mathbf{0 . 6 2 5}$, looking upstream from back [44].

Figure 66 shows a zoom in of Figure 65 at $\mathrm{x} / \mathrm{c}=0.625$.

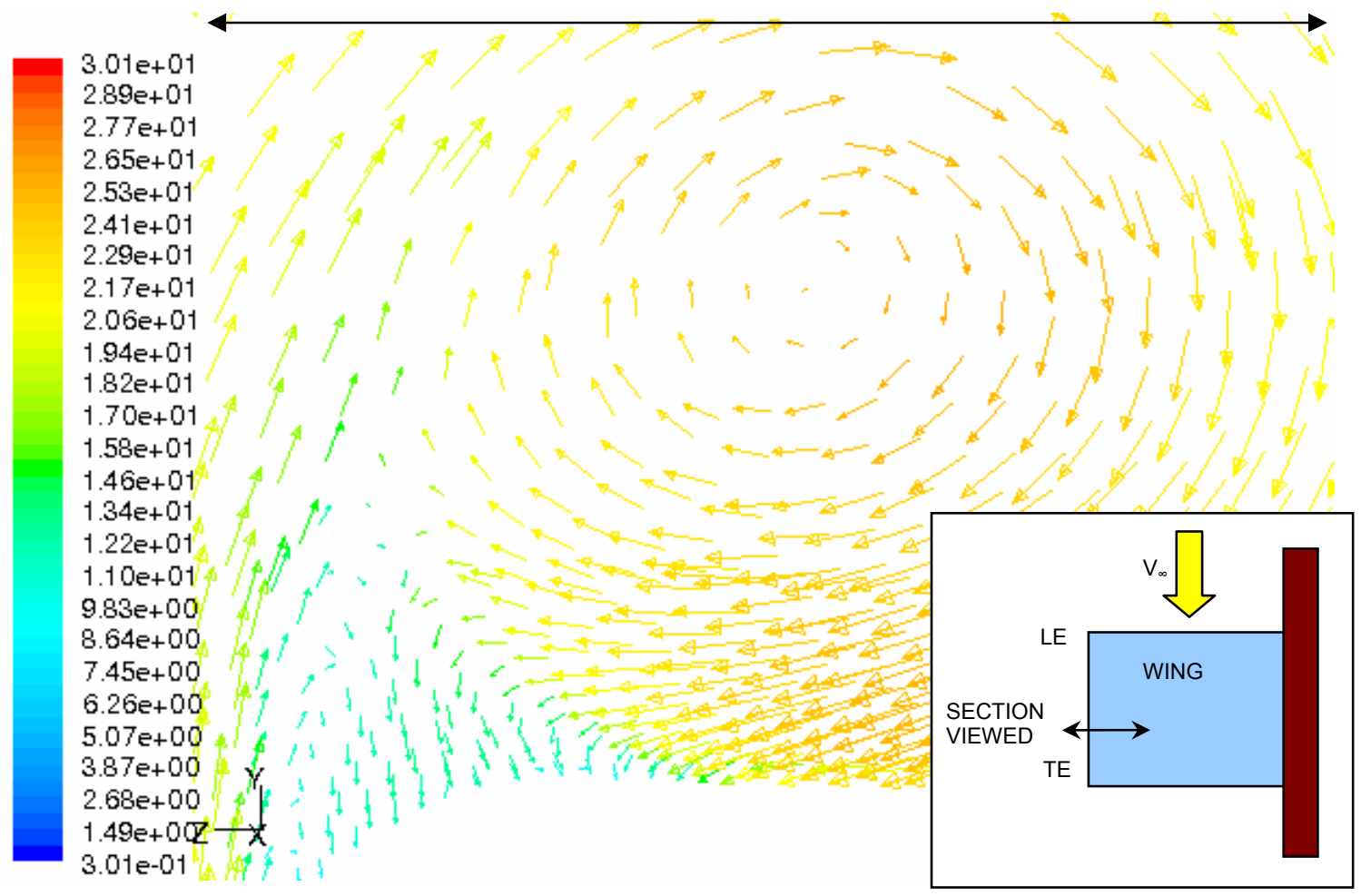

Figure 66. Velocity vectors and magnitude ( $\mathrm{ft} / \mathrm{s})$ at $\mathrm{x} / \mathrm{c}=\mathbf{0 . 6 2 5}$, looking upstream from back [44].

Figure 67 shows the velocity vectors at $\mathrm{x} / \mathrm{c}=0.704$. 


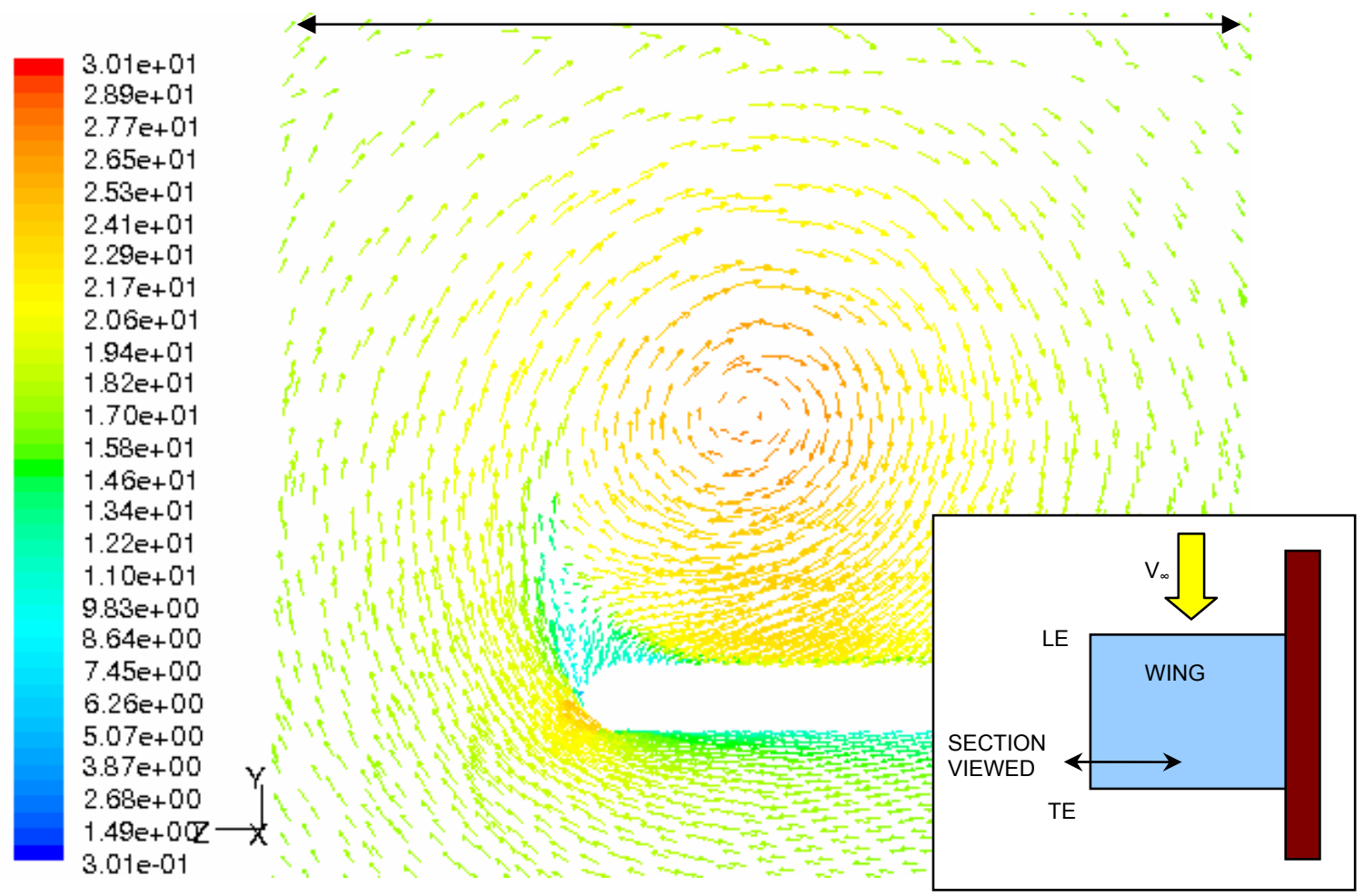

Figure 67. Velocity vectors and magnitude $(\mathrm{ft} / \mathrm{s})$ at $\mathrm{x} / \mathrm{c}=0.704$, looking upstream from back [44].

Figure 68 shows a zoom in of Figure 67 at x/c=0.704.

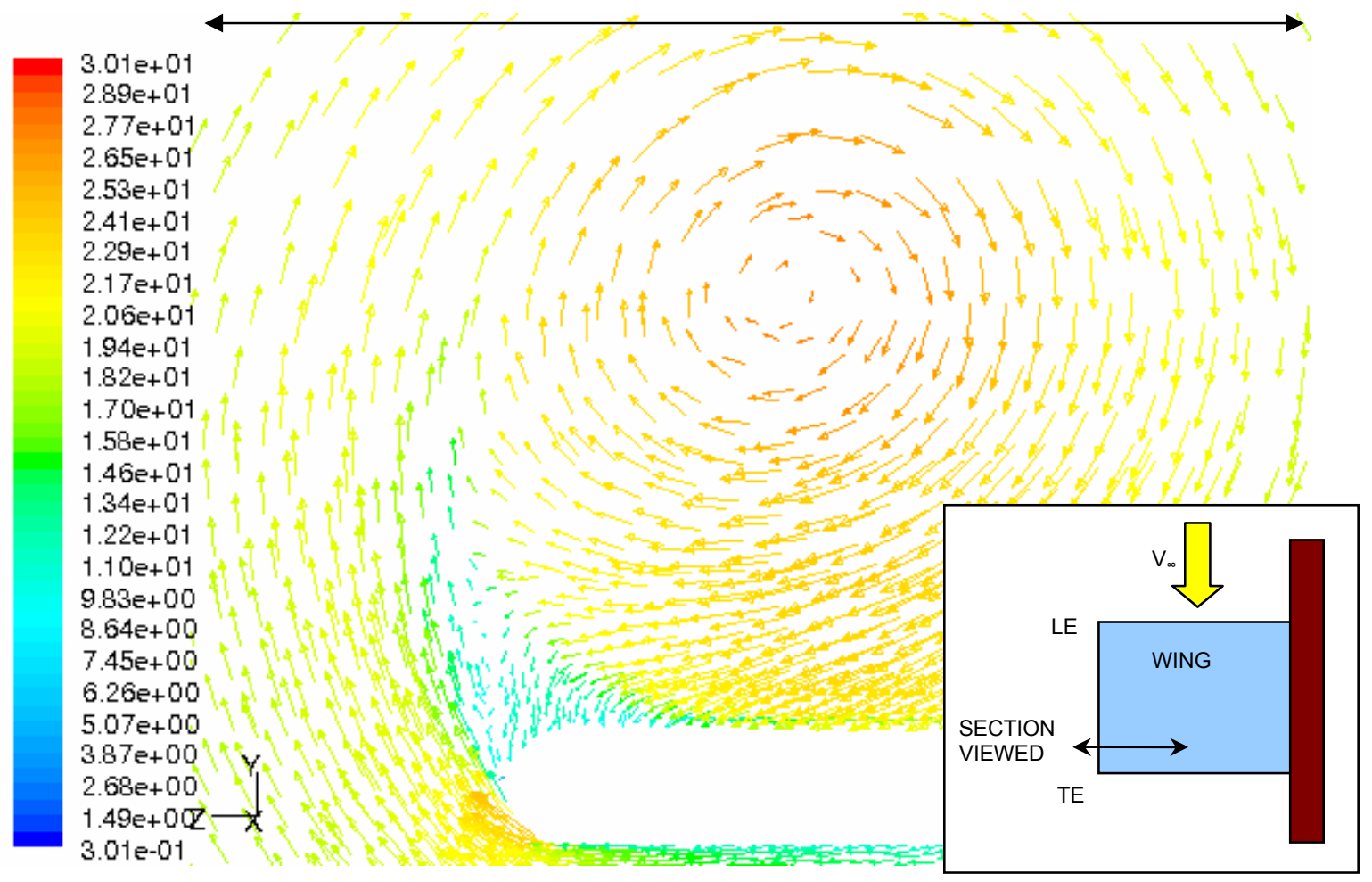

Figure 68. Velocity vectors and magnitude $(\mathrm{ft} / \mathrm{s})$ at $\mathrm{x} / \mathrm{c}=\mathbf{0 . 7 0 4}$, looking upstream from back [44].

The velocity vectors at $\mathrm{x} / \mathrm{c}=0.729$ are shown in Figure 69 . 
$3.01 \mathrm{e}+01$

$2.89 \mathrm{e}+01$

$2.77 \mathrm{e}+01$

$2.65 e+01$

$2.53 \mathrm{e}+01$

$2.41 \mathrm{e}+01$

$2.29 \mathrm{e}+01$

$2.17 \mathrm{e}+01$

$2.06 \mathrm{e}+01$

$1.94 \mathrm{e}+01$

$1.82 \mathrm{e}+01$

$1.70 \mathrm{e}+01$

$1.58 \mathrm{e}+01$

$146 e+01$

$1.34 \mathrm{e}+01$

$1.22 \mathrm{e}+01$

$1.10 \mathrm{e}+01$

$9.83 e+00$

$8.64 \mathrm{e}+00$

$7.45 \mathrm{e}+00$

$6.26 \mathrm{e}+00$

$5.07 e+00$

$3.87 e+00$

$2.68 \mathrm{e}+00$

$1.49 \mathrm{e}+0 \mathrm{R} \longrightarrow \mathrm{x}$

3.01e-01

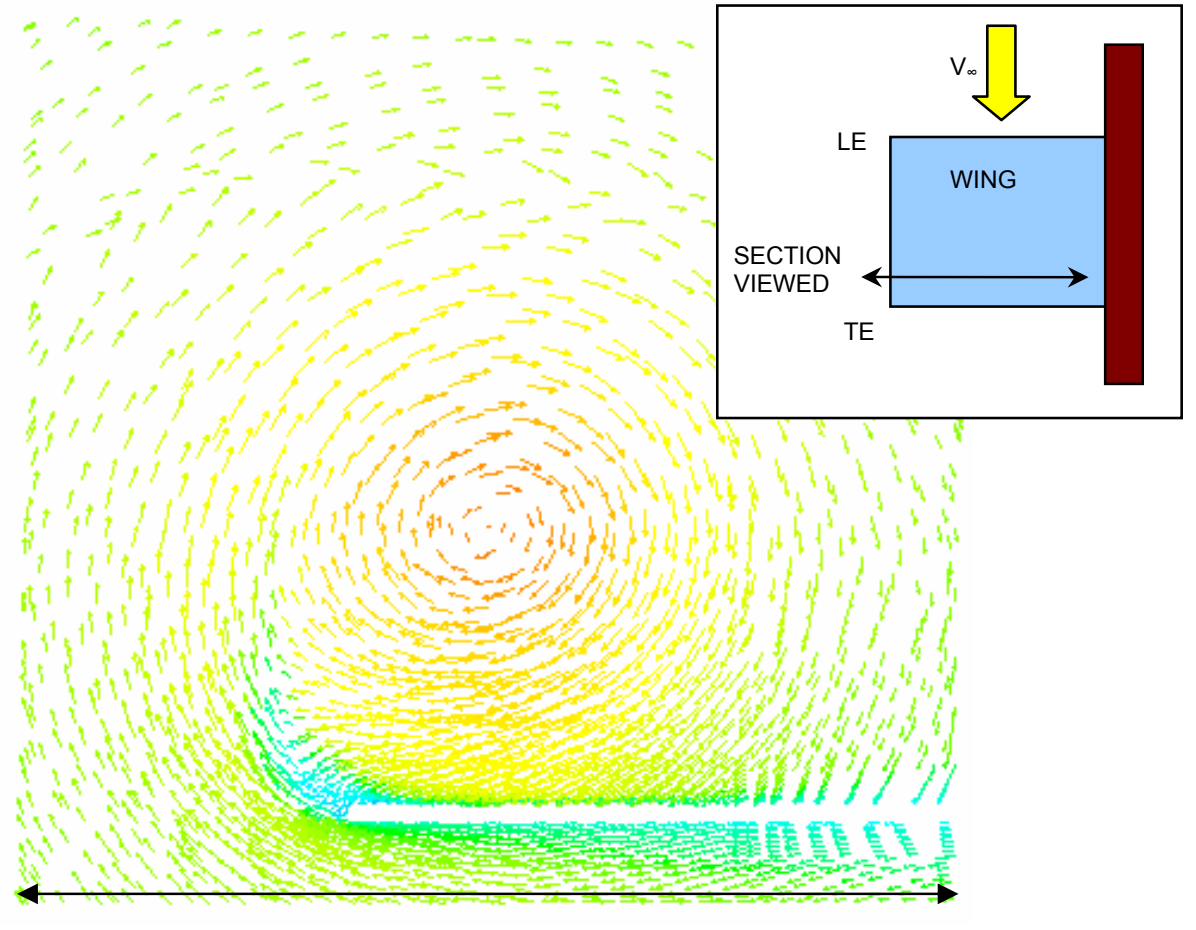

Figure 69. Velocity vectors and magnitude (ft/s) at $\mathbf{x} / \mathrm{c}=0.729$, looking upstream from back [44].

Figure 70 shows a zoom in of Figure 69 at $\mathrm{x} / \mathrm{c}=0.729$.

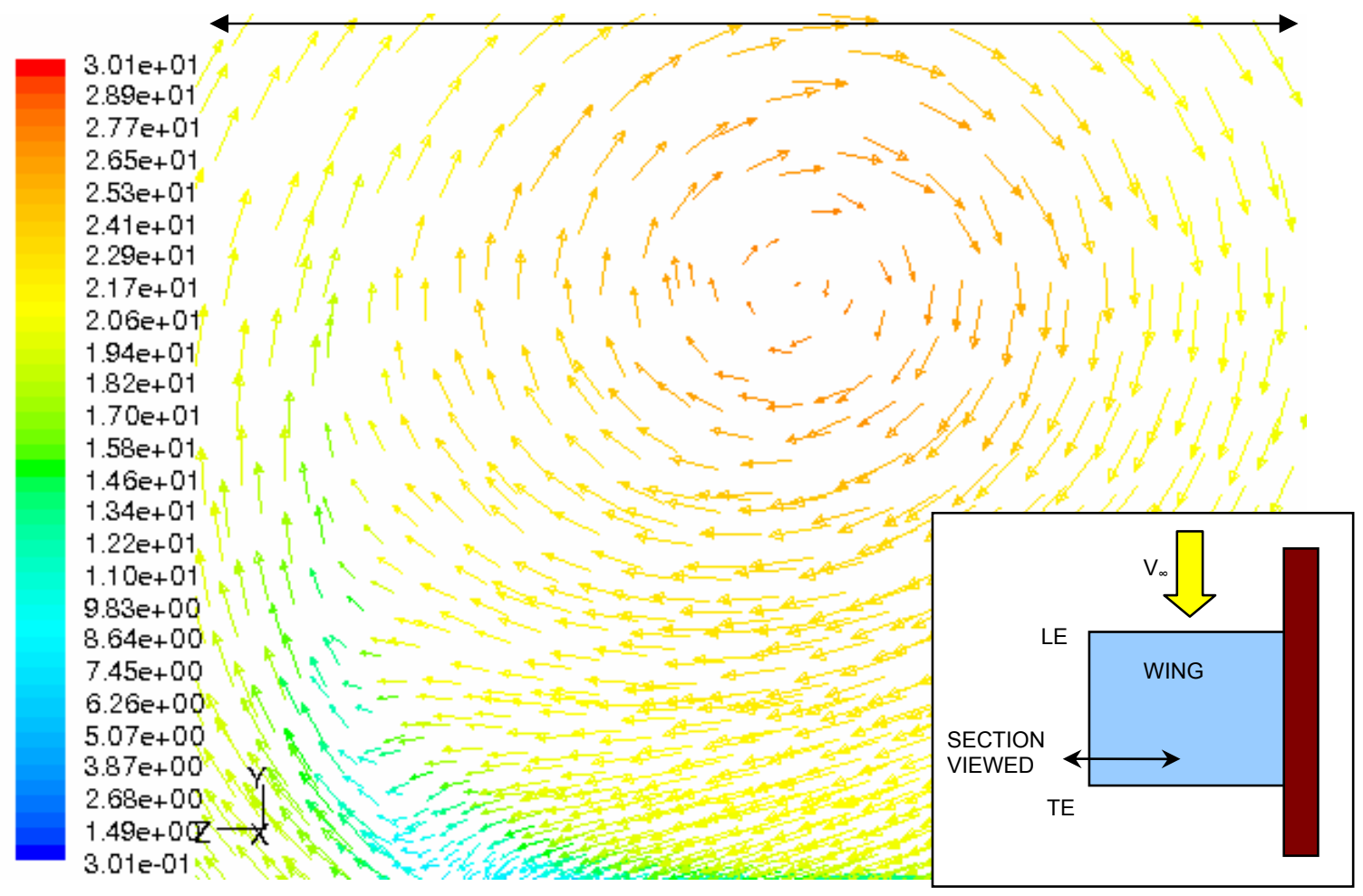

Figure 70. Velocity vectors and magnitude ( $\mathrm{ft} / \mathrm{s})$ at $\mathrm{x} / \mathrm{c}=\mathbf{0 . 7 2 9}$, looking upstream from back [44].

Figure 71 shows the velocity vectors at $\mathrm{x} / \mathrm{c}=0.735$. 


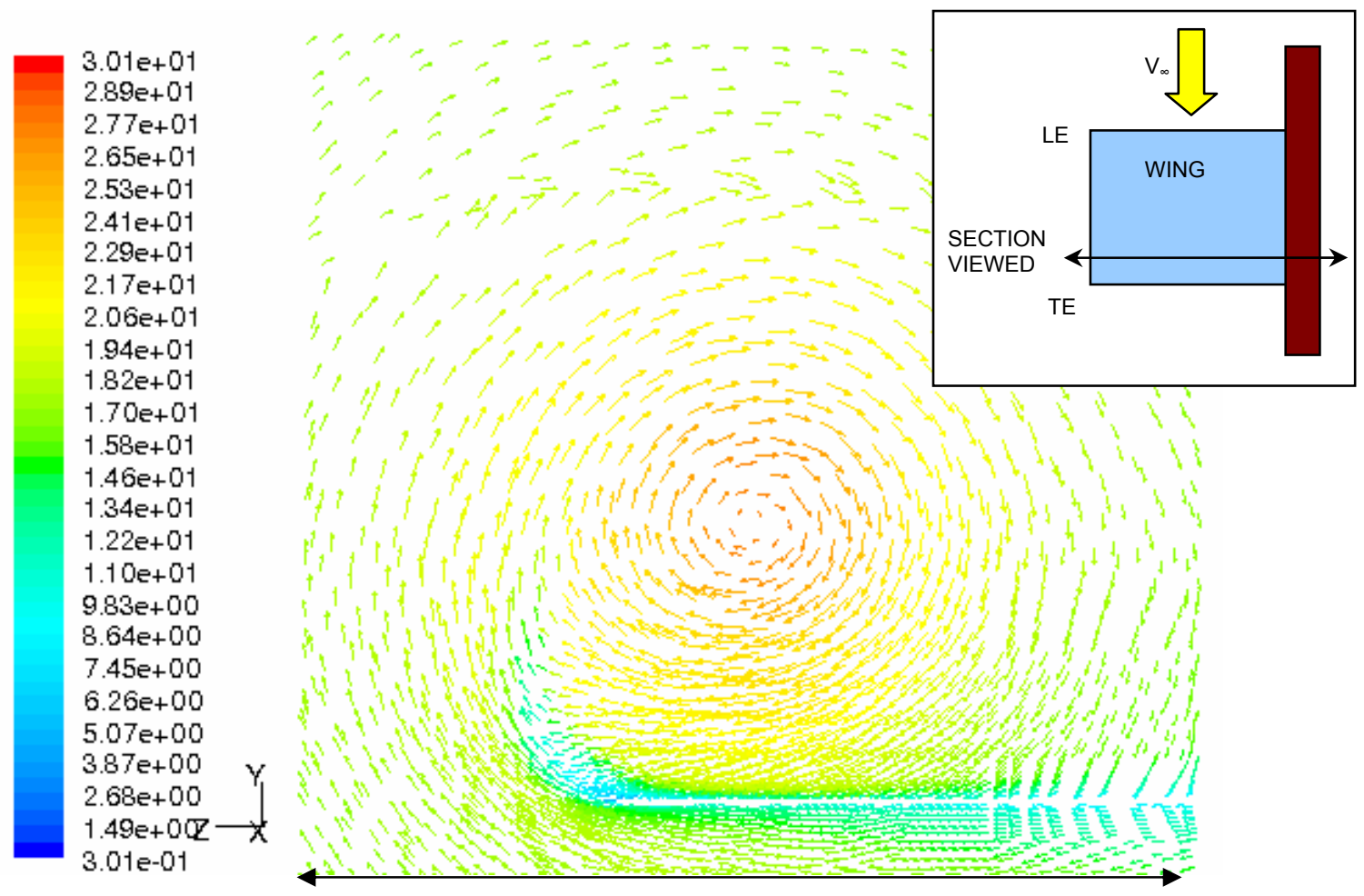

Figure 71. Velocity vectors and magnitude $(\mathrm{ft} / \mathrm{s})$ at $\mathrm{x} / \mathrm{c}=\mathbf{0 . 7 3 5}$, looking upstream from back [44].

Figure 72 shows the velocity vectors at $\mathrm{x} / \mathrm{c}=0.744$.
$3.01 \mathrm{e}+01$
$2.89 \mathrm{e}+01$
$2.77 e+01$
$2.65 \mathrm{e}+01$
$2.53 \mathrm{e}+01$
$2.41 \mathrm{e}+01$
$2.29 \mathrm{e}+01$
$2.17 \mathrm{e}+01$
$2.06 \mathrm{e}+01$
$1.94 \mathrm{e}+01$
$1.82 \mathrm{e}+01$
$1.70 \mathrm{e}+01$
$1.58 \mathrm{e}+01$
$1.46 \mathrm{e}+01$
$1.34 \mathrm{e}+01$
$1.22 \mathrm{e}+01$
1.10e+01
$9.83 \mathrm{e}+00$
$8.64 \mathrm{e}+00$
$7.45 e+00$
$6.26 \mathrm{e}+00$
$5.07 e+00$
$3.87 e+00$
$2.68 \mathrm{e}+00$
$1.49 e+0 Q \longrightarrow x$
3.01e-01

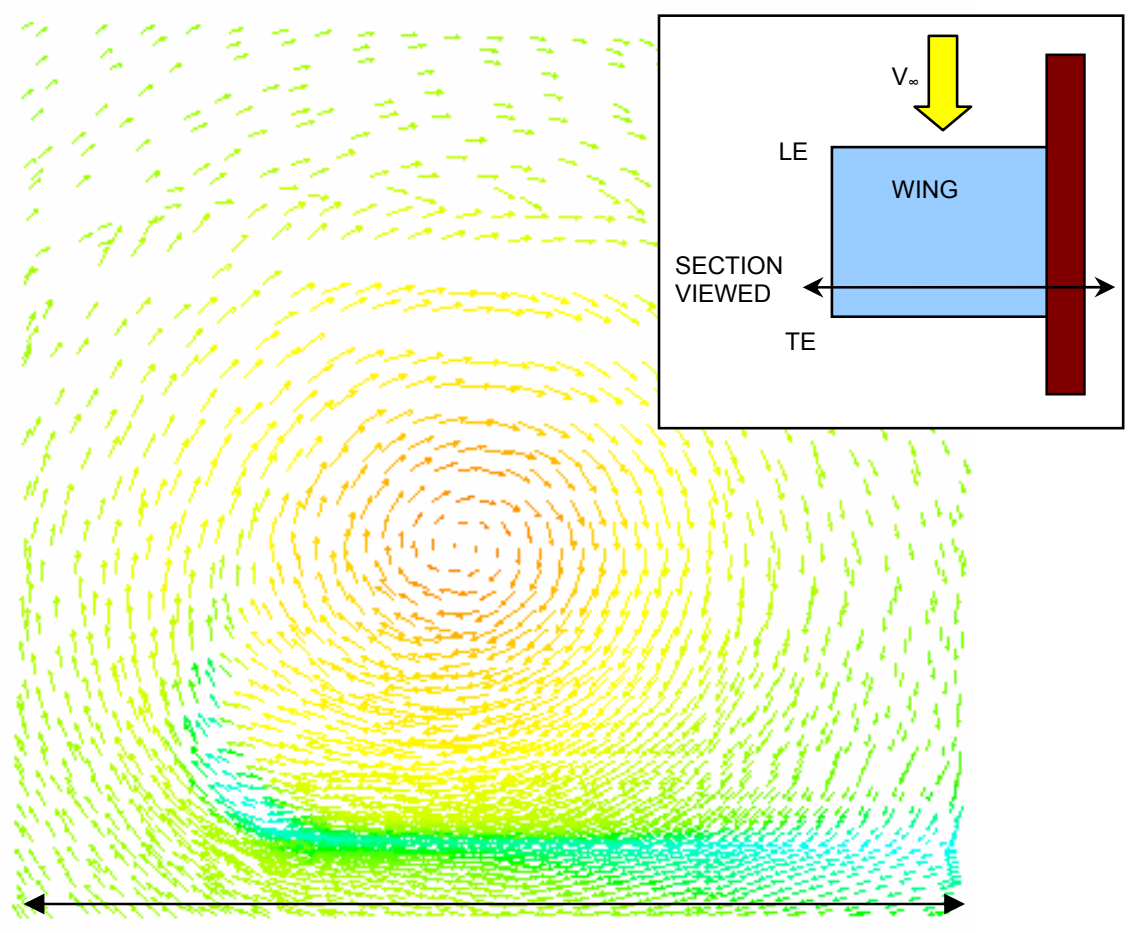

Figure 72. Velocity vectors and magnitude (ft/s) at $\mathrm{x} / \mathrm{c}=\mathbf{0 . 7 4 4}$, looking upstream from back [44].

Figure 73 shows the velocity vectors at $\mathrm{x} / \mathrm{c}=0.803$. 
$3.01 \mathrm{e}+01$

$2.89 \mathrm{e}+01$

$2.77 \mathrm{e}+01$

$2.65 \mathrm{e}+01$

$2.53 \mathrm{e}+01$

$2.41 \mathrm{e}+01$

$2.29 \mathrm{e}+01$

$2.17 \mathrm{e}+01$

$2.06 \mathrm{e}+01$

$1.94 \mathrm{e}+01$

$1.82 \mathrm{e}+01$

$1.70 \mathrm{e}+01$

$1.58 \mathrm{e}+01$

$1.46 \mathrm{e}+01$

$1.34 \mathrm{e}+01$

$1.22 \mathrm{e}+01$

$1.10 \mathrm{e}+01$

$9.83 e+00$

$8.64 \mathrm{e}+00$

$7.45 \mathrm{e}+00$

$6.26 \mathrm{e}+00$

$5.07 e+00$

$3.87 e+00$

$2.68 \mathrm{e}+00$

$1.49 \mathrm{e}+0 \mathrm{QR}$

3.01e-01

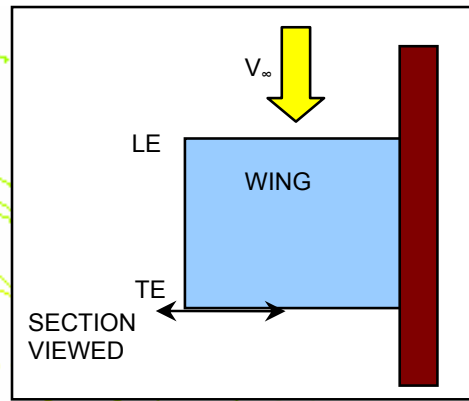

Figure 73. Velocity vectors and magnitude (ft/s) at $\mathbf{x} / \mathbf{c}=\mathbf{0 . 8 0 3}$, looking upstream from back [44].

The velocity vectors at $\mathrm{x} / \mathrm{c}=0.864$ are shown in Figure 74.
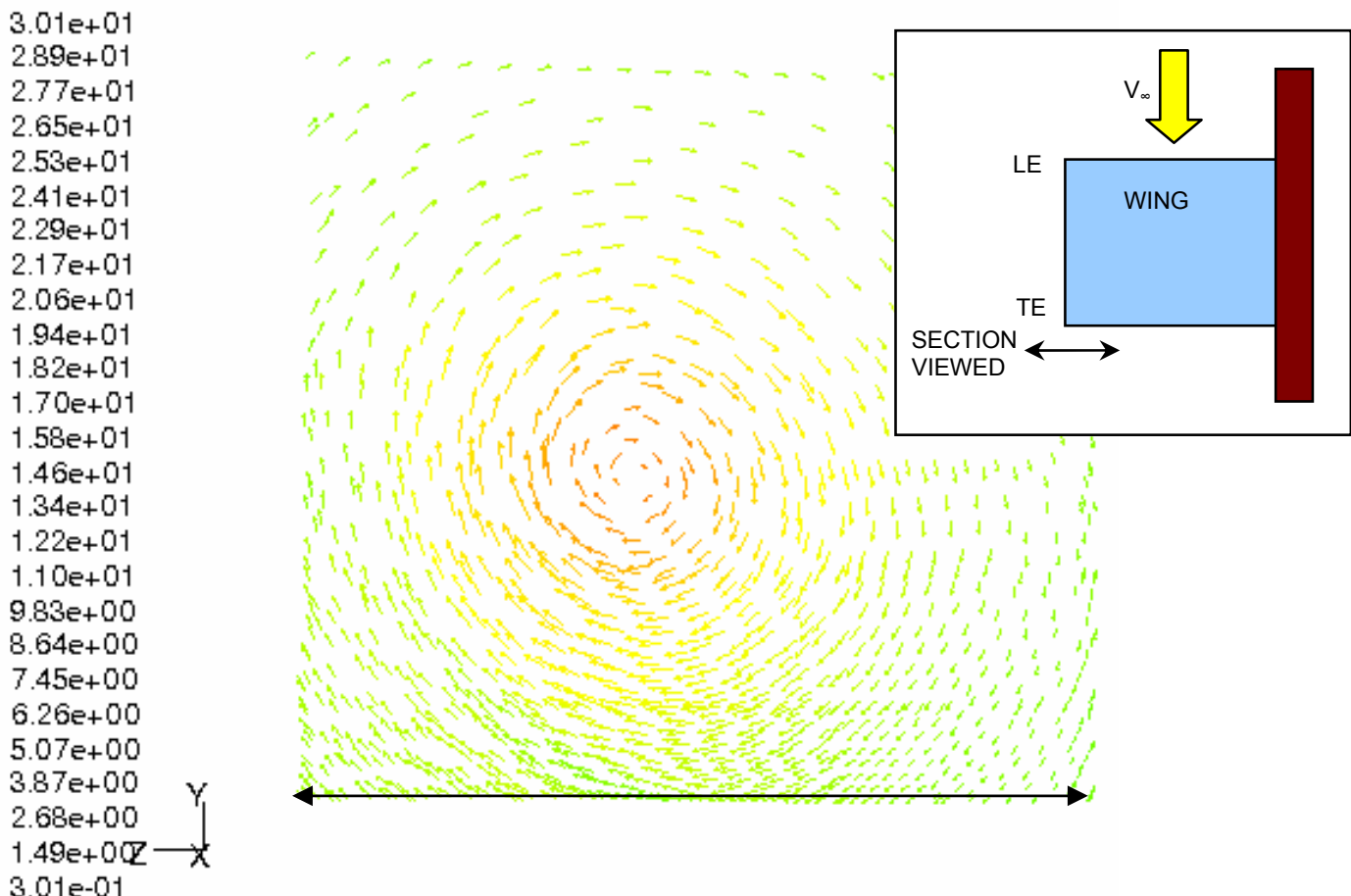

$1.34 \mathrm{e}+01$

$1.22 \mathrm{e}+01$

$1.10 \mathrm{e}+01$

$9.83 \mathrm{e}+00$

$8.64 \mathrm{e}+00$

$6.26 \mathrm{e}+00$

$5.07 \mathrm{e}+00$

$3.87 e+00$

$3.01 \mathrm{e}-01$

Figure 74. Velocity vectors and magnitude (ft/s) at $\mathrm{x} / \mathrm{c}=\mathbf{0 . 8 6 4}$, looking upstream from back [44].

Figure 75 shows the velocity vectors at $\mathrm{x} / \mathrm{c}=0.886$. 
$3.01 \mathrm{e}+01$

$2.89 \mathrm{e}+01$

$2.77 e+01$

$2.65 e+01$

$2.53 \mathrm{e}+01$

$2.41 \mathrm{e}+01$

$2.29 \mathrm{e}+01$

$2.17 \mathrm{e}+01$

$2.06 \mathrm{e}+01$

$1.94 \mathrm{e}+01$

$1.82 \mathrm{e}+01$

$1.70 \mathrm{e}+01$

$1.58 \mathrm{e}+01$

$146 e+01$

$1.34 \mathrm{e}+01$

$1.22 \mathrm{e}+01$

$1.10 \mathrm{e}+01$

$9.83 e+00$

$8.64 \mathrm{e}+00$

$7.45 \mathrm{e}+00$

$6.26 \mathrm{e}+00$

$5.07 e+00$

$3.87 e+00$

$2.68 \mathrm{e}+00$

$1.49 e+0 Q 2 \longrightarrow$

3.01e-01

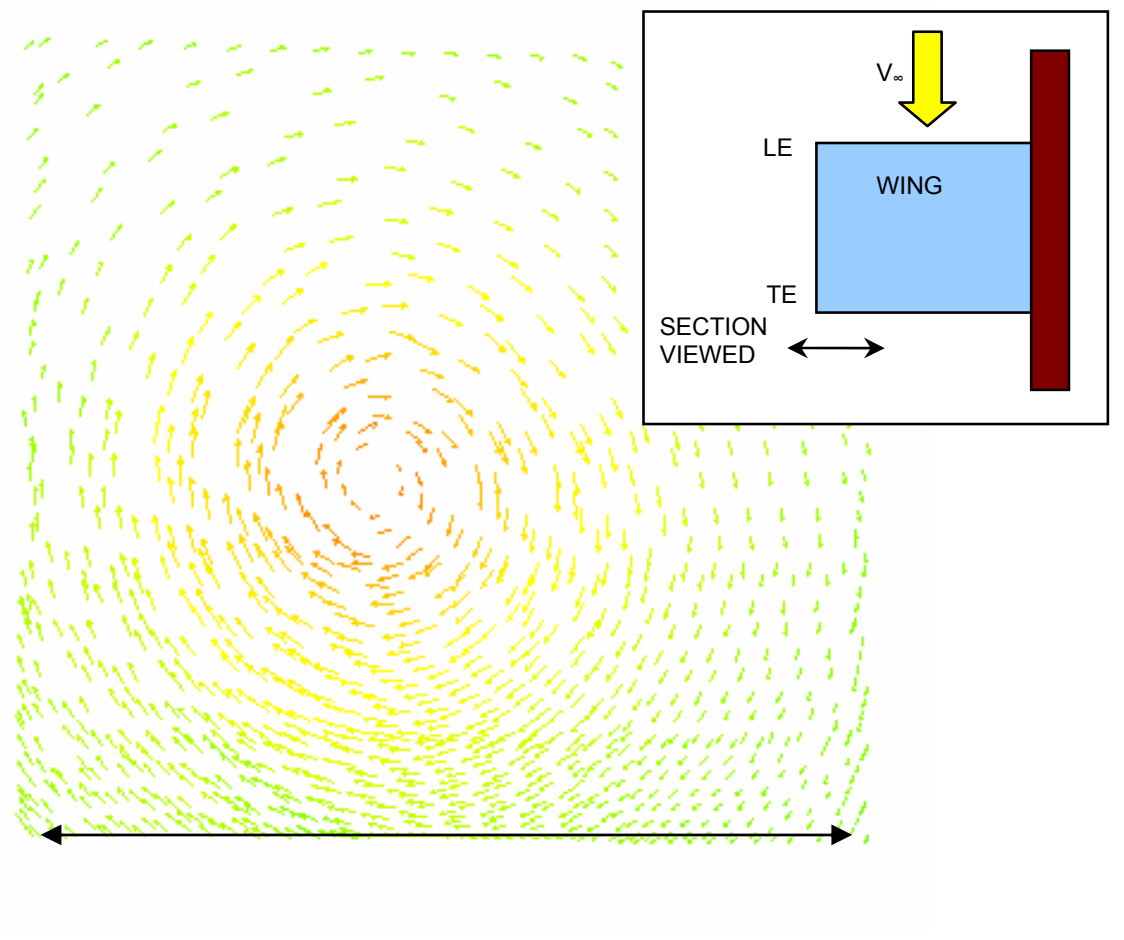

Figure 75. Velocity vectors and magnitude (ft/s) at $\mathbf{x} / \mathbf{c}=\mathbf{0 . 8 8 6}$, looking upstream from back [44].

Figure 76 shows the velocity vectors at $\mathrm{x} / \mathrm{c}=1.005$.
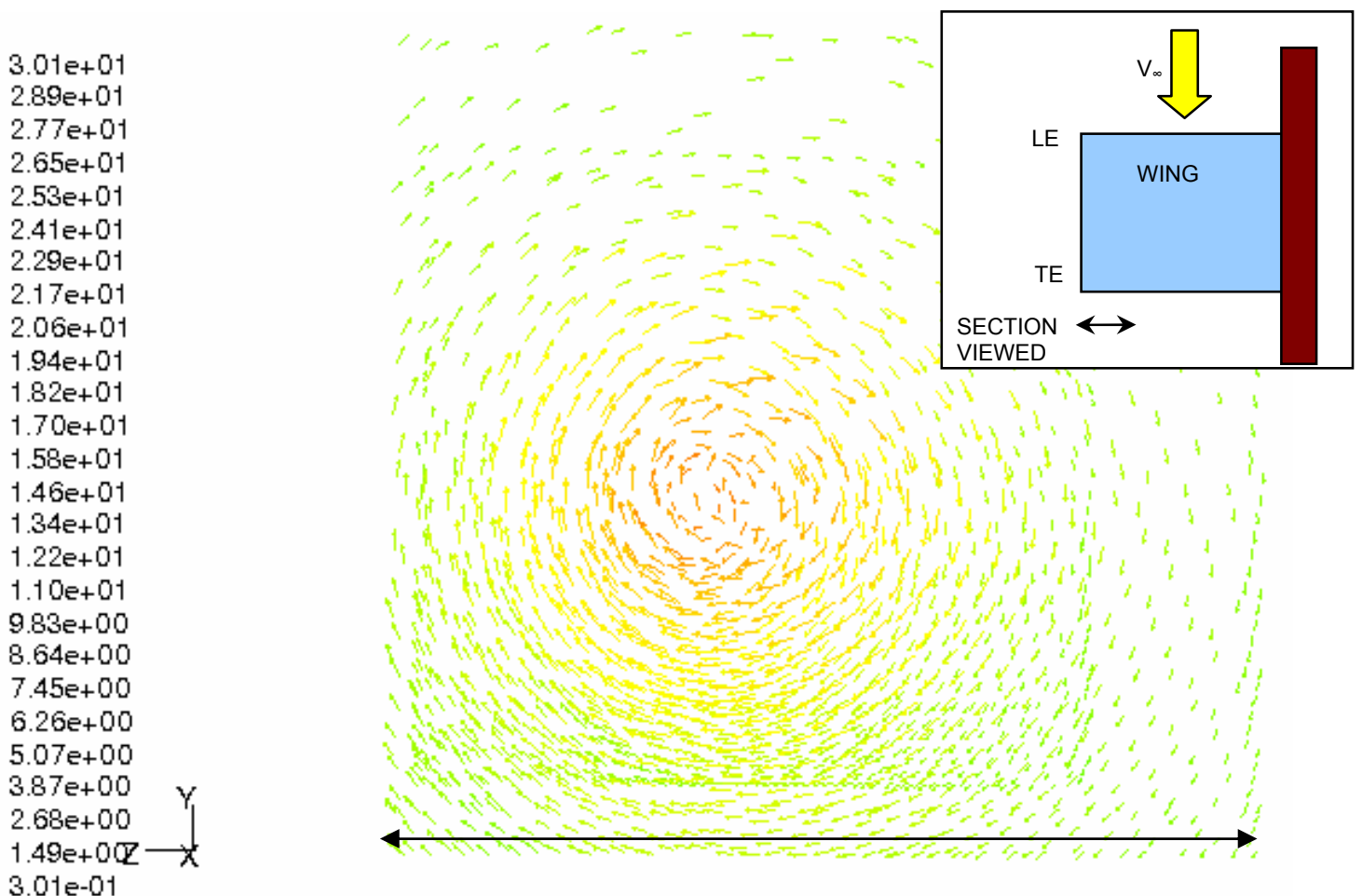

$1.70 e+01$

$1.58 \mathrm{e}+01$

$1.46 \mathrm{e}+01$

$1.34 \mathrm{e}+01$

$1.22 \mathrm{e}+01$

$1.10 \mathrm{e}+01$

$9.83 \mathrm{e}+00$

$8.64 \mathrm{e}+00$

$7.45 \mathrm{e}+00$

$6.26 \mathrm{e}+00$

$5.07 \mathrm{e}+00$

$3.87 e+00$

$2.68 \mathrm{e}+00$

$3.01 \mathrm{e}-01$

igure 76. Velocity vectors and magnitude (ft/s) at $x / c=1.005$, looking upstream from back [44].

The velocity vectors at $\mathrm{x} / \mathrm{c}=1.678$ are shown in Figure 77 . 

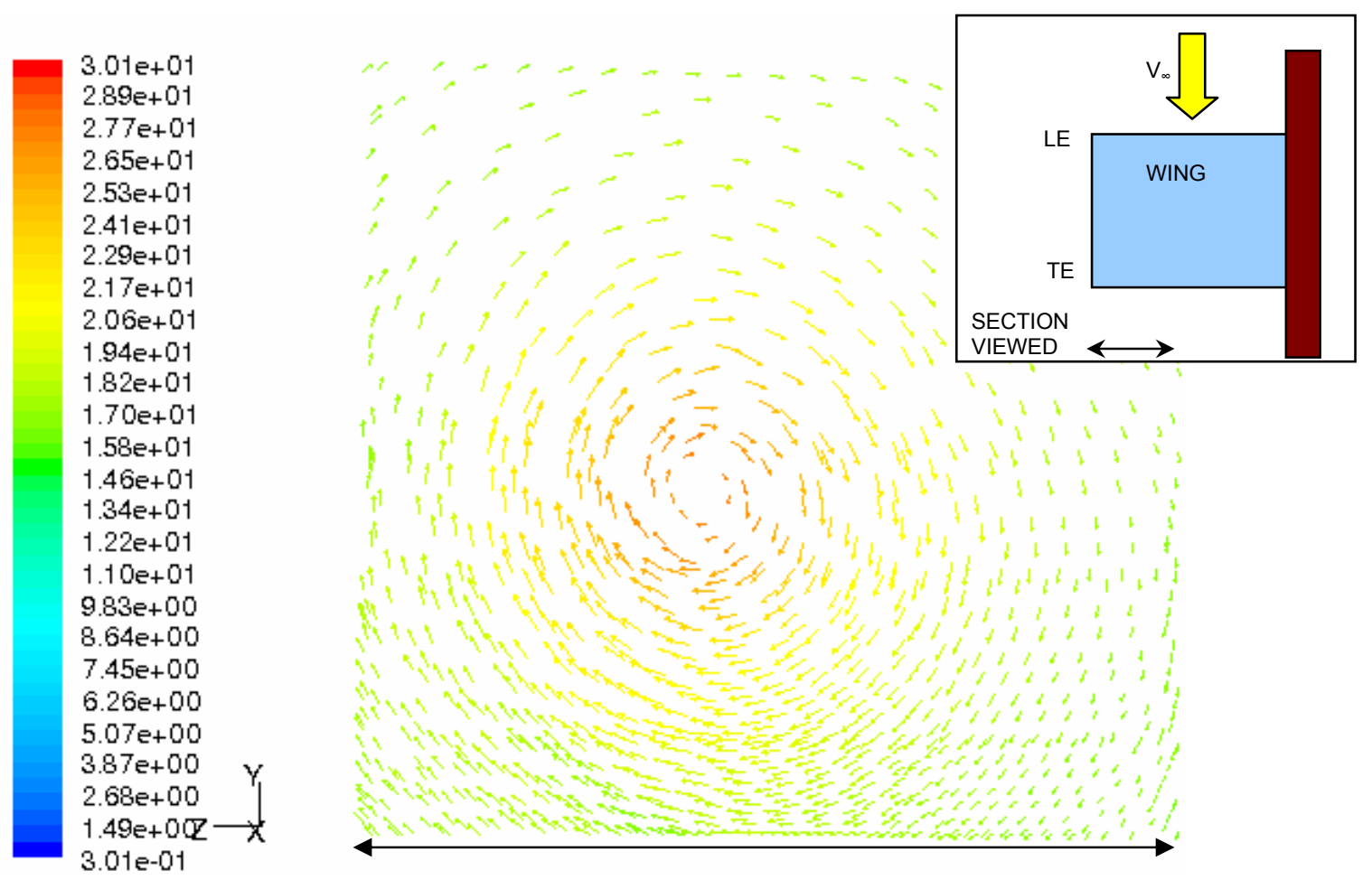

Figure 77. Velocity vectors and magnitude (ft/s) at $x / c=1.678$, looking upstream from back [44]. 


\section{Chapter 4: Numerical Study of the Wingtip Vortex}

\section{Overview}

This chapter presents a comparison between CFD simulations of a wingtip vortex flow field done by the author, experimental measurements done at NASA by Chow et al. [43], and the numerical studies done by Dacles-Mariani et al. [37] and Kim and Rhee [44], as a validation of the present numerical CFD simulations.

\section{Computational Resources}

The simulations were run on the WVU Math Department Beowulf High Performance Computing cluster. The cluster consists of 42 nodes. The node processors are dual core Opteron 270 with a clock speed $2 \mathrm{GHz}$. The network interface is a gigabit copper Ethernet. Each node has a 4 GB DDR SDRAM memory card and a 80 GB 7200 RPM SATA hard drive [45].

\section{Procedure}

\section{Navier Stokes Solver}

The present simulations were run in Fluent 6.2.16, which models fluid flow and heat transfer problems in complex geometries. This commercial CFD software solves the general transport equations using the finite volume method. Steady-state, transient, incompressible, compressible, inviscid, viscid, laminar, and turbulent flows can be solved with Fluent.

\section{Complete Geometry Case}

The measurement and computational domain includes a half-wing inside a wind tunnel (Figure 78) such as the one used by Dacles-Mariani et al. [37] and Chow et al. [43]. The model is a rectangular wing with an aspect ratio of 1.5: $4 \mathrm{ft}$ chord and $3 \mathrm{ft}$. halfspan. The airfoil section is a NACA 0012 at $10^{\circ}$ angle of attack. The dimensions of the wind tunnel test section are $32 \times 48$ in. Free stream velocity is $170 \mathrm{ft} / \mathrm{s}$ yielding a chord Reynolds number of $4.6 \times 10^{6}$. 


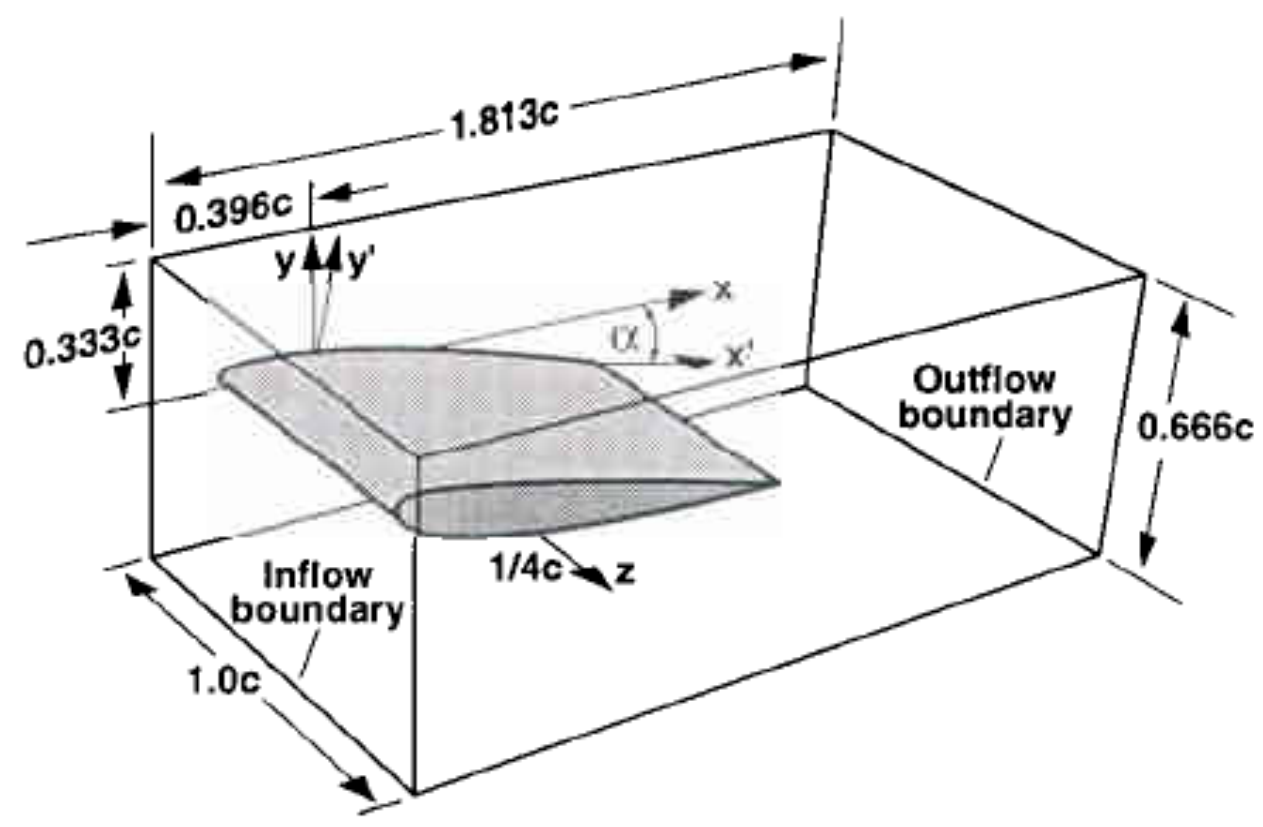

Figure 78. Measurement and Computational Domain [37].

\section{Grid Generation}

Several grid-generation strategies were explored in order to accurately resolve the flow on the wing and that of the wing tip vortex. Multiblock zonal grids were used in the grid convergence study. Tetrahedral meshes followed very well the contours of the model and yielded very good results.

The grid was continuously refined as far as the computer resources allowed. The final mesh used for the simulations had around seven million cells and 1.3 million nodes. Figure 79 shows a mesh with two million cells and 0.4 million nodes.

Fluent grid adaptation capability was used during the simulations. This feature allows refining the mesh when the value of a selected variable at the cells falls in the specified range. Two variables were selected for this numerical study: the $\mathrm{y}^{+}$value, and the pressure. The results with the grid adaptation do not differ too much from the results without grid adaptation. 


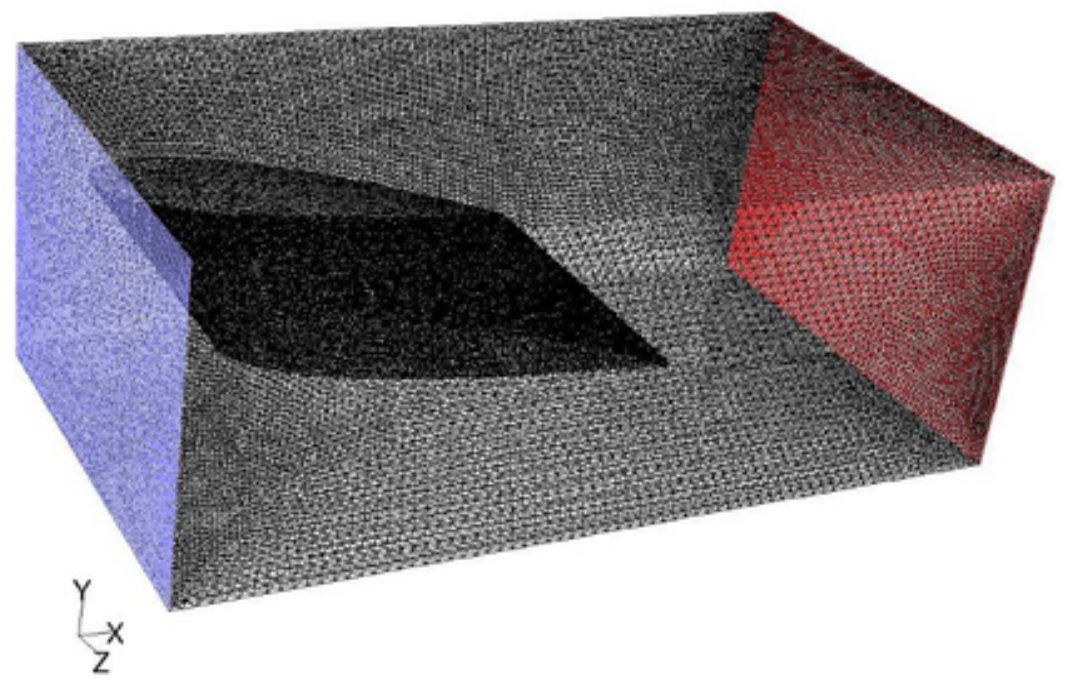

Figure 79. Grid Topology with two million cells and 0.4 million nodes.

The grid used by Dacles-Mariani et al [37], contained 1.5 million grid points and similarly used a single zone approach (Figure 80 ). The grid was a hybrid grid C-O topology consisting of an inner hyperbolic grid surrounding the wing matched with an elliptic grid near the wind-tunnel walls.

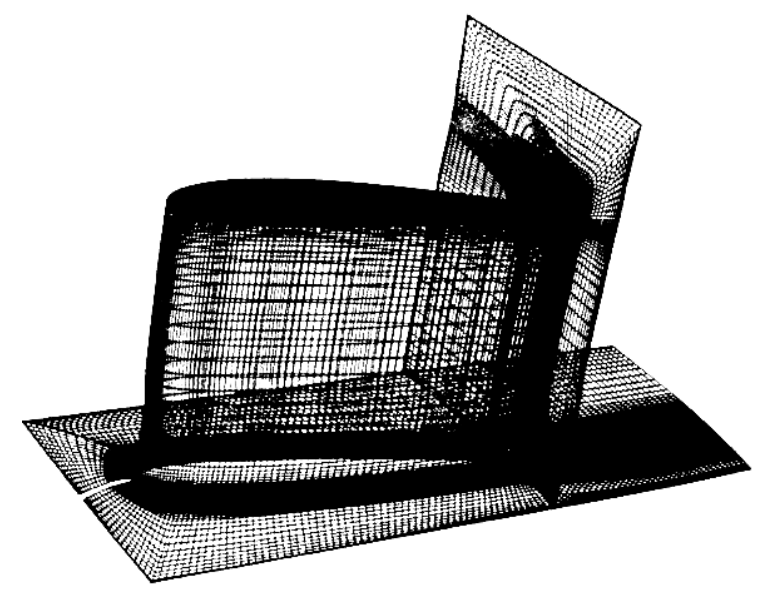

Figure 80. Grid Topology used by Dacles-Mariani et al [3].

\section{Turbulence Modeling}

According to the literature review, the Spalart-Allmaras model is a very inexpensive turbulence model computationally and it has shown very good results in wing tip vortex flows [44]. This model includes a non-viscous destruction term which depends on distance from the wall. This equation is local; this means that the equation at one point does not depend on the solution at other points which is good for grids of any 
structure. This turbulence model yields a relatively smooth laminar-turbulent transition [39]. It performs well in the near wake, which is what is desired in this wing tip problem.

\section{Solver Configuration}

The solver was set to segregated with an implicit formulation. Segregated schemes solve each governing equation separately and sequentially; linking the pressure, density and velocity together by a Poisson-type pressure equation or pressure correction equation.

The pressure-based method used is SIMPLE (Semi Implicit Method for Pressure Linked Equations) by Patankar [46] because of its simplicity and success.

The discretization of the pressure was realized by a second order scheme [47]. The discretization of the momentum and modified turbulent viscosity was done by QUICK [47]. The QUICK discretization scheme may provide better accuracy than the second order scheme for rotating or swirling flows as in this case.

\section{Boundary Conditions}

The inlet boundary condition was defined as a uniform inlet velocity $=170 \mathrm{ft} / \mathrm{s}$. The outlet boundary condition was based on a pressure outlet condition similar to DaclesMariani's paper. The wind tunnel walls were defined as slip walls with a uniform velocity of $\mathrm{V}_{\infty}$, except on the wall of symmetry, where the normal velocity component were set equal to zero. The wing surface was defined as a stationary surface.

\section{Results}

The simulations ran over 6000 iterations and the residuals, the lift coefficient, and the drag coefficient monitors are plotted in Figures 81, 82, and 83, respectively. The percent variation in lift coefficient was $4 \times 10^{-4}$ and in drag was $7 \times 10^{-4}$. 


\begin{tabular}{|l|}
\hline Residuals \\
\hline continuity \\
\hline x-velocity \\
y-velocity \\
z-velocity \\
nut \\
\hline
\end{tabular}

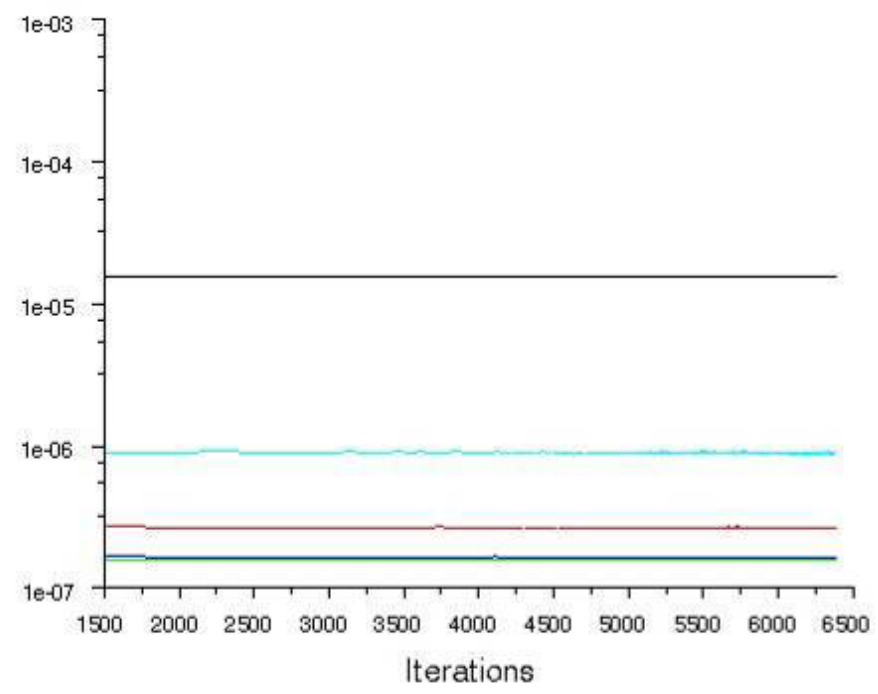

Figure 81. Residuals for mesh with 7 million cells and 1.3 million nodes.

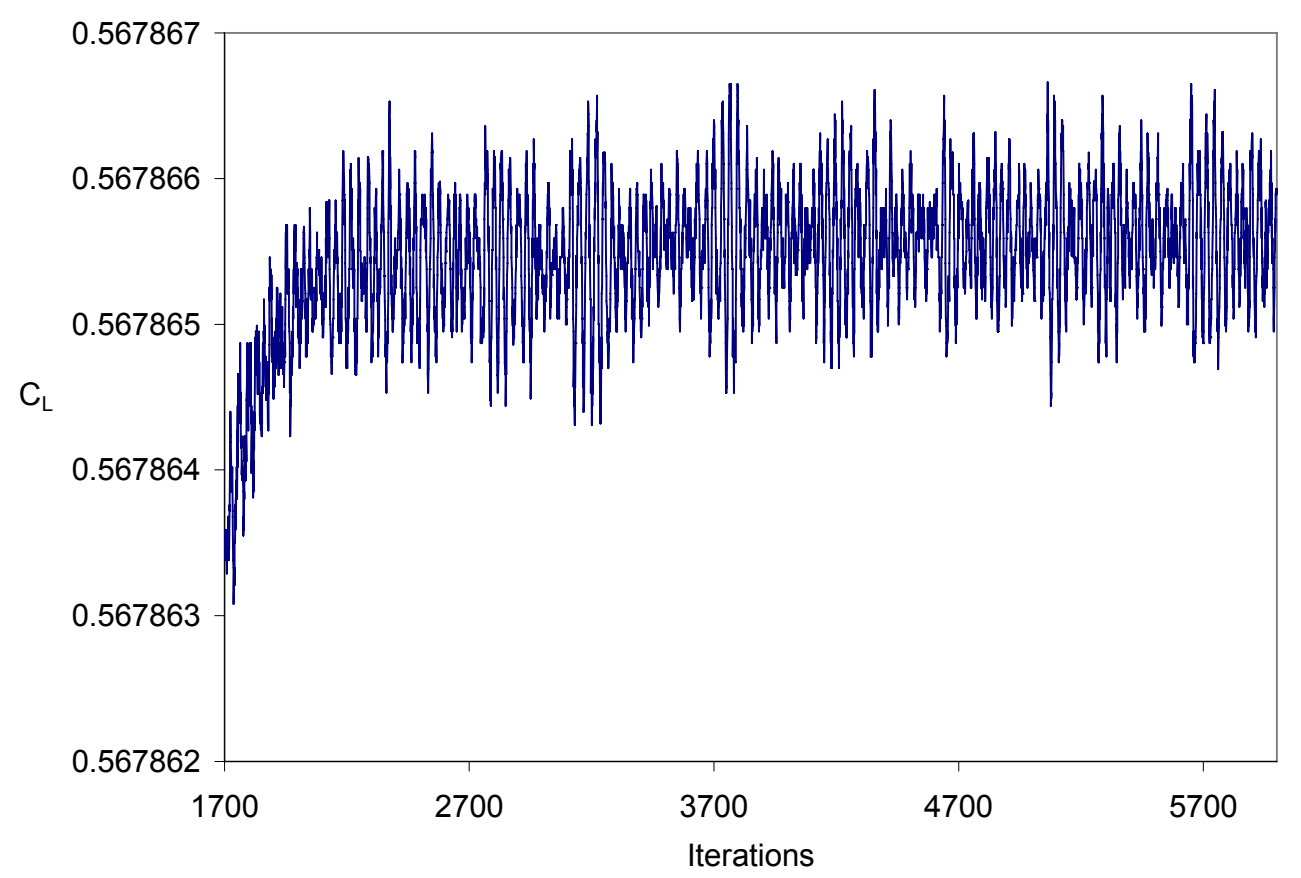

Figure 82. Lift coefficient monitor for mesh with 7 million cells and 1.3 million nodes. 


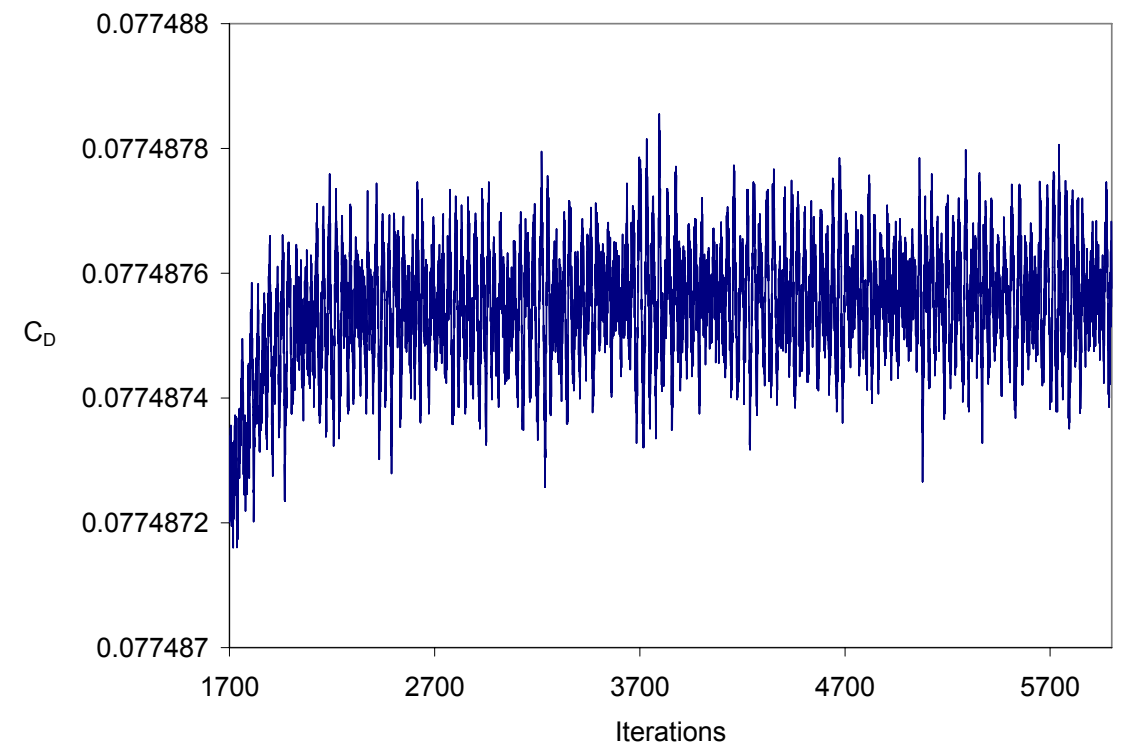

Figure 83. Drag coefficient monitor for mesh with 7 million cells and 1.3 million nodes.

\section{Pressure Coefficient Contours}

The measured and computed values of the pressure coefficient obtained by Chow et al. [43] and Dacles-Mariani et al. [37], are shown respectively in Figure 84.
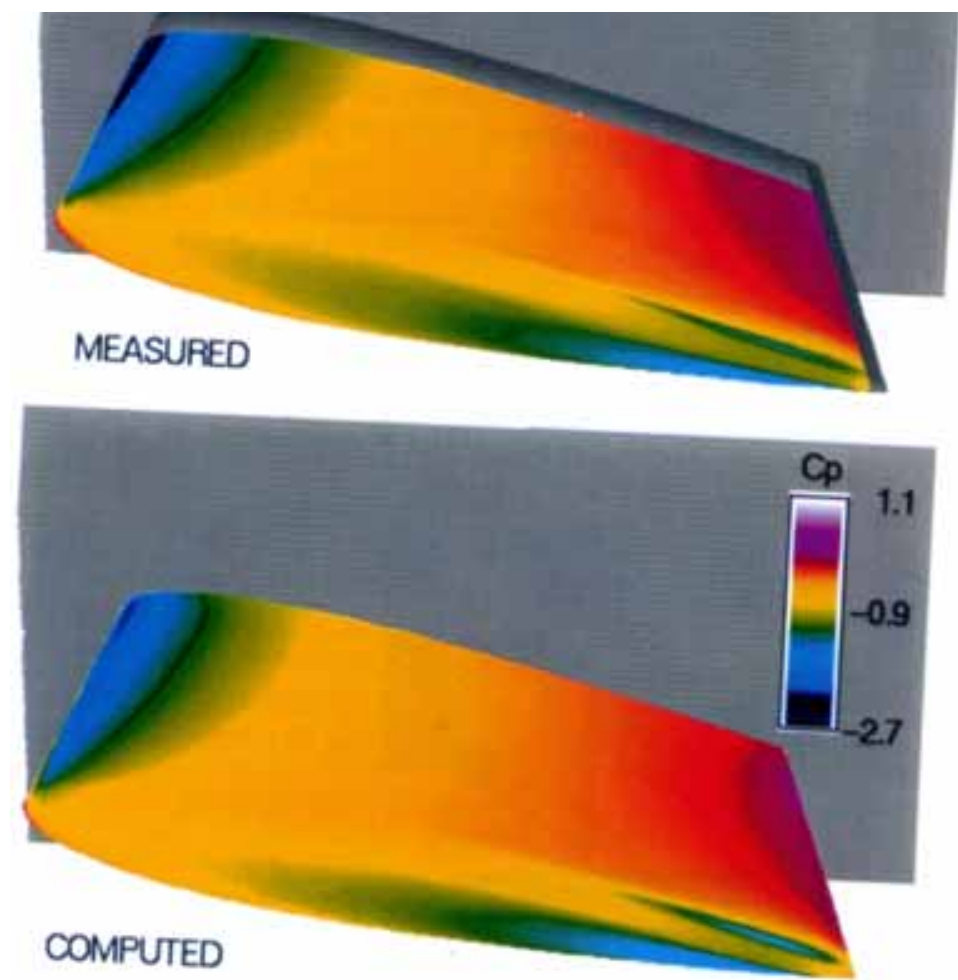

Figure 84. Pressure Coefficients measured and computed by Chow et al. [43] and Dacles-Mariani et al. [37]. 
The next figure (Figure 85) shows the pressure coefficient $(\mathrm{Cp})$ contours obtained by this author. These results are similar to those in Figure 84, with a low pressure zone at the leading edge peaking around $\mathrm{Cp}=-2.8$. The high pressure zone in the trailing edge peaked at $\mathrm{Cp}=0.15$ as is shown in Figure 84. Similar data are shown in Figure 85, with a $\mathrm{Cp}$ value of about 0.1 . The $\mathrm{Cp}$ value of 0.15 was also obtained from $\mathrm{Cp}$ measurements table done by Chow et al (see appendix B). The yellow zone on Figure 84 agrees with the green zone on Figure 85, their $\mathrm{Cp}$ values are about -0.9 compared to -0.8 in Figure 84 . Figure 85 shows the low pressure zone in the wingtip (blue zone in Figure 84) generated by the wingtip vortex.

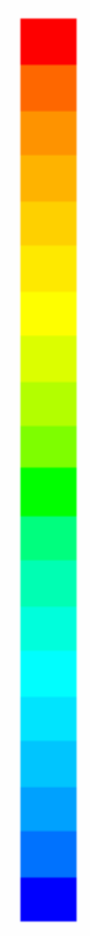
$1.08 \mathrm{e}+00$
$8.71 \mathrm{e}-01$
$6.64 \mathrm{e}-01$
4.58e-01
2.52e-01
4.53e-02
$-1.61 \mathrm{e}-01$
$3.67 \mathrm{e}-01$
$-5.74 \mathrm{e}-01$
$-7.80 \mathrm{e}-01$
$-9.86 \mathrm{e}-01$
$-1.19 e+00$
$-1.40 \mathrm{e}+00$
$-1.61 \mathrm{e}+00$
$-1.81 e+00$
$-2.02 e+00$
$-2.22 \mathrm{e}+00$
$-2.43 e+00$
$-2.64 e+00$
$-2.84 e+00$
$3.05 e+00$

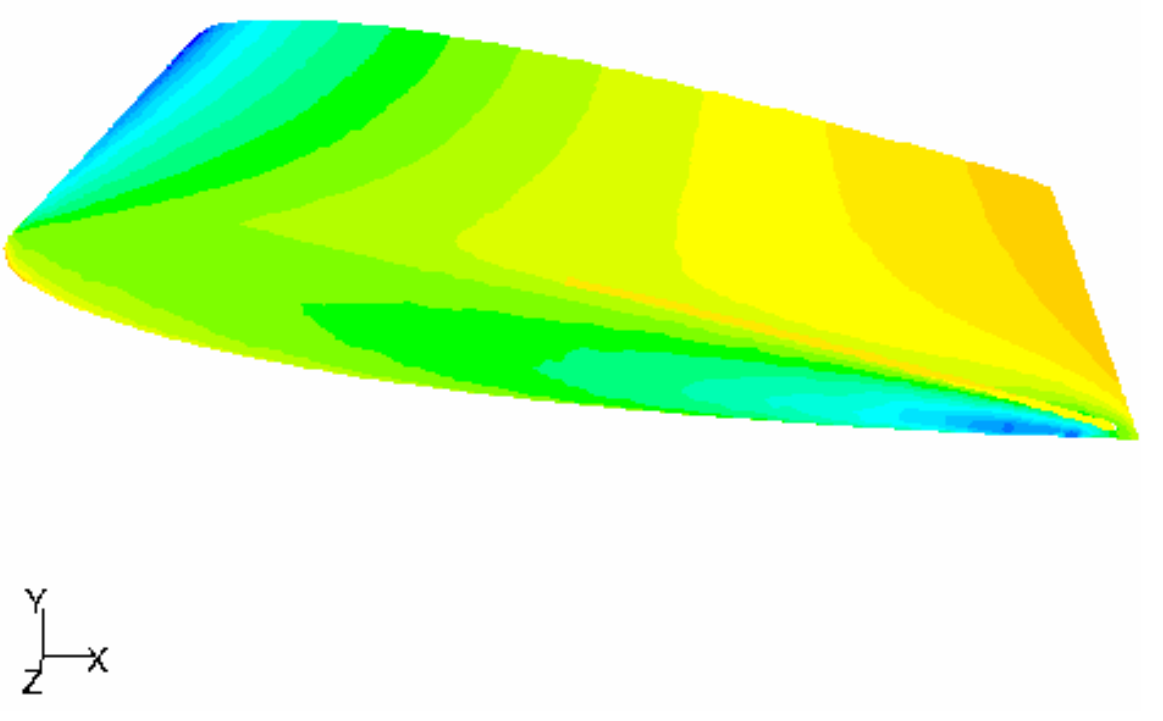

Figure 85. Pressure Coefficient contours on the wing.

\section{Velocity Magnitude Contours}

Figure 86 shows the velocity contours measured by Chow et al. [43] and predicted by Dacles-Mariani et al. [37]. The simulations done by Dacles-Mariani et al. [37] showed good agreement with the measurements. 


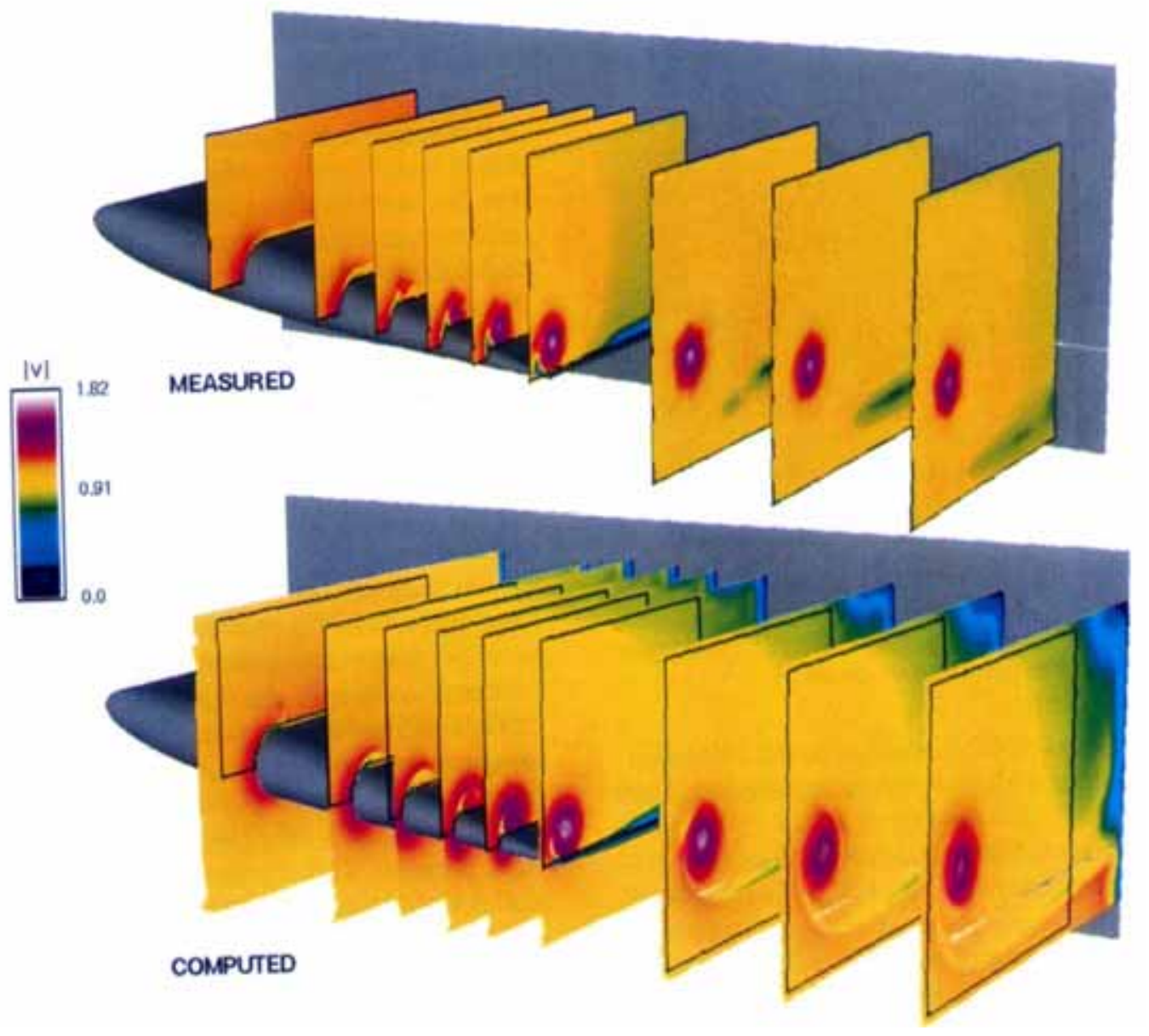

Figure 86. Measured and Computed Velocity Contours by Chow et al. [43] and Dacles-Mariani et al. [37].

Figure 87 shows an example of the results obtained in this numerical study. This simulation predicts the wing tip vortex appearance. It also shows a high velocity in the wing tip core of around 1.15 compared to about 1.5 in Figure 87. Figure 86 shows a low velocity zone (blue) in the upper right corner, similar to what was obtained by DaclesMariani et al. [37]. A high velocity region in Figure 87 (red) is possibly caused by blockage of the flow by the bottom wind tunnel wall, as also predicted by Dacles-Mariani et al. [37] (Figure 86). 


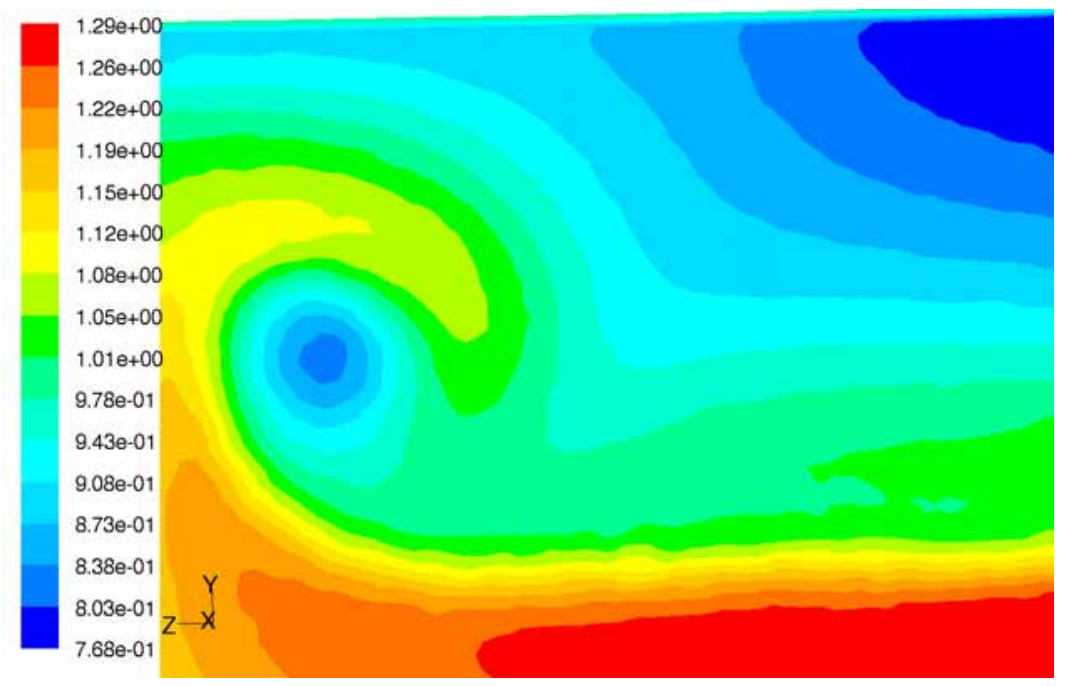

Figure 87. Velocity Contours at the outflow boundary.

\section{Surface Pathlines}

Figure 88 shows the pathlines obtained during the experiments and the numerical studies done presented in the literature review. 

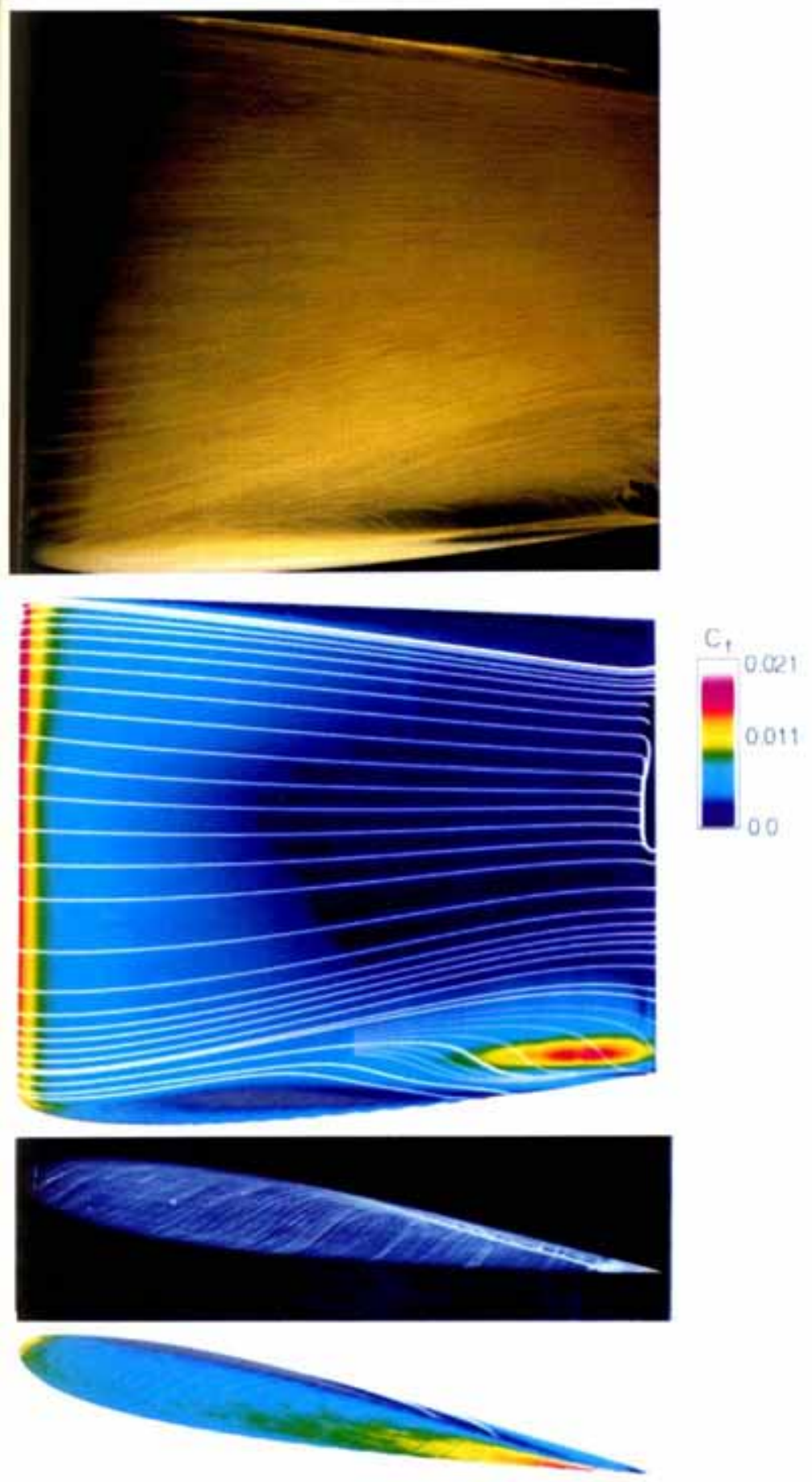

Figure 88. Surface Pathlines and Friction Coefficient measured and computed previously [43].

Figure 89 shows the pathlines on the wing obtained in this study. This figure shows clearly the wing tip vortex. It also shows the pathlines on top of the wing flowing inboard, similar to what was visualized by the experiments of Chow et al. [43] (Figure 88). 


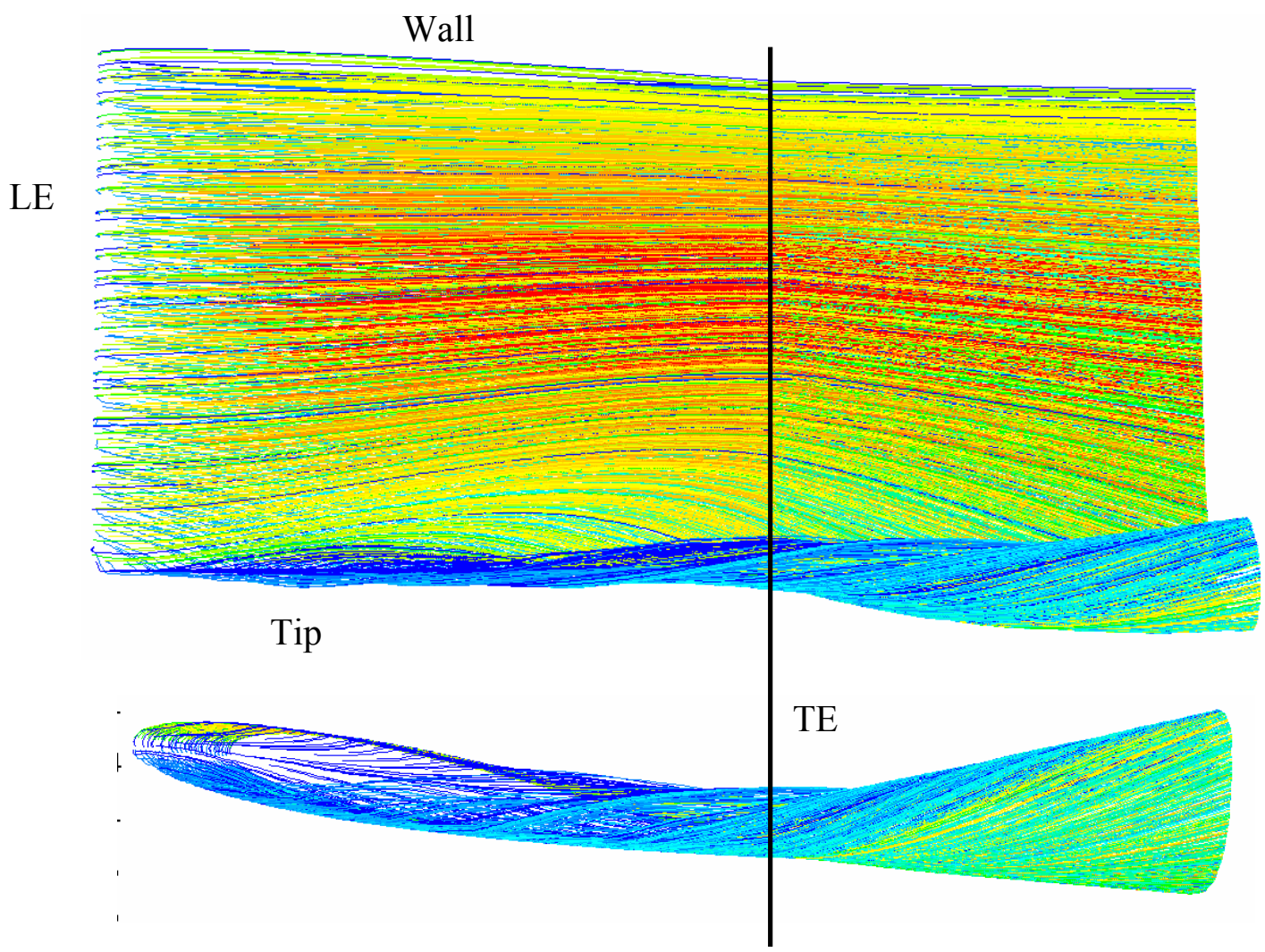

Figure 89. Pathlines computed on the top wing surface and the wing tip.

\section{Pressure Coefficient Values}

Figure 90 shows a comparison between pressure coefficient measurements done at NASA by Chow et al. and simulations done in Fluent by Kim and Rhee [44] and at WVU for meshes with 2.3 and 7 million cells. The $\mathrm{Cp}$ values are measured at a spanwise position $\mathrm{z} / \mathrm{c}$ equal to 0.125 . The numerical simulations show reasonably good agreement with the experimental measurements, especially on the lower wing surface. The agreement at this spanwise position is better than at other positions. 


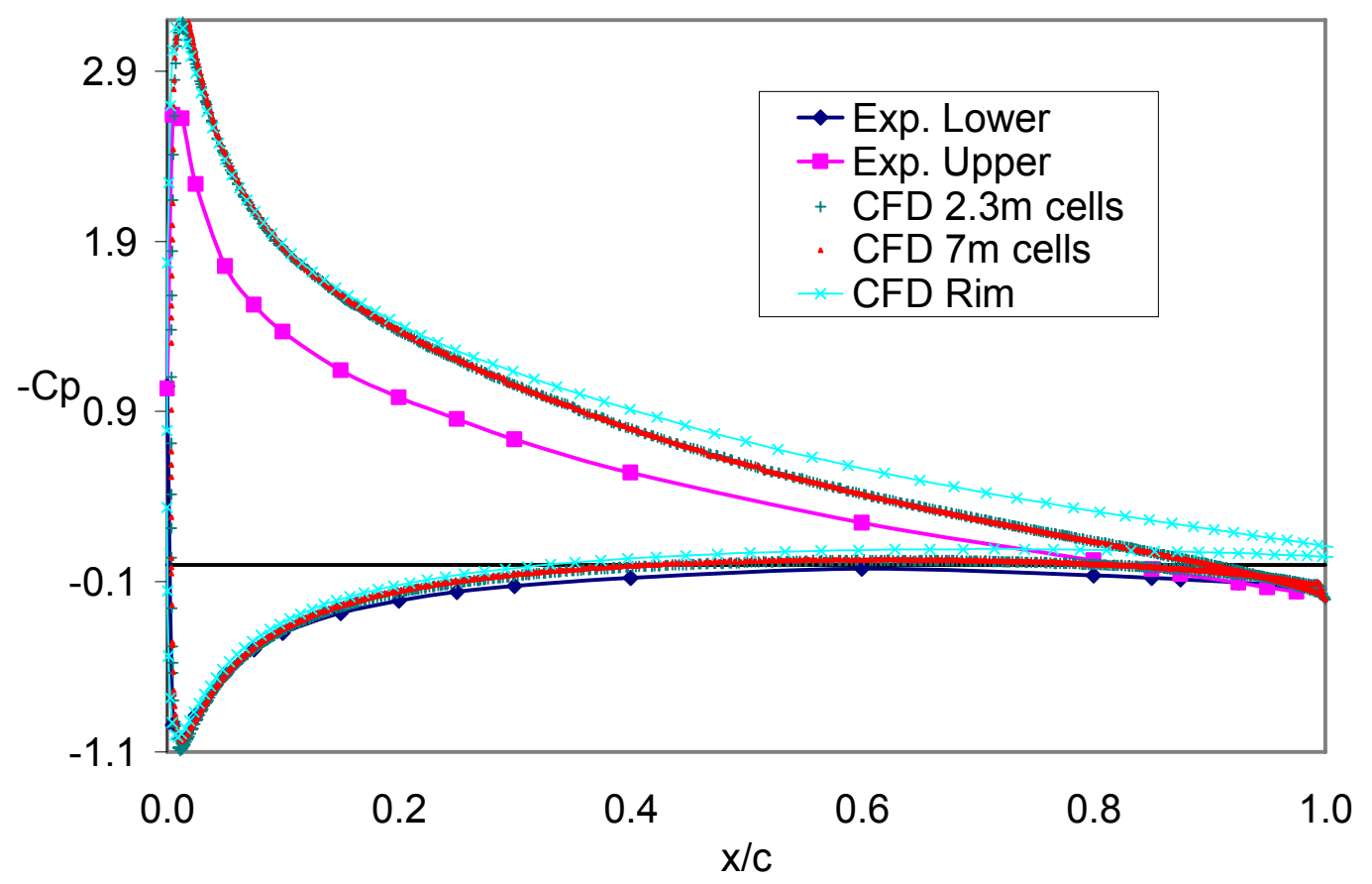

Figure 90. Comparison between experimental and CFD pressure coefficients at $\mathrm{z} / \mathrm{c}=\mathbf{0 . 1 2 5}$.

\section{Circulation across the Wingtip Vortex Core}

Circulation variation with distance from the center of the vortex core on a NACA 0012 wing with rounded wingtip is presented in this section. The simulation was obtained from Kim and Rhee at an angle of attack of $10^{\circ}$ and an $\mathrm{AR}=1.5$ [44]. The circulation value was obtained from the surface integral.

$$
\Gamma=-\oint V \cdot d s=-\iint(\nabla \times \vec{V}) \cdot d A
$$

For a plane it becomes:

$$
\Gamma=-\oint(u d x+v d y)=-\iint\left(\frac{\partial v}{\partial x}-\frac{\partial u}{\partial y}\right) d x d y
$$

Where $\mathrm{u}$ is the velocity component in the $\mathrm{x}$-direction and $\mathrm{v}$ is the velocity component in the y-direction.

Several square contours were drawn around the wingtip vortex core in order to compute the circulation in each of them (Figure 91). 


\begin{tabular}{|c|c|}
\hline 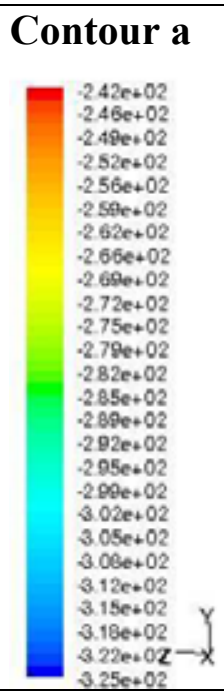 & 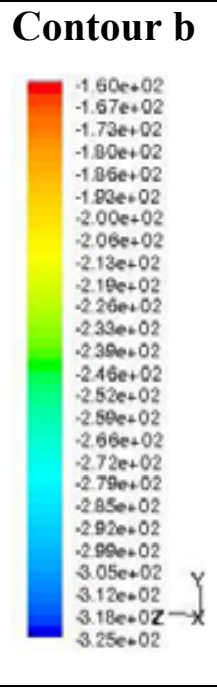 \\
\hline 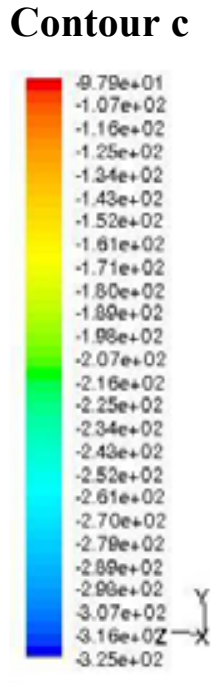 & 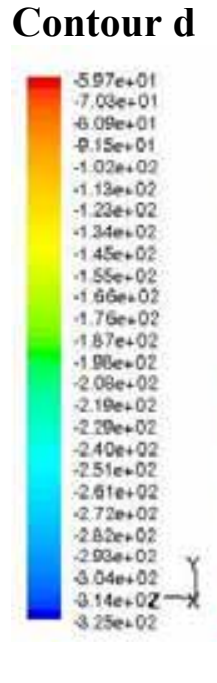 \\
\hline 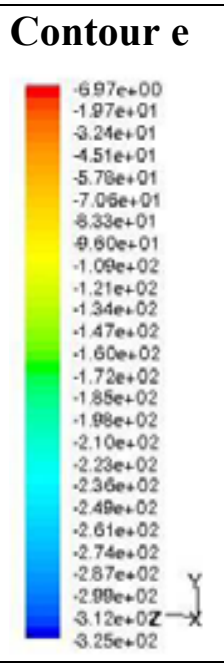 & 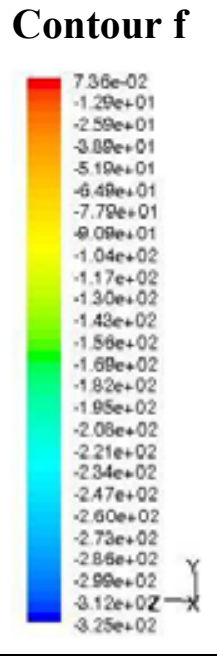 \\
\hline
\end{tabular}




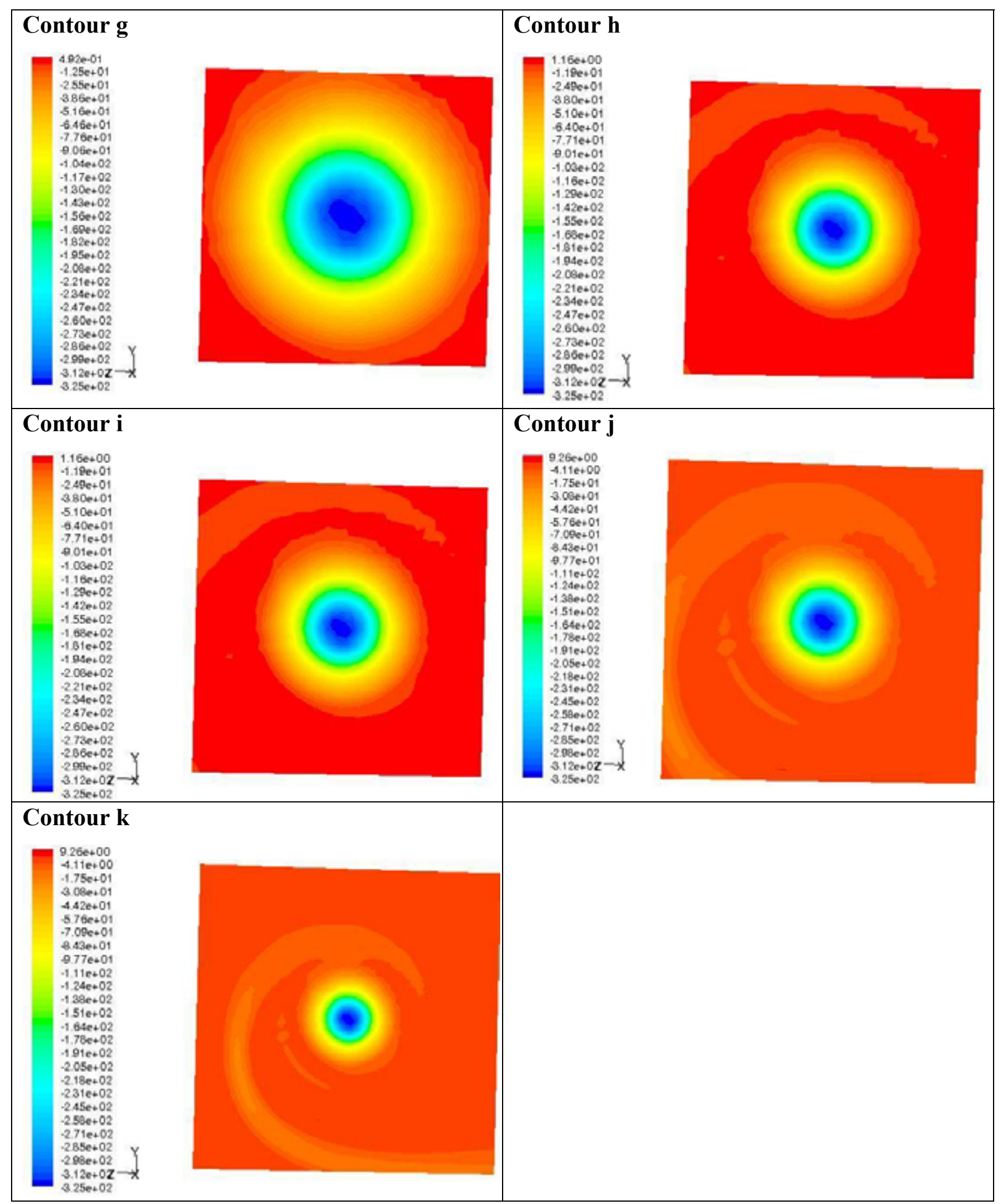

Figure 91. Square contours around the wingtip vortex core to compute the circulation [44]. 
The circulation value for Contour A (Row 1 Table 3) was verified by evaluating the line integral. Velocity values were exported to Excel and then integrated. The circulation by the line integral method was equal to $2.93 \mathrm{ft}^{2} / \mathrm{s}$, very close to the surface integral value $3.04 \mathrm{ft}^{2} / \mathrm{s}$. The small difference between these two values is caused by the approximation done to calculate the line integral by knowing the velocity values at certain points, but not knowing the actual velocity function.

Table 3. Wingtip Vortex Circulation at various distances from the core.

\begin{tabular}{|c|c|c|c|c|c|c|}
\hline \multirow{2}{*}{ Contour } & \multicolumn{2}{|c|}{$\mathbf{Y}(\mathbf{f t})$} & \multicolumn{2}{c|}{$\mathbf{Z}(\mathbf{f t})$} & Circulation & Distance \\
\cline { 2 - 7 } & $\min$ & $\max$ & $\min$ & $\max$ & $\mathbf{f t}^{2} / \mathbf{s}$ & $\mathbf{f t}$ \\
\hline $\mathrm{a}$ & -0.263 & -0.16 & 2.585 & 2.68 & 3.04 & 0.05 \\
\hline $\mathrm{b}$ & -0.283 & -0.14 & 2.565 & 2.70 & 5.50 & 0.07 \\
\hline $\mathrm{c}$ & -0.303 & -0.12 & 2.545 & 2.72 & 7.95 & 0.09 \\
\hline $\mathrm{d}$ & -0.323 & -0.10 & 2.525 & 2.74 & 10.11 & 0.11 \\
\hline $\mathrm{e}$ & -0.363 & -0.06 & 2.485 & 2.78 & 13.19 & 0.15 \\
\hline $\mathrm{f}$ & -0.403 & -0.02 & 2.445 & 2.82 & 14.77 & 0.19 \\
\hline $\mathrm{g}$ & -0.443 & 0.02 & 2.405 & 2.86 & 15.23 & 0.23 \\
\hline $\mathrm{h}$ & -0.503 & 0.08 & 2.345 & 2.92 & 15.60 & 0.29 \\
\hline $\mathrm{i}$ & -0.603 & 0.18 & 2.245 & 3.02 & 16.43 & 0.39 \\
\hline $\mathrm{j}$ & -0.703 & 0.28 & 2.145 & 3.12 & 17.36 & 0.49 \\
\hline $\mathrm{k}$ & -0.903 & 0.48 & 1.945 & 3.32 & 19.01 & 0.69 \\
\hline
\end{tabular}

Figure 92 shows the wingtip vortex variation as the squares contours drawn around the vortex core are increased. Notice that the curve approaches an asymptotic value when it reaches a distance of $0.29 \mathrm{ft}$ from the vortex core (contour h). However when the distance continues to increase, the circulation likewise increases. This is because the contours are affected by the wake circulation of the wing. It is more clearly seen in Figure 91, Contour k. 


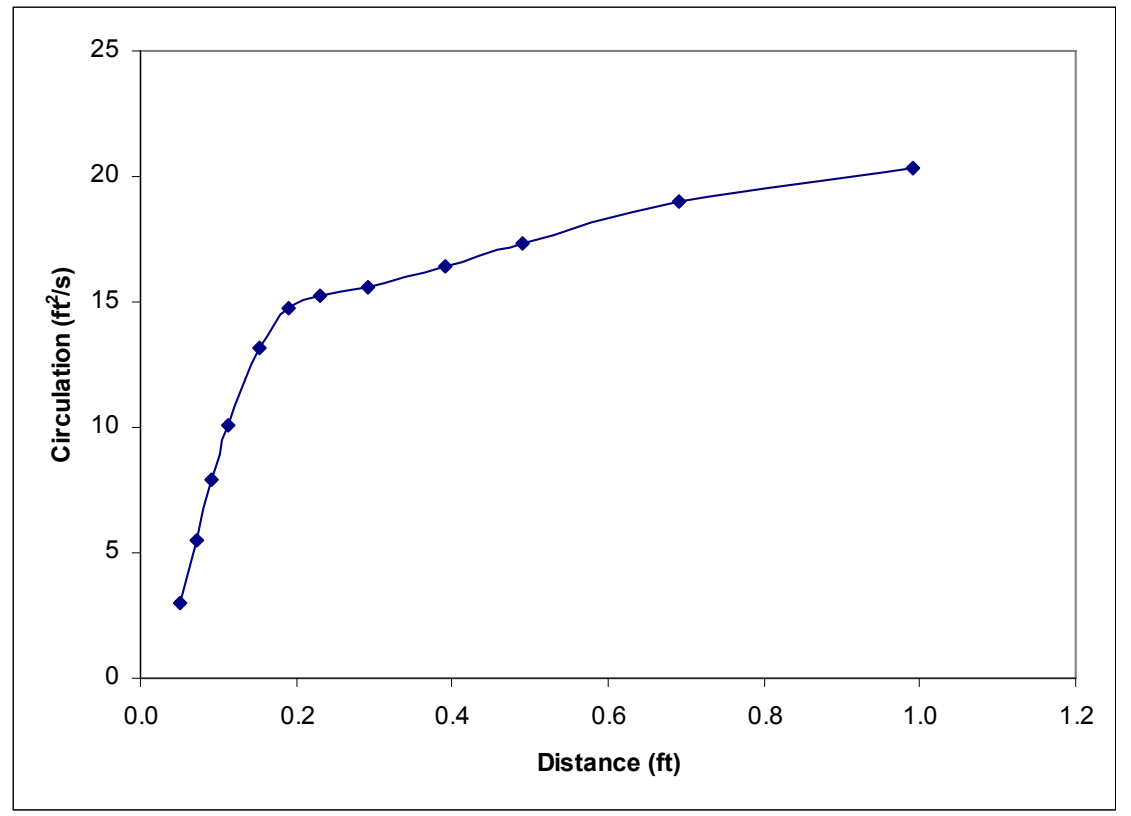

Figure 92. Wingtip vortex variation with distance from the vortex core.

The whole vorticity contours of a NACA 0012 wing at one chord downstream the trailing edge can be seen in Figure 93. It includes the vorticity in the wing tip vortex and the vorticity in the wake of the wing.
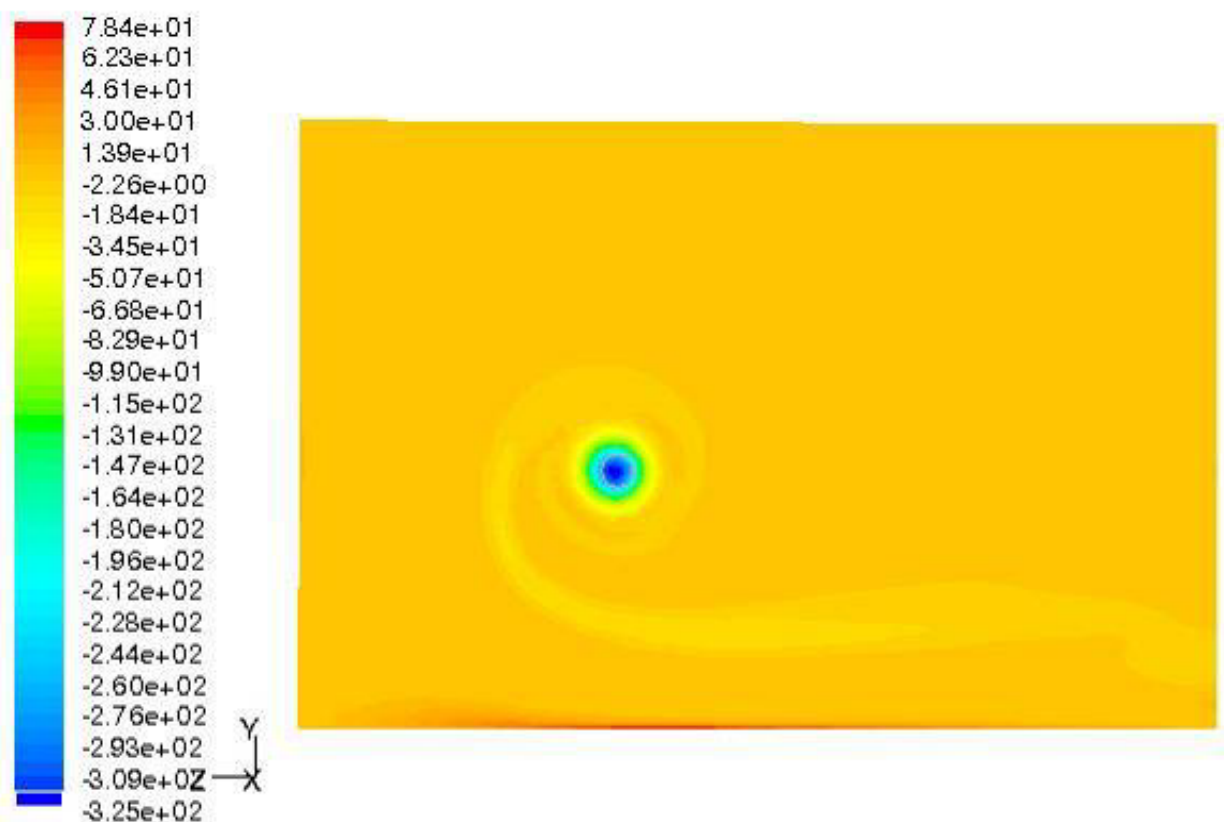

Figure 93. Vorticity at one chord downstream of a NACA 0012 wing [44]. 
Figure 94 shows the tangential velocity variation across the wingtip vortex core of a NACA 0012 wing at one chord downstream the trailing edge. The right side of the curve agrees very well with the ideal Rankine vortex, but the left side does not. This disagreement in the left side of the curve is due to the air still flowing from the bottom region of the vortex to the top one, or it could be due to the close proximity to the wind tunnel wall.

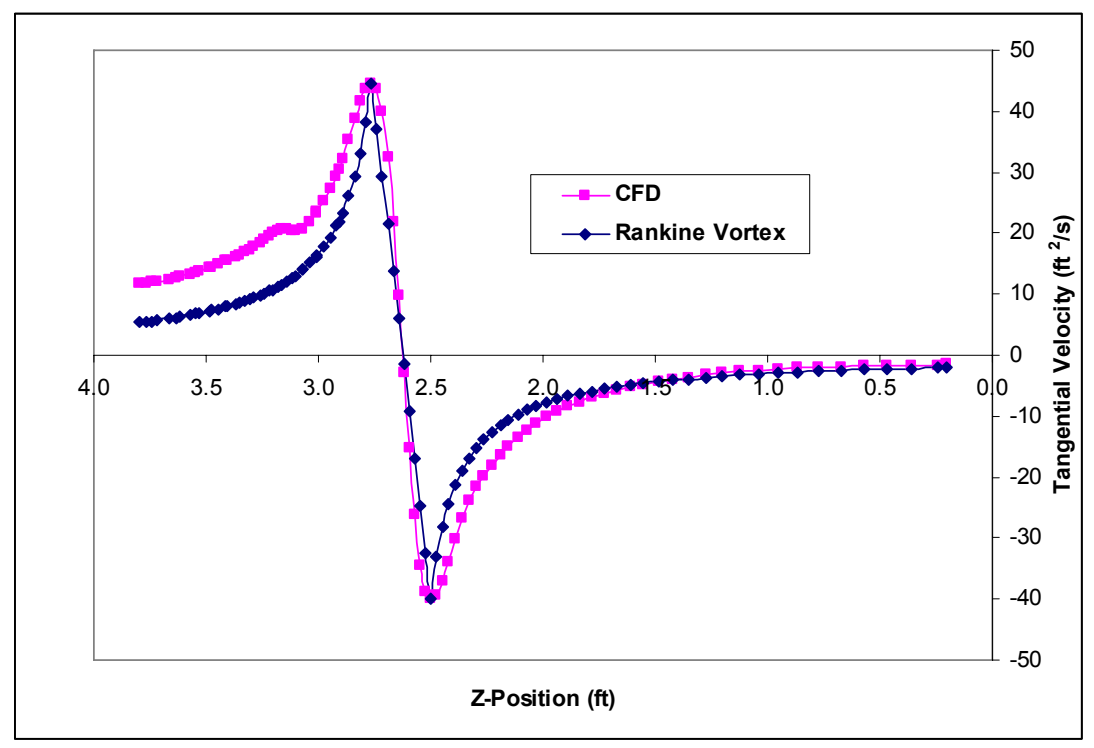

Figure 94. Tangential velocity variation across the wingtip vortex core compared to an ideal Rankine vortex.

Some rectangles were drawn to the right of the wingtip vortex core to compute the circulation and know how it varies (Figure 95). Table 4 shows the circulation values at different areas to the right of the vortex core at one chord downstream of the TE of a NACA 0012 wing trailing edge. 


\begin{tabular}{|c|c|c|}
\hline $\begin{array}{l}\text { a) Contour } 105 \\
2.24 \mathrm{e}-01 \\
-1.27 \mathrm{e}+01 \\
-2.56 \mathrm{e}+01 \\
-3.86 \mathrm{e}+01 \\
-5.15 \mathrm{e}+01 \\
-6.44 \mathrm{e}+01 \\
-7.74 \mathrm{e}+01 \\
-9.03 \mathrm{e}+01 \\
-1.03 \mathrm{e}+02 \\
-1.16 \mathrm{e}+02 \\
-1.29 \mathrm{e}+02 \\
-1.42 \mathrm{e}+02 \\
-1.55 \mathrm{e}+02 \\
-1.68 \mathrm{e}+02 \\
-1.81 \mathrm{e}+02 \\
-1.94 \mathrm{e}+02 \\
-2.07 \mathrm{e}+02 \\
-2.20 \mathrm{e}+02 \\
-2.33 \mathrm{e}+02 \\
-2.46 \mathrm{e}+02 \\
-2.58 \mathrm{e}+02 \\
-2.71 \mathrm{e}+02 \\
-2.84 \mathrm{e}+02 \\
-2.97 \mathrm{e}+02 \\
-3.10 \mathrm{e}+0 \mathrm{z} \\
3.23 \mathrm{e}+02\end{array}$ & $\begin{array}{l}\text { b) Contour } 104 \\
2.54 \mathrm{e}-01 \\
-1.27 \mathrm{e}+01 \\
-2.56 \mathrm{e}+01 \\
-3.86 \mathrm{e}+01 \\
-5.15 \mathrm{e}+01 \\
-6.44 \mathrm{e}+01 \\
-7.74 \mathrm{e}+01 \\
-9.03 \mathrm{e}+01 \\
-1.03 \mathrm{e}+02 \\
-1.16 \mathrm{e}+02 \\
-1.29 \mathrm{e}+02 \\
-1.42 \mathrm{e}+02 \\
-1.55 \mathrm{e}+02 \\
-1.68 \mathrm{e}+02 \\
-1.81 \mathrm{e}+02 \\
-1.94 \mathrm{e}+02 \\
-2.07 \mathrm{e}+02 \\
-2.20 \mathrm{e}+02 \\
-2.33 \mathrm{e}+02 \\
-2.46 \mathrm{e}+02 \\
-2.58 \mathrm{e}+02 \\
-2.71 \mathrm{e}+02 \\
-2.84 \mathrm{e}+02 \\
-2.97 \mathrm{e}+02 \\
-3.10 \mathrm{e}+0 \mathrm{z} \\
3.23 \mathrm{e}+02\end{array}$ & $\begin{array}{l}\text { c) Contour } 103 \\
3.63 e-01 \\
-1.26 e+01 \\
-2.55 e+01 \\
-3.85 e+01 \\
-5.14 e+01 \\
-6.43 e+01 \\
-7.73 e+01 \\
-9.02 e+01 \\
-1.03 e+02 \\
-1.16 e+02 \\
-1.29 e+02 \\
-1.42 e+02 \\
-1.55 e+02 \\
-1.68 e+02 \\
-1.81 e+02 \\
-1.94 e+02 \\
-2.07 e+02 \\
-2.20 e+02 \\
-2.33 e+02 \\
-2.45 e+02 \\
-2.58 e+02 \\
-2.71 e+02 \\
-2.84 e+02 \\
-2.97 e+02 \\
3.10 e+02 \\
3.23 e+02\end{array}$ \\
\hline $\begin{array}{l}\text { d) Contour } 102 \\
6.41 \mathrm{e}-01 \\
-1.23 \mathrm{e}+01 \\
-2.53 \mathrm{e}+01 \\
-3.82 \mathrm{e}+01 \\
-5.12 \mathrm{e}+01 \\
-6.41 \mathrm{e}+01 \\
-7.71 \mathrm{e}+01 \\
-9.00 \mathrm{e}+01 \\
-1.03 \mathrm{e}+02 \\
-1.16 \mathrm{e}+02 \\
-1.29 \mathrm{e}+02 \\
-1.42 \mathrm{e}+02 \\
-1.55 \mathrm{e}+02 \\
-1.68 \mathrm{e}+02 \\
-1.81 \mathrm{e}+02 \\
-1.94 \mathrm{e}+02 \\
-2.07 \mathrm{e}+02 \\
-2.20 \mathrm{e}+02 \\
-2.32 \mathrm{e}+02 \\
-2.45 \mathrm{e}+02 \\
-2.58 \mathrm{e}+02 \\
-2.71 \mathrm{e}+02 \\
-2.84 \mathrm{e}+02 \\
-2.97 \mathrm{e}+02 \\
-3.10 \mathrm{e}+02 \\
-3.23 \mathrm{e}+02\end{array}$ & $\begin{array}{l}9.75 e-01 \\
-1.20 e+01 \\
-2.50 e+01 \\
-3.79 e+01 \\
-5.09 e+01 \\
-6.38 e+01 \\
-7.68 e+01 \\
-8.98 e+01 \\
-1.03 e+02 \\
-1.16 e+02 \\
-1.29 e+02 \\
-1.42 e+02 \\
-1.55 e+02 \\
-1.68 e+02 \\
-1.81 e+02 \\
-1.93 e+02 \\
-2.06 e+02 \\
-2.19 e+02 \\
-2.32 e+02 \\
-2.45 e+02 \\
-2.58 e+02 \\
-2.71 e+02 \\
-2.84 e+02 \\
-2.97 e+02 \\
3.10 e+02 \\
-3.23 e+02\end{array}$ & \\
\hline
\end{tabular}




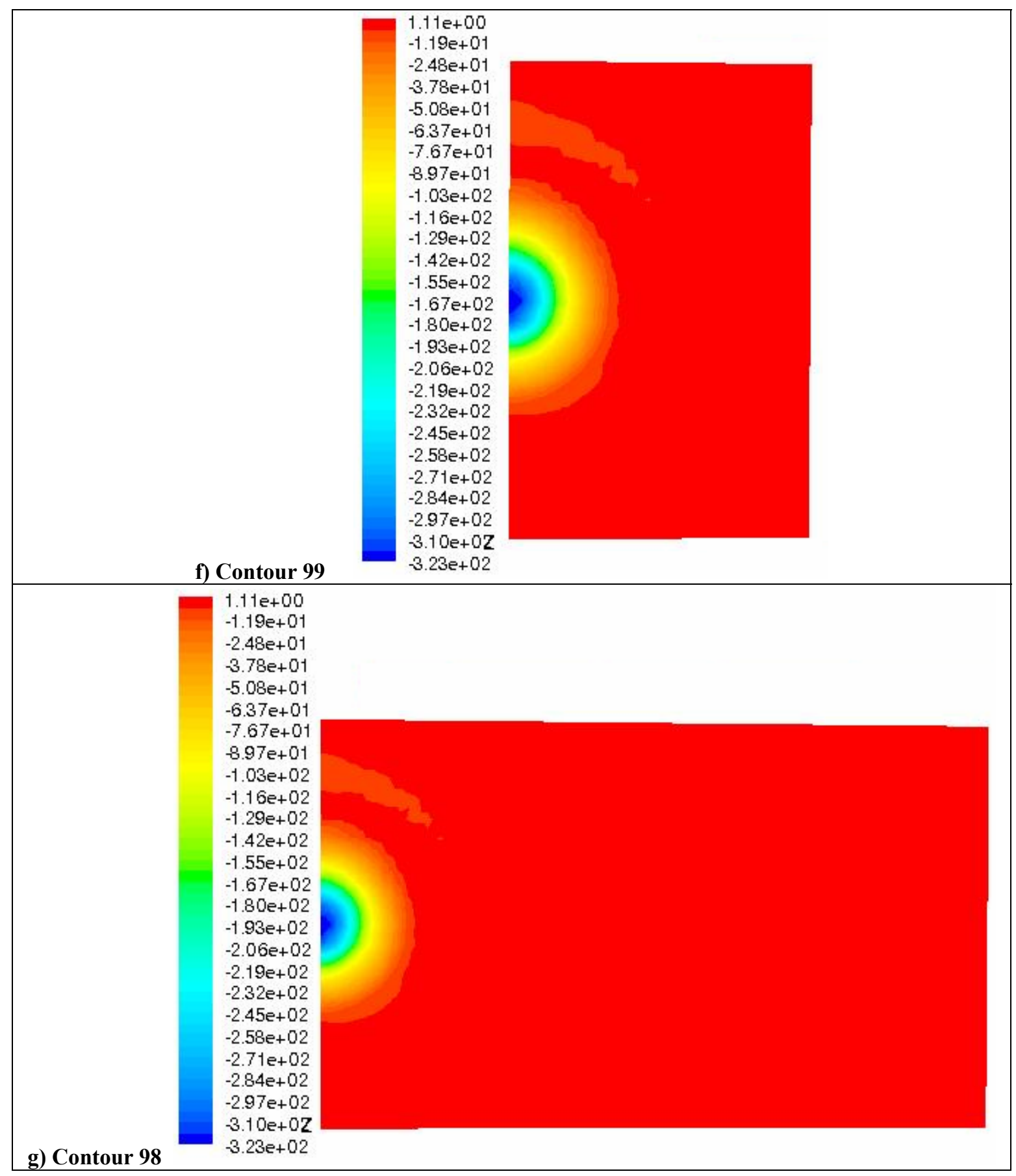




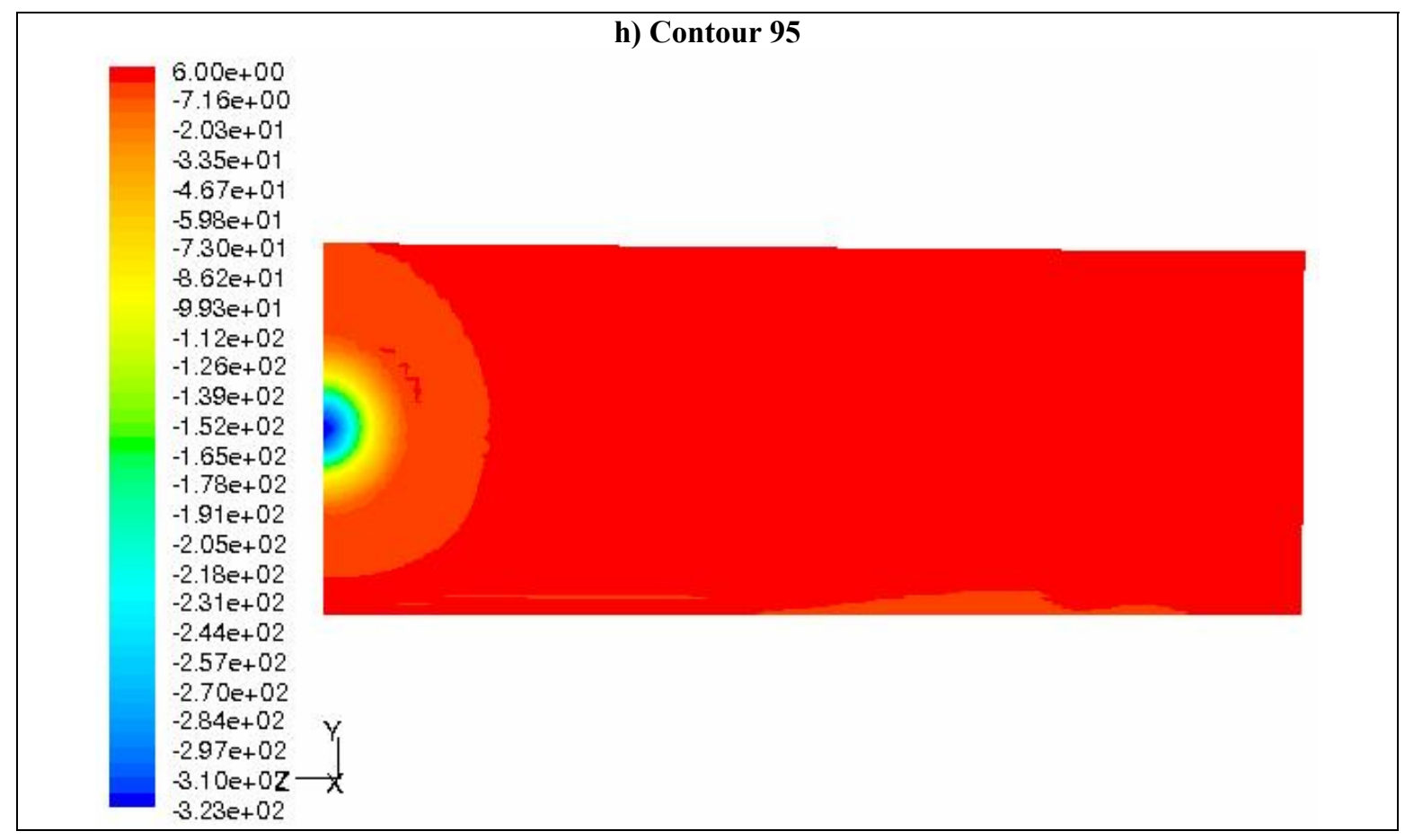

Figure 95. Rectangles drawn to the right of the vortex core to compute the circulation.

Table 4. Circulation variation at different areas at the right of the vortex core.

\begin{tabular}{|c|c|c|c|c|c|c|}
\hline \multirow{2}{*}{ Contour } & \multicolumn{2}{|c|}{$\mathbf{z}(\mathbf{f t})$} & \multicolumn{2}{|c|}{$\mathbf{y}(\mathbf{f t})$} & Distance \\
\cline { 2 - 5 } & $\min$ & $\max$ & $\min$ & $\mathbf{m a x}$ & $\begin{array}{c}\text { Circulation } \\
\left(\mathbf{f t}^{2} / \mathbf{s}\right)\end{array}$ \\
\hline 93 & 0.00 & 2.62 & 0.70 & 0.28 & 2.623 & 7.14 \\
\hline 95 & 1.00 & 2.62 & 0.70 & 0.28 & 1.623 & 7.66 \\
\hline 98 & 2.00 & 2.62 & 0.70 & 0.28 & 0.623 & 7.62 \\
\hline 99 & 2.20 & 2.62 & 0.70 & 0.28 & 0.423 & 7.63 \\
\hline 101 & 2.40 & 2.62 & 0.70 & 0.28 & 0.223 & 7.36 \\
\hline 102 & 2.50 & 2.62 & 0.70 & 0.28 & 0.123 & 6.44 \\
\hline 103 & 2.55 & 2.62 & 0.70 & 0.28 & 0.073 & 4.73 \\
\hline 104 & 2.58 & 2.62 & 0.70 & 0.28 & 0.048 & 3.35 \\
\hline 105 & 2.60 & 2.62 & 0.70 & 0.28 & 0.023 & 1.67 \\
\hline
\end{tabular}

Figure 96 shows the variation of the circulation at different areas to the right of the wingtip vortex core at one chord downstream of the trailing edge of a NACA 0012 wing. It shows the circulation approaches an asymptotic value when the area gets bigger. When the area nears the wall, the circulation begins to decrease due to wall effects. 


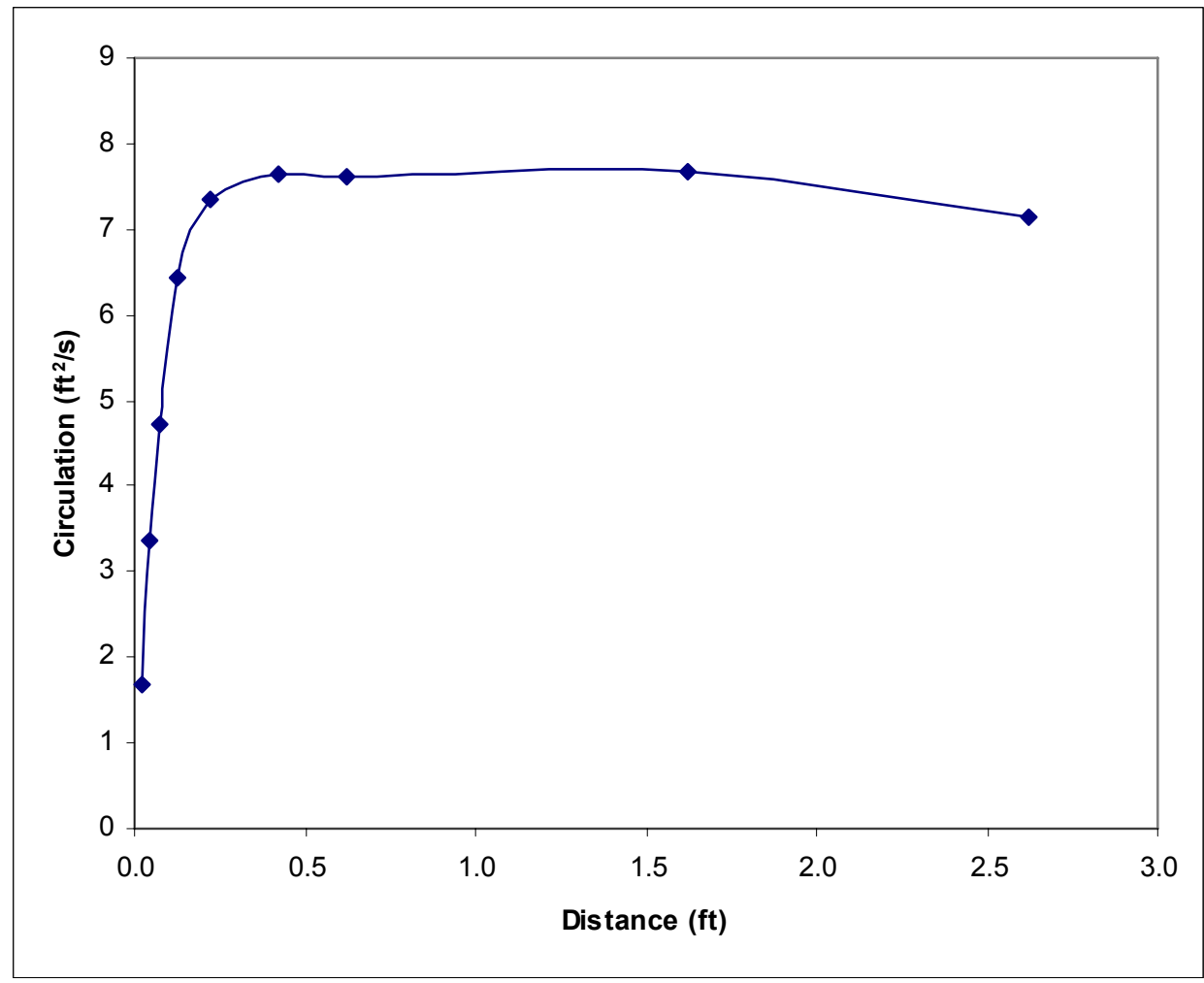

Figure 96. Circulation at different areas at the right of a wingtip vortex core. 


\section{Chapter 5: Application of Boundary Layer Control}

\section{Overview}

High pressure air on the bottom of a wing accelerates to the low pressure region on top of a wing near the wing tip. This flow is shed as a wing tip vortex. The particles on the bottom moving to the top cause an outboard movement of the adjacent streamlines. Similarly, this flow around the tip also pushes the streamlines on top of the wing in the inboard direction. The application of BLC by suction slots along the wing tip trailing edge helps to reduce these spanwise pressure gradients. To further reduce these spanwise pressure gradients, one or more suction slots are required in the vicinity of the wing tip. The mass flow captured at this slot can be vented either at a circulation control slot or at any jet slot into the free stream direction.

\section{Wing Tip Vortex Reduction by BLC}

Several different configurations have been evaluated computationally to explore their potential to reduce the wing tip vortex. The goal of these simulations is to show that the wing tip vortex can be reduced qualitatively.

It should be recognized that the low aspect ratio of the wing simulated (1.5) does not represent a realistic wing, since aspect ratio usually ranges from 6 to 20. However, this wing does allow to see the wingtip vortex formation to be easily visualized.

The wing model used in these simulations is similar to the one used by Chow et al. [43] and Dacles-Mariani et al. [37]. The model was a rectangular wing with $\mathrm{AR}=1.5,4$ $\mathrm{ft}$. chord, and $3 \mathrm{ft}$. half-span. The airfoil section was a NACA 0012 at $10^{\circ}$ angle of attack. The free-stream velocity was $170 \mathrm{ft} / \mathrm{s}$ and the Reynolds number $4.6 \times 10^{6}$. The computational domain was shown in Figure 78.

\section{Blowing air at the Wing Tip}

The boundary condition for this wing tip was obtained by defining a uniform inlet velocity of magnitude $V_{\infty}=170 \mathrm{ft} / \mathrm{s}$. Figure 97 shows two separate wing tip vortices as predicted by other authors for a straight wing tip. The inner blue vortex is produced by 
the separation of the upper boundary layer, while the outer green vortex is a result of the separation of the bottom boundary layer. The colors represent the particle id.

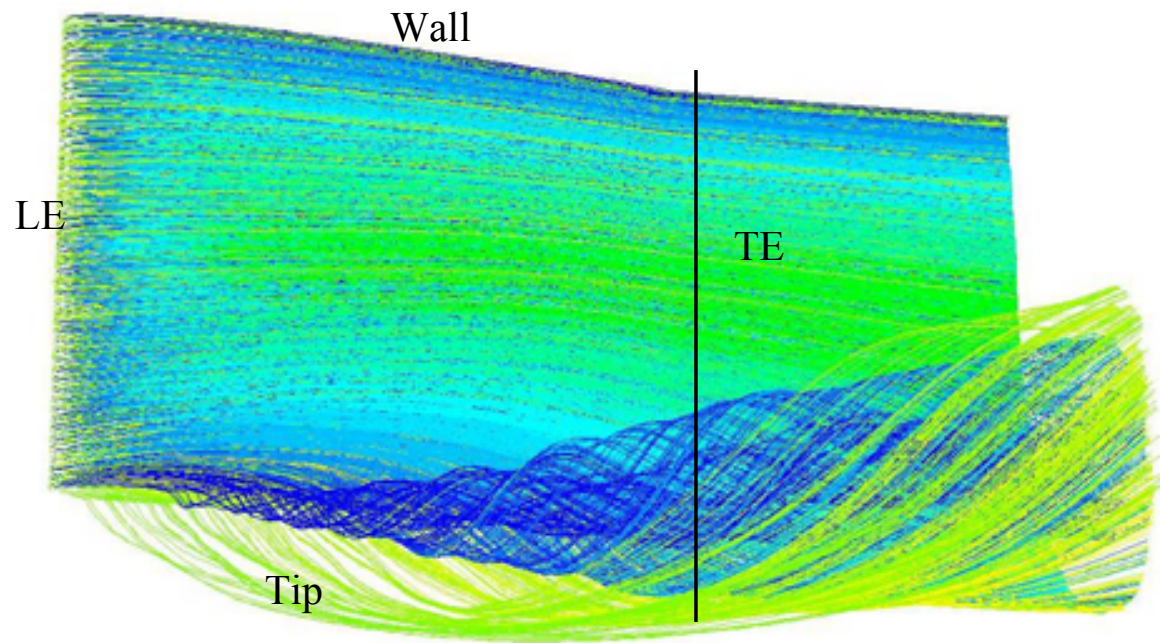

Figure 97. Streamlines on a wing with blowing at $V_{\infty}$ through a slot at the wing tip.

Blowing at the wing tip with this slot geometry does not counteract the wing tip vortex; rather, it splits the vortex in two and increases their diameter.

\section{Applying Suction at the Wing Tip}

Suction is applied at the wing tip slot by introducing an opening suction inlet velocity equal to $\mathrm{V}_{\infty}$. The magnitude of the suction is not enough to cancel the inboard movement of the top streamlines, as can be seen in Figure 98.

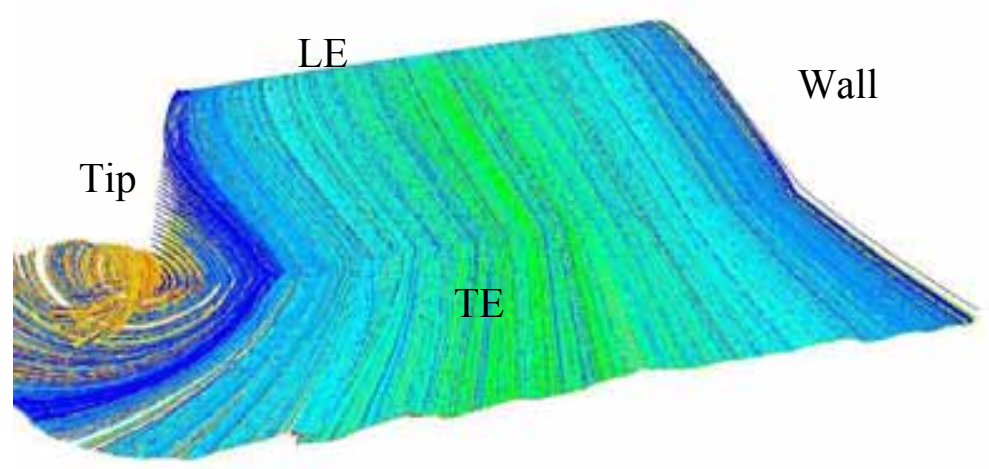

Figure 98. Streamlines on a wing with suction at the wing tip, as viewed from downstream.

However, suction at $\mathrm{V}_{\infty}=170 \mathrm{ft} / \mathrm{s}$ is sufficient to make the pressure coefficient contours more parallel on top of the wing (Figure 99). 


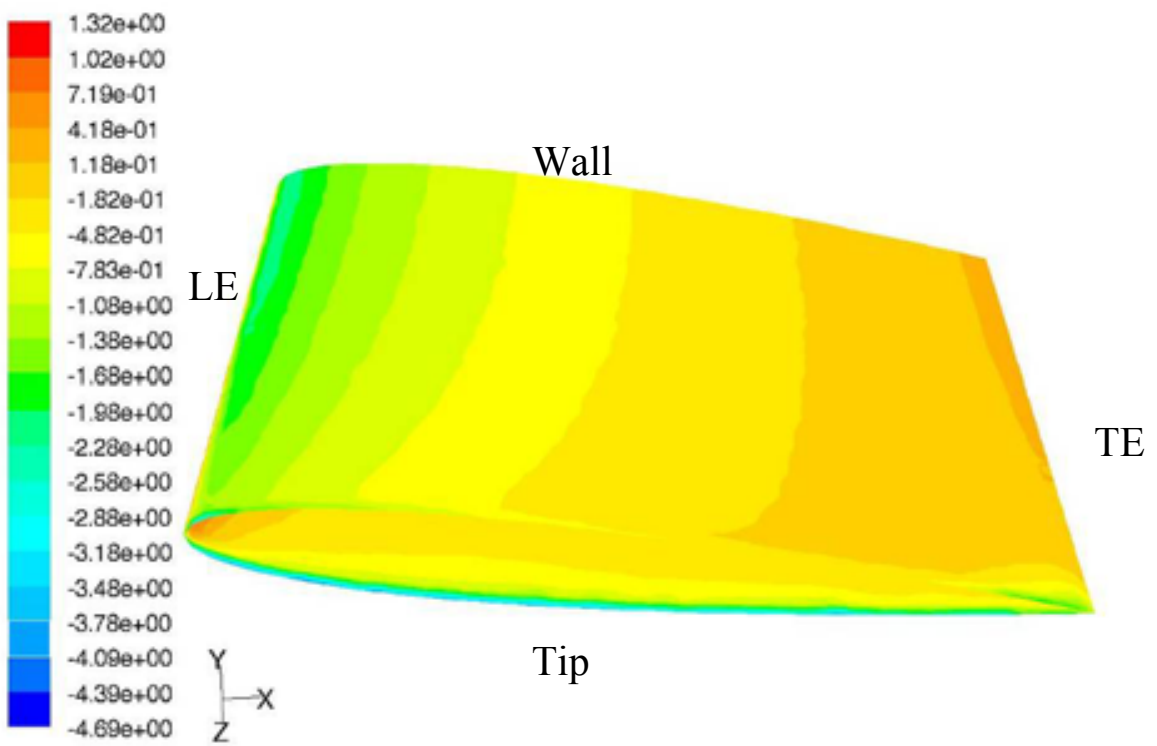

Figure 99. Pressure coefficient contours on top of the wing when wing tip suction is applied.

Then, the wing tip slot suction velocity is increased to twice $\mathrm{V}_{\infty}$, and now the streamlines on top of the wing are almost straight (Figure 100). Unfortunately, the wing tip vortex is still present as shown by the outboard movement of the bottom streamlines (Figure 101).

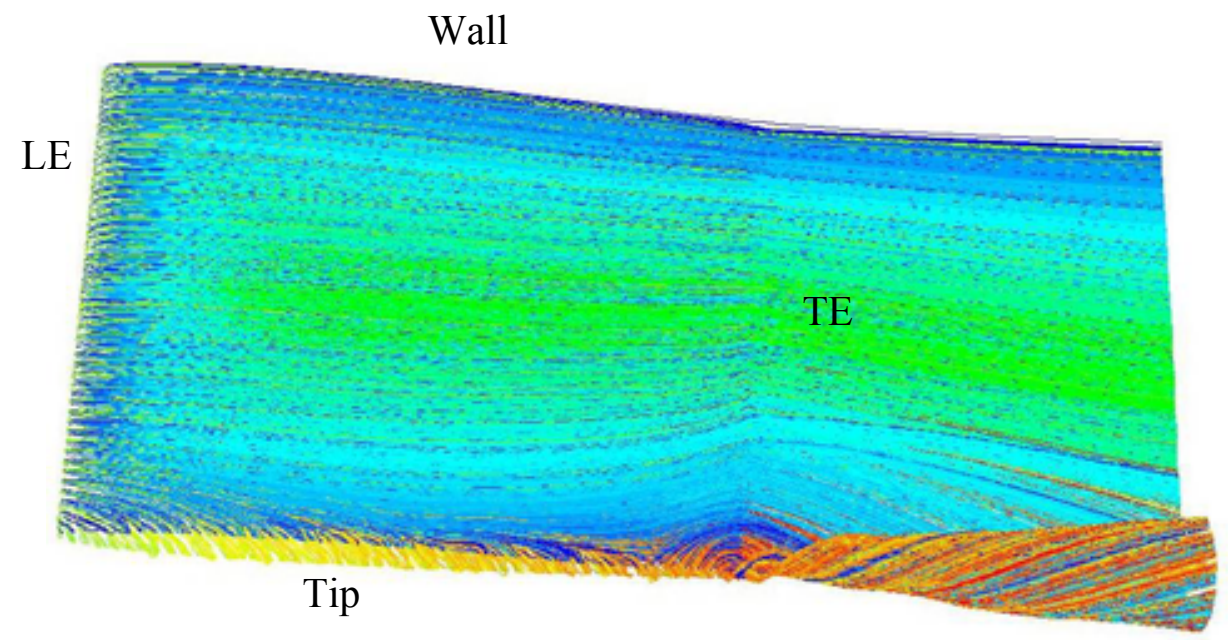

Figure 100. Streamlines on top of the wing when suction is applied at the wing tip. 


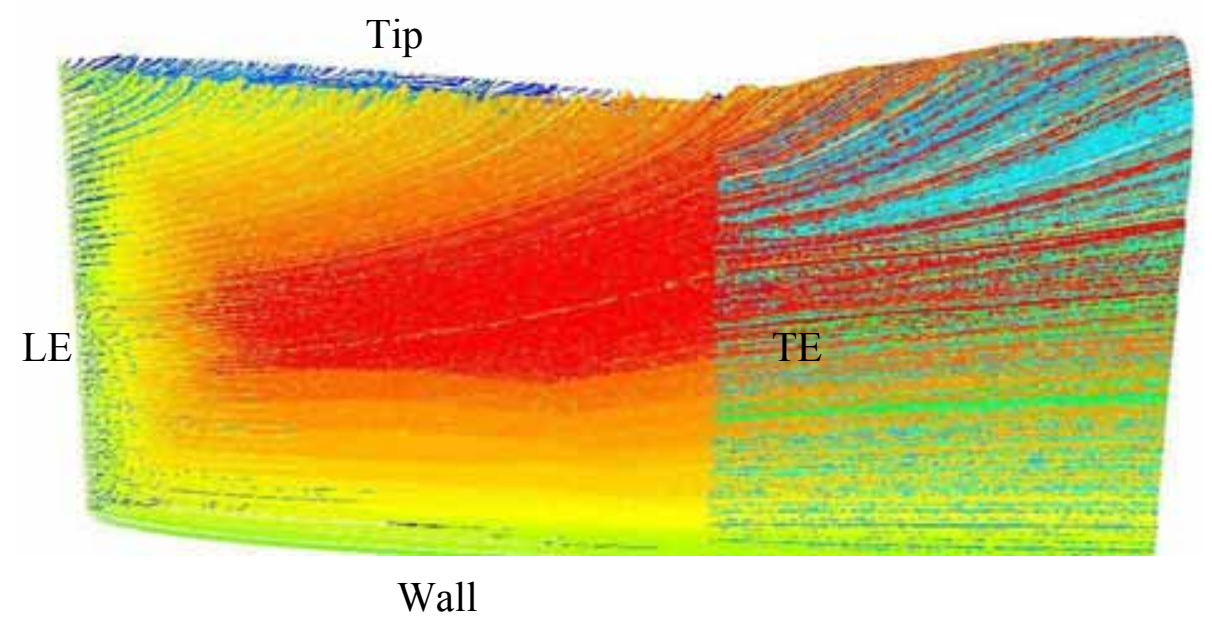

Figure 101. Streamlines on the bottom of the wing when suction is applied.

A suction velocity of twice $V_{\infty}$ at the wing tip is enough to avoid the top streamlines from forming a shed wing tip vortex. Another method is required to counteract the bottom streamlines from producing a shed vortex.

\section{Suction on upper surface and blowing on lower surface}

In this geometry, suction was used to avoid the inboard movement of the top streamlines and air blowing to avoid the outboard movement of the bottom streamlines. Figure 102 shows the same grid with two zones of suction on top and blowing on the bottom of the wing. These slots were $0.3 \mathrm{ft}$ width and the mesh had six million cells.

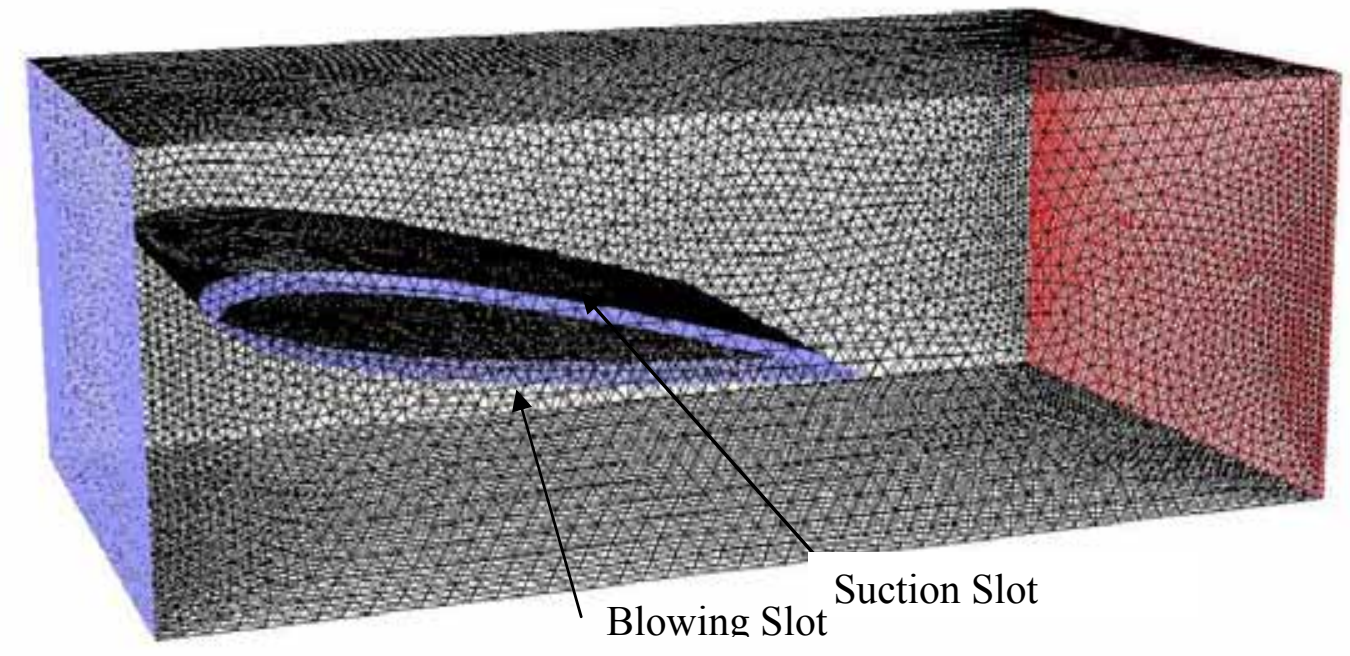

Figure 102. Grid when suction is applied on top and blowing on the bottom. 
This geometry was quickly discarded because the air injected at the bottom surface was flowing directly into the suction slot on top, thereby producing an even bigger wing tip vortex.

\section{Applying Suction on the Bottom wing surface}

A small slot of $0.5 \mathrm{ft}$ width and $0.1 \mathrm{ft}$ high was placed on the bottom of the wing to suck the boundary layer, and to redirect the bottom streamlines. Figure 103 shows the same grid when looking from below the wing.

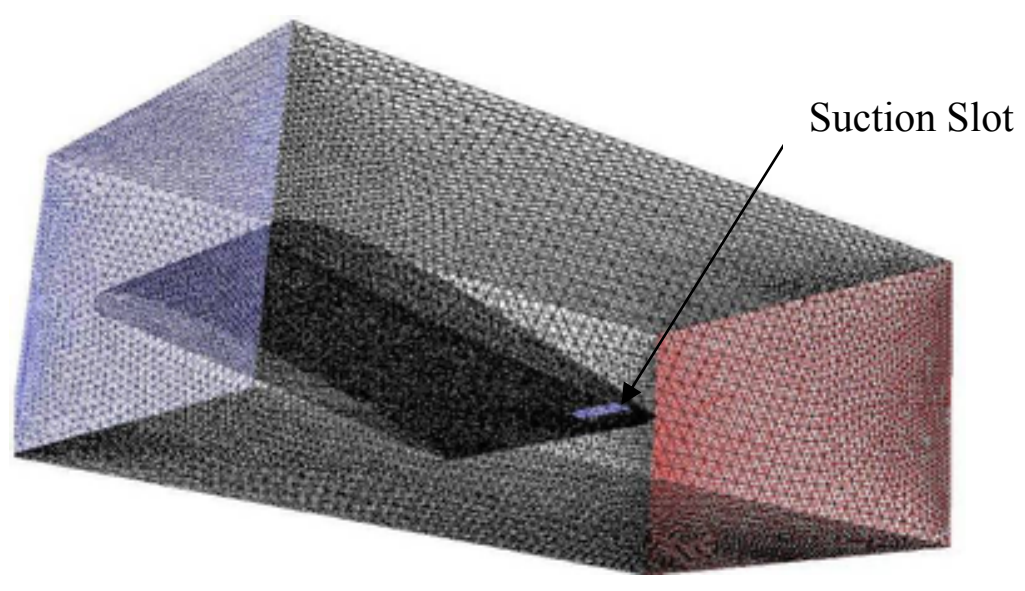

Figure 103. Grid when a small suction slot is placed on the bottom of the wing.

A velocity magnitude equal to $\mathrm{V}_{\infty}$ was first applied to the suction slot, but it was insufficient to make the bottom streamlines straight (Figure 104).

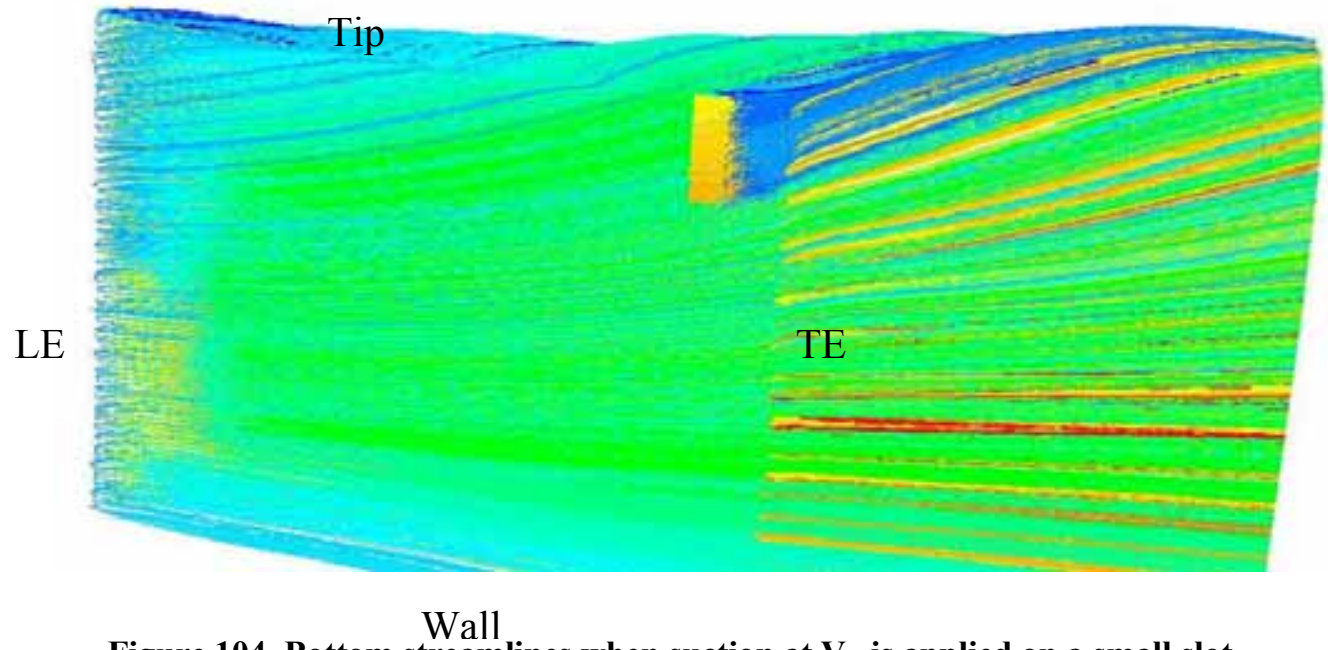

Figure 104. Bottom streamlines when suction at $V_{\infty}$ is applied on a small slot. 


\section{Applying Suction at wingtip Trailing Edge.}

In this configuration, suction is applied at the trailing edge with a velocity of 2 times $\mathrm{V}_{\infty}$ whereupon this air is also then ejected near the leading edge with the same velocity. Figure 105 shows the grid for this configuration, similar to previous grids.

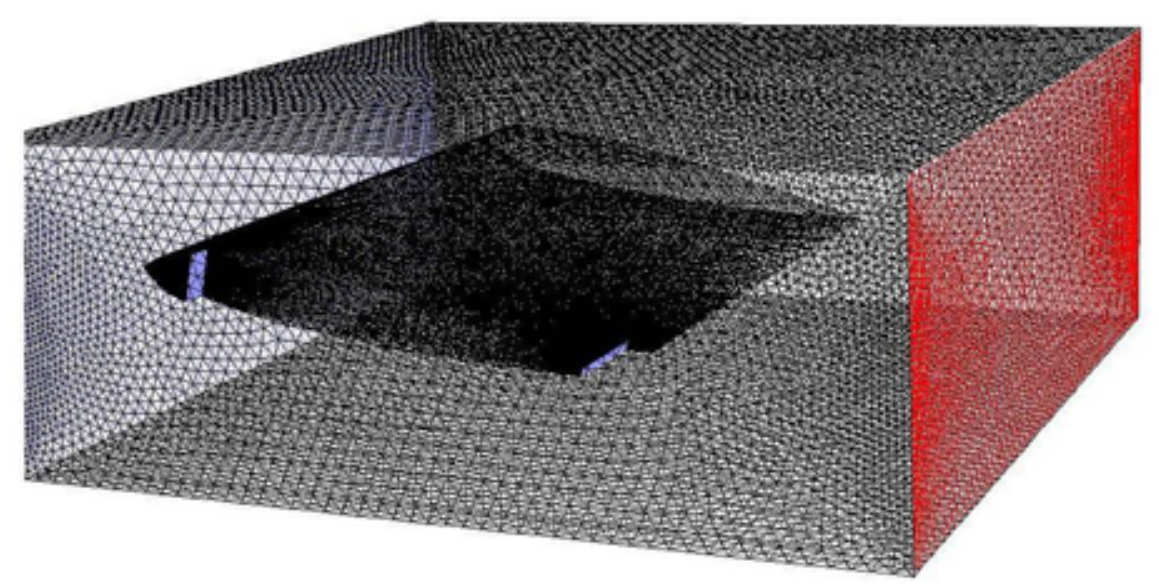

Figure 105. Grid used when suction and blowing are applied at the trailing edge.

The streamlines at the top and the bottom of the wing flow straight (Figure 106 and 107).

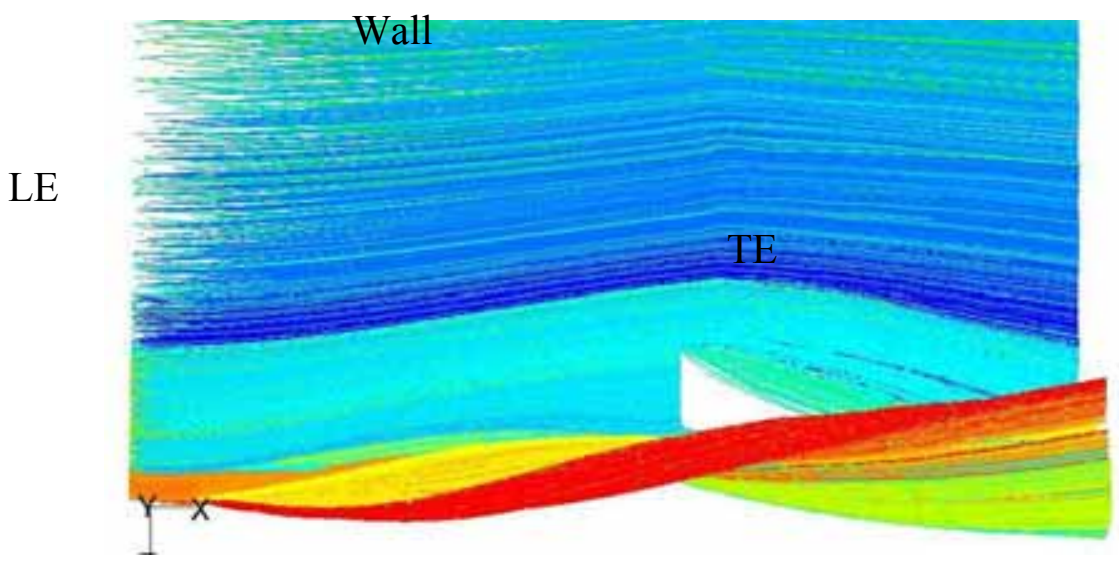

Figure 106. Streamlines at theipop of the wing when suction and blowing are applied at the TE. 


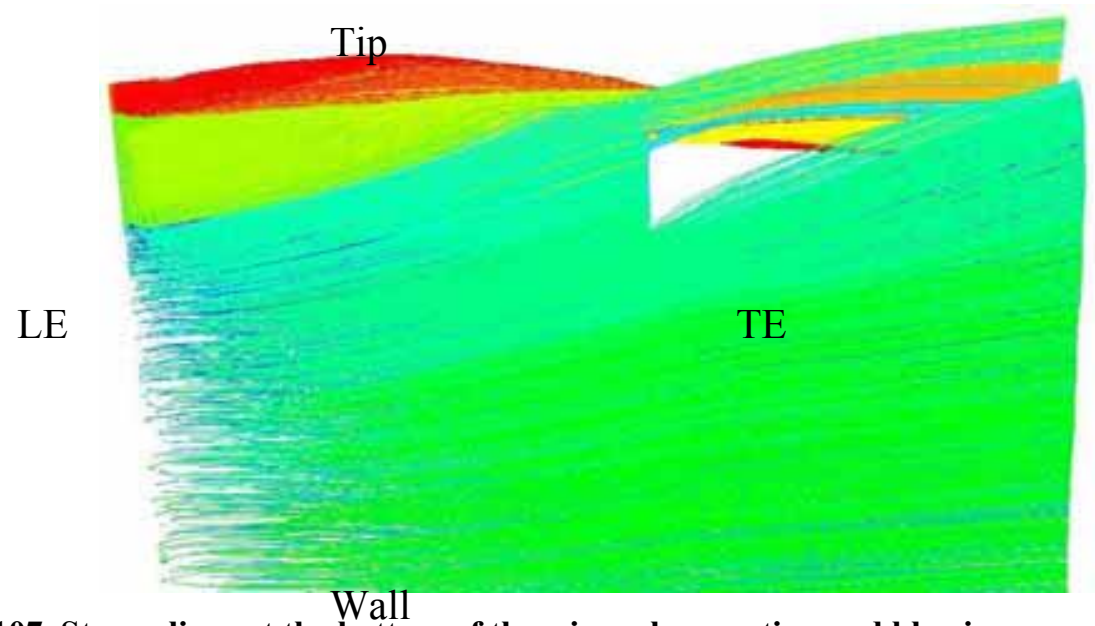

Figure 107. Streamlines at the bottom of the wing when suction and blowing are applied at the TE.

Figure 108 shows the streamlines viewed from the back of the wing.

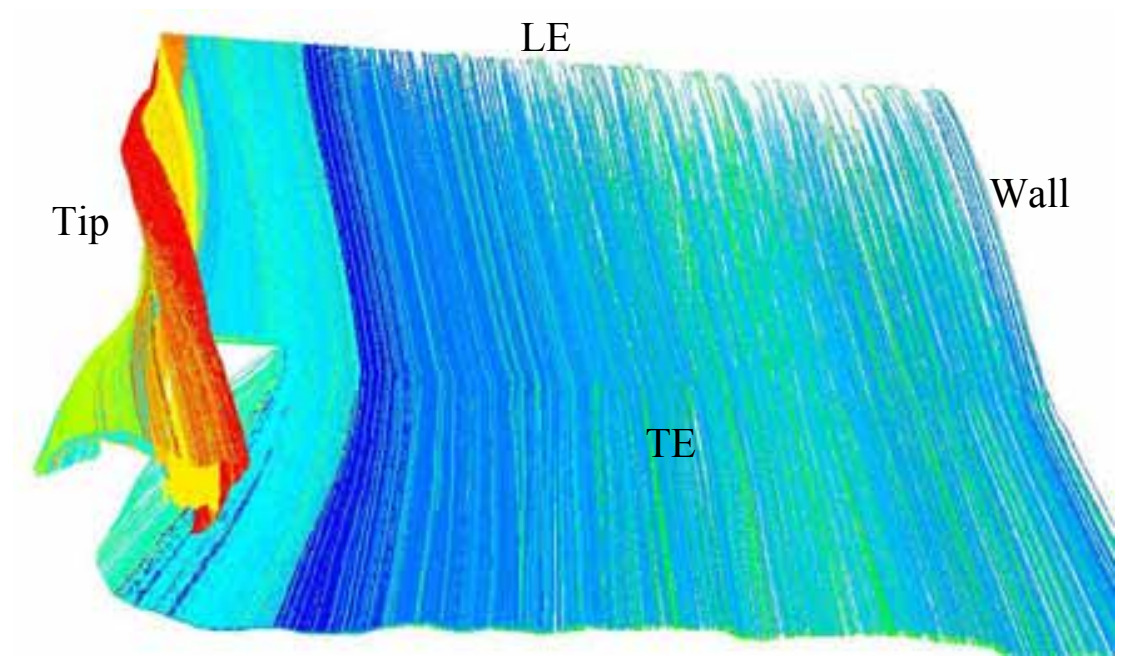

Figure 108. Streamlines when suction and blowing are applied at the TE.

\section{Applying Suction on the Wing Tip and on a Slot on the Bottom wing surface}

Suction equal to $\mathrm{V}_{\infty}$ is applied on the wing tip to cancel the inboard movement of the upper surface streamlines, while the outboard movement is avoided by a suction slot along the bottom part of the wing (Figure 109). The suction slot on the bottom surface of the wing is $3 \mathrm{ft}$ long by $0.2 \mathrm{ft}$ high. The magnitude of the suction velocity in this slot is three times $\mathrm{V}_{\infty}$. Figure 110 shows that the wing tip shed vortex was canceled and the upper surface streamlines are straight. BLC suction along the trailing edge was capable of eliminating wake vorticity but at the expense of bound vorticity and lift. 


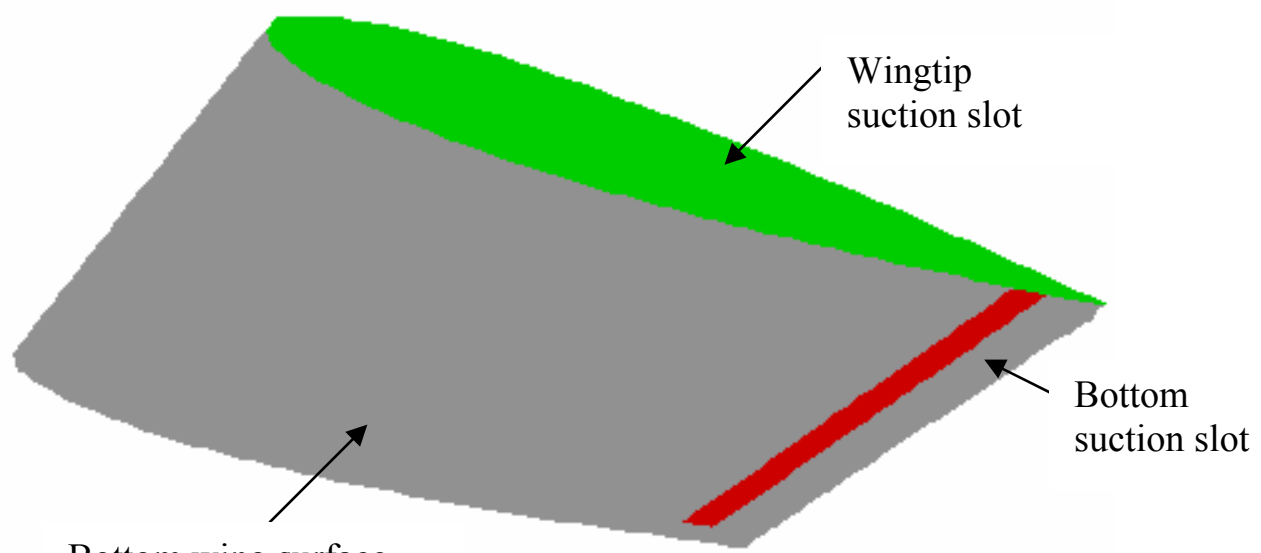

Bottom wing surface

Figure 109. Suction slots on a rectangular wing $A R=1.5$.

Wall

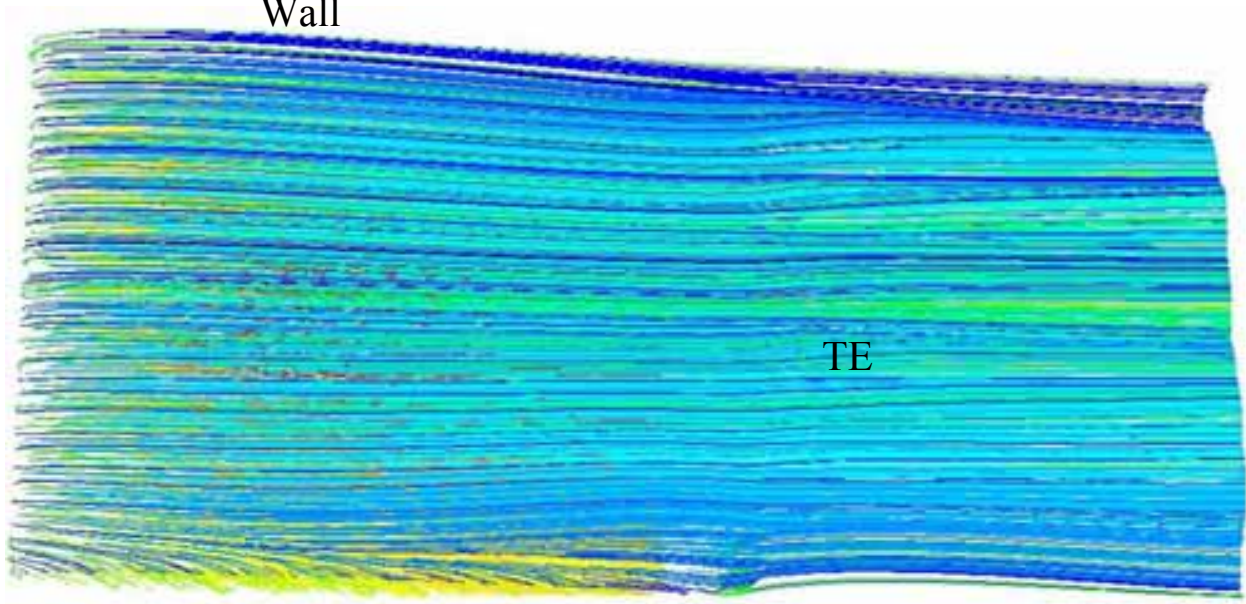

Tip

Figure 110. Wing tip vortex cancellation by wing tip suction and suction slot along the bottom.

Figure 111 shows that the bottom streamlines are also straight.

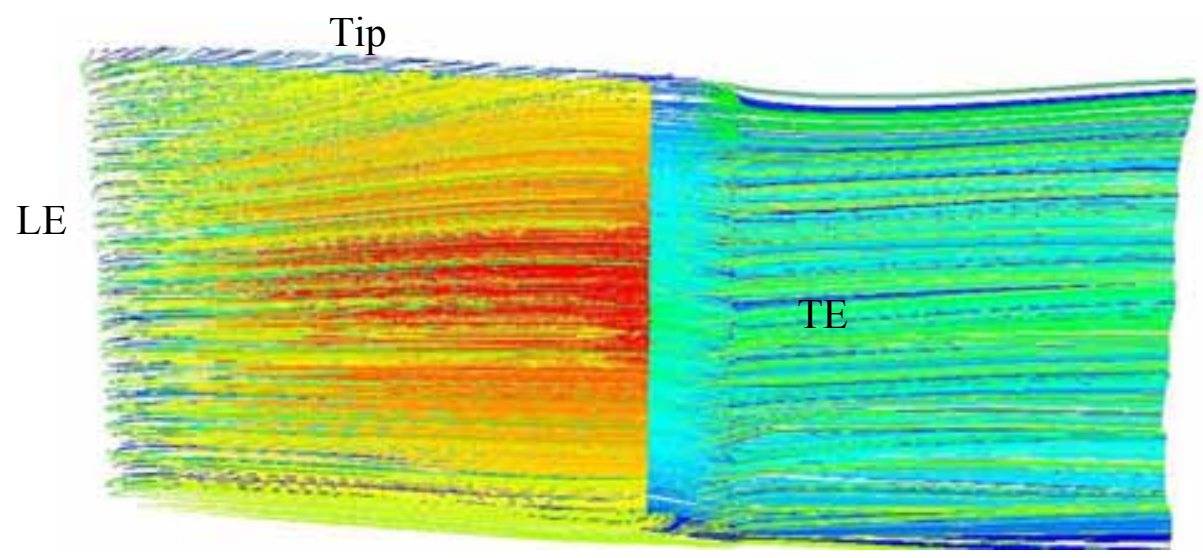

Wall

Figure 111. Bottom streamlines when suction is applied at the wing tip and along a slot on the bottom. 
Figure 112 shows the streamlines when looking from the back.

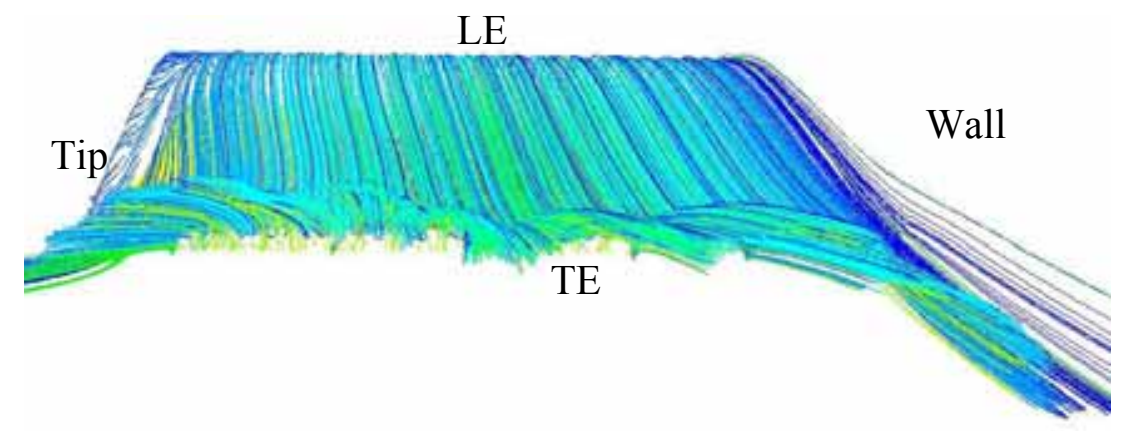

Figure 112. Streamlines when suction is applied at the wing tip and along the bottom of the wing.

Surface pressure contours for this case are shown in Figure 113 and 114.

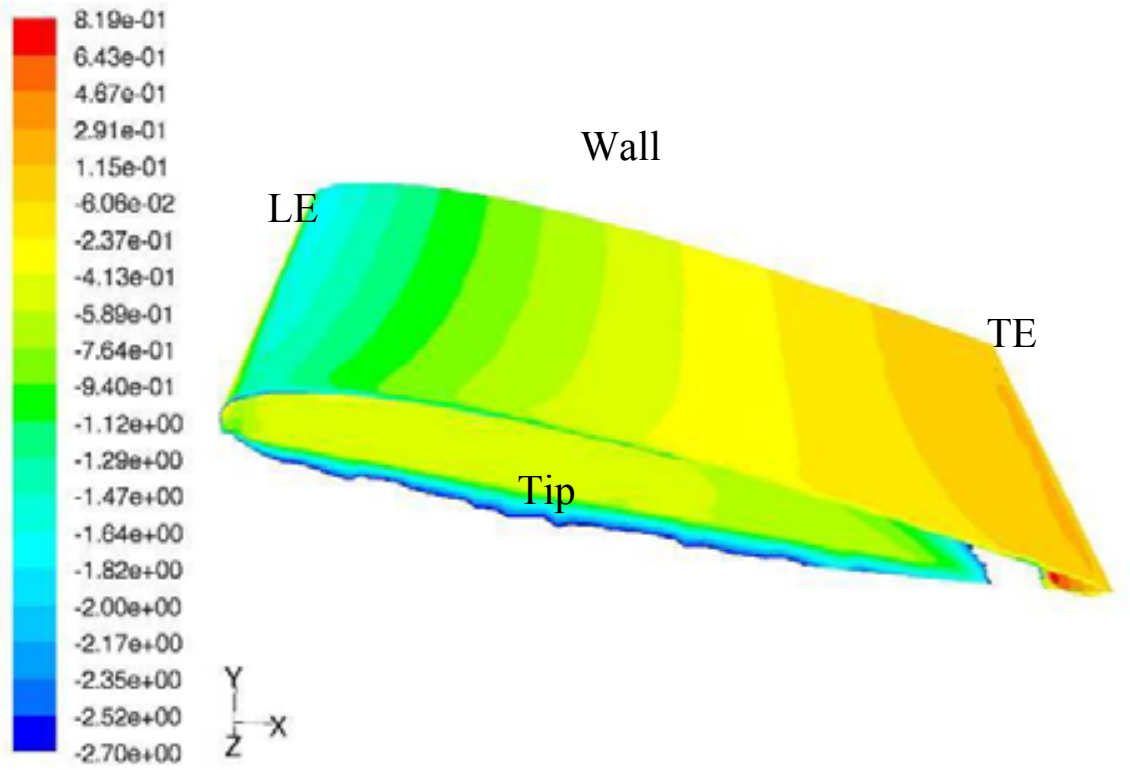

Figure 113. Surface pressure contours on top of the wing when suction is applied on the wing tip and along the bottom of the wing. 


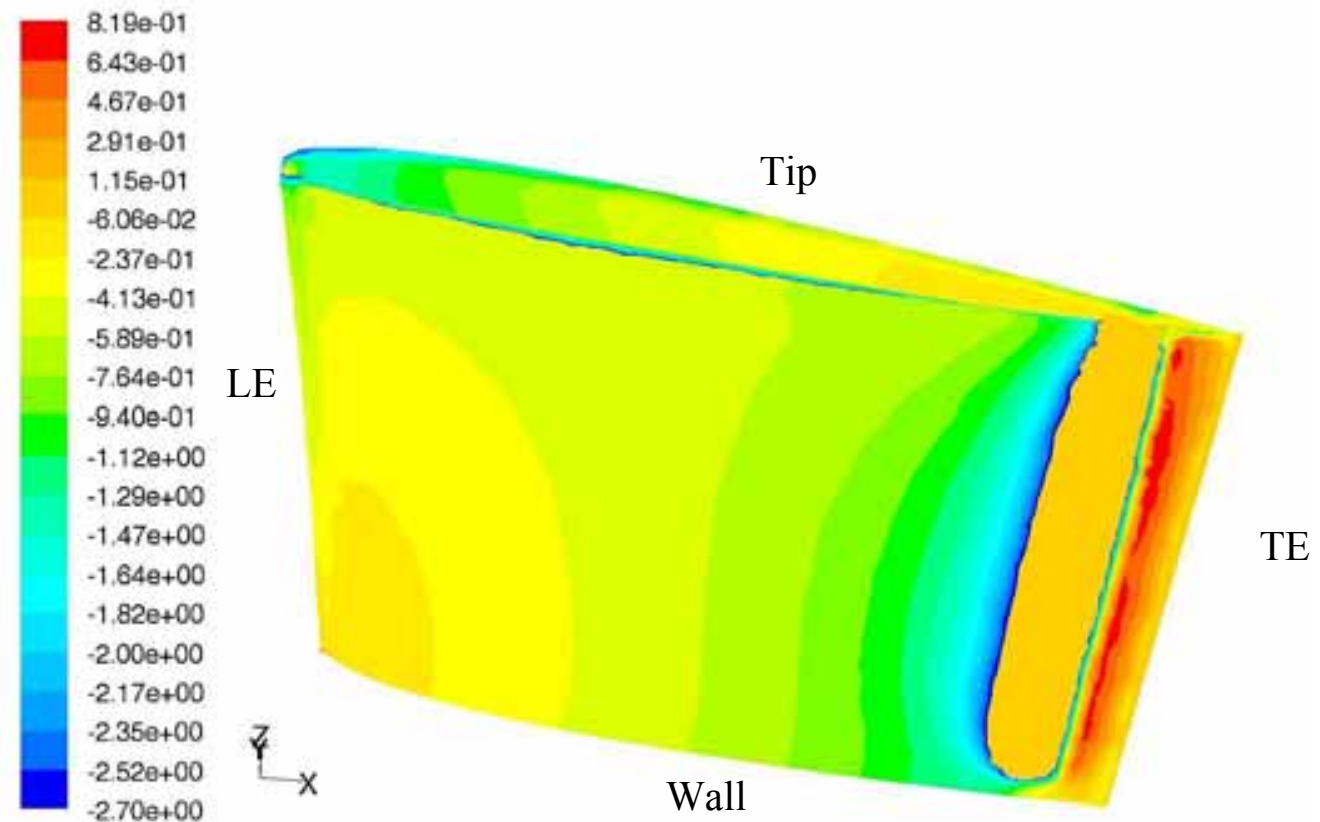

Figure 114. Surface pressure contours on the bottom of the wing when suction is applied on the wing tip and along the bottom of the wing.

\section{Vorticity Results}

The surface integral of the vorticity in the x-direction has been computed for three different configurations: a two dimensional wing, the finite wing from the previous section, and the finite wing with suction on the wing tip and on a slot on the bottom wing surface as presented in the previous section. The integral has been calculated on nine surfaces parallel to the inlet across the computational domain. The surfaces are at the inlet, leading edge, $1 / 4$ chord, $1 / 2$ chord, $3 / 4$ chord, trailing edge, $1 / 4$ chord behind the TE, $1 / 2$ chord behind the TE, and at the outlet. Figure 115 shows that the vorticity is significantly reduced when suction is applied on the wing tip and along a slot on the bottom surface, as presented in Figure 109. 


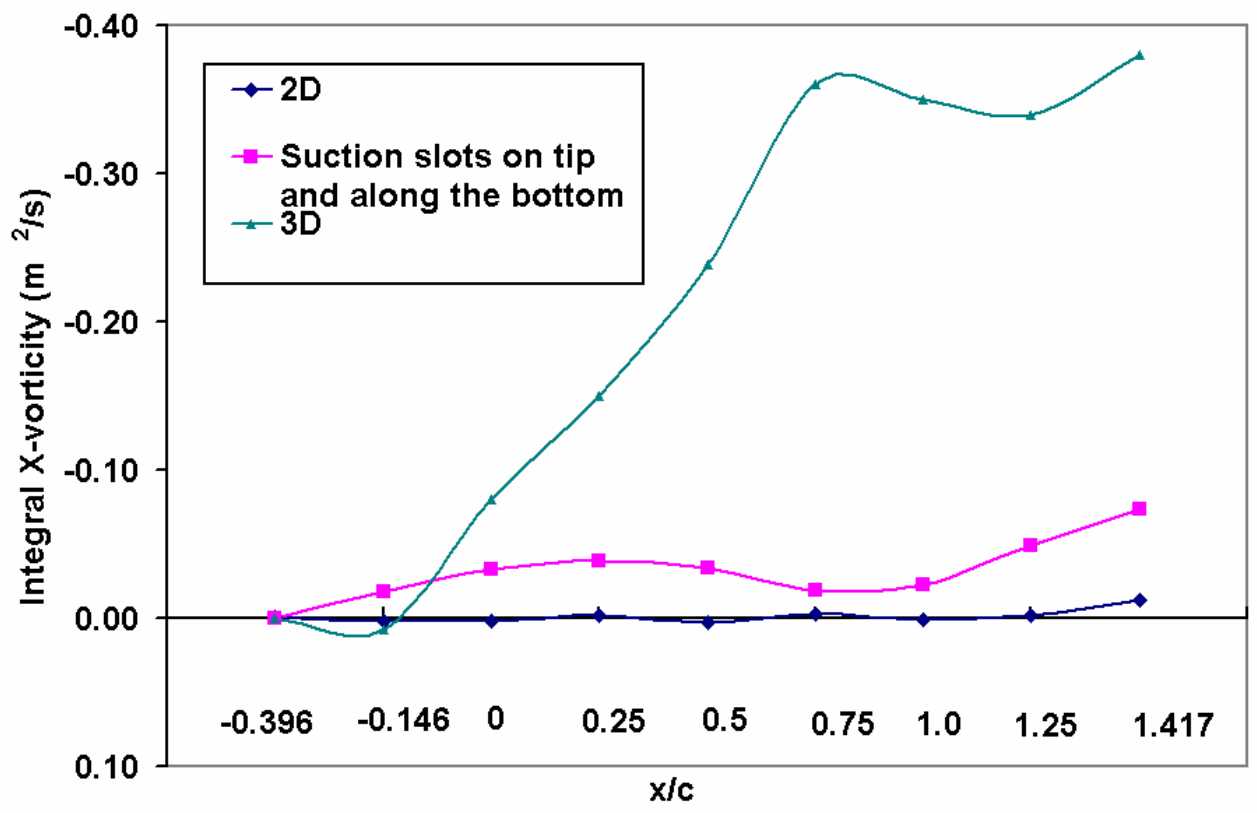

Figure 115. Surface Integral of the X-Vorticity for three wings. 


\section{Chapter 6: Lift and Drag Prediction}

\section{Overview}

This chapter presents a comparison between lift and drag coefficients obtained numerically by the author and experimental measurements done by Chometon and Laurent on a NACA $64_{3}-018$ rectangular wing with $\mathrm{AR}=4$ [48]. These authors used wake survey and balance measurements to obtain lift, profile and induced drag coefficient values.

\section{Drag Prediction using Wake Measurements}

The most common technique to compute the drag of a wing is the integration of the pressure and shear stress distribution acting on its surface. This technique can lead to inaccuracies in the drag prediction but it continues to be the technique used to predict total drag in CFD codes and in some experimental measurements [49]. The determination of the pressure-drag contribution to the total drag involves subtraction of a large component in the thrust direction from a slightly larger force component in the drag direction [49]. Thus the form drag can be accurately predicted if the pressure distribution is known with great accuracy and in great detail. An alternative to the surface integration is the wake integration technique which is based on the principle that the aerodynamic drag is equal to the momentum deficit in the wake.

Several authors have used wake measurements to accurately predict the drag components, e.g.: profile and induced drag. These results have been validated by force balance measurements $[48,50]$. The benefit of using flow surveys of the wake is that it provides separate measurements of induced drag, profile drag, and lift which are valuable for CFD validation [50]. The reason why it has not been accepted as a standard technique is because wake surveys are relatively expensive compared to balance measurements and very little was known about the accuracy of this technique [50].

Chometon and Laurent [48] conducted several experiments in the S2 wind tunnel of the Institut Aérotechnique de Saint-Cyr. This tunnel is of the Eiffel type, with a constant rectangular semi-open test section $2.2 \mathrm{~m}$. wide, and $1.8 \mathrm{~m}$. high. The wing used in their experiments was a rectangular wing with an aspect ratio of 4 , a span of $1.2 \mathrm{~m}$ and 
$0.3 \mathrm{~m}$ chord using an NACA $64_{3}-018$ airfoil. The free stream velocity was $55 \mathrm{~m} / \mathrm{s}$ and the angle of attack $10^{\circ}$.

A six-component balance was used to measure the total drag coefficient and the lift coefficient. Drag components of the wing were obtained by measurements of total pressure and velocity at one span downstream of the trailing edge. The measurements of total pressure were realized using a Kiel probe, and the measurements of the velocity components were performed with a six-hole cone probe. The results are summarized in Table 5. Their total drag coefficient measured by the wake survey method is very close to the one measured by the balance.

Table 5. Wake and Balance measurements for a NACA $64_{3}-018$ at $\operatorname{Re}=1.1 \times 10^{6}$ and $\alpha=10^{\circ}[48]$.

\begin{tabular}{|l|c|}
\hline$C_{D}$ due to pressure losses & 0.034 \\
\hline$C_{D}$ due to momentum deficit & -0.001 \\
\hline$C_{D}$ due to induced drag & 0.018 \\
\hline Total $C_{D}$ by wake survey & 0.051 \\
\hline Total $C_{D}$ by balance data & 0.054 \\
\hline
\end{tabular}

Drag due to total pressure losses, due to component of momentum deficit, and due to $\mathrm{V}$ and $\mathrm{W}$ components of induced drag were computed by equations $6.1,6.2$, and 6.3 , respectively. The drag coefficients were then obtained by dividing the drag by the dynamic pressure and the wing planform area.

$$
\begin{aligned}
& D_{p p}=\iint\left(P_{T \infty}-P_{T}\right) d s \\
& D_{p m}=\frac{\rho}{2} \iint\left(U_{\infty}^{2}-U^{2}\right) d s \\
& D_{i}=\frac{\rho}{2} \iint\left(V^{2}+W^{2}\right) d s
\end{aligned}
$$

\section{Procedure}

The simulations were run in Fluent 6.2.16 on a Beowulf HPC as described in Chapter 4. The Reynolds numbers, the angles of attack and the wings used in the simulations were identical to those used in experiments by Chometon and Laurent [48]. 
Several grids were generated to identify which one gives results closest to the measured ones. The dimensions of the domain and the number of cells and nodes are presented in the results section. Tetrahedral elements were used for all of the grids. Grids up to eleven million cells and 2.1 million nodes were generated. The solver configuration and the turbulence model used were the same as for the numerical study of the wing tip vortex case presented in Chapter 4.

\section{Lift and Drag Coefficients Results}

The simulations required 1700 iterations to converge. The residuals, the lift and drag coefficient monitors are plotted in Figures 116, 117, and 118. Note that the lift coefficient was converged to $\pm 0.0003 \%$, while the drag coefficient was converged to $\pm 0.0012 \%$.
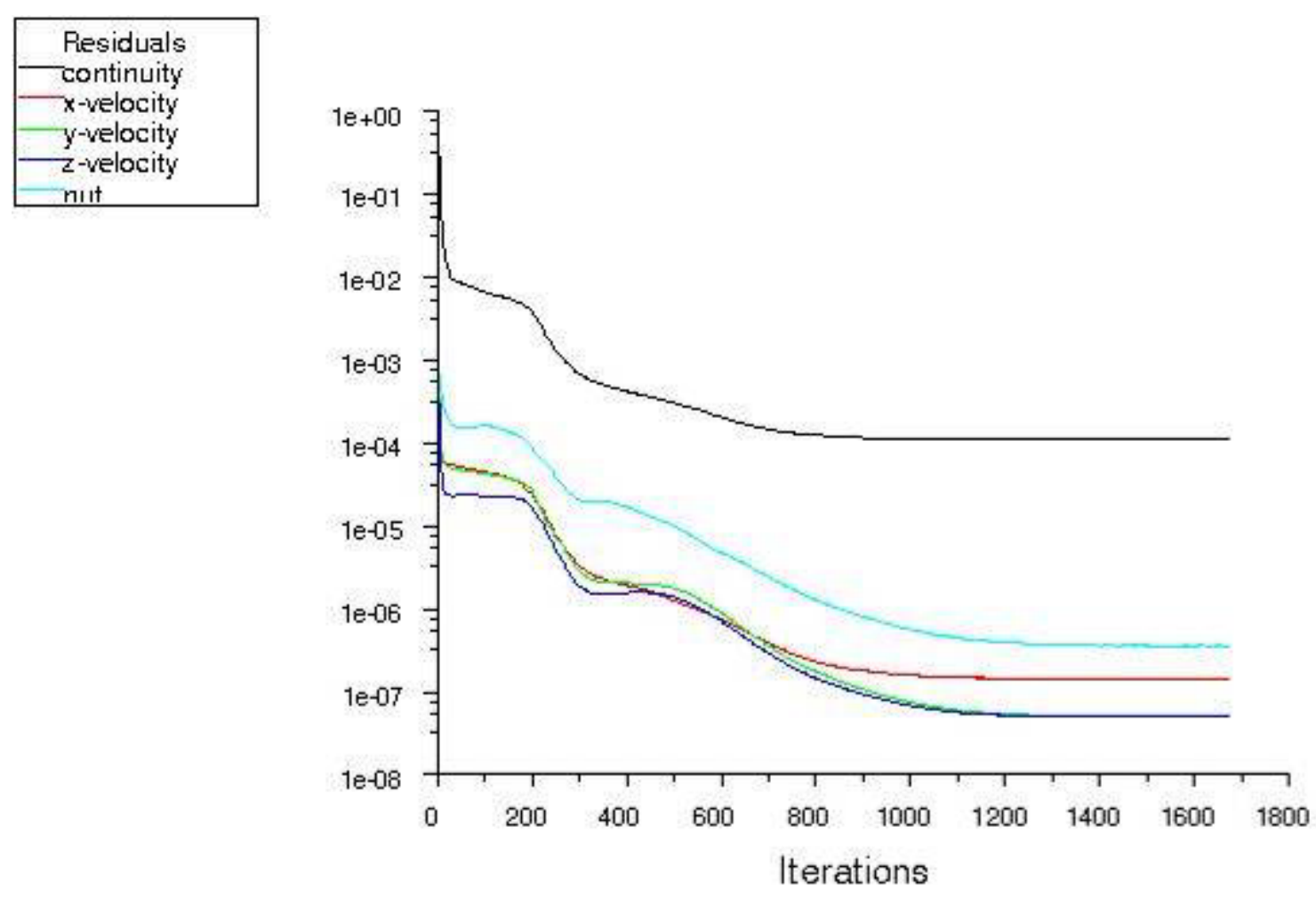

Figure 116. Iterations convergence. 


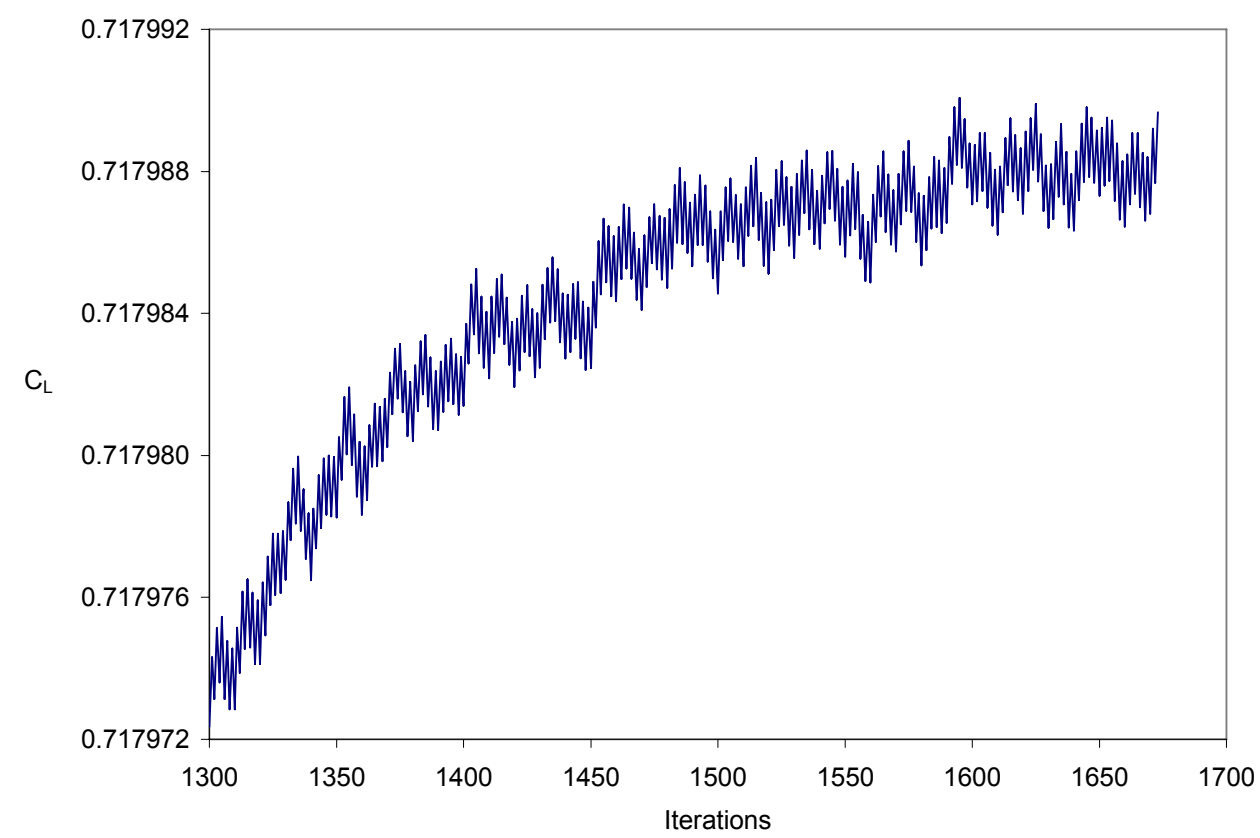

Figure 117. Lift coefficient convergence.

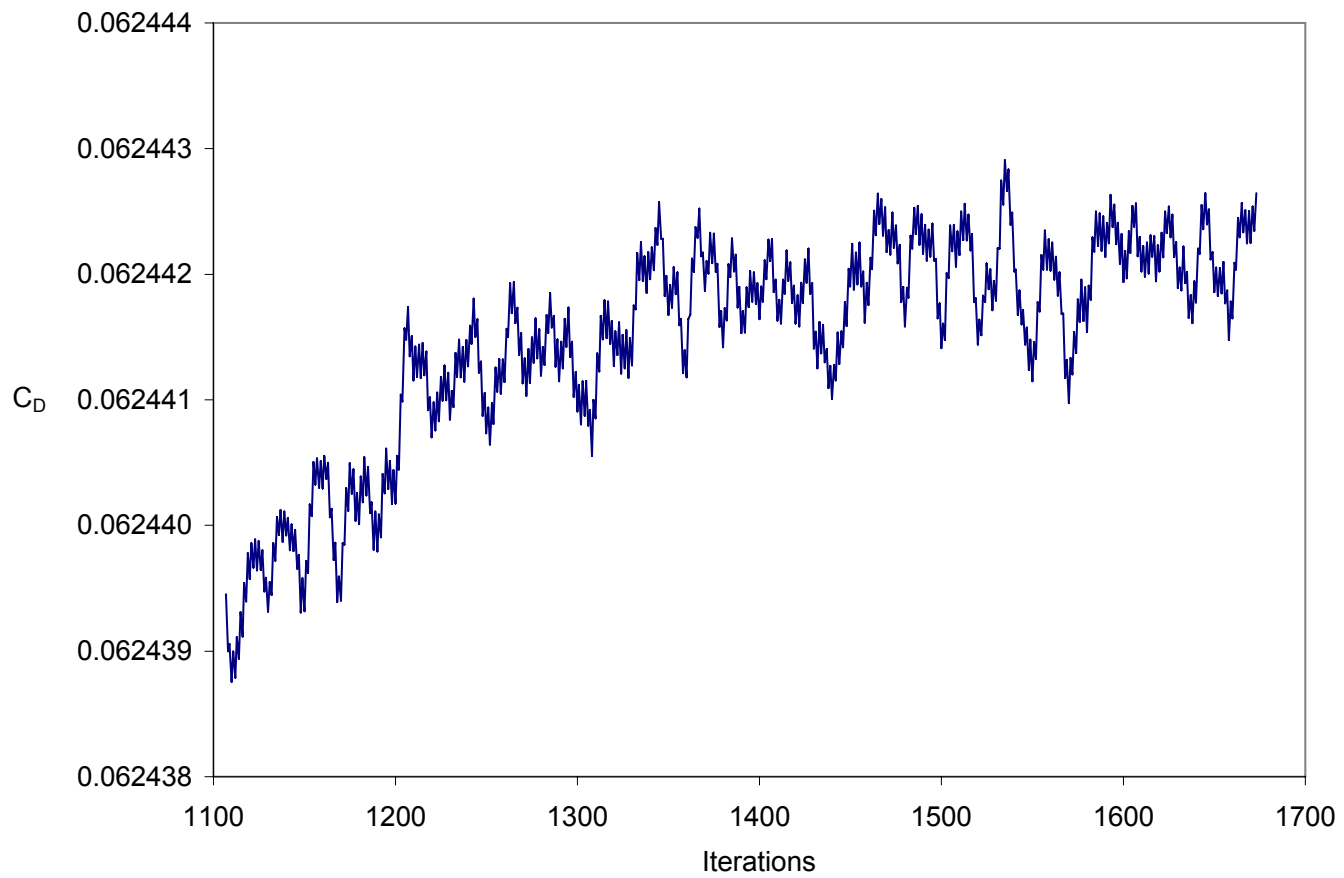

Figure 118. Drag Coefficient Convergence.

Chometon and Laurent obtained coefficients of lift, induced drag, drag due to total pressure losses, and drag due to momentum losses (Table 6). The numerical simulations done at WVU predict accurately Chometon and Laurent's experiment. The 
total drag coefficient column was obtained by summing the induced drag, the drag due to pressure losses, and the drag due to momentum losses.

Table 6. Experimental and Numerical Drag Coefficients on a NACA $64_{3}-018$.

\begin{tabular}{|c|c|c|c|c|c|c|c|c|c|c|}
\hline $\begin{array}{c}\text { Domain } \\
X x Y x Z(m)\end{array}$ & $\begin{array}{l}\text { Cells } \\
\left(\times 10^{6}\right)\end{array}$ & $\begin{array}{l}\text { Nodes } \\
\left(\times 10^{6}\right)\end{array}$ & $C_{L}$ & $\begin{array}{l}\% \mathrm{C}_{\mathrm{L}} \\
\text { Error }\end{array}$ & $C_{D i}$ & $\begin{array}{l}\% C_{D i} \\
\text { Error }\end{array}$ & $C_{D p m}$ & $C_{D p p}$ & $C_{D}$ & $\begin{array}{l}\% C_{D} \\
\text { Error }\end{array}$ \\
\hline Experimental & & & 0.690 & & 0.018 & & -0.0010 & 0.034 & 0.051 & \\
\hline $4 \times 1.8 \times 1.1$ & 1.7 & 0.3 & 0.705 & 2.2 & 0.020 & $\overline{11}$ & -0.0005 & 0.044 & 0.064 & 25 \\
\hline $4 \times 1.8 \times 1.1$ & 3.0 & 0.6 & 0.710 & 2.9 & 0.022 & 22 & -0.0009 & 0.041 & 0.062 & 22 \\
\hline $4 \times 1.8 \times 1.1$ & 6.0 & 1.1 & 0.715 & 3.6 & 0.020 & 11 & -0.0011 & 0.037 & 0.056 & 10 \\
\hline
\end{tabular}

\section{BLC Application Results}

\section{NACA 0016 Wing}

An aspect ratio six unswept rectangular wing using a NACA 0016 airfoil has been used herein to explore the potential of various BLC configurations to reduce induced drag. The computational domain selected was $3 \times 8 \times 6 \mathrm{ft}$. in $\mathrm{x}, \mathrm{y}$, and $\mathrm{z}$-directions, respectively. The wing was at $8.22^{\circ}$ angle of attack and it had $1 \mathrm{ft}$ chord and $6 \mathrm{ft}$ span. The grid contains 7.9 million cells and 1.5 million nodes. The inlet velocity $\mathrm{V}_{\infty}$ was 205 $\mathrm{ft} / \mathrm{s}$ and the Reynolds number was 1.27 million based on the chord length. Figure 119 and 120 show the wing with the four suction slots on the wing tip and the blowing slot next to the mid-wing chord. The top and bottom chord suction slots are $0.02 \mathrm{ft}$ width and $1.02 \mathrm{ft}$ long, while the top and bottom TE suction slots are $0.002 \mathrm{ft}$ width and $0.42 \mathrm{ft}$ long.

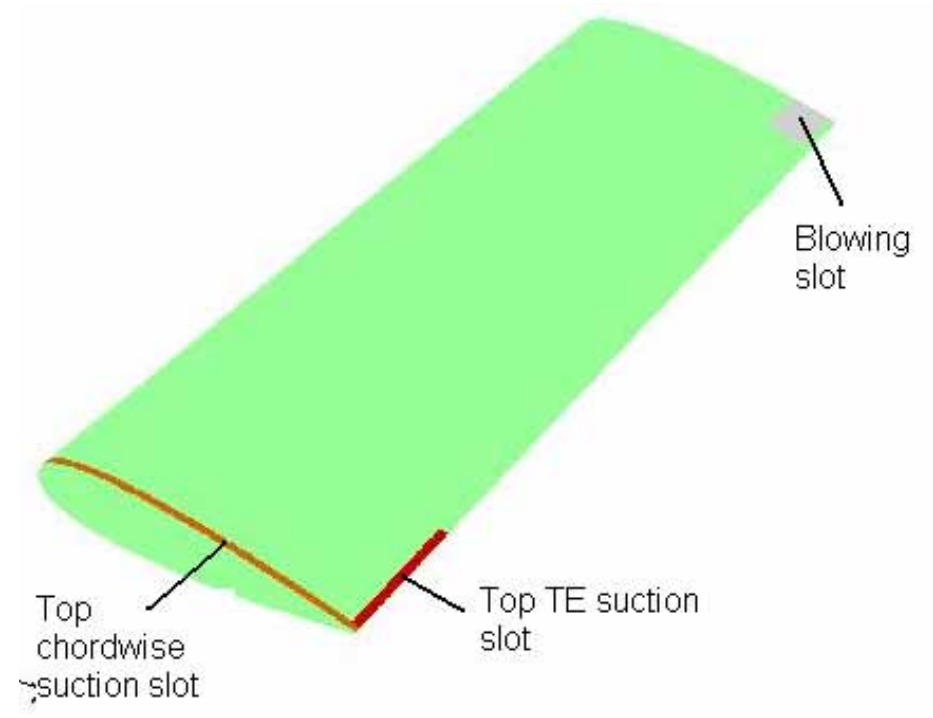

Figure 119. Top Wing Surface. 


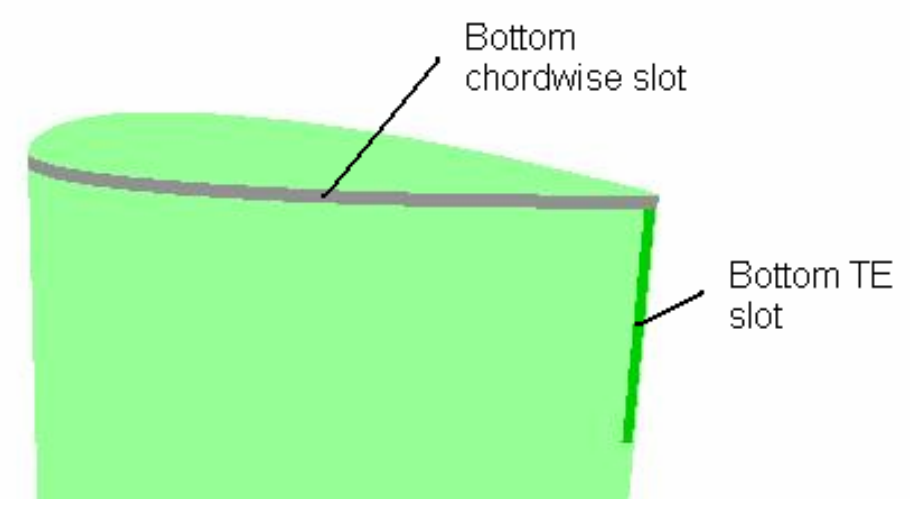

Figure 120. Bottom wing surface.

Table 7 provides the results obtained with various BLC blowing configurations. The first row shows the reference values without blowing or suction. The location and velocity of the blowing and suction slots are shown in the first two columns. The next-tolast column called $\% \mathrm{D}_{\mathrm{i}}$ computes the percent reduction in induced drag. The last column shows the increment in the lift-to-drag-ratio as a percentage.

Table 7. Parametric Study of BLC application on a Wing.

\begin{tabular}{|c|c|c|c|c|c|c|c|c|c|}
\hline $\begin{array}{c}\text { Blowing } \\
(\mathrm{ft} / \mathrm{s})\end{array}$ & Suction (ft/s) & $C_{D i}$ & $C_{D p m}$ & $C_{D p p}$ & $C_{D}$ & $C_{L}$ & L/D & $\begin{array}{c}\% D_{1} \\
\text { reduction }\end{array}$ & $\begin{array}{c}\% \text { L/D } \\
\text { increase }\end{array}$ \\
\hline & & 0.019 & -0.005 & 0.033 & 0.047 & 0.580 & 12.3 & & \\
\hline & all-205 & 0.018 & 0.032 & 0.026 & 0.076 & 0.556 & 7.3 & 5 & -40 \\
\hline & top-205 & 0.024 & 0.013 & 0.033 & 0.070 & 0.626 & 8.9 & -26 & -27 \\
\hline & bot-100 & 0.018 & 0.004 & 0.033 & 0.055 & 0.553 & 10.1 & 5 & -18 \\
\hline & bot-205 & 0.017 & 0.014 & 0.033 & 0.064 & 0.525 & 8.2 & 11 & -33 \\
\hline & bot TE-205 & 0.018 & 0.001 & 0.033 & 0.052 & 0.533 & 10.2 & 5 & -17 \\
\hline & bot chord-205 & 0.019 & 0.008 & 0.033 & 0.060 & 0.572 & 9.5 & 0 & -22 \\
\hline $\mathrm{V} \times 205$ & & 0.018 & -0.011 & 0.032 & 0.039 & 0.566 & 14.5 & 5 & $\overline{18}$ \\
\hline $\mathrm{V} \times 100$ & all-100 & 0.018 & 0.011 & 0.033 & 0.062 & 0.556 & 9.0 & 5 & -26 \\
\hline Vx 205 & all-205 & 0.017 & 0.025 & 0.033 & 0.075 & 0.542 & 7.2 & 11 & -41 \\
\hline Vx 205 & bot-205 & 0.017 & 0.008 & 0.033 & 0.058 & 0.511 & 8.8 & 11 & -28 \\
\hline $\mathrm{V} \times 205$ & bot TE-205 & $\begin{array}{l}0.017 \\
\end{array}$ & -0.005 & 0.033 & 0.045 & 0.518 & 11.5 & 11 & -6 \\
\hline$V \times 205$ & bot TE-100 & 0.017 & -0.008 & 0.031 & 0.040 & 0.541 & 13.5 & 11 & 10 \\
\hline $\mathrm{V} \times 205$ & bot TE-50 & 0.017 & -0.009 & 0.031 & 0.039 & 0.553 & 14.2 & 11 & 16 \\
\hline
\end{tabular}

Table 7 shows very encouraging results. When providing suction to all suction slots (with velocity $\mathrm{V}_{\infty}=205 \mathrm{ft} / \mathrm{s}$ ), there is a decrease of $5 \%$ in induced drag. The bottom suction slots are more effective than the top suction slots. The highest induced drag reduction occurs when applying suction of magnitude $\mathrm{V}_{\infty}$ on the bottom slots $(11 \%)$. The suction slots at the trailing edge are more effective than the chord-wise slots. Returning the mass flow entrained in the suction slots by means of blowing at $\mathrm{V}_{\infty}$ in the downstream 
direction causes a 5\% induced drag reduction. Three cases showed 10 to $18 \%$ increase in $\mathrm{L} / \mathrm{D}$ ratio. Five other cases also had $11 \%$ reduction in induced drag using various suction, along with blowing at $\mathrm{V}_{\mathrm{x}}=205 \mathrm{ft} / \mathrm{s}$.

\section{NACA $64_{3}-018$ Wing}

The computational domain for these simulations is $4 \mathrm{x} 1.8 \mathrm{x} 1.1 \mathrm{~m}$. in the $\mathrm{x}, \mathrm{y}$, and $\mathrm{z}$ directions, respectively. The mesh had 3.6 million cells and 0.7 million nodes. Figure 121 and 122 shows the blowing and suction slots on top and bottom wing surfaces. The area of the blowing slots was $0.0015 \mathrm{~m}^{2}$, the area of the bottom suction slot at the TE was $0.0004 \mathrm{~m}^{2}$, and the area of the bottom chord-wise suction slot was $0.0015 \mathrm{~m}^{2}$.

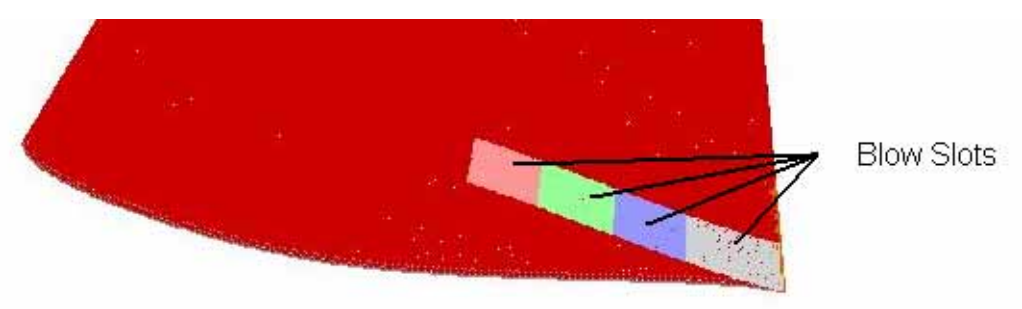

Figure 121. Top Surface.

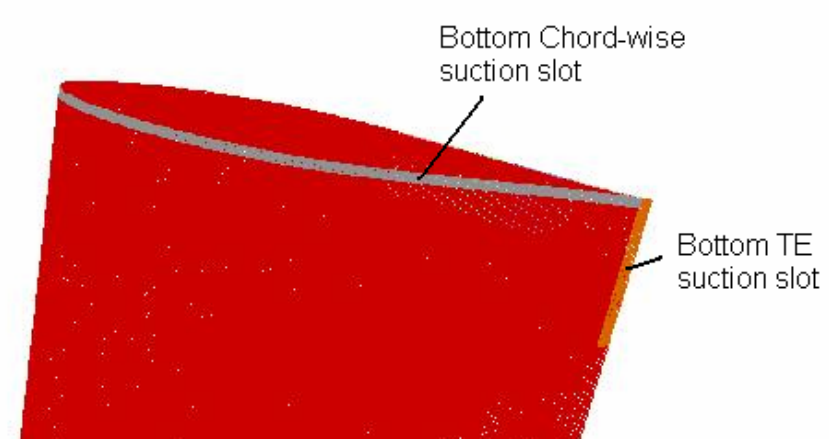

Figure 122. Bottom Surface.

Results of the parametric study of this wing are presented in Table 8. As seen from this table, bottom suction is more effective in reducing induced drag. The optimum suction velocity is $\mathrm{V}_{\infty}(146 \mathrm{~m} / \mathrm{s})$. The other evaluated alternative was the blowing at the bottom chord-wise suction slot, but it was unsuccessful. Different suction velocities at the bottom slots were evaluated with the same blowing velocity $\left(\mathrm{V}_{\infty}\right)$. When blowing at $\mathrm{V}_{\infty}$, higher induced drag reductions were obtained than when suction was once or twice $\mathrm{V}_{\infty}$, but the highest lift to drag ratio was obtained when suction was $0.33 \mathrm{~V}_{\infty}$. Mass 
conservation was taken into account in the last three configurations; this is clearly recognized by noticing that drag coefficient due to momentum deficit is almost zero. Suction without blowing creates a sink drag because some momentum is removed. The best configuration is obtained when suction is applied at $\mathrm{V}_{\infty}$ using an ejector with exit velocity 1.26 times $\mathrm{V}_{\infty}$. In that case, induced drag is reduced by $8 \%$ and the lift to drag ratio is increased by $13 \%$. Even though higher $\mathrm{L} / \mathrm{D}$ increases of $38 \%$ are obtained when blowing at $\mathrm{Vx}=146 \mathrm{~m} / \mathrm{s}$, this is not considered the best case because suction is not applied and thus, mass is not conserved.

The volume flow rate required to apply suction on bottom slots at $\mathrm{V}_{\infty}=146 \mathrm{~m} / \mathrm{s}$ is $0.27 \mathrm{~m}^{3} / \mathrm{s}$. The dynamic pressure at this free stream velocity $\left(\mathrm{V}_{\infty}=146 \mathrm{~m} / \mathrm{s}\right)$ is $13056 \mathrm{~Pa}$. Therefore, the suction power required is $4.72 \mathrm{HP}$.

Table 8. Parametric Study of BLC application on a NACA $64_{3}-018$ Wing.

\begin{tabular}{|c|c|c|c|c|c|c|c|c|c|}
\hline $\begin{array}{c}\text { Blowing } \\
(\mathrm{m} / \mathrm{s})\end{array}$ & $\begin{array}{l}\text { Suction } \\
(\mathrm{m} / \mathrm{s})\end{array}$ & $C_{D i}$ & $C_{D p m}$ & $C_{D p p}$ & $C_{D}$ & $C_{L}$ & L/D & $\begin{array}{c}\% D_{1} \\
\text { reduction }\end{array}$ & $\begin{array}{c}\% \text { L/D } \\
\text { increase }\end{array}$ \\
\hline & & 0.024 & -0.001 & 0.039 & 0.062 & 0.715 & 11.5 & & \\
\hline & bot-75 & 0.023 & 0.010 & 0.037 & 0.070 & 0.690 & 9.9 & 4 & -14 \\
\hline & bot-115 & 0.023 & 0.016 & 0.036 & 0.075 & 0.678 & 9.0 & 4 & -21 \\
\hline & bot-146 & 0.022 & 0.021 & 0.036 & 0.079 & 0.669 & 8.5 & 8 & -26 \\
\hline & bot TE-146 & 0.022 & 0.004 & 0.038 & 0.064 & 0.679 & 10.6 & 8 & -8 \\
\hline Vx146 & & 0.023 & -0.017 & 0.038 & 0.044 & 0.697 & 15.8 & 4 & 38 \\
\hline bot chord 146 & & 0.024 & -0.018 & 0.058 & 0.064 & 0.730 & 11.4 & 0 & -1 \\
\hline bot chord 146 & bot TE-146 & 0.023 & -0.013 & 0.057 & 0.067 & 0.699 & 10.4 & 4 & -9 \\
\hline Vx146 & bot-50 & 0.023 & -0.010 & 0.036 & 0.049 & 0.683 & 13.9 & 4 & 21 \\
\hline Vx146 & bot-146 & 0.022 & 0.004 & 0.035 & 0.061 & 0.658 & 10.8 & 8 & -6 \\
\hline $\mathrm{Vx} 40$ & bot TE-146 & 0.022 & -0.001 & 0.040 & 0.062 & 0.670 & 10.9 & 8 & -5 \\
\hline Vx184 & bot-146 & 0.022 & 0.000 & 0.029 & 0.051 & 0.660 & 12.9 & 8 & 13 \\
\hline
\end{tabular}

Span-wise blowing was also studied. The grid for this configuration had five million cells and one million nodes. Figures 123 and 124 show top and bottom wing surfaces of the wing. Table 9 shows the results of the parametric study. Span-wise blowing decreases the induced drag and the drag due to momentum deficit, and increases the lift coefficient. However, it increases the drag due to total pressure losses and the total drag, thereby decreasing the lift to drag ratio. Blowing at $\mathrm{V}_{\infty}$ gives the highest induced drag reduction with the lowest blowing velocity. Applying suction at the bottom while blowing span-wise at $\mathrm{V}_{\infty}$ further decreases the induced drag but the drag due to momentum deficit increases and the lift is reduced. This configuration provides the highest induced drag reduction (13\%) but the lift to drag ratio has decreased. The best 
blowing location on the top surface was also investigated. If there is any reduction in induced drag from using a particular blowing slot, there is usually a slight increase in liftto-drag-ratio when blowing occurs closer to the trailing edge.

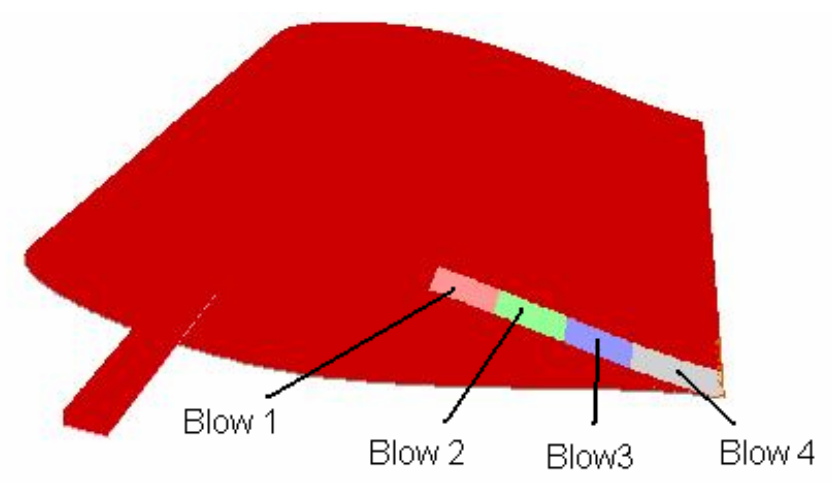

Figure 123. Wing Top Surface with Span-wise Blowing.

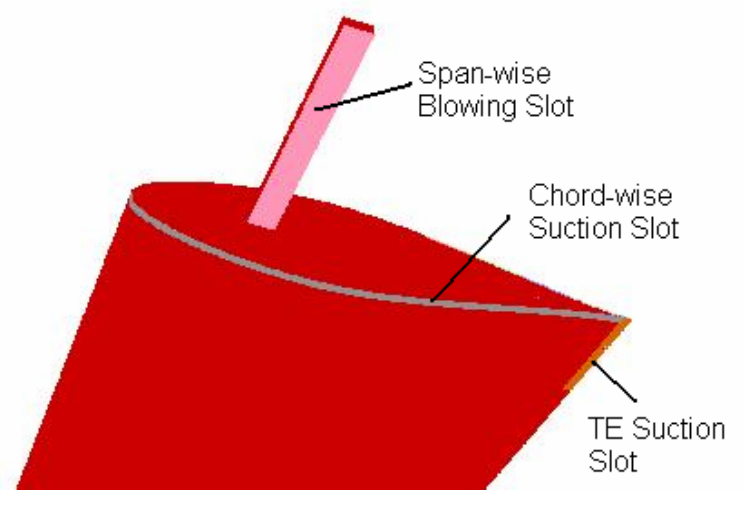

Figure 124. Wing Bottom Surface with Span-wise Blowing

Table 9. Parametric Study of BLC application on a NACA 643-018 Wing with Span-wise blowing.

\begin{tabular}{|l|c|c|c|c|c|c|r|r|r|}
\hline $\begin{array}{c}\text { Blowing } \\
(\mathbf{m} / \mathbf{s})\end{array}$ & $\begin{array}{c}\text { Suction } \\
(\mathbf{m} / \mathbf{s})\end{array}$ & $\mathbf{C}_{\mathbf{D} \text { i }}$ & $\mathbf{C}_{\mathrm{Dpm}}$ & $\mathbf{C}_{\mathrm{Dpp}}$ & $\mathbf{C}_{\mathbf{D}}$ & $\mathbf{C}_{\mathrm{L}}$ & $\mathbf{L} / \mathbf{D}$ & $\begin{array}{c}\text { \%D } \mathbf{I} \\
\text { reduction }\end{array}$ & $\begin{array}{c}\text { \% L/D } \\
\text { increase }\end{array}$ \\
\hline & & $\mathbf{0 . 0 2 3}$ & $-\mathbf{0 . 0 0 1}$ & $\mathbf{0 . 0 5 0}$ & $\mathbf{0 . 0 7 2}$ & $\mathbf{0 . 7 3 1}$ & $\mathbf{1 0 . 1}$ & & \\
\hline span 75 & & 0.022 & -0.017 & 0.070 & 0.075 & 0.744 & 9.9 & 4 & -2 \\
\hline span 100 & & 0.022 & -0.022 & 0.077 & 0.077 & 0.749 & 9.7 & 4 & -4 \\
\hline span 146 & & 0.021 & -0.033 & 0.093 & 0.081 & 0.760 & 9.4 & 9 & -7 \\
\hline span 146 & bot-146 & 0.020 & -0.011 & 0.091 & 0.100 & 0.721 & 7.2 & 13 & -29 \\
\hline blow1 V146 & & 0.022 & -0.012 & 0.061 & 0.071 & 0.720 & 10.1 & 4 & 0 \\
\hline blow2 V146 & & 0.022 & -0.013 & 0.060 & 0.069 & 0.717 & 10.4 & 4 & 3 \\
\hline blow3 V146 & & 0.022 & -0.013 & 0.058 & 0.067 & 0.712 & 10.6 & 4 & 5 \\
\hline blow4 V146 & & 0.022 & -0.016 & 0.059 & 0.065 & 0.696 & 10.7 & 4 & 6 \\
\hline
\end{tabular}

Suction areas were split in two equal parts to investigate the effect of the reduction in the area. The mesh for this geometry had five million cells and one million nodes. Figure 125 and 126 show the suction and blowing slot positions in the top and 
bottom wing surfaces. Table 10 shows the results of various blowing and suction alternatives. There is a decrease in the drag due to total pressure losses because of the smaller span-wise blowing slot. Applying suction at $\mathrm{V}_{\infty}$ with a suction slot half the area as the one used in Table 7 produces half the induced drag reduction. Increasing the suction velocity to twice $\mathrm{V}_{\infty}$ does not further reduce the induced drag and it actually decreases the lift.

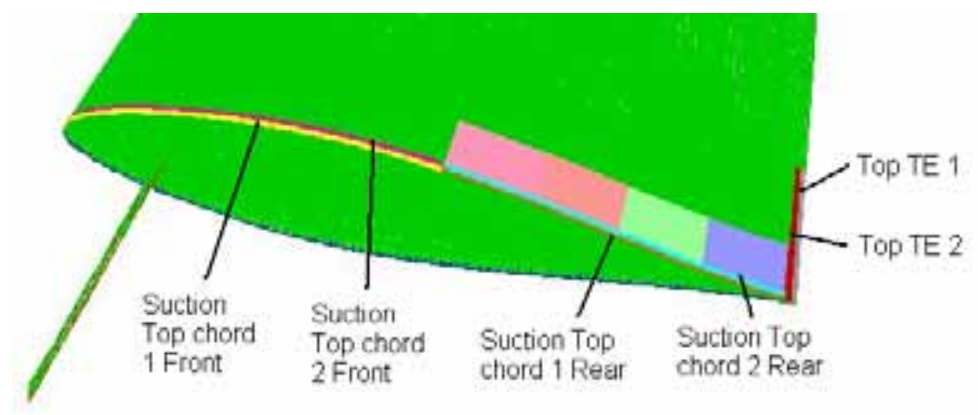

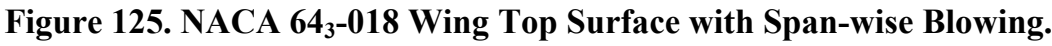

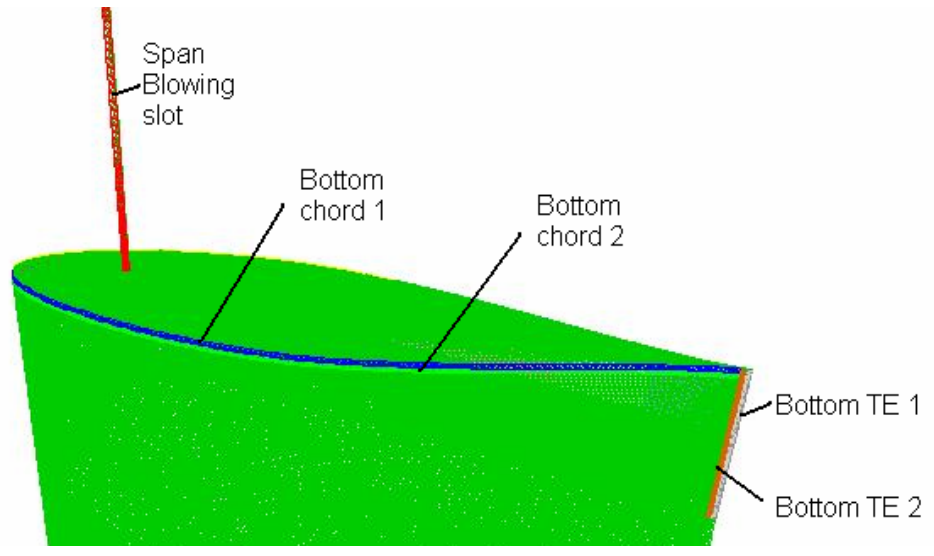

Figure 126. NACA 64 -018 Wing Bottom Surface with Span-wise Blowing

Table 10. Parametric Study of BLC application on a NACA 64,-018 Wing with Span-wise blowing.

\begin{tabular}{|c|c|c|c|c|c|c|c|c|c|}
\hline $\begin{array}{c}\text { Blowing } \\
(\mathrm{m} / \mathrm{s})\end{array}$ & Suction (m/s) & $C_{D i}$ & $C_{D p m}$ & $C_{D p p}$ & $C_{D}$ & $C_{L}$ & L/D & $\begin{array}{c}\% D_{\text {I }} \\
\text { reduction }\end{array}$ & $\begin{array}{c}\% \text { L/D } \\
\text { increase }\end{array}$ \\
\hline & & 0.024 & -0.001 & 0.040 & 0.063 & 0.712 & 11.2 & & \\
\hline & bot chord1-146 & 0.024 & 0.008 & 0.038 & 0.070 & 0.706 & 10.1 & 0 & -10 \\
\hline & bot TE1-146 & 0.023 & 0.002 & 0.037 & 0.062 & 0.691 & $\overline{11.2}$ & 4 & $\overline{0}$ \\
\hline & bot TE1\&2-146 & 0.023 & 0.004 & 0.037 & 0.064 & 0.68 & $\overline{10.6}$ & 4 & $\overline{-6}$ \\
\hline & top TE1-146 & 0.024 & 0.002 & 0.042 & 0.068 & 0.733 & 10.8 & 0 & -3 \\
\hline & top chord1R-146 & 0.024 & 0.003 & 0.022 & 0.049 & 0.702 & 14.2 & 0 & 27 \\
\hline & top chord1F-146 & 0.024 & 0.004 & 0.039 & 0.067 & 0.706 & 10.6 & 0 & -6 \\
\hline blow span146 & & 0.023 & -0.006 & 0.046 & 0.063 & 0.722 & 11.4 & 4 & 2 \\
\hline blow Vx146 & & 0.023 & -0.016 & 0.039 & 0.046 & 0.699 & 15.2 & 4 & 35 \\
\hline blow span146 & bot TE1\&2 -164 & 0.022 & -0.001 & 0.043 & 0.065 & 0.686 & $\overline{10.6}$ & 8 & -5 \\
\hline
\end{tabular}


Figures 127 and 128 present the top and bottom wing surface of a rectangular wing which uses a NACA $64_{3}-018$ airfoil with its suction and blowing slots. The mesh for this configuration had seven million cells and 1.4 million nodes. The results of the parametric study are shown in Table 11. Induced drag reductions up to $19 \%$ and lift-todrag-ratio increases of up to $11 \%$ were obtained.

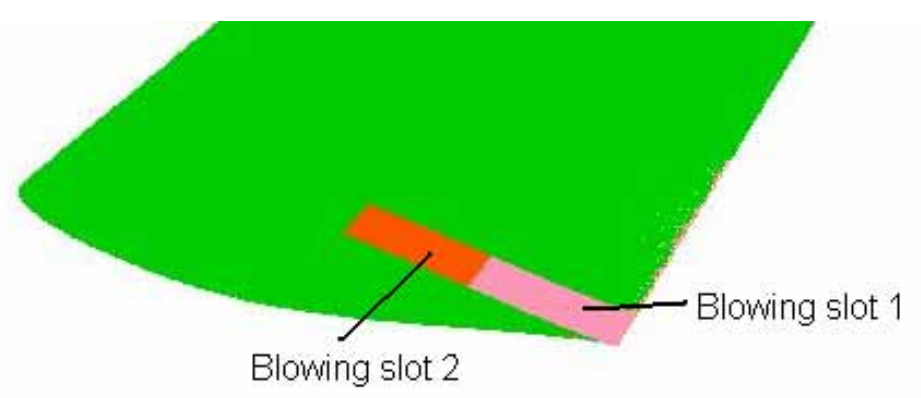

Figure 127. Blowing slots on Wing Top Surface.

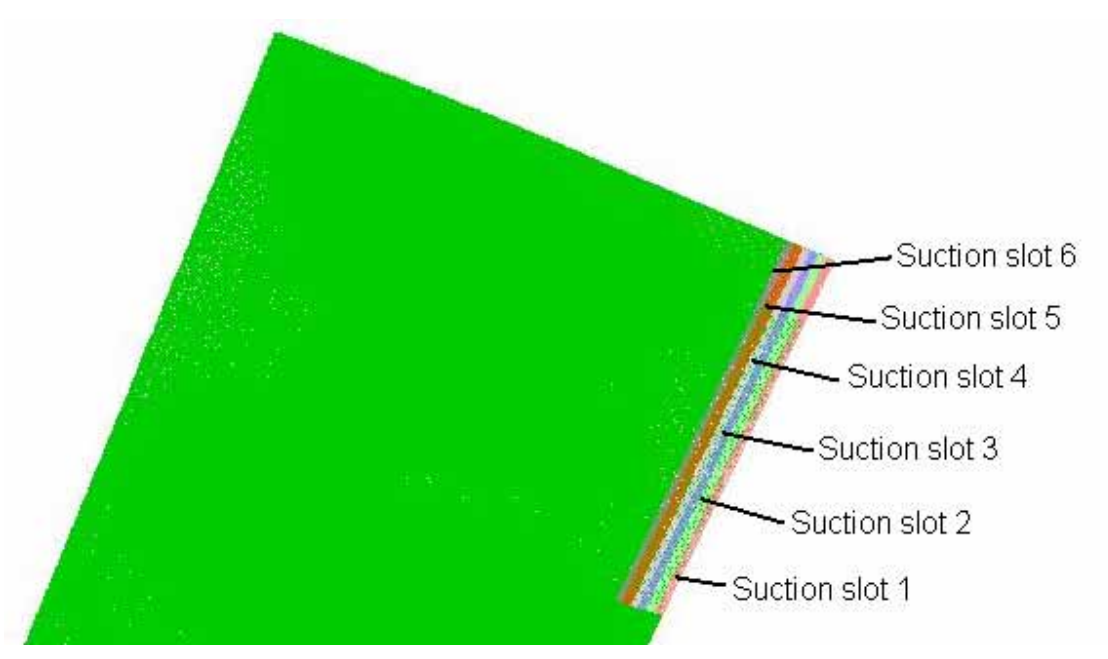

Figure 128. Suction slots on Wing Bottom surface.

Table 11. Parametric Study of BLC application on a NACA 64 $4_{3}-018$ Wing.

\begin{tabular}{|c|c|c|c|c|c|c|c|r|r|}
\hline $\begin{array}{c}\text { Blowing } \\
(\mathbf{m} / \mathbf{s})\end{array}$ & $\begin{array}{c}\text { Suction } \\
(\mathbf{m} / \mathbf{s})\end{array}$ & $C_{D i}$ & $C_{D p m}$ & $C_{D p p}$ & $C_{D}$ & $C_{L}$ & L/D & $\begin{array}{c}\text { \%D } \\
\text { reduction }\end{array}$ & $\begin{array}{c}\text { \% L/D } \\
\text { increase }\end{array}$ \\
\hline & & $\mathbf{0 . 0 2 5}$ & $\mathbf{0 . 0 0 0}$ & $\mathbf{0 . 0 3 7}$ & $\mathbf{0 . 0 6 1}$ & $\mathbf{0 . 7 0 9}$ & $\mathbf{1 1 . 6}$ & & \\
\hline$V \times 146$ & bot1 -146 & 0.020 & -0.003 & 0.031 & 0.048 & 0.594 & 12.4 & 19 & 7 \\
\hline$V \times 146$ & bot186-70 & 0.021 & -0 & 0.032 & 0.049 & 0.631 & 12.9 & 15 & 11 \\
\hline
\end{tabular}




\section{BLC suction on top and bottom surfaces of a rectangular wing with $A R=4$}

Three different $\mathrm{BLC}$ cases were run on a rectangular wing with $\mathrm{AR}=4$ which uses a NACA 64-018 airfoil. The free stream velocity was $\mathrm{V}_{\infty}=55 \mathrm{~m} / \mathrm{s}$ and the grid had 4.6 million cells. The first case is the baseline and BLC was not applied. In the second case, suction was applied on the bottom and top trailing edge slots at two times $\mathrm{V}_{\infty}$. In the third case, suction was only applied on the bottom trailing edge slot at two times $\mathrm{V}_{\infty}$. The drag coefficient and the integral of the vorticity on a plane one foot downstream the wing are shown in Table 12.

Table 12. BLC suction on top and bottom surfaces of a rectangular wing with $A R=4$.

\begin{tabular}{|c|c|c|c|c|c|}
\hline Case & Description & $\mathbf{C}_{\mathbf{D}}$ & $\begin{array}{c}\text { Vorticity } \\
\left(\mathrm{m}^{2} / \mathbf{s}\right)\end{array}$ & $\mathbf{C}_{\mathrm{L}}$ & $\mathbf{L} / \mathbf{D}$ \\
\hline 1 & No BLC & 0.068 & -5.35 & 0.705 & 10.4 \\
\hline 2 & Suction on bottom and top TE at $2 \mathrm{~V}_{?}$ & 0.071 & -5.37 & 0.711 & 10.0 \\
\hline 3 & Suction on bottom TE at $2 \mathrm{~V}_{?}$ & 0.063 & -5.25 & 0.660 & 10.5 \\
\hline
\end{tabular}

Figures 129, 130, and 131 show the vorticity contours on cases 1, 2, and 3, respectively. In case 2 the vorticity in the center of the wing tip vortex increases with respect to case 1 . The white region means the vorticity value was larger than the higher limit. When suction is applied only on the bottom trailing edge slot, the high vorticity area has decreased to just a small point.
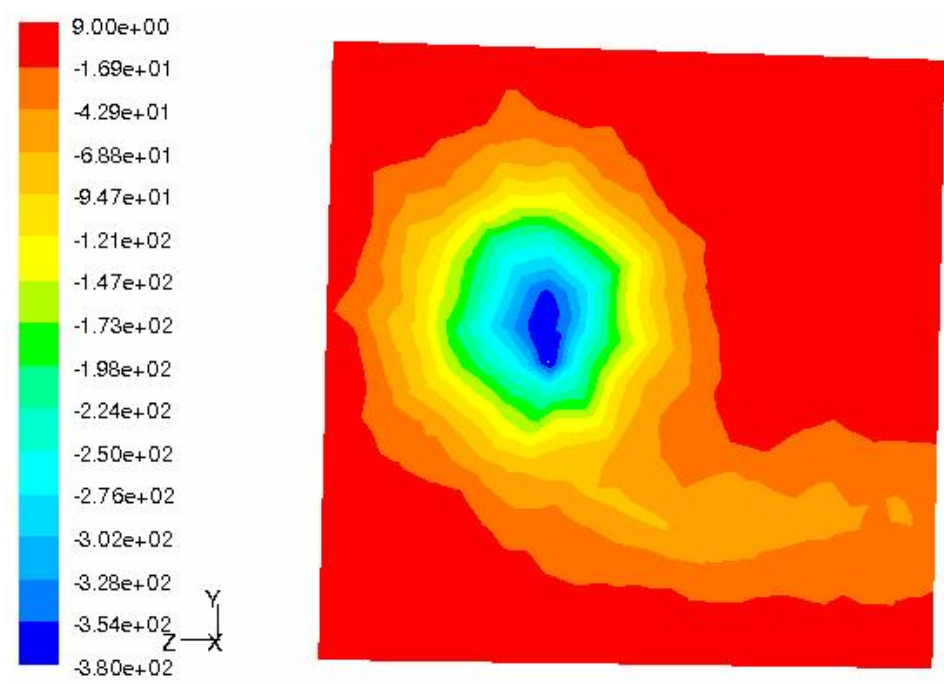

Figure 129. Vorticity contours behind a rectangular wing of $A R=4$ without $B L C$. 

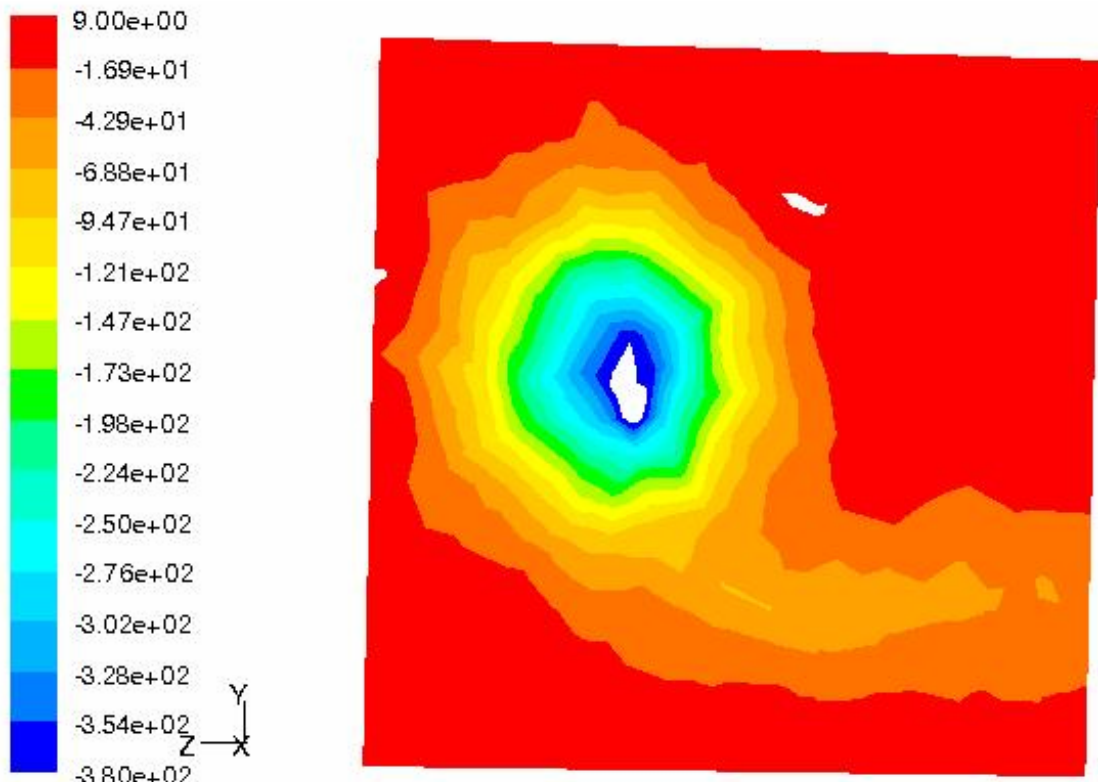

Figure 130. Vorticity contours behind a rectangular wing of $A R=4$ with suction at the TE on both top and bottom surfaces at two times $\mathrm{V}_{\infty}$.
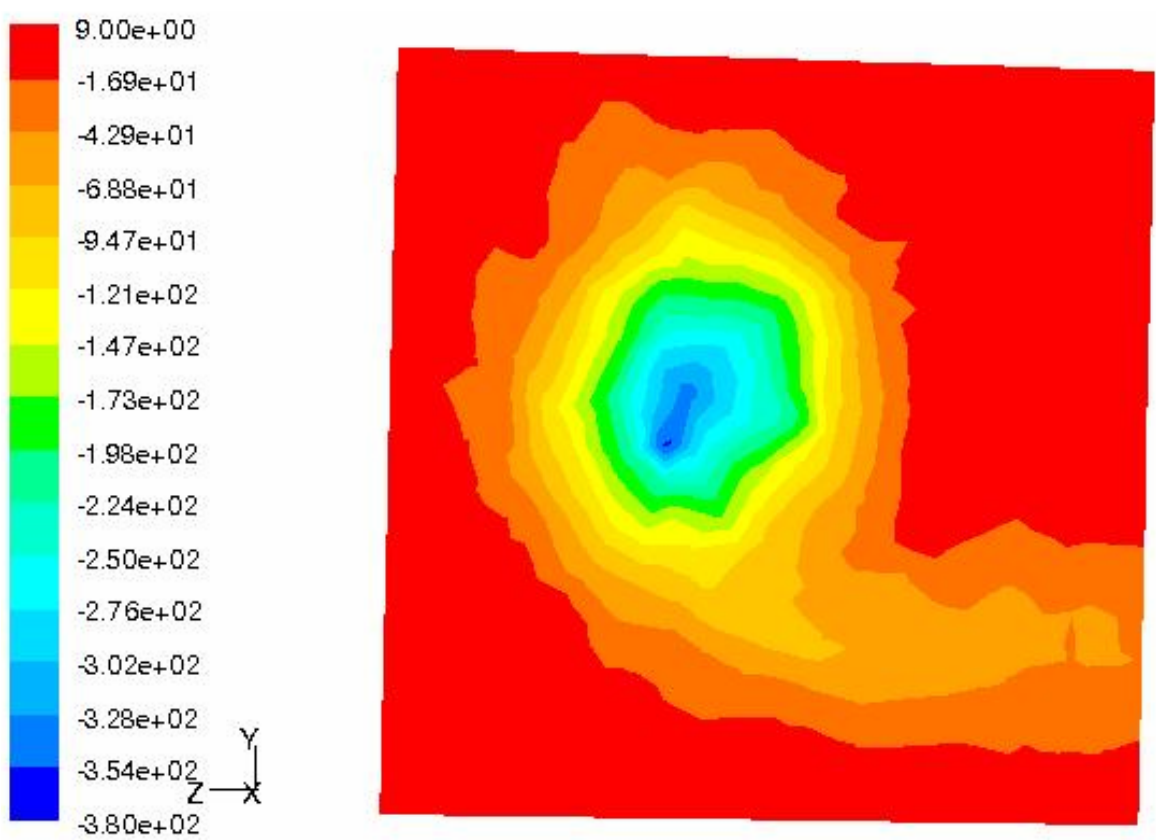

Figure 131. Vorticity contours behind a rectangular wing of $A R=4$ with suction at the TE on bottom surface at two times $\mathrm{V}_{\infty}$.

\section{BLC suction on three slots on bottom surface of a rectangular wing of $A R=4$}

Three suction slots on the bottom surface of a rectangular wing of $A R=4$ which uses a NACA 64-018 airfoil were used to prevent the bottom boundary layer from going to the upper surface (Figure 132). The mesh had 5.8 million cells and 1.1 million nodes. 
First, the wing without BLC was simulated, then suction was applied at the three slots at $\mathrm{V}_{\infty}$ and then at two times $\mathrm{V}_{\infty}$. The drag coefficients and vorticity values are shown in Table 13. Vorticity contours were computed at $0.9 \mathrm{~m}$. downstream of the trailing edge for three different cases (Figures 133, 134 and 135). Suction on the bottom decreases the vorticity. The drag coefficient was obtained by the pressure and shear integration method.

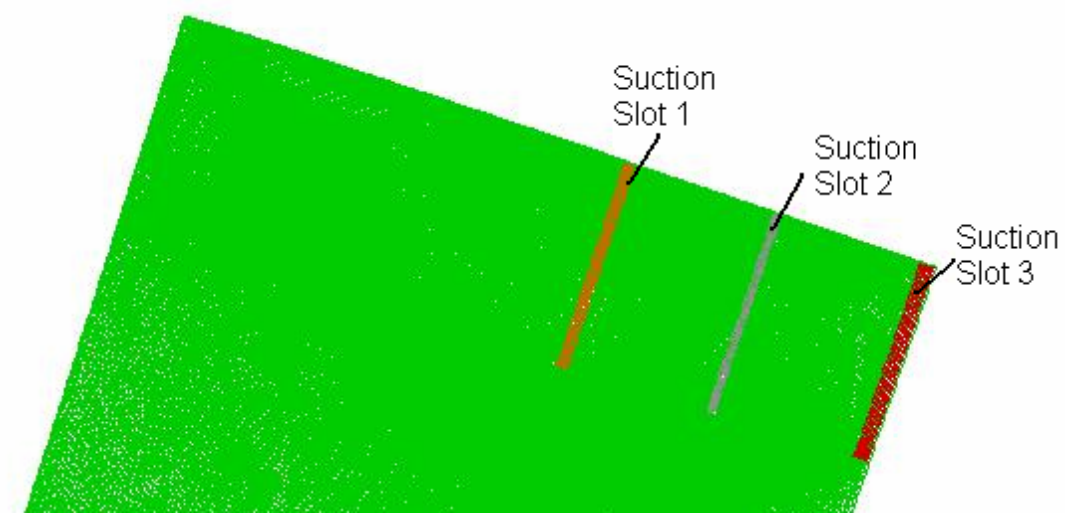

Figure 132. Bottom wing surface of a rectangular wing $A R=4$ with suction slots.

Table 13. BLC suction on three slots on bottom surface of a rectangular wing of $A R=4$.

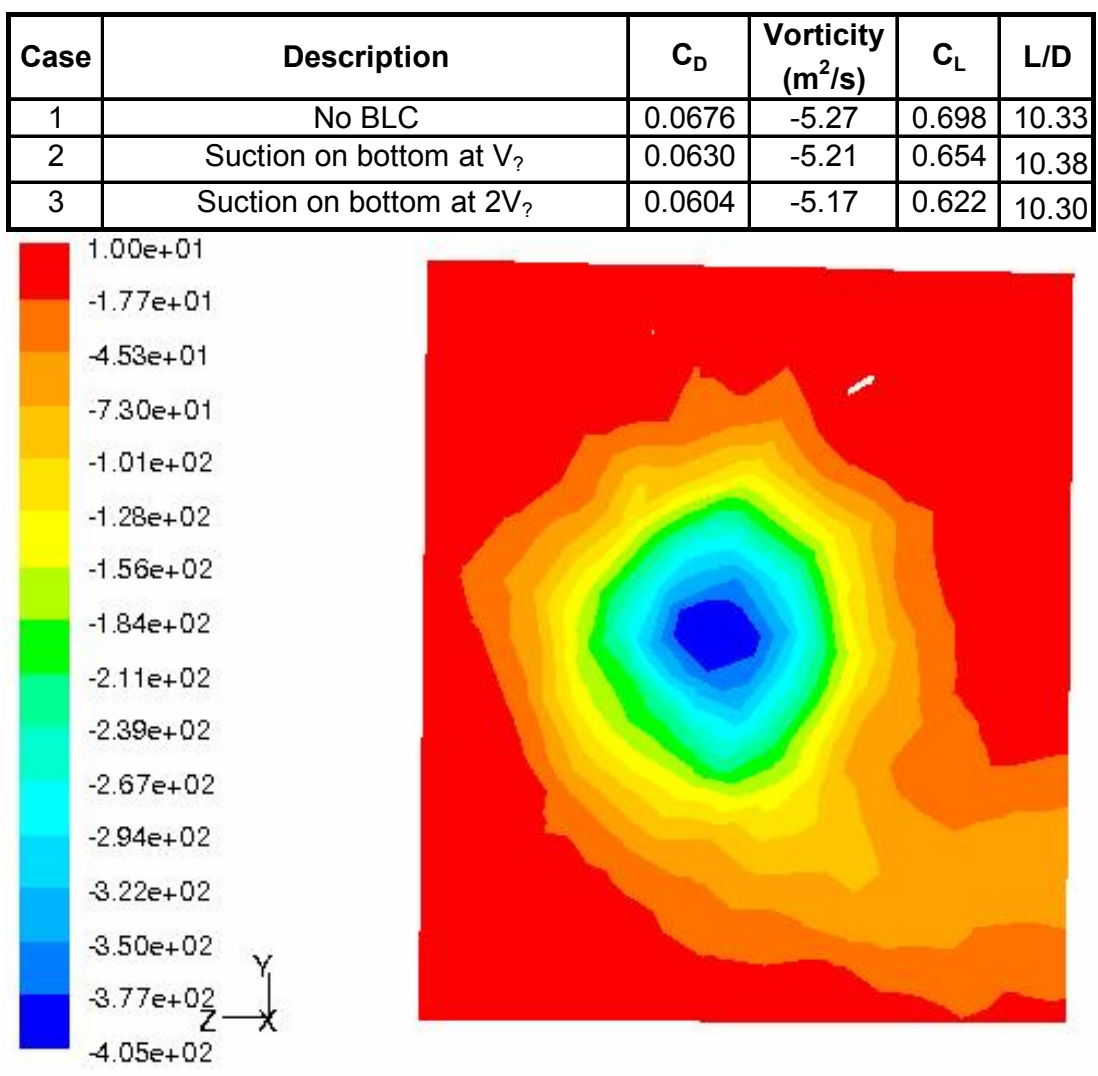

Figure 133. Vorticity contours behind an $A R=4$ rectangular wing without $B L C$. 

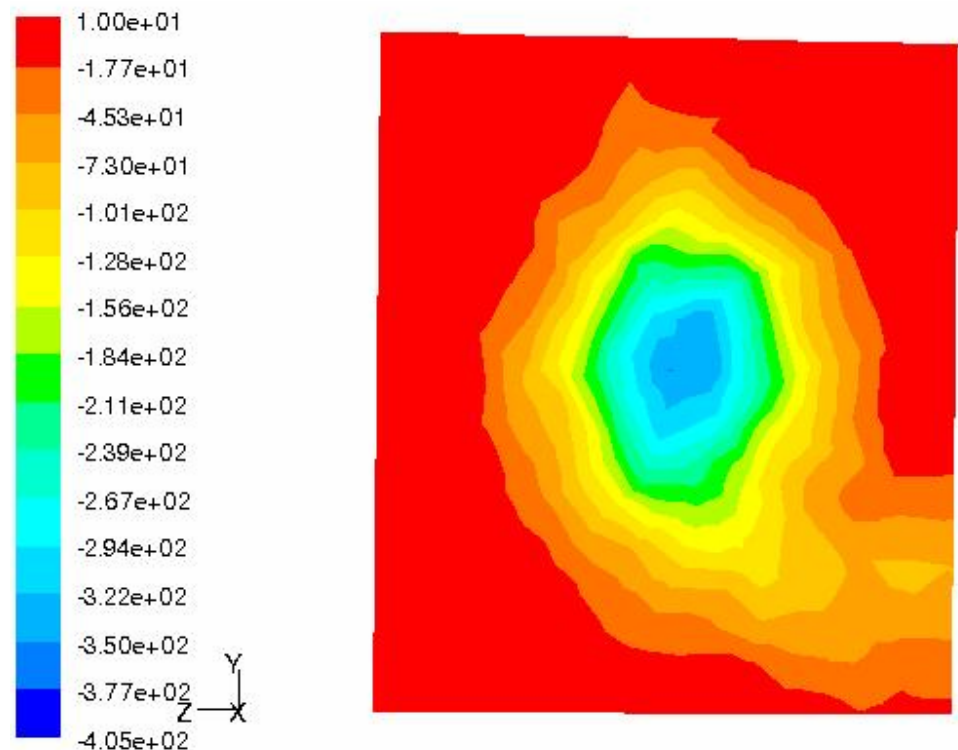

Figure 134. Vorticity contours behind an $A R=4$ rectangular wing with suction on bottom surface at $V_{\infty}$.
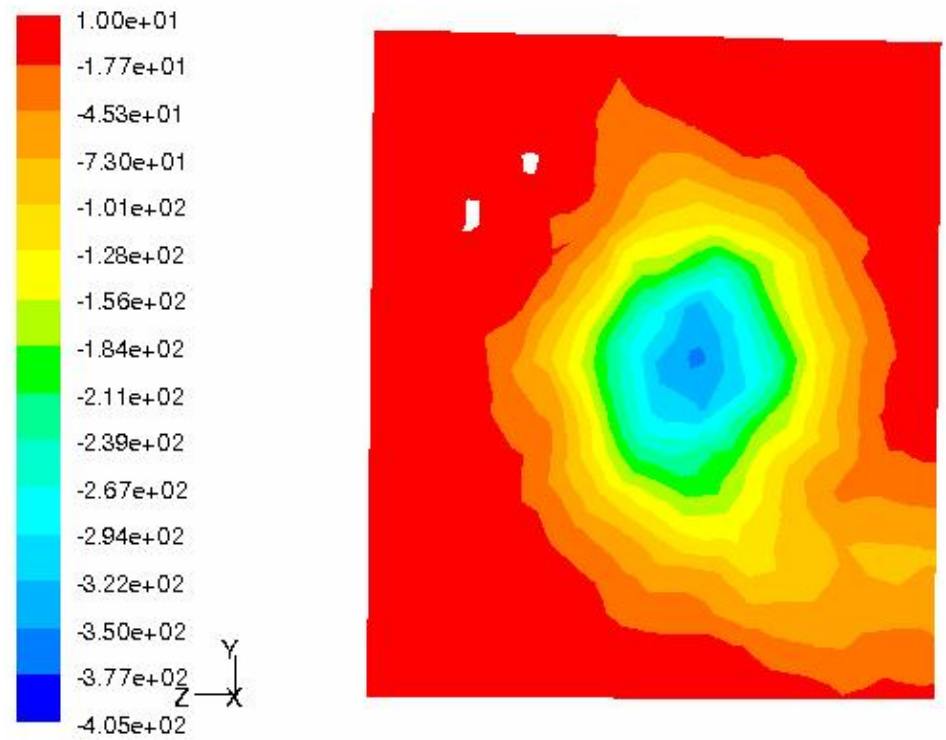

Figure 135. Vorticity contours behind an $A R=4$ rectangular wing with suction on bottom surface at $2 V_{\infty}$.

\section{BLC suction on bottom surface and wing tip on an $A R=1.5$ rectangular wing}

A rectangular wing with an $\mathrm{AR}=1.5$ and an NACA 0012 airfoil like the one shown in Figure 78 was simulated using a mesh with 0.9 million nodes and 5.2 million cells. The wing had a suction slot at the bottom TE and at the wing tip. Table 14 shows the drag coefficients and vorticity at the outlet boundary for various BLC cases. Figure 136 shows the vorticity contours for the baseline configuration without BLC, while 
Figure 137 shows the contours when suction was applied at the TE slot and at the wing tip at $0.5 \mathrm{~V}_{\infty}$. Figure 138 shows the vorticity contours when suction was applied only to the bottom slot at $0.5 \mathrm{~V}_{\infty}$.

Table 14. BLC Suction on bottom surface and wingtip on an $A R=1.5$ rectangular wing.

\begin{tabular}{|c|c|c|c|c|r|}
\hline Case & Description & $C_{D}$ & $\begin{array}{c}\text { Vorticity } \\
\left(\mathbf{f t}^{2} / \mathbf{s}\right)\end{array}$ & $\mathbf{C}_{\mathbf{L}}$ & L/D \\
\hline 1 & No BLC & 0.116 & -279 & 0.842 & 7.3 \\
\hline 2 & Suction on bottom and tip at $0.5 \mathrm{~V}_{?}$ & 0.032 & -118 & 0.280 & 8.8 \\
\hline 3 & Suction on bottom at $0.5 \mathrm{~V}_{?}$ & 0.069 & -153 & 0.459 & 6.7 \\
\hline
\end{tabular}

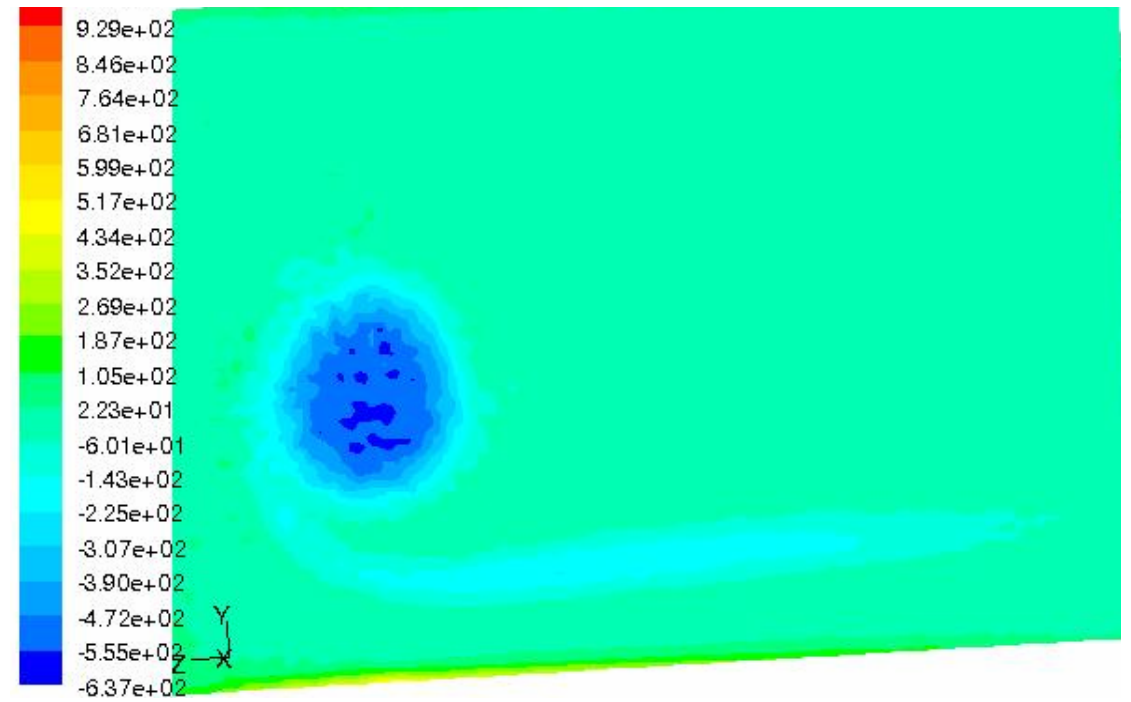

Figure 136. Vorticity contours on an $A R=1.5$ rectangular wing without $B L C$ suction.

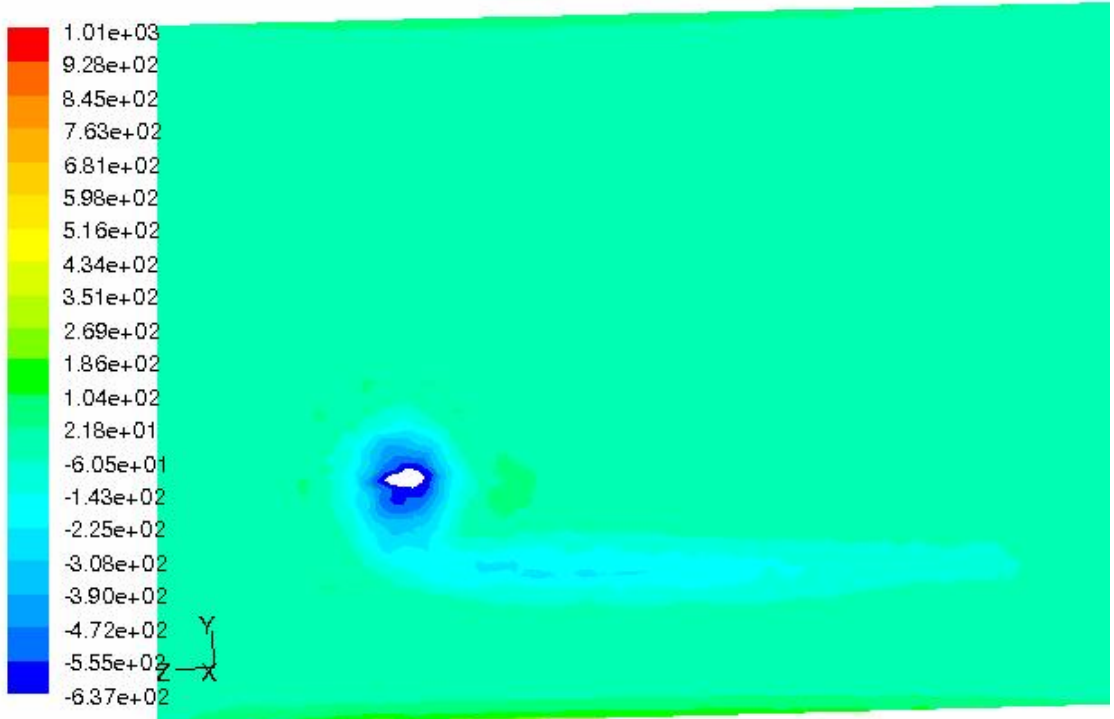

Figure 137. Vorticity contours on an $A R=1.5$ rectangular wing with suction at $0.5 V_{\infty}$ on bottom $T E$ and wing tip. 


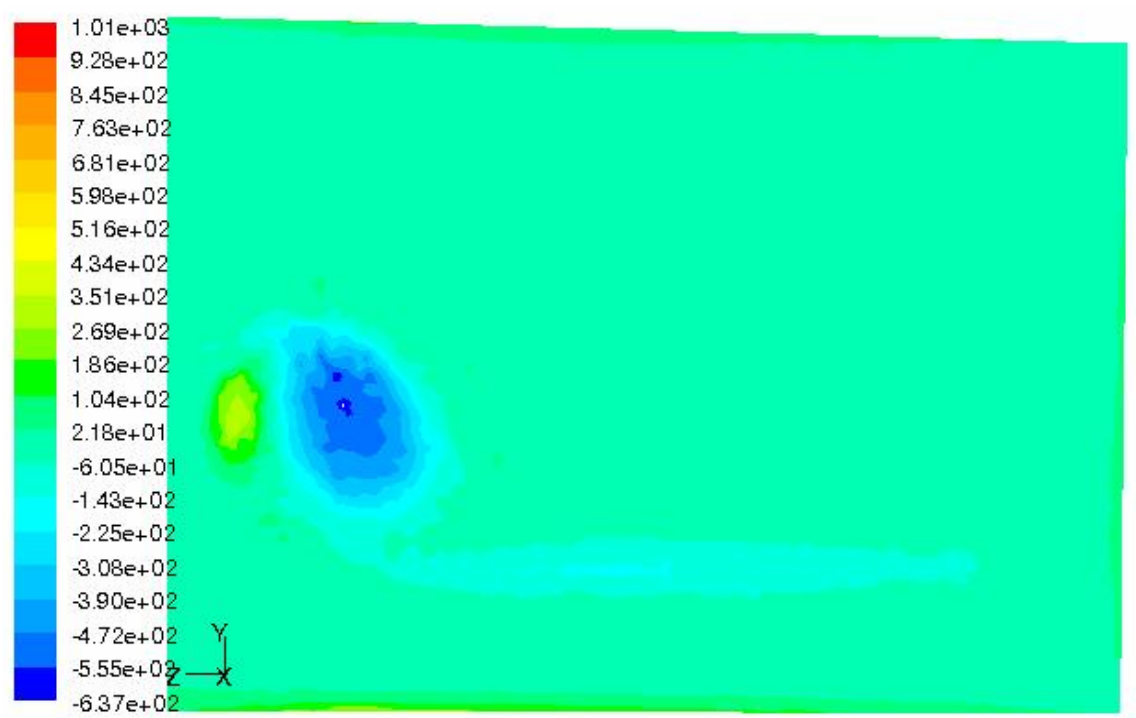

Figure 138. Vorticity contours on an $A R=1.5$ rectangular wing with suction at $0.5 V_{\infty}$ on bottom $\mathrm{TE}$.

\section{Grid Convergence Study}

A grid convergence study has been performed on the best BLC configurations obtained in the previous simulations. Suction on the bottom wing surface at half chord and at the trailing edge is applied in the next simulations over an area of $0.038 \mathrm{ft}^{2}$ each one (Figure 139). Applying suction on the bottom wing surface at $0.5 \mathrm{~V}_{\infty}$ has shown induced drag reductions up to $15 \%$ and increase in lift-to-drag-ratios up to $11 \%$ (Table 11). This reduction is due to the removal of the bottom wing surface boundary layer before it goes to the top wing surface generating the wing tip vortex. The suction on the bottom surface also helps to straighten the bottom streamlines. Also, suction on the top wing surface along the rear chord is going to be applied over an area of $0.16 \mathrm{ft}^{2}$ (Figure 140). This configuration increases the lift-to-drag-ratio up to $27 \%$ (Table 10), and decreases the drag coefficient due to pressure losses from 0.040 to 0.022 . It seems that the suction on the top surface along the rear chord reattaches the wingtip boundary layer decreasing the formation of a recirculation region, and therefore, a low pressure region at the wingtip vortex core. This region is responsible for keeping the wingtip vortex downstream. 


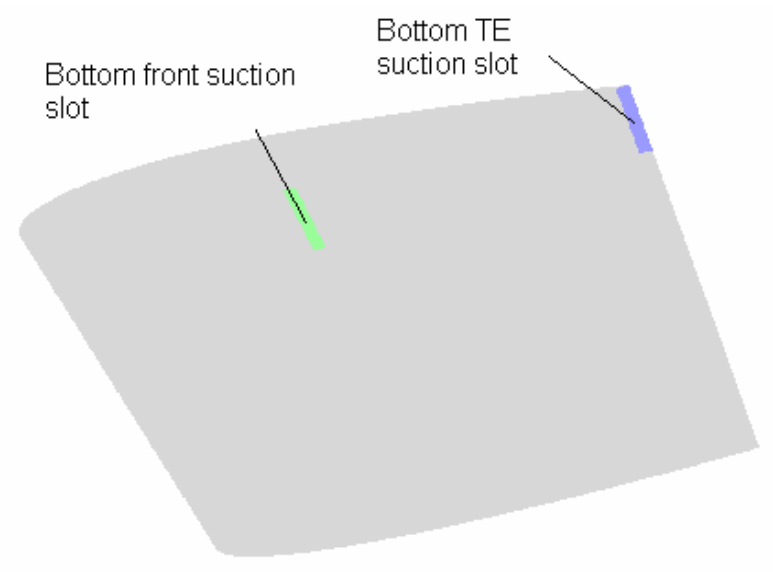

Figure 139. Bottom suction slots on bottom wing surface.

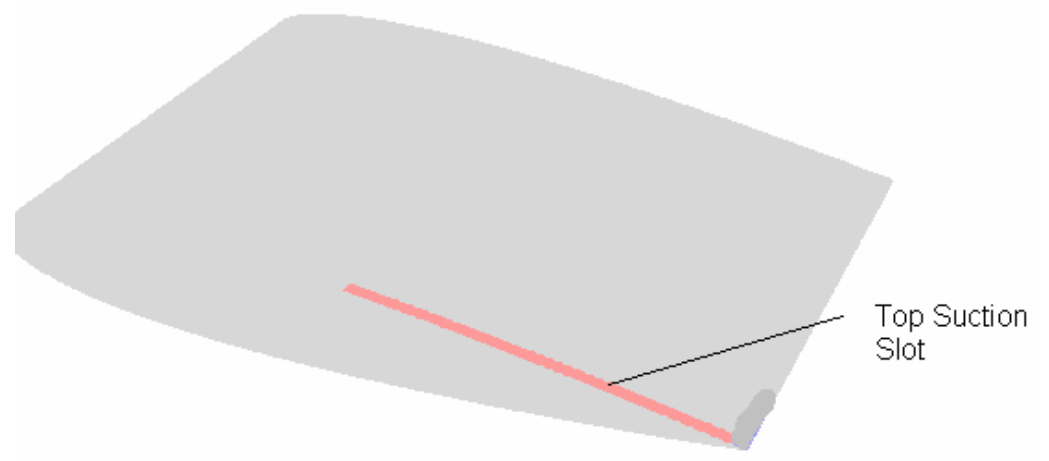

Figure 140. Top Suction slot on top wing surface.

\section{Computation Domain, Meshes and Simulation Setup}

Three grids similar to the one used by Kim and Rhee [44] were built. The external dimensions are $20 \times 2.66 \times 4 \mathrm{ft}$. (Figure 141). The model is an $\mathrm{AR}=1.5$ rectangular wing with half-span=3 ft, chord $4 \mathrm{ft}$ and uses a NACA 0012 airfoil. Table 15 shows the number of cells and nodes used for each of the three grids. Tetrahedral elements were used to build these meshes. Figure 141 shows the topology of the coarse mesh. Meshes with more than 2.2 million nodes could not be built due to the fact that Gambit, the mesh generator, cannot run in parallel and the limitation of 32-bit-processors to address more than 4 gigabytes of RAM. 


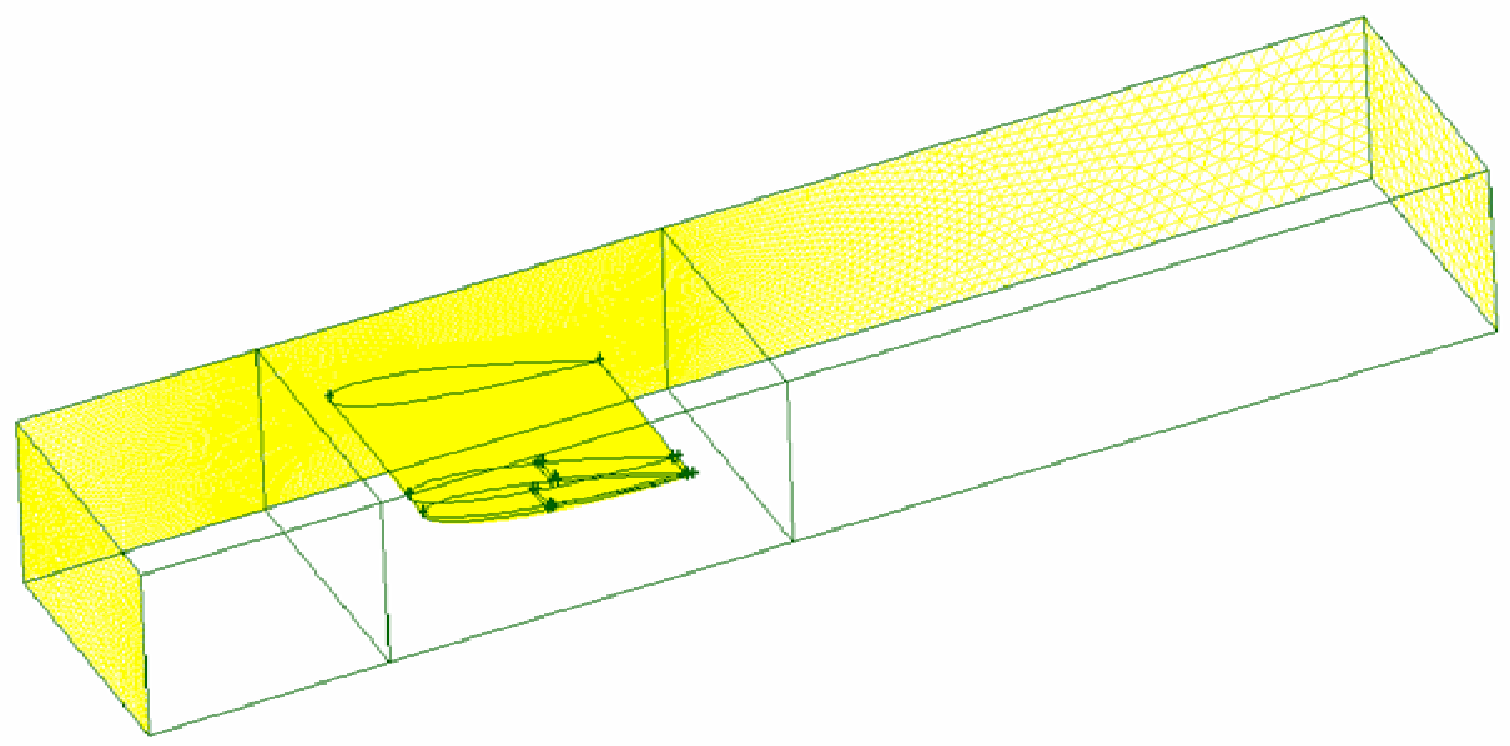

Figure 141. Solution Domain and Partial View of the Coarse mesh.

Table 15. Number of Cells and Nodes for the three grids used for the grid convergence study.

\begin{tabular}{ccc}
\hline Grid & Number of Cells & Number of Nodes \\
\hline Coarse mesh & 5.2 million & 0.9 million \\
Fine mesh & 8.6 million & 1.5 million \\
Finest mesh & 12.2 million & 2.2 million \\
\hline
\end{tabular}

The simulations were run on a Beowulf HPC. Each simulation required around 4000 iterations and the average time per simulation was eight hours. The turbulence model used was Spalart-Allmaras. For the pressure-velocity coupling a SIMPLE method was used, and a second order scheme was used for the pressure discretization. The momentum and modified turbulent viscosity used a QUICK scheme. The boundary condition for the inlet was a velocity inlet with a magnitude equal to $V_{\infty}=170 \mathrm{ft} / \mathrm{s}$. The top, bottom and side walls were modeled as moving walls with a velocity equal to $\mathrm{V}_{\infty}$. The outlet face was set to a pressure outlet and the other surfaces were set to fixed walls.

\section{Base-line Wing Results}

The residuals and the drag-coefficient monitor for the coarse mesh simulation are shown in Figures 142 and 143, respectively. The pressure contours, the velocity magnitude contours, and the vorticity contours in a plane at $\mathrm{x}=9 \mathrm{ft}$. in the downstream 
direction are shown in Figures 144, 145, and 146; respectively. Force and integral of vorticity comparison will be presented subsequently. Figure 144 shows a low pressure coefficient in the vortex core.
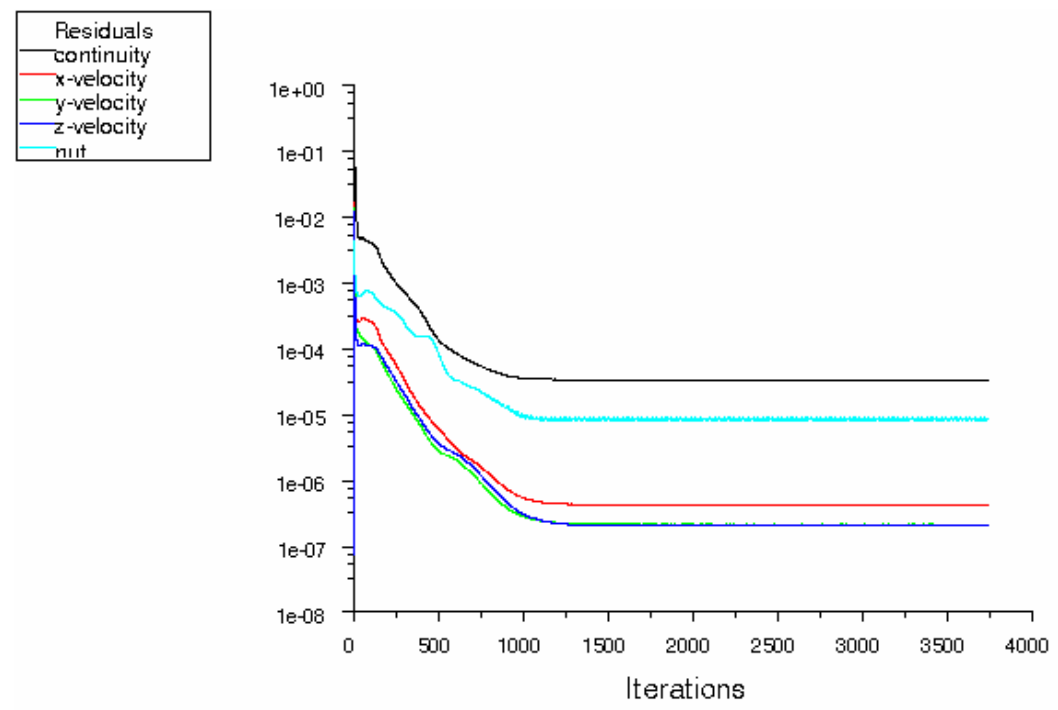

Figure 142. Continuity and Velocity residuals for the coarse mesh.

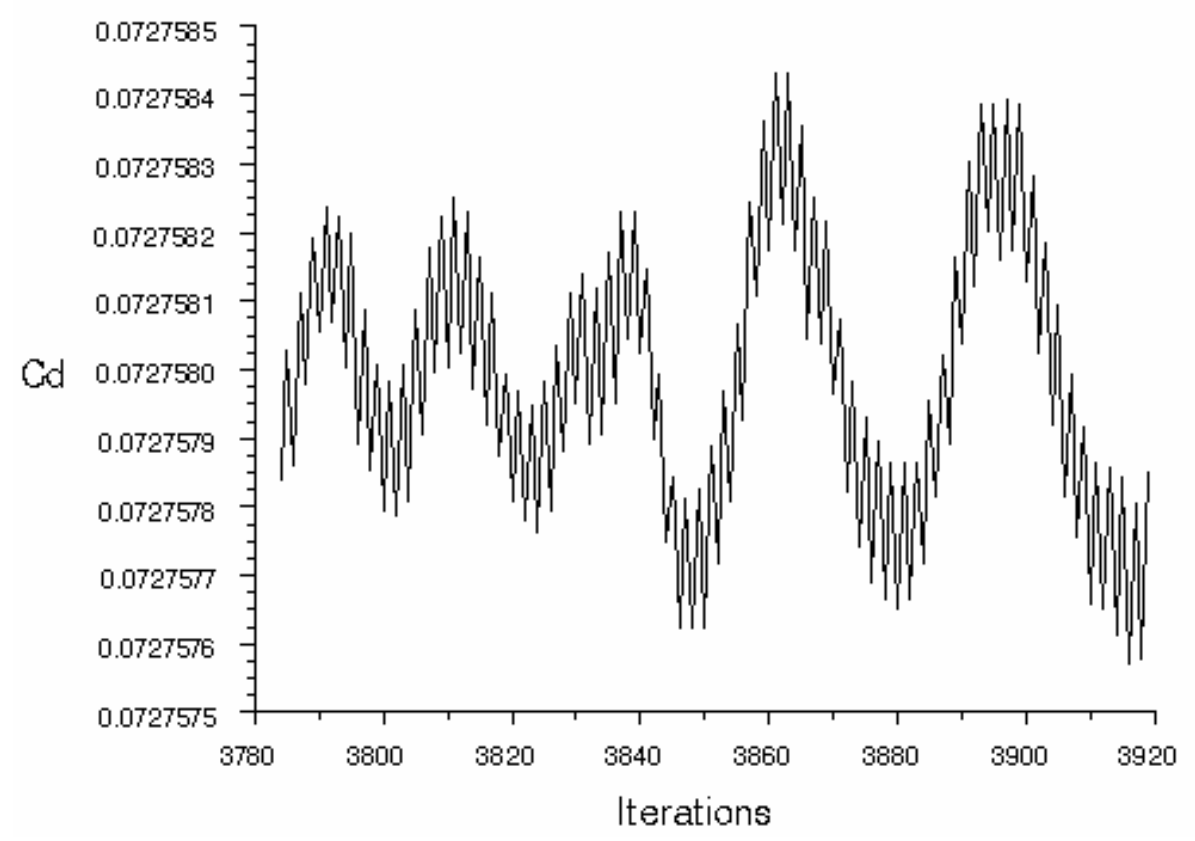

Figure 143. Drag Coefficient monitors for the coarse mesh. 


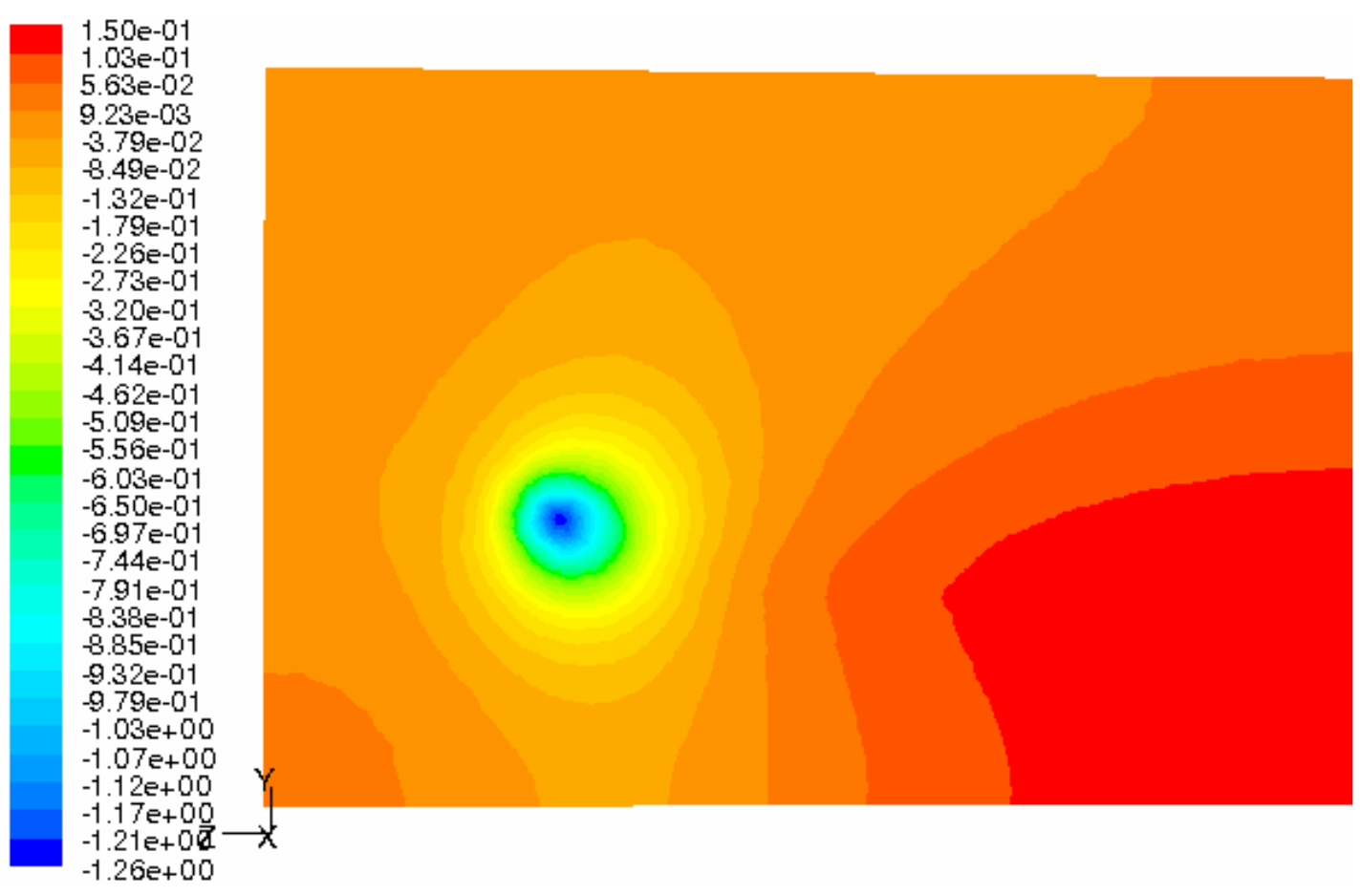

Figure 144. Pressure coefficient contours at a plane $x=9 \mathrm{ft}$ for the coarse mesh.

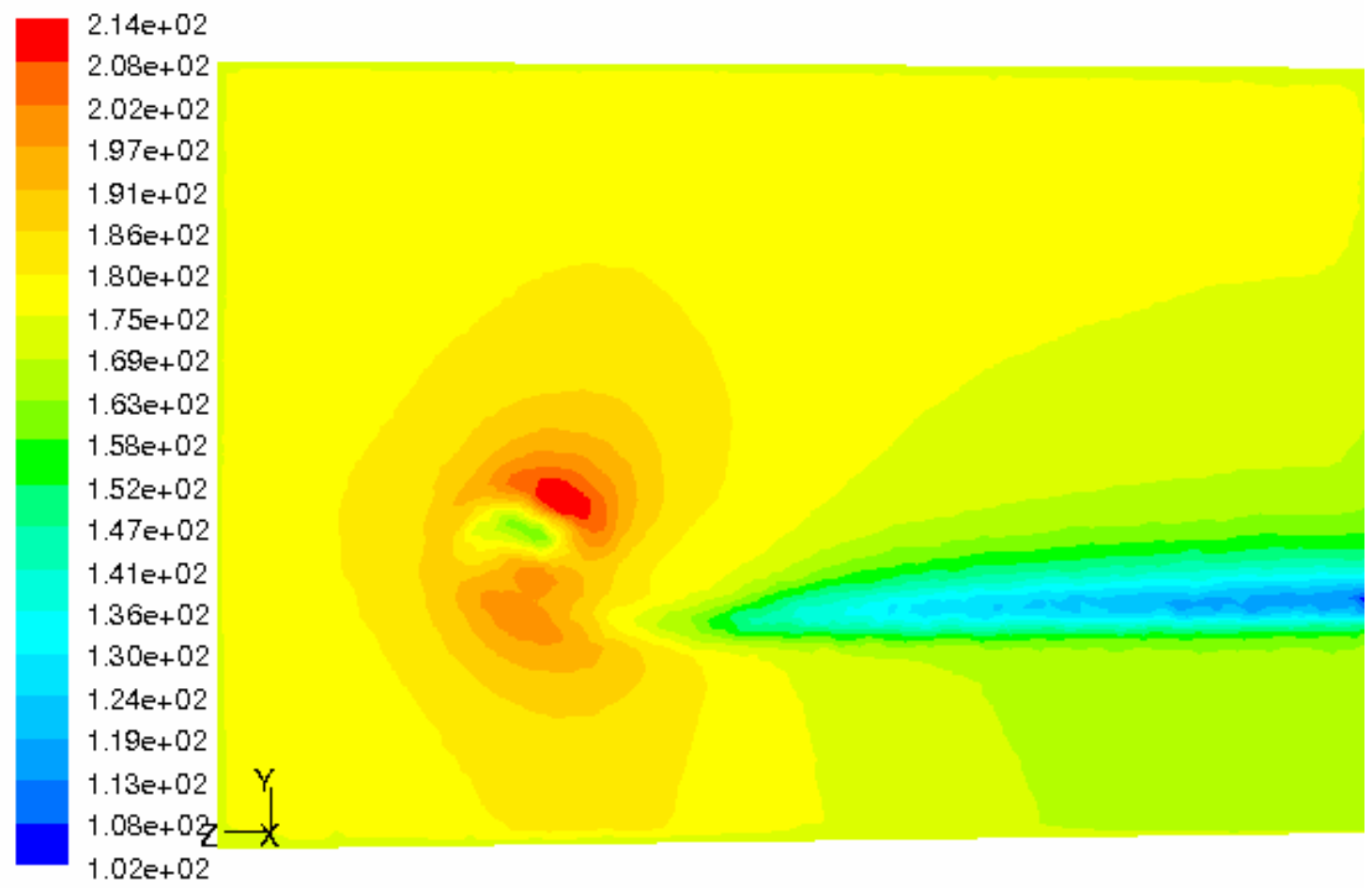

Figure 145. Velocity magnitude (ft/s) contours at a plane $x=9 \mathrm{ft}$ for the coarse mesh. 


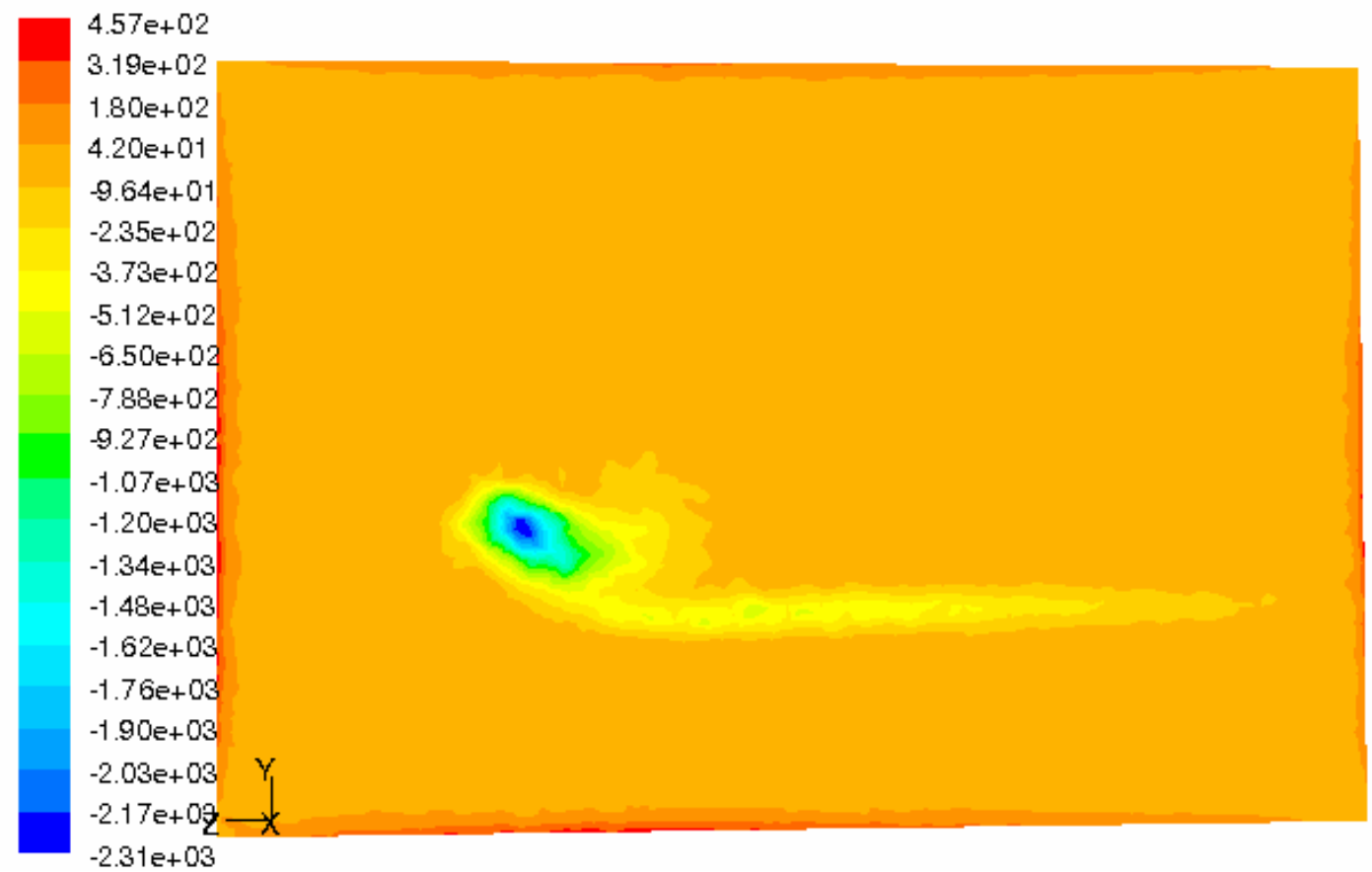

Figure 146. Vorticity contours $(1 / \mathrm{s})$ at a plane $x=9 \mathrm{ft}$. for the coarse mesh.

The pressure coefficient, velocity magnitude and vorticity contours in a plane at $\mathrm{x}=9 \mathrm{ft}$. downstream for the fine mesh are shown in Figures 147, 148, and 149, respectively.

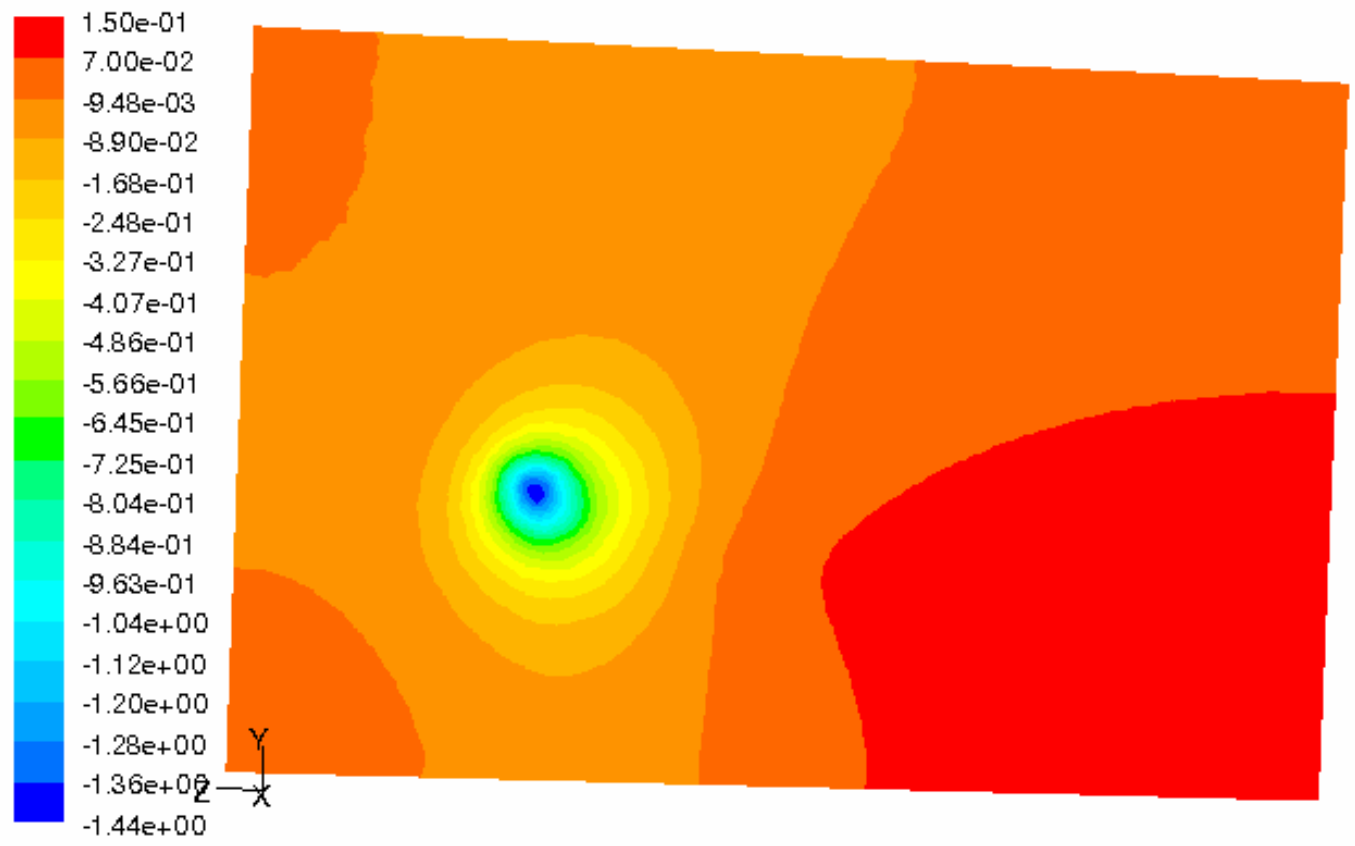

Figure 147. Pressure coefficient contours at $x=9$ ft. for the fine mesh. 


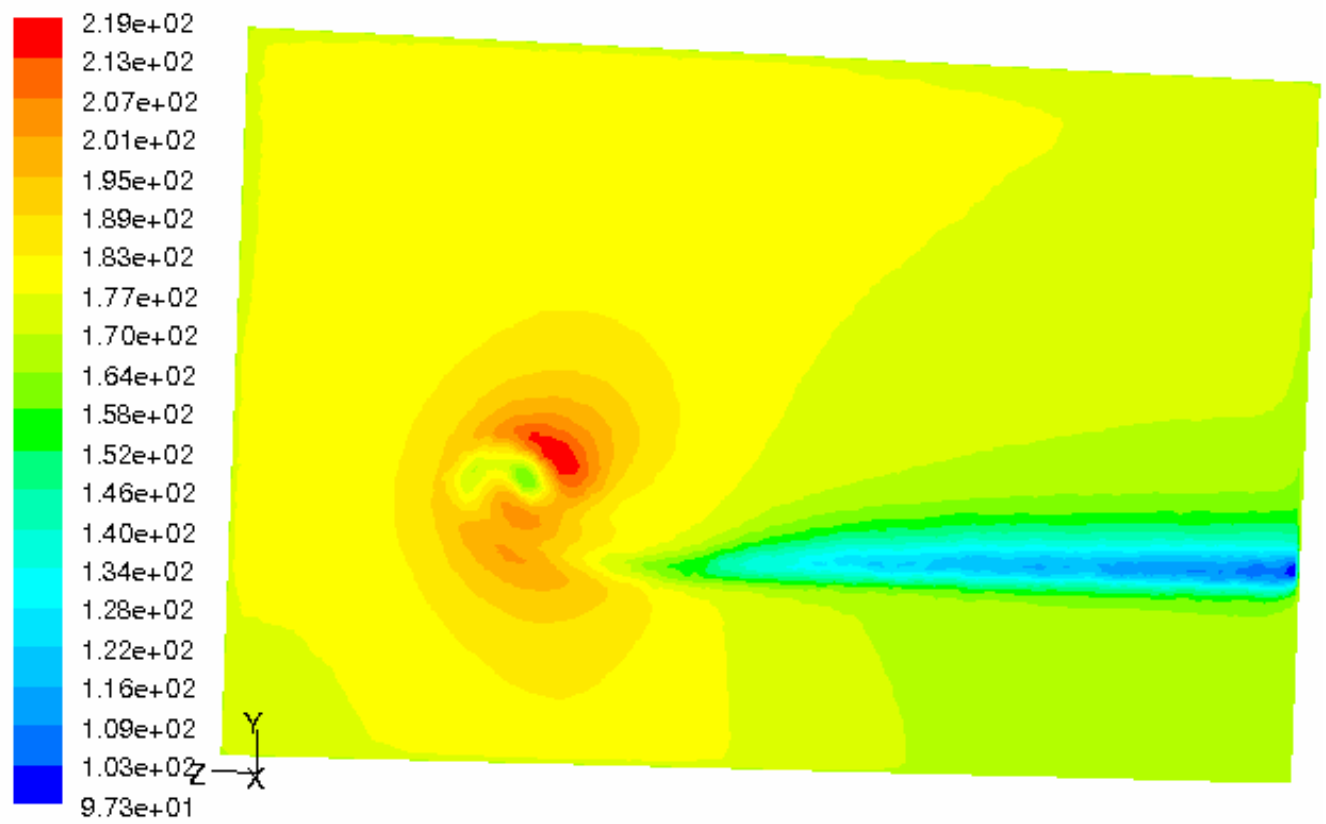

Figure 148. Velocity magnitude ( $\mathrm{ft} / \mathrm{s}$ ) contours at $\mathrm{x}=9 \mathrm{ft}$ for the fine mesh.

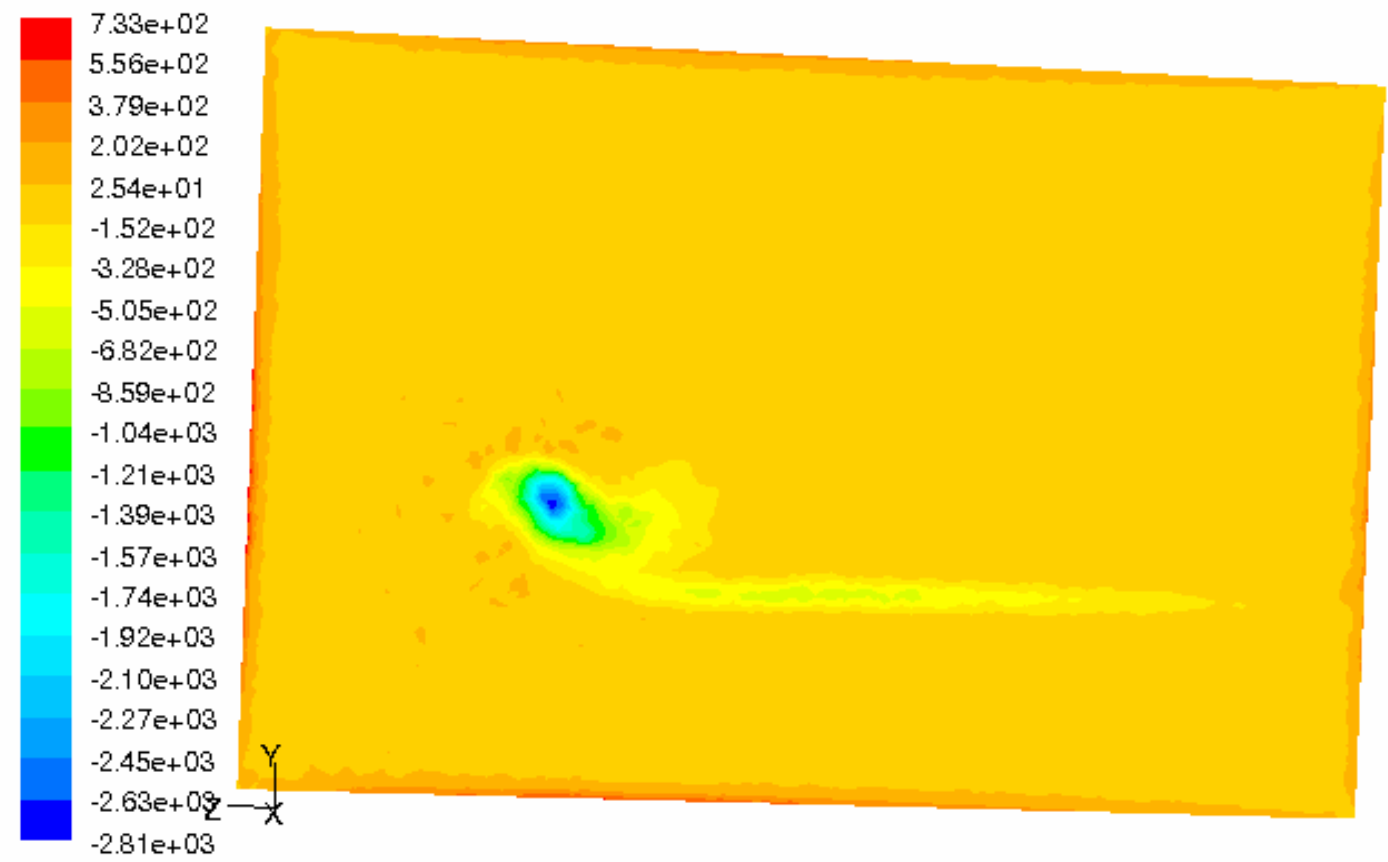

Figure 149. Vorticity (1/s) contours at $\mathrm{x}=9 \mathrm{ft}$ for the fine mesh.

The pressure coefficient, velocity magnitude and vorticity contours in a plane at $\mathrm{x}=9 \mathrm{ft}$. downstream for the finest mesh are shown in Figures 150, 151, and 152, respectively. 


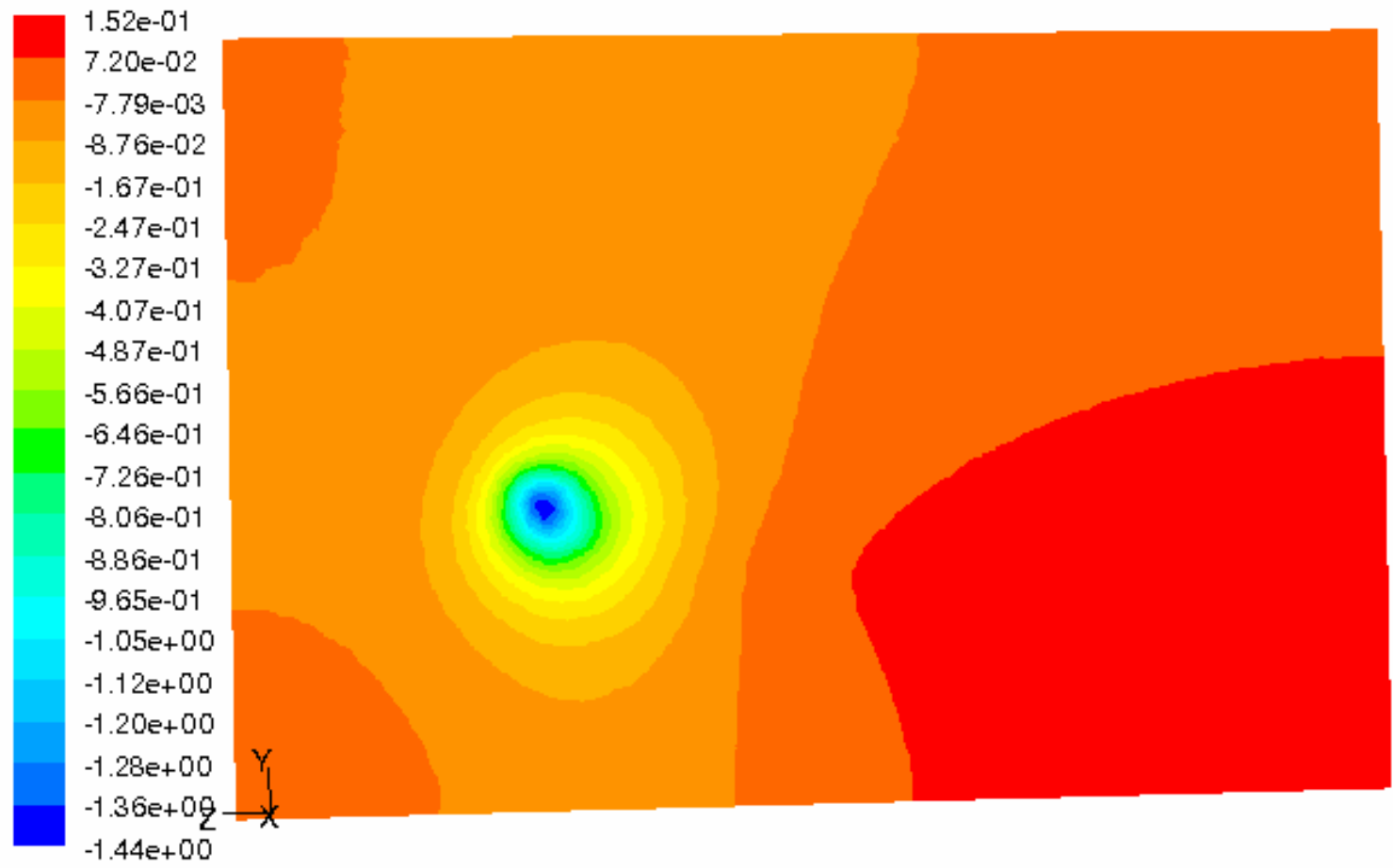

Figure 150. Pressure coefficient contours at $x=9 \mathrm{ft}$. for the finest mesh.

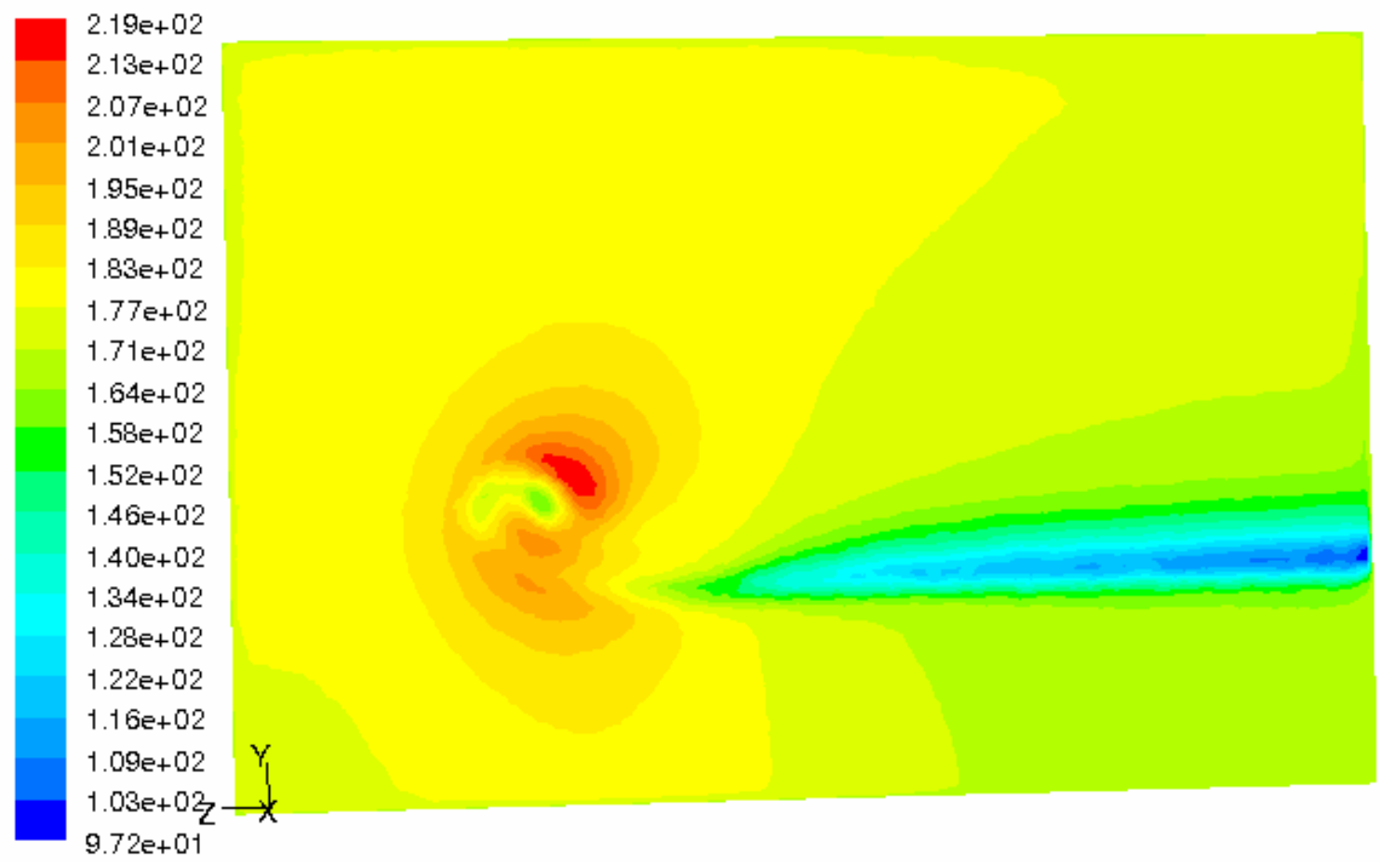

Figure 151. Velocity magnitude (ft/s) contours at $x=9 \mathrm{ft}$ for the finest mesh. 


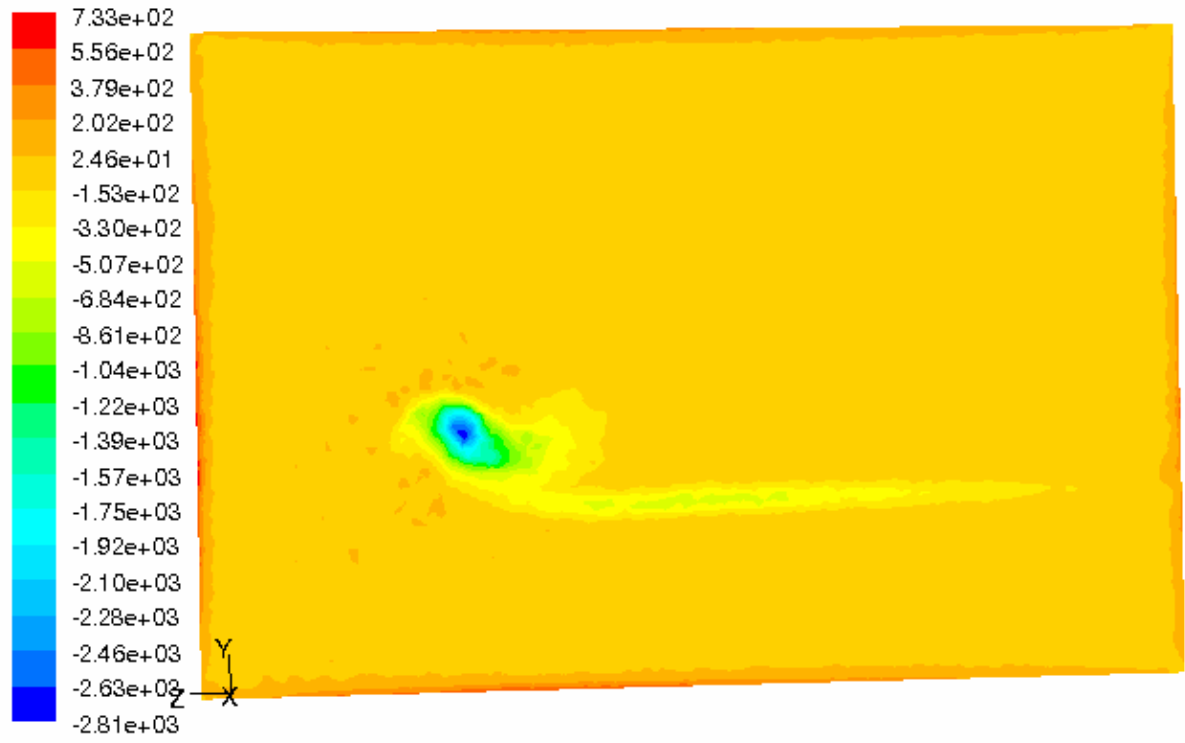

Figure 152. Vorticity (1/s) contours at $x=9 \mathrm{ft}$ for the finest mesh.

\section{BLC Application at $0.5 \mathrm{~V}_{\infty}$}

BLC suction was applied on the three suction slots at $0.5 \mathrm{~V}_{\infty}(85 \mathrm{ft} / \mathrm{s})$ for the three meshes to verify grid convergence.

The pressure coefficient, velocity magnitude and vorticity contours in a plane at $\mathrm{x}=9 \mathrm{ft}$. downstream for the coarse mesh, when suction is applied at $0.5 \mathrm{~V}_{\infty}(85 \mathrm{ft} / \mathrm{s})$, are shown in Figures 153, 154, and 155, respectively.

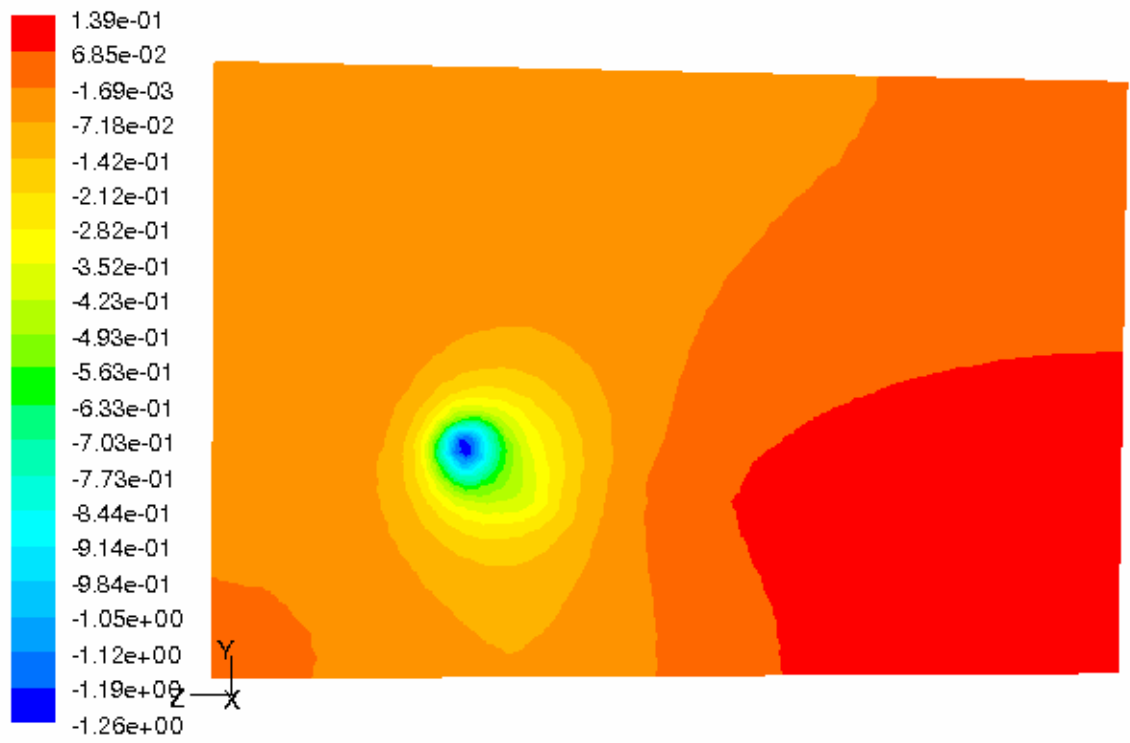

Figure 153. Pressure coefficient contours at $x=9$ ft. for the coarse mesh with suction $=0.5 \mathrm{~V}_{\infty}$. 


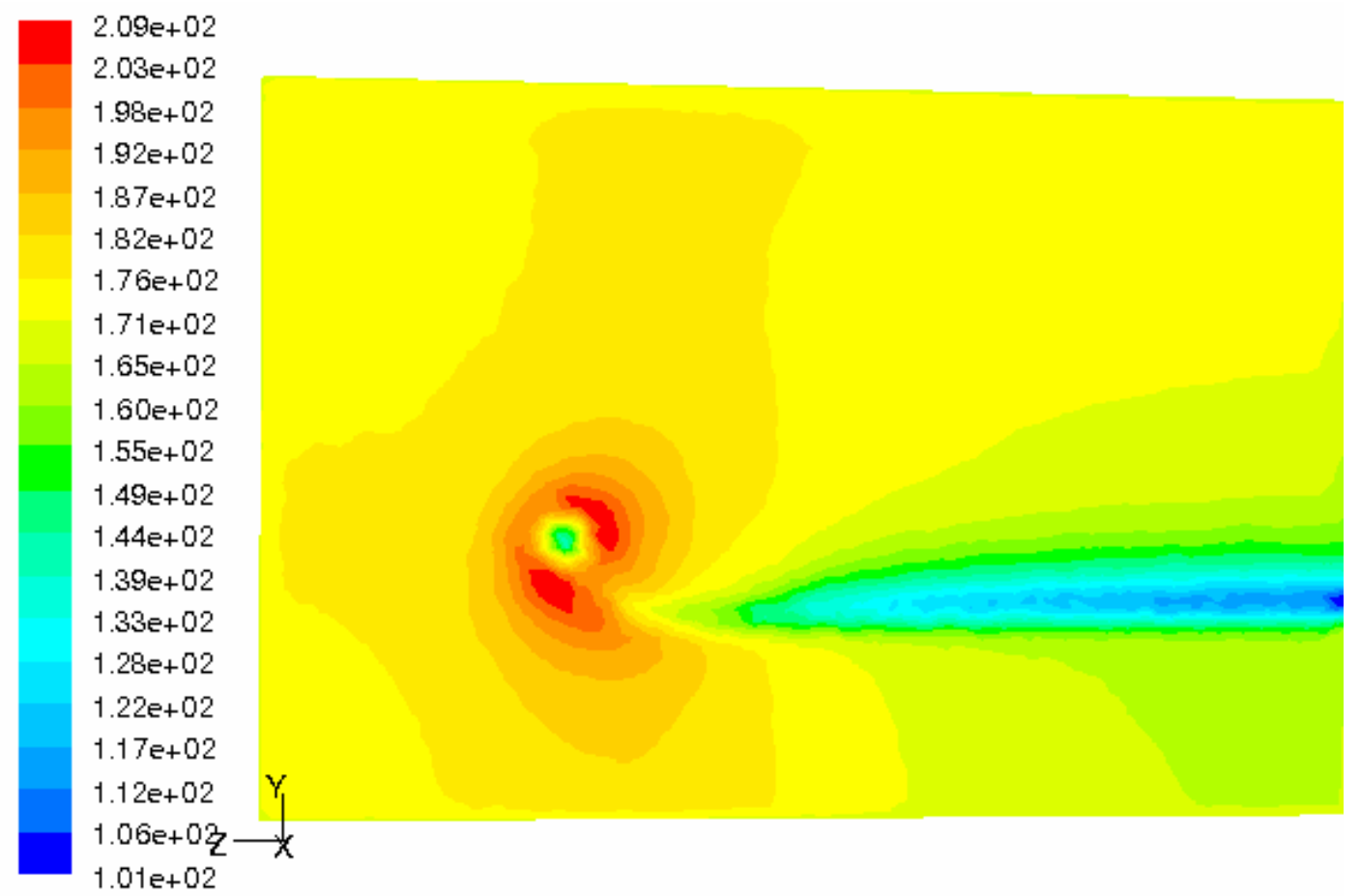

Figure 154. Velocity magnitude (ft/s) contours at $x=9 \mathrm{ft}$ for the coarse mesh with suction $=0.5 \mathrm{~V}_{\infty}$.

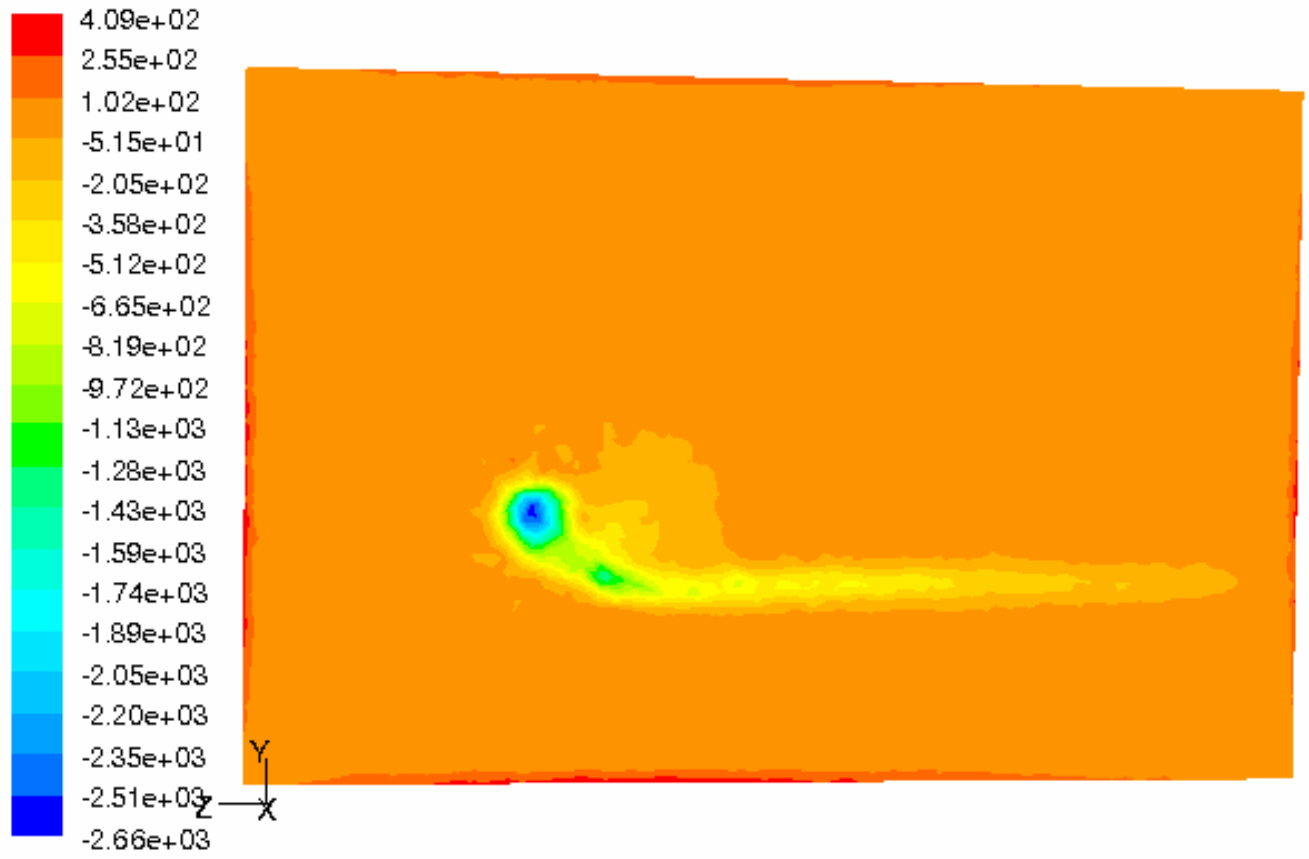

Figure 155. Vorticity $(1 / \mathrm{s})$ contours at $x=9 \mathrm{ft}$ for the coarse mesh with suction $=0.5 V_{\infty}$.

The pressure coefficient, velocity magnitude and vorticity contours in a plane at $\mathrm{x}=9 \mathrm{ft}$. downstream for the fine mesh, when suction is applied at $0.5 \mathrm{~V}_{\infty}(85 \mathrm{ft} / \mathrm{s})$, are shown in Figures 156, 157, and 158, respectively. 


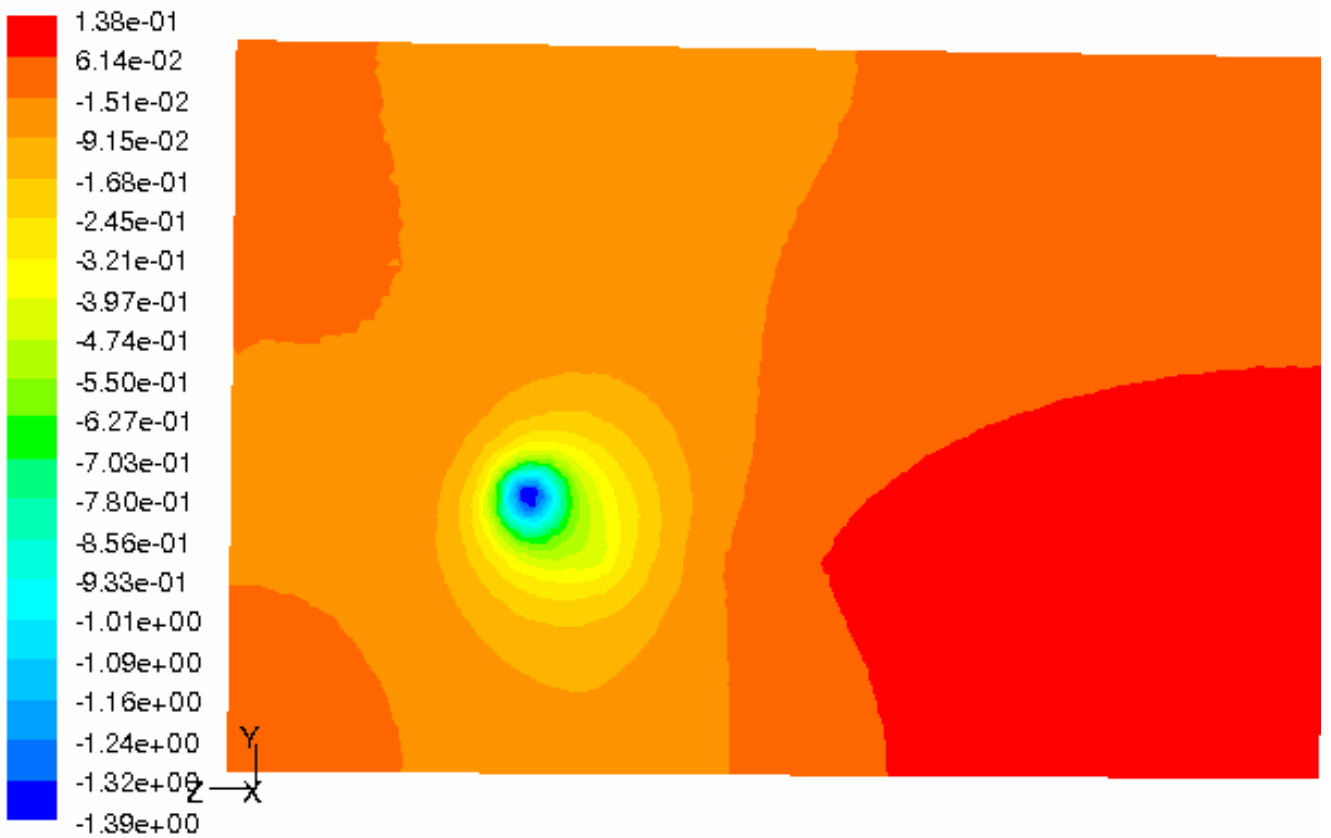

Figure 156. Pressure coefficient contours at $x=9$ ft. for the fine mesh with suction $=0.5 V_{\infty}$.

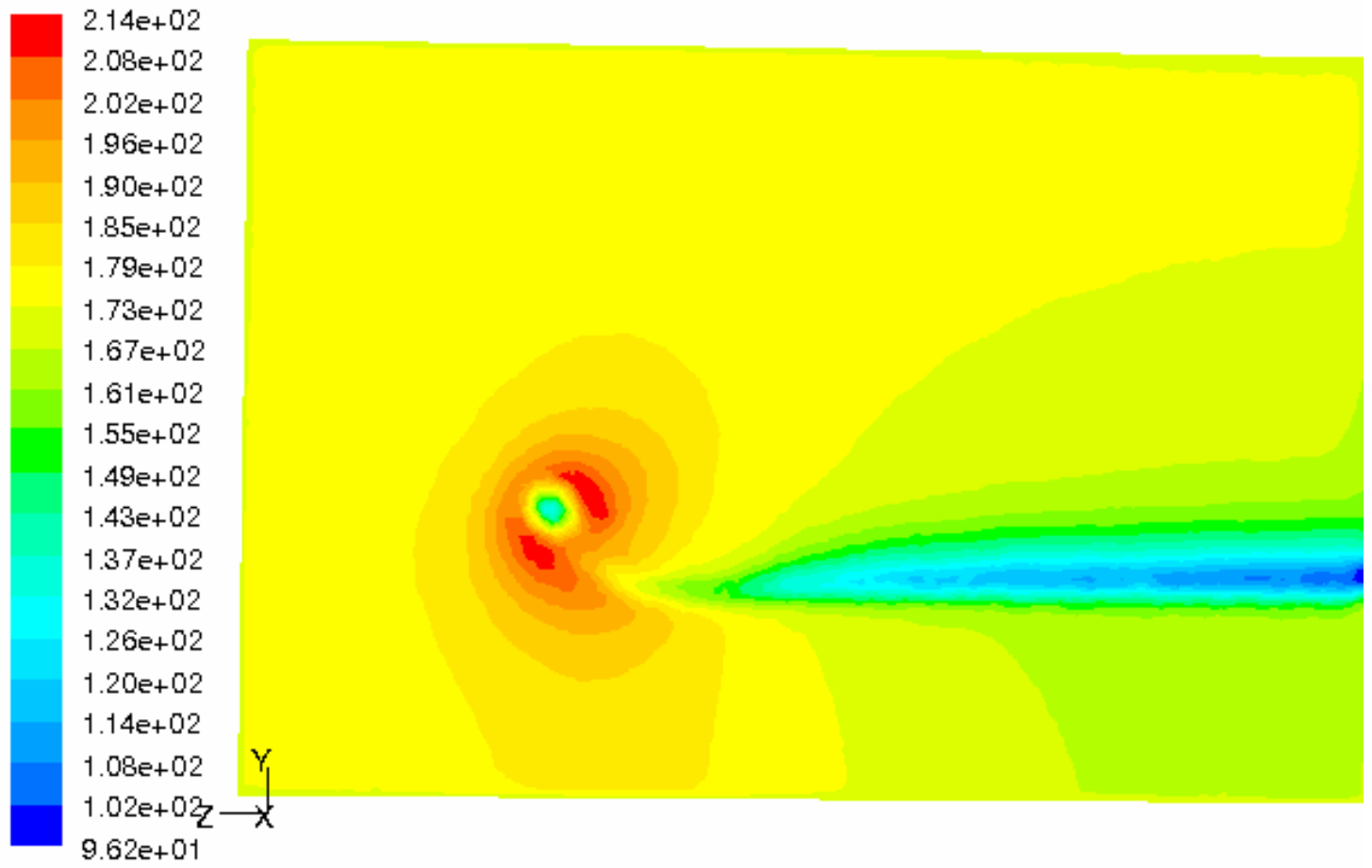

Figure 157. Velocity magnitude (ft/s) contours at $x=9 \mathrm{ft}$ for the fine mesh with suction $=0.5 \mathrm{~V}_{\infty}$. 


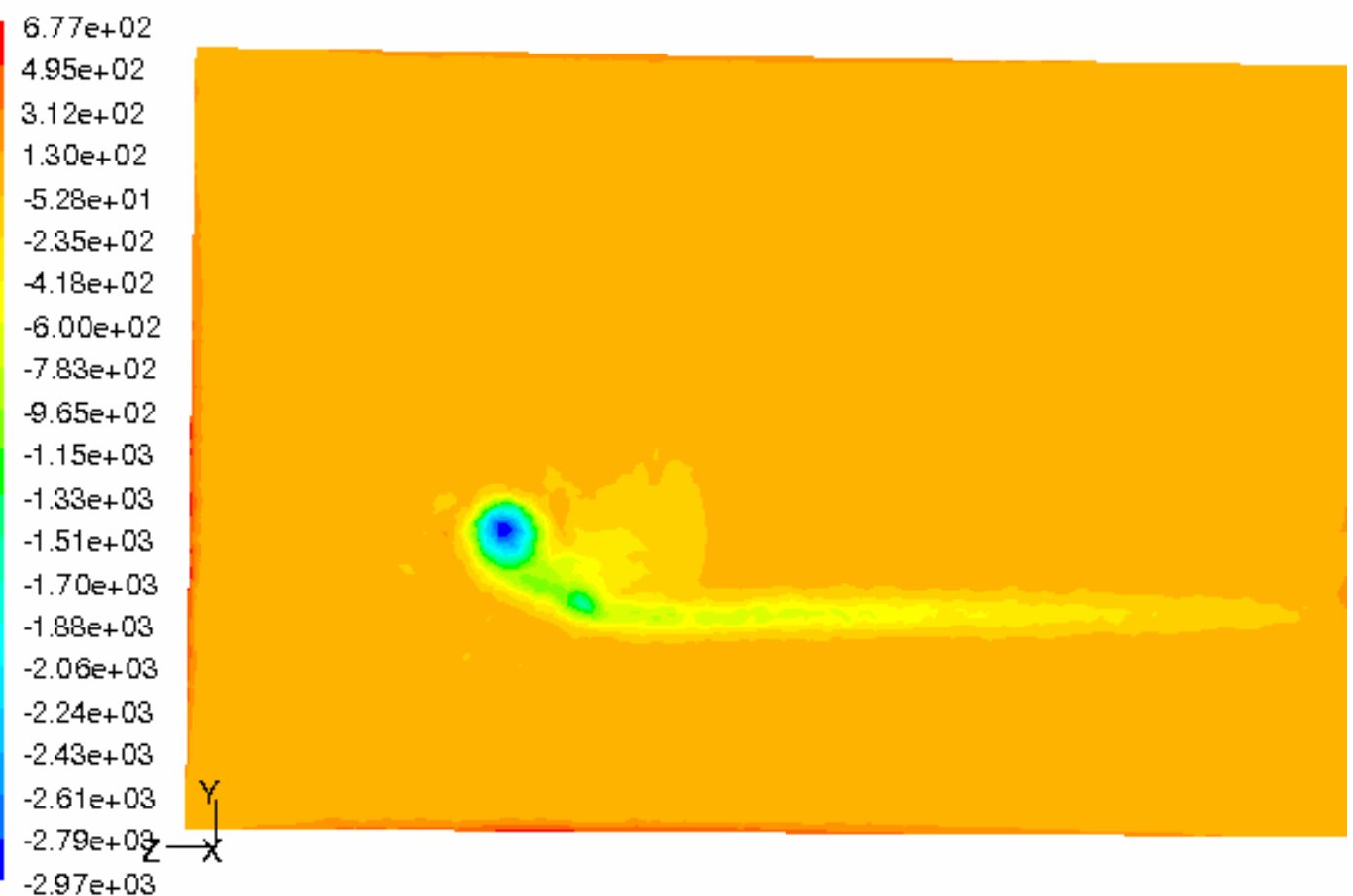

Figure 158. Vorticity $(1 / \mathrm{s})$ contours at $\mathrm{x}=9 \mathrm{ft}$ for the fine mesh with suction $=0.5 \mathrm{~V}_{\infty}$.

The pressure coefficient, velocity magnitude and vorticity contours in a plane at $\mathrm{x}=9 \mathrm{ft}$. downstream for the finest mesh, when suction is applied at $0.5 \mathrm{~V}_{\infty}(85 \mathrm{ft} / \mathrm{s})$, are shown in Figures 159, 160, and 161, respectively.

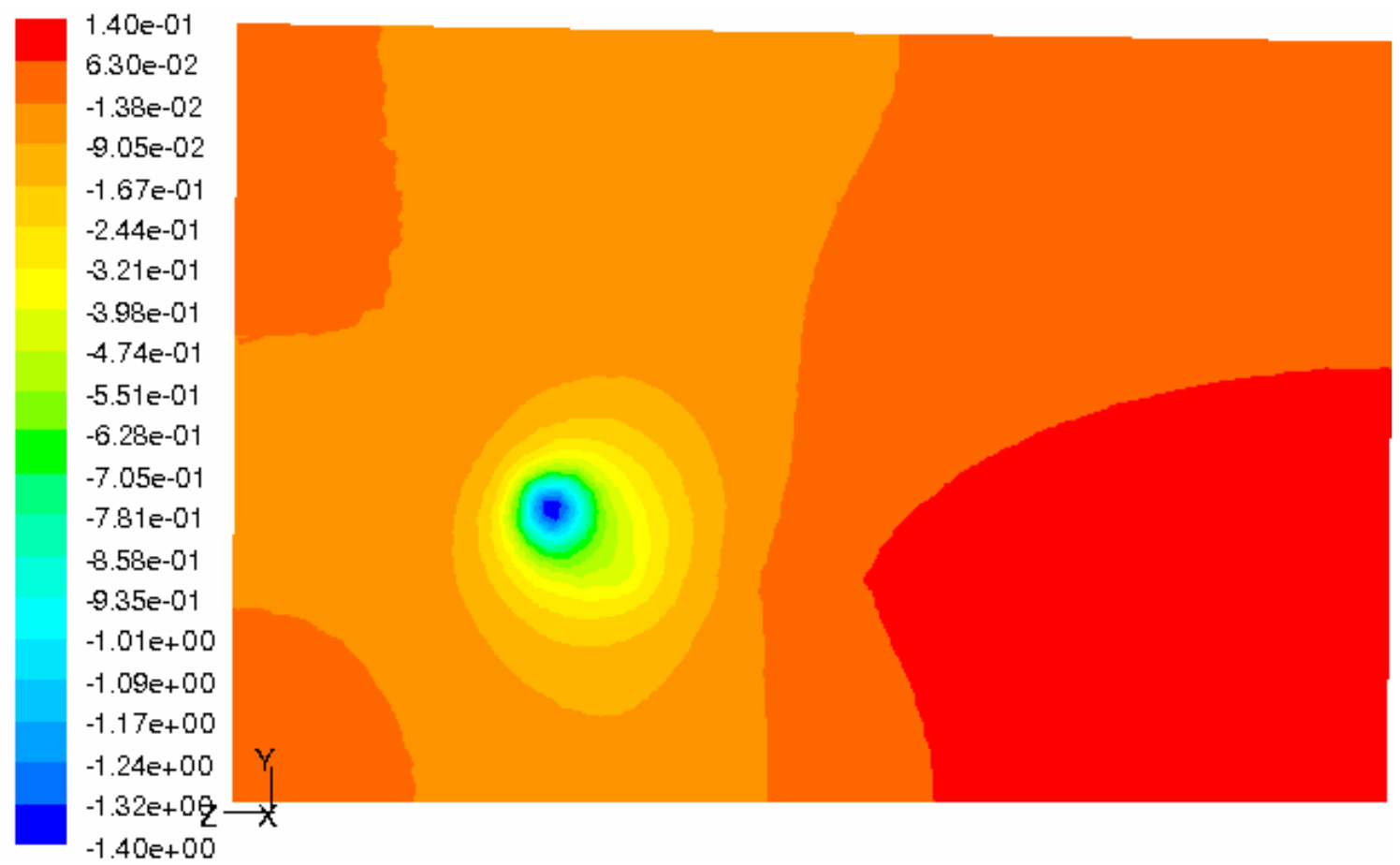

Figure 159. Pressure coefficient contours at $x=9$ ft. for the finest mesh with suction $=0.5 V_{\infty}$. 


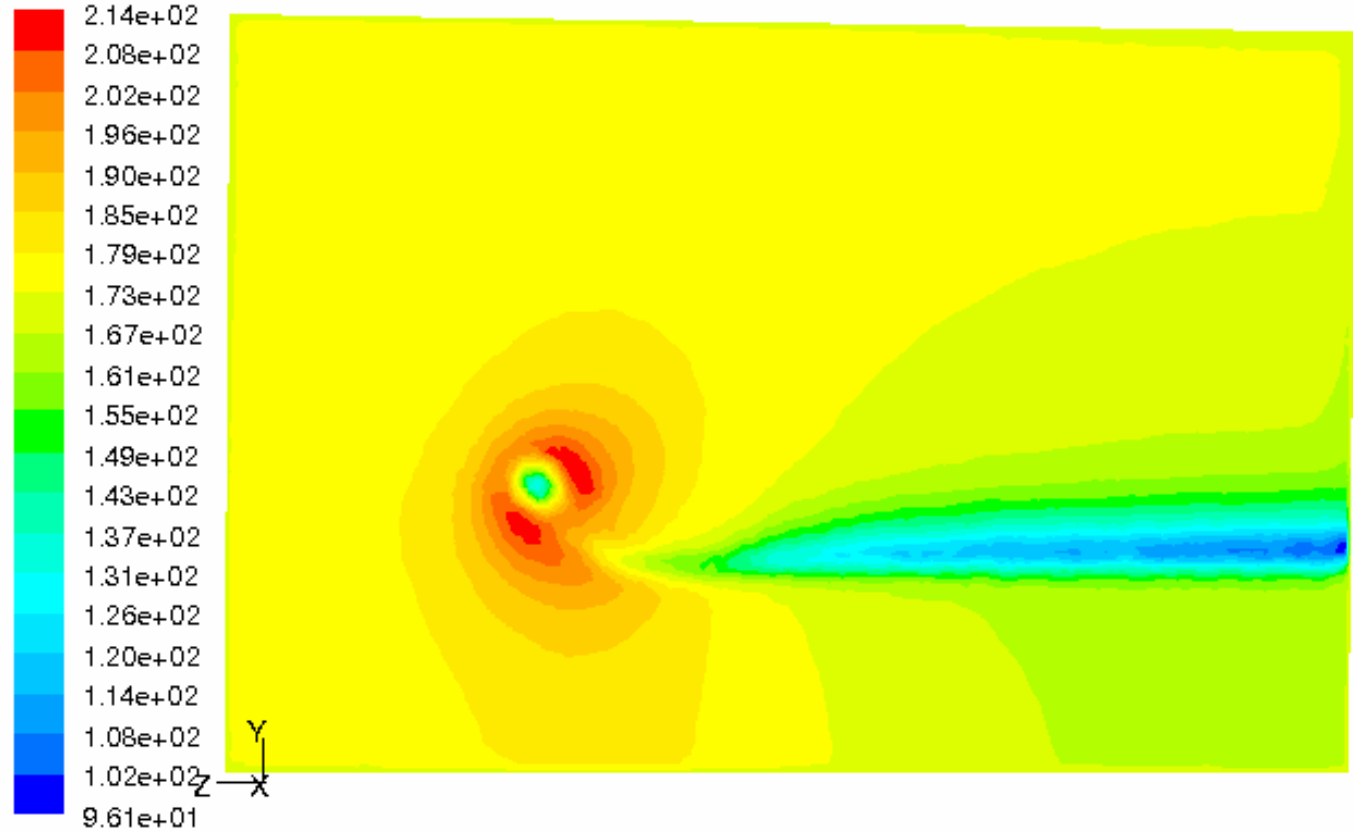

Figure 160. Velocity magnitude $(\mathrm{ft} / \mathrm{s})$ contours at $\mathrm{x}=9 \mathrm{ft}$ for the finest mesh with suction $=0.5 \mathrm{~V}_{\infty}$.

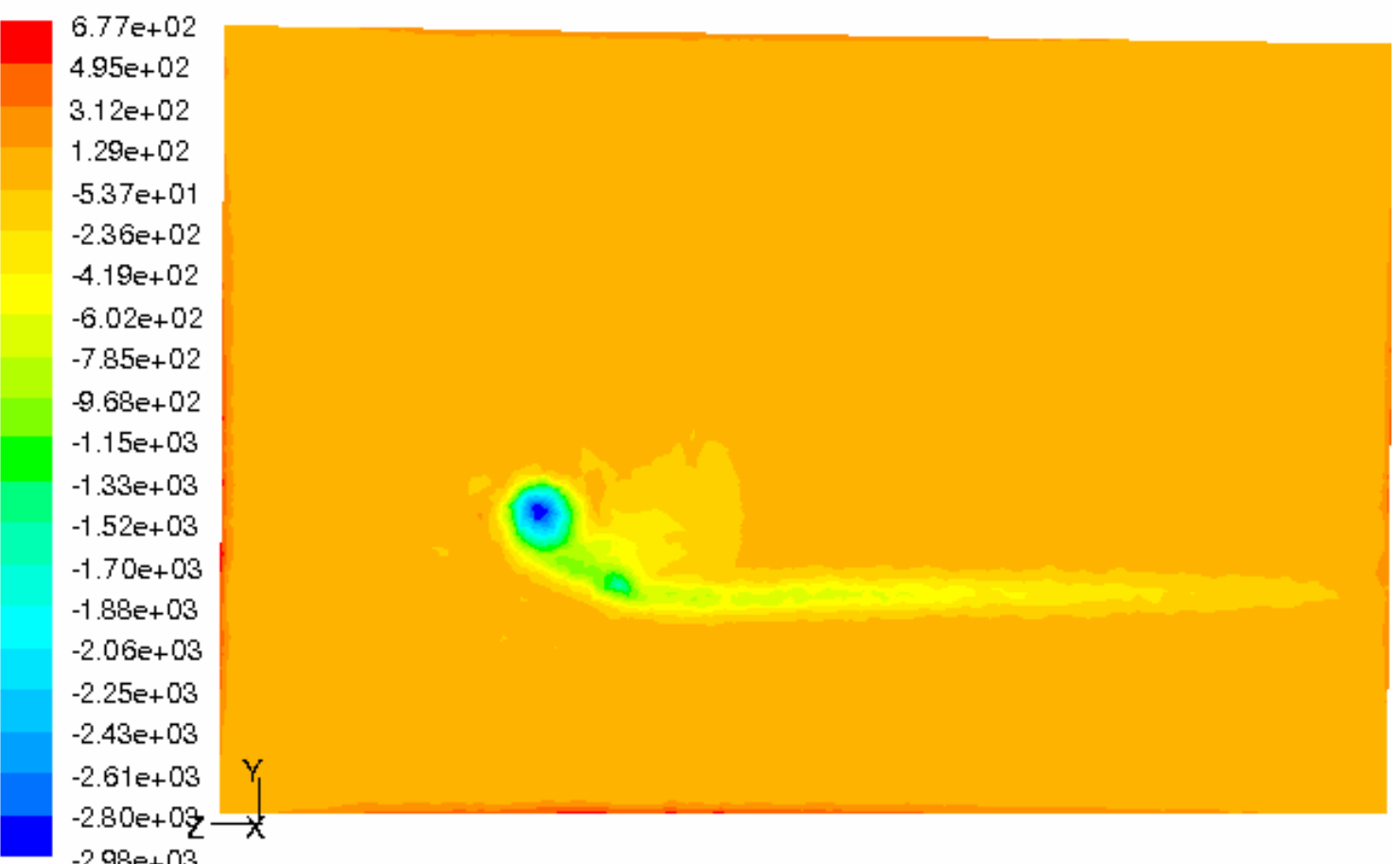

$-2.98 \mathrm{e}+03$

Figure 161. Vorticity $(1 / \mathrm{s})$ contours at $x=9 \mathrm{ft}$ for the finest mesh with suction $=0.5 \mathrm{~V}_{\infty}$.

\section{Grid Convergence Study Results}

Table 16 shows the results of the grid convergence study for the wing without BLC and when BLC suction was applied on the three slots at $0.5 \mathrm{~V}_{\infty}(85 \mathrm{ft} / \mathrm{s})$. Vorticity 
and Drag coefficient results are shown for the three different meshes generated for this study. It can be seen how grid convergence is obtained at the fine mesh. Vorticity decrements up to $3.6 \%$ and drag coefficient decrements up to $7.6 \%$ are obtained when BLC suction is applied.

Table 16. Grid Convergence Study Results with and without BLC application at $0.5 \mathrm{~V}_{\infty}$.

\begin{tabular}{|c|c|c|c|c|c|c|c|c|c|c|}
\hline \multirow[b]{2}{*}{ Grid } & \multicolumn{2}{|c|}{ Vorticity $\left(\mathrm{ft}^{2} / \mathrm{s}\right)$} & \multirow{2}{*}{$\begin{array}{c}\text { Vorticity } \\
\text { Decrease } \\
(\%)\end{array}$} & \multicolumn{2}{|c|}{$\mathrm{C}_{\mathrm{D}}$} & \multirow{2}{*}{$\begin{array}{c}C_{D} \\
\text { Decrease } \\
(\%) \\
\end{array}$} & \multicolumn{2}{|c|}{$\mathrm{C}_{\mathrm{L}}$} & \multicolumn{2}{|c|}{ L/D } \\
\hline & \begin{tabular}{|c|} 
Without \\
BLC
\end{tabular} & $\begin{array}{c}\text { BLC } \\
\text { applied }\end{array}$ & & \begin{tabular}{|c|} 
Without \\
BLC
\end{tabular} & $\begin{array}{c}\text { BLC } \\
\text { applied }\end{array}$ & & $\begin{array}{c}\text { Without } \\
\text { BLC }\end{array}$ & $\begin{array}{c}\text { BLC } \\
\text { applied }\end{array}$ & $\begin{array}{c}\text { Without } \\
\text { BLC }\end{array}$ & $\begin{array}{c}\text { BLC } \\
\text { applied }\end{array}$ \\
\hline Coarse & .77 & -208.77 & 3.24 & 0.0727 & 0.0672 & 7.57 & 0.7296 & 0.6870 & 10.04 & 10.22 \\
\hline Fine & -217.04 & .15 & 3.64 & 21 & 0.0666 & 7.63 & 47 & $\overline{0.6}$ & 5 & 10.26 \\
\hline Finest & -217.05 & -209.15 & 3.64 & 0.0721 & 0.0666 & 7.63 & 0.7246 & 0.6830 & 10.05 & 10.26 \\
\hline
\end{tabular}

\section{BLC Suction Effectiveness Study}

In the previous study, all the suction slots (top, bottom front, and bottom rear) were operating but the effect of each of them individually cannot be assessed. Each of the slots is evaluated singly so it can be determined which of them is more effective on reducing wingtip wake vorticity. BLC suction is applied at $0.5 \mathrm{~V}_{\infty}(85 \mathrm{ft} / \mathrm{s})$ and $\mathrm{V}_{\infty}(170$ $\mathrm{ft} / \mathrm{s}$ ). All of the following simulations are computed using the finest mesh.

The pressure coefficient, velocity magnitude and vorticity contours in a plane at $\mathrm{x}=9 \mathrm{ft}$. downstream when suction is applied on top slot at $0.5 \mathrm{~V}_{\infty}(85 \mathrm{ft} / \mathrm{s})$ are shown in Figures 162, 163, and 164, respectively. 


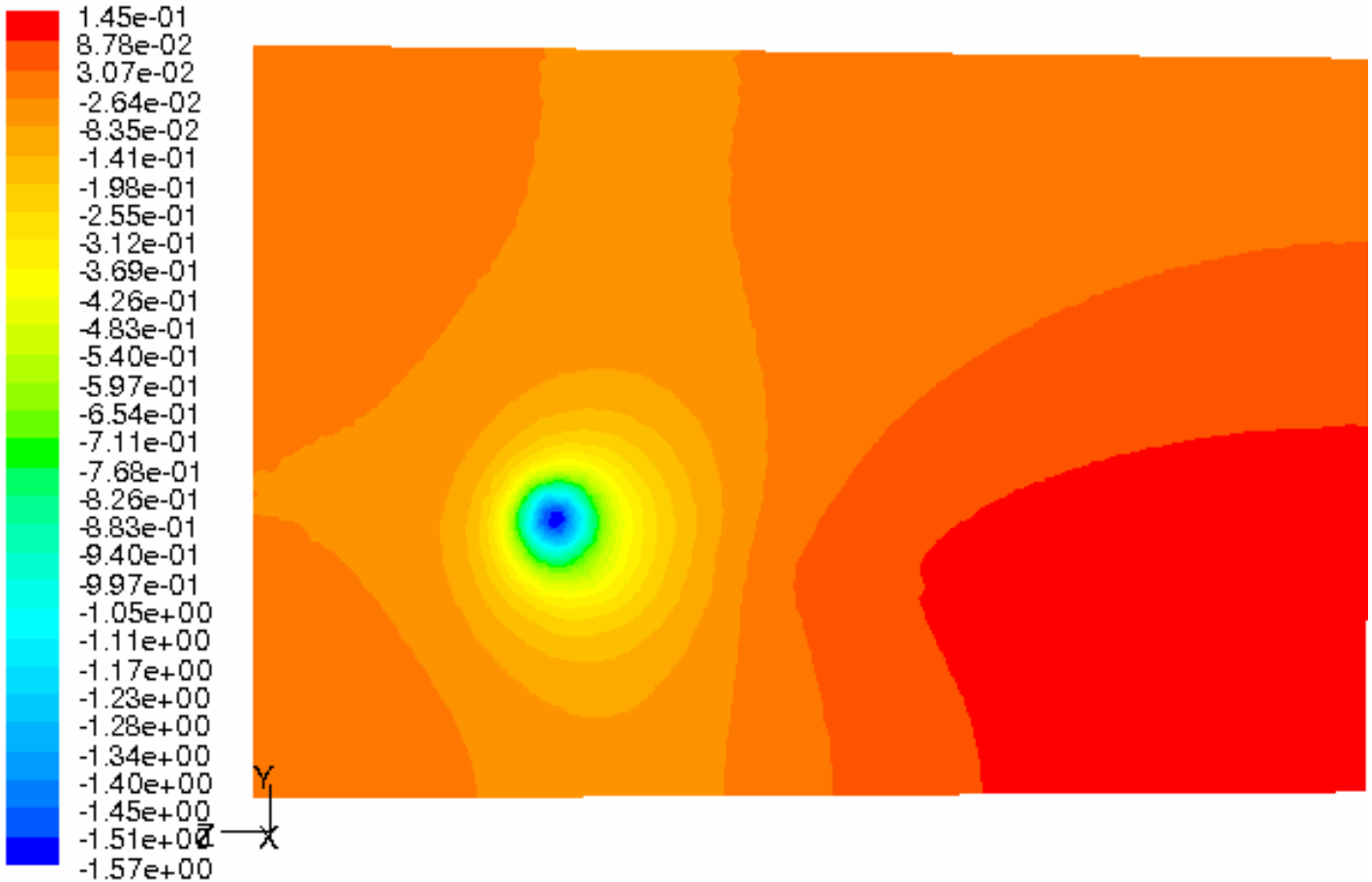

Figure 162. Pressure coefficient contours at $x=9 \mathrm{ft}$. for the finest mesh with suction $=0.5 \mathrm{~V}_{\infty}$ on top slot.

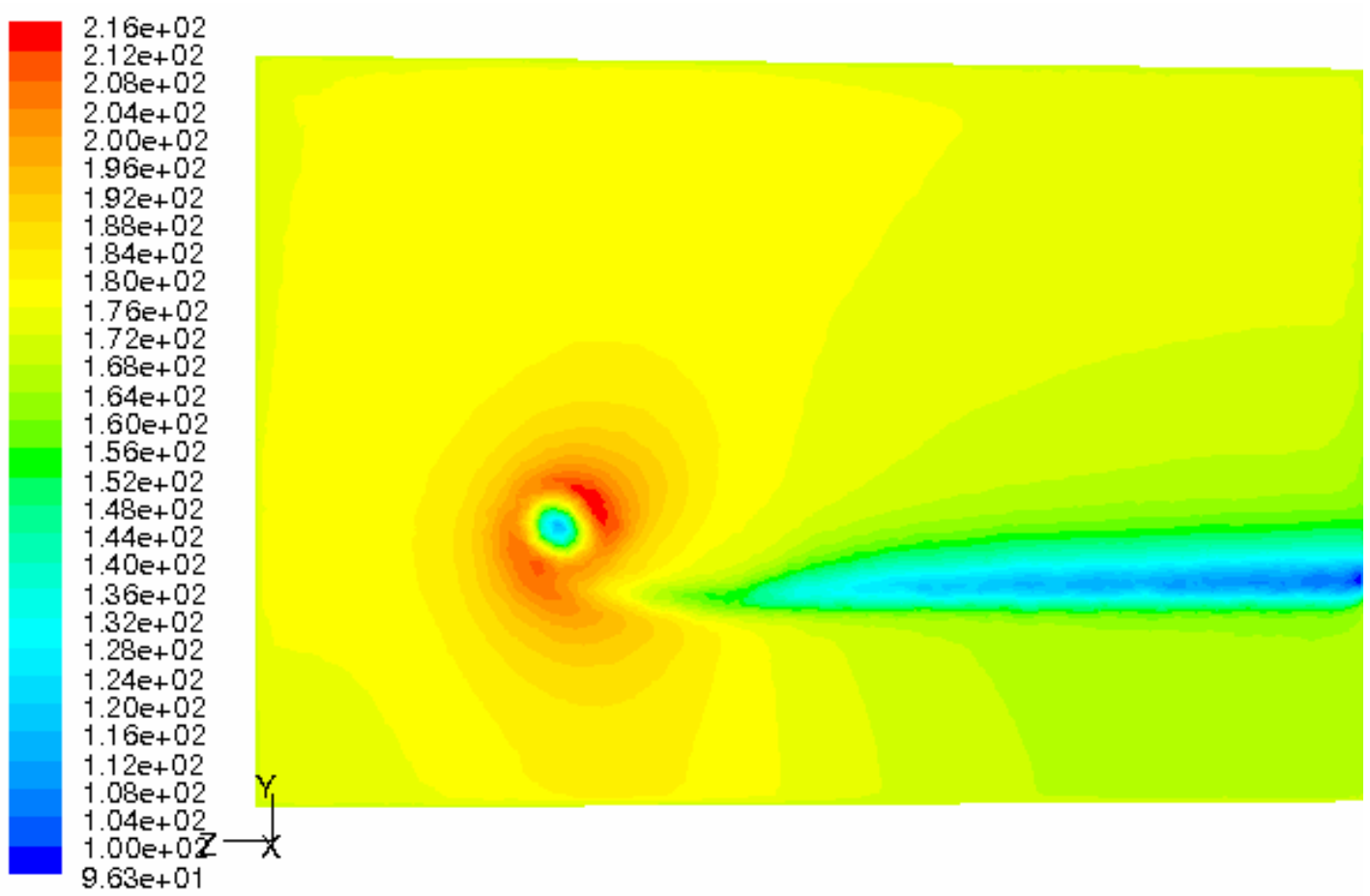

Figure 163. Velocity magnitude ( $\mathrm{ft} / \mathrm{s})$ contours at $\mathrm{x}=9 \mathrm{ft}$ for the finest mesh with suction $=0.5 \mathrm{~V}_{\infty}$ on top slot. 


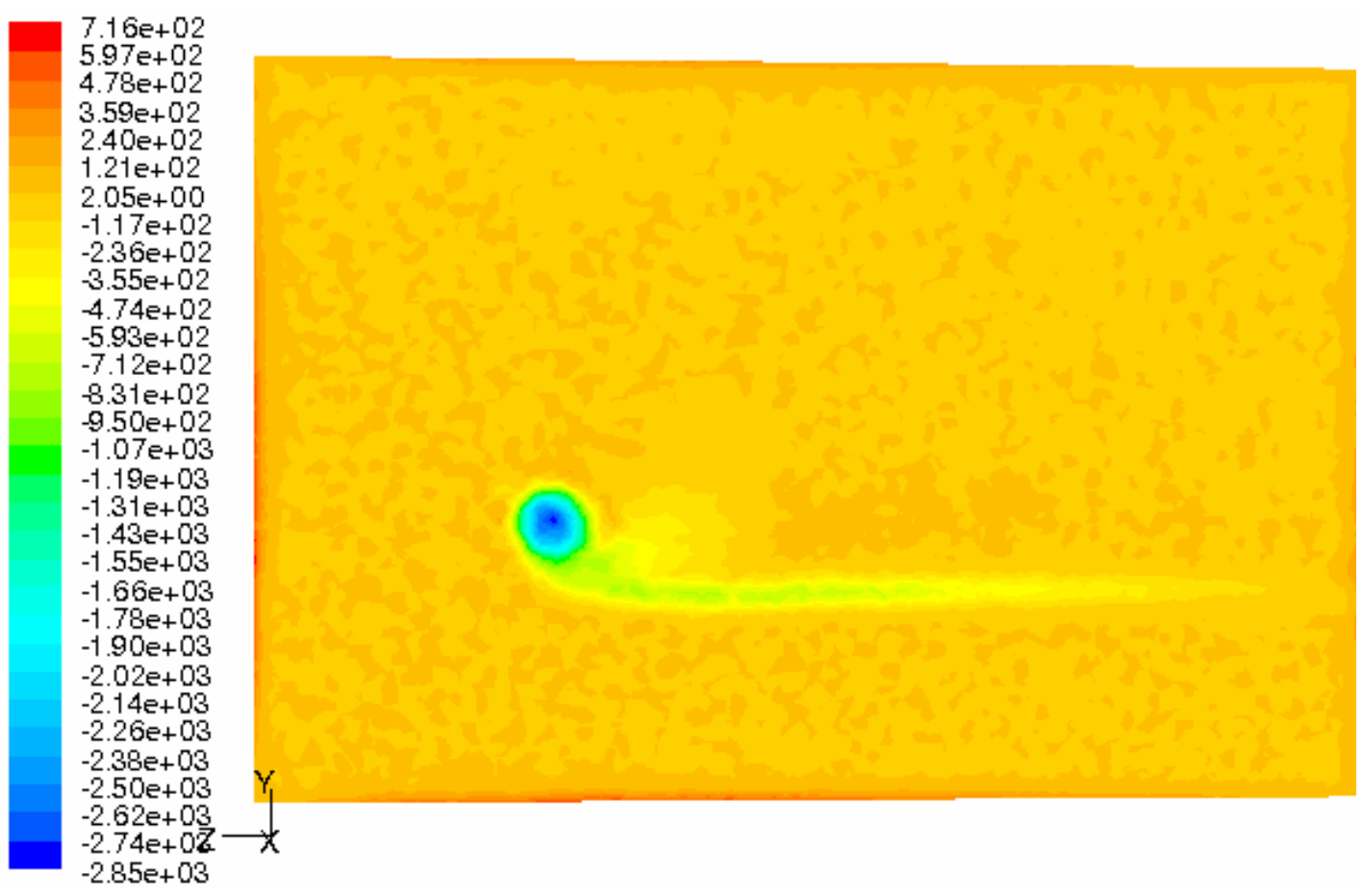

Figure 164. Vorticity $(1 / \mathrm{s})$ contours at $x=9 \mathrm{ft}$ for the finest mesh with suction $=0.5 \mathrm{~V}_{\infty}$ on top slot.

The pressure coefficient, velocity magnitude and vorticity contours in a plane at $\mathrm{x}=9 \mathrm{ft}$. downstream when suction is applied on top slot at $\mathrm{V}_{\infty}(170 \mathrm{ft} / \mathrm{s})$ are shown in Figures 165, 166, and 167, respectively.

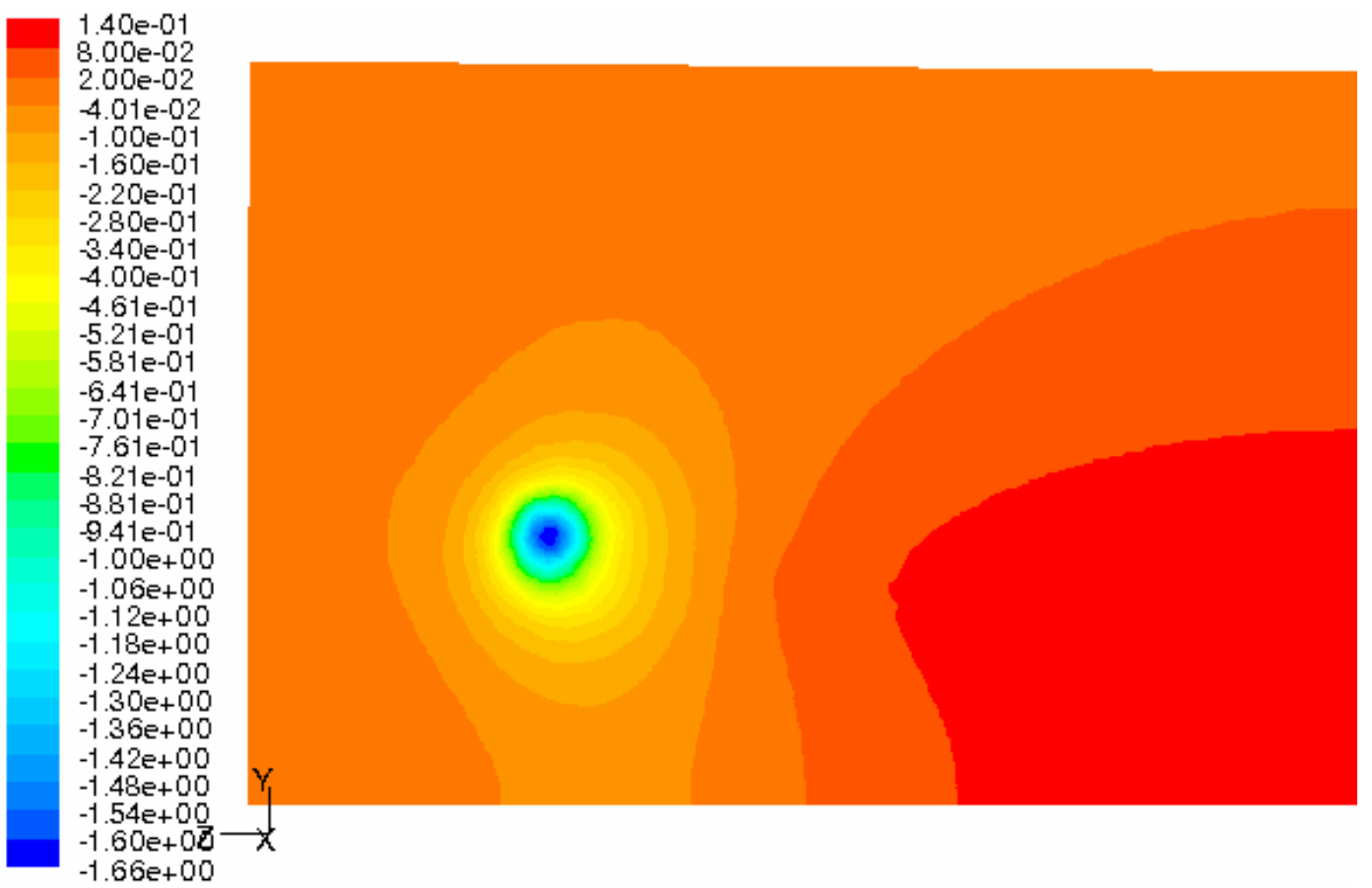

Figure 165. Pressure coefficient contours at $x=9 \mathrm{ft}$. for the finest mesh with suction $=V_{\infty}$ on top slot. 


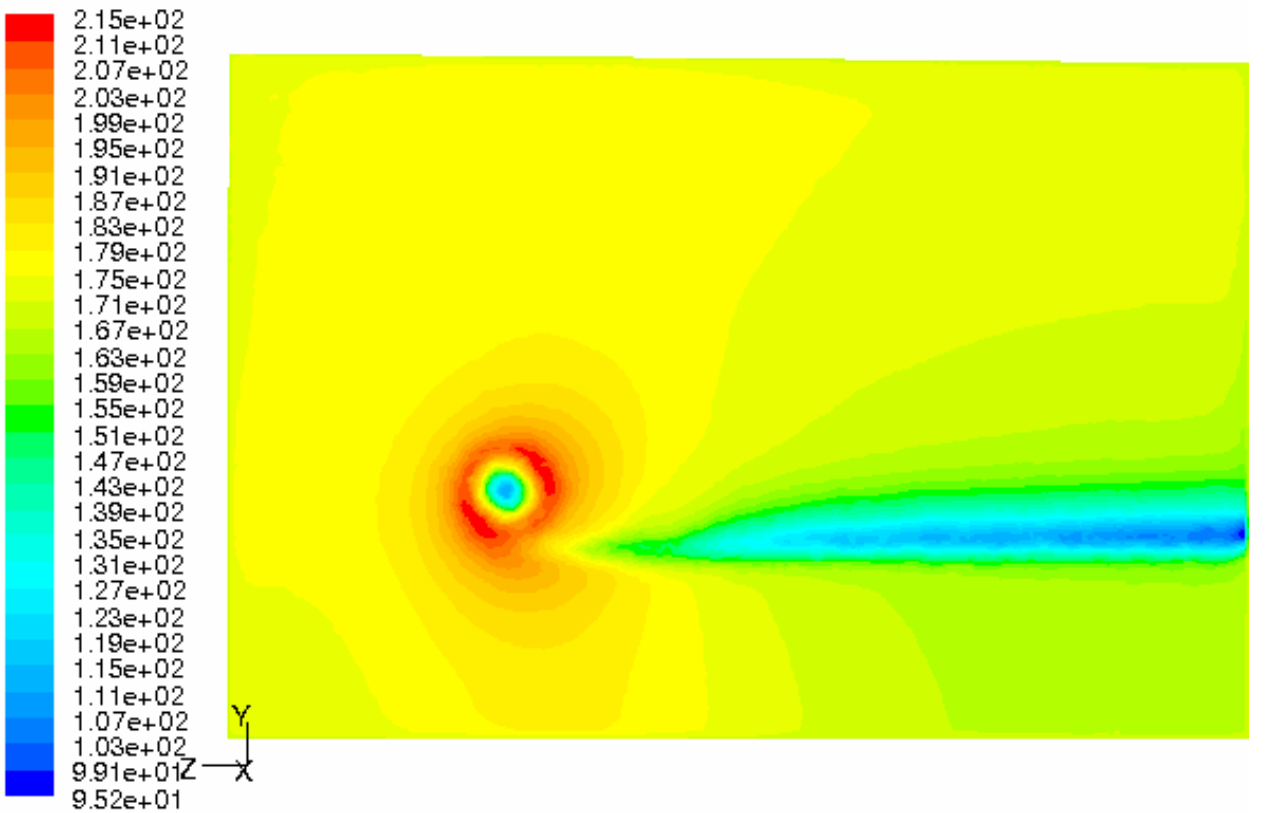

Figure 166. Velocity magnitude ( $\mathrm{ft} / \mathrm{s})$ contours at $\mathrm{x}=9 \mathrm{ft}$ for the finest mesh with suction $=\mathrm{V}_{\infty}$ on top slot.

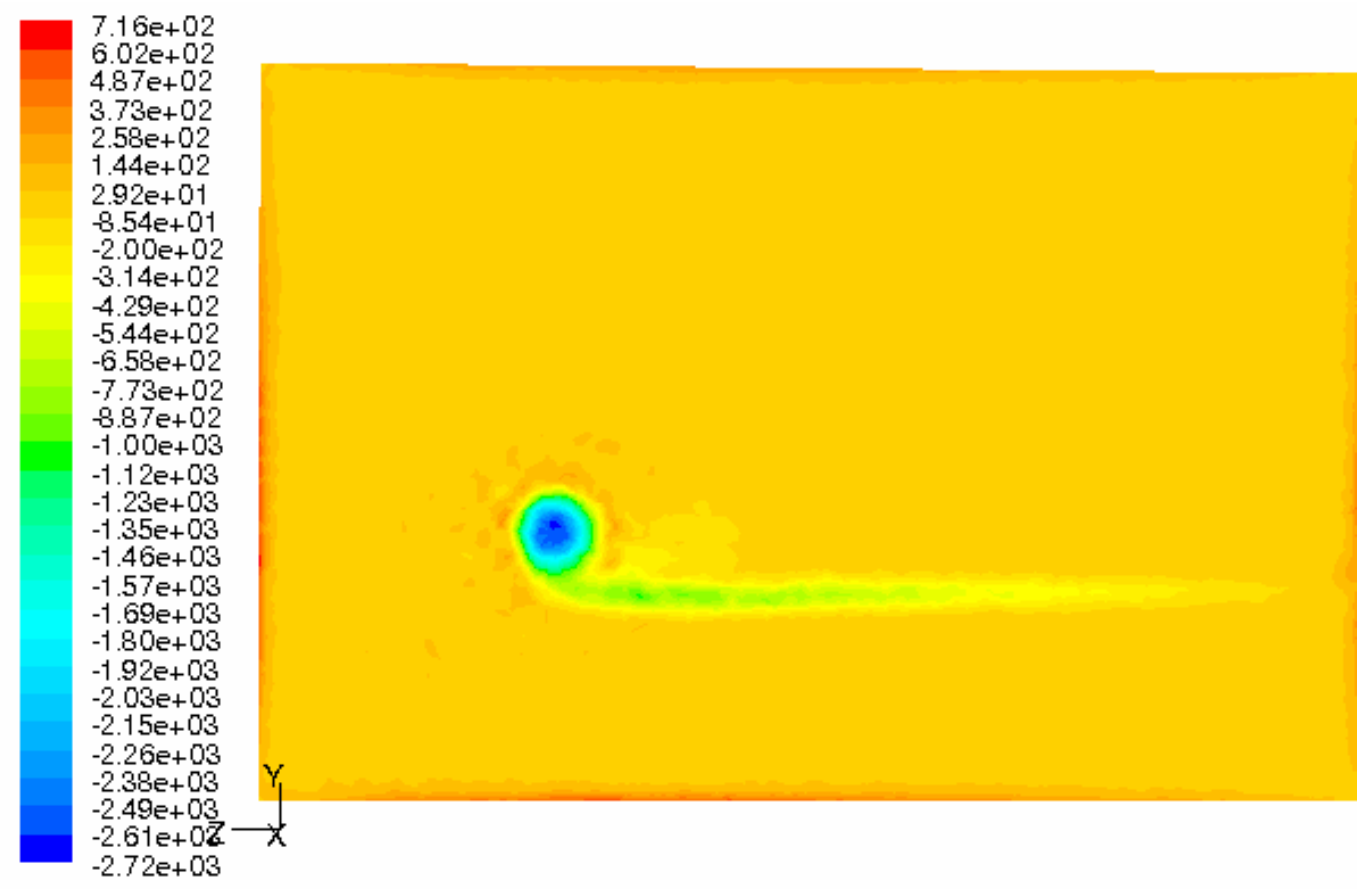

Figure 167. Vorticity (1/s) contours at $x=9 \mathrm{ft}$ for the finest mesh with suction $=V_{\infty}$ on top slot.

The pressure coefficient, velocity magnitude and vorticity contours in a plane at $\mathrm{x}=9 \mathrm{ft}$. downstream when suction is applied on bottom slots at $0.5 \mathrm{~V}_{\infty}(85 \mathrm{ft} / \mathrm{s})$ are shown in Figures 168, 169, and 170, respectively. 


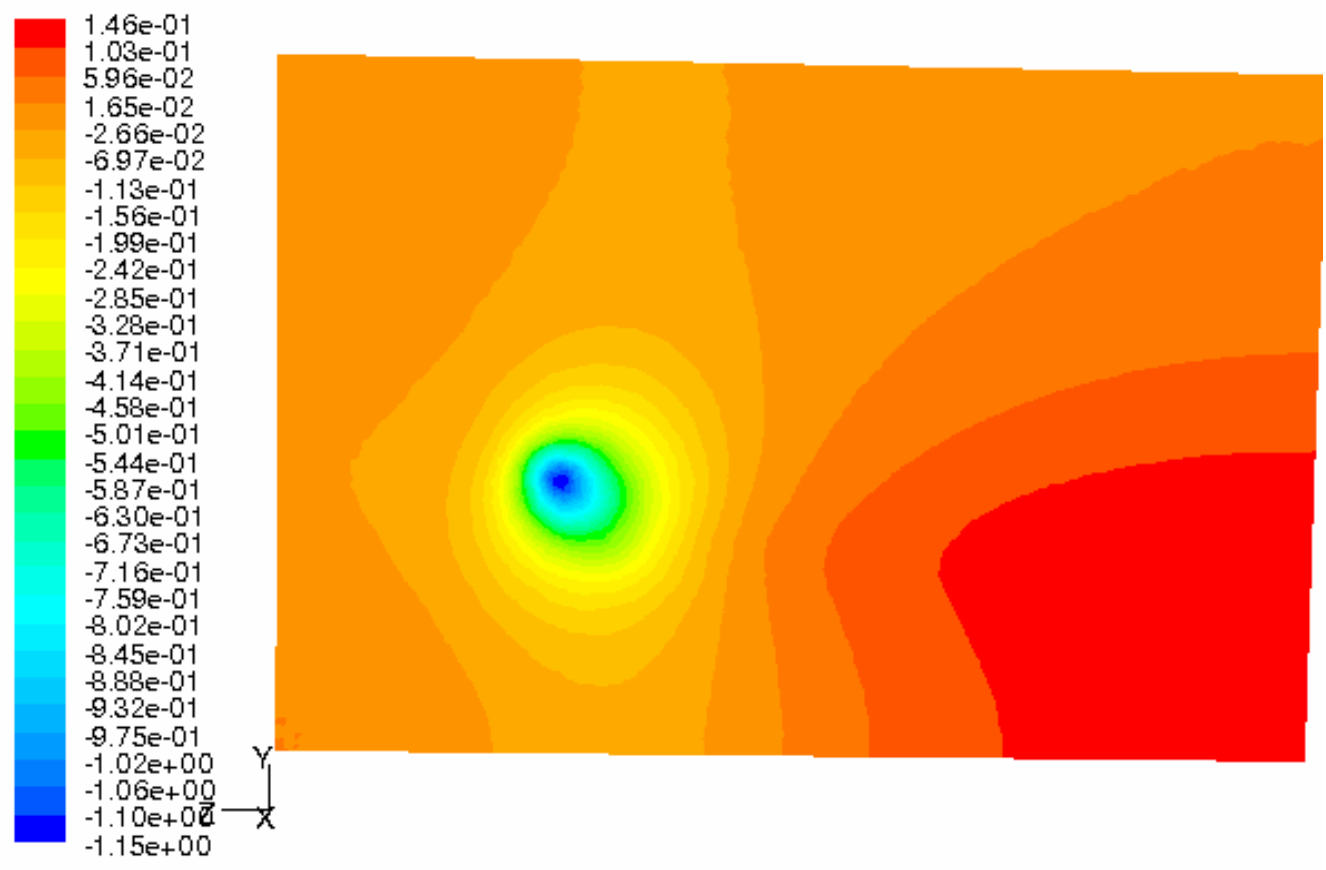

Figure 168. Pressure coefficient contours at $x=9$ ft. for the finest mesh with suction $=0.5 V_{\infty}$ on bottom slots.

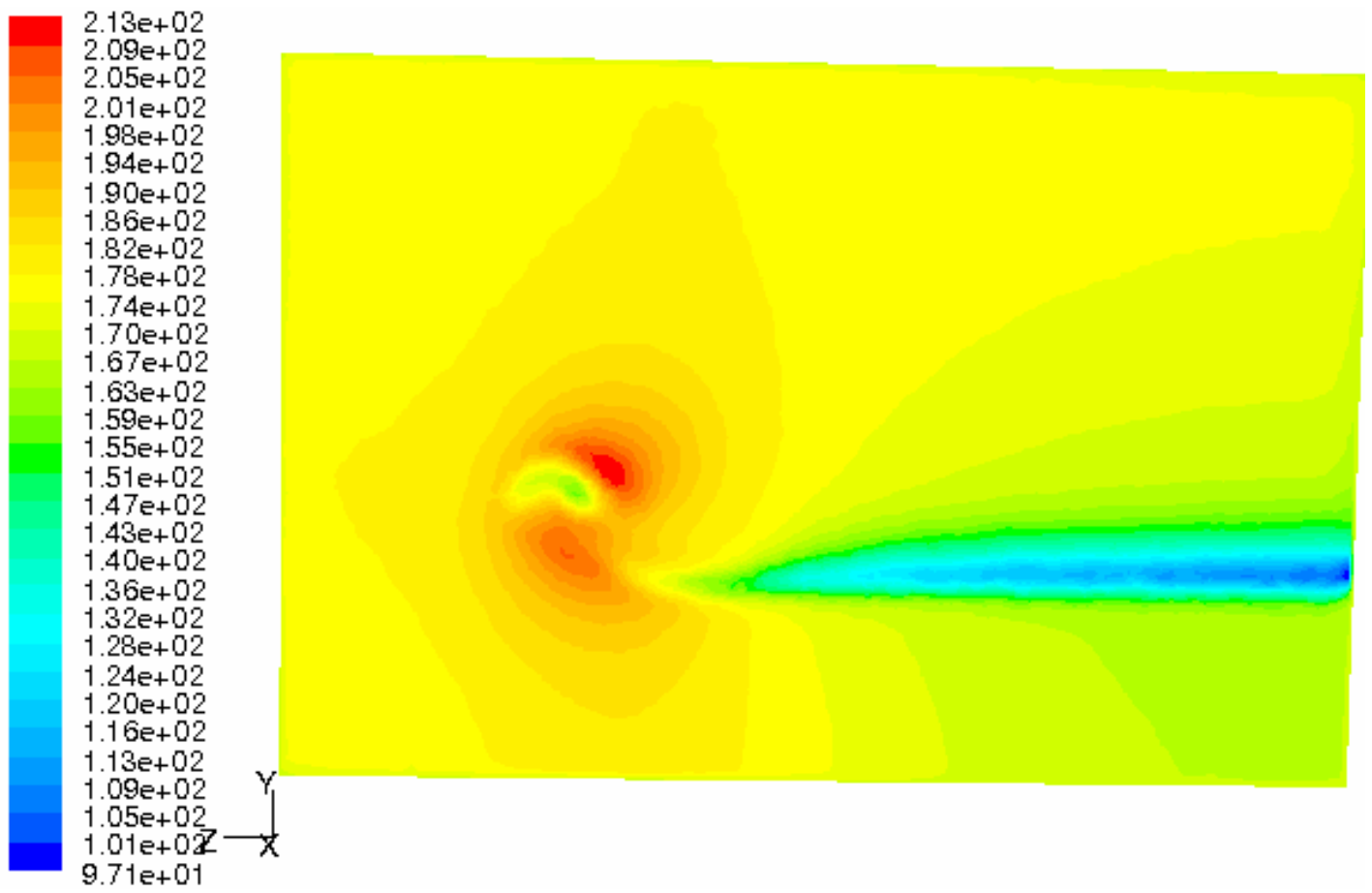

Figure 169. Velocity magnitude ( $\mathrm{ft} / \mathrm{s})$ contours at $\mathrm{x}=9 \mathrm{ft}$ for the finest mesh with suction $=0.5 \mathrm{~V}_{\infty}$ on bottom slots. 


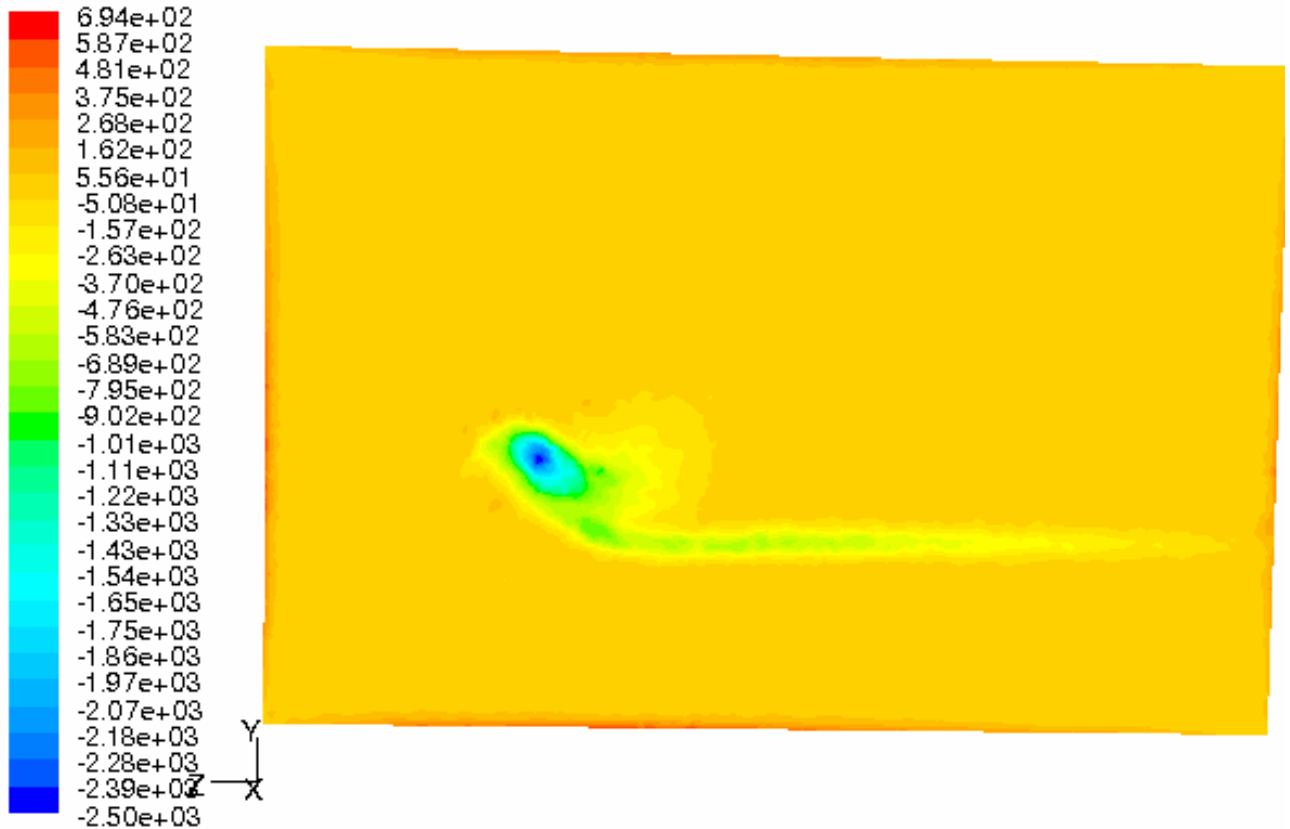

Figure 170. Vorticity (1/s) contours at $x=9 \mathrm{ft}$ for the finest mesh with suction $=0.5 \mathrm{~V}_{\infty}$ on bottom slots.

The pressure coefficient, velocity magnitude and vorticity contours in a plane at $x=9 \mathrm{ft}$. downstream when suction is applied on the bottom slots at $V_{\infty}(170 \mathrm{ft} / \mathrm{s})$ are shown in Figures 171, 172, and 173, respectively.

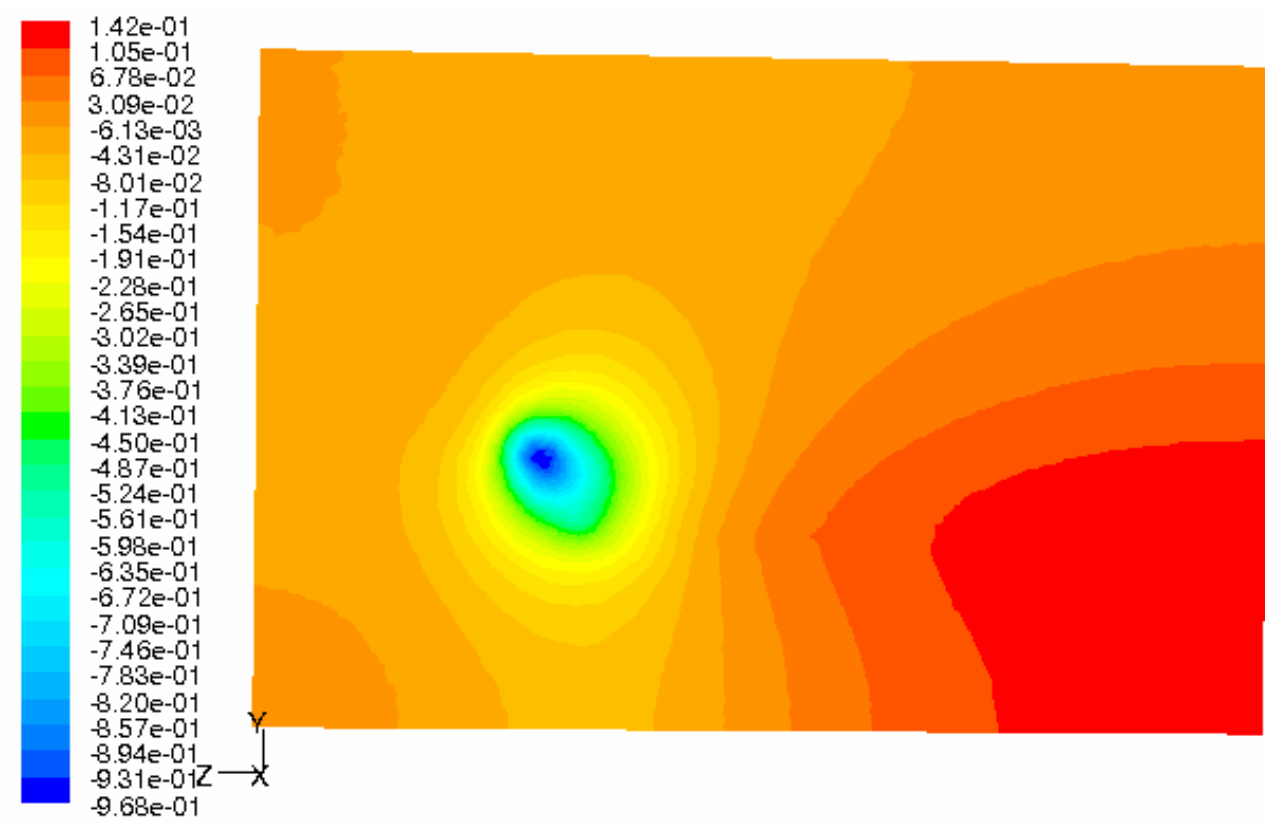

Figure 171. Pressure coefficient contours at $x=9$ ft. for the finest mesh with suction $=V_{\infty}$ on bottom slots. 


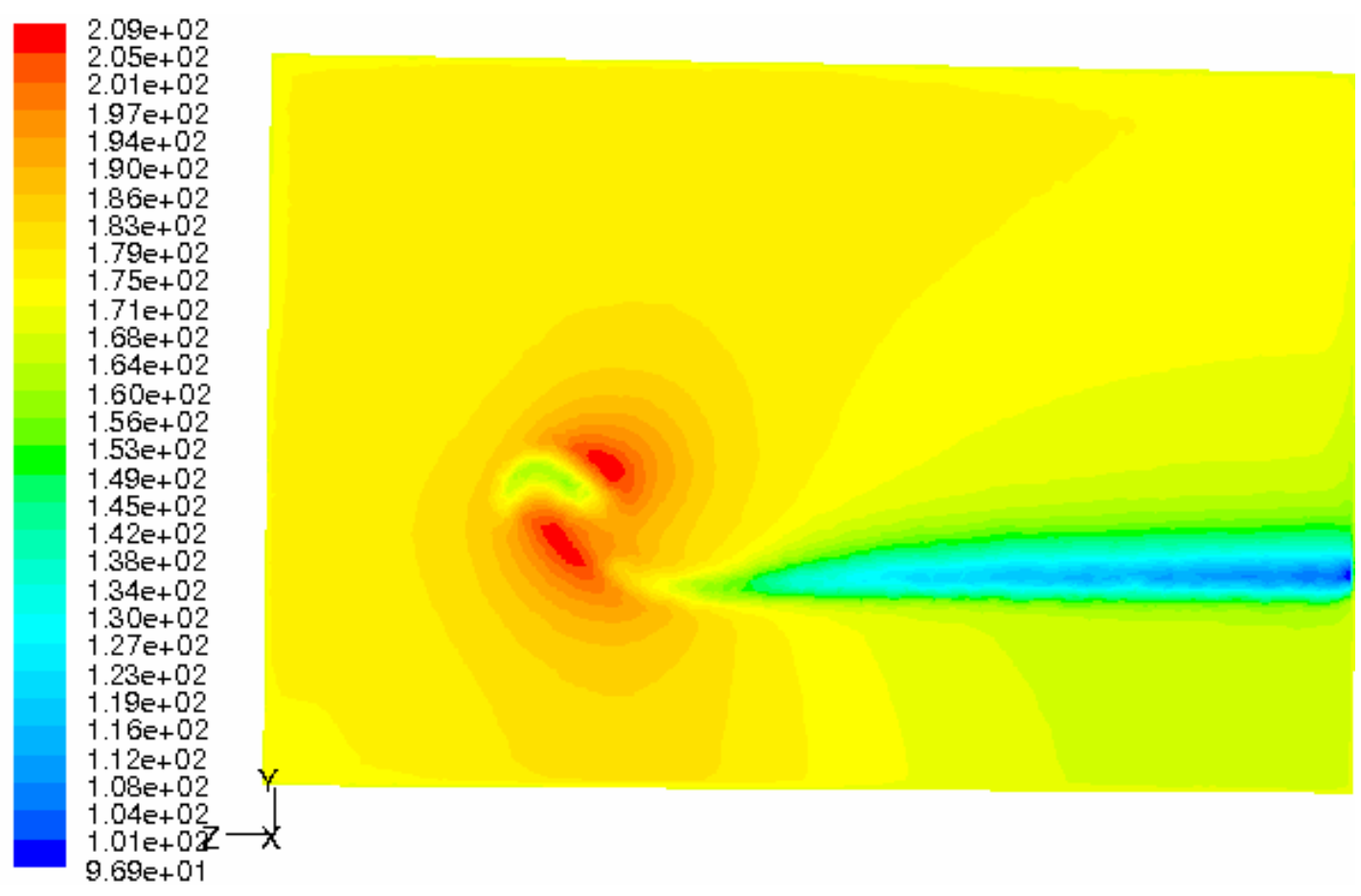

Figure 172. Velocity magnitude (ft/s) contours at $x=9 \mathrm{ft}$ for the finest mesh with suction $=\mathbf{V}_{\infty}$ on bottom slots.

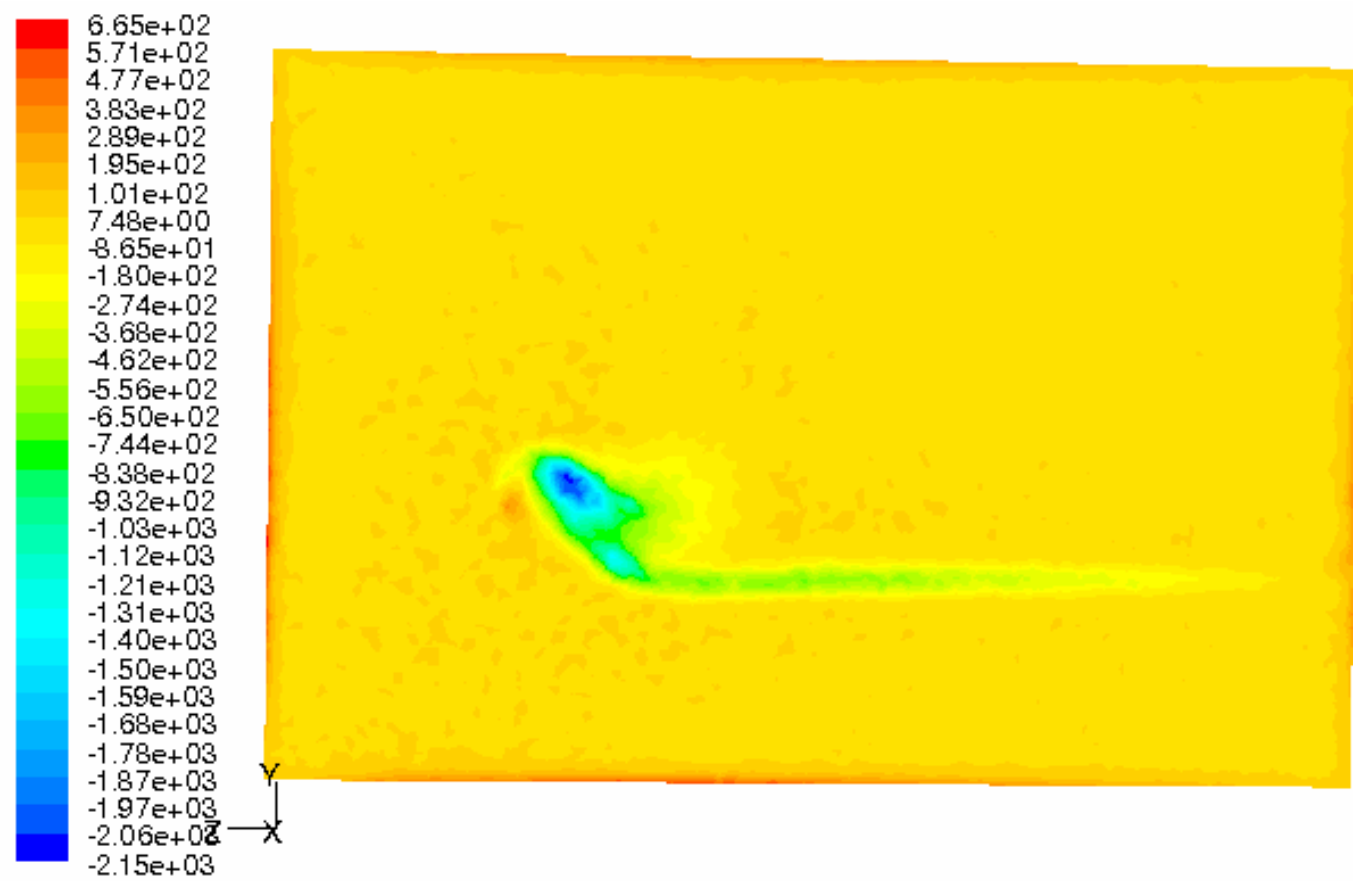

Figure 173. Vorticity (1/s) contours at $x=9 \mathrm{ft}$ for the finest mesh with suction $=V_{\infty}$ on bottom slots.

The pressure coefficient, velocity magnitude and vorticity contours in a plane at $\mathrm{x}=9 \mathrm{ft}$. downstream when suction is applied on bottom front slot at $0.5 \mathrm{~V}_{\infty}(85 \mathrm{ft} / \mathrm{s})$ are shown in Figures 174, 175, and 176, respectively. 


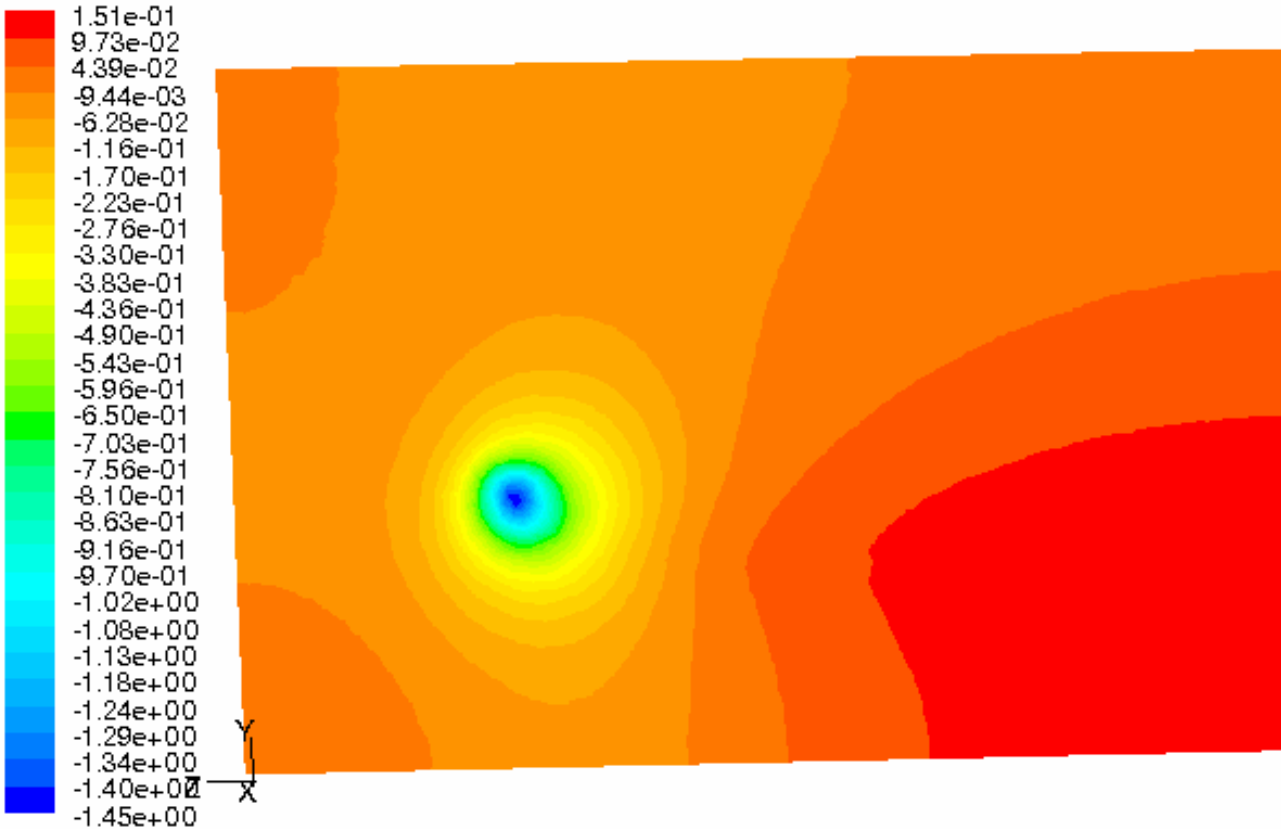

Figure 174. Pressure coefficient contours at $x=9 \mathrm{ft}$. for the finest mesh with suction $=0.5 \mathrm{~V}_{\infty}$ on bottom front slot.

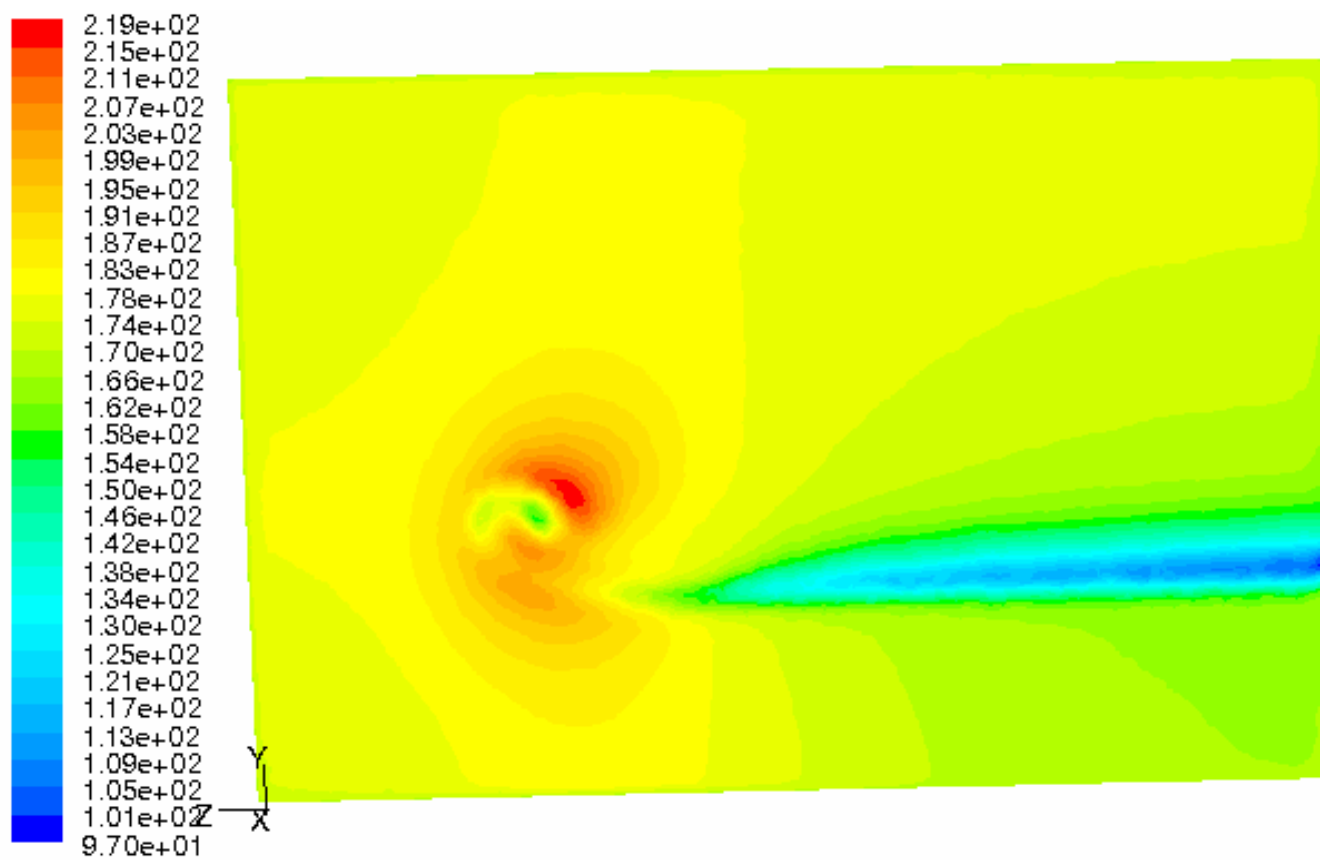

Figure 175. Velocity magnitude $(\mathrm{ft} / \mathrm{s})$ contours at $\mathrm{x}=9 \mathrm{ft}$ for the finest mesh with suction $=0.5 \mathrm{~V}_{\infty}$ on bottom front slot. 


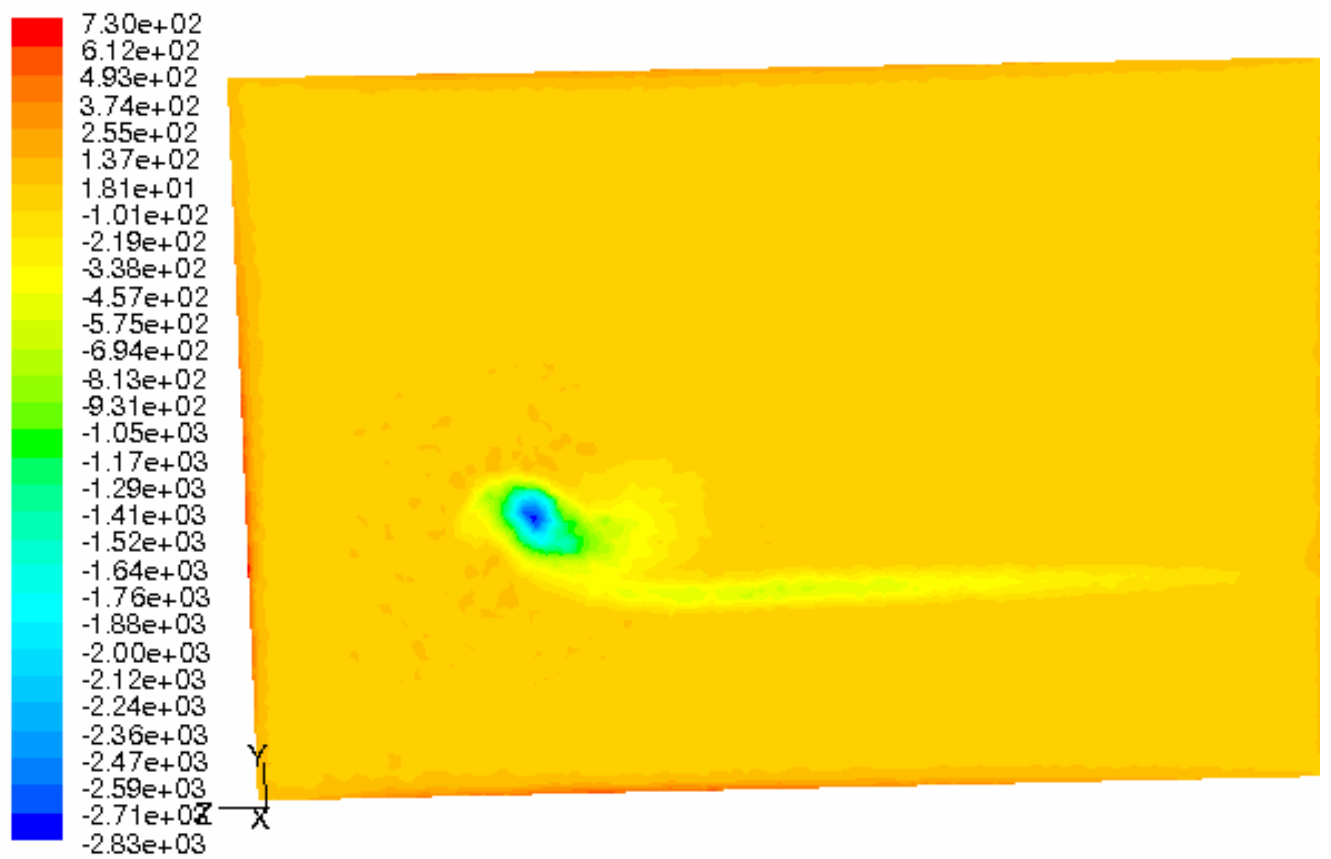

Figure 176. Vorticity $(1 / \mathrm{s})$ contours at $x=9 \mathrm{ft}$ for the finest mesh with suction $=0.5 \mathrm{~V}_{\infty}$ on bottom front slot.

The pressure coefficient, velocity magnitude and vorticity contours in a plane at $\mathrm{x}=9 \mathrm{ft}$. downstream when suction is applied on bottom front slot at $\mathrm{V}_{\infty}(170 \mathrm{ft} / \mathrm{s})$ are shown in Figures 177, 178, and 179, respectively.

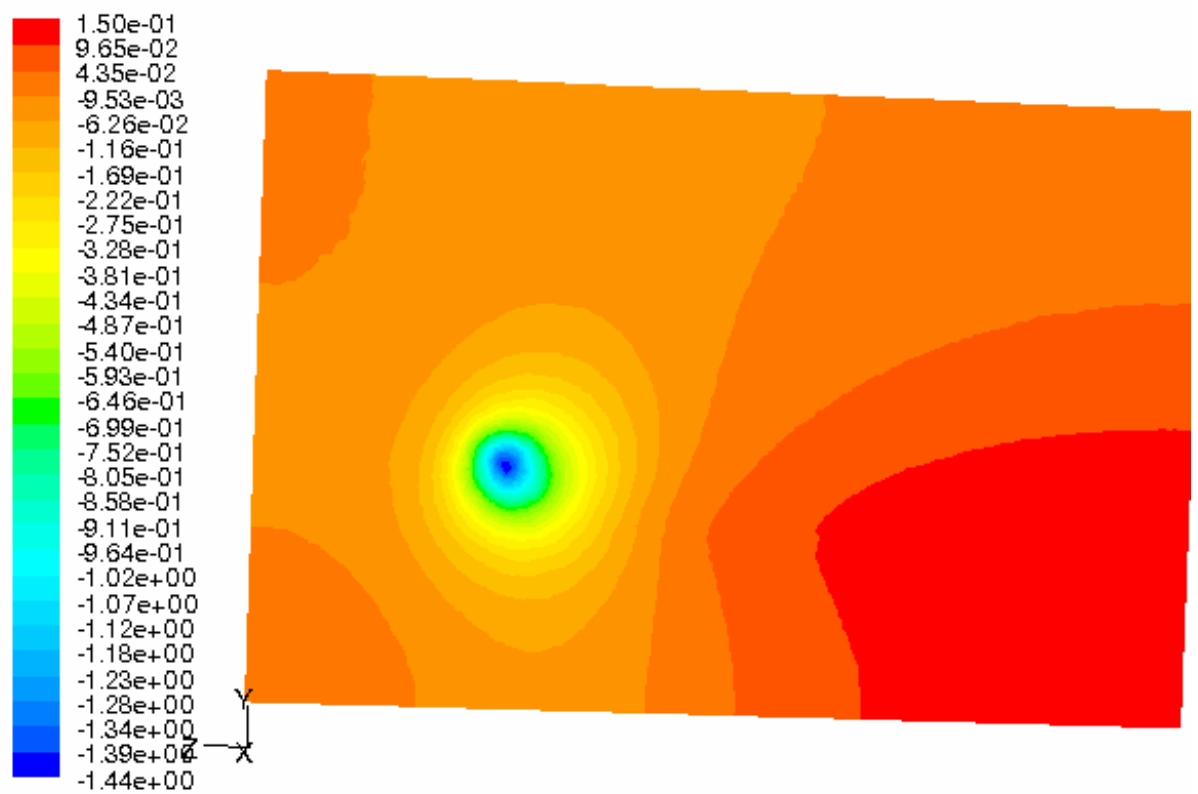

Figure 177. Pressure coefficient contours at $x=9$ ft. for the finest mesh with suction $=V_{\infty}$ on bottom front slot. 


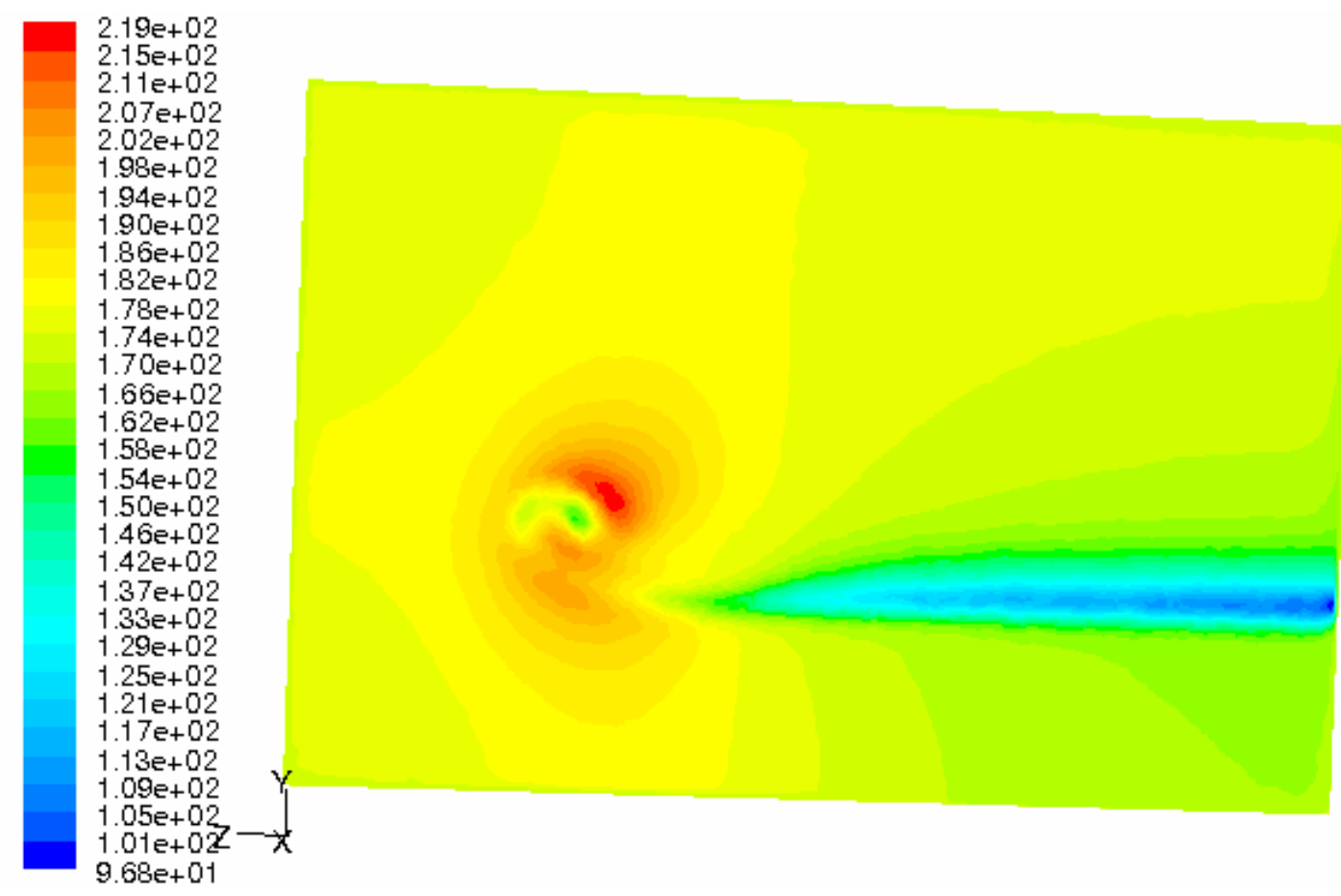

Figure 178. Velocity magnitude (ft/s) contours at $x=9 \mathrm{ft}$ for the finest mesh with suction $=\mathrm{V}_{\infty}$ on bottom front slot.
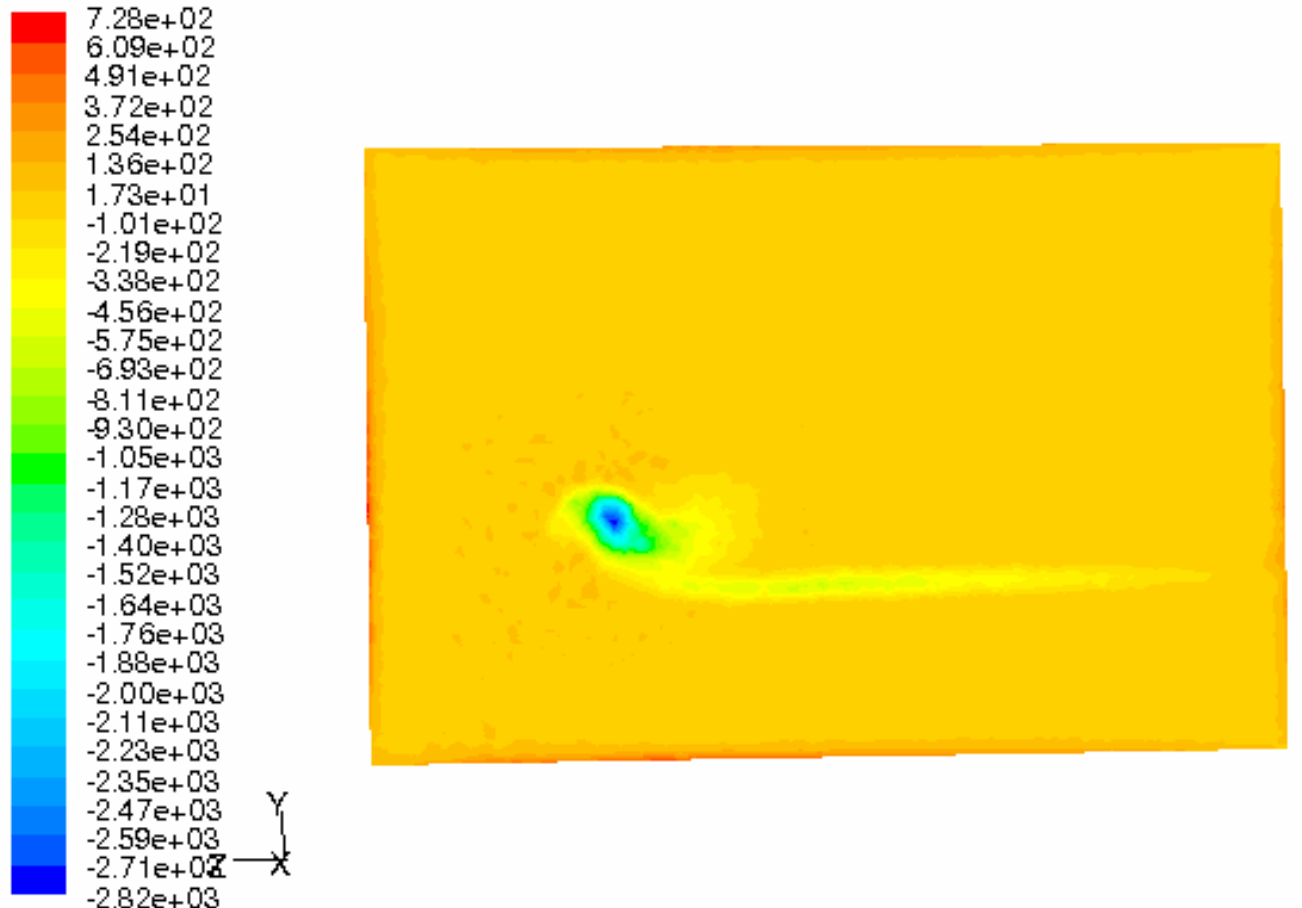

Figure 179. Vorticity (1/s) contours at $x=9 \mathrm{ft}$ for the finest mesh with suction= $V_{\infty}$ on bottom front slot. 
The pressure coefficient, velocity magnitude and vorticity contours in a plane at $\mathrm{x}=9 \mathrm{ft}$. downstream when suction is applied on bottom rear slot at $0.5 \mathrm{~V}_{\infty}(85 \mathrm{ft} / \mathrm{s})$ are shown in Figures 180, 181, and 182, respectively.

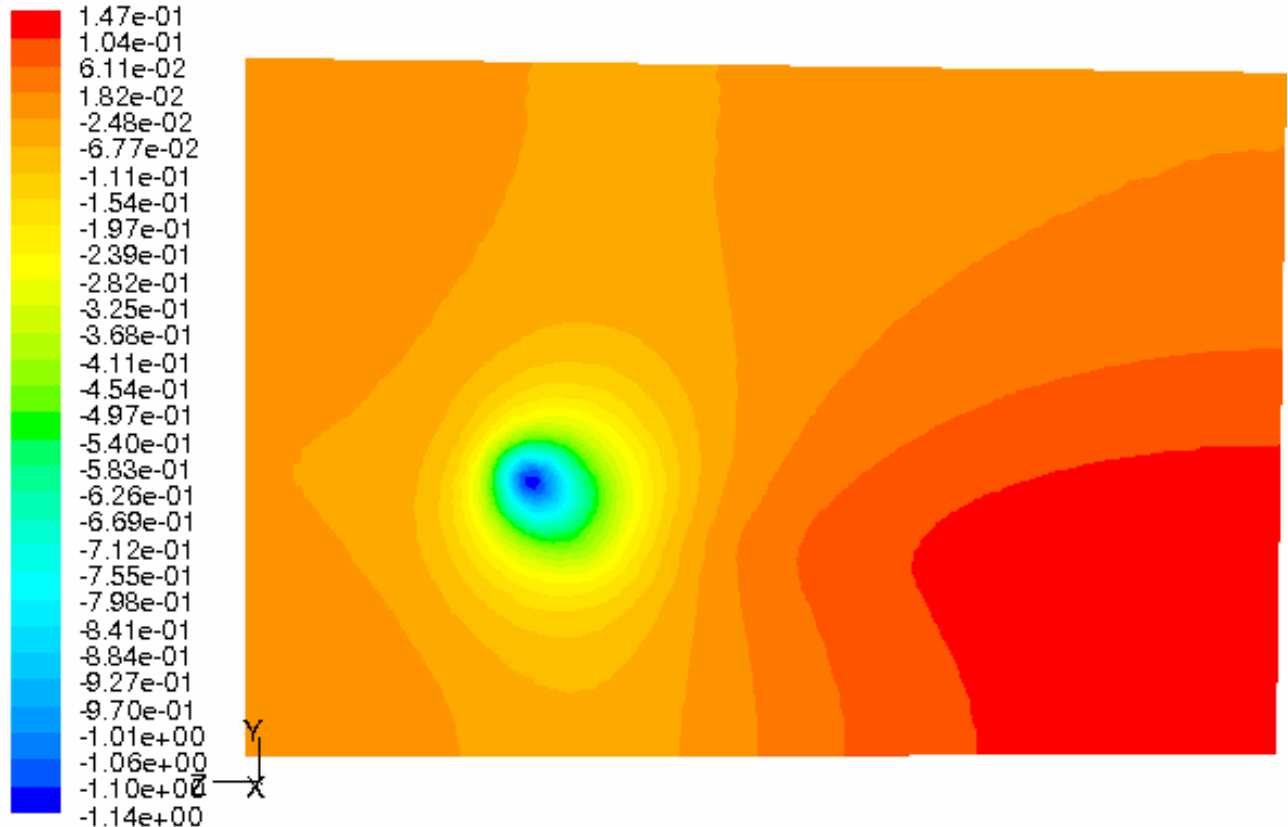

Figure 180. Pressure coefficient contours at $x=9 \mathrm{ft}$. for the finest mesh with suction $=0.5 \mathrm{~V}_{\infty}$ on bottom rear slot.

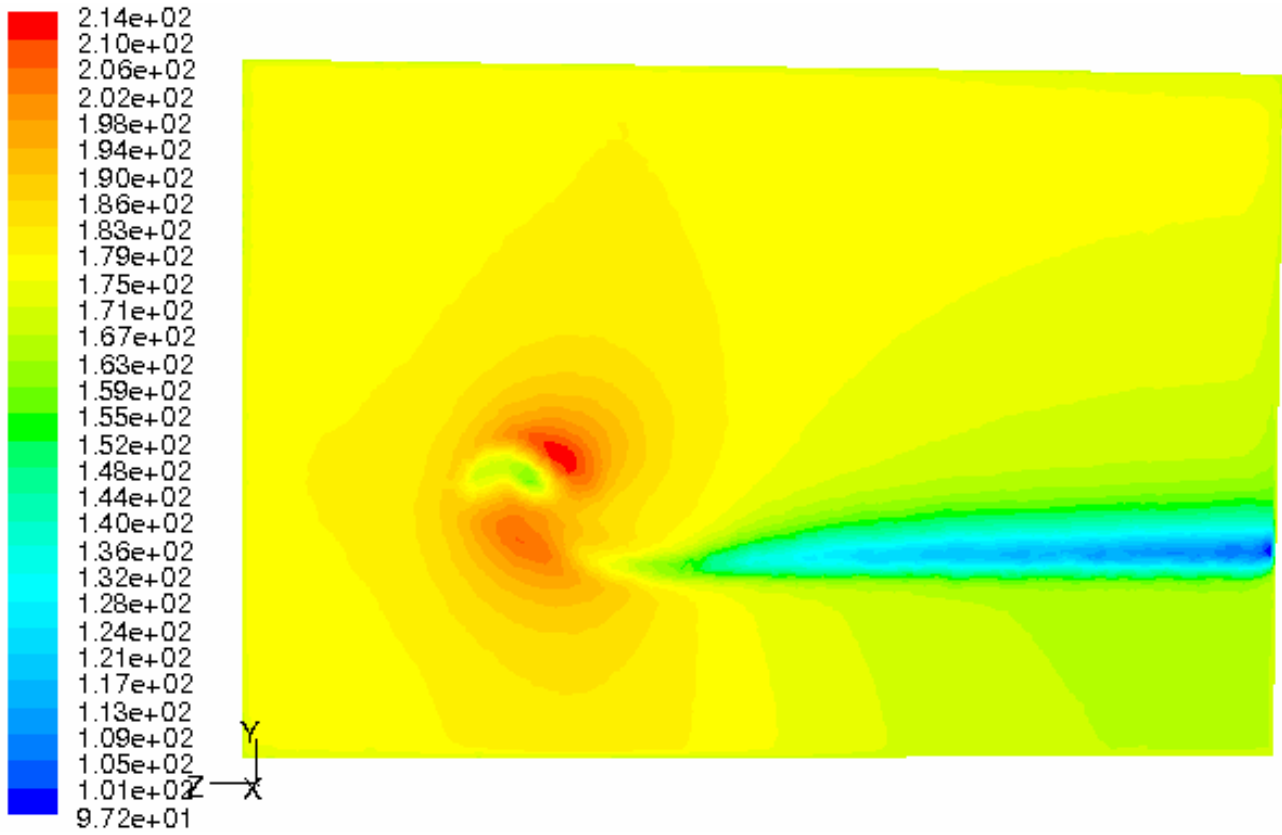

Figure 181. Velocity magnitude ( $\mathrm{ft} / \mathrm{s}$ ) contours at $\mathrm{x}=9 \mathrm{ft}$ for the finest mesh with suction $=0.5 \mathrm{~V}_{\infty}$ on bottom rear slot. 


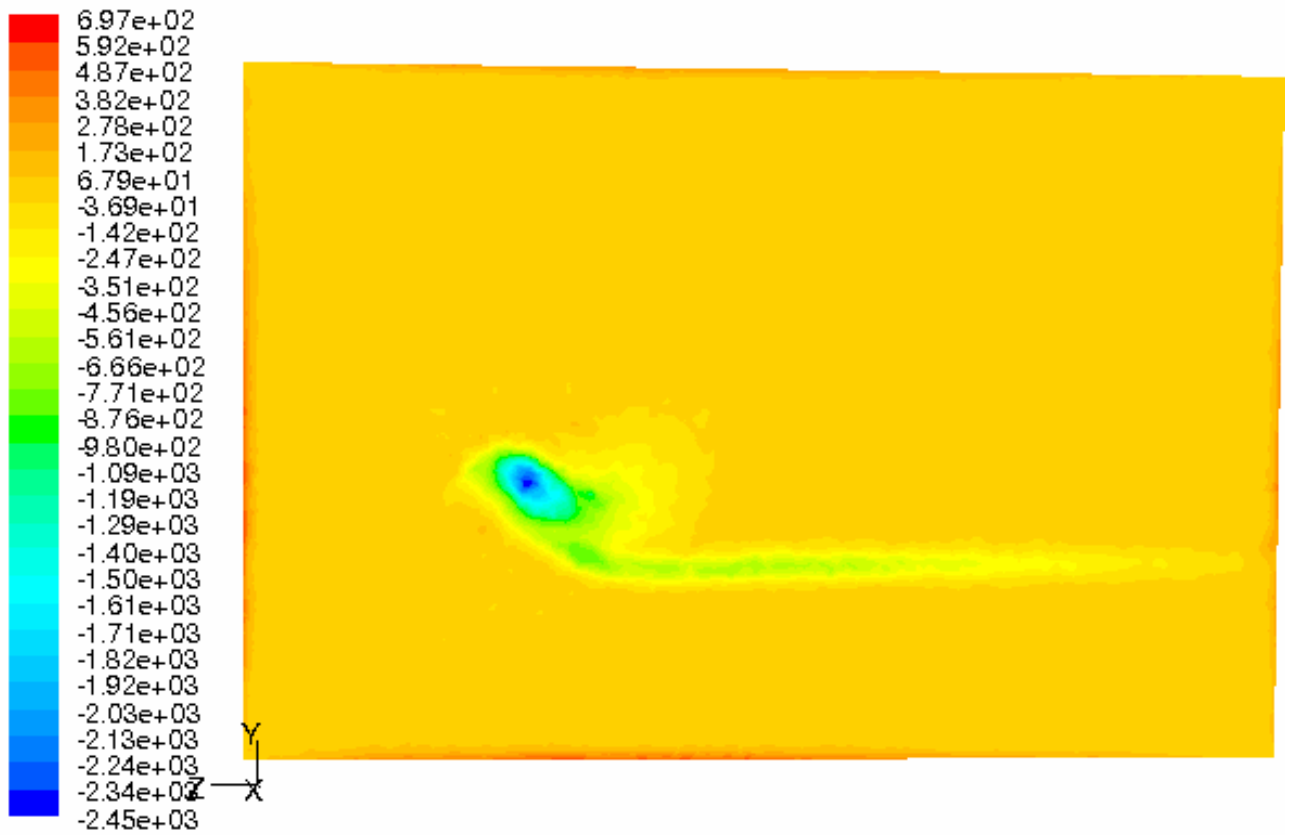

Figure 182. Vorticity (1/s) contours at $x=9 \mathrm{ft}$ for the finest mesh with suction $=0.5 \mathrm{~V}_{\infty}$ on bottom rear slot.

The pressure coefficient, velocity magnitude and vorticity contours in a plane at $\mathrm{x}=9 \mathrm{ft}$. downstream when suction is applied on bottom rear slot at $\mathrm{V}_{\infty}(170 \mathrm{ft} / \mathrm{s})$ are shown in Figures 183, 184, and 185, respectively.

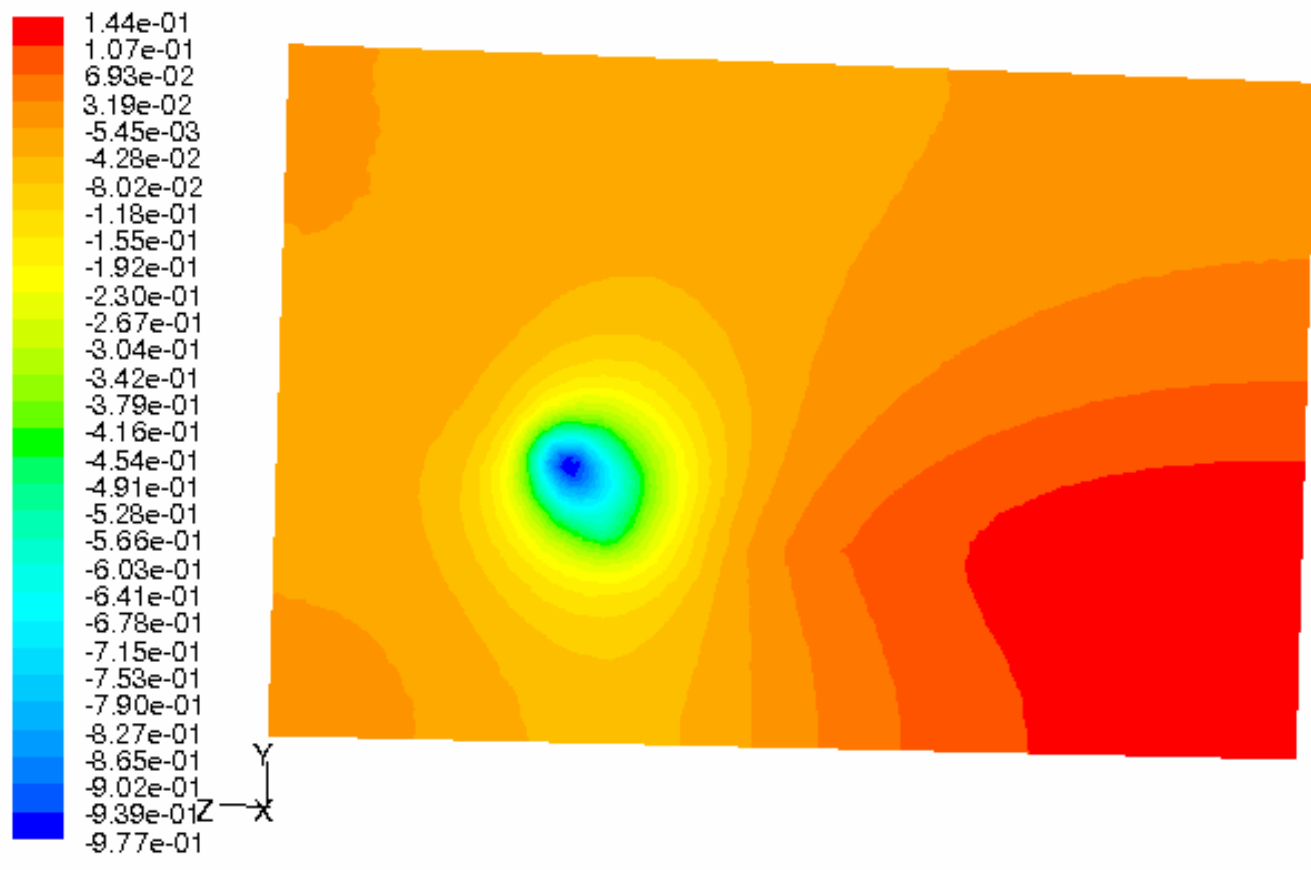

Figure 183. Pressure coefficient contours at $x=9$ ft. for the finest mesh with suction $=V_{\infty}$ on bottom rear slot. 


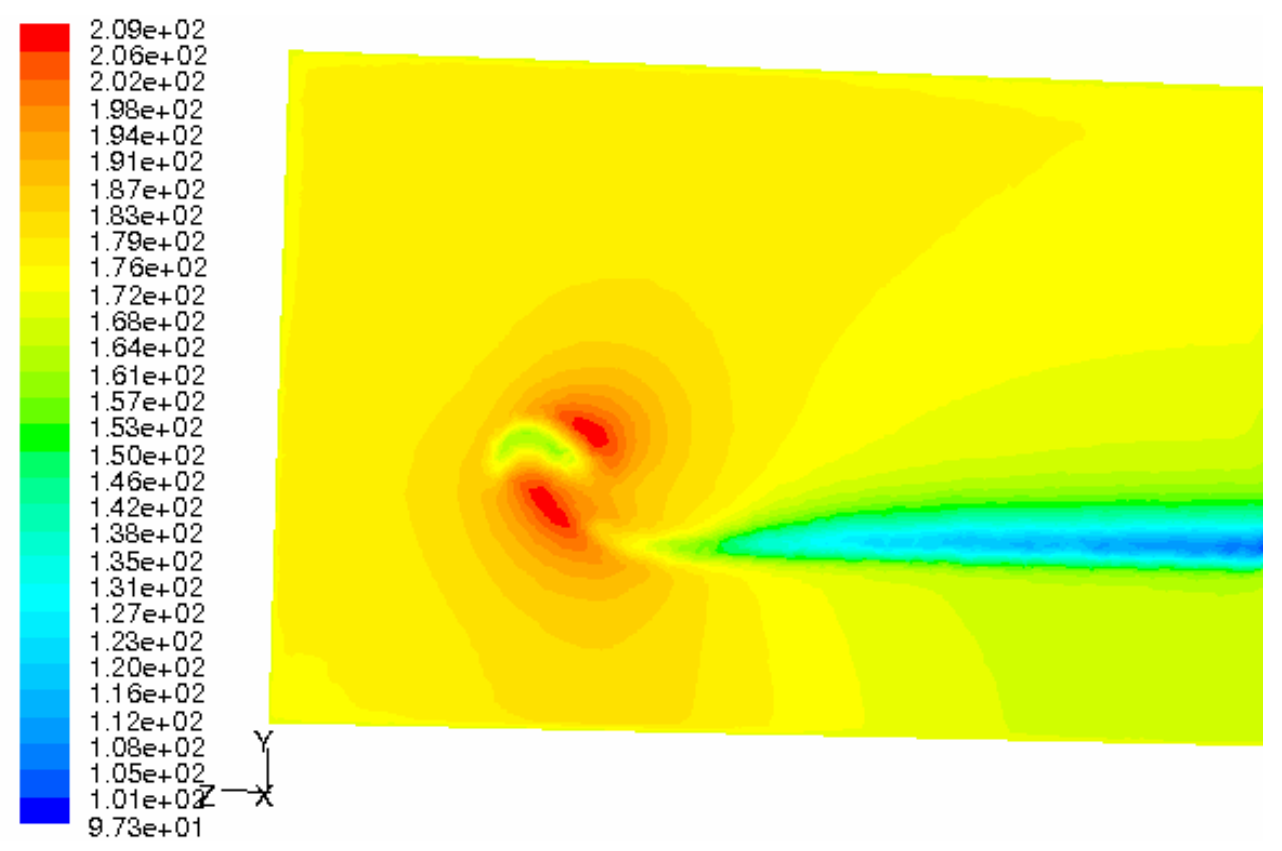

Figure 184. Velocity magnitude (ft/s) contours at $x=9$ ft for the finest mesh with suction $=V_{\infty}$ on bottom rear slot.

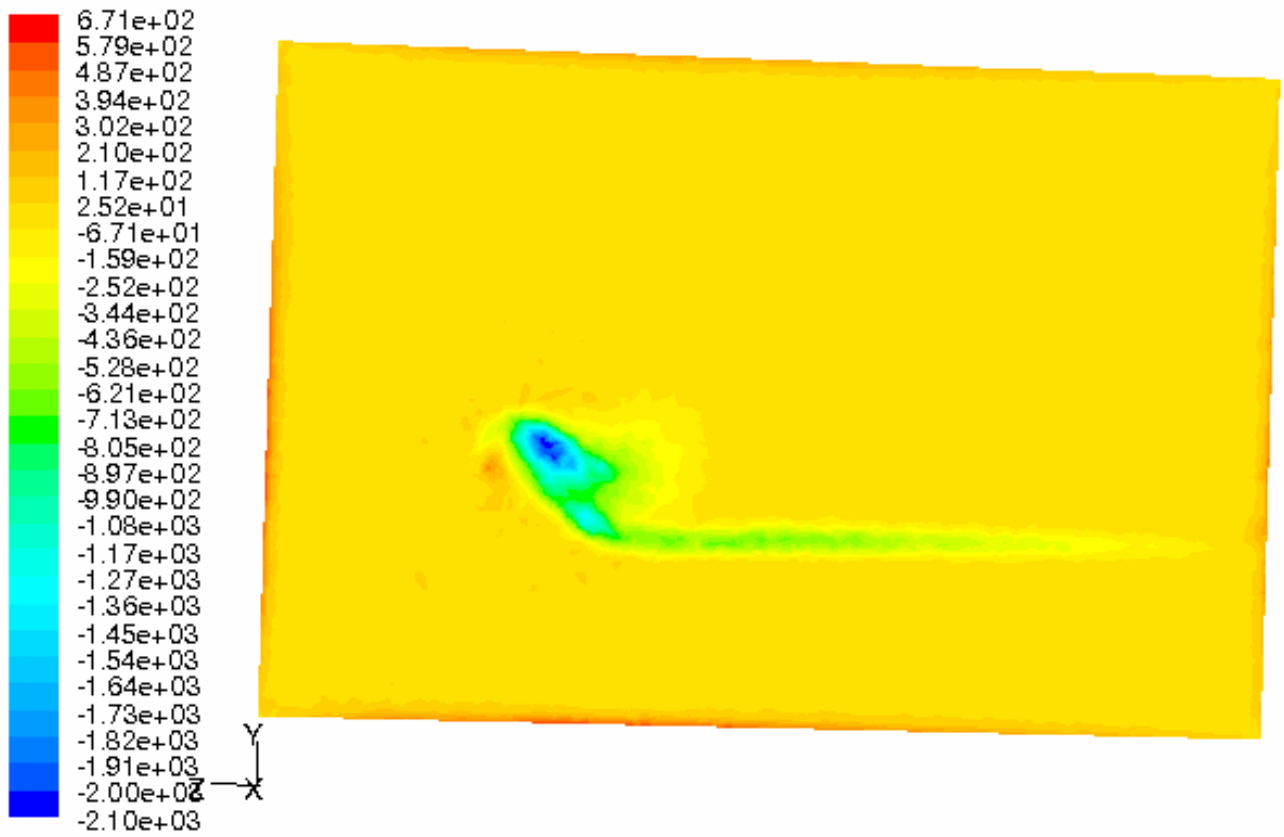

Figure 185. Vorticity (1/s) contours at $x=9$ ft for the finest mesh with suction $=V_{\infty}$ on bottom rear slot.

Drag coefficient and vorticity values for the previous configurations are shown in Table 17. Applying suction on the top slot is more effective than on bottom slots. It requires more suction velocity to remove the bottom boundary layer and straighten the bottom streamlines than attaching the boundary layer to the top wing surface. 
Table 17. Effectiveness Study Results of drag coefficient and vorticity.

\begin{tabular}{|l|r|c|c|r|r|r|r|}
\hline $\begin{array}{c}\text { BLC } \\
\text { suction slot }\end{array}$ & $\begin{array}{c}\text { BLC Suction } \\
\text { Velocity (ft/s) }\end{array}$ & $\mathbf{C}_{\mathrm{L}}$ & $\mathbf{C}_{\mathbf{D}}$ & $\begin{array}{l}\text { Vorticity } \\
\left.\mathbf{( f t}^{2} / \mathbf{s}\right)\end{array}$ & L/D & $\begin{array}{c}\text { CD Decrease } \\
\mathbf{( \% )}\end{array}$ & $\begin{array}{c}\text { Vorticity } \\
\text { Decrease (\%) }\end{array}$ \\
\hline Finest & & 0.7246 & 0.0721 & -217.05 & 10.05 & & \\
\hline Top & 85 & 0.7023 & 0.0695 & -211.00 & 10.11 & 3.74 & 2.79 \\
\hline Top & 170 & 0.6993 & 0.0701 & -209.43 & 9.98 & 2.85 & 3.51 \\
\hline Bottom & 85 & 0.7043 & 0.0690 & -214.29 & 10.21 & 4.49 & 1.27 \\
\hline Bottom & 170 & 0.6872 & 0.0664 & -211.32 & 10.35 & 8.58 & 2.64 \\
\hline Bottom front & 85 & 0.7206 & 0.0713 & -216.08 & 10.11 & 1.12 & 0.45 \\
\hline Bottom front & 170 & 0.7164 & 0.0703 & -215.34 & 10.19 & 2.56 & 0.79 \\
\hline Bottom rear & 85 & 0.7084 & 0.0699 & -215.22 & 10.13 & 3.15 & 0.84 \\
\hline Bottom rear & 170 & 0.6953 & 0.0682 & -212.56 & 10.20 & 5.72 & 2.07 \\
\hline
\end{tabular}

\section{Top BLC Suction Velocity Study}

Now that the effectiveness of the top suction slot has been verified against bottom suction slots, we know evaluate the effect of the suction velocity on the wingtip vorticity. Suction velocities ranging from 0 to $170 \mathrm{ft} / \mathrm{s}$ are applied to the top slot in increments of $21 \mathrm{ft} / \mathrm{s}$.

The pressure coefficient, velocity magnitude and vorticity contours in a plane at $\mathrm{x}=9 \mathrm{ft}$. downstream when suction is not applied on any slot have been shown in Figures 150,151 , and 152 , respectively.

The pressure coefficient, velocity magnitude and vorticity contours in a plane at $\mathrm{x}=9 \mathrm{ft}$. downstream when suction is applied on top slot at $21 \mathrm{ft} / \mathrm{s}$ are shown in Figures 186,187 , and 188 , respectively. 


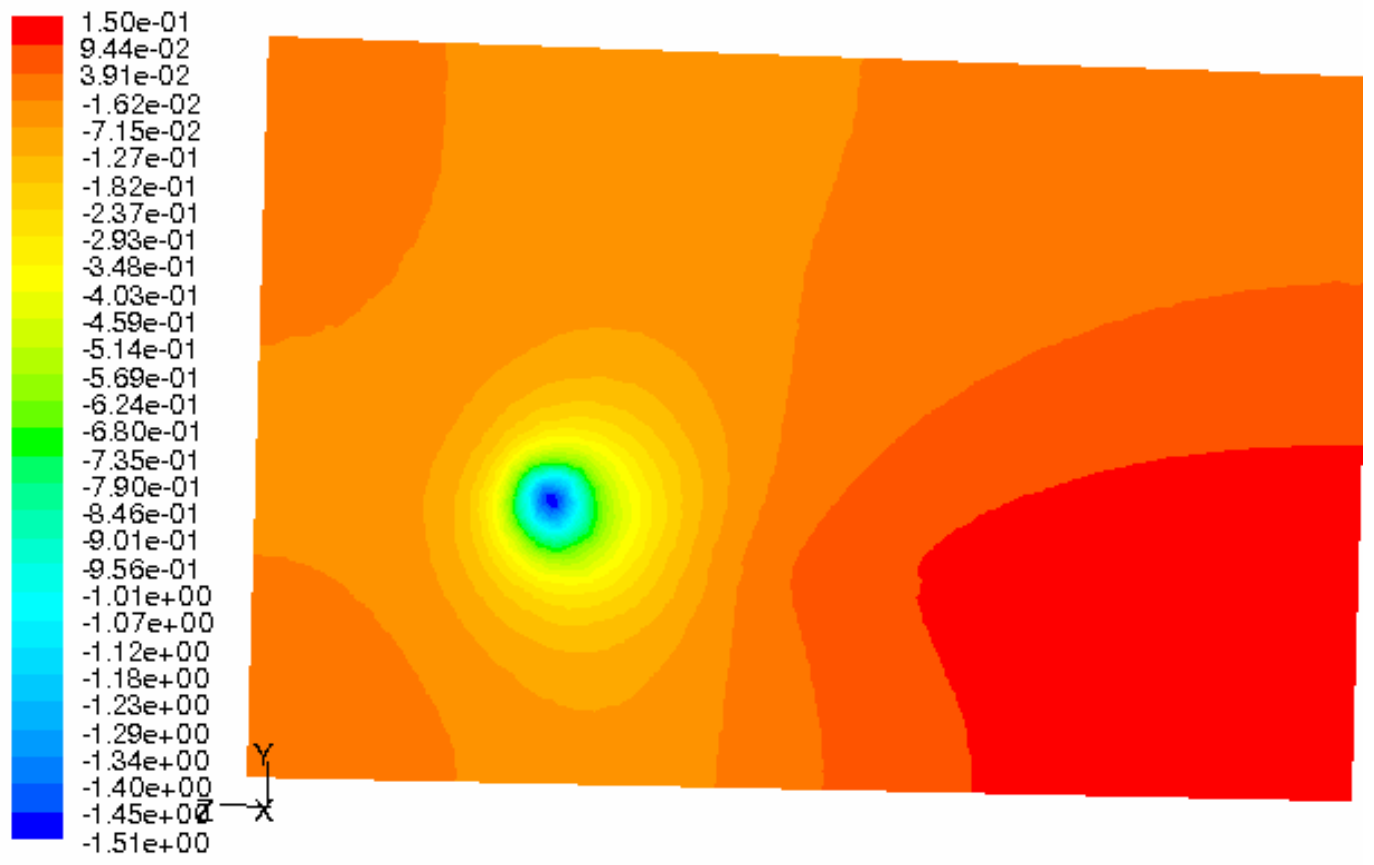

Figure 186. Pressure coefficient contours at $\mathrm{x}=9 \mathrm{ft}$. for the finest mesh with suction=21 ft/s on top slot.

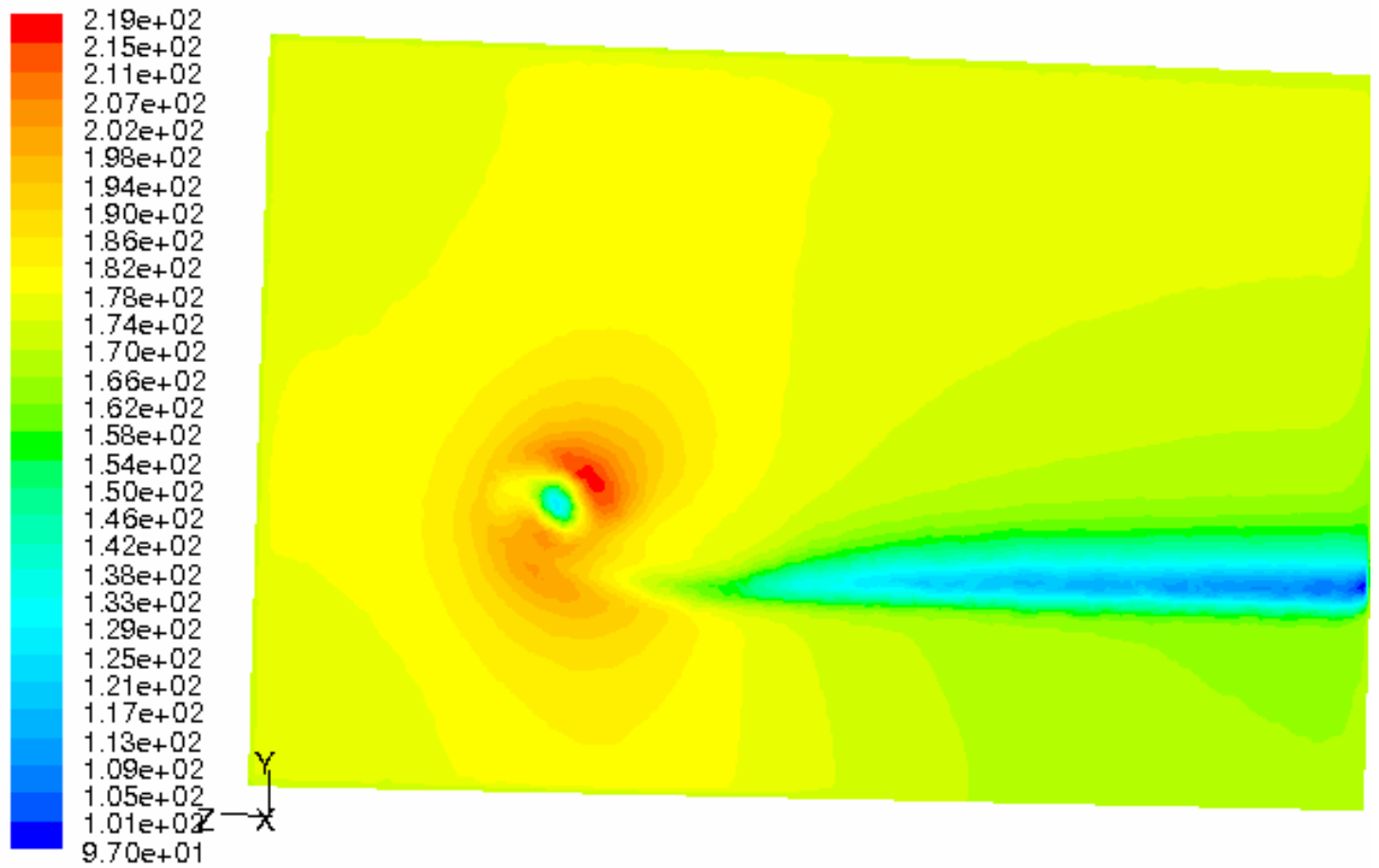

Figure 187. Velocity magnitude (ft/s) contours at $x=9 \mathrm{ft}$ for the finest mesh with suction=21 ft/s on top slot. 


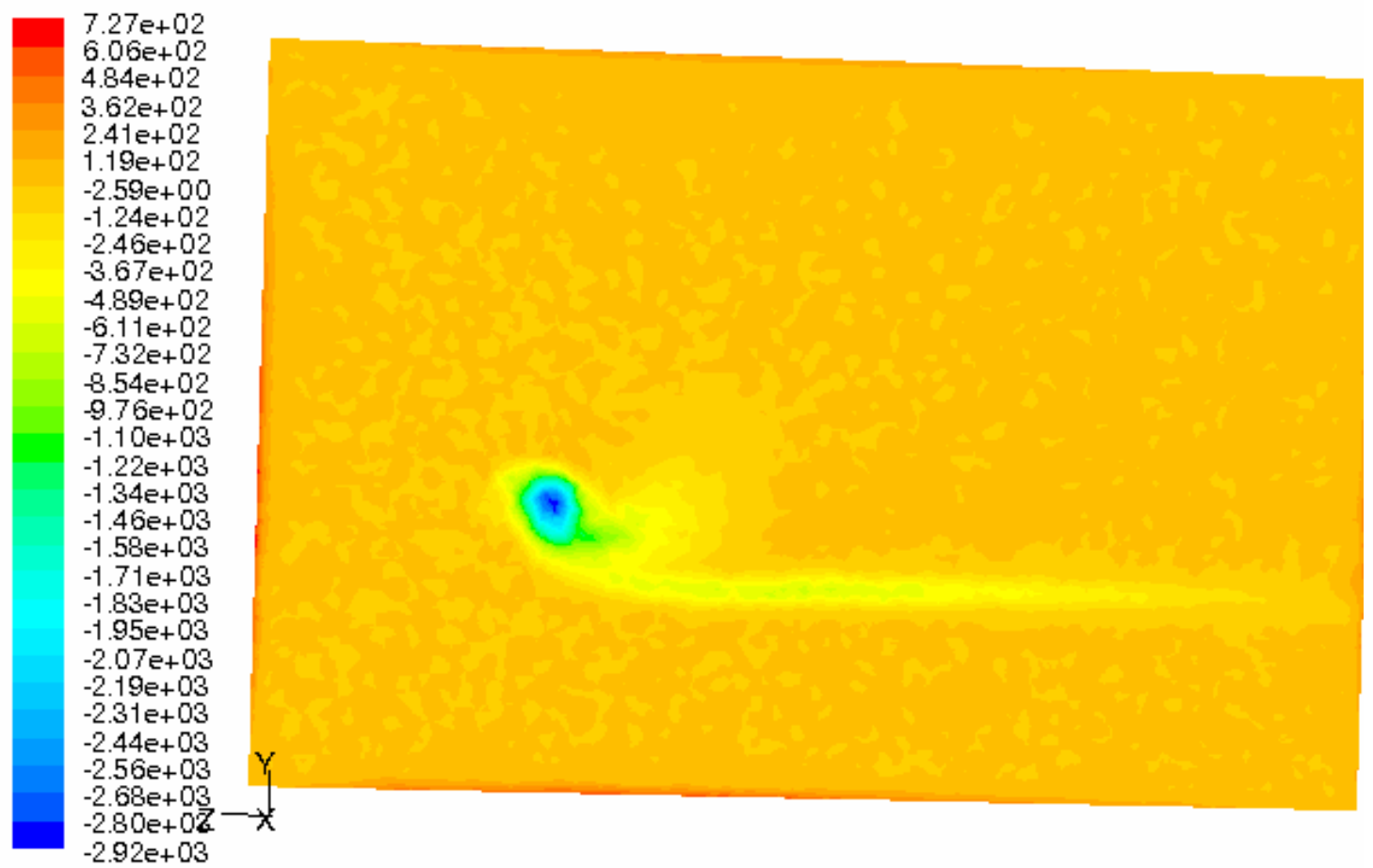

Figure 188. Vorticity (1/s) contours at $x=9 \mathrm{ft}$ for the finest mesh with suction=21 $\mathrm{ft} / \mathrm{s}$ on top slot.

The pressure coefficient, velocity magnitude and vorticity contours in a plane at $\mathrm{x}=9 \mathrm{ft}$. downstream when suction is applied on top slot at $42 \mathrm{ft} / \mathrm{s}$ are shown in Figures 189 , 190, and 191, respectively.

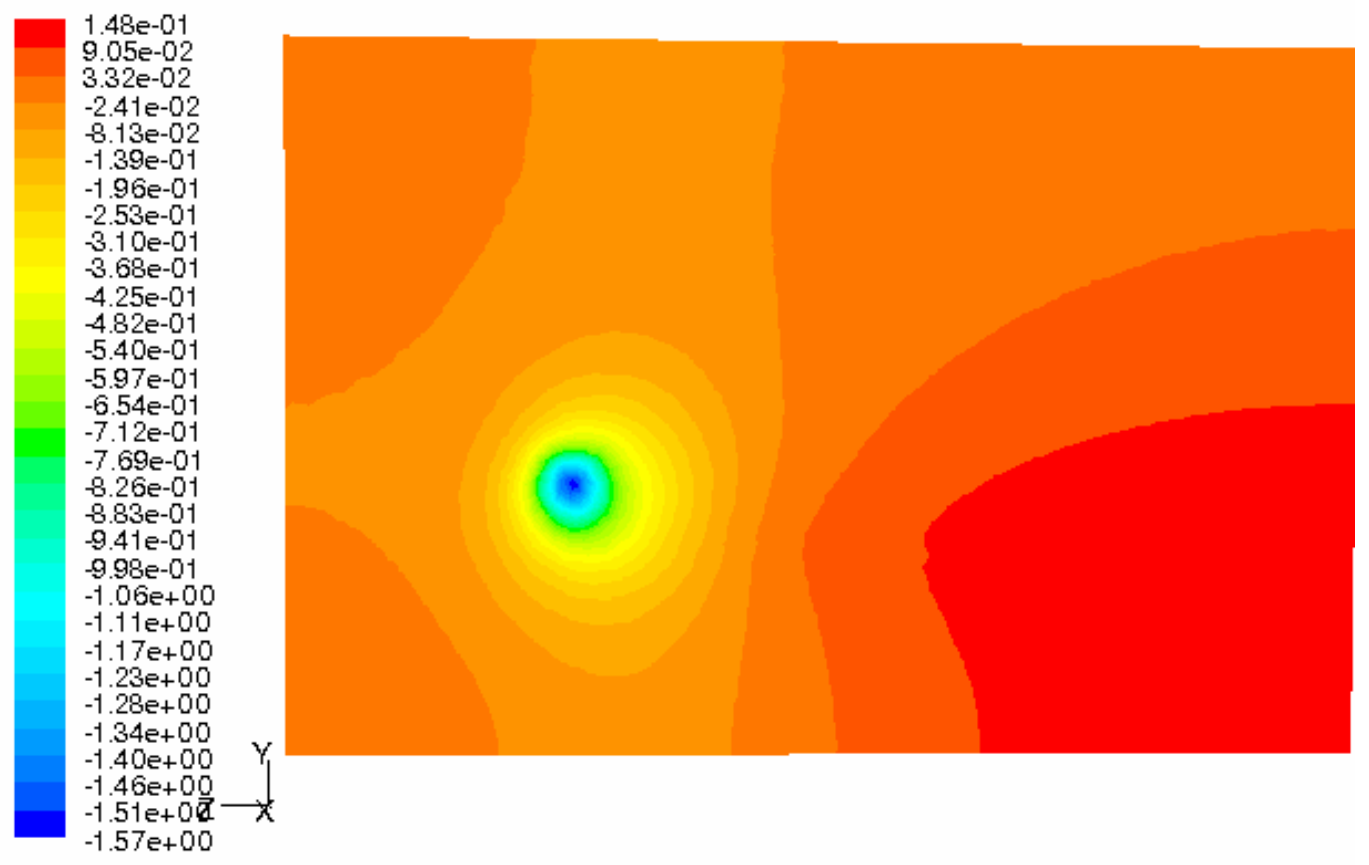

Figure 189. Pressure coefficient contours at $x=9 \mathrm{ft}$. for the finest mesh with suction=42 ft/s on top slot. 


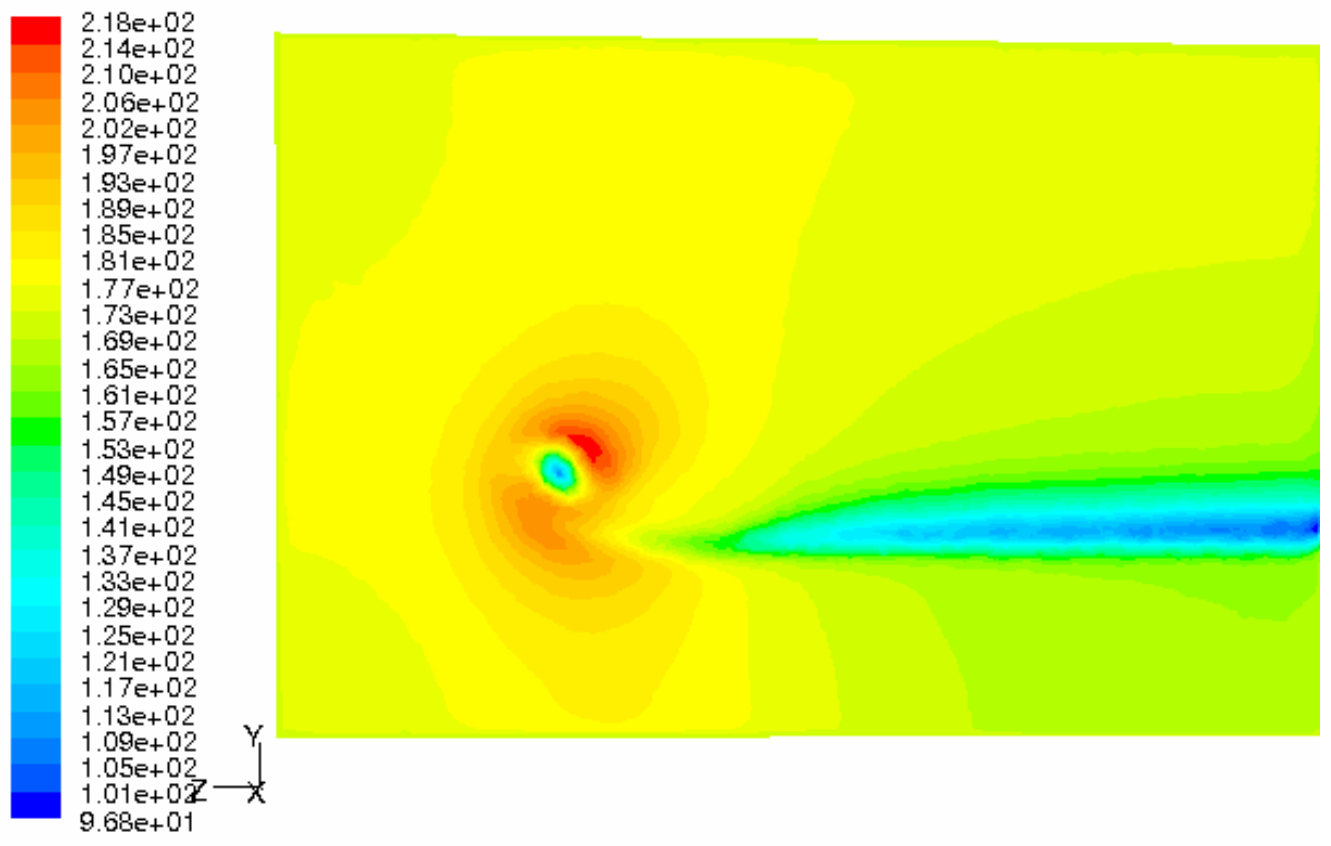

Figure 190. Velocity magnitude ( $\mathrm{ft} / \mathrm{s}$ ) contours at $\mathrm{x}=9 \mathrm{ft}$ for the finest mesh with suction $=42 \mathrm{ft} / \mathrm{s}$ on top slot.

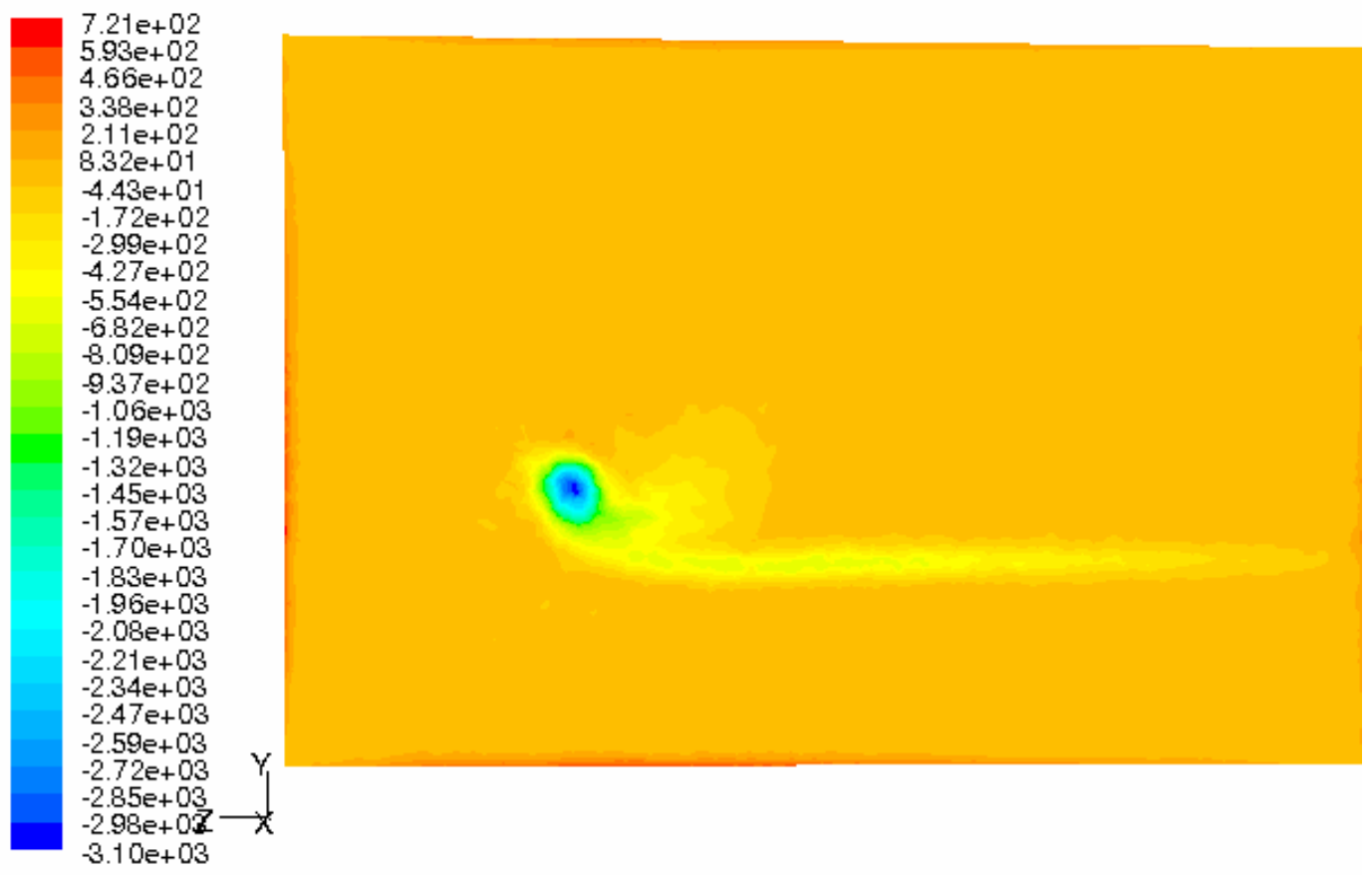

Figure 191. Vorticity (1/s) contours at $x=9 \mathrm{ft}$ for the finest mesh with suction=42 ft/s on top slot.

The pressure coefficient, velocity magnitude and vorticity contours in a plane at $\mathrm{x}=9 \mathrm{ft}$. downstream when suction is applied on top slot at $63 \mathrm{ft} / \mathrm{s}$ are shown in Figures 192, 193, and 194, respectively. 


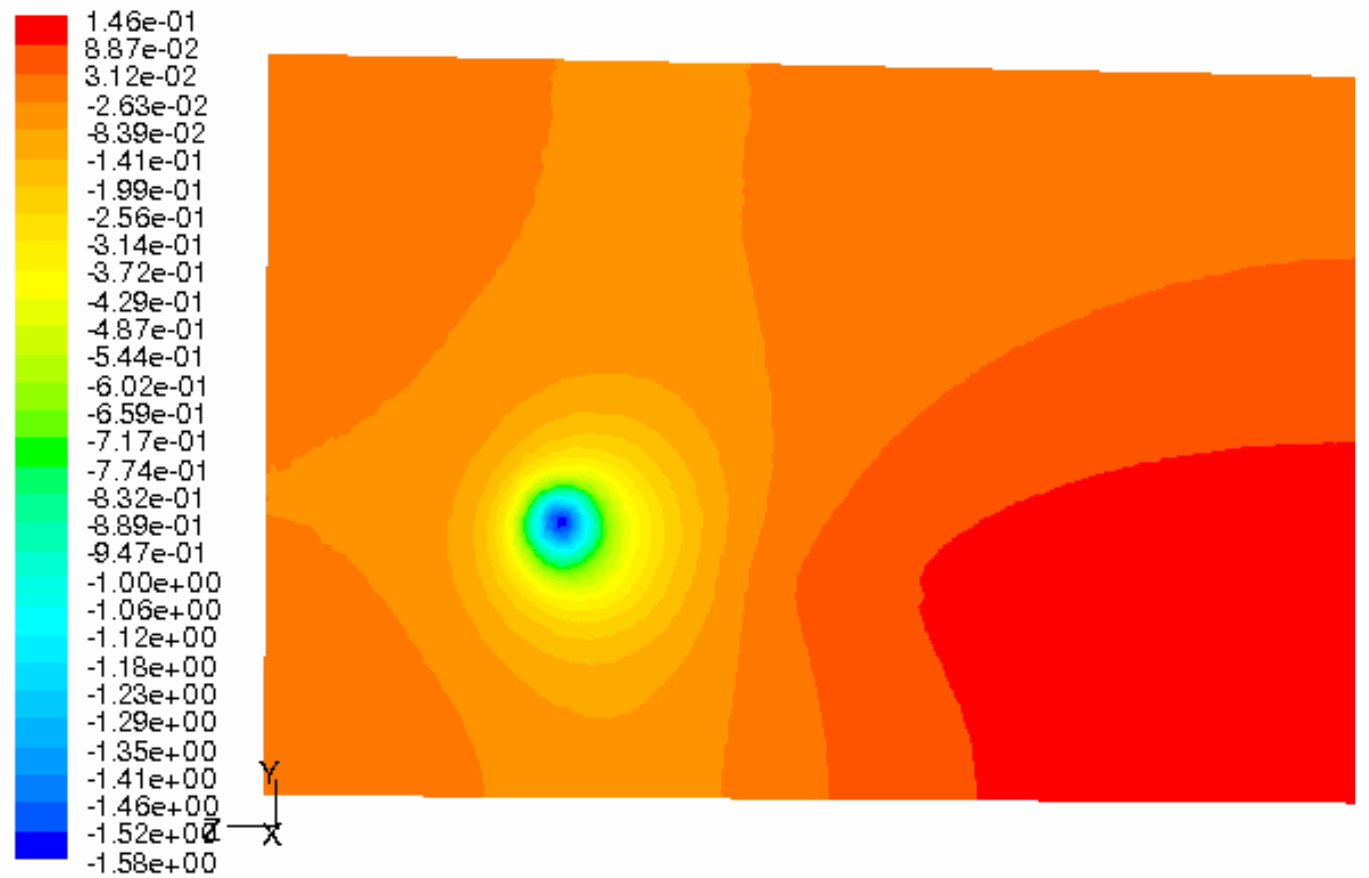

Figure 192. Pressure coefficient contours at $x=9 \mathrm{ft}$. for the finest mesh with suction=63 ft/s on top slot.

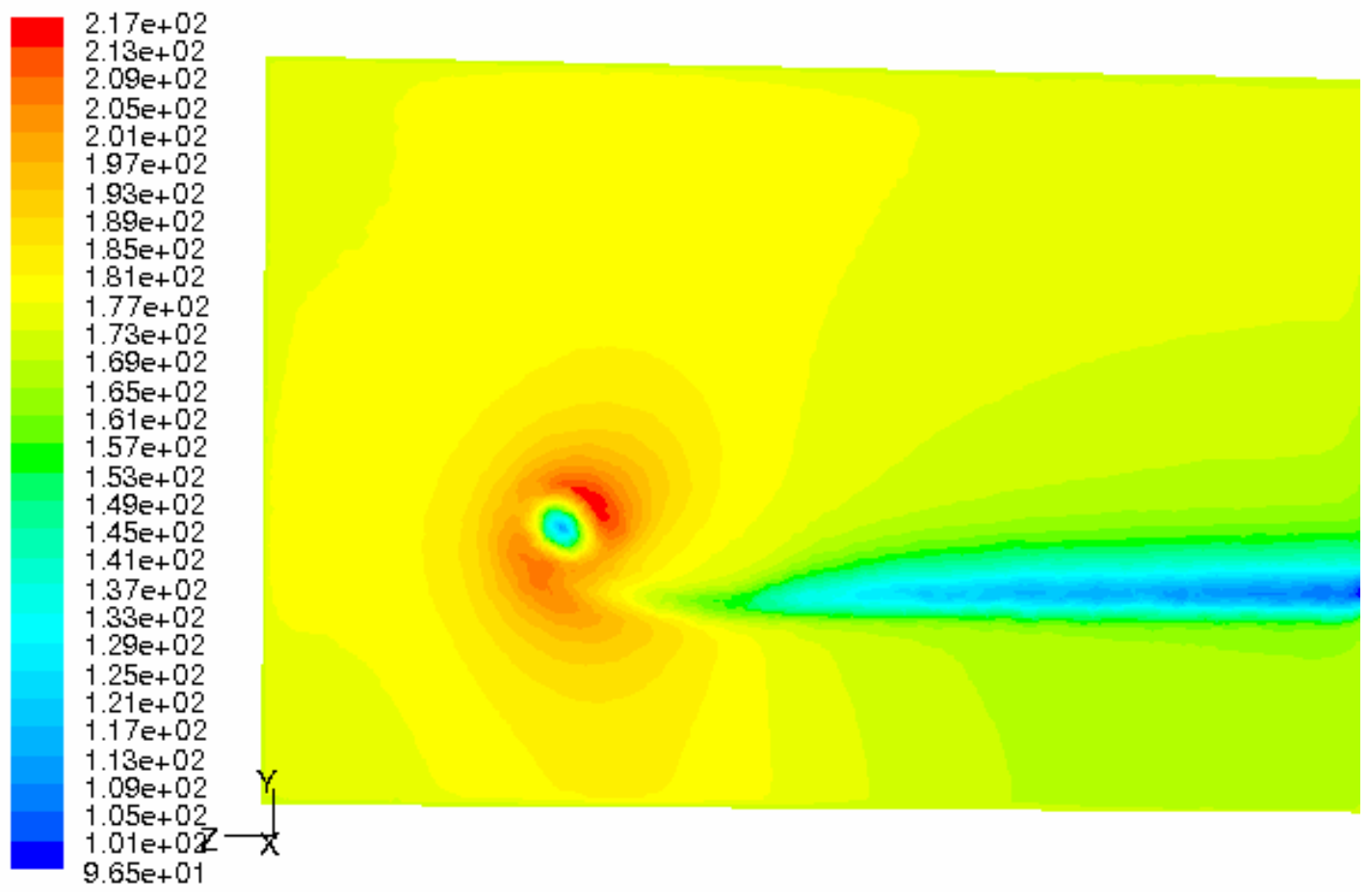

Figure 193. Velocity magnitude ( $\mathrm{ft} / \mathrm{s}$ ) contours at $\mathrm{x}=9 \mathrm{ft}$ for the finest mesh with suction $=63 \mathrm{ft} / \mathrm{s}$ on top slot. 


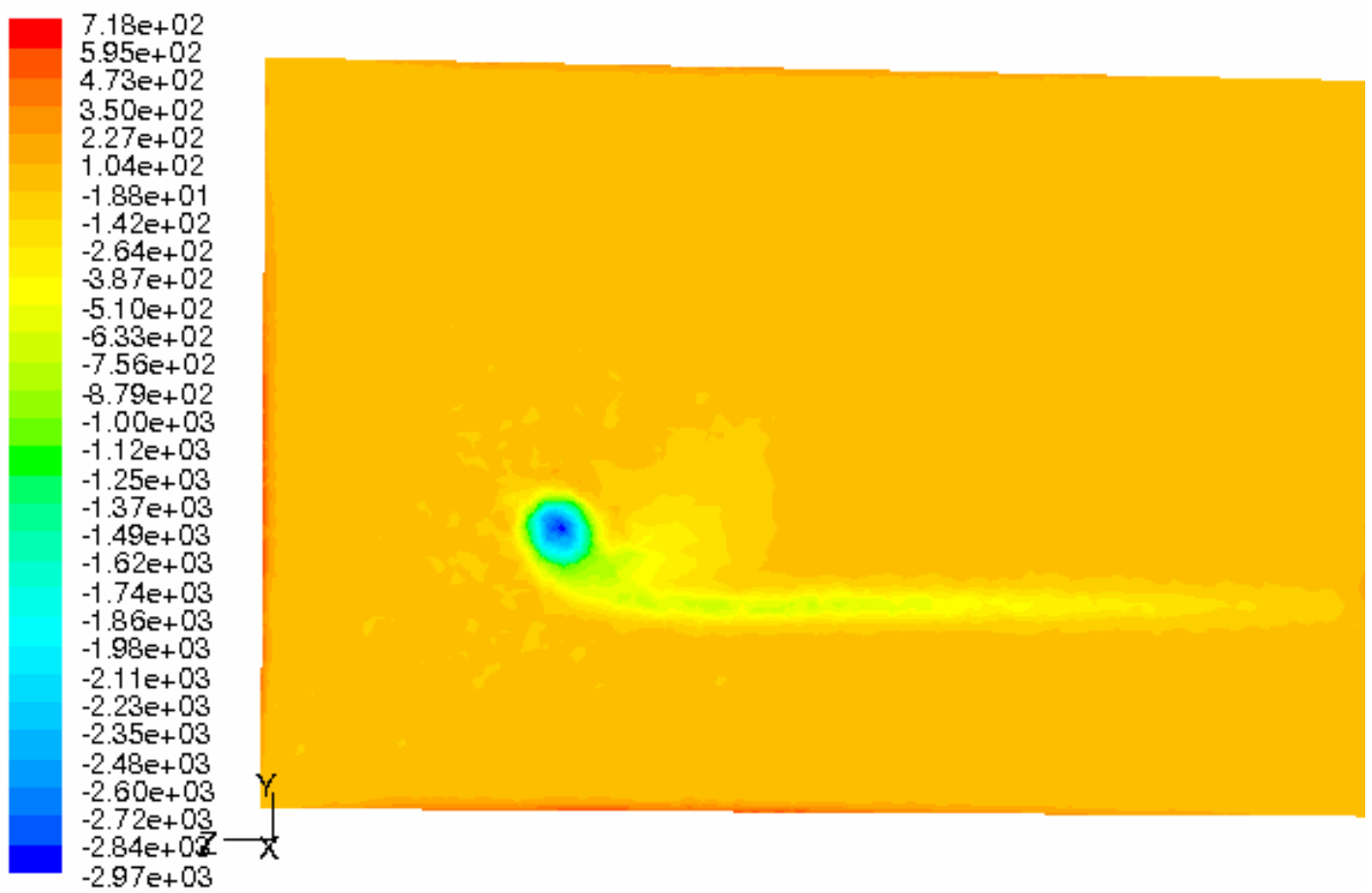

Figure 194. Vorticity (1/s) contours at $x=9 \mathrm{ft}$ for the finest mesh with suction=63 ft/s on top slot.

The pressure coefficient, velocity magnitude and vorticity contours in a plane at $\mathrm{x}=9 \mathrm{ft}$. downstream when suction is applied on top slot at $85 \mathrm{ft} / \mathrm{s}$ have been shown in Figures 159, 160, and 161, respectively.

The pressure coefficient, velocity magnitude and vorticity contours in a plane at $\mathrm{x}=9 \mathrm{ft}$. downstream when suction is applied on top slot at $106 \mathrm{ft} / \mathrm{s}$ are shown in Figures 195, 196, and 197, respectively. 


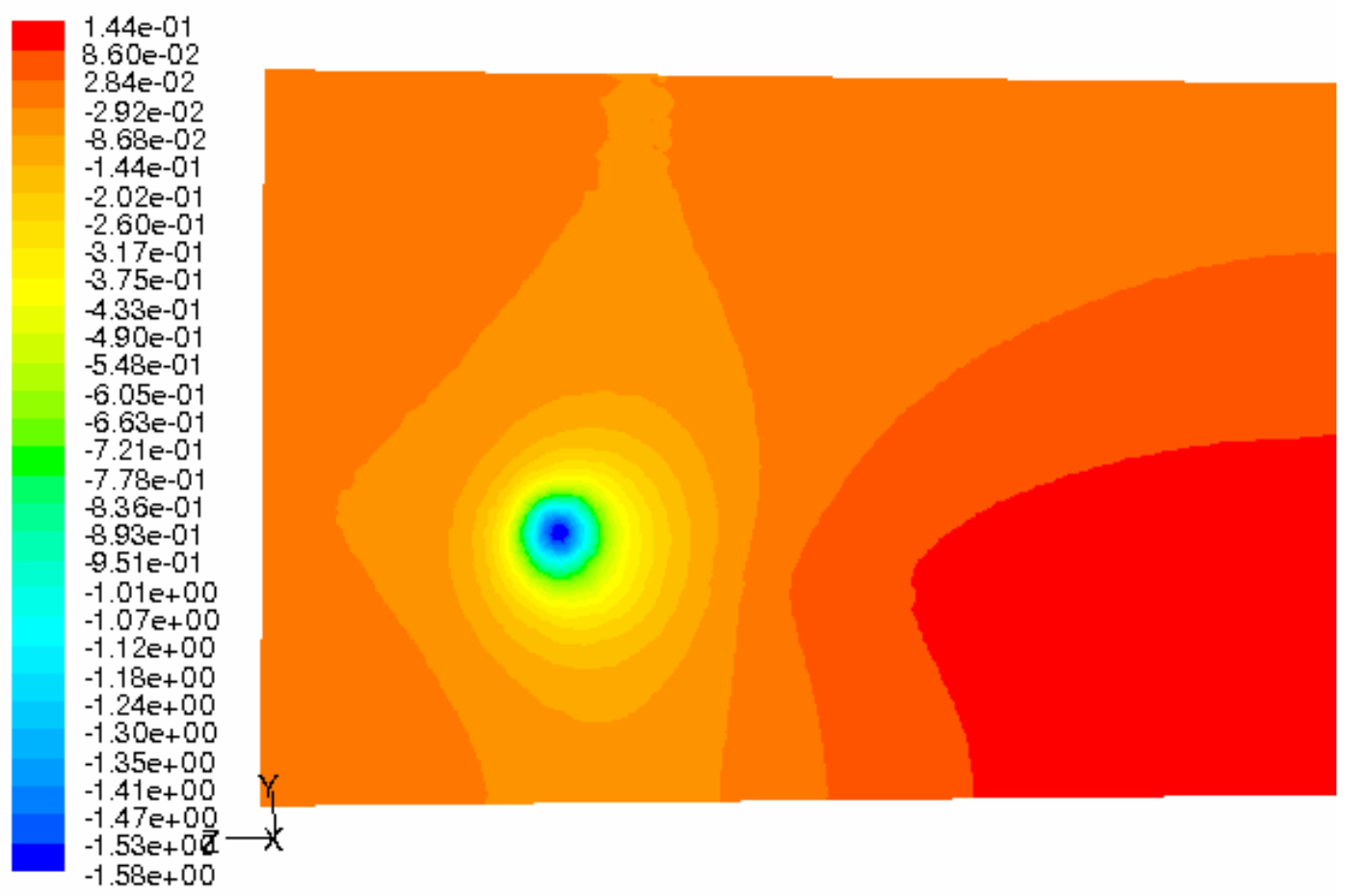

Figure 195. Pressure coefficient contours at $x=9 \mathrm{ft}$. for the finest mesh with suction=106 ft/s on top slot.

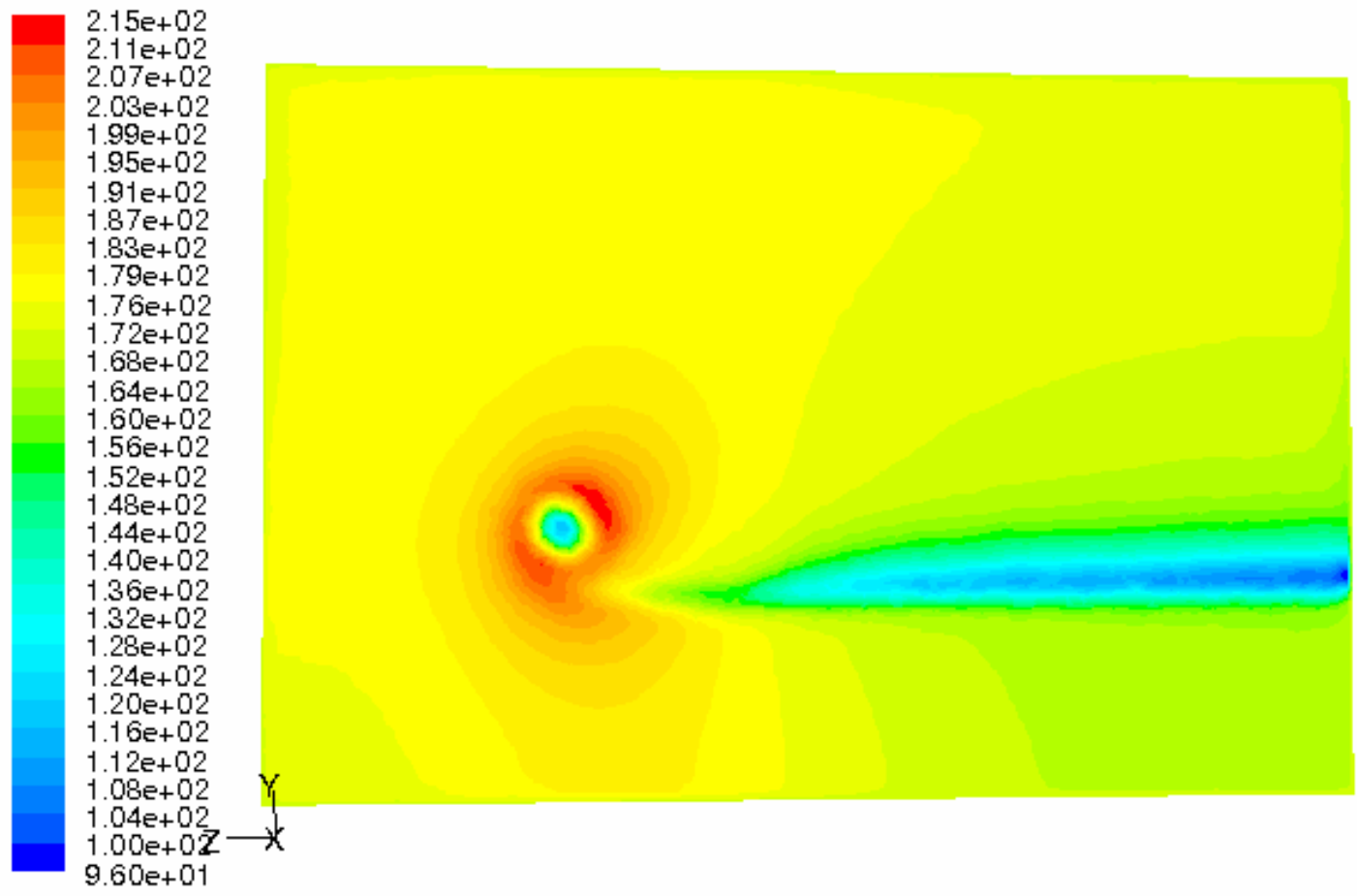

Figure 196. Velocity magnitude (ft/s) contours at $x=9 \mathrm{ft}$ for the finest mesh with suction=106 ft/s on top slot. 


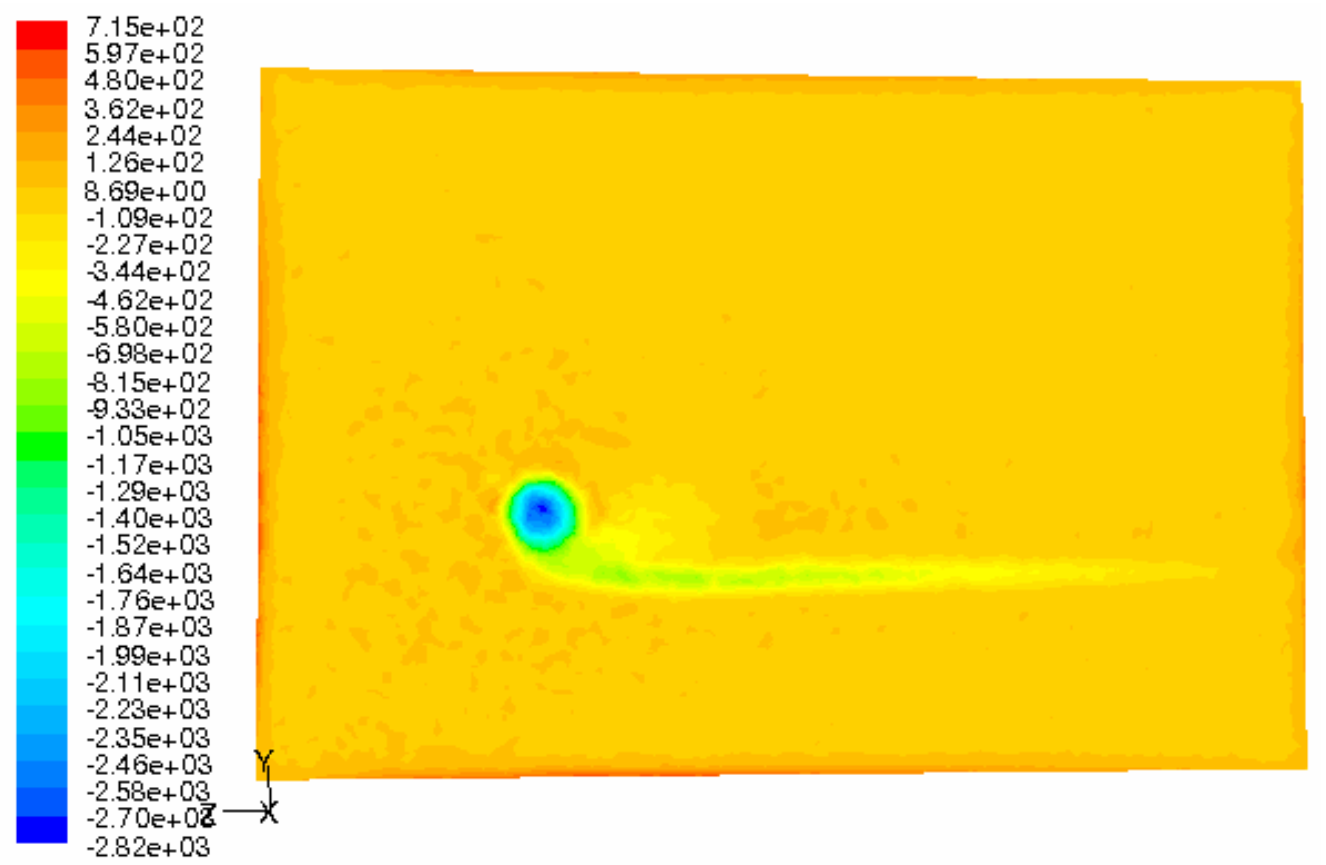

Figure 197. Vorticity (1/s) contours at $x=9 \mathrm{ft}$ for the finest mesh with suction=106 ft/s on top slot.

The pressure coefficient, velocity magnitude and vorticity contours in a plane at $\mathrm{x}=9 \mathrm{ft}$. downstream when suction is applied on top slot at $127 \mathrm{ft} / \mathrm{s}$ are shown in Figures 198, 199, and 200, respectively.

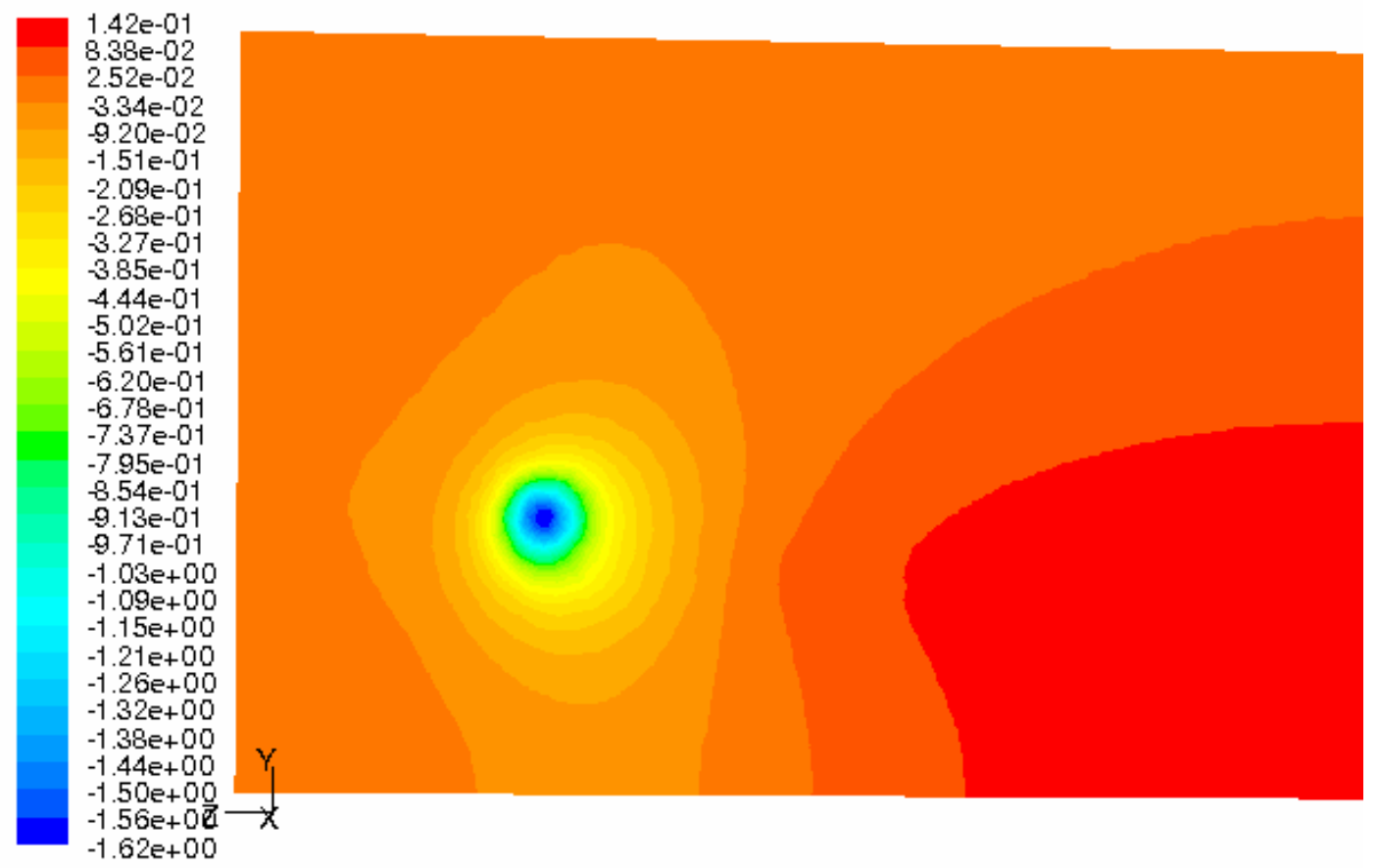

Figure 198. Pressure coefficient contours at $x=9 \mathrm{ft}$. for the finest mesh with suction=127 ft/s on top slot. 


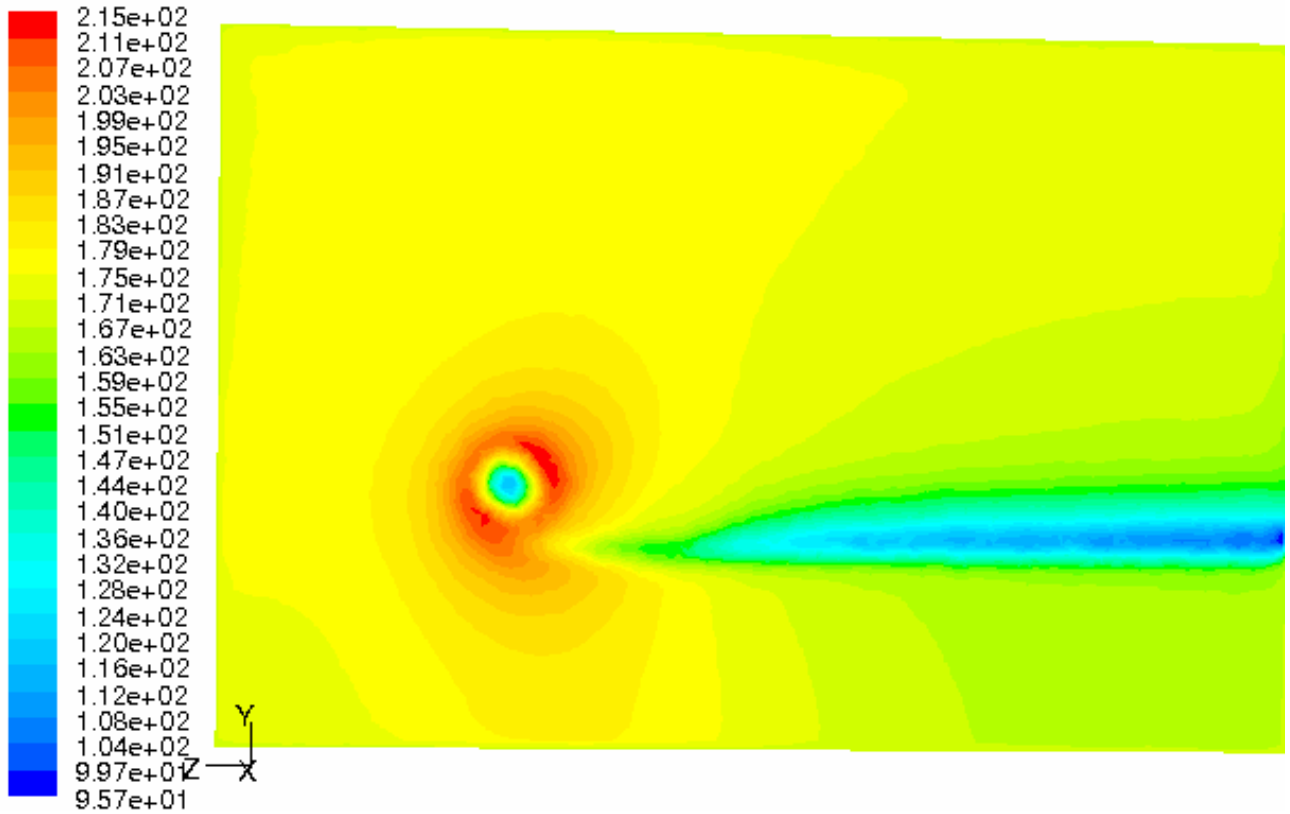

Figure 199. Velocity magnitude ( $\mathrm{ft} / \mathrm{s})$ contours at $x=9 \mathrm{ft}$ for the finest mesh with suction $=127 \mathrm{ft} / \mathrm{s}$ on top slot.

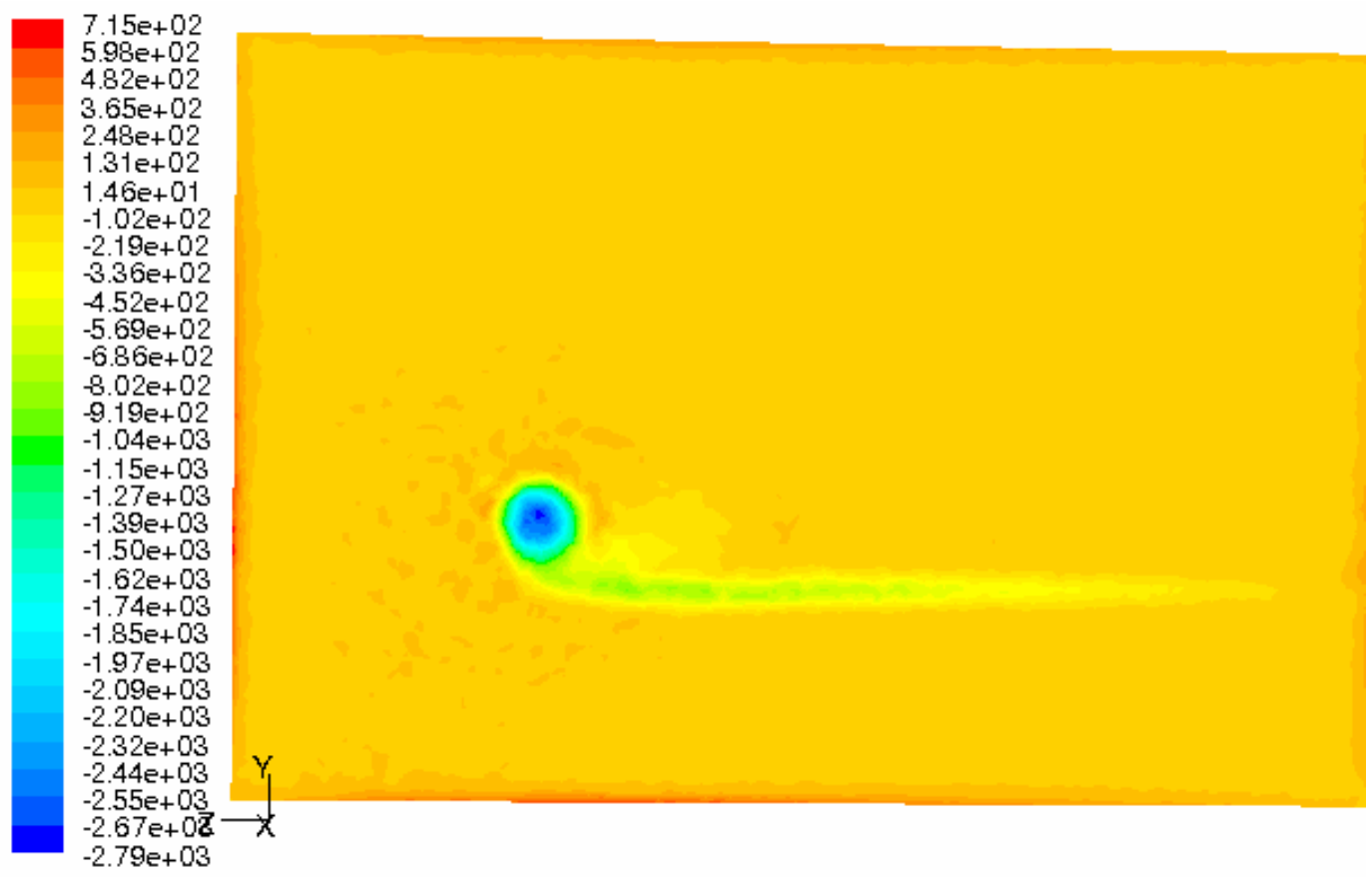

Figure 200. Vorticity (1/s) contours at $\mathrm{x}=9 \mathrm{ft}$ for the finest mesh with suction=127 $\mathrm{ft} / \mathrm{s}$ on top slot.

The pressure coefficient, velocity magnitude and vorticity contours in a plane at $\mathrm{x}=9 \mathrm{ft}$. downstream when suction is applied on top slot at $148 \mathrm{ft} / \mathrm{s}$ are shown in Figures 201, 202, and 203, respectively. 


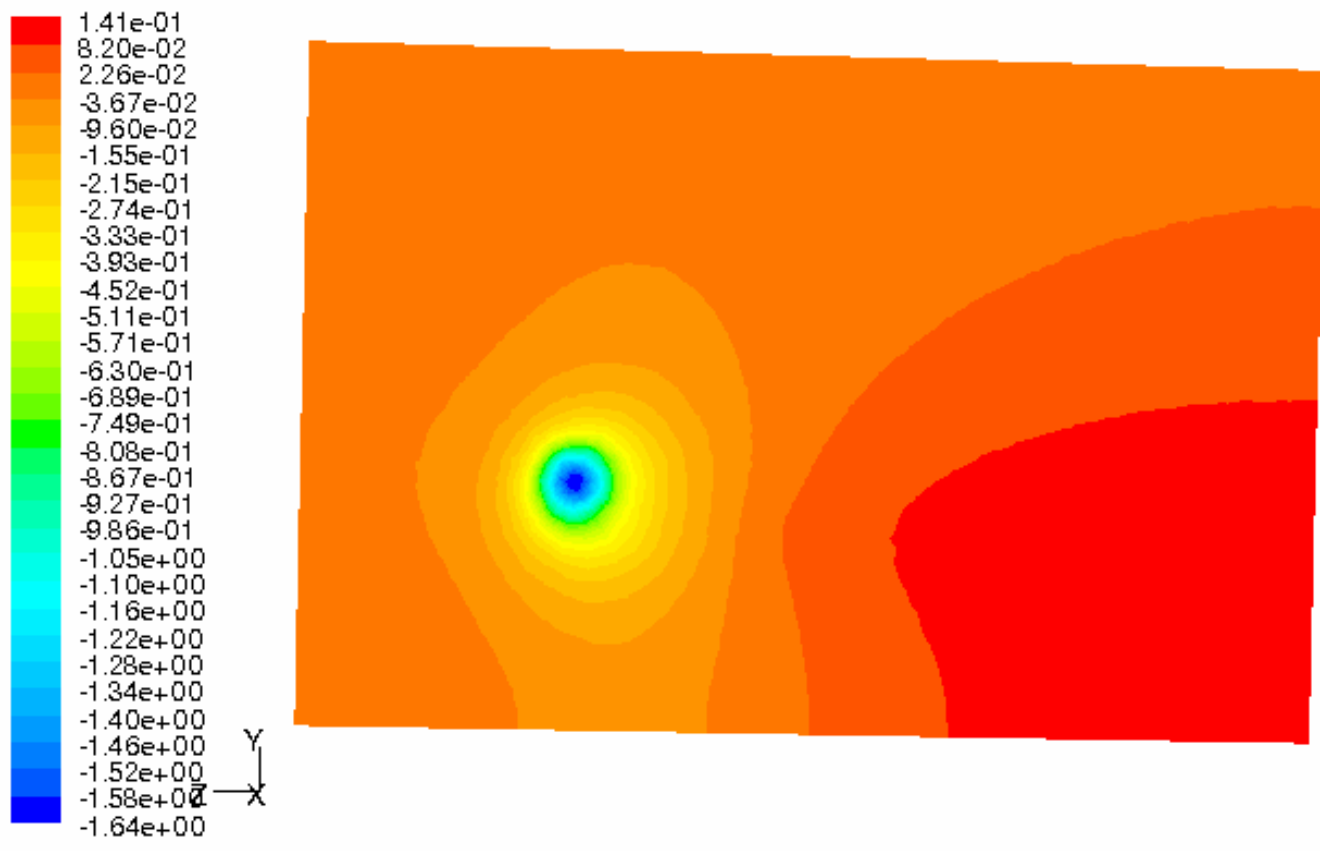

Figure 201. Pressure coefficient contours at $x=9 \mathrm{ft}$. for the finest mesh with suction=148 ft/s on top slot.

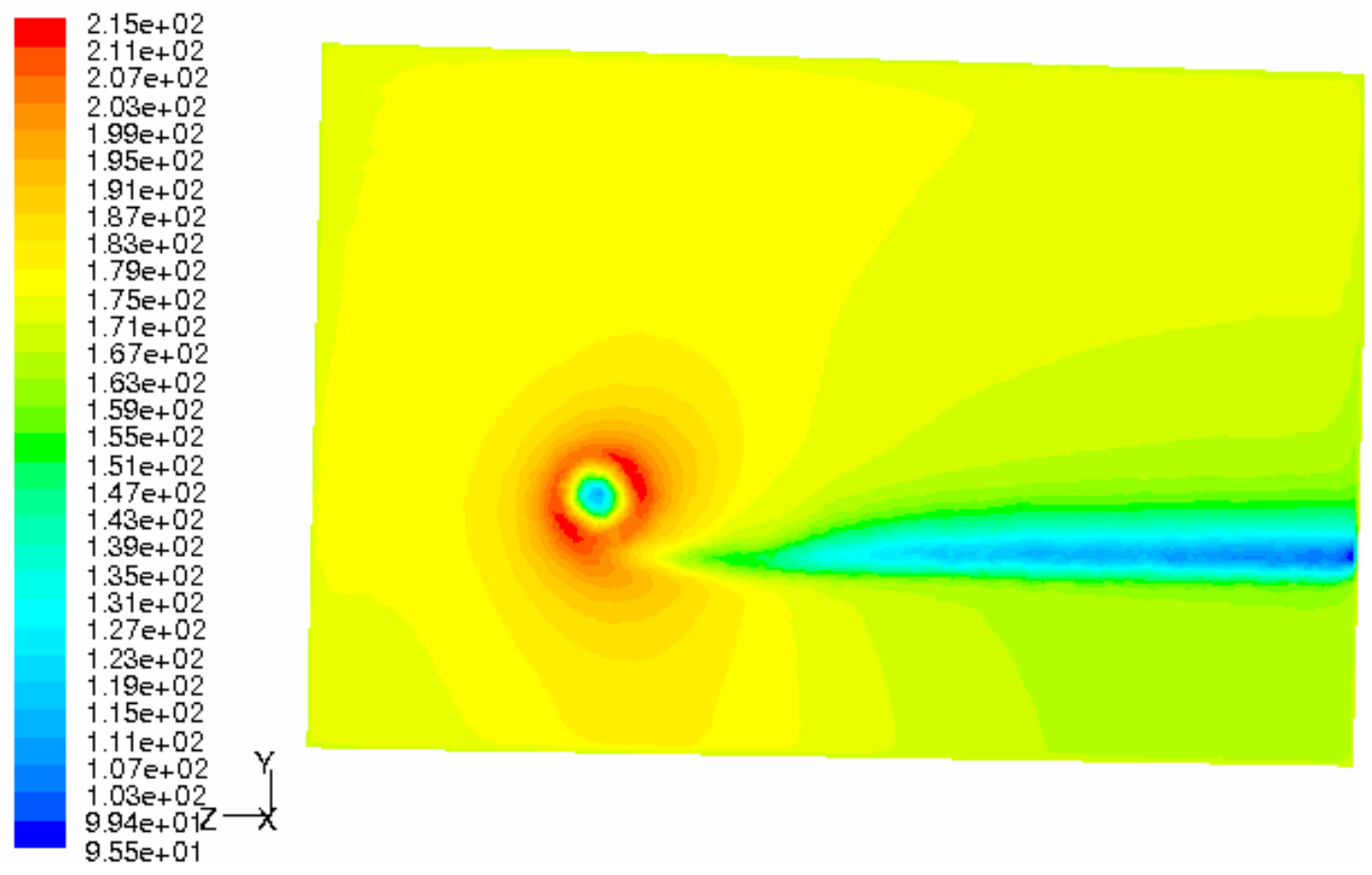

Figure 202. Velocity magnitude ( $\mathrm{ft} / \mathrm{s})$ contours at $\mathrm{x}=9 \mathrm{ft}$ for the finest mesh with suction=148 $\mathrm{ft} / \mathrm{s}$ on top slot. 

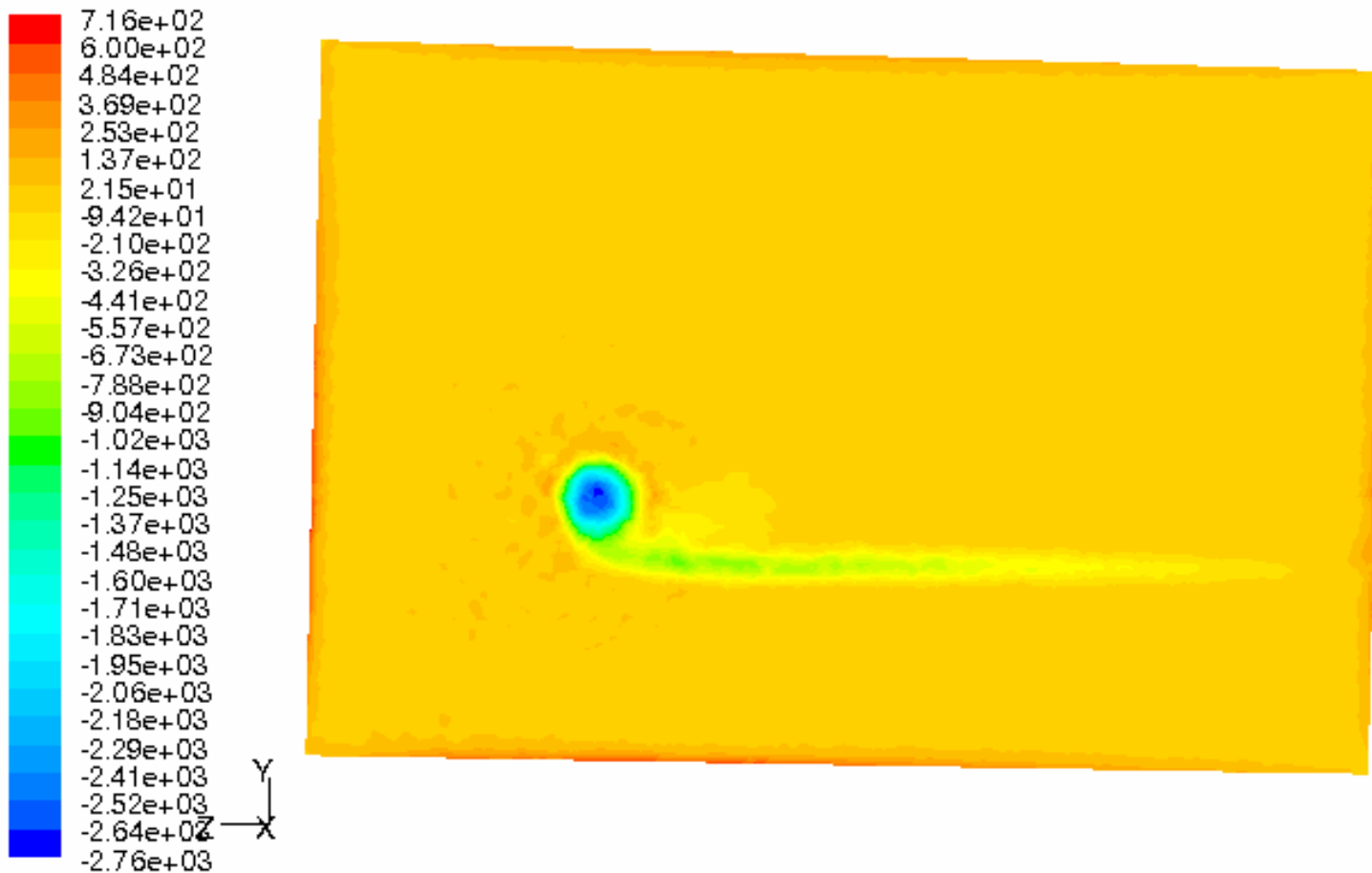

Figure 203. Vorticity (1/s) contours at $x=9 \mathrm{ft}$ for the finest mesh with suction=148 $\mathrm{ft} / \mathrm{s}$ on top slot.

The pressure coefficient, velocity magnitude and vorticity contours in a plane at $\mathrm{x}=9 \mathrm{ft}$. downstream when suction is applied on top slot at $170 \mathrm{ft} / \mathrm{s}$ are shown in Figures 165,166 , and 167, respectively.

Table 18 shows the drag coefficient and vorticity results for this study. The table shows that drag coefficient and vorticity decrease when suction velocity increases. Figure 220 shows a plot of the wingtip vorticity versus the suction velocity. Vorticity decreases almost linearly up to a suction velocity of $85 \mathrm{ft} / \mathrm{s}$, and then the curve starts declining to an asymptotic value. It seems that this velocity is enough to attach the boundary layer to the top wing surface. 
Table 18. Drag Coefficient and Vorticity variation when the suction velocity on top slot increases.

\begin{tabular}{|r|c|c|r|r|r|r|}
\hline $\begin{array}{c}\text { Velocity } \\
\text { (ft/s) }\end{array}$ & $C_{L}$ & $C_{D}$ & $\begin{array}{c}\text { Vorticity } \\
\left.\text { (ft }{ }^{2} / \mathbf{s}\right)\end{array}$ & L/D & $\begin{array}{c}\text { CD Decrease } \\
\text { (\%) }\end{array}$ & $\begin{array}{c}\text { Vorticity } \\
\text { Decrease (\%) }\end{array}$ \\
\hline 0 & 0.7246 & 0.0721 & -217.05 & 10.05 & & \\
\hline 21 & 0.7160 & 0.0710 & -214.90 & 10.08 & 1.55 & 0.99 \\
\hline 42 & 0.7094 & 0.0701 & -213.22 & 10.12 & 2.85 & 1.76 \\
\hline 63 & 0.7053 & 0.0697 & -211.59 & 10.12 & 3.44 & 2.52 \\
\hline 85 & 0.7023 & 0.0695 & -211.00 & 10.11 & 3.74 & 2.79 \\
\hline 106 & 0.7004 & 0.0695 & -210.00 & 10.08 & 3.74 & 3.25 \\
\hline 127 & 0.6993 & 0.0696 & -209.90 & 10.05 & 3.59 & 3.29 \\
\hline 148 & 0.6990 & 0.0698 & -209.62 & 10.01 & 3.30 & 3.42 \\
\hline 170 & 0.6993 & 0.0701 & -209.43 & 9.98 & 2.85 & 3.51 \\
\hline
\end{tabular}

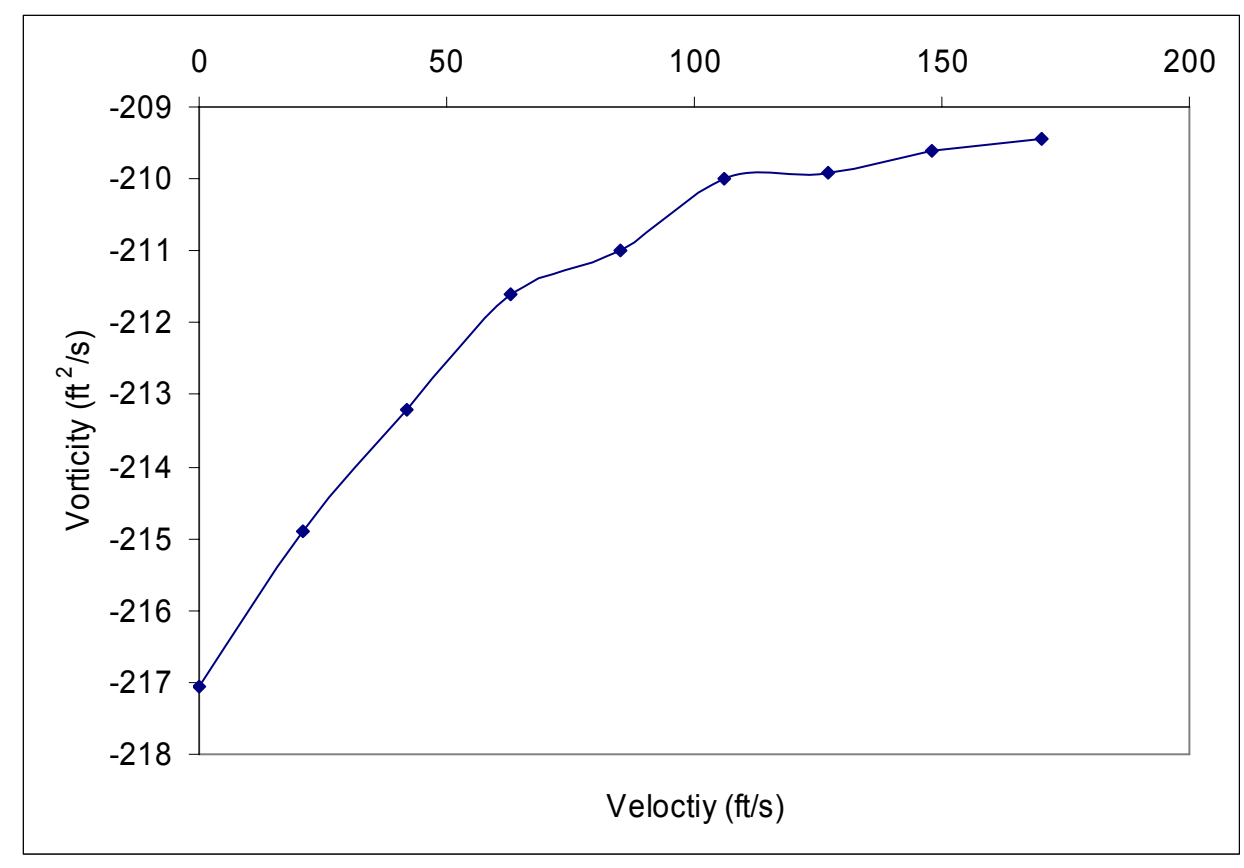

Figure 204. Wingtip vorticity variation with the BLC suction velocity.

The volume flow rate required to apply suction on top at $85 \mathrm{ft} / \mathrm{s}$ is $13.6 \mathrm{ft}^{3} / \mathrm{s}$. Flying at $\mathrm{V}_{\infty}=170 \mathrm{ft} / \mathrm{s}$ produces a dynamic pressure of $34.3 \mathrm{lbf} / \mathrm{ft}^{2}$. Therefore, the suction power required to apply this suction velocity is $0.85 \mathrm{HP}$. 


\section{Chapter 7: Conclusions}

CFD simulations done in the current study to reproduce the experimental measurements done by Chow et al. [43] at NASA has shown very good agreement to predict the formation of wing tip vortices, and to obtain agreement for pressure coefficient values.

Drag prediction by wake measurement results agree with experimental data Beside this, the wake integral approach provides a way to quantify each individual component of drag separately, i.e.: drag due to total pressure losses, drag due to momentum deficit, and drag due to lift. The experimental data taken as reference was the wake survey measurements done by Chometon and Laurent on an $\mathrm{AR}=4$ rectangular wing which uses a NACA $64_{3}-018$ airfoil [48].

BLC application to bottom suction slots is effective to straighten the streamlines. The suction slots at the trailing edge are more effective than the chord-wise slots for the cases tested. The optimum suction velocity on the bottom suction slot is $\mathrm{V}_{\infty}$. The best configuration is obtained when suction is applied at $0.25 \mathrm{~V}_{\infty}$ using an ejector with exit velocity equal to $\mathrm{V}_{\infty}$. In that case, induced drag is reduced by $11 \%$ and the lift to drag ratio is increased by $16 \%$. Based on the Cessna 206 characteristics, the induced drag power saved is $6.4 \mathrm{HP}$. The volume flow rate required to apply suction on bottom slots at $\mathrm{V}_{\infty}=50 \mathrm{ft} / \mathrm{s}$ is $8.95 \mathrm{ft}^{3} / \mathrm{s}$ and the suction power required is $0.78 \mathrm{HP}$. The power saved is much higher than the power required.

Span-wise blowing decreases the induced drag and the drag due to momentum deficit; it also increases the lift coefficient. However, it increases the drag due to total pressure losses and the total drag, thereby decreasing the lift to drag ratio.

Wingtip vorticity decreases up to $3.6 \%$ and total drag up to $7.6 \%$ when suction is applied on bottom half-chord, bottom trailing edge and top suction slots at $0.5 \mathrm{~V}_{\infty}(85$ $\mathrm{ft} / \mathrm{s})$. The top suction slot is more effective in reducing wingtip vorticity than the other configurations. This can be caused by BLC suction application on the top slot having a reattachment of the boundary layer thereby causing a recirculation region on wing upper surface. Vorticity decreases almost linearly up to a suction velocity of $85 \mathrm{ft} / \mathrm{s}$, and then 
declines to an asymptotic value. This velocity is enough to attach the boundary layer to the top wing surface. The volume flow rate required to apply suction on top at this velocity is $13.6 \mathrm{ft}^{3} / \mathrm{s}$ and the suction power required is $0.85 \mathrm{HP}$. These conclusions were obtained from this numerical study and they have not been validated experimentally.

The ejectors have shown many benefits in prior work when applied in combination with BLC and $\mathrm{CC}$, which are: reduction in mass flow, decrease in duct pressure loss, CC flap cooling, increase in lift by CC blowing, better CC blowing to free stream velocity ratio, and reduction in air supply duct size required. The ejector area ratio should be chosen with the goals of the application in mind. High dimensionless nozzle area ejectors produce higher suction vacuum, higher nozzle volume flow rate, higher total exit pressure, higher exit velocity, and lower total pressure losses. The increased range of error between theoretical and experimental values for low area ratio nozzles indicate that more precise methods incorporating viscous effects should be used when such types of ejectors are required in a design.

Based on experience with boundary layer control by blowing and suction and the use of ejectors [51], it appear to be possible to dissipate all vorticity captured in a suction slot by means of mixing inside an ejector.

If one can discover a BLC suction slot configuration capable of preventing the bound vorticity generating boundary layer, in the wingtip region from escaping into the wake, by capturing and dissipating its vorticity inside an ejector, then it will:

- Reduce vorticity inside the wake

- Reduce downwash velocity inside the wake, and

- Reduce upwash flow field beyond the wing span.

Future work should be done to validate experimentally the results obtained in this numerical study. 


\section{References}

1. Thomas, A. S., "Aircraft Drag Reduction Technology - A Summary," Advisory Group for Aerospace Research and Development (AGARD), Report 723, Belgium, 1985.

2. Airguide, "Jet Aircraft World Fleet Summary," Air Guide Online. 2006. $<\mathrm{http}: / /$ www.airguideonline.com/aircr_wfleet.htm> (4 May 2006).

3. Loth, J. L. et al., "Flight Performance of a Circulation Control STOL Aircraft," Journal of Aircraft, Vol. 13, March 1976, pp.169-173.

4. Joslin, R.D., and Jones, G.S., Applications of Circulation Control Technology, Progress in Astronautics and Aeronautics, AIAA, 2006.

5. Barnett, A., "Pace hots up in a World forever $\mathrm{n}$ the move," Guardian Unlimited. 2006. http://observer.guardian.co.uk/carbontrust/story/0,16099,1511925,00.html> (4 May 2006).

6. Loth, J., Engineering Approach to Aerodynamics and Aircraft Performance $2^{\text {nd }}$ Edition, McGraw Hill, 2005.

7. Munk, M., "The Minimum Induced Drag of Aerofoils," National Advisory Committee for Aeronautics, Report No. 121, pp. 373-390.

8. Whitcomb, R., "A Design Approach and Selected Wind-Tunnel Results at High Subsonic Speeds for Wing Tip mounted Winglets,” NASA TN-D-8260, 1976.

9. Anderson, J., Introduction to Flight $5^{\text {th }}$ Edition, McGraw Hill, Boston, MA, 2005.

10. Anderson, J., Fundamentals of Aerodynamics $4^{\text {th }}$ Edition, McGraw Hill, Boston, MA 2007.

11. Bertin, J. J. and Smith, M. L., Aerodynamics for Engineers $3^{\text {rd }}$ Edition, Prentice Hall, Upper Saddle River, NJ 1998.

12. Stinton, D., The Design of the Aeroplane 2nd Edition, Blackwell publishing, London, Great Britain 2003.

13. Hoerner, S.F., Fluid Dynamic Drag Theoretical, Experimental and Statistical Information, Published by the author, Brick Town, N.J. 1965.

14. McCormick, B.W., Aerodynamics of V/STOL Flight, Academic press, London, 1967.

15. Saffman, P.G., Vortex Dynamics, Cambridge University Press, Cambridge, 1992. 
16. Webber, G.W. and Dansby, T., "Wing Tip Devices for Energy Conservation and Other Purposes," Canadian Aeronautics and Space Journal, Vol. 29, No. 2, June 1983, pp. 105-200.

17. Kroo, I. "Nonplanar Wing Concepts for Increased Aircraft Efficiency," VKI Lecture Series on Innovative Configurations and Advanced Concepts for Future Civil Aircraft, Stanford, 2005.

18. Whitcomb, R.T., "Methods for reducing subsonic drag due to lift," Special course on concepts for drag reduction, AGARD, France 1977, pp. 2-1 to 2-11.

19. Gerontakos, P., and Lee, T. "Effects of Winglet Dihedral on a Tip Vortex," AIAA Journal of Aircraft, Vol. 43, No. 1, January, 2006.

20. Wu, J.M. et al., "Wing tip jets aerodynamics performance," $13^{\text {th }}$ Congress of the International Council of the Aeronautical Sciences/AIAA Aircraft Systems and Technology Conference, ICAS proceedings, Vol. 2, 1982, pp. 1115-1121.

21. Loth, J.L., "Betz Type Limitation of Vortex Wind Machines," Journal of Wind Engineering, Vol. 1, No. 3, 1977, pp. 169-185, London ELT-IDJ, England.

22. Spillman, J.J., "The Use of Wing Tip Sails to reduce Vortex Drag," Aeronautical Journal, September 1978, pp. 387-395.

23. Traub, L.W., "Aerodynamic Effects of Delta Planform Tip Sails on Wing Performance," Journal of Aircraft, Vol. 31, No. 5, September-October 1994.

24. Aerospace Web, "Boeing 767 Raked Wing tips," 2006. $<$ http://www.aerospaceweb.org/question/aerodynamics/q0148.shtml $>$ (1 Aug. 2006).

25. Dunham, R.E., "Unsuccessful Concepts for Aircraft Wake Vortex Minimization," NASA SP-409, Wake Vortex Minimization Conference, Washington D.C., February 25-26 1976.

26. Rossow, V., "Lift generated vortex wakes of subsonic transport aircraft," Progress in Aerospace Sciences, Vol. 35, No. 6, 1999, pp. 507-560.

27. Patterson, J.C. and Flechner, S.G., "An exploratory wind tunnel investigation of the wake effect of a panel tip-mounted fan-jet engine on the lift-induced vortex," NASA TN D-5729, Washington D.C., May 1970.

28. Nazarinia, M., Soltani, M., and Sharif, K., "Flow Analysis over and Behind a Wing with Different Winglet Shapes," $42^{\text {nd }}$ AIAA Aerospace Sciences Meeting and Exhibit, Jan 5-8, Reno, Nevada, 2004.

29. Lachmann, G.V., Boundary Layer and Flow Control, Pergamon Press, Vol. 1, Bristol, 1961. 
30. Mansour, N.N., "Numerical Simulation of the Tip Vortex Off a Low Aspect Ratio Wing at Transonic Speed," AIAA Journal, Vol. 23, No. 8, 1985, pp. 1143-1149.

31. Hsiao, C. T., and Pauley, L. L., "Numerical Study of the Steady-State Tip Vortex Flow Over a Finite-Span Hydrofoil," ASME Journal of Fluids Engineering, Vol. 120, No. 6, 1998, pp. 345-353.

32. Baldwin, B.S. and Lomax, H., "Thin Layer Approximation and Algebraic Model for Separated Turbulent Flows," AIAA Paper 78-257, 1978.

33. Srinivasan, G.R., McCroskey, W.J., Baeder, J.D., and Edwards, T.A., " Numerical Simulation of Tip Vortices of Wings in Subsonic and Transonic Flows," AIAA Journal, Vol. 26, No. 10, 1988, pp. 1153-1162.

34. Kramer, E., Hertel, J., and Wagner, S., "Computation of Subsonic and Transonic Helicopter Rotor Flow Using Euler Equations," Vertica, Vol. 12, No. 3, 1988, pp. 279-291.

35. Strawn, R.C., "Wing Tip Vortex Calculations with an Unstructured AdaptiveGrid Euler Solver," 47th Annual Forum of the American Helicopter Society, Phoenix, AZ, May 1991.

36. McAlister, K. W., and Takahashi, T. K., "NACA 0015 Wing Pressure and Trailing Vortex Measurements," NASA TP 3151, 1991.

37. Dacles-Mariani, J., Zilliac, G., Chow, J., and Bradshaw, P., "Numerical/Experimental Study of a Wingtip Vortex in the Near Field," AIAA Journal, Vol. 33, No. 9, 1995, pp. 1561-1568.

38. Baldwin, B. S., and Barth, T. J., "A One-Equation Transport Model for High Reynolds Number Wall-Bounded Flows,” NASA TM 102847, 1990.

39. Spalart, P. R., and Allmaras, S. R., "A One-Equation Turbulence Model for Aerodynamic Flows," La Recherche Aerospatiale, 1994-1, 1994, pp. 5-21.

40. Lockard, D. P., and Morris, P. J., "Wing-Tip Vortex Calculations Using a HighAccuracy Scheme," Journal of Aircraft, Vol. 35, No. 5, 1998, pp. 728-738.

41. Devenport, W.J., Rife, M., Liapis, S., and Follin, G., "Structures and Development of a Wing-Tip Vortex,' Journal of Fluid Mechanics, Vol. 312, 1996, pp. 67-106.

42. Spall, R. E., "Numerical Study of a Wing-Tip Vortex Using the Euler Equations," Journal of Aircraft, Vol. 38, No. 1, January-February 2001, pp. 22-27.

43. Chow, J., Zilliac, G., and Bradshaw, P., "Turbulence Measurements in the Nearfield of a Wingtip Vortex," NASA Technical Memorandum 110418, February 1997. 
44. Kim, S.-E., and Rhee, S.H., "Prediction of Tip-Vortex Flow Past a Finite Wing," 43rd AIAA Aerospace Sciences Meeting and Exhibit, Reno, USA, Jan 10-13, 2005.

45. Christey, D. "Beowulf Cluster for WVU Department of Mathematics" summer, 2006.

46. Patankar, S.V., Numerical Heat Transfer and Fluid Flow, Hemisphere Publishing Corporation, New York, 1980.

47. Fluent Inc., "Fluent User's Guide,” Fluent, 2006

$<$ http://www.fluentusers.com/fluent/doc/ori/html/ug/main_pre.htm> (15 October 2006).

48. Chometon, F., and Laurent, J., "Study of Three Dimensional Separated Flows, Relation between Induced Drag and Vortex Drag," European Journal of Mechanics, B/Fluids, Vol. 9, No. 5, 1990, pp. 437-455.

49. Chao, D.D., and Van Dam, C.P., "Airfoil Drag Prediction and Decomposition," AIAA $16^{\text {th }}$ Applied Aerodynamics Conference, Paper No. 98-2783, Albuquerque, NM, June, 1998.

50. Brune, G.W., and Bogataj, P.W., "Induced Drag of a Simple Wing from Wake Measurements," SAE Aerospace Technology Conference and Exposition, Paper No. 901934, Long Beach, CA, 1990.

51. Posada, J.A. and Browning, P. "Low Velocity Ejector Test Results for CC Blowing with BLC Suction," $3^{\text {rd }}$ AIAA Mid-Atlantic Mini-Conference, Baltimore, MD, November 52005.

52. Boasson, M., and Loth, J. L., "Combination of Suction and Blowing in Boundary Layer Control" $27^{\text {th }}$ Israel Annual Conference on Aviation and Astronautics, Haifi, Israel, 1986.

53. Loth, J. "Ejector Development for Seawater Desalination," West Virginia University, Report prepared under contract with Colt Industries, June 1971.

54. Loth, J. L., Fanucci, J., and Roberts, S., "Flight Performance of a Circulation Control STOL Aircraft," AIAA paper No. 74-994, $6^{\text {th }}$ Aircraft Design, flight Test and Operations Meeting, Los Angeles, California, August 12-14, 1974

55. Loth, J. L., "Some Aspects of STOL Aircraft Aerodynamics," SAE Business Aircraft Meeting, 730328, SAE, Wichita, KS, 1973.

56. Loth, J. L., and Boasson, M., "Circulation Controlled STOL Wing Optimization," AIAA Journal of Aircraft, Vol. 21, No. 2, 1984, pp. 128-134. 


\section{Appendixes}

\section{Appendix A: Ejectors to Provide Wingtip Trailing Edge Suction}

Ejectors are devices used to produce suction by entrainment by a high velocity jet inside a flow mixing tube. Ejectors are expected to be a practical means for generating the required boundary layer suction, for the BLC strategies explored in Chapter 5. A small amount of mass flow at high pressure is supplied through a supersonic nozzle. The nozzle discharges into a mixing tube, where it entrains the ambient air to provide the desired suction. The entrained gas or liquid is accelerated to form a near uniform pressure and velocity region near the outlet of the mixing tube. This is followed by diffusion to a pressure higher than the suction pressure provided. The ejector outlet to suction pressure ratio is a function of geometry and supply air mass flow rate.

Ejectors are used for a wide range of industrial processes where large volume flow rates of gas or liquid are handled. They are used to evacuate chemical reactors, lowdensity hypersonic velocity tunnels, rocket test cells, and numerous other devices. The installation, operating and maintenance cost is usually lower than for mechanical compressors due to the absence of moving parts. This is even more true when the fluid to be pumped is chemically active, contaminated, or at a high temperature and the operating time is very short [53]. However, their isentropic compression efficiency is usually less than $20 \%$ which is much less than that of a mechanical compressor [54].

In the design of the first flight of a Circulation Control (CC) High Lift System in 1974, using a CC Technology Demonstrator STOL Aircraft [3, 55], ejectors were used extensively for structural cooling, preventing flow separation at the flap hinge, improving the CC blowing efficiency by optimizing the Coanda jet velocity to flight velocity ratio, and to increase aileron effectiveness by upper surface blowing at low flight speed.

This aircraft employed a retractable CC flap, which is shown deployed in Figure 205. The flap could be rotated forward inside the wing by using bell-cranks visible adjacent to the fuselage. CC compressor bleed air was supplied by a GTC-85 jet-engine (its intake-grille can be seen on the side of the fuselage). The aircraft's Model B wing [56] increased wing chord length by $20 \%$ in the STOL mode. Its performance was a big 
improvement over that of the 1972 wind tunnel model A wing [56], shown in Figure 206, which lowered the chord length by $12 \%$ in the $\mathrm{CC}$ mode.

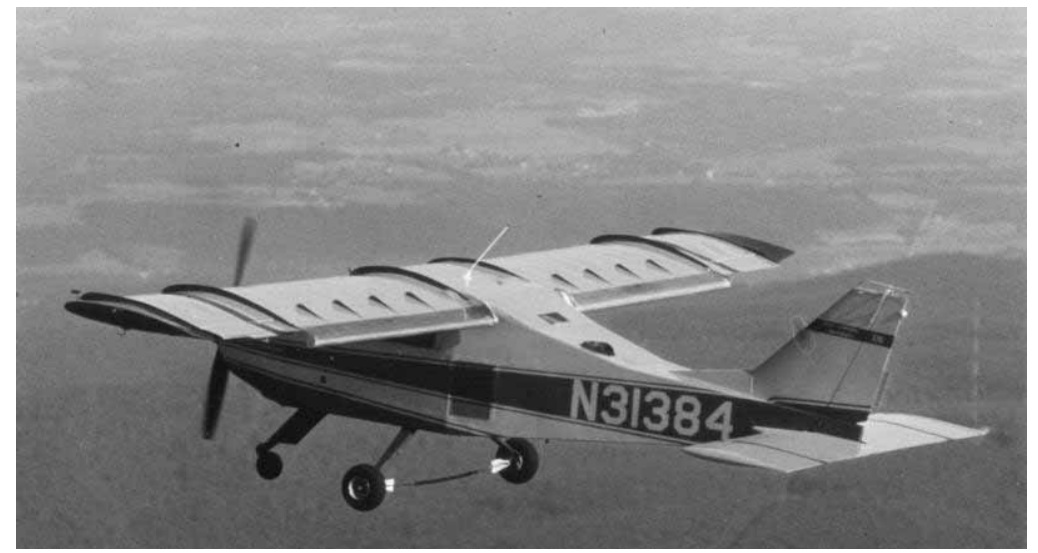

Figure 205. WVU CC Technology Demonstrator STOL Aircraft in flight, 1974.

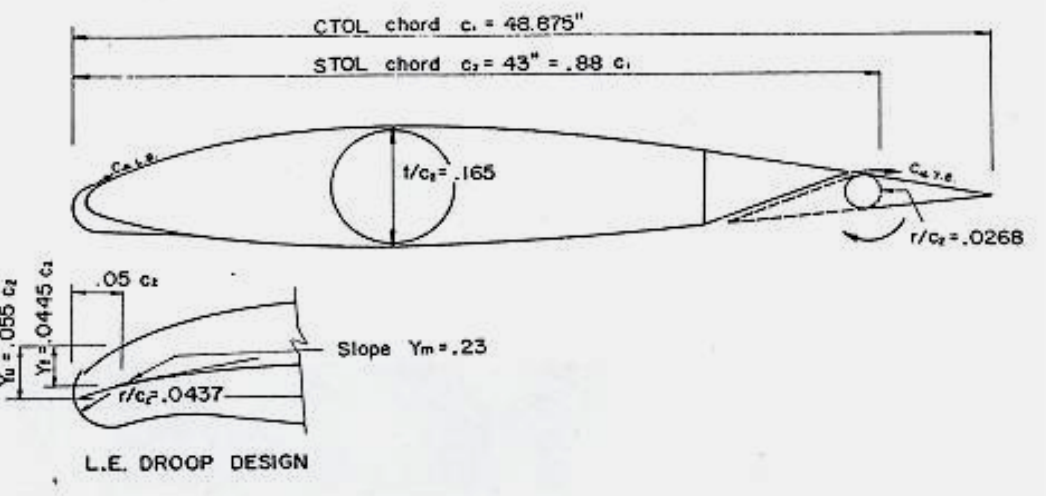

Figure 206. WVU CC Wind Tunnel Model A Wing, convertible form round to sharp trailing edge.

The Model B wing shown in Figure 207 provided a 20\% increase in wing chord length when its retractable CC flap was deployed. An enlarged view of this CC flap is shown in Figure 208 to make the geometry of its two-dimensional internal ejector clearly visible. Its supersonic nozzle provided the momentum to entrain air for BLC suction at the $\mathrm{CC}$ flap hinge. This internal ejector reduced the velocity ratio $\mathrm{V}_{\mathrm{j}} / \mathrm{V}_{\infty}$ by a factor of two, thereby increasing $\mathrm{CC}$ lift augmentation, as proven by Boasson $[57,53]$. The available BLC control by suction at the flap hinge allowed further increasing the lift augmentation by flap deflection. WVU tests [56] showed an $8 \%$ increase in $\mathrm{C}_{\mathrm{Lmax}}$ due to BLC. The ejector reduced the air mass flow from the compressor, which in turn lowered air duct size and pressure loss. 


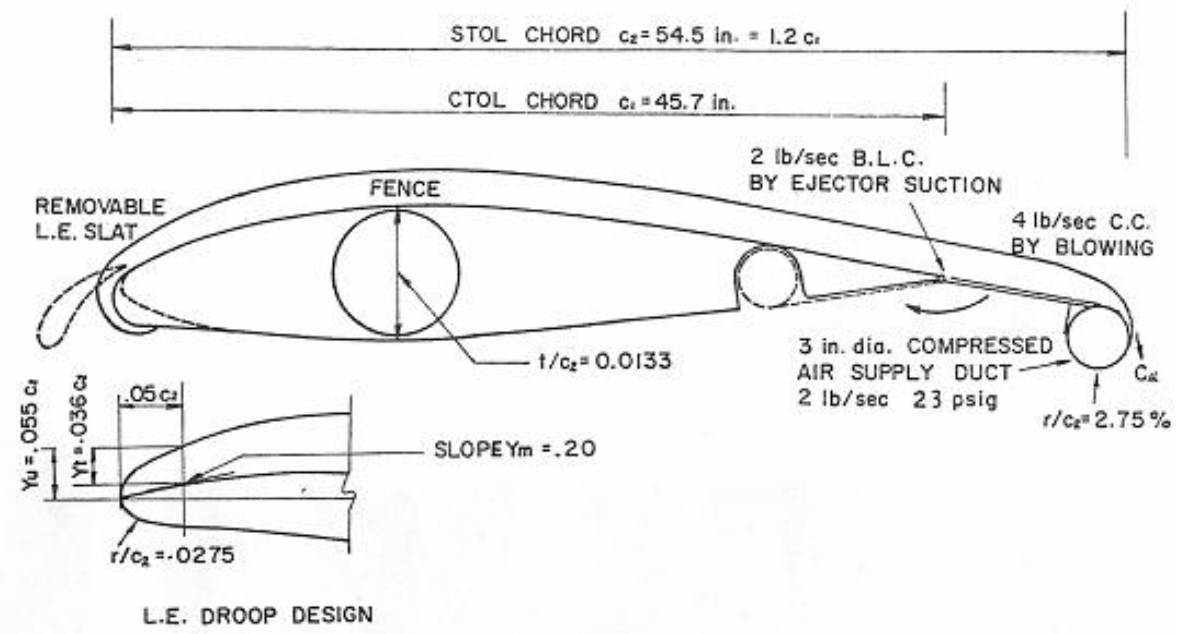

Figure 207. WVU CC Technology Demonstrator STOL Aircraft Model B Wing [55].

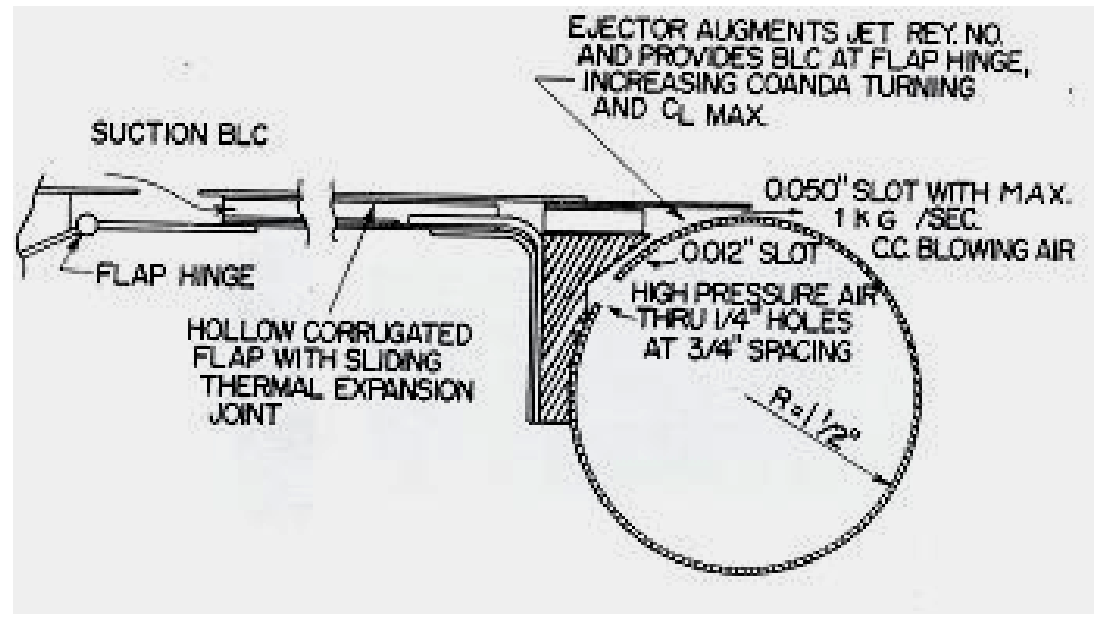

Figure 208. . WVU Model B CC flap with details of the internal ejector and Coanda surface [3].

\section{One-Dimensional Compressible Flow Ejector Equations}

An ejector is used to entrain mass flow without moving components. Figure 209 shows a CAD model of a simple ejector. This ejector consists of a constant area mixing duct of area $A_{e}$, nozzle area $A_{n}$ and remaining suction area $A_{s}=A_{e}-A_{n}$. To satisfy subsonic equilibrium, appropriate boundary condition at the exit of the nozzle is that the static pressure of the nozzle should self adjust to the static suction pressure at the nozzle exit. 


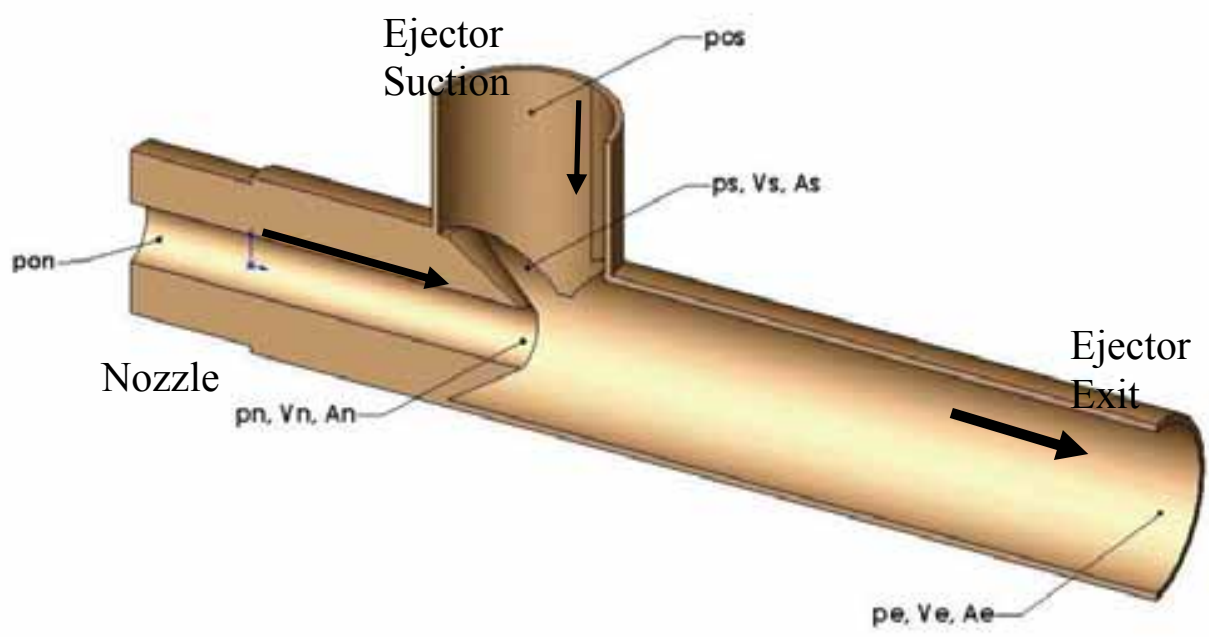

Figure 209. One-dimensional ejector configuration with nozzle, ejector suction and exit.

The governing equations for a one-dimensional incompressible flow ejector are the continuity equation, the momentum equation, and the energy conservation (Bernoulli) equation. Compressible flow has two additional unknowns: density, $\rho$, and temperature, T. Two more equations are required to solve for all the variables of the system: the equation of state, and the enthalpy equation for calorically perfect ideal gases.

The continuity equation states

$$
\dot{m}_{n}+\dot{m}_{s}=\dot{m}_{e}
$$

where

$$
\begin{aligned}
& \dot{m}_{n(\text { nozzle })}=\rho_{n} V_{n} A_{n} \\
& \dot{m}_{s(\text { suction })}=\rho_{s} V_{s} A_{s} \\
& \dot{m}_{e(\text { exit })}=\rho_{e} V_{e} A_{e}
\end{aligned}
$$

Neglecting wall friction, the momentum equation becomes the impulse function

$$
F_{n}+F_{s}=F_{e}
$$

where:

$$
\begin{aligned}
& F_{n}=\dot{m}_{n} V_{n}+\left(p_{n}-p_{\infty}\right) A_{n} \\
& F_{s}=\dot{m}_{s} V_{s}+\left(p_{s}-p_{\infty}\right) A_{s}
\end{aligned}
$$




$$
F_{e}=\dot{m}_{e} V_{e}+\left(p_{e}-p_{\infty}\right) A_{e}
$$

noting that all pressures are measured in absolute pressures.

The energy conservation equation for steady, adiabatic, inviscid flow is

$$
\dot{m}_{e}\left(h_{e}+\frac{V_{e}^{2}}{2}\right)=\dot{m}_{n}\left(h_{n}+\frac{V_{n}^{2}}{2}\right)+\dot{m}_{s}\left(h_{s}+\frac{V_{s}^{2}}{2}\right)
$$

The enthalpy equation for a calorically perfect gas is

$$
h=c_{p} T
$$

Substituting in the energy conservation equation

$$
\dot{m}_{e}\left(c_{p} T_{e}+\frac{V_{e}^{2}}{2}\right)=\dot{m}_{n}\left(c_{p} T_{n}+\frac{V_{n}^{2}}{2}\right)+\dot{m}_{s}\left(c_{p} T_{s}+\frac{V_{s}^{2}}{2}\right)
$$

The equation of state is $p=\rho R T$ but here use instead

$$
\rho R=p / T
$$

By multiplying the continuity equation (Eq. (A.1)) by $\left(R / A_{e}\right)$, then replacing $(\rho R)$ with $(p / T)$ gives:

$$
D=\frac{p_{e}}{T_{e}} V_{e}=\frac{p_{n}}{T_{n}} V_{n} \frac{A_{n}}{A_{e}}+\frac{p_{s}}{T_{s}} V_{s} \frac{A_{s}}{A_{e}}
$$

Performing the same algebraic manipulation to the momentum equation (Eq. (A.2)) yields

$$
\frac{p_{e}}{T_{e}} V_{e}^{2}+p_{e} R=\frac{p_{n}}{T_{n}} V_{n}^{2} \frac{A_{n}}{A_{e}}+p_{n} R \frac{A_{n}}{A_{e}}+\frac{p_{s}}{T_{s}} V_{s}^{2} \frac{A_{s}}{A_{e}}+p_{s} R \frac{A_{s}}{A_{e}}
$$

recalling that $p_{s}=p_{n}$ and $A_{n}+A_{s}=A_{e}$, gives

$$
B=\frac{p_{e}}{T_{e}} V_{e}^{2}+p_{e} R=\frac{p_{n}}{T_{n}} V_{n}^{2} \frac{A_{n}}{A_{e}}+p_{n} R+\frac{p_{s}}{T_{s}} V_{s}^{2} \frac{A_{s}}{A_{e}}
$$

Doing the same manipulation to the energy equation (Eq. (A.3)) gives 


$$
C=\frac{p_{e}}{T_{e}} V_{e}\left(T_{e}+\frac{V_{e}^{2}}{2 c_{p}}\right)=\frac{p_{n}}{T_{n}} V_{n} \frac{A_{n}}{A_{e}}\left(T_{n}+\frac{V_{n}^{2}}{2 c_{p}}\right)+\frac{p_{s}}{T_{s}} V_{s} \frac{A_{s}}{A_{e}}\left(T_{s}+\frac{V_{s}^{2}}{2 c_{p}}\right) \text { (A.7) }
$$

Combining Eqs. (A.5) and (A.6) gives

$$
\begin{aligned}
& \mathrm{B}=\mathrm{DV}_{\mathrm{e}}+\mathrm{p}_{\mathrm{e}} \mathrm{R} \\
& \mathrm{BV} \mathrm{V}_{\mathrm{e}} / \mathrm{R}=\mathrm{p}_{\mathrm{e}} \mathrm{V}_{\mathrm{e}}+\mathrm{DV}_{\mathrm{e}}{ }^{2} / \mathrm{R}
\end{aligned}
$$

Combining Eqs. (A.5) and (A.7) gives

$$
\mathrm{C}=\mathrm{p}_{\mathrm{e}} \mathrm{V}_{\mathrm{e}}+\mathrm{DV}_{\mathrm{e}}^{2} /\left(2 \mathrm{c}_{\mathrm{p}}\right)
$$

Subtracting Eqs. (A.8) and (A.9) gives a quadratic equation

$$
0=V_{e}^{2}\left(\frac{D}{R}-\frac{D}{2 c_{p}}\right)-V_{e} \frac{B}{R}+C
$$

The positive square root gives an imaginary value, so $\mathrm{V}_{\mathrm{e}}$ is:

$$
V_{e}=\frac{\frac{B}{R}-\sqrt{\left(\frac{B}{R}\right)^{2}-4 C\left(\frac{D}{R}-\frac{D}{2 c_{p}}\right)}}{2\left(\frac{D}{R}-\frac{D}{2 c_{p}}\right)}
$$

With $\mathrm{V}_{\mathrm{e}}$ known, $\mathrm{p}_{\mathrm{e}}$ can be found from Eq. (A.8)

$$
\mathrm{p}_{\mathrm{e}}=\mathrm{B} / \mathrm{R}-\mathrm{V}_{\mathrm{e}} \mathrm{D} / \mathrm{R}
$$

From Eq. (6.5)

$$
\mathrm{T}_{\mathrm{e}}=\mathrm{p}_{\mathrm{e}} \mathrm{V}_{\mathrm{e}} / \mathrm{D}
$$

and $\rho_{\mathrm{e}}$ from equation of state

$$
\rho_{\mathrm{e}}=\mathrm{p}_{\mathrm{e}} /\left(\mathrm{RT}_{\mathrm{e}}\right)
$$

\section{One-Dimensional Incompressible Flow Ejector Equations}

As long as the Mach number at any location of the flow does not exceed 0.3, then density throughout the flow can be considered a constant within $\pm 5 \%$ error. Flow analysis can be further simplified in these cases using incompressible flow equations. 
Dimensionless areas of less than unity (designated by $\bar{A}$ ) are obtained by dividing by exit area $\mathrm{A}_{\mathrm{e}}$, which gives $\bar{A}_{\mathrm{s}}=\mathrm{A}_{\mathrm{s}} / \mathrm{A}_{\mathrm{e}}$ and $\bar{A}_{\mathrm{n}}=\mathrm{A}_{\mathrm{n}} / \mathrm{A}_{\mathrm{e}}$, thus $\bar{A}_{\mathrm{s}}+\bar{A}_{\mathrm{n}}=\bar{A}_{\mathrm{e}}=1$. Dimensionless velocities of less than unity (designated as $\bar{V}$ ) are obtained by dividing by the nozzle velocity, which gives $\bar{V} \mathrm{e}_{\mathrm{e}}=\mathrm{V}_{\mathrm{e}} / \mathrm{V}_{\mathrm{n}}$ and $\bar{V}_{\mathrm{s}}=\mathrm{V}_{\mathrm{s}} / \mathrm{V}_{\mathrm{n}}$, and $\bar{V}{ }_{\mathrm{n}}=1$. Finally, pressures are non-dimensionalized by dividing the corresponding pressure by the nozzle total pressure, $p_{o n}$.

Applying continuity and momentum equations through the device, the following is found:

One-dimensional ejector continuity equation

$$
\begin{aligned}
& \rho A_{s} V_{s}+\rho A_{n} V_{n}=\rho A_{e} V_{e} \\
& \frac{A_{s}}{A_{e}} \frac{V_{s}}{V_{n}}+\frac{A_{n}}{A_{e}} \frac{V_{n}}{V_{n}}=\frac{A_{e}}{A_{e}} \frac{V_{e}}{V_{n}} \\
& \bar{A}_{s} \bar{V}_{s}+\bar{A}_{n}=\bar{V}_{e}
\end{aligned}
$$

One-dimensional ejector momentum equation, ignoring wall shear and using $\mathrm{p}_{\mathrm{s}}=$ $p_{n}$ and the exit pressure $p_{e}$ to be equal to the atmospheric pressure

$$
\rho \mathrm{A}_{\mathrm{s}} \mathrm{V}_{\mathrm{s}}^{2}+\left(p_{s}-p_{\infty}\right) A_{s}+\rho \mathrm{A}_{\mathrm{n}} \mathrm{V}_{\mathrm{n}}^{2}+\left(p_{n}-p_{\infty}\right) A_{n}=\rho \mathrm{A}_{\mathrm{e}} \mathrm{V}_{\mathrm{e}}^{2}+\left(p_{e}-p_{\infty}\right) A_{e}
$$

Assuming BLC suction applied at ambient pressure is $\mathrm{p}_{\infty}$ then the stagnation pressures are:

$$
\begin{aligned}
& p_{o s}=p_{\infty} \\
& p_{o s}=p_{s}+q_{s} \\
& p_{s}=p_{n}=p_{o s}-q_{s}=p_{\infty}-\frac{1}{2} \rho V_{s}^{2}
\end{aligned}
$$

Combining Eqs. (A.15) and (A.16):

$$
\begin{aligned}
& \bar{A}_{S} \bar{V}_{s}^{2}-\left(\frac{\rho V_{s}^{2} \bar{A}_{s}}{2 \rho V_{n}^{2}}\right)+\bar{A}_{n}-\left(\frac{\rho V_{s}^{2} \bar{A}_{n}}{2 \rho V_{n}^{2}}\right)=\bar{V}_{e}^{2} \\
& \bar{A}_{s} \bar{V}_{s}^{2}-0.5 \bar{A}_{s} \bar{V}_{s}^{2}+\bar{A}_{n}-0.5 \bar{A}_{n} \bar{V}_{s}^{2}=\bar{V}_{e}^{2} \\
& \bar{A}_{s} \bar{V}_{s}^{2}+2 \bar{A}_{n}-\bar{A}_{n} \bar{V}_{s}^{2}=2 \bar{V}_{e}^{2}
\end{aligned}
$$


Combining Eqs. (A.14) and (A.17)

$$
\begin{aligned}
& \bar{A}_{s} \bar{V}_{s}^{2}+2 \bar{A}_{n}-\bar{A}_{n} \bar{V}_{s}^{2}=2\left(\bar{A}_{s} \bar{V}_{s}+\bar{A}_{n}\right)^{2} \\
& \bar{V}_{s}^{2}\left(\bar{A}_{s}-\bar{A}_{n}-2 \bar{A}_{s}^{2}\right)-4 \bar{A}_{s} \bar{A}_{n} \bar{V}_{s}+2 \bar{A}_{n}-2 \bar{A}_{n}^{2}=0 \\
& A \bar{V}_{s}^{2}+B \bar{V}_{s}+C=0 \\
& A=\bar{A}_{s}-\bar{A}_{n}-2 \bar{A}_{s}^{2} \\
& B=-4 \bar{A}_{s} \bar{A}_{n} \\
& C=2 \bar{A}_{n}-2 \bar{A}_{n}^{2}
\end{aligned}
$$

$\bar{V}_{e}$ can be found from Eq. (A.14)

The volume flow ratios are

$$
\begin{aligned}
& \mathrm{Vol}_{n}=A_{n} V_{n} \\
& \frac{\mathrm{Vol}_{n}}{\mathrm{Vol}_{e}}=\frac{A_{n} V_{n}}{A_{e} V_{e}}=\frac{\bar{A}_{n}}{\bar{V}_{e}} \\
& \mathrm{Vol}_{s}+\mathrm{Vol}_{n}=\mathrm{Vol}_{e} \\
& \frac{\mathrm{Vol}_{s}}{\mathrm{Vol}_{e}}+\frac{\mathrm{Vol}_{n}}{\mathrm{Vol}_{e}}=1 \\
& \frac{\mathrm{Vol}_{s}}{\mathrm{Vol}_{e}}=1-\frac{\mathrm{Vol}_{n}}{\mathrm{Vol}_{e}}
\end{aligned}
$$

Pressure ratios can be found from Eq. (A.16)

$$
\begin{aligned}
& \frac{p_{s}}{p_{\text {on }}}=\frac{\frac{p_{s}}{q_{n}}}{\frac{p_{n}}{q_{n}}+\frac{q_{n}}{q_{n}}}=\frac{\bar{V}_{s}^{2}}{\bar{V}_{s}^{2}-1} \\
& \frac{p_{o e}}{p_{o n}}=\frac{\frac{p_{e}}{q_{n}}+\frac{q_{e}}{q_{n}}}{\frac{p_{n}}{q_{n}}+\frac{q_{n}}{q_{n}}}=\frac{\bar{V}_{e}^{2}}{-\bar{V}_{s}^{2}+1}
\end{aligned}
$$

Applying these equations to different ejector area ratios, the parameters in Table 19 are obtained. 
Table 19. Theoretical non-dimensional parameters for one-dimensional incompressible flow ejectors at various area ratios.

\begin{tabular}{c|ccccc}
\hline $\mathbf{A}_{\mathbf{n}} / \mathbf{A}_{\mathbf{e}}$ & $\mathbf{1 / 3}$ & $\mathbf{1 / 4}$ & $\mathbf{1 / 8}$ & $\mathbf{1 / 1 6}$ & $\mathbf{1 / 2 0}$ \\
\hline $\mathrm{A}_{\mathrm{n}} / \mathrm{A}_{\mathrm{e}}$ & 0.340 & 0.250 & 0.174 & 0.063 & 0.049 \\
$\mathrm{~A}_{\mathrm{s}} / \mathrm{A}_{\mathrm{e}}$ & 0.660 & 0.750 & 0.826 & 0.938 & 0.951 \\
$\mathrm{~A}$ & -0.551 & -0.625 & -0.713 & -0.883 & -0.906 \\
$\mathrm{~B}$ & -0.898 & -0.750 & -0.574 & -0.234 & -0.188 \\
$\mathrm{C}$ & 0.449 & 0.375 & 0.287 & 0.117 & 0.094 \\
$\mathrm{~V}_{\mathrm{s}} / \mathrm{V}_{\mathrm{n}}$ & 0.401 & 0.380 & 0.349 & 0.255 & 0.235 \\
$\mathrm{~V}_{\mathrm{e}} / \mathrm{V}_{\mathrm{n}}$ & 0.605 & 0.535 & 0.462 & 0.302 & 0.272 \\
$\mathrm{Vol}_{\mathrm{n}} / \mathrm{Vol}_{\mathrm{e}}$ & 0.562 & 0.467 & 0.376 & 0.207 & 0.181 \\
$\mathrm{Vol}_{\mathrm{s}} / \mathrm{Vol}_{\mathrm{e}}$ & 0.438 & 0.533 & 0.624 & 0.793 & 0.819 \\
$\mathrm{p}_{\mathrm{s}} / \mathrm{p}_{\text {on }}$ & -0.192 & -0.169 & -0.139 & -0.070 & -0.058 \\
$\mathrm{p}_{\text {oe }} / \mathrm{p}_{\text {on }}$ & 0.436 & 0.334 & 0.243 & 0.097 & 0.078 \\
\hline
\end{tabular}

\section{Test Apparatus and Procedures}

Five nozzles were made for one ejector, each with a different area ratio between the nozzle and the ejector exit (Figure 210). The ejector exit area was $0.0017 \mathrm{ft}^{2}$. The sketch of the experiment setup is shown in Figure 211. The actual setup is shown in Figure 212 (note the digital scale used to verify the calculated exit flow momentum).

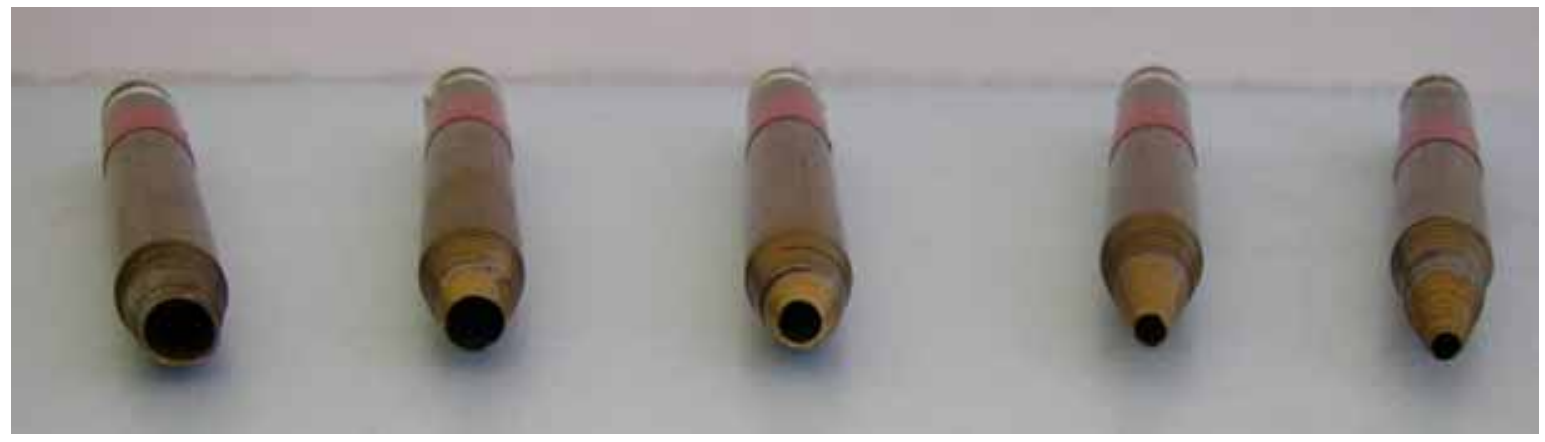

Figure 210. Nozzles fabricated for the ejector tests. 
Air

Compressor

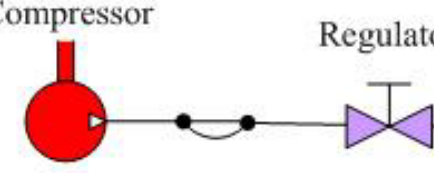

\section{Pressure gauge}
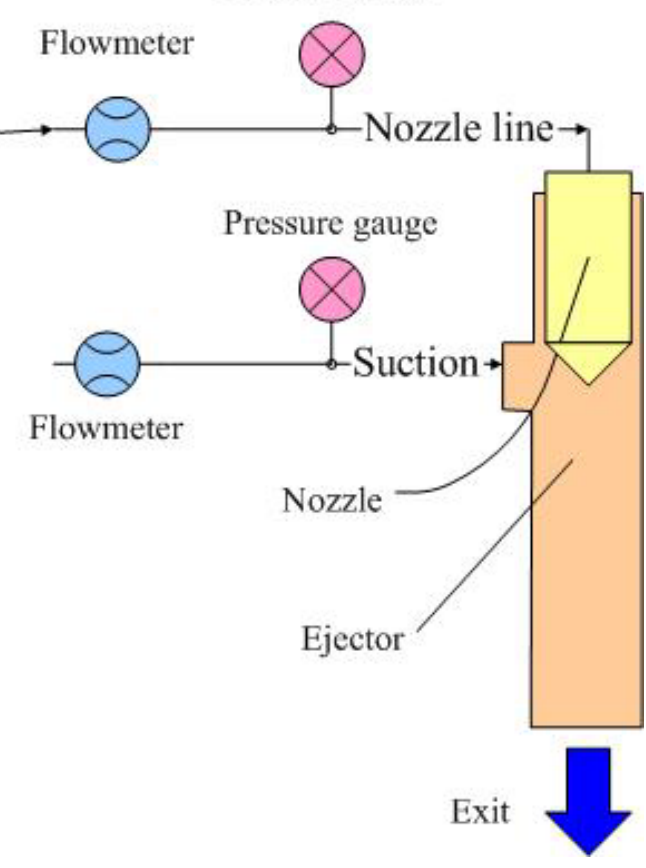

Figure 211. Sketch of the test apparatus used for ejector experiments.

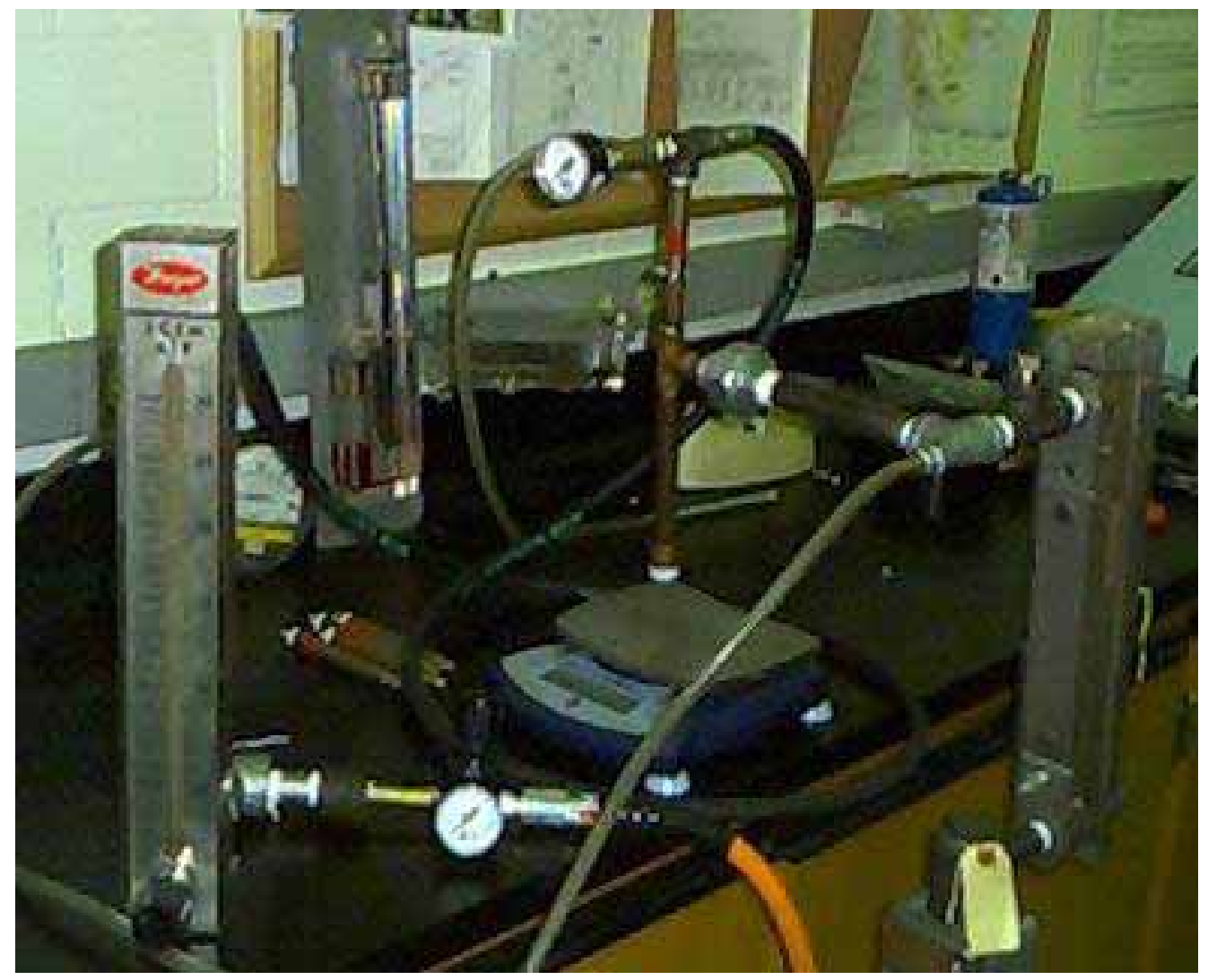

Figure 212. Test apparatus used for ejector experiments. 
Figure 213 shows the theoretical curve and the test data for the non-dimensional exit velocity $\left(\mathrm{V}_{\mathrm{e}} / \mathrm{V}_{\mathrm{n}}\right)$ vs. the non-dimensional nozzle area. Figure 214 does the same for the non-dimensional suction velocity $\left(\mathrm{V}_{\mathrm{s}} / \mathrm{V}_{\mathrm{n}}\right)$, and Figure 215 for the non-dimensional total exit pressure $\left(\mathrm{p}_{\mathrm{oe}} / \mathrm{p}_{\mathrm{on}}\right)$, Figure 216 for the non-dimensional suction pressure $\left(\mathrm{p}_{\mathrm{s}} / \mathrm{p}_{\mathrm{on}}\right)$, and Figure 217 for the non-dimensional volume flow rate at the suction $\left(\mathrm{Vol}_{\mathrm{s}} / \mathrm{Vol}_{\mathrm{e}}\right)$.

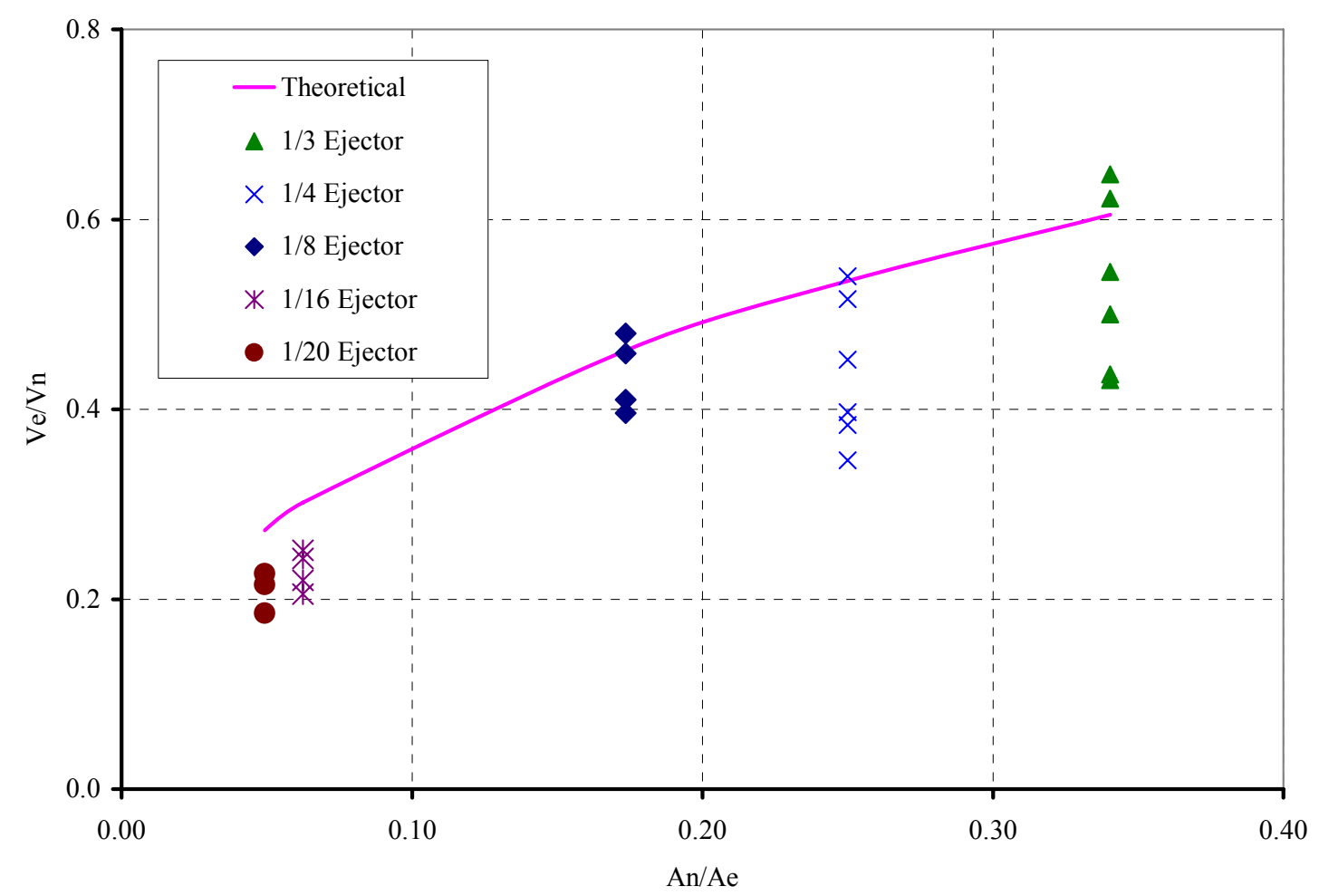

Figure 213. Dimensionless exit velocity increases with an increase in nozzle-to-exit area ratio. 


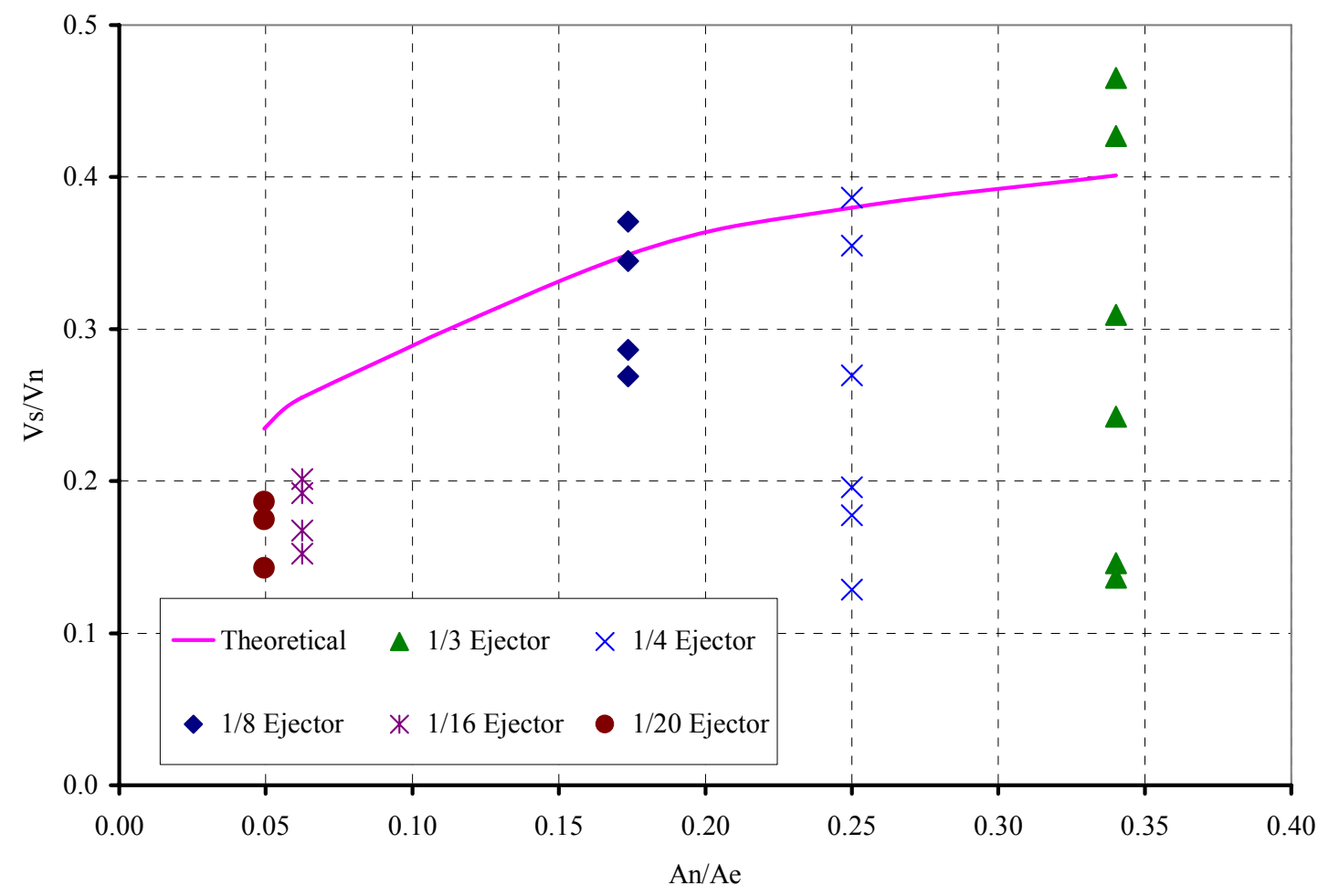

Figure 214. Dimensionless suction velocity increases with an increase in nozzle-to-exit area ratio.

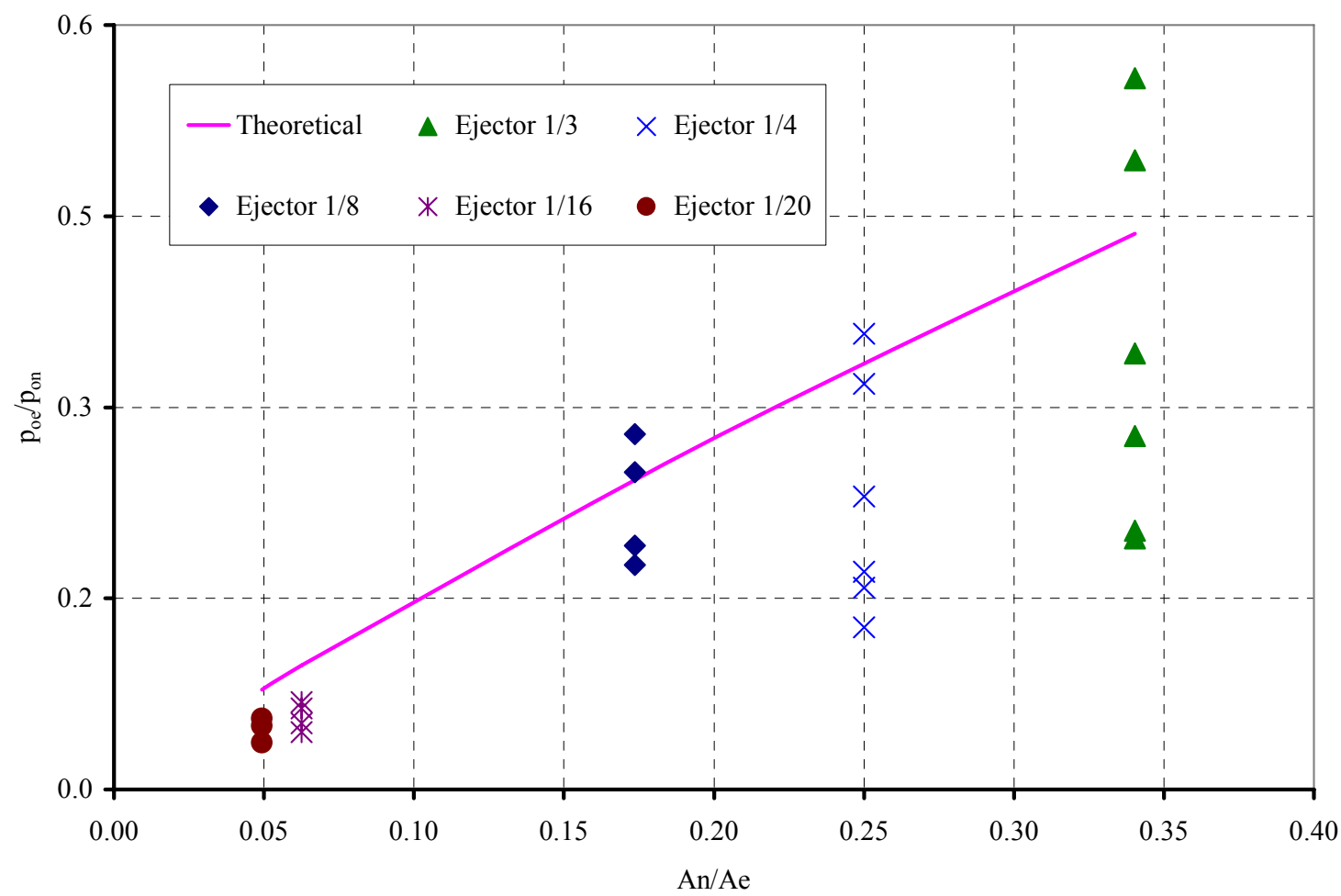

Figure 215. Dimensionless total exit pressure increases with an increase in nozzle-to-exit area ratio. 


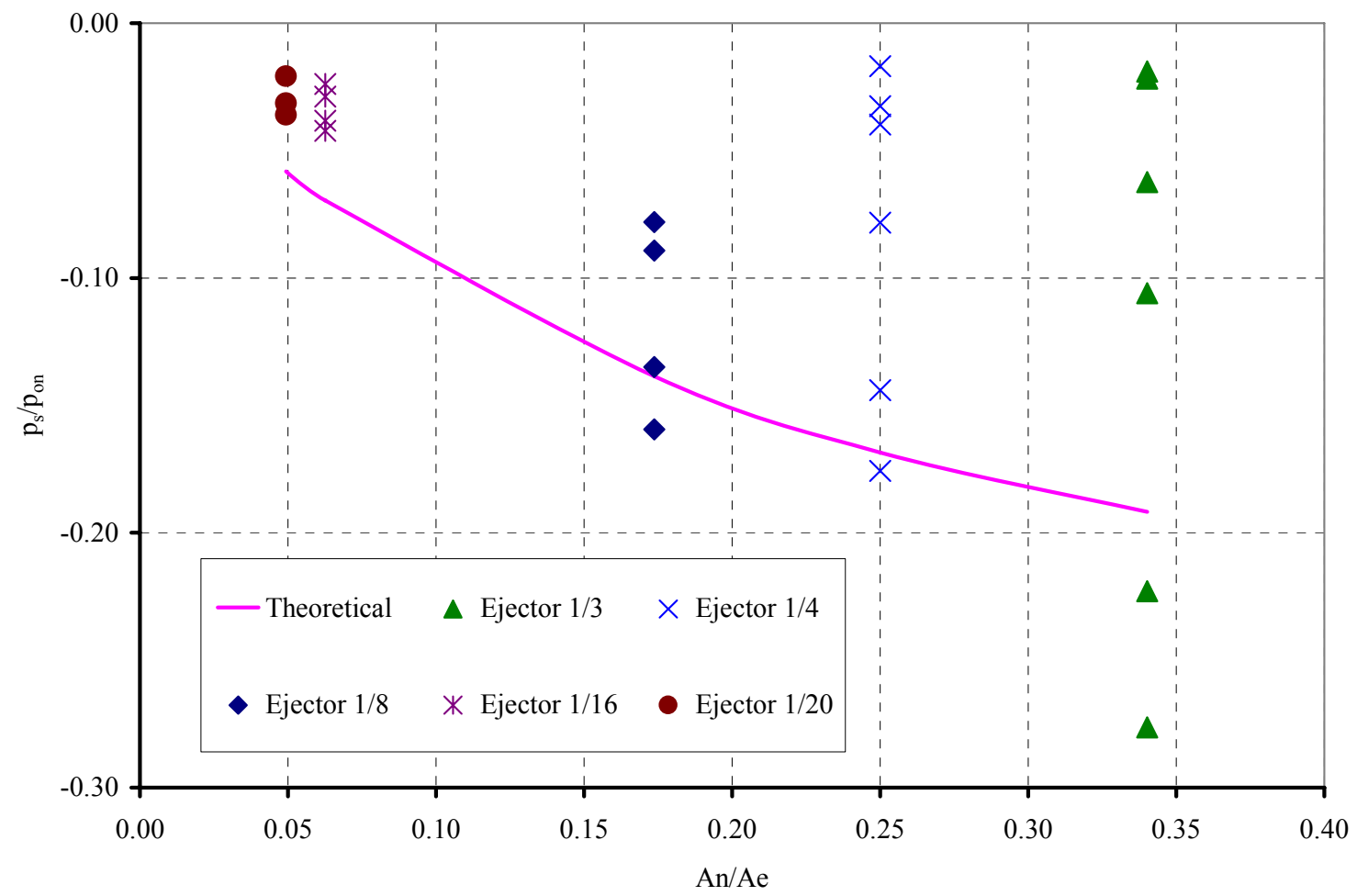

Figure 216. Dimensionless suction pressure decreases with an increase in nozzle-to-exit area ratio.

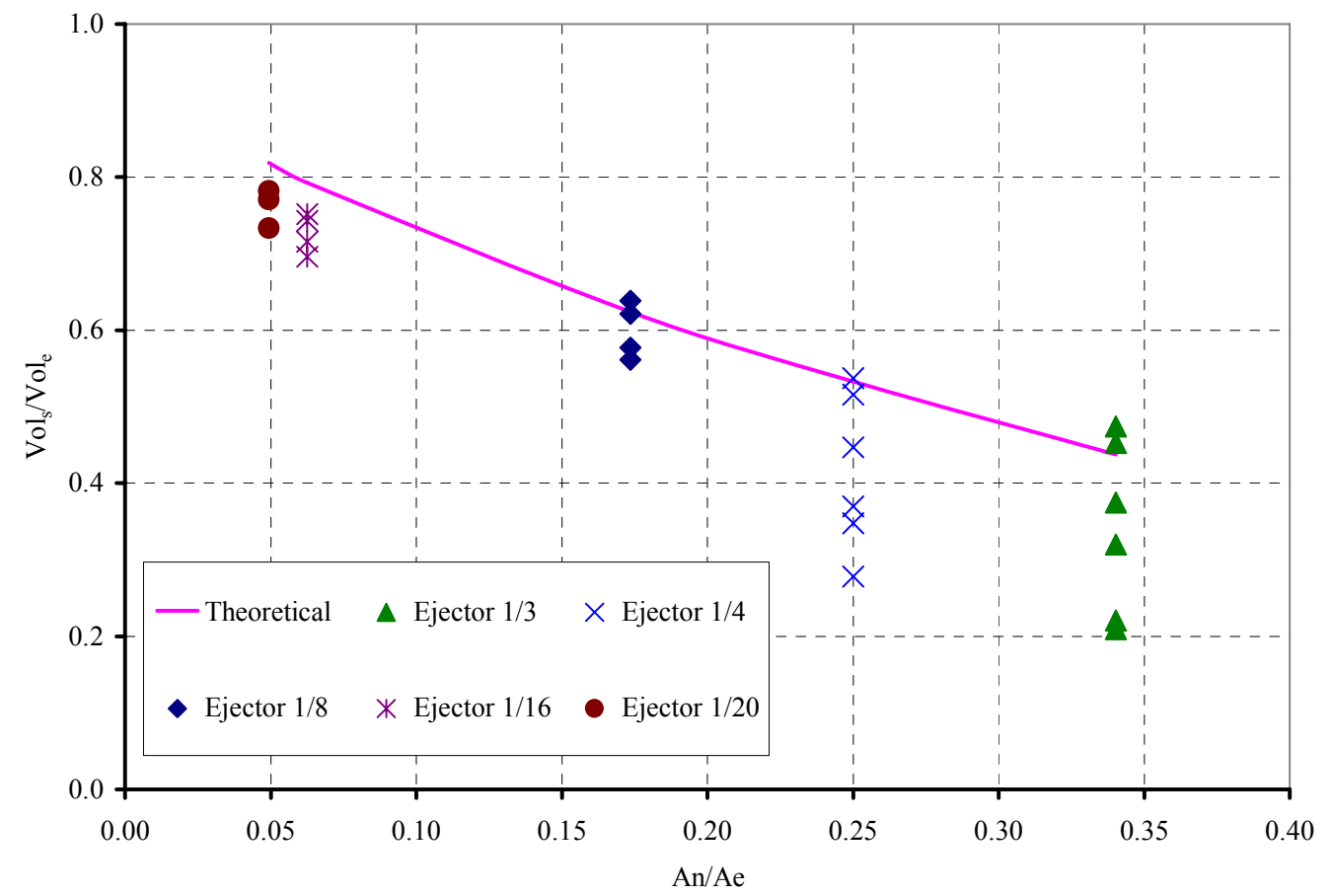

Figure 217. Dimensionless suction volume flow rate decreases with an increase in nozzle-to-exit area ratio. 


\section{Ejector Performance Graphs}

The experimental data for the ejector area ratios of 1/3,1/4 and 1/8 are closer to the theoretically predicted values, while the results for the ejector area ratios of 1/16 and $1 / 20$ stray further from the expected values. This error may be caused by the high total

pressures inside the nozzle, due to viscous effects not having been taken into account by the inviscid continuity and momentum equations used in the theoretical derivation.

When the relative nozzle area becomes smaller; there is an increase in the dimensionless values of nozzle velocity, suction velocity, exit velocity, and suction volume flow rate. Static pressure at the suction port (which is the same as the static pressure at the nozzle) decreases, while the total pressure at the nozzle increases.

The $V_{e} / V_{n}$ ratio increases as the $A_{n} / A_{e}$ ratio increases.

As can be seen from the $p_{o e} / p_{o n}$ vs. $A_{n} / A_{e}$ curve, the bigger the area of the nozzle with respect to the exit area, the lower the total pressure losses of the system are, indicating a more efficient design.

Higher relative suction pressures are achieved by larger nozzle to exit area ratios. However, large nozzle to exit area ratio ejectors cause a small mass flow ratio at the suction. In other words, if high suction pressure and low total pressure losses are desired, then low nozzle to exit area ratios are recommended. Conversely, if high suction mass flow rates are required, then high nozzle to exit area ratios are the best selection. Experimentally determined values of $p_{s} / p_{\text {on }}$ range from $-2 \%$ to $-27 \%$ for $1 / 20$ to $1 / 3$ nozzle to exit area ratios, respectively. Pressure losses ( $\mathrm{p}_{\mathrm{oe}} / \mathrm{p}_{\mathrm{on}}$ ) range from $63 \%$ to $95.5 \%$ for $1 / 3$ to $1 / 20$ nozzle to exit area ratios, respectively. Suction mass flow ratios $\left(m_{s} / m_{e}\right)$ range from $21 \%$ to $78 \%$ for $1 / 3$ to $1 / 20$ nozzle to exit area ratios, respectively. 
Appendix B: Chow et al. [43] Pressure Coefficient Tables

\begin{tabular}{|c|c|c|c|}
\hline$x / c$ & $z / b_{o}$ or & Upper $/$ Lower & $C_{p}$ \\
& $\gamma(\mathrm{deg})$ & Surface & \\
\hline-0.250 & 0.181 & Lower & -1.048 \\
-0.245 & 0.181 & Lower & 0.942 \\
-0.237 & 0.181 & Lower & 1.022 \\
-0.225 & 0.181 & Lower & 0.885 \\
-0.200 & 0.181 & Lower & 0.640 \\
-0.175 & 0.181 & Lower & 0.496 \\
-0.150 & 0.181 & Lower & 0.401 \\
-0.100 & 0.181 & Lower & 0.285 \\
-0.050 & 0.181 & Lower & 0.211 \\
0.000 & 0.181 & Lower & 0.158 \\
0.050 & 0.181 & Lower & 0.125 \\
0.150 & 0.181 & Lower & 0.077 \\
0.350 & 0.181 & Lower & 0.022 \\
0.550 & 0.181 & Lower & 0.062 \\
0.600 & 0.181 & Lower & 0.078 \\
0.625 & 0.181 & Lower & 0.084 \\
0.675 & 0.181 & Lower & 0.106 \\
0.700 & 0.181 & Lower & 0.117 \\
0.725 & 0.181 & Lower & 0.141 \\
-0.250 & 0.181 & Upper & -1.033 \\
-0.245 & 0.181 & Upper & -2.642 \\
-0.237 & 0.181 & Upper & -2.621 \\
-0.225 & 0.181 & Upper & -2.236 \\
-0.200 & 0.181 & Upper & -1.753 \\
-0.175 & 0.181 & Upper & -1.527 \\
-0.150 & 0.181 & Upper & -1.370 \\
-0.100 & 0.181 & Upper & -1.143 \\
-0.050 & 0.181 & Upper & -0.984 \\
0.000 & 0.181 & Upper & -0.855 \\
0.050 & 0.181 & Upper & -0.736 \\
0.150 & 0.181 & Upper & -0.540 \\
0.350 & 0.181 & Upper & -0.247 \\
0.550 & 0.181 & Upper & -0.024 \\
0.600 & 0.181 & Upper & 0.027 \\
0.625 & 0.181 & Upper & 0.053 \\
0.675 & 0.181 & Upper & 0.106 \\
0.700 & 0.181 & Upper & 0.133 \\
0.725 & 0.181 & Upper & 0.158 \\
\hline & & & \\
\hline
\end{tabular}




\begin{tabular}{|c|c|c|c|}
\hline$x / c$ & $\begin{array}{c}z / b_{o} \text { or } \\
(\mathbf{d e g})\end{array}$ & $\begin{array}{c}\text { Upper/Lower } \\
\text { Surface }\end{array}$ & $C_{p}$ \\
\hline-0.250 & 0.966 & Lower & 0.194 \\
-0.245 & 0.966 & Lower & 0.983 \\
-0.237 & 0.966 & Lower & 0.799 \\
-0.225 & 0.966 & Lower & 0.569 \\
-0.200 & 0.966 & Lower & 0.299 \\
-0.175 & 0.966 & Lower & 0.170 \\
-0.150 & 0.966 & Lower & 0.092 \\
-0.100 & 0.966 & Lower & -0.005 \\
-0.050 & 0.966 & Lower & -0.066 \\
0.000 & 0.966 & Lower & -0.115 \\
0.050 & 0.966 & Lower & -0.152 \\
0.150 & 0.966 & Lower & -0.201 \\
0.350 & 0.966 & Lower & -0.264 \\
0.550 & 0.966 & Lower & -0.280 \\
0.600 & 0.966 & Lower & -0.269 \\
0.625 & 0.966 & Lower & -0.262 \\
0.675 & 0.966 & Lower & -0.214 \\
0.700 & 0.966 & Lower & -0.180 \\
0.725 & 0.966 & Lower & -0.163 \\
-0.250 & 0.966 & Upper & 0.238 \\
-0.245 & 0.966 & Upper & -1.080 \\
-0.237 & 0.966 & Upper & -1.239 \\
-0.225 & 0.966 & Upper & -1.089 \\
-0.200 & 0.966 & Upper & -0.939 \\
-0.175 & 0.966 & Upper & -0.840 \\
-0.150 & 0.966 & Upper & -0.767 \\
-0.100 & 0.966 & Upper & -0.672 \\
-0.050 & 0.966 & Upper & -0.603 \\
0.000 & 0.966 & Upper & -0.551 \\
0.050 & 0.966 & Upper & -0.508 \\
0.150 & 0.966 & Upper & -0.453 \\
0.350 & 0.966 & Upper & -0.617 \\
0.550 & 0.966 & Upper & -1.247 \\
0.600 & 0.966 & Upper & -1.309 \\
0.625 & 0.966 & Upper & -1.290 \\
0.675 & 0.966 & Upper & -1.178 \\
0.700 & 0.966 & Upper & -1.074 \\
0.725 & 0.966 & Upper & -0.836 \\
\hline & & & \\
\hline
\end{tabular}

